\title{
Cosmological implications of spectator and dynamical fields during inflation
}

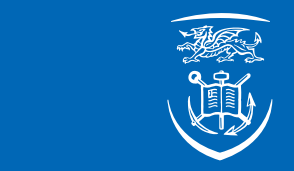

Swansea University

Prifysgol Abertawe

Jonathan Holland

Department of Physics

Swansea University

Submitted to Swansea University in fulfilment of the requirements for the degree of

Doctor of Philosophy 


\begin{abstract}
Some possible cosmological implications of the inclusion of spectator gauge fields in the inflationary action are considered. In this context, a spectator field is a field that is not directly responsible for inflation but can affect cosmological observables. Spectator gauge fields can lead to novel and distinguishable features in inflationary models (as compared to singlefield inflation) that may be detectable. A model of anisotropic inflation is considered where universal anisotropy is maintained through a vector field that is coupled to both the inflaton and its derivative. The derivative coupling reduces the anisotropy induced when compared to the non-derivative case, reducing tension with observations. $S U(N)$ gauge fields appear naturally in the actions of string inflation models. The inclusion of an $S U(N)$ gauge field coupled both to scalars and pseudo-scalars (axions) is considered in two models of string inflation whose inflatons are Kähler moduli: Kähler moduli inflation and fibre inflation. It is shown that the coupling between the axion and the gauge field can lead to a large (chiral) enhancement of the tensor spectrum of these two models. In the case of Kähler moduli inflation, a model that generically predicts an unobservable value for the tensor-to-scalar ratio $\left(r \ll 10^{-3}\right)$, this enhancement is capable of boosting the tensor-to-scalar ratio to values that will be potentially observable at next generation detectors. However, the parameters required to achieve this goal may present a challenge for its successful realisation in string theory. In the case of fibre inflation, a model that generically predicts an observable value for the tensor-to-scalar ratio, it is shown that this enhancement can render the tensor spectrum of fibre inflation almost entirely chiral, giving it a distinguishable feature that may be detectable.
\end{abstract}




\section{Declaration}

This work has not previously been accepted in substance for any degree and is not being concurrently submitted in candidature for any degree.

Signcd:

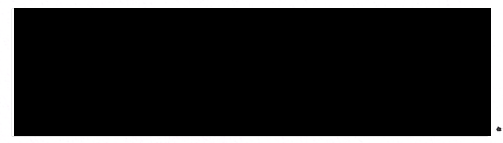

(candidatc)

Date: $25 / 09 / 2020$

\section{Statement 1}

This thesis is the result of my own investigations, except where otherwise stated. Where correction scrvices have becn used, the extent and nature of the correction is clearly marked in a footnote(s). Other sources are acknowledged by footnotes giving explicit references. A bibliography is appended.

Signed:

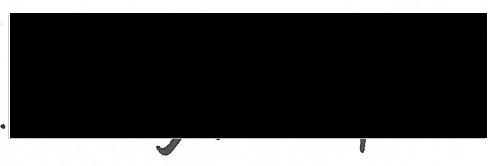
(candidate)

Date: $25 / 09 / 2020$

\section{Statement 2}

I hereby give consent for my thesis, if accepted, to be available for photocopying and for inter-library loan, and for the title and summary to be made available to outside organisations.

Signed:

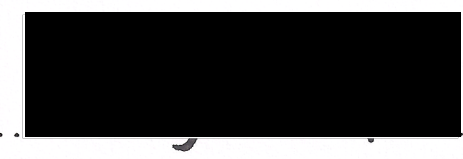
(candidate)

Date: $\quad 25 / 09 / 2.620$ 


\section{Conventions}

I attempt to adhere as closely as possible to the conventions as stated on this page.

The speed of light, $c$, and the reduced Planck's constant, $\hbar$, are set to unity throughout, $c=\hbar=1$. The spacetime metric signature is $(-,+,+,+)$. I use Greek letters, $\mu, \nu, \rho, \sigma \ldots$, to denote spacetime indices of coordinate $x^{\mu}$, and 'middle' Latin letters, $i, j, k, l \ldots$, to denote spatial indices of coordinate $x^{i}$. For example the Minkowski line element is

$$
d s^{2}=\eta_{\mu \nu} d x^{\mu} d x^{\nu}=-d t^{2}+\delta_{i j} d x^{i} d x^{j}
$$

'Early' Latin letters, $a, b, c, d \ldots$, will be used to denote indices of scalar field coordinates, $\phi^{a}$. While capital Latin letters, $A, B, C, D \ldots$, denote the gauge field index for some gauge field $A_{\mu}^{A}$. The reduced Planck mass

$$
M_{\mathrm{pl}}=\sqrt{\frac{\hbar c}{8 \pi G}}=\sqrt{\frac{1}{8 \pi G}},
$$

with $G$ Newton's constant, will be routinely referred to as the Planck mass, and will be used instead of $G$ where appropriate. For example Einstein's equations are written

$$
G_{\mu \nu}=\frac{1}{M_{\mathrm{pl}}^{2}} T_{\mu \nu}
$$




\section{Contents}

List of Figures

1 Introduction 1

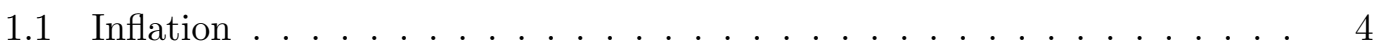

1.1.1 Problems with standard big bang theory . . . . . . . . . . 4

$1.1 .2 \quad$ A possible solution . . . . . . . . . . . . . 8

1.1.3 Inflationary dynamics . . . . . . . . . . . . 11

1.1.4 Perturbations . . . . . . . . . . . . . . . . . 13

1.1.5 Multi-field inflation . . . . . . . . . . . . 24

1.1.6 Non-standard cosmologies and non-scalar fields . . . . . . . . 26

1.2 Structure of this thesis . . . . . . . . . . . . . . 26

2 Anisotropic Inflation 22

2.1 Introduction . . . . . . . . . . . . . . . . . . . 29 29

2.2 Scalar-vector-tensor action with general derivative couplings . . . . . 33

2.3 Anisotropic power-law inflation with derivative couplings . . . . . . . 35

2.3.1 Stability of the anisotropic solutions . . . . . . . . . . 42

2.4 More general solutions . . . . . . . . . . . . . . . . . . . . 45

2.4.1 DBI inflation with monomial, derivative coupling solutions . . . 46

2.5 Discussion . . . . . . . . . . . . . . . . . . . . . 4 48

3 Enhancement of the primordial gravitational wave spectrum 51

3.1 Motivations . . . . . . . . . . . . . . . . . . . . 5 51

3.2 Basic model . . . . . . . . . . . . . . . . 5 52

3.3 Using an isotropic gauge field . . . . . . . . . . . . . . 559

3.3 .1 Background evolution . . . . . . . . . . . 60 
3.3 .2 Tensor perturbations . . . . . . . . . . . . . . . 65

3.3 .3 Scalar perturbations . . . . . . . . . . . . . . 69 69

3.3 .4 Backreaction . . . . . . . . . . . . . . . 70

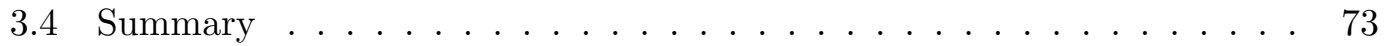

4 Introduction to Kähler moduli inflation 75

4.1 Basics of Kähler moduli inflation . . . . . . . . . . . . . . . 776

4.1.1 Kähler modulus inflation potential . . . . . . . . . . 78

$4.1 .2 \quad$ Fibre inflation potential . . . . . . . . . . . . . . . 84

4.2 Bog-standard Kähler moduli inflation . . . . . . . . . . . . 86

4.3 Kähler moduli inflation with a higher energy scale (example 1) . . . . . 94

4.4 Including an extra modulus (example 2$) \ldots \ldots$. . . . . . . . . .

4.5 Fibre inflation . . . . . . . . . . . . . . . . . . 105

4.6 Summary . . . . . . . . . . . . . . . . . . . . . . 112

5 Primordial Gravitational Waves in String Inflation 113

5.1 Introduction . . . . . . . . . . . . . . . . . . . . . 113

5.2 Gauge fields in general multi-field inflation . . . . . . . . . . . . 118

5.2.1 Background evolution and slow-roll inflation . . . . . . . . . . 120

$5.2 .2 \quad$ Slow-roll Dynamics . . . . . . . . . . . . . . . . . . . . . . . 122

5.2.3 Large Chern-Simons couplings are needed in spectator chromonatural inflation . . . . . . . . . . . . . . . 124

5.3 Examples of (spectator) chromo-natural Kähler inflation . . . . . . . . 125

5.3.1 Background evolution of example 1 . . . . . . . . . . . . 128

5.3.2 Background evolution of example 2 . . . . . . . . . . . . 135

5.4 Perturbations . . . . . . . . . . . . . . . . . . . . . . 141

5.4 .1 Set-up . . . . . . . . . . . . . . . . . 143

5.4 .2 Analytic approximation . . . . . . . . . . . . . . 145

5.4 .3 Numerical results . . . . . . . . . . . . . . . . . . . . . . . . . . . 148

5.5 Scalar perturbations . . . . . . . . . . . . . . . . . . 149

5.6 Backreaction . . . . . . . . . . . . . . . 155

5.7 Justifying the large Chern-Simons coupling (example 3) . . . . . . . 159

5.7.1 Background evolution and cosmological parameters . . . . . . . 163

5.7 .2 Tensors . . . . . . . . . . . . . . . . . . . 168 
$5.7 .3 \quad$ Scalars . . . . . . . . . . . . . . . . . 170

5.7 .4 Backreaction . . . . . . . . . . . . . . . 173

5.8 Discussion of viability of example $3 \ldots \ldots \ldots \ldots$. . . . . . . . . .

5.9 Fibre inflation $($ example 4$) \ldots \ldots \ldots \ldots$. . . . . . . . . . . . . . . .

5.9 .1 Background evolution . . . . . . . . . . . . . . . 180

5.9 .2 Tensors . . . . . . . . . . . . . . . . . . . . . 184

$5.9 .3 \quad$ Scalars . . . . . . . . . . . . . . . . . . . 186

5.9 .4 Backreaction . . . . . . . . . . . . . . . . 188

5.10 Summary . . . . . . . . . . . . . . . . . . . 188

6 Summary and discussions 191

6.1 Anisotropic inflation . . . . . . . . . . . . . . . . . . . 191

6.2 Gauge field-induced enhancement of the tensor power spectrum . . . . . 193

6.3 Final thoughts . . . . . . . . . . . . . . . . 200

A Calabi-Yau compactification and the moduli space 201

A.1 Maths . . . . . . . . . . . . . . . . . . . . . 201

A.1.1 Differential forms and boundaries . . . . . . . . . . . 201

A.1.2 Cohomology . . . . . . . . . . . . . . . . . 202

A.1.3 Homology . . . . . . . . . . . . . . . . . . . . . . 203

A.2 Calabi-Yau compactifications . . . . . . . . . . . . . . 204

A.2.1 Preserving supersymmetry on $\mathcal{M} \ldots \ldots$. . . . . . . . 204

A.2.2 Calabi-Yau manifolds and their moduli . . . . . . . . . 205

B Second order action for action with isotropic gauge field 211

C Full perturbation equations for chapter 5

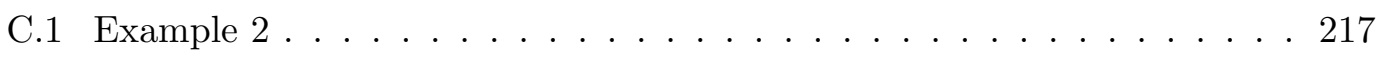

C.2 Example $3 \ldots \ldots \ldots \ldots \ldots$. . . . . . . . . . . . . . . . . . . . . . . . .

C.3 Example $4 \ldots \ldots \ldots \ldots \ldots \ldots \ldots \ldots$

References 


\section{Acknowledgements}

I would like to thank my parents; the members of the Swansea University Physics Department; my collaborators, Sugumi Kanno and Gianmassimo Tasinato; and especially my supervisor, Ivonne Zavala, without whom this thesis would not have been possible. 


\section{List of Figures}

1.1 The anisotropies of the CMB as measured by Planck and WMAP. This image is taken from [1] and is shown here with thanks to ESA and the Planck Collaboration. . . . . . . . . . . . . . 5

1.2 A typical inflationary potential given by $V=\Lambda^{4}\left(1-m_{1} \phi e^{-m_{2} \phi}\right)$ with $\Lambda=3.62 \times 10^{-3} M_{\mathrm{pl}}, m_{1}=2.17 M_{\mathrm{pl}}^{-1}$ and $m_{2}=0.8 M_{\mathrm{pl}}^{-1}$. This is an example of a large-field model in which the inflaton spans super-Planckian values during its evolution. . . . . . . . . . . . . . . . . . . . 13

2.1 In these plots we show how the slow-roll parameter, $\epsilon$, varies with $\lambda$ (for $\rho=20)$ and $\rho$ (for $\lambda=0.1$ ), for the values of $n$ shown. . . . . . 41

2.2 In these plots we show how the anisotropy, $\frac{\Sigma}{H}$, varies with $\lambda($ for $\rho=20$ ) and $\rho$ (for $\lambda=0.1$ ), for negative values of $n$ as shown. . . . . . 41

3.1 Plots (on a log-log scale) of $A+$ against $x=-k \tau=k / a H$ with different normalisation factors. We have used values from [2] of $\delta=0.2$ and $\xi_{*}=5$ and chosen the minimisation point of the axion, $x_{*}=-k \tau_{*}=1$, to be horizon-crossing. $A_{+}$is greatly enhanced as inflation proceeds. . . . . .

3.2 Plots (on a log-log scale) of $E+$ (left) and $B_{+}$(right) against $x=-k \tau=$ $k / a H$ with different normalisation factors. We have used values from [2] of $\delta=0.2$ and $\xi_{*}=5$ and chosen the minimisation point of the axion, $x_{*}=-k \tau_{*}=1$, to be horizon-crossing. Both are greatly enhanced as inflation proceeds despite the accelerated expansion of the universe. . .

3.3 Plots of the evolution of the scalar inflaton, $\phi$, in units of $M_{\mathrm{pl}}$ during the last 60 e-folds and last few e-folds of inflation in the left and right figure, respectively. . . . . . . . . . . . . . . . . . . . 


\section{LIST OF FIGURES}

3.4 Plots of the evolution of the spectator axion (left figure) and $\xi_{\chi}=\frac{\lambda}{2 H f} \dot{\chi}$ (right figure) during the last 60 e-folds of inflation. . . . . . . . 63

3.5 Plots of the evolution of the gauge field, $Q$ (left figure), and its effective mass, $\xi_{Q}=\frac{\mathrm{gQ}}{H}$ (right figure), during the last 60 e-folds of inflation. . . 63

3.6 Plot (on a log scale) of the energy densities of the inflaton (blue), $\rho_{\phi}=$ $\frac{1}{2} \dot{\phi}^{2}+V$, the axion (orange), $\rho_{\chi}=\frac{1}{2} \dot{\chi}^{2}+U$, and the gauge field (green), $\rho_{Q}=\frac{3}{2}(\dot{Q}+H Q)^{2}+\frac{3}{2} \mathrm{~g}^{2} Q^{4}$. The energy densities of the axion and the gauge field are vastly sub-dominant to the energy density in $\phi$, which allows $\phi$ to cause inflation.

3.7 Plot (on a $\log$ scale) of $\epsilon=-\frac{\dot{H}}{H^{2}}$ (blue), and its components: $\epsilon_{\phi}=\frac{\dot{\phi}^{2}}{2 M_{\mathrm{pl}}^{2} H^{2}}$ (orange), $\epsilon_{\chi}=\frac{\dot{\chi}^{2}}{2 M_{\mathrm{pl}}^{2} H^{2}}$ (green), $\epsilon_{B}=\frac{\mathrm{g}^{2} Q^{4}}{M_{\mathrm{pl}}^{2} H^{2}}$ (red), and $\epsilon_{E}=\frac{(\dot{Q}+H Q)^{2}}{M_{\mathrm{pl}}^{2} H^{2}}$ during the last 60 e-folds of inflation. . . . . . . . . . . . .

3.8 Plot of $\sqrt{2 k} x\left|\psi_{R}\right|$ and $\sqrt{2 k} x\left|t_{R}\right|$ against $x=k / a H$ with horizon-crossing, $x=1$, taken to be 60 e-folds before the end of inflation for the background solution shown in FIGs 3.33 .7 . The right-helicity mode of the gravitational wave tensor receives a modest enhancement. For reference the non-sourced solution for $\psi_{R}$ is also plotted (dashed line). . . . . . 68

3.9 Plot of $\sqrt{2 k} x\left|\Delta_{\phi}\right|, \sqrt{2 k} x\left|\Delta_{\chi}\right|, \sqrt{2 k} x\left|\Delta_{1}\right|$ and $\sqrt{2 k} x\left|\Delta_{2}\right|$ against $x=$ $k / a H$ with horizon-crossing, $x=1$, taken to be 60 e-folds before the end of inflation for the background solution shown in FIGs 3.33 .7 . .

3.10 The backreaction, $\mathcal{T}_{B R}^{Q}$, (red), along with the dominant contributions of (3.3.7): $2 \mathrm{~g} H \xi_{\chi} Q^{2}$ (blue), $2 \mathrm{~g}^{2} Q^{3}$ (orange), and $2 H^{2} Q$ plotted against $x=\frac{k}{a H}$ for the background solution shown in FIGs 3.33 .7 and horizoncrossing, $x=1$, chosen to be 60 e-folds before the end of inflation. . . .

4.1 A plot of the potential, $V$, near its minimum with respect to the volume, $\mathcal{V}$, for three different values of $\tau_{3}$ with all other fields set to their values at the global minimum. Blue corresponding to the global minimum; and orange and green corresponding to values of $\tau_{3}$ just below and above its value at the global minimum, respectively. The minimum for the volume is highly sensitive to changes in $\tau_{3}$, and shifting it away from the global minimum destroys this minimum for the volume, which then has a minimum at $\mathcal{V} \rightarrow \infty$. 
4.2 A plot of the potential, $V$, near its minimum with respect to the volume, $\mathcal{V}$, for three different values of $b_{3}$ with all other fields set to their values at the global minimum. Blue corresponding to the global minimum; and orange and green corresponding to values of $b_{3}$ shifted slightly away from the minimum. The minimum for the volume is highly sensitive to changes in $b_{3}$ and shifting it away from the global minimum destroys this minimum for the volume, which then has a minimum at $\mathcal{V} \rightarrow \infty$. . . 91

4.3 A plot of the potential, $V$, against the inflaton, $\tau_{2}$, with all other fields set to their values at the global minimum. Starting inflation with $\tau_{2}^{i n}=$ 0.802 , the inflaton first rolls slowly down the flat part of the potential before exiting slow-roll as it nears the minimum and picks up speed. . .

4.4 The evolution of the inflaton modulus, $\tau_{2}$, during the last 60 e-folds of inflation (left figure) and at the end of inflation when it decays (right figure) for the parameters given in 4.2 .13 . . . . . . . . . .

4.5 The evolution of the axionic partner to the inflaton modulus, $b_{2}$, during the last 60 e-folds of inflation (left figure) and at the end of inflation when it decays (right figure) for the parameters given in 4.2.13 . . . .

4.6 The evolution of the stabiliser modulus, $\tau_{3}$, and its axionic partner, $b_{3}$, during the last 60 e-folds of inflation for the parameters given in 4.2.13. Both fields are extremely stable during inflation. . . . . . . . . . . .

4.7 The evolution of the volume modulus, $\mathcal{V}$, during the last 60 e-folds of inflation for the parameters given in 4.2.13. $\mathcal{V}$ is extremely stable during inflation despite the relatively large change in $\tau_{2} \ldots \ldots \ldots$

4.8 The evolution of the inflaton modulus, $\tau_{2}$, during the last 60 e-folds of inflation (left figure) and at the end of inflation when it decays (right figure) for the parameters given in 4.3.1) (example 1). . . . . . . .

4.9 The evolution of the axionic partner to the inflaton modulus, $b_{2}$, during the last 60 e-folds of inflation (left figure) and at the end of inflation when it decays (right figure) for the parameters given in 4.3.1) (example 1). 


\section{LIST OF FIGURES}

4.10 The evolution of volume modulus, $\mathcal{V}$, during the last 60 e-folds of inflation (left figure), and at the end of inflation when it decays (right figure) for the parameters given in 4.3.1) (example 1). The volume modulus is stable while the inflaton, $\tau_{2}$, is slowly rolling but decays quickly in line with the decay of the inflaton. . . . . . . . . . . . 96

4.11 The evolution of the stabilising modulus, $\tau_{3}$, during the last 60 e-folds of inflation (left figure) and at the end of inflation when it decays (right figure) for the parameters given in (4.3.1) (example 1). . . . . . . . 97

4.12 The evolution of the inflaton modulus, $\tau_{2}$, during the last 60 e-folds of inflation (left figure) and at the end of inflation when it decays (right figure) for the parameters given in 4.3.1 with the volume modulus, $\mathcal{V}$, and stabiliser, $\tau_{3}$, excluded from the evolution. The evolution proceeds almost identically to that of FIG. 4.8 for which $\mathcal{V}$ and $\tau_{3}$ were included in the evolution.

4.13 The evolution of the axionic partner to the inflaton modulus, $b_{2}$, during the last 60 e-folds of inflation (left figure) and at the end of inflation when it decays (right figure) for the parameters given in 4.3.1) with the volume modulus, $\mathcal{V}$, and stabiliser, $\tau_{3}$, excluded from the evolution. The evolution proceeds almost identically to that of FIG. 4.8 for which $\mathcal{V}$ and $\tau_{3}$ were included in the evolution.

4.14 The evolution of the inflaton modulus, $\tau_{2}$, during the last 60 e-folds of inflation (left figure) and at the end of inflation when it decays (right figure) for the extra modulus example with the parameters given in 4.3.1

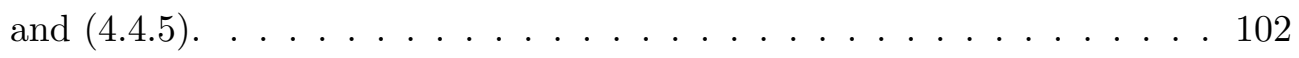

4.15 The evolution of the axion, $b_{4}$ (left figure), and its scalar superpartner, the $\tau_{4}$ modulus, during the last 60 e-folds of inflation for the extra modulus example with the parameters given in 4.3.1 and 4.4.5). Because $b_{4}$ has no gauge field to support it, it rapidly falls to its minimum and does not evolve. . . . . . . . . . . . . . . . . . . 102 
4.16 The evolution of volume modulus, $\mathcal{V}$, during the last 60 e-folds of inflation (left figure), and at the end of inflation when it decays (right figure) for the extra modulus example with the parameters given in (4.3.1) and (4.4.5). The volume modulus is stable while the inflaton, $\tau_{2}$, is slowly rolling but decays quickly in line with the decay of the inflaton. . . . . . 102

4.17 The evolution of the stabilising modulus, $\tau_{3}$, during the last 60 e-folds of inflation (left figure) and at the end of inflation when it decays (right figure) for the extra modulus example with the parameters given in 4.3.1)

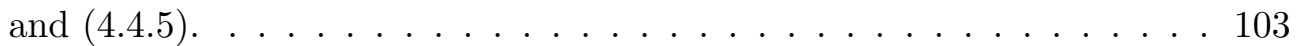

4.18 The evolution of the inflaton modulus, $\tau_{2}$, during the last 60 e-folds of inflation (left figure) and at the end of inflation when it decays (right figure) for the extra modulus example with the parameters given in 4.3.1 and (4.4.5) with the volume modulus, $\mathcal{V}$, and stabiliser, $\tau_{3}$, excluded from the evolution. The evolution proceeds almost identically to that of FIG. 4.14 for which $\mathcal{V}$ and $\tau_{3}$ were included in the evolution. . . . . . 104

4.19 The evolution of the axion, $b_{4}$ (left figure), and its scalar superpartner, the $\tau_{4}$ modulus, during the last 60 e-folds of inflation for the extra modulus example with the parameters given in $(4.3 .1)$ and 4.4 .5 with the volume modulus, $\mathcal{V}$, and stabiliser, $\tau_{3}$, excluded from the evolution. The evolution proceeds almost identically to that of FIG. 4.8 for which $\mathcal{V}$ and $\tau_{3}$ were included in the evolution. Because $b_{4}$ has no gauge field to support it, it rapidly falls to its minimum and does not evolve. . . . . . 105

4.20 The evolution of the principal inflaton modulus, $\tau_{1}$, during the last 60 e-folds of inflation (left figure) and at the end of inflation when it decays (right figure) for fibre inflation with an additional, spectator, modulus, $T_{4}$, and parameters given in 4.5.20-4.5.21 . . . . . . . 111

4.21 The evolution of the volume modulus, $\mathcal{V}$, during the last 60 e-folds of inflation (left figure) and at the end of inflation when it decays (right figure) for fibre inflation with an additional, spectator, modulus, $T_{4}$, and parameters given in 4.5.20-4.5.21 . . . . . . . . . . 111 


\section{LIST OF FIGURES}

4.22 The evolutions of the axion, $b_{4}$ (left figure), and its superpartner modulus, $\tau_{4}$ (right figure), during the last 60 e-folds for fibre inflation with an additional, spectator, modulus, $T_{4}$, and parameters given in 4.5.20 4.5.21. Because there is no gauge field to act to slow it, the axion immediately goes to its minimum and stays there for the duration of inflation. . . . . . . . . . . . . . . . . . .

5.1 Parametric plot of the cosmological evolution of $\tau_{2}$ and $b_{2}$, during the last 60 e-folds on the left and the last e-fold of inflation in example 1. . 130

5.2 Cosmological evolution of $\tau_{2}$ and $b_{2}$, during the last 61 e-folds in example 1. Inflation ends at $N=60 . \ldots \ldots \ldots \ldots \ldots \ldots$

5.3 Cosmological evolution of $\tau_{2}$ and $b_{2}$, during the last e-fold in example 1 . Inflation ends at $N=60 \ldots \ldots \ldots \ldots \ldots \ldots$

5.4 Cosmological evolution of the gauge field $Q$ (left figure) and $\xi_{Q}=\frac{g Q}{H}$ (right figure) during the last 60 e-folds of inflation in example 1. The gauge field is supported by its coupling to the axion determined by $\xi_{h}$. . 131

5.5 Cosmological evolution of the effective coupling terms $\xi_{f}=\frac{\dot{\tau}_{2}}{2 H \tau_{2}}$ (left figure) and $\xi_{h}=\frac{c_{2} \dot{b_{2}}}{2 c_{1} H \tau_{2}}$ (right figure) during the last 60 e-folds of inflation in example $1 . \ldots \ldots \ldots \ldots$. . . . . . . . . . . . . . . . . . . . . . . . .

5.6 The evolution of the slow-roll parameter, $\epsilon=-\frac{\dot{H}}{H^{2}}$ (left figure), and the proportion of $\epsilon$ made up by $\epsilon_{\varphi}=\frac{1}{2 H^{2}} \gamma_{a b} \dot{\phi}^{a} \dot{\phi}^{b}$ (right figure) during the last 60 e-folds of inflation in example 1. $\epsilon_{\varphi}$ dominates over the other components of the slow-roll parameter. . . . . . . . . . . . 131

5.7 The evolution of the electric and magnetic components of the slow roll parameter, $\epsilon_{E}=\frac{c_{1}}{2 \pi} \tau_{2} \frac{(\dot{Q}+H Q)^{2}}{H^{2} M_{\mathrm{pl}}^{2}}$ (left figure) and $\epsilon_{B}=\frac{c_{1}}{2 \pi} \tau_{2} \frac{\mathrm{g}^{2} Q^{4}}{H^{2} M_{\mathrm{pl}}^{2}}$ (right figure) respectively during the last 60 e-folds of inflation in example 1. They contribute only a small amount to the overall slow-roll parameter, FIG. $5.6 . \ldots \ldots \ldots 132$ 
5.8 A comparison of the contributions to $b_{2}$ 's equation of motion 5.2.12 from the term provided by the potential, $\frac{4 \mathcal{V} \sqrt{\tau_{2}}}{3 \alpha \lambda_{2}} \frac{d V}{d b_{2}}$; and the term provided by the gauge field, $-3 \mathrm{~g} \frac{c_{2}}{2 \pi} \frac{4 \mathcal{V} \sqrt{\tau_{2}}}{3 \alpha \lambda_{2}}(H Q+\dot{Q})$ for example 1. Unlike the examples shown in [3] and [4], this is not a "slow-roll solution" whereby the axion, $b_{2}$ is slowed by the backreaction of the gauge field, $Q$. In this example $b_{2}$ 's evolution is unaffected by the presence of $Q$ but $Q$ is supported nonetheless. . . . . . . . . . . . . . . . 132

5.9 A quick comparison of the backreaction, $\mathcal{T}_{B R}^{Q}$, on 5.2.13 (solid lines), and the largest term in 5.2 .13$), 2 H g Q^{2} \xi_{h}$ (dashed lines) against $\xi_{Q}$ for different values of $\mathrm{g}$. Reducing $\mathrm{g}$ decreases the backreaction and increases the largest term in $(5.2 .13) \ldots \ldots$. . . . . . . . . . . . . . 134

5.10 The evolution of the inflaton, $\tau_{2}$ during the last 60 e-folds of inflation (left) and during the last few e-folds (right) for example $2 . \ldots . . .137$

5.11 The evolution of the secondary modulus, $\tau_{4}$ and its axion partner $b_{4}$ in example 2. Both fields decay well before the end of inflation.

5.12 The contributions of (left) the term $3 \mathrm{~g} \frac{c_{2}}{2 \pi} \frac{4 \mathcal{V} \sqrt{\tau_{4}}}{3 \alpha \lambda_{4}}(H Q+\dot{Q})$ provided by the gauge field, $Q$; and (right) the term $\frac{4 \mathcal{V} \sqrt{\tau_{4}}}{3 \alpha \lambda_{4}} \frac{d V}{d b_{4}}$ provided by the potential, to the equation of motion for $b_{4}$ given by the form in 5.2.12 in example 2. The contribution from the gauge field almost exactly cancels the contribution from the potential leading to a slow-roll evolution for $b_{4}$.138

5.13 The evolution of the gauge field, $Q$ (left) and $\xi_{Q}=\frac{\mathrm{g} Q}{H}$ (right) during the last 60 e-folds of inflation in example 2. The evolution of $Q$ is tied to the evolution of $b_{4} \ldots \ldots \ldots \ldots \ldots \ldots \ldots$

5.14 The evolution of the effective coupling constants of $\tau_{4}$ and $b_{4}$ to the gauge field, $\xi_{f}$ (left) and $\xi_{h}$ (right) during the last 60 e-folds in example 2. . . 139

5.15 The evolution of the slow-roll parameter, $\epsilon$ (left), and the proportion of $\epsilon$ made up by $\epsilon_{\varphi}$, during the last 60 e-folds of inflation in example 2 .

5.16 The evolution of the electric and magnetic components of the slow roll parameter, $\epsilon_{E}$ (left figure) and $\epsilon_{B}$ (right figure) respectively during the last 60 e-folds of inflation in example $2 \ldots \ldots$. . . . . . . . 139

5.17 Comparing $\epsilon_{B}$ with the overall slow-roll parameter, $\epsilon . \epsilon_{B}$ provides the largest contribution to $\epsilon$ for the majority of the last 60 e-folds of inflation.140 


\section{LIST OF FIGURES}

5.18 Analytic estimate of the sourced tensor-to-scalar ratio, $r$, against $\xi_{Q}=$ $\frac{\mathrm{g} Q}{H}$ for different values of $\mathrm{g}$. The dashed lines correspond to the (nonsourced) background tensor-to-scalar ratio for our example $r_{b}=7.10 \times$ $10^{-7}$ (orange) and the observational cut-off $r_{\text {obs }} \gtrsim 10^{-3}$ (blue). . . . . . 142

5.19 The backreaction of the tensor fluctuations on the equation of motion for $Q, \mathcal{T}_{B R}^{Q}$ (solid lines) along with the largest term in the equation of motion for $Q, 2 \mathrm{~g} Q^{2} H \xi_{h}$ (dashed lines) plotted against $\xi_{Q}$ for different values of $\mathrm{g}$. Increasing $\xi_{Q}$ increases $\mathcal{T}_{B R}^{Q}$ much faster than $2 \mathrm{~g} Q^{2} H \xi_{h}$, but decreasing g reduces $\mathcal{T}_{B R}^{Q}$ whilst increasing $2 \mathrm{~g} Q^{2} H \xi_{h}$. . . . . . . . 143

5.20 The amplification factor, $\mathcal{F}^{2}$, of the tensor power spectrum for righthelicity modes in our analytic estimate plotted against the effective mass of the gauge field, $\xi_{Q}$. The left figure uses $\xi_{f}=9.91 \times 10^{-5}$ and shows the function $e^{3.6 \xi_{Q}}$ which gives an idea of how the enhancement scales with $\xi_{Q}$. The right figure shows two cases, $\xi_{f}=9.91 \times 10^{-5}$ and $\xi_{f}=$ 0 demonstrating that $\xi_{f}$ is too small to have any effect on the tensor perturbations. This plot is therefore completely equivalent to that given

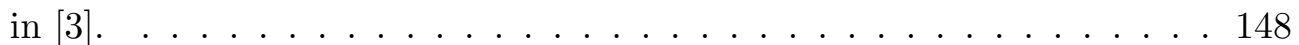

5.21 The evolution of the (right-helicity) tensor modes for the gauge field, $t_{R}$, and the gravity sector, $\psi_{R}$ for example 2 plotted against $x=k / a H$. . 150

5.22 The evolution of the scalar perturbations defined in $(5.5 .12)$ with (left figure) and without (right figure) the inclusion of the metric perturbations $\alpha, \beta$ found through (5.5.11) and (5.5.10) for example 2 plotted against $x=k / a H$. The metric perturbations have a negligible effect on the evolution and can safely be set to zero. . . . . . . . . . . . 153

5.23 The evolution of the scalar perturbations defined in (5.5.12) and (5.5.15) plotted against $x=k / a H$. After horizon-crossing, $x<1$, the scalar perturbation associated with the scalar fields, $\Delta_{s}$ is consistently much larger than the scalars associated with the gauge field, $\Delta_{1}, \Delta_{2} \ldots \ldots$. . 154

5.24 The full evolution of the scalar perturbations defined in $(5.5 .12)$ and 5.5.15 plotted against $x=k / a H$ including the decays of all the perturbations which take place concurrently with the decays of the corresponding background fields. . . . . . . . . . . . . . . . 154 
5.25 The evolution of the leading terms in the equation of motion for $Q$ (5.6.4), $2 \mathrm{~g} Q^{2} H \xi_{h}$ (blue), $2 \mathrm{~g}^{2} Q^{3}$ (orange), $2 H^{2} Q$ (green) and the backreaction induced by the gauge tensor perturbation, $\mathcal{T}_{B R}^{Q}$ (red) in example 2 plotted

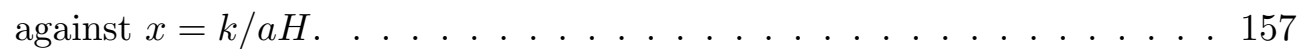

5.26 The evolution of the leading terms in the equation of motion for $Q$ 5.6.4), $2 \mathrm{~g} Q^{2} H \xi_{h}$ (blue), $2 \mathrm{~g}^{2} Q^{3}$ (orange), $2 H^{2} Q$ (green) and the backreaction induced by the gauge tensor perturbation, $\mathcal{T}_{B R}^{Q}$ (red) in example 1 plotted

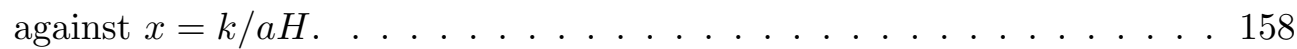

5.27 The evolution of the energy densities in $\phi^{a}, \rho_{\varphi} \simeq V$ (blue); in $Q$, $\rho_{Q}=\frac{3}{2} f\left[(H Q+\dot{Q})^{2}+\mathrm{g}^{2} Q^{4}\right]$ (orange); and in $t_{R}, 5.6 .8$ (green), in example 2 plotted against $x=k / a H \ldots \ldots \ldots \ldots \ldots$

5.28 A schematic of the set-up used in this section. . . . . . . . . . . . 161

5.29 The evolution of the inflaton, $\tau_{2}$ during the last 60 e-folds of inflation (left) and during the last few e-folds (right) for example 3.

5.30 The evolution of the spectator modulus, $\tau_{4}$ and the axion $b$ during the last 60 e-folds of inflation for example 3 . Both fields reach their minima well before the end of inflation.

5.31 The contributions of (left) the term $-3 \mathrm{~g} m \frac{\sqrt{\zeta} \mathcal{V}}{2 g_{s} \sqrt{\tau_{4}}} Q^{2}(H Q+\dot{Q})$ provided by the gauge field, $Q$; and (right) the term $\frac{\sqrt{\zeta} \mathcal{V}}{2 g_{s} \sqrt{\tau_{4}}} \frac{\partial V}{\partial b}$ provided by the potential, to the equation of motion for $b$ given by the form in (5.2.12) for example 3. The contribution from the gauge field almost exactly cancels the contribution from the potential leading to a slow-roll evolution for $b$

5.32 The evolution of the gauge field, $Q$ (left) and $\xi_{Q}=\frac{\mathrm{g} Q}{H}$ (right) during the last 60 e-folds of inflation for example 3. The evolution of $Q$ is tied to the evolution of $b \ldots \ldots \ldots \ldots \ldots \ldots \ldots \ldots$

5.33 Left figure: The two dominant terms in the equation of motion for $Q$, 5.2.13), satisfy $2 \mathrm{~g}^{2} Q^{3} \sim \mathrm{g} H Q^{2} \xi_{h}$ for example 3 . The term introduced through the coupling to the axion almost cancels $2 \mathrm{~g}^{2} Q^{3}$, a term that sends $Q$ to zero. In this way the axion-gauge coupling supports the gauge field. Right figure: The evolution of the $b_{4}$ modulus for example 3 - started at its minimum, it moves only negligibly during inflation, and can be safely ignored in the numerics. 


\section{LIST OF FIGURES}

5.34 The evolution of the effective couplings of $\tau_{4}$ and $b$ to the gauge field, $\xi_{f}$ (left) and $\xi_{h}$ (right) during the last 60 e-folds of example $3 . \ldots \ldots$

5.35 The evolution of the slow-roll parameter, $\epsilon$ (left), and the proportion of $\epsilon$ made up by $\epsilon_{\varphi}$, during the last 60 e-folds of inflation for example 3.

5.36 The evolution of the electric and magnetic components of the slow roll parameter, $\epsilon_{E}$ (left figure) and $\epsilon_{B}$ (right figure) respectively during the last 60 e-folds of inflation for example $3 \ldots \ldots \ldots$. . . . . . . . 167

5.37 Left figure: Comparing $\epsilon_{B}$ with the overall slow-roll parameter, $\epsilon . \epsilon_{B}$ provides the largest contribution to $\epsilon$ for a substantial part of the last 60 e-folds of inflation in example 3. Right figure: Plot of the instantaneous decay constant, $f_{c}=\frac{\sqrt{\gamma_{b b}}}{a m n}$, during the last 60 e-folds of inflation

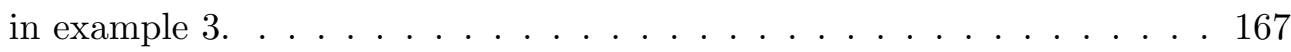

5.38 Plot of how the effective gauge coupling $g$ constrains the value of the gauge degree $N$ in example 3 . The example given $\mathrm{g}=\frac{1}{2000}$ is marked with a blue dot. . . . . . . . . . . . . . . . . 169

5.39 The evolution of the (right-helicity) tensor modes for the gauge field, $t_{R}$, and the gravity sector, $\psi_{R}$ for example 3 plotted against $x=k / a H$. . . 169

5.40 The evolution of the scalar perturbations defined in (5.7.19) with (left figure) and without (right figure) the inclusion of the metric perturbations $\alpha, \beta$ found through (5.5.10), 5.5.11) for the example 3 plotted against $x=k / a H$. As we can see, the metric perturbations have a negligible effect on the evolution and can safely be set to zero. . . . . . . . 171

5.41 The evolution of the scalar perturbations defined in (5.7.19) and 5.7.22 for example 3 plotted against $x=k / a H$. After horizon-crossing, $x<1$, the scalar perturbation associated with the scalar fields, $\Delta_{s}$ is consistently much larger than the scalars associated with the gauge field, $\Delta_{1}, \Delta_{2} .172$

5.42 The full evolution of the scalar perturbations defined in 5.7.19 and 5.7.22 plotted against $x=k / a H$ for example 3 including the decays of all the perturbations which take place concurrently with the decays of the corresponding background fields. . . . . . . . . . 172 
5.43 The evolution of the leading terms in the equation of motion for $Q$ (5.6.4), $2 \mathrm{~g} Q^{2} H \xi_{h}$ (blue), $2 \mathrm{~g}^{2} Q^{3}$ (orange), $2 H^{2} Q$ (green) and the backreaction induced by the gauge tensor perturbation, $\mathcal{T}_{B R}^{Q}$ (red) for example 3 plotted against $x=k / a H \ldots \ldots \ldots \ldots \ldots \ldots \ldots \ldots$

5.44 The evolution of the energy densities in $\phi^{a}, \rho_{\varphi} \simeq V$ (blue); in $Q$, $\rho_{Q}=\frac{3}{2} f\left[(H Q+\dot{Q})^{2}+\mathrm{g}^{2} Q^{4}\right]$ (orange); and in $t_{R}, 5.6 .8$ (red), for the example 3 plotted against $x=k / a H \ldots \ldots \ldots \ldots$. . . . . . . .

5.45 The value of $\epsilon_{B}=f \frac{\mathrm{g}^{2} Q^{4}}{M_{\mathrm{pl}}^{2} H^{2}}=f \frac{H^{2} \xi_{Q}^{4}}{\mathrm{~g}^{2} M_{\mathrm{pl}}^{2}}$ plotted against $\xi_{Q}=\frac{\mathrm{gQ}}{H}$. If one wishes to achieve the same value of $\xi_{Q}$ after reducing $g$ to mitigate the backreaction, $\epsilon_{B}$ will be larger. The dashed line shows the value of $\epsilon_{\varphi}$ 60 e-folds before the end of inflation for example 3. Similarly the dot corresponds to the values for $\xi_{Q}$ and $\epsilon_{B}$ in example 360 e-folds before the end of inflation. . . . . . . . . . . . . . . . 176

5.46 Two plots showing how the enhancement factor, $\frac{r_{s}+r_{b}}{r_{b}}$, varies relative to the ratio of $\frac{\epsilon_{B}}{\epsilon_{\varphi}}$ where $\epsilon_{\varphi}=2.80 \times 10^{-8}$, the value it takes 60 e-folds before the end of inflation in example 3 . The left plot corresponds to $\mathrm{g}=\frac{1}{2000}$, the value of $\mathrm{g}$ used in example 3 and the right plot shows $\mathrm{g}=\frac{1}{100}$. The dot corresponds to the values in example 3 . The red part of the lines corresponds to parameter space where the backreaction is too large. It can be seen immediately that with $g=\frac{1}{100}$, it is impossible to get a substantial enhancement without incurring excessive backreaction. Because of the low value of $\epsilon_{\varphi}$ in Kähler inflation, in order to get a large enhancement with controlled backreaction, we require $g$ small, which leads to a large value of $\epsilon_{B}$ relative to $\epsilon_{\varphi} \ldots \ldots \ldots$. . . . . . 177

5.47 The evolution of the inflaton field, $\tau_{1}$, during the last 60 e-folds (left figure) and its decay as inflation ends (right figure) for example 4 plotted against $N$ the e-fold number. . . . . . . . . . . . . . . . . 181

5.48 The evolution of the volume modulus, $\mathcal{V}$, during the last 60 e-folds (left figure) and its decay as inflation ends (right figure) for example 4 plotted against $N$ the e-fold number. . . . . . . . . . . 18

5.49 The evolution of the spectator fields, $\tau_{4}$ (left figure) and $b$ (right figure), during the last 60 e-folds of inflation for example 4 plotted against $N$ the e-fold number 


\section{LIST OF FIGURES}

5.50 The evolution of the gauge field, $Q$ (left figure), and its effective mass, $\xi_{Q}$ (right figure), during the last 60 e-folds of inflation for example 4 plotted against $N$ the e-fold number. . . . . . . . . . . . . . . 182

5.51 The evolution of the effective couplings from the spectator fields to the gauge field, $\xi_{f}=\frac{\dot{f}}{2 H f}$ (left figure) and $\xi_{h}=\frac{\dot{h}}{2 H f}$ (right figure), during the last 60 e-folds of inflation for example 4 plotted against $N$ the e-fold number.

5.52 The contributions of (left) the term $-3 \mathrm{~g} m \frac{\sqrt{\zeta} \mathcal{V}}{2 g_{s} \sqrt{\tau_{4}}} Q^{2}(H Q+\dot{Q})$ provided by the gauge field, $Q$; and (right) the term $\frac{\sqrt{\zeta} V}{2 g_{s} \sqrt{\tau_{4}}} \frac{\partial V}{\partial b}$ provided by the potential, to the equation of motion for $b$ given by the form in 5.2.12 for example 4. The contribution from the gauge field almost exactly cancels the contribution from the potential leading to a slow-roll

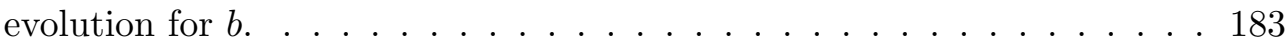

5.53 Left figure: The two dominant terms in the equation of motion for $Q$, 5.2.13), satisfy $2 \mathrm{~g}^{2} Q^{3} \sim \mathrm{g} H Q^{2} \xi_{h}$ for example 4 . The term introduced through the coupling to the axion almost cancels $2 \mathrm{~g}^{2} Q^{3}$, a term that sends $Q$ to zero. In this way the axion-gauge coupling supports the gauge field. Right figure: The evolution of the $b_{4}$ modulus for example 4 - started at its minimum, it moves only negligibly during inflation, and can be safely ignored in the numerics.

5.54 The evolution of the slow-roll parameter, $\epsilon=-\frac{\dot{H}}{H^{2}}$ (left figure) and its scalar component $\epsilon_{\varphi}=\gamma_{a b} \frac{\dot{\phi}^{a} \dot{\phi}^{b}}{2 H^{2}}$ (right figure), during the last 60 e-folds of inflation for example 4 plotted against $N$ the e-fold number. . . . . . 183

5.55 The evolution of the magnetic component of slow-roll parameter, $\epsilon_{B}=$ $f \frac{\mathrm{g}^{2} Q^{4}}{M_{\mathrm{pl}}^{2} H^{2}}$ (left figure) and the electric component of the slow-roll parameter $\epsilon_{E}=f \frac{(H Q+\dot{Q})^{2}}{M_{\mathrm{pl}}^{2} H^{2}}$ (right figure), during the last 60 e-folds of inflation for example 4 plotted against $N$ the e-fold number. . . . . . . . . . . . 184

5.56 The evolution of the (right-helicity) tensor modes for the gauge field, $t_{R}$, and the gravity sector, $\psi_{R}$ for example 4 plotted against $x=k / a H$. . 185 
5.57 The evolution of the scalar perturbations defined in 5.9.13) and 5.9.16 for example 4 plotted against $x=k / a H$. After horizon-crossing, $x<1$, the scalar perturbation associated with the scalar fields, $\Delta_{s}$ is consistently much larger than the scalars associated with the gauge field, $\Delta_{1}, \Delta_{2}$.187

5.58 The full evolution of the scalar perturbations defined in 5.9.13 and 5.9.16 plotted against $x=k / a H$ for example 4 including the decays of all the perturbations which take place concurrently with the decays of the corresponding background fields. . . . . . . . . . . 187

5.59 The evolution of the leading terms in the equation of motion for $Q$ 5.6.4), $2 \mathrm{~g} Q^{2} H \xi_{h}$ (blue), $2 \mathrm{~g}^{2} Q^{3}$ (orange), $2 H^{2} Q$ (green) and the backreaction induced by the gauge tensor perturbation, $\mathcal{T}_{B R}^{Q}$ (red) for example 4 plotted against $x=k / a H \ldots \ldots \ldots \ldots \ldots \ldots \ldots$

5.60 The evolution of the energy densities in $\phi^{a}, \rho_{\varphi} \simeq V$ (blue); in $Q$, $\rho_{Q}=\frac{3}{2} f\left[(H Q+\dot{Q})^{2}+\mathrm{g}^{2} Q^{4}\right]$ (orange); and in $t_{R}$, 5.6.8) (red), for the example 4 plotted against $x=k / a H \ldots \ldots \ldots \ldots$

A.1 Hodge diamond for a Calabi-Yau three-fold with $S U(3)$ holonomy. . . . 208 


\section{Chapter 1}

\section{Introduction}

In 1949, during a talk on BBC radio, British astronomer Sir Fred Hoyle used the term 'big bang' [5, 6] to describe the creation of the universe at one particular time in the past. The term 'Big Bang Theory' has become widespread in popular culture and is the de facto name for the current most-widely accepted cosmological model of the universe. Despite accidentally creating its name, Hoyle was not a proponent of the Big Bang theory, instead preferring the 'Steady state' model of the universe [6], and it was not until the discovery of the Cosmic Microwave Background (CMB) that the term 'big bang' really started to become popular with cosmologists [6].

Some 27 years prior, in 1922, Soviet physicist Alexander Friedmann had derived the Friedmann equations that govern a cosmological universe. Friedmann used general relativity and the assumption that the universe is both homogeneous and isotropic on extremely large scales and therefore has metric given by

$$
d s^{2}=-d t^{2}+a(t)^{2} d \Sigma^{2},
$$

with

$$
d \Sigma^{2}=\frac{d r^{2}}{1-k r^{2}}+r^{2}\left(d \theta^{2}+\sin ^{2} \theta d \phi^{2}\right)
$$

in polar coordinates where $k=0, \pm 1$ is the constant of curvature of the universe, determining whether the universe is perfectly flat, positively curved or negatively curved, respectively. This metric is the so-called Friedmann-Lemaitre-Robertson-Walker met- 


\section{INTRODUCTION}

ric ${ }^{1}$ and $a(t)$ is the scale factor of the universe that gives scales to the distances between objects at cosmological distances. Using this metric, Friedmann derived the equations that govern the scale factor [7]

$$
\begin{gathered}
H^{2}=\frac{\dot{a}^{2}}{a^{2}}=\frac{\rho}{3 M_{\mathrm{pl}}^{2}}-\frac{k}{a^{2}} \\
\frac{\ddot{a}}{a}=-\frac{\rho+3 p}{6 M_{\mathrm{pl}}^{2}}-\frac{k}{a^{2}}
\end{gathered}
$$

where $\rho$ is the energy density, $p$ is the pressure, $H=\frac{\dot{a}}{a}$ is the Hubble parameter and we have ignored the cosmological constant.

In 1929, American astronomer, Edwin Hubble, released an article in which an observed relationship between distance and the recession speed of galaxies was described [8, 9], which later came to be known as Hubble's law. Hubble's law can be written simply as

$$
v=H_{0} d
$$

where $v$ is the recession speed of a galaxy a distance $d$ away and $H_{0}$ is the current rate of cosmic expansion, known as the Hubble constant. The simple law tells us that not only are galaxies receding from us, the further away they are, the faster they recede. A simple explanation for this law is that the universe is expanding, as can be reconciled with the Friedmann equations.

A natural possible consequence of this is that some time in the distant past, the scale factor (which increases in time) was zero, suggesting that the universe had zero size, and there was an initial singularity. This hypothesis was first suggested by Lemaître in 1931 [10, and the 'big bang theory' was formed. This theory was developed and championed by Soviet-American physicist, George Gamow, as well as many others including Gamow's students: American physicists Ralph Asher Alpher and Robert Herman. In 1948, Alpher and Herman presented the first estimate of the temperature of leftover radiation from the big bang [11, 12, nowadays referred to as the Cosmic Microwave Background (CMB). The logic of the big bang model requiring the presence of leftover radiation is thus: if the universe started at some extremely dense state,

\footnotetext{
${ }^{1}$ After Belgian physicist and Reverend, Georges Lemaître; American physicist, Howard Robertson; and British physicist, Arthur Walker. Although we include Lemaître in the name here, this metric is customarily referred to as the FRW metric.
} 
the matter (protons and electrons) of the early universe must have been extremely energetic. When conditions are sufficiently hot, protons and electrons do not bind to form atoms because the electrons are too energetic: this is a state of matter that is commonly referred to as a fully-ionised plasma. Fully-ionised plasmas are extremely opaque to radiation due to scattering of photons with electrons: estimates for the amount of time it takes a photon formed at the centre of the Sun (an example of a fully-ionised plasma) to reach its surface and escape suggest a timescale of $\sim 100,000$ years [13]. This opacity meant that the early universe did not permit the travel of radiation over great distances. However as the universe expanded, it cooled, eventually cooling enough for atoms to form at a time known as recombination, approximately 400,000 years after the big bang. With the electrons now bound into atoms, photons decoupled and free-streamed. Some of this radiation is just reaching us now from the distant universe, and this is known as the CMB. It is light that continually arrives at us from the far reaches of the universe, and it is the furthest back in time we can see, giving us a picture of what the early universe was like.

The problem for those who advocated big bang theory was that no such radiation had been discovered despite the fact that - by its very definition - it should be everywhere. The main challenger to the big bang theory was the 'steady-state' model, largely inspired by British-Austrian physicist, Sir Hermann Bondi and BritishAmerican-Austrian physicist, Thomas Gold [14]; as well as Hoyle [15]. The steady-state theory proposes that, although the universe is expanding, the density of matter in the universe remains constant. This requires that there be a 'creation field' responsible for the continuous creation of matter to leave the universe at constant density. The principal reason for the popularity of this model was that it adheres to the perfect cosmological principle: not only would a steady-state universe be isotropic and homogeneous on large scales, it would also be time-independent, asserting that we occupy no special place in space or time.

The steady-state model was delivered a blow in 1965 when American physicists, Arno Penzias and Robert Wilson, detected the CMB as background noise in their experiment built to detect radio waves reflected off Echo balloon satellites? which

\footnotetext{
${ }^{2}$ Project Echo was the world's first communications satellite project [16].
} 


\section{INTRODUCTION}

provided the first concrete evidence for the big bang theory [17]渄. Over the subsequent decades, the CMB has been measured to greater and greater accuracy through the Cosmic Background Explorer (COBE) [21], the Wilkinson Microwave Anisotropy Probe (WMAP) [22] and, most recently, Planck [1, 23, 24].

\subsection{Inflation}

In this section I will review cosmological inflation. Inflation is the subject of countless lecture notes and textbook sections, but I particularly recommend [25-27] for detailed discussions.

\subsubsection{Problems with standard big bang theory}

\section{Horizon problem}

In 1992, COBE discovered anisotropies in the CMB [21, 28], that is to say, small fluctuations from the mean temperature (the CMB is a blackbody spectrum of mean temperature $2.73 \mathrm{~K}$ ), dependent on the direction that is viewed in the sky, were observed. What is remarkable from a physics point of view about this discovery is that it took 27 years from the original discovery of the CMB to find fluctuations to the background temperature. The reason for this is that these fluctuations are very small $\left(\Delta T / T \sim 10^{-5}\right.$ [29]) relative to the background temperature, which poses an intriguing problem. These anisotropies (see FIG. 1.1) tell us about inhomogeneities in the density of the universe when it was only 400,000 years old. Light we receive from regions that were denser than the average density of the universe appears redshifted because it has had to escape from a greater gravitational well - likewise, light we receive from under dense regions appears blueshifted ${ }^{6}$ The fact that the light we receive is exceptionally isotropic tells

\footnotetext{
${ }^{3}$ Penzias and Wilson searched hard for a possible source for the noise they were detecting, and even removed a bird's nest they had found in the antenna. After a year of searching they could find no reason for the noise [18. The explanation that they were detecting leftover radiation from the early universe was given by Jim Peebles, David Wilkinson, Robert Dicke and Peter Roll [19.

${ }^{4}$ Penzias and Wilson were awarded the Nobel Prize in 1978 for this discovery.

${ }^{5}$ Although this is widely regarded as the discovery of the CMB, in 1941 Andrew McKellar had found evidence suggesting that space had a blackbody temperature of a few Kelvin [20]. The significance of this was perhaps lost because the CMB would not be predicted as a consequence of the big bang by Alpher and Herman for another seven years.

${ }^{6}$ See 30 , for a review of CMB physics - in reality there are many effects at play, and the physics is extremely complex.
} 


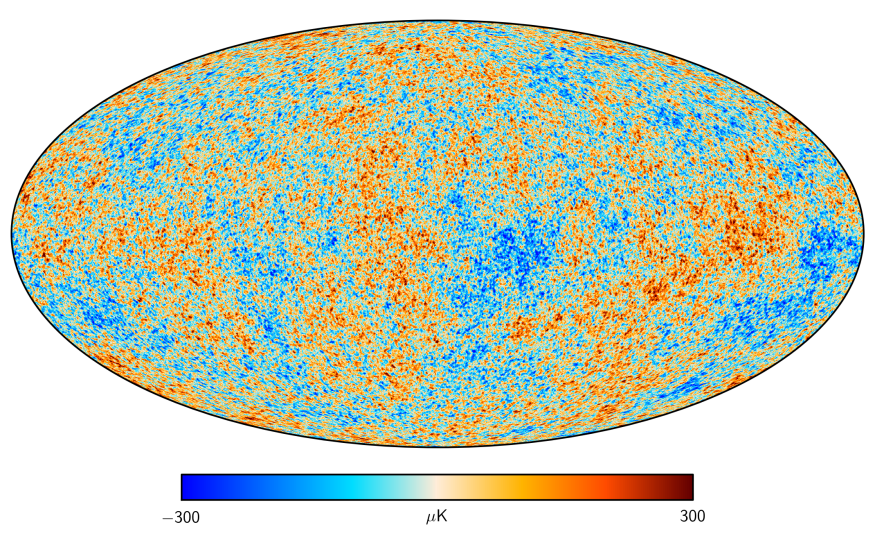

Figure 1.1: The anisotropies of the CMB as measured by Planck and WMAP. This image is taken from [1] and is shown here with thanks to ESA and the Planck Collaboration.

us that the universe was exceptionally homogeneous when it was only 400,000 years old.

The Friedmann equations (1.3) 1.4 can be recast as a continuity equation in the energy density of the universe

$$
\frac{d \rho}{d t}+3 H(\rho+p)=0
$$

which can be written as

$$
\frac{d \ln \rho}{d N}=-3(1+\omega)
$$

where $N=\ln a$ is known as the e-folding number and

$$
\omega=\frac{p}{\rho}
$$

is the equation of state of the universe. Equation (1.7) tells us

$$
\rho=\rho_{0} a^{-3(1+\omega)}
$$

and with this the Friedmann equation (1.3) (with negligible curvature, $k=0$ ) tells us that

$$
(a H)^{-1}=H_{0}^{-1} a^{\frac{1}{2}(1+3 \omega)}
$$

where $(a H)^{-1}$ is called the comoving Hubble radius. The comoving Hubble radius is of interest because the maximum (comoving) distance light can travel between two times 


\section{INTRODUCTION}

$t=0$ and $t$ (the comoving horizon) is given by

$$
\chi:=\int_{0}^{t} \frac{d t^{\prime}}{a\left(t^{\prime}\right)}=\int_{0}^{a} \frac{d \tilde{a}}{H \tilde{a}^{2}}=\int_{0}^{a} d \tilde{a} H_{0}^{-1} \tilde{a}^{\frac{1}{2}(3 \omega-1)}
$$

which tells us that the comoving horizon

$$
\chi \propto a^{\frac{1}{2}(1+3 \omega)}
$$

grows monotonically with time whenever the equation of state $\omega=\frac{p}{\rho}>-\frac{1}{3}$. Conventional wisdom of non-inflationary big bang theory would say that the energy density of the universe is made up of radiation and cold matter. In early times when particles had high energy, the vast majority of the energy density and pressure of the universe was made up of what could be considered radiation. But later (after about 47,000 years after the big bang), the universe cooled and its energy density and pressure were dominated by cold matter. The reason it is useful to split the universe into epochs like this is that we know the equation of state for both radiation and cold matter, and can therefore evolve the scale factor. Matter is assumed to be pressureless: $\omega_{m}=0$; while radiation satisfies $\omega_{r}=\frac{1}{3}$.

Given that both the radiation and matter-dominated periods of the universe had $\omega>-\frac{1}{3}$, we can see immediately that, in standard cosmology, the comoving horizon (1.12) has only grown in time. Light we see from the CMB introduces us to a scale that has just entered our comoving horizon, and if the comoving horizon has only grown in time, this is the first time the scale has entered the horizon, and in fact would have been far outside the horizon at the time of the CMB ( 14 billion years ago). This suggests that regions we see of the $\mathrm{CMB}$ were not causally connected at the time of recombination 7 . We can roughly estimate the number of causally disconnected regions by comparing the horizon today with that of the $\mathrm{CMB}$

$$
\left(\frac{\chi_{\text {today }}}{\chi_{c m b}}\right)^{3}=\left(\frac{a_{\text {today }}}{a_{c m b}}\right)^{\frac{3}{2}(1+3 \omega)}=\left(1+z_{c m b}\right)^{\frac{3}{2}(1+3 \omega)}
$$

\footnotetext{
${ }^{7}$ That is to say there had literally not been enough time, only 400,000 years after the big bang, for light to have travelled between two causally-disconnected regions. This is a consequence of the fact that in standard big bang cosmology, the universe started expanding at some extraordinary speed and has only been slowing down since.
} 
where we have used the definition of the redshift, $z$ :

$$
1+z=\frac{a_{\text {today }}}{a}
$$

By assuming pure matter domination from the time since recombination to today ${ }^{8}$, we have $\omega=0$ and therefore

$$
\left(\frac{\chi_{\text {today }}}{\chi_{c m b}}\right)^{3}=\left(1+z_{c m b}\right)^{3 / 2} \simeq(1100)^{3 / 2} \sim 3.7 \times 10^{4}
$$

using $z_{c m b} \simeq 1100$. This estimate tells us that the universe at the time of the CMB was made up of over 30,000 causally-disconnected regions, so how is it possible that the CMB appears so uniform when this tells us that the universe was highly homogeneous at the time of the CMB? This is the horizon problem. In standard cosmology, the comoving horizon always increases with time and this suggests that, at the time of the $\mathrm{CMB}$, the universe was made up of many regions which could never have interacted with one another, and yet, these regions had roughly the same density. How could these regions 'know' to be the same without ever having come into contact and equilibrating?

\section{Flatness problem}

The horizon problem is not the only issue that arises in standard big bang cosmology. The Friedmann equation 1.3 can be rewritten as

$$
1-\Omega(a)=-k(a H)^{-2}
$$

where $\Omega(a):=\frac{\rho(a)}{\rho_{c}(a)}$ is the ratio of the energy density of the universe to the energy density of a perfectly flat universe with $\rho_{c}=3 M_{\mathrm{pl}}^{2} H^{2}$ the critical (flat) density, with a flat universe satisfying $\Omega=1$. As we have seen (eqn. $(1.10)$ ), the Hubble radius

$$
(a H)^{-1}=H_{0}^{-1} a^{\frac{1}{2}(1+3 \omega)}
$$

will only grow in time when $\omega>-\frac{1}{3}$, a condition that always holds when the dominant energy of the universe is in radiation or matter. Since $k$ is just a constant, the RHS of (1.16) will grow in magnitude, and consequently $\Omega(a)-1$ will grow in time during

\footnotetext{
${ }^{8}$ In reality of course we are now in a period of dark-energy dominated expansion, but for the purposes of a rough calculation, this approximation will do.
} 


\section{INTRODUCTION}

both radiation and matter domination. This suggests that the universe will always tend away from being flat and will become more curved as time passes. Interestingly however, it seems that the universe is flat or very close to being flat: $\Omega(a)=1$ is consistent with data 31. This is the flatness problem of standard big bang cosmology. If the universe only becomes more curved during the radiation and matter-dominated epochs, then it must have been considerably flatter in the past. Estimates suggest that at the time of big bang nucleosynthesis ( $\sim 3$ minutes after the big bang) [25]

$$
\left|\Omega\left(a_{b b n}\right)-1\right| \leq \mathcal{O}\left(10^{-16}\right)
$$

and that earlier still the universe had to be considerably flatter. Without the assumption of an extremely specific initial condition for the curvature of the universe, how is it possible that the universe is currently so flat?

\subsubsection{A possible solution}

The horizon and flatness problems can be seen as initial conditions problems in that very specific initial conditions, namely an extremely flat and homogeneous universe, are required to lead to the universe we see today. It would be highly preferable instead if the universe we see today could have arisen from very general initial conditions. This can be achieved with a period of inflation.

As has been hinted, the key is that the Hubble radius and consequently the comoving horizon only grow in time when $\omega>-\frac{1}{3}$. Therefore, let us postulate a period of time when the universe was dominated by a field with equation of state satisfying $\omega<-\frac{1}{3}$. Then the Hubble radius

$$
(a H)^{-1}=H_{0}^{-1} a^{\frac{1}{2}(1+3 \omega)}
$$

shrinks. An epoch with a shrinking Hubble sphere

$$
\frac{d}{d t}\left((a H)^{-1}\right)<0
$$

is known as inflation where the universe undergoes a period of accelerated expansion:

$$
\frac{d}{d t}(a H)^{-1}<0 \Longrightarrow \frac{d}{d t}(\dot{a})^{-1}<0 \Longrightarrow-\frac{\ddot{a}}{\dot{a}^{2}}<0 \Longrightarrow \ddot{a}>0
$$


i.e. the scale factor is accelerating. The condition of accelerated expansion can be cast in terms of $\epsilon=-\frac{\dot{H}}{H^{2}}$, which we will refer to as the slow-roll parameter:

$$
\epsilon=-\frac{\dot{H}}{H^{2}}=1-\frac{\ddot{a}}{a H^{2}}
$$

therefore

$$
\ddot{a}>0 \Longrightarrow \epsilon<1
$$

where perfect exponential expansion (de Sitter space) requires

$$
\epsilon=0 \Longrightarrow \dot{H}=0 \Longrightarrow a \propto e^{H t}
$$

Generally during inflation, we assume what is known as slow-roll with $\epsilon \ll 1$ and $\omega \sim-1$ which implies the universe undergoes exponential expansion $a \propto e^{H t}=e^{N}$.

So how does a period of accelerated expansion solve the horizon and flatness problems? Starting with the horizon problem we first note that if two particles are separated by a distance greater than the comoving horizon, they have never been in causal contact, however if they are separated by a distance greater than the Hubble radius, they cannot communicate now, but may have been in causal contact in the past. This difference is at the heart of the resolution of the horizon problem - with an inflationary epoch, the Hubble radius was exponentially larger before inflation than after. Effectively inflation solves the horizon problem by allowing the universe to be smaller for a slightly longer period of time than is predicted by the big bang model. During this period, different patches that we now see as causally disconnected regions in the CMB were within the Hubble radius and consequently were able to communicate and equilibrate before inflation took place. During inflation they then receded from one another exponentially quickly. The acceleration is necessary because the scale factor has to be smaller for longer than would be predicted by standard big bang theory - it then enters a period of acceleration to 'catch up' to the value standard big bang theory would predict. To be more explicit, note that the physical distance light can travel between two times $t_{1}$ and $t_{2}$ is given by

$$
d_{p}=a_{2} \chi=a_{2} \int_{a_{1}}^{a_{2}} d \tilde{a} \frac{1}{\tilde{a}^{2} H}
$$

where $a_{1}=a\left(t_{1}\right)$ and $a_{2}=a\left(t_{2}\right)$. The physical distance light possibly could have 


\section{INTRODUCTION}

travelled at recombination (the $\mathrm{CMB}$ ) is

$$
\begin{gathered}
d_{r}=a_{r} \chi_{r} \simeq a_{r} \int_{a_{i}}^{a_{e}} d \tilde{a} \frac{1}{\tilde{a}^{2} H}=a_{r} \int_{a_{i}}^{a_{e}} d \tilde{a} H_{I}^{-1} \tilde{a}^{\frac{1}{2}(3 \omega-1)} \\
=\frac{a_{r}}{H_{I}}\left(a_{i}^{-1}-a_{e}^{-1}\right) \simeq \frac{a_{r}}{H_{I}} a_{i}^{-1}=\frac{a_{r}}{a_{e} H_{I}} e^{N}
\end{gathered}
$$

where we have assumed that the vast majority of the integral will made up by the inflationary epoch because both $(a H)^{-1}$ and $a^{-1}$ will be exponentially bigger at the start of inflation than at the end; we use $\omega=-1 \sqrt[9]{9}$; we have used that $a_{e} \gg a_{i}=a_{e} e^{-N}$ where $a_{i}$ and $a_{e}$ are the scale factors at the start and end of inflation, respectively; $a_{r}$ is the scale factor at recombination and $H_{I}$ is the Hubble parameter during inflation. To solve the horizon problem, we require that, at the very least, $d_{r}$ is larger than the area we see as the CMB today. This is just given by the distance between two points that are now one Hubble radius apart (the current size of the horizon) scaled by the scale factor at the time of recombination, $a_{r}$, so:

$$
d_{h u b}=a_{r}\left(a_{0} H_{0}\right)^{-1} .
$$

Therefore to solve the horizon problem, we require that

$$
\frac{a_{r} e^{N}}{a_{e} H_{I}}>\frac{a_{r}}{a_{0} H_{0}} \Longrightarrow N>\ln \left(\frac{a_{e} H_{I}}{a_{0} H_{0}}\right)
$$

The original estimate given by American physicist Alan Guth in his seminal paper - in which the idea of inflation to solve the horizon and flatness problems was introduced was that $N \sim 64$ [32, i.e. the universe would have to scale by a factor of at least $e^{64}$ to solve the horizon problem.

The way inflation solves the flatness problem is much simpler. Recall that the Friedmann equation implies that

$$
|\Omega-1|=\left|k(a H)^{-2}\right|
$$

where $\Omega=\frac{\rho}{\rho_{\text {crit }}}$ is a measure of how close to the universe is to being flat, $\Omega=1$. The flatness problem stemmed from the fact that in standard cosmology, the Hubble radius $(a H)^{-1}$ only grows in time, so unless the universe started with some extraordinary

\footnotetext{
${ }^{9}$ This implies exponential expansion: $a=e^{N}, N=H_{I} t=\ln a$.
} 
degree of flatness, we would expect it to be highly curved now. We can see immediately that inflation solves this - even if $\Omega-1$ is far from 0 as an initial condition, a period of inflation, where $(a H)^{-2} \propto e^{-2 N}$ sends the RHS to zero and consequently $\Omega-1 \rightarrow 0$. The exponential expansion of the universe shrinks the Hubble sphere, which leads to the universe becoming flat.

\subsubsection{Inflationary dynamics}

We have seen how a period of exponential expansion can solve both the horizon and flatness problems. But what field could dominate the energy density and crucially satisfy the equation of state, $\omega=\frac{p}{\rho} \leq-\frac{1}{3}$, i.e. have negative pressure. The simplest answer is a scalar field whose energy density is dominated by its potential energy. A universe dominated by a canonically-normalised scalar field, $\phi$, has action

$$
S=\int d^{4} x \sqrt{-g}\left\{\frac{M_{\mathrm{pl}}^{2}}{2} R-\frac{1}{2} \partial_{\mu} \phi \partial^{\mu} \phi-V(\phi)\right\}
$$

where $V(\phi)$ is the potential of the scalar field, $g$ is metric determinant and $R$ the Ricci scalar for what we assume is a flat FRW metric

$$
g_{\mu \nu}=a^{2}(t) \operatorname{diag}\left(-a(t)^{-2}, 1,1,1\right)
$$

which gives the Ricci scalar as $R=6\left(\dot{H}+2 H^{2}\right)$. From this action, assuming that $\phi=\phi(t)$ is homogeneous at the background level, we arrive at the scalar equation of motion

$$
\ddot{\phi}+3 H \dot{\phi}+\frac{d V}{d \phi}=0
$$

For a scalar field, the energy momentum tensor is given by

$$
\begin{aligned}
T_{\mu \nu} & =\frac{-2}{\sqrt{-g}} \frac{\delta S_{\text {matter }}}{\delta g^{\mu \nu}} \\
& =\operatorname{diag}\left(\frac{1}{2} \dot{\phi}^{2}+V, a^{2}\left(\frac{1}{2} \dot{\phi}^{2}-V\right), a^{2}\left(\frac{1}{2} \dot{\phi}^{2}-V\right), a^{2}\left(\frac{1}{2} \dot{\phi}^{2}-V\right)\right)
\end{aligned}
$$

so its energy density is

$$
\rho=T_{00}=\frac{1}{2} \dot{\phi}^{2}+V
$$




\section{INTRODUCTION}

and its pressure is given by

$$
p=\frac{1}{3} \delta_{j}^{i} T_{i}^{j}=\frac{1}{2} \dot{\phi}^{2}-V
$$

This gives us the (flat) Friedmann equations as

$$
3 M_{\mathrm{pl}}^{2} H^{2}=\frac{1}{2} \dot{\phi}^{2}+V
$$

and

$$
M_{\mathrm{pl}}^{2} \dot{H}=-\frac{1}{2} \dot{\phi}^{2}
$$

From this we can constrain our scalar field so that it is a viable inflaton. For inflation we require $\epsilon=-\frac{\dot{H}}{H^{2}}<1$ and for quasi-exponential expansion, we want $\epsilon \ll 1$ which tells us

$$
\frac{3 \dot{\phi}^{2}}{2 V+\dot{\phi}^{2}} \ll 1 \Longrightarrow V \gg \dot{\phi}^{2}
$$

i.e. the energy density of the scalar field is dominated by its potential. For inflation to last a sufficient amount of time, we require that derivatives of $\epsilon$ are also small so that the universe does not leave its phase of accelerated expansion too quickly. We define

$$
\eta=\frac{1}{H} \frac{d \ln \epsilon}{d t}
$$

and require that $|\eta| \ll 1$. In single field inflation, this can be recast as

$$
\eta=2\left(\frac{\ddot{\phi}}{H \dot{\phi}}+\epsilon\right)
$$

where we have used that $\epsilon=\epsilon_{\phi}:=\frac{\dot{\phi}^{2}}{2 M_{\mathrm{pl}}^{2} H^{2}}$ in single field inflation. For a sustained period of inflation, it is therefore necessary that

$$
H \dot{\phi} \gg \ddot{\phi}
$$

so that 1.32 can be simplified to

$$
3 H \dot{\phi} \simeq-\frac{d V}{d \phi} .
$$




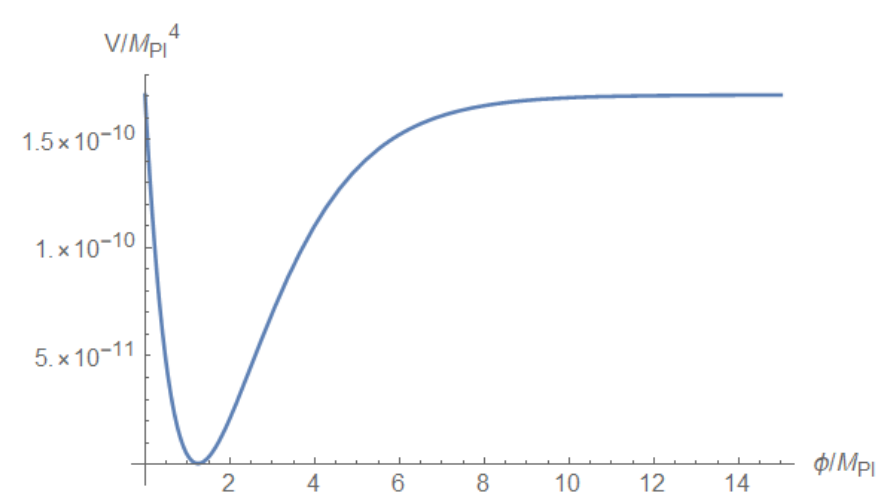

Figure 1.2: A typical inflationary potential given by $V=\Lambda^{4}\left(1-m_{1} \phi e^{-m_{2} \phi}\right)$ with $\Lambda=3.62 \times 10^{-3} M_{\mathrm{pl}}, m_{1}=2.17 M_{\mathrm{pl}}^{-1}$ and $m_{2}=0.8 M_{\mathrm{pl}}^{-1}$. This is an example of a large-field model in which the inflaton spans super-Planckian values during its evolution.

If both $\epsilon, \eta \ll 1$, the scalar field rolls slowly, the Hubble parameter $H \simeq \sqrt{\frac{V}{3 M_{\mathrm{pl}}^{2}}} \sim$ const. is dominated by the potential energy, which is roughly constant, and the scale factor increases exponentially, $a \propto e^{H t}$. If these conditions are satisfied, the universe is in a phase of slow-roll inflation. A typical inflationary potential is plotted in FIG. 1.2. The inflaton starts at some high value, $\phi_{\max }$, and rolls slowly down the flat part of the potential - during this part of its evolution the potential and therefore the Hubble parameter are roughly constant. Eventually the inflaton nears its minimum and speeds up dramatically, exits slow-roll and $\epsilon \rightarrow 1$ marks the end of inflation. The inflaton of course settles in its minimum at a much lower energy scale and converts its energy to standard model degrees of freedom through a process called reheating.

\subsubsection{Perturbations}

Everything in the previous section assumed $\phi=\phi(t)$ is perfectly homogeneous. In reality, the inflaton has small perturbations, $\delta \phi=\delta \phi\left(t, x^{i}\right)$, that depend on position as well as time. These perturbations prove to be extremely useful: small fluctuations in the inflaton field leave under and over-densities that evolve to become both the source of the anisotropies of the CMB and large-scale structure (galaxies and so on). A key point of course is that these perturbations must be small relative to the background inflaton so that the results of the previous chapter are still valid, and also so that the over and under-densities are not large enough to seed a universe that is not homogeneous and isotropic on large scales. 


\section{INTRODUCTION}

Since the inflaton dominates the energy density of the universe, a fluctuation in the inflaton field leads to a fluctuation in the energy-momentum tensor and, through that, it leads to a fluctuation in the metric, $\delta g_{\mu \nu}\left(t, x^{i}\right)$. We first set up the system as follows: we have the perturbations

$$
\phi\left(t, x^{i}\right)=\phi^{0}(t)+\delta \phi\left(t, x^{i}\right), \quad g_{\mu \nu}\left(t, x^{i}\right)=g_{\mu \nu}^{0}(t)+\delta g_{\mu \nu}\left(t, x^{i}\right)
$$

where 0 stands for background quantities. The most general scalar-tensor decomposition of the metric is

$$
d s^{2}=-(1+2 \Phi) d t^{2}+2 a \partial_{i} B d x^{i} d t+a^{2}\left\{(1-2 \Psi) \delta_{i j}+E_{i j}\right\} d x^{i} d x^{j}
$$

where the traceless, symmetric tensor $E_{i j}$ (which has 5 degrees of freedom) can be decomposed as (via scalar-vector-tensor decomposition) $E_{i j}=\partial_{i} \partial_{j} E-\frac{1}{3} \delta_{i j} \nabla^{2} E+h_{i j}$ with $\Phi, \Psi$ and $E$ scalars and $h_{i j}$ are the (traceless) tensor degrees of freedom ${ }^{10}$. We have completely ignored the vector part of the metric since this is uninteresting both because vectors are not sourced by the scalar inflaton and because they are naturally diluted by the exponential expansion of space 11 . We now define a gauge-invariant quantity known as the comoving curvature perturbation of single-field inflation:

$$
\mathcal{R}:=\Psi+H \frac{\delta \phi}{\dot{\phi}^{0}}
$$

It is defined as such because, under a transformation on constant-time hypersurfaces ${ }^{12}$

$$
t \rightarrow t+\xi
$$

the scalar spatial curvature perturbation (see (1.44) $), \Psi$, and the inflaton perturbation

\footnotetext{
${ }^{10} \mathrm{~A}$ vector, $w_{i}$, can always be decomposed into a parallel and a perpendicular part, $w_{i}=w_{i}^{p a}+w_{i}^{p e}$ where $\nabla \times \vec{w}^{p a}=\nabla \cdot \vec{w}^{p e}=0$. Given that the curl of a gradient is always zero, the parallel part may expressed $\vec{w}^{p a}=\nabla S$, i.e. the gradient of a scalar, $S$, so can actually be considered one scalar degree of freedom. The remaining (2) vector degrees of freedom are tied up in the divergenceless perpendicular part. Analogously a tensor can decomposed into a parallel (1 scalar d.o.f.), perpendicular (2 vector d.o.f.) and a transverse and traceless tensor part, which has the 2 remaining d.o.f. corresponding to the two polarisations of gravitational waves.

${ }^{11}$ See chapter 3 for details on how vectors dilute during inflation.

${ }^{12}$ See 33 for all the gauge transformations and (lots of) details.
} 
transform as 33 ]

$$
\begin{gathered}
\Psi \rightarrow \Psi+H \xi \\
\delta \phi \rightarrow \delta \phi-\xi \dot{\phi}^{0}
\end{gathered}
$$

We wish to move to the comoving frame where the inflationary perturbation is zero, $\delta \phi_{\text {com }}=0$. In this frame, $\mathcal{R}=\Psi$. Since $\delta \phi \rightarrow \delta \phi_{\text {com }}=\delta \phi-\xi \dot{\phi}^{0}=0$, we see that the coordinate change that moves us to the comoving slicing is $\xi=\frac{\delta \phi}{\dot{\phi}^{0}}$. We see therefore that $\mathcal{R}$ is the curvature perturbation, $\Psi$, in the comoving gauge. Furthermore, it is gauge-invariant through (1.47).

$\mathcal{R}$ is particularly useful because it is conserved on super-Hubble scales, $k<a H$ [25, 33], when the fluctuations in the matter content are adiabatic, i.e. they satisfy

$$
\delta p-\frac{\dot{p^{0}}}{\rho^{0}} \delta \rho=0 .
$$

Single field models of inflation always produce adiabatic fluctuations [34], and therefore $\mathcal{R}$ is conserved after its scale becomes bigger than the Hubble scale which shrinks during inflation. The metric as defined in (1.44) has 6 non-vector degrees of freedom (plus 4 vector d.o.f. that we have left out) as well as our scalar, $\delta \phi$, leaving 11 total degrees of freedom ( 5 scalar, 4 vector and 2 tensor). Of course the gauge freedom of general relativity allows us to immediately remove two scalar degrees of freedom, and another two appear only as constraints. A nice way of dealing with the constraints is to recast the metric in terms of the $\mathrm{ADM}^{13}$ formalism 35

$$
d s^{2}=-N^{2} d t^{2}+g_{i j}\left(d x^{i}+N^{i} d t\right)\left(d x^{j}+N^{j} d t\right)
$$

which leaves our scalar field action 1.30 as

$$
\begin{aligned}
S_{a d m}=\frac{1}{2} \int d^{4} x & \sqrt{-g}\left\{N R_{3}-2 N V+N^{-1}\left(E^{i j} E_{i j}-E^{2}\right)\right. \\
& \left.+N^{-1}\left(\dot{\phi}-N^{i} \partial_{i} \phi\right)^{2}-N g^{i j} \partial_{i} \phi \partial_{j} \phi-2 V\right\}
\end{aligned}
$$

\footnotetext{
${ }^{13}$ After American physicists, Richard Arnowitt, Stanley Deser and Charles W Misner.
} 


\section{INTRODUCTION}

where $N$ is the lapse, $R_{3}$ is the spatial Ricci scalar of $g_{i j}$

$$
E_{i j}=\frac{1}{2}\left(\dot{g_{i j}}-\nabla_{i}^{(3)} N_{j}-\nabla_{j}^{(3)} N_{i}\right), \quad E=g^{i j} E_{i j}
$$

with $N_{i}$ the shift, which can be decomposed into one scalar and two vector d.o.f. $N_{i}=\partial_{i} \beta+v_{i}$ where $\nabla \cdot \vec{v}=0$. Our two scalar degrees of freedom $N$ and $\beta$ are in fact non-dynamical (their equations of motion have no time derivatives) and are therefore constraints leaving us with only one dynamical scalar degree of freedom as we would expect given the content of our theory. This remaining scalar degree of freedom depends on the gauge. Of course any gauge can be chosen, and here we will follow [25] and use the gauge where the inflaton perturbation is zero (the comoving gauge):

$$
\delta \phi=0, \quad g_{i j}=a^{2}\left\{(1-2 \mathcal{R}) \delta_{i j}+h_{i j}\right\}
$$

where $h_{i j}$ is transverse and traceless $h_{i i}=\partial_{i} h_{i j}=0$. The action 1.50 needs to be perturbed to second order with the set-up given by (1.52). This is done by first solving for the constraints $N$ and $\beta$ and substituting these back into the action. For the time being, we set $M_{\mathrm{pl}}=1$ for simplicity, and then the scalar part of the second order action is given by (see [25] Appendix B)

$$
S_{\mathcal{R}^{2}}=\int d^{4} x a^{3} \epsilon\left\{\dot{\mathcal{R}}^{2}-a^{-2}(\nabla \mathcal{R})^{2}\right\}
$$

using $\epsilon=\epsilon_{\phi}=\frac{\dot{\phi}^{2}}{2 H^{2}}$. This action is easier to work with after defining a new variable $v=z \mathcal{R}$ with $z^{2}=a^{2} \frac{\dot{\phi}^{2}}{H^{2}}=2 a^{2} \epsilon$ and moving to conformal time, which is defined through

$$
\frac{d t}{d \tau}=a(t)
$$

The action becomes

$$
S_{v^{2}}=\frac{1}{2} \int d \tau d^{3} x\left\{v^{\prime 2}-\left(\partial_{i} v\right)^{2}+\frac{z^{\prime \prime}}{z} v^{2}\right\}
$$

where primes stand for derivatives with respect to conformal time, $\partial / \partial \tau$. To take account of the inherently quantum nature of the fluctuations, $v$ must be promoted to 
a quantum operator:

$$
\hat{v}(\tau, \vec{x})=\int \frac{d^{3} k}{(2 \pi)^{3}}\left\{v_{k}(\tau) \hat{a}(\vec{k}) e^{i \vec{k} \cdot \vec{x}}+v_{k}^{*}(\tau) \hat{a}^{\dagger}(\vec{k}) e^{-i \vec{k} \cdot \vec{x}}\right\} .
$$

The canonical quantisation condition 14

$$
\left[\hat{v}(\tau, \vec{x}), \hat{v}^{\prime}(\tau, \vec{y})\right]=i \delta^{3}(\vec{x}-\vec{y})
$$

can be satisfied with

$$
\left[\hat{a}(\vec{p}), \hat{a}^{\dagger}(\vec{q})\right]=(2 \pi)^{3} \delta^{3}(\vec{p}-\vec{q})
$$

and

$$
v_{k} v_{k}^{* \prime}-v_{k}^{\prime} v_{k}^{*}=i
$$

The mode functions, $v_{k}(\tau)$, satisfy the equation of motion found through the action (1.55):

$$
v_{k}^{\prime \prime}+\left(k^{2}-\frac{z^{\prime \prime}}{z}\right) v_{k}=0 .
$$

In the distant past, the mode functions had wavelengths well inside the Hubble radius (recall that inflation shrinks the Hubble sphere) $\frac{k}{a H}=-k \tau \gg 1$. Now use that

$$
\frac{z^{\prime \prime}}{z}=2 a^{2} H^{2}
$$

to zeroth order in slow-roll parameters, $\epsilon, \eta$, and therefore $k^{2} \gg \frac{z^{\prime \prime}}{z}$ so that in the distant past of the inflationary period the mode equation becomes

$$
v_{k}^{\prime \prime}+k^{2} v_{k} \simeq 0
$$

which is simply a harmonic oscillator and has solutions

$$
v_{k}=c_{1} e^{i k \tau}+c_{2} e^{-i k \tau}
$$

We wish to normalise solutions to the full equation 1.60 in this limit $k \gg a H$ using 1.59). The vacuum solution, which satisfies $\hat{a}(\vec{k})|0\rangle=\langle 0| \hat{a}^{\dagger}(\vec{k})=0$, should be an eigenvector of the Hamiltonian, $\hat{H}|0\rangle=E_{0}|0\rangle$. The Hamiltonian for this system is

\footnotetext{
${ }^{14}$ The canonical condition is defined through $[v, \Pi]=i \delta^{3}(\vec{x}-\vec{y})$ where (remembering to switch back $\left.d \tau=a^{-1} d t\right) \Pi=\frac{\partial \mathcal{L}}{\partial \dot{v}}=a(\tau) \dot{v}=v^{\prime}$.
} 


\section{INTRODUCTION}

given by

$$
\hat{H}=\frac{1}{2} \int d^{3} x a^{-1}\left\{\hat{v}^{\prime 2}+\left(\partial_{i} \hat{v}\right)^{2}-\frac{z^{\prime \prime}}{z} \hat{v}^{2}\right\}
$$

using $H=\Pi \dot{v}-L$ with $L=\int d^{3} x \mathcal{L}$ the Lagrangian and $\Pi=\frac{\partial \mathcal{L}}{\partial \dot{v}}$. Applying this to our ground state, and using $\hat{a}|0\rangle=0$ as well as 1.58 gives us

$$
\begin{aligned}
\hat{H}|0\rangle= & \frac{1}{2 a} \int \frac{d^{3} k}{(2 \pi)^{3}}\left\{\left|v_{k}^{\prime}\right|^{2}+\left(k^{2}-\frac{z^{\prime \prime}}{z}\right)\left|v_{k}\right|^{2}\right\}|0\rangle \\
& +\frac{1}{2 a} \int \frac{d^{3} k}{(2 \pi)^{3}}\left\{v_{k}^{\prime 2}+\left(k^{2}-\frac{z^{\prime \prime}}{z}\right) v_{k}^{2}\right\}^{*} \hat{a}^{\dagger}(\vec{k}) \hat{a}^{\dagger}(-\vec{k})|0\rangle .
\end{aligned}
$$

Since we require that $|0\rangle$ be an eigenstate of the Hamiltonian, the second term must vanish. This gives us an extra condition. Again assuming that in the distant past, scales were well inside the horizon, $\frac{z^{\prime \prime}}{z} \ll k^{2}$, we get

$$
v_{k}^{\prime 2}+k^{2} v_{k}^{2}=0 \Longrightarrow v_{k}^{\prime}= \pm i k v_{k} .
$$

This is important because it constrains our early-time solution 1.63 so that either $c_{1}$ or $c_{2}$ must be zero. Furthermore, using (1.59), we get the condition

$$
2 k\left(\left|c_{2}\right|^{2}-\left|c_{1}\right|^{2}\right)=1
$$

Satisfying these conditions simultaneously leaves us with $c_{1}=0, c_{2}=\frac{1}{\sqrt{2 k}}$, and we arrive at the early-time solution

$$
\lim _{k \tau \rightarrow-\infty} v_{k}=\frac{e^{-i k \tau}}{\sqrt{2 k}}
$$

which is known as the Bunch-Davie ${ }^{15}$ initial condition for quantum fields in (quasi-) de Sitter spacetimes. With an initial condition, we can solve the full equation for $v_{k}$ given in 1.60 . Assuming true de Sitter $\left(H^{\prime}=0, a=-\frac{1}{H \tau}\right)$, this equation becomes

$$
v_{k}^{\prime \prime}+\left(k^{2}-\frac{2}{\tau^{2}}\right) v_{k}=0
$$

where we have used that $\frac{z^{\prime \prime}}{z}=2 a^{2} H^{2}=\frac{2}{\tau^{2}}$ when $H^{\prime}=0$. This equation of motion has

\footnotetext{
${ }^{15}$ After Timothy Bunch and Paul Davies $\underline{36}$.
} 
general solution

$$
v_{k}=A e^{-i k \tau}\left(1-\frac{i}{k \tau}\right)+B e^{i k \tau}\left(1+\frac{i}{k \tau}\right)
$$

and using our Bunch-Davies initial condition, we get

$$
v_{k}=\frac{e^{-i k \tau}}{\sqrt{2 k}}\left(1-\frac{i}{k \tau}\right) \text {. }
$$

When it comes to constraining inflationary models, a key prediction is the scalar power spectrum. Although this scalar power spectrum is not directly seen in the CMB or in the large-scale structure of the universe, it acts as an initial condition for wellunderstood (but highly complex) physics that can be used to predict what is seen in the CMB and through this, models of inflation can be constrained. We define the scalar power spectrum through the comoving curvature perturbation:

$$
\left\langle\mathcal{R}_{\vec{p}} \mathcal{R}_{\vec{q}}\right\rangle=(2 \pi)^{3} \delta^{3}(\vec{p}+\vec{q}) P_{\mathcal{R}}(p), \quad \mathcal{P}_{s}(p):=\frac{p^{3}}{2 \pi^{2}} P_{\mathcal{R}}(p)
$$

where $\mathcal{P}_{s}(p)$ is the dimensionless scalar power spectrum, which is of interest to us. In terms of our mode function, $v_{k}$ (recall that $\mathcal{R}=\frac{v}{\sqrt{2 a^{2} \epsilon}}$ )

$$
\left\langle\mathcal{R}_{\vec{p}} \mathcal{R}_{\vec{q}}\right\rangle=(2 \pi)^{3} \delta^{3}(\vec{p}+\vec{q}) \frac{\left|v_{p}\right|^{2}}{2 a^{2} \epsilon}
$$

and then using 1.71

$$
\left\langle\mathcal{R}_{\vec{p}} \mathcal{R}_{\vec{q}}\right\rangle=(2 \pi)^{3} \delta^{3}(\vec{p}+\vec{q}) \frac{H^{2}}{4 p^{3} \epsilon}\left(1+\left(\frac{p}{a H}\right)^{2}\right)
$$

where we have used that $-k \tau=\frac{k}{a H}$. From this we can read off the scalar power spectrum

$$
\mathcal{P}_{s}(k)=\frac{H^{2}}{8 \pi^{2} \epsilon}\left(1+\left(\frac{k}{a H}\right)^{2}\right) .
$$

To simplify this we can use that $\mathcal{R}$ is constant on super-Hubble scales and therefore we can take $\frac{k}{a H}<<1$, which gives the standard form for the power spectrum

$$
\mathcal{P}_{s}(k)=\frac{H^{2}}{8 \pi^{2} \epsilon}
$$

which is evaluated at horizon-crossing $k=a H$ (usually taken to be 50-60 e-folds before 


\section{INTRODUCTION}

the end of inflation, $\epsilon \rightarrow 1$ ), after which conservation of $\mathcal{R}$ guarantees that the power spectrum will remain constant till the scale $k$ re-enters the horizon and is seen today in the CMB. The latest results from Planck [37] suggest that the inflationary power spectrum should satisfy

$$
\mathcal{P}_{s} \sim 2 \times 10^{-9}
$$

This condition effectively constrains the scale of the potential (since $3 H^{2} \sim V$ ) relative to the value of $\epsilon$ 50-60 e-folds before the end of inflation. This fact has an important consequence we will consider shortly. First however, we discuss another inflationary observable, the scalar spectral index, $n_{s}$, which determines the scale-dependence of the inflationary power spectrum - that is, it determines the degree to which the density of two points in the inflationary universe correlate depends on their distance. A simple derivation for $n_{s}$ in terms of basic inflationary parameters is given here. Recall $\epsilon=$ $-\frac{\dot{H}}{H^{2}}=-\frac{1}{H} \frac{d H}{d N}$ and $\eta=\frac{d \ln \epsilon}{d N}$ with $N=\ln a$. The scalar spectral index is defined through

$$
n_{s}-1=\frac{d \ln \mathcal{P}_{s}}{d \ln k}
$$

where

$$
\mathcal{P}_{s}=\frac{H^{2}}{8 \pi^{2} \epsilon}, \quad \ln k=N+\ln H
$$

because $x=k / a H=1$ at horizon-crossing. Using the chain rule

$$
\frac{d \ln \mathcal{P}_{s}}{d \ln k}=\frac{d \ln \mathcal{P}_{s}}{d N} \frac{d N}{d \ln k}
$$

and

$$
d \ln \mathcal{P}_{s}=d \ln \left(\frac{H^{2}}{8 \pi^{2} \epsilon}\right)=2 d \ln H-d \ln \epsilon \Longrightarrow \frac{d \ln \mathcal{P}_{s}}{d N}=2 \frac{d \ln H}{d N}-\frac{d \ln \epsilon}{d N}
$$

but

$$
\epsilon=-\frac{1}{H} \frac{d H}{d N}=-\frac{d \ln H}{d N} \Longrightarrow \frac{d \ln \mathcal{P}_{s}}{d N}=-2 \epsilon-\eta
$$

Also

$$
\begin{array}{r}
\frac{d N}{d \ln k}=\left(\frac{d \ln k}{d N}\right)^{-1}=\left(1+\frac{d \ln H}{d N}\right)^{-1} \approx 1+\epsilon \\
\Longrightarrow n_{s}-1=\frac{d \ln \mathcal{P}_{s}}{d \ln k}=\frac{d \ln \mathcal{P}_{s}}{d N} \frac{d N}{d \ln k}=(-2 \epsilon-\eta)(1+\epsilon) \approx-2 \epsilon-\eta
\end{array}
$$


leads us to

$$
n_{s}=1-2 \epsilon-\eta
$$

Constraints from Planck place $0.9607 \leq n_{s} \leq 0.9691$ [37], suggesting the inflationary power spectrum is nearly scale-invariant. In all the models considered in this thesis, $\epsilon$ is much smaller than 1 , usually $\epsilon<10^{-3}$, and therefore this constraint on $n_{s}$ suggests that $\eta \sim 0.03$ and this strongly constrains the type of inflationary potential that can be used. We note that in (slow-roll) single-field inflation, the slow-roll parameters can be written in terms of the potential. First

$$
\epsilon=-\frac{\dot{H}}{H^{2}}=\frac{\dot{\phi}^{2}}{2 H^{2} M_{\mathrm{pl}}^{2}} \simeq \frac{1}{2} \frac{V^{\prime}(\phi)^{2}}{9 M_{\mathrm{pl}}^{2} H^{4}} \simeq \frac{M_{\mathrm{pl}}^{2}}{2}\left(\frac{V^{\prime}}{V}\right)^{2}
$$

where $V^{\prime}=\frac{d V}{d \phi}$, and we have used 1.42 so that $\dot{\phi} \simeq-\frac{1}{3 H} V^{\prime}$ and $3 H^{2} M_{\mathrm{pl}}^{2} \simeq V$ during slow-roll. With this form for $\epsilon$, we can equally estimate $\eta$ in terms of the potential:

$$
\eta=\frac{d \ln \epsilon}{d N}=\frac{1}{\epsilon H} \frac{d \epsilon}{d t}=2 M_{\mathrm{pl}}^{2}\left(\frac{V^{\prime}}{V}\right)^{2}-2 M_{\mathrm{pl}}^{2} \frac{V^{\prime \prime}}{V} \simeq 4 \epsilon-2 M_{\mathrm{pl}}^{2} \frac{V^{\prime \prime}}{V} \Longrightarrow \eta \sim-2 M_{\mathrm{pl}}^{2} \frac{V^{\prime \prime}}{V} .
$$

Since we require $\eta$ to be positive to satisfy the constraint on $n_{s}$, this immediately tells us that

$$
\frac{V^{\prime \prime}}{V}<0
$$

i.e. we require a concave potential.

\section{Tensors}

We consider now the other form of perturbation that is sourced during inflation: tensors. Using again 1.52 as our metric with tensor perturbation $h_{i j}$, the tensor part of the second order action can be found to be (see chapter 3 for a related derivation)

$$
S_{h_{\mu \nu}^{2}}=\frac{M_{\mathrm{pl}}^{2}}{8} \int d \tau d^{3} x a^{2}\left\{\hat{h}_{i j}^{\prime} \hat{h}_{i j}^{\prime}-\partial_{i} \hat{h}_{j k} \partial_{i} \hat{h}_{j k}\right\}
$$

The operator $h_{i j}$ can be expanded in terms of mode functions as

$$
\hat{h}_{i j}=\sum_{s} \int \frac{d^{3} k}{(2 \pi)^{3}}\left\{\epsilon_{i j}^{s}(k) h_{k}^{s}(\tau) e^{i \vec{k} \cdot \vec{x}} \hat{a}(\vec{k})+\epsilon_{i j}^{s *}(k) h_{k}^{s *}(\tau) e^{-i \vec{k} \cdot \vec{x}} \hat{a}^{\dagger}(\vec{k})\right\}
$$


where $\epsilon_{i j}^{s}(k)$ is a polarisation tensor, $h_{k}^{s}(\tau)$ is for a mode function, $s$ is an index of the two helicities of a gravitational wave, while $\hat{a}$ and $\hat{a}^{\dagger}$ satisfy the usual commutation relations. The system can be simplified by making the substitution $h_{k}^{s}=\frac{2}{a M_{\mathrm{pl}}} u_{k}^{s}$. The action for the mode functions then takes a very similar form to that of the scalar equation 1.55

$$
S_{u^{2}}=\sum_{s} \frac{1}{2} \int d \tau d^{3} x\left\{\left(\partial_{\tau} u^{s}\right)^{2}-\left(\partial_{i} u^{s}\right)^{2}+\frac{a^{\prime \prime}}{a} u^{s}\right\}
$$

which gives equations of motion

$$
u_{k}^{s \prime \prime}+\left(k^{2}-\frac{2}{\tau^{2}}\right) u_{k}^{s}=0
$$

where we have used $\frac{a^{\prime \prime}}{a}=\frac{2}{\tau^{2}}$. This is the same equation of motion as we had for the scalars, $v_{k}$, satisfied by each helicity mode function separately. Therefore in the super-Hubble limit, we know

$$
\left|u_{k}^{s}\right|^{2} \simeq \frac{1}{2 k} \Longrightarrow\left|h_{k}^{s}\right|^{2} \simeq \frac{2}{a^{2} M_{\mathrm{pl}}^{2} k}=\frac{2 H^{2}}{M_{\mathrm{pl}}^{2} k^{3}}
$$

at horizon-crossing, $a=\frac{k}{H}$. As we did for the scalars, we can define a power spectrum for $h$ through

$$
\left\langle h_{\vec{p}}^{s} h_{\vec{q}}^{s}\right\rangle=(2 \pi)^{3} \delta^{3}(\vec{p}+\vec{q}) P_{h}(p), \quad \mathcal{P}_{h}(p):=\frac{p^{3}}{2 \pi^{2}} P_{h}(p)
$$

Then since

$$
\left\langle h_{\vec{p}}^{s} h_{\vec{q}}^{s}\right\rangle=(2 \pi)^{3} \delta^{3}(\vec{p}+\vec{q}) \frac{2 H^{2}}{M_{\mathrm{pl}}^{2} k^{3}}
$$

we can read off

$$
\mathcal{P}_{h}(k)=\frac{H^{2}}{\pi^{2} M_{\mathrm{pl}}^{2}} .
$$

The full dimensionless tensor power spectrum is therefore

$$
\mathcal{P}_{t}(k)=2 \mathcal{P}_{h}(k)=\frac{2 H^{2}}{\pi^{2} M_{\mathrm{pl}}^{2}}
$$

where the factor of two arises to account for the two helicities of the mode functions. A crucial inflationary observable can now be defined through the ratio of the tensor and 
scalar power spectra. The tensor-to-scalar ratio is given by

$$
r:=\frac{\mathcal{P}_{t}}{\mathcal{P}_{s}} \simeq 16 \epsilon
$$

where the final equality is true for single-field inflation. The value of $r$ can also be tested through the CMB. Light, like gravity, has two polarisations, and in CMB physics, the polarisations are chosen to be split into what are called $E$ - and $B$-modes: ${ }^{16}$, $E$ mode polarisation can be sourced by both scalar and tensor perturbations, however, importantly, $B$-mode polarisation can only be sourced by tensor perturbation 17 - and therefore a detection of $B$-modes in the $\mathrm{CMB}$ would be strong evidence for tensor perturbations from inflation, often referred to as primordial gravitational waves. The current constraint (due to the non-detection of $B$-modes) on the tensor-to-scalar ratio is [37]

$$
r \lesssim 0.07
$$

\section{$r$ is limited by $V$}

As mentioned earlier, the fact that the power spectrum

$$
\mathcal{P}_{s} \simeq \frac{H^{2}}{8 \pi^{2} \epsilon} \simeq \frac{V}{24 M_{\mathrm{pl}}^{2} \pi^{2} \epsilon} \sim 2 \times 10^{-9}
$$

constrains $\epsilon$ relative to the scale of the potential, $V$, has an important consequence. Since $r \sim 16 \epsilon$, we see immediately that lower-energy inflation leads to a lower value for the tensor-to-scalar ratio. Future experiments proposed to search for $B$-modes expect to have sensitivity down to $r \sim 10^{-3}$ [39 44]. We can thus parametrise whether a particular potential can predict an observable value for the tensor-to-scalar ratio, namely $r \gtrsim 10^{-3}$, through

$$
r=\frac{\mathcal{P}_{t}}{\mathcal{P}_{s}}=\mathcal{P}_{s}^{-1} \frac{2 H^{2}}{\pi^{2} M_{\mathrm{pl}}^{2}} \simeq 10^{-3}\left(\frac{V^{1 / 4}}{5.68 \times 10^{15} \mathrm{GeV}}\right)^{4},
$$

\footnotetext{
${ }^{16}$ The " $E$ " and " $B$ " come from parallels with electromagnetism - the $E$-mode (electric field) has vanishing curl while the $B$-mode (magnetic field) is divergenceless.

${ }^{17}$ This is a slight simplification - $B$-mode polarisation can be sourced by gravitational lensing and galactic foregrounds as well, but this can distinguished from $B$-modes sourced by pure inflationary tensor modes [38.
} 


\section{INTRODUCTION}

i.e. we require a potential satisfying $V^{1 / 4} \gtrsim 5.68 \times 10^{15} \mathrm{GeV}$ to lead to an observable gravitational wave spectrum during vanilla inflation. However, as we will see in this thesis, there are ways in which a gravitational wave source can arise during inflation, and with an additional source, this bound can circumvented. In particular, the introduction of a vector or gauge field suitably coupled to an axion during inflation can introduce a large source term for the tensor modes under the right conditions. Applying this mechanism to a low-energy model of inflation called Kähler moduli inflation is the main concern of this thesis.

\subsubsection{Multi-field inflation}

Kähler moduli inflation is in general a multi-field model of inflation - we therefore quickly note here some features of multi-field inflation. In particular, an important feature is that it is easy to generalise the single-field results above to the case where there are multiple scalar fields. Consider a set of scalars fields, $\phi^{a}=\left(\phi_{1}, \phi_{2}, \ldots\right)$. The space spanned by this set of fields can be endowed with a (field-space) metric, $\gamma_{a b}$, that in practice accounts for non-trivial couplings between the fields in their kinetic terms. An action equivalent to 1.30 can then be written

$$
S=\int d^{4} x \sqrt{-g}\left\{\frac{M_{\mathrm{pl}}^{2}}{2} R-\frac{1}{2} \gamma_{a b} \partial_{\mu} \phi^{a} \partial^{\mu} \phi^{b}-V\left(\phi^{a}\right)\right\}
$$

where $V\left(\phi^{a}\right)$ is a function of all the scalar fields. Notice that in the case of trivial fieldspace metric, $\gamma_{a b}=\delta_{a b}$, the kinetic terms for the fields become canonical $\left(\frac{1}{2} \partial_{\mu} \phi_{1} \partial^{\mu} \phi_{1}+\right.$ ...). In fact if the field space has zero curvature, defined by the Ricci scalar for the field-space: $R_{\gamma_{a b}}=0$, then the fields can always be redefined so that we end up with canonical kinetic terms. This action gives equations of motion:

$$
\begin{gathered}
\ddot{\phi}^{a}+3 H \dot{\phi}^{a}+\Gamma_{b c}^{a} \dot{\phi}^{b} \dot{\phi}^{c}+\gamma^{a b} \frac{\partial V}{\partial \phi^{b}}=0, \\
3 M_{\mathrm{pl}}^{2} H^{2}=\frac{1}{2} \dot{\varphi}^{2}+V
\end{gathered}
$$

and

$$
M_{\mathrm{pl}}^{2} \dot{H}=-\frac{1}{2} \dot{\varphi}^{2}
$$


where $\Gamma_{b c}^{a}$ are the Christoffel symbols for metric $\gamma_{a b}$ and $\dot{\varphi}=\sqrt{\gamma_{a b} \dot{\phi}^{a} \dot{\phi^{b}}}$. Through this we can define equivalent slow-roll parameters, e.g. in this system

$$
\epsilon:=-\frac{\dot{H}}{H^{2}}=\epsilon_{\varphi}:=\frac{1}{2 M_{\mathrm{pl}}^{2} H^{2}} \dot{\varphi}^{2}
$$

Crucially the scalar perturbations $\delta \phi^{a}$, of which there are now many, can be cast in terms of one tangential perturbation 18

$$
\delta_{s}=\frac{\gamma_{a b} \dot{\phi}^{a} \delta \phi^{b}}{\dot{\varphi}}=\frac{\Delta_{s}}{a}
$$

where $\Delta_{s}$ is completely equivalent to $v$ in the single-field case, and is related to the comoving curvature perturbation through $\mathcal{R}=H \frac{\Delta_{s}}{a \dot{\varphi}}$. For this reason we know that if $\left|\Delta_{s}\right| \rightarrow \frac{1}{\sqrt{2 k} x}$ for $x=\frac{k}{a H}<1$ (as is true for $v_{k}$ ), then the curvature perturbation behaves in the exact same way as in single field inflation, and the power spectrum and scalar spectral index are both well-approximated by their standard forms:

$$
\mathcal{P}_{s}=\frac{H^{2}}{8 \pi^{2} \epsilon}, \quad n_{s}=1-2 \epsilon-\eta .
$$

In addition to this, the equation of motion for the tensor modes is unchanged and therefore the tensor-to-scalar ratio is again given by

$$
r=16 \epsilon .
$$

In other words, all the results from single-field inflation nicely generalise to the multifield case as long as

$$
\left|\Delta_{s}\right| \rightarrow \frac{1}{\sqrt{2 k x}}, \quad x=\frac{k}{a H}<1
$$

is satisfied.

\footnotetext{
${ }^{18} \mathrm{Of}$ course, since this is a multi-field system, there are perturbations that correspond to the directions orthogonal to this. These perturbations are called entropic or isocurvature perturbations. As we have noted, single-field models predict that the curvature perturbation is purely adiabatic (i.e. it induces no isocurvature perturbation) and this is consistent with data from Planck that put strong constraints on the magnitude of isocurvature perturbations. Therefore in a full analysis of the multi-field inflationary model, it is necessary to demonstrate that the isocurvature perturbations of the model are under control. However, in the work presented in this thesis, isocurvature perturbations are assumed to be negligible and we leave their study to a future work.
} 


\section{INTRODUCTION}

\subsubsection{Non-standard cosmologies and non-scalar fields}

We end this review of inflation by quickly introducing an anisotropic inflationary metric that will be used in chapter 2, So far we have considered inflation with a flat FRW metric. During slow-roll, when $H \sim$ const, the inflationary metric is

$$
d s^{2}=-d t^{2}+e^{2 H t} \delta_{i j} d x^{i} d x^{j}
$$

However in chapter 2, we will consider an anisotropic variation of this metric. Defining the anisotropy to be along the $x$-axis, we can write the anisotropic metric as

$$
d s^{2}=-d t^{2}+e^{2 H t}\left[e^{-4 \Sigma t} d x^{2}+e^{2 \Sigma t}\left(d y^{2}+d z^{2}\right)\right]
$$

where $\Sigma$ parametrises the degree of anisotropy, determining the difference in the Hubble parameter along the $x$-axis relative to the other two axes. Normally during inflation anisotropy is quickly diluted since the scalar field dominating the universe's energy density is isotropic. However in chapter 2, we consider an example where a gauge field, supported through its coupling to the inflaton, can induce and support a small universal anisotropy that leaves an observable imprint on the CMB in the form of a small departure from perfect rotational invariance of the power spectrum.

We note finally that although the actions we have considered in this section have contained only scalar fields, it is quite possible for there to be additional fields besides the scalars. The important point is that the energy density of the universe is completely dominated by the scalar field(s) $3 M_{\mathrm{pl}}^{2} H^{2} \simeq \rho_{\phi} \simeq V(\phi)$ so that the Hubble parameter is roughly constant and inflation can take place. The title of this thesis refers to "spectator fields" and the main candidates for that role in this thesis are gauge fields which, despite being massively sub-dominant in energy density relative to the inflaton and thus not stopping the universe from inflating, can still have non-trivial effects like increasing the tensor-to-scalar ratio (see chapter 3 and chapter 5) and inducing non-negligible universal anisotropy (see chapter 2).

\subsection{Structure of this thesis}

The main subject of this thesis is the role that gauge fields can play in introducing non-trivial dynamics during inflation that can lead to interesting, observable features 
that can distinguish a model. With this in mind, we start off in chapter 2 by expanding on a model [45] in which a coupling between the inflaton of a simplistic inflationary model and a $U(1)$ vector field can sustain a small cosmological anisotropy that could be detected in the CMB. Applying this first to a model with canonically-normalised inflaton, we show that it is capable of producing small enough anisotropy so as not to be at odds with observations, but still potentially detectable in the future. We then discuss a non-canonical, DBI-inspired ${ }^{19}$ case as first studied in [47] and although we find anisotropic solutions, we are unable to find solutions that satisfy constraints on anisotropy.

In chapter 3 , we discuss a mechanism by which a gauge field can be supported during inflation. This gauge field generates an extra source term for the tensor perturbations of the metric and, under the right conditions, can artificially enhance the gravitational wave spectrum, circumventing the need for a higher-energy potential. In this chapter we first discuss why vectors are diluted during the exponential expansion of the universe. We then look at the main features of one of the first models in which a vector field [2, 48], supported through its coupling to an axion, is used to add an additional source term for the primordial gravitational waves. Finally we consider in detail a toy example of a more refined model [3] in which, instead of a vector field, an $S U(2)$ gauge field is used to provide a gravitational wave source term.

In chapter 4, we show how the potentials for Kähler moduli inflation [49] and a related model, called fibre inflation [50, arise before discussing some explicit models of Kähler moduli inflation and fibre inflation with a particular interest in models that could conceivably contain an axion capable of supporting an $S U(N)$ gauge field ${ }^{20}$ and thus lead to an enhancement of the gravity wave sector of these models. This is of particular interest in Kähler moduli inflation because it predicts a very small tensor-to-scalar ratio, $r \lesssim 10^{-6}$, and thus, if evidence is found for primordial tensors, Kähler moduli inflation would be effectively ruled out without the addition of a way of enhancing its gravitational wave spectrum. Much of this chapter is also concerned with simplifying

\footnotetext{
${ }^{19}$ Dirac-Born-Infeld inflation 46 is a model of inflation derived from string theory in which the inflaton is associated with the movement of a relativistic brane whose action is the DBI action.

${ }^{20}$ In the enhancement model of $[3$. (which will be discussed in chapter 3), the gauge field is $S U(2)$. We will show in chapter 5 how starting with an $S U(N)$ gauge field, the system can become equivalent to using an $S U(2)$ gauge field instead.
} 
these models as much as possible before introducing a gauge field, allowing for more feasible numerics, particularly when considering perturbations.

In chapter 5, we round off the main focus of the thesis by actually considering models of Kähler inflation coupled to a gauge field as discussed in chapter 3. Finally, in chapter 6 , we summarise and discuss the work of the previous chapters. 


\section{Chapter 2}

\section{Anisotropic Inflation}

This chapter is a modified version of a paper published in Physical Review D titled Anisotropic Inflation with Derivative Couplings [51, written with Sugumi Kanno and Ivonne Zavala.

\section{$2.1 \quad$ Introduction}

In the post-Planck [37] era, models of inflation have been constrained considerably by observational data, particularly data from the CMB. However, there remains a plethora of inflationary models that satisfy observational constraints [52]. A reason for this is that inflationary models are constrained almost exclusively by their predictions of the tensor-to-scalar ratio, $r$ and the scalar spectral index, $n_{s}$. With this in mind, it is perhaps now important to look into models that predict novel features. A possibility for one such novel feature is universal anisotropy sustained after inflation. Although quite heavily constrained in its own right through the primordial power spectrum required to explain the CMB [53, 54], universal anisotropy can still be non-negligible and a future detection of it would provide an interesting test of inflationary models.

Universal anisotropy is most easily defined through the metric - consider an infla- 


\section{ANISOTROPIC INFLATION}

tionary metri ${ }^{1}$

$$
d s^{2}=-d t^{2}+e^{2 H t}\left[e^{-4 \Sigma t} d x^{2}+e^{2 \Sigma t}\left(d y^{2}+d z^{2}\right)\right]
$$

where we naturally assume, $H \sim$ const and equivalently $\Sigma \sim$ const. The $x$-direction is clearly the direction of the anisotropy and the degree of anisotropy is given by $\Sigma / H$ which generally follows the law:

$$
\frac{\Sigma}{H}=\frac{1}{3} I \epsilon
$$

where $\epsilon$ is the slow-roll parameter, $\epsilon=-\frac{\dot{H}}{H^{2}}$ and $I$ is a model-dependent parameter taking values between $0 \leq I \leq 1$. This equation for the anisotropy arises after finding power-law solutions for the inflaton, isotropic and anisotropic scale factors in an anisotropic set-up. We will show this explicitly in the next section. This anisotropy imprints itself on the statistics of the power spectrum [55]:

$$
\mathcal{P}(\vec{k})=\mathcal{P}(k)\left(1+g_{*} \sin ^{2} \theta\right)
$$

where $g_{*}$ characterises the degree to which $P(\vec{k})$ fails to be rotationally invariant and $\theta$ is the angle between $\vec{k}$ and the direction of anisotropy. $g_{*}$ is found through perturbation theory and for the inflationary scalar power spectrum is given by

$$
g_{*}=24 I N_{e}^{2}
$$

where $N_{e}$ is the number of e-folds after horizon exit to the end of inflation. The most recent bounds on $g_{*}$ are 53

$$
g_{*}=0.002 \pm 0.016 \text {. }
$$

It was thought that anisotropy would be impossible to sustain during inflation due to the cosmic no-hair conjecture [56, 57]. In the context of early universe cosmology, the cosmic no-hair conjecture effectively states that inflation can proceed from very general initial conditions and that the assumption that a flat FRW metric can be used to

\footnotetext{
${ }^{1}$ Note that during the rest of this chapter we will rewrite this metric in terms of e-folds, $\alpha \sim H t$, $\sigma \sim \Sigma t$. We deliberately distinguish this here from the usual notation of $N=\ln a$ so it is clear there is an isotropic part given by $\alpha$ and an anisotropic part given by $\sigma$. The choice that we have $e^{-4 \Sigma t}$ along the $x$-direction and $e^{2 \Sigma t}$ along the $y$ and $z$-directions is to allow for the average expansion rate of the universe to be independent of $\Sigma$ and to be given by $H$.
} 
describe the universe during the inflationary epoch is valid. In other words, a universe that undergoes inflation will successfully approach quasi-de Sitter spacetime even if there exists an initial non-negligible anisotropic component of the energy density. This is due to the fact that the inflationary component of the energy density will remain, by necessity, roughly constant, whereas any other energy densities will dilute quickly due to the expansion of the universe. In this way, even if to start with the universe has some large anisotropy, after a period of time, the quasi-vacuum energy of the inflaton will come to dominate the energy density of the universe and this universe will be well approximated by an FRW metric. In the same way, any anisotropic field, for example a gauge field, will be rapidly diluted during inflation.

Multi-field models of inflation arise naturally in extensions of the simplest singlefield inflationary framework, for example in models derived from fundamental theories such as supergravity and string theory. Usually, models with more than one scalar field are considered in these extensions. However, fields with other spins, such as gauge fields, may play an interesting and testable role during inflation as well [54, 55, 58]. Gauge fields are not commonly considered in the study of inflation, due to the cosmic no-hair conjecture. However, [59] found the first working model (free from ghosts) of inflation with a spectator vector field that can produce persistent anisotropy in the background spacetime. The argument presented above is circumvented by coupling the inflaton to the gauge field through the term $f^{2}(\phi) F^{2}$ where $\phi$ is the scalar inflaton: this coupling means that the energy densities of the gauge and scalar field cannot be truly separated, and therefore the isotropic and anisotropic energy densities cannot be properly separated. This model brought forward the interesting possibility that light gauge fields may affect cosmological observations by generating some observable amount of statistical anisotropy (for reviews on anisotropic inflation see [54, [55, 58]).

Another possibility for generating observable statistical anisotropies in the presence of vector fields is the vector curvaton scenario $[60,62$. In this scenario, the inflaton is a scalar driving inflation, while the vector field becomes important after inflation, when it may dominate the universe and imprint its perturbation spectrum before it decays, as in the scalar curvaton scenario (for a review of the vector curvaton see [63]).

In the context of D-brane inflationary models, the inflaton is typically identified with the scalar field parametrising the transverse fluctuations of the D-brane (that is, its position in the internal compact six-dimensional space). Such a brane features a 


\section{ANISOTROPIC INFLATION}

world-volume two-form field $F_{\mu \nu}$, associated with the longitudinal fluctuations of the Dbrane. Therefore, it is natural to investigate the role of this brane field in the dynamics of inflation. Indeed, in [64], a D-brane vector curvaton realisation was discussed, while in [65] a Wilson line inflationary model was studied with interesting predictions. In this case, it is precisely one of the D-brane vector internal components which drives inflation.

In a D-brane scenario, the scalar field associated with brane position, identified with the inflaton, and the vector field couple disformally via the DBI action that describes the D-brane dynamiç2 2 , In particular, the gauge kinetic function, $f$, depends on the scalar field $\phi$ and its derivative $X:=\frac{1}{2}(\partial \phi)^{2}, f(\phi, X)$. This coupling can thus alter the anisotropic background evolution and the amount of statistical anisotropy produced.

Furthermore, $f(\phi, X)$ represents a general parametrisation of a generic inflatonmatter coupling. This has recently been used in studies in which the inflationary universe is viewed as a cosmological collider [67]. It is thus important to know what effect the derivative coupling has on the inflationary evolution. This derivative coupling also appeared in the recently proposed EFT of anisotropic inflation [68, 69,

Motivated by the D-brane scenario and the more generic nature of a derivative coupling between the inflaton and a vector field (and even more generally, with matter), we study anisotropic inflation with derivative couplings. We start by considering a phenomenological model where the gauge kinetic function has a monomial dependence on $X$ and an exponential dependence on $\phi$. For the power-law cases we consider, no stable solutions exist for $f=f(X)$ only. On the other hand, for $f=f(\phi, X)$, stable anisotropic solutions exist and the anisotropy is considerably reduced in comparison to the non-derivative case. This is interesting in view of the latest constraints on anisotropy [53, 70] (where the anisotropy is constrained through $g_{*}$ as in (2.1.5)). We next use our general equations to explore more general solutions. Finally we conclude in section 2.5 with a discussion of our results and prospects for future work.

\footnotetext{
${ }^{2}$ For an example of a theory where two scalar fields couple disformally via the DBI action, see [66].
} 


\subsection{Scalar-vector-tensor action with general derivative cou- plings}

Although later we will only look at power-law inflationary solutions, in this section we will keep the discussion as general as possible when presenting the set-up and equations of motion. This is useful in describing a variety of power-law models as we discuss later. In most of the chapter we will concentrate on a simple field theory model that will serve to illustrate the consequences of taking derivative couplings into account.

Our starting point is the general scalar-vector-tensor action of the form:

$$
S=\int d^{4} x \sqrt{-g}\left\{\frac{M_{\mathrm{pl}}^{2}}{2} R-P(\phi, X)-\frac{f^{2}(\phi, X)}{4} F_{\mu \nu} F^{\mu \nu}\right\},
$$

where $F_{\mu \nu}=\partial_{\mu} A_{\nu}-\partial_{\nu} A_{\mu}, 2 X=(\partial \phi)^{2}$, thus we see that the gauge kinetic function depends both on the inflaton, $\phi$ and its derivative, $X$. This action is motivated from Dbrane actions in string theory models of inflation, where $P(\phi, X)$ and $f(\phi, X)$ take very specific forms and arise from the Dirac-Born-Infeld (DBI) action (see 64] for details). Here we keep these functions general, in order to cover other possibilities 3 .

The equations of motion derived from (2.2.1) are given by

$$
\begin{aligned}
& R_{\mu \nu}-\frac{1}{2} g_{\mu \nu} R=\frac{1}{M_{\mathrm{pl}}^{2}}\left(T_{\mu \nu}^{A}+T_{\mu \nu}^{\phi}\right), \\
& \frac{1}{\sqrt{-g}} \partial_{\mu}\left[\sqrt{-g}\left(\frac{F^{2}}{2} f f_{X}+P_{X}\right) \partial^{\mu} \phi\right]=\frac{F^{2}}{2} f f_{\phi}+P_{\phi}, \\
& \partial_{\mu}\left[\sqrt{-g} f^{2}(\phi, X) F^{\mu \nu}\right]=0,
\end{aligned}
$$

where we have denoted the derivatives as $f_{i}=\partial_{i} f$ and similarly for $P$, for $i=\phi, X$. The energy-momentum tensors for the vector and the scalar fields are given by

$$
\begin{aligned}
T_{\mu \nu}^{\phi} & =\partial_{\mu} \phi \partial_{\nu} \phi\left(\frac{1}{2} f f_{X} F^{2}+P_{X}\right)-g_{\mu \nu} P, \\
T_{\mu \nu}^{A} & =f^{2}\left[F_{\nu}^{\alpha} F_{\mu \alpha}-g_{\mu \nu} \frac{F^{2}}{4}\right] .
\end{aligned}
$$

\footnotetext{
${ }^{3}$ Anisotropic inflationary solutions with a DBI kinetic term for the scalar field and a pure inflatondependent gauge kinetic function $f$ were considered in [47]. More general forms for $P(\phi, X)$ were further considered in 71 .
} 


\section{ANISOTROPIC INFLATION}

We are interested in anisotropic solutions and therefore, without loss of generality, we consider the following anisotropic metric:

$$
d s^{2}=-d t^{2}+e^{2 \alpha(t)}\left[e^{-4 \sigma(t)} d x^{2}+e^{2 \sigma(t)}\left(d y^{2}+d z^{2}\right)\right]
$$

where $e^{\alpha(t)}$ is identified with the isotropic scale factor, and $e^{\sigma(t)}$ characterises the anisotropy. Furthermore, we use gauge invariance to choose $A_{0}=0$ and, for concreteness, we consider homogeneous fields of the form [45, 59]:

$$
\phi=\phi(t), \quad A_{\mu}=(0, v(t), 0,0) !^{4}
$$

With these Ansätze, the equation of motion for the vector field takes the simple form:

$$
\frac{d}{d t}\left[f^{2} e^{\alpha+4 \sigma} \dot{v}\right]=0
$$

which can be readily solved to give:

$$
f^{2} e^{\alpha+4 \sigma} \dot{v}=p_{A}
$$

where $p_{A}$ is a constant of integration. Since $-\dot{v}=F_{x 0}=E_{x}, p_{A}$ is the electric field modulated by the expansion of the universe.

The Einstein equations, on the other hand, can be arranged into the following set of equations

$$
\begin{aligned}
& \ddot{\alpha}=-3 \dot{\alpha}^{2}+\frac{1}{6 M_{\mathrm{pl}}^{2}}\left[6 P+f^{2} \dot{v}^{2} e^{-2 \alpha+4 \sigma}+3 \dot{\phi}^{2}\left(P_{X}-\frac{f_{X}}{f} f^{2} \dot{v}^{2} e^{-2 \alpha+4 \sigma}\right)\right], \\
& \ddot{\sigma}=-3 \dot{\alpha} \dot{\sigma}+\frac{f^{2} \dot{v}^{2}}{3 M_{\mathrm{pl}}^{2}} e^{-2 \alpha+4 \sigma}, \\
& \dot{\alpha}^{2}=\dot{\sigma}^{2}+\frac{1}{3 M_{\mathrm{pl}}^{2}}\left[P+\frac{f^{2} \dot{v}^{2}}{2} e^{-2 \alpha+4 \sigma}+\dot{\phi}^{2}\left(P_{X}-\frac{f_{X}}{f} f^{2} \dot{v}^{2} e^{-2 \alpha+4 \sigma}\right)\right],
\end{aligned}
$$

\footnotetext{
${ }^{4}$ Notice that the vector is directed along the same direction as the anisotropy in the metric.
} 
where we have used 2.2 .10 . Finally, the equation of motion for the scalar field becomes:

$$
\begin{aligned}
\ddot{\phi}\left[-\dot{\phi}^{2} P_{X X}+P_{X}-f^{2} \dot{v}^{2} e^{-2 \alpha+4 \sigma}\left(\frac{f_{X}}{f}+3 \frac{f_{X}^{2}}{f^{2}} \dot{\phi}^{2}-\frac{f_{X X}}{f} \dot{\phi}^{2}\right)\right] \\
+\dot{\phi}\left[\dot{\phi} P_{X \phi}+3 \dot{\alpha} P_{X}+f^{2} \dot{v}^{2} e^{-2 \alpha+4 \sigma} \frac{f_{X}}{f}\left(\dot{\phi}\left(3 \frac{f_{\phi}}{f}-\frac{f_{X \phi}}{f_{X}}\right)+4 \dot{\sigma}+\dot{\alpha}\right)\right] \\
+P_{\phi}-\frac{f_{\phi}}{f} f^{2} \dot{v}^{2} e^{-2 \alpha+4 \sigma}=0 .
\end{aligned}
$$

From these equations, it is easy to recover the various examples studied in the literature, for which $f_{X}=0$ [45, 47, [59, 71, 72].

In what follows we use these equations to look for stable anisotropic solutions. We start by looking at a phenomenological example that serves as a prototype to understand the effect of the derivative coupling between the inflaton and the vector field, and then we explore more general cases.

\subsection{Anisotropic power-law inflation with derivative cou- plings}

In this section we start the analysis of power-law anisotropic inflation with derivative couplings, providing the first explicit example of the situation described in the EFT description of 68]. We start with a canonically normalised inflaton:

$$
P(\phi, X)=\frac{1}{2}(\partial \phi)^{2}+V(\phi)=X+V
$$

and thus replace, $P_{\phi}=V_{\phi}, P_{X}=1$ in the equations of motion above, 2.2.11-2.2.14. Note that eqs. 2.2.11) and 2.2.13 depend only on the derivative of $f$ w.r.t. $X$. One then immediately sees that a suitable choice of functional form is given by setting:

$$
X \frac{f_{X}}{f}=-n
$$

where $n=$ const. This has the solution:

$$
f(X, \phi)=(-X)^{-n} g(\phi)
$$




\section{ANISOTROPIC INFLATION}

for some function $g(\phi)$. On the other hand, we can also see that a suitable choice of $\phi$ dependence is given when $f_{\phi} / f=$ const. that is, an exponential dependence. So we find that a suitable Ansatz for the gauge kinetic function's dependence on the scalar and its derivative is given by

$$
f(\phi, X)=M_{\mathrm{pl}}^{4 n} f_{0} \frac{e^{\frac{\rho}{M_{\mathrm{pl}}} \phi}}{(-X)^{n}} .
$$

In addition to this, we also consider an exponential potential for the scalar field

$$
V(\phi)=V_{0} e^{\frac{\lambda}{M_{\mathrm{pl}}} \phi}
$$

We are now ready to look for power-law solutions of the form:

$$
\alpha=\zeta \log \left(M_{\mathrm{pl}} t\right), \quad \sigma=\beta \log \left(M_{\mathrm{pl}} t\right), \quad \frac{\phi}{M_{\mathrm{pl}}}=\xi \log \left(M_{\mathrm{pl}} t\right)+\phi_{0}
$$

where $\zeta, \beta, \xi$ and $\phi_{0}$ are constants. Using this Ansatz with the Hamiltonian constraint 2.2 .13 , we obtain the conditions:

$$
\lambda \xi=-2, \quad \rho \xi+2 \zeta+2 \beta+2 n=1 .
$$

We arrive at these two conditions 2.3.7) by requiring that, after substitution of 2.3.6 into (2.2.11)-2.2.14), powers in $t$ balance in all equations (i.e., we end up with equations of the form $C t^{x}=K t^{x}$ where $C, K$ are independent of $t$ ). The remaining conditions (below) come from ensuring that $C=K$ in 2.2.11 2.2.14, i.e. that the equations are satisfied (the amplitudes balance) after substitution of 2.3.6). For the amplitudes to balance in 2.2.13 (the Hamiltonian constraint), it is required that:

$$
-\zeta^{2}+\beta^{2}+\frac{1}{6} \xi^{2}+\frac{1}{3} u+(1-4 n) 4^{-n} \xi^{4 n} \frac{w}{6}=0,
$$

where we have defined $u, w$ as:

$$
u=\frac{V_{0}}{M_{\mathrm{pl}}^{4}} e^{\lambda \phi_{0}}, \quad w=\frac{p_{A}^{2}}{M_{\mathrm{pl}}^{4}} f_{0}^{-2} e^{-2 \rho \phi_{0}} .
$$

\footnotetext{
${ }^{5}$ In a string theory scenario, the effective $4 \mathrm{D}$ action can be written in terms of the 4D $M_{\mathrm{pl}}$, which would be a function of the string scale and coupling, as well as the compactification volume.
} 
From the equation for the scale factor (2.2.11) we then obtain:

$$
3 \zeta^{2}-\zeta-u+4^{-n}(6 n-1) \xi^{4 n} \frac{w}{6}=0,
$$

Similarly from the anisotropy equation $(2.2 .12)$ we get:

$$
-\beta(3 \zeta-1)-4^{-n} \xi^{4 n} \frac{w}{3}=0 .
$$

Finally from the equation for the inflaton we obtain:

$$
-\xi+3 \zeta \xi+\lambda u+4^{-n} \xi^{4 n-1} w[2 n(\zeta+4 \beta+4 n-1)+\rho \xi(4 n-1)]=0 .
$$

Using these equations, we can solve for $u$ and $w$ to get:

$$
u=-\zeta+3 \zeta^{2}+\frac{1}{2} \beta-3 n \beta-\frac{3}{2} \zeta \beta+9 n \zeta \beta, \quad w=3 \beta 4^{n} \xi^{-4 n}(3 \zeta-1) .
$$

Substituting these into the inflaton equation 2.3.12), and using the constraints for $\xi$ and $\beta$ from 2.3.7 gives:

$$
\begin{aligned}
(-1+3 \zeta)\left[8+\lambda^{2}(1-6 \zeta+2 n(-1+9 \zeta(-1+2 n+2 \zeta)))\right. \\
\left.-4 \lambda \rho(-2+3 \zeta+3 n(1+3 \zeta))+12 \rho^{2}\right]=0 .
\end{aligned}
$$

In contrast with [45], we now obtain a cubic, rather than a quadratic equation for $\zeta$. As in [45], we have the solution $\zeta=\frac{1}{3}$ which gives $u=w=0$, implying that there is no anisotropy and no potential driving inflation. We hence discard this solution and focus on the other two:

$$
\zeta_{+}=\frac{A+\sqrt{B}}{72 n \lambda^{2}}, \quad \zeta_{-}=\frac{A-\sqrt{B}}{72 n \lambda^{2}},
$$

where

$$
A=6 \lambda^{2}+18 n \lambda^{2}-36 n^{2} \lambda^{2}+12 \lambda \rho+36 n \lambda \rho,
$$

and

$$
B=A^{2}-144 n \lambda^{2}\left(8+\lambda^{2}-2 n \lambda^{2}+8 \lambda \rho-12 n \lambda \rho+12 \rho^{2}\right) .
$$

These solutions trivially satisfy 2.3.8, and it is important to remember that they are constrained from the requirement that $w, u$ must be positive by definition, 2.3.9. 


\section{ANISOTROPIC INFLATION}

To look for inflationary solutions we define the average slow-roll parameter $\epsilon$ in terms of the Hubble parameter defined by $H=\dot{\alpha}$, as:

$$
\epsilon:=-\frac{\dot{H}}{H^{2}}=\frac{1}{\zeta}
$$

Hence there are two branches of solutions for $\epsilon$ corresponding to $\zeta_{ \pm}$. In order to have inflation, we need $\epsilon \ll 1$, that is, we are looking for regions in the parameter space where $\zeta_{ \pm} \gg 1$

The anisotropy is characterised by:

$$
\frac{\Sigma}{H}:=\frac{\dot{\sigma}}{\dot{\alpha}}=\frac{\beta}{\zeta},
$$

where $\beta$ is given by:

$$
\beta=\frac{1}{2}+\frac{\rho}{\lambda}-\zeta-n
$$

As for $\epsilon$, there are two possible branches of solutions, associated to $\zeta_{ \pm}$.

Let us now discuss two cases of interest. Firstly, $\rho=0$, which corresponds to a gauge kinetic function that depends only on the derivative of the scalar field. And secondly, $\rho \neq 0$, when it depends on both.

Shift symmetric coupling, $\rho=0$. A purely shift symmetric coupling of the inflaton with the vector field arises for $\rho=0$ (see (2.3.4) $)$. This type of coupling of the inflaton to matter was considered recently in [67]. In order for inflationary solutions to arise, we need $\zeta \gg 1$. Moreover, the solutions should satisfy $u, w>0$ (see 2.3.13)). In terms of $\zeta$ (with $\rho=0), w$ and $u$ are given by:

$$
\begin{gathered}
w=-3 \times 2^{-2 n-1}(3 \zeta-1)\left(-\frac{1}{\lambda}\right)^{-4 n}(2 \zeta+2 n-1), \\
u=-\frac{1}{4}(2 n-1)(3 \zeta-1)(6 \zeta+6 n-1) .
\end{gathered}
$$

Therefore, in order for $w, u$ to be positive, $n$ must be negative and $|n| \gg 1$, so that $n<1 / 2-\zeta$. From the expressions for $\zeta_{ \pm}$2.3.15 we see that for $|n| \gg 1, \zeta_{ \pm} \sim$

\footnotetext{
${ }^{6}$ This is the average (of the three spatial directions) slow-roll parameter in the sense that it is defined in terms of $H$ which gives us the average rate of expansion.
} 
$\frac{n}{2}(-1 \pm 1) \cdot 7$ Thus in principle there are anisotropic inflationary solutions that satisfy all necessary conditions for sufficiently large $|n|$. For example, $n=-10^{5}, \lambda=1$, gives $\epsilon \sim 10^{-4}, \Sigma / H \sim 10^{-9}$. Note that requiring sufficiently small anisotropy requires very large values of $|n|$. That being said, we find no stable solutions in this case (when $\rho=0) 8$

More general coupling, $\rho \neq 0$. Let us now consider the case when the gauge kinetic function depends on both the inflaton and its derivative, that is $\rho \neq 0$. As before, inflationary solutions require $\zeta \gg 1$. Furthermore, the conditions for positive $w$ (and $u$ ) can be obtained by looking at 2.3.13, which takes the form:

$$
w=-3 \times 2^{-2 n}(3 \zeta-1)\left(-\frac{1}{\lambda}\right)^{-4 n}\left(\zeta+n-\frac{\rho}{\lambda}-\frac{1}{2}\right),
$$

Therefore for sufficiently large $\zeta, w$ can be positive for positive or negative $n$ and large values of $\rho / \lambda$, which is also required to obtain large values of $\zeta$ (see eq. 2.3.15)). In the limit $\rho / \lambda \gg 1$, the solutions $\zeta_{ \pm}$become:

$$
\zeta_{ \pm} \simeq \frac{\rho\left(1+3 n \pm \sqrt{(3 n-1)^{2}-\frac{8 n}{\rho^{2}}}\right)}{6 n \lambda} .
$$

Examining this, we see that for $n>0$, the numerator is always positive since $(1+3 n)>$ $\sqrt{(3 n-1)^{2}-(8 n) / \rho^{2}}$ meaning $\zeta_{ \pm}$are both positive. Similarly for $n<0$, we see that $|1+3 n|<\sqrt{(3 n-1)^{2}-(8 n) / \rho^{2}}$ which tells us that $\zeta_{+}$is negative while $\zeta_{-}$is positive. That is, in this limit, there are positive solutions for both $\zeta_{ \pm}$for positive $n$, while for negative $n$ only one solution is positive. We are also interested in small anisotropy, $\Sigma / H \ll 12.3 .19$, where in terms of the parameters we have:

$$
\frac{\Sigma}{H}=\frac{1}{\zeta}\left(\frac{\rho}{\lambda}+\frac{1}{2}-n-\zeta\right)
$$

which, using both that $\epsilon=\frac{1}{\zeta}$ and our solutions for $\zeta$ 2.3.15, can be written in the form of 2.1.2

$$
\frac{\Sigma_{ \pm}}{H}=\frac{1}{3} I_{ \pm} \epsilon
$$

\footnotetext{
${ }^{7}$ Notice that to see that $u$ is positive in this limit (as required) one needs to include the next to leading order term in the large $n$ expansion for $\zeta_{ \pm}$.

${ }^{8}$ This has been confirmed recently in [47.
} 


\section{ANISOTROPIC INFLATION}

with

$$
I_{ \pm}=\frac{1}{24 n \lambda^{2}}\left\{-6 \lambda\left(\lambda-3 n \lambda+6 n^{2} \lambda+2 \rho-6 n \rho\right) \pm \sqrt{B}\right\}
$$

where $B$ is defined in 2.3.17) and is function of $\rho, \lambda$ and $n$. In this way, the anisotropy can be written in terms of the free parameters of the system 9 . This further selects the appropriate solution for $\zeta^{10}$. With these conditions, one can check that there is a range of values for which anisotropic solutions exist with $n>0$. A stability analysis shows that for these cases, both the isotropic and anisotropic solutions are attractors. Therefore the evolution of the system depends on the initial conditions. Since we are interested in the case where the anisotropic solution is the only attractor, in what follows we focus our search for anisotropic stable solutions to the case $n<0$.

In Figure 2.1 we show the behaviour of the slow-roll parameter, $\epsilon$, as a function of the parameters $\lambda$ and $\rho$ for different values of $n$. As one can see, the slow-roll parameter $\epsilon$ decreases very slightly as the magnitude of $n$ increases. Conversely, as can be seen in Figure 2.2 the anisotropy, $\Sigma / H$, can be reduced by the introduction of a derivative coupling: the greater the magnitude of $n$, the smaller the magnitude of anisotropy.

We can understand the decrease in the anisotropy as follows. Since we are only interested in solutions where the anisotropic point is a single attractor (and since the only observable anisotropic effects come from the final value of the anisotropy), we do not have to worry about the initial value for the gauge field. If the anisotropy converges to a number, its final value is given by the ratio of energy density of the vector field to that of the scalar field [73]. From eq. 22.2.12, we can define this ratio $\mathcal{R}[11$ as:

$$
\frac{\Sigma}{H} \simeq \frac{2}{3} \mathcal{R}, \quad \mathcal{R}=\frac{\rho_{v}}{V(\phi)} \sim \frac{\frac{f^{2}}{2} \dot{v}^{2} e^{-2 \alpha+4 \sigma}}{V(\phi)} .
$$

Using (2.2.10, 2.3.4) and 2.3.5, this ratio can be written as

$$
\mathcal{R} \sim \dot{\phi}^{4 n} e^{-2 \rho \phi-4 \alpha-4 \sigma-\lambda \phi} \sim \xi^{4 n} t^{-2 \rho \xi-4 \zeta-4 \beta-\lambda \xi-4 n},
$$

where in the second expression we used (2.3.6). Furthermore, using the conditions

\footnotetext{
${ }^{9}$ As a reference, in the non-derivative coupling case $(n=0)$ of 45$], I=\frac{\lambda^{2}+2 \rho \lambda-4}{\lambda^{2}+2 \rho \lambda}$.

${ }^{10}$ We focus only on solutions with $|n| \geq 1$, which guarantees real values for $w$ in the $\lambda>0$ we are interested in.

${ }^{11}$ Not to be confused with the curvature perturbation.
} 


\subsection{Anisotropic power-law inflation with derivative couplings}
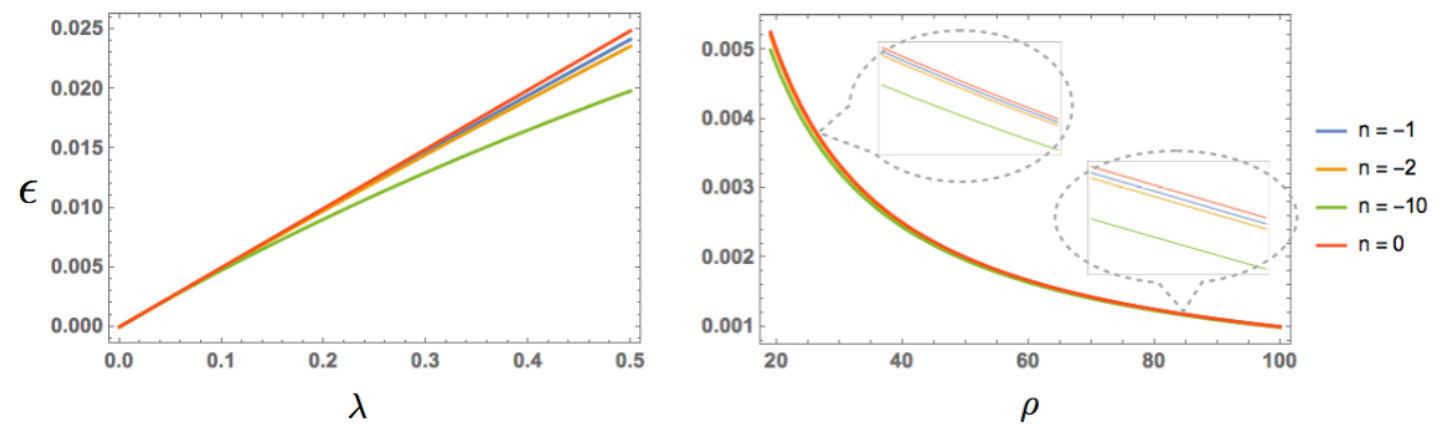

Figure 2.1: In these plots we show how the slow-roll parameter, $\epsilon$, varies with $\lambda$ (for $\rho=20$ ) and $\rho$ (for $\lambda=0.1$ ), for the values of $n$ shown. Recall that $\lambda, \rho$ and $n$ are merely parameters of the potential and gauge kinetic function which are defined, respectively as $V(\phi)=V_{0} e^{\frac{\lambda}{M_{\mathrm{pl}}} \phi}$ and $f(\phi, X)=M_{\mathrm{pl}}^{4 n} f_{0} e^{\frac{\rho}{M_{\mathrm{pl}}} \phi}(-X)^{-n}$.
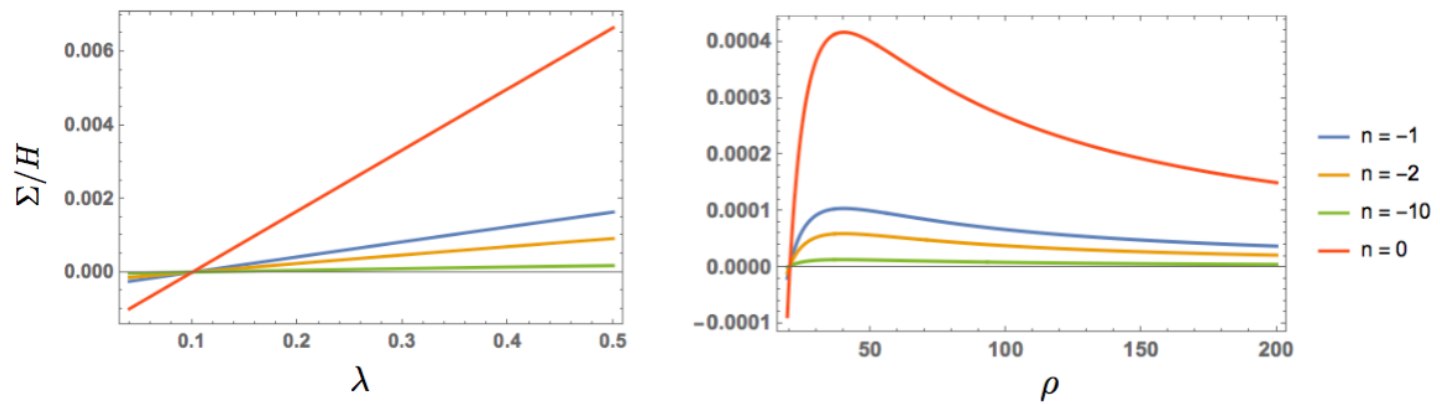

Figure 2.2: In these plots we show how the anisotropy, $\frac{\Sigma}{H}$, varies with $\lambda$ (for $\rho=20$ ) and $\rho$ (for $\lambda=0.1$ ), for negative values of $n$ as shown. Recall that $\lambda, \rho$ and $n$ are merely parameters of the potential and gauge kinetic function which are defined, respectively as $V(\phi)=V_{0} e^{\frac{\lambda}{M_{\mathrm{pl}}} \phi}$ and $f(\phi, X)=M_{\mathrm{pl}}^{4 n} f_{0} e^{\frac{\rho}{M_{\mathrm{pl}}} \phi}(-X)^{-n}$.

2.3.7, we find that the ratio becomes a constant given by

$$
\mathcal{R} \sim \xi^{4 n}=\frac{1}{\xi^{4|n|}}, \quad n<0,
$$

and thus we see why the anisotropy decreases with $|n|$ in the case with derivative couplings. Since $\xi^{4 n}$ is coming from the energy density of the vector field, we see that the anisotropy is reduced because the energy density of the vector field becomes small during inflation. 


\subsubsection{Stability of the anisotropic solutions}

We now study the stability of the solutions above using a dynamical system analysis. For this, we define the dimensionless variables:

$$
W=\frac{\dot{\sigma}}{\dot{\alpha}}, \quad Y=\frac{1}{M_{\mathrm{pl}}} \frac{\dot{\phi}}{\dot{\alpha}}, \quad Z=\frac{f(\phi, X) e^{-\alpha+2 \sigma}}{M_{\mathrm{pl}}} \frac{\dot{v}}{\dot{\alpha}},
$$

where we use the e-fold number as time coordinate, $d \alpha=\dot{\alpha} d t$. Using these variables, the Hamiltonian constraint 2.2.13 can be written as

$$
-\frac{V}{M_{\mathrm{pl}}^{2} \dot{\alpha}^{2}}=3\left(W^{2}-1\right)+\frac{Y^{2}}{2}+\frac{Z^{2}}{2}(1-4 n) .
$$

Since the inflationary potential is positive definite, we immediately see that 2.3 .32 implies

$$
W^{2}+\frac{Y^{2}}{6}+\frac{Z^{2}}{6}(1-4 n)<1 .
$$

In terms of the variables 2.3.31, the slow-roll parameter $\epsilon$, becomes:

$$
\epsilon=3 W^{2}+\frac{Y^{2}}{2}+\frac{Z^{2}}{3}(1-3 n) .
$$

Using the Hamiltonian constraint 2.3.32, the equations of motion in terms of 2.3.31 can be written as:

$$
\begin{gathered}
\frac{d W}{d \alpha}=\frac{1}{3} Z^{2}(W+1-3 n W)+W\left(3\left(W^{2}-1\right)+\frac{1}{2} Y^{2}\right) \\
\frac{d Y}{d \alpha}=\frac{1}{6} Y\left\{18 W^{2}+3 Y^{2}+2 Z^{2}-6 n Z^{2}+3 C(Y, Z)\left[-4 n Z^{2}(1+4 W)\right.\right. \\
\left.\left.+Y\left(-6 Y+6 \lambda\left(W^{2}-1\right)+\lambda Y^{2}-Z^{2}(-1+4 n)(\lambda+2 \rho)\right)\right]\right\} \\
\frac{d Z}{d \alpha}=\frac{1}{6} Z\left\{-12-12 W+18 W^{2}+3 Y^{2}+2 Z^{2}-6 n Z^{2}-6 \rho Y\right. \\
\quad-6 n C(Y, Z)\left[4 n Z^{2}(1+4 W)\right. \\
\left.\left.\quad-Y\left(-6 Y+6 \lambda\left(W^{2}-1\right)+\lambda Y^{2}-Z^{2}(4 n-1)(2 \rho+\lambda)\right)\right]\right\}
\end{gathered}
$$


where

$$
C(Y, Z)=\frac{1}{Y^{2}+2 n Z^{2}(1-4 n)} .
$$

We can now find the fixed points of the system by setting $d W / d \alpha=d Y / d \alpha=d Z / d \alpha=$ 0. From (2.3.35), we find:

$$
Z^{2}=\frac{3 W\left(-6+6 W^{2}+Y^{2}\right)}{2(-1-W+3 n W)}
$$

These equations are solved numerically for suitable values of the parameters $\lambda, \rho$, and $n$ (chosen so that $w$ is positive) such that $W, Y$ and $Z$ are all non-zero and real. To choose appropriate solutions, we perform a linear stability analysis. The isotropic fixed point solution is located at $W=Z=0, Y=-\lambda$, corresponding the coupling $f(\phi, X)$ being switched off. The linearised equations of motion around this point reduce to:

$$
\begin{aligned}
\frac{d \delta W}{d \alpha} & =\left(\frac{1}{2} \lambda^{2}-3\right) \delta W \\
\frac{d \delta Y}{d \alpha} & =\left(\frac{1}{2} \lambda^{2}-3\right) \delta Y \\
\frac{d \delta Z}{d \alpha} & =\left[\left(\frac{1}{2}-n\right) \lambda^{2}+\rho \lambda-2\right] \delta Z .
\end{aligned}
$$

When $\lambda$ is small, the LHS of these equations are all negative (corresponding to the isotropic fixed point being an attractor solution) if $\lambda^{2}(1-2 n)+2 \rho \lambda<4$. If however $\lambda^{2}(1-2 n)+2 \rho \lambda>4$, the isotropic fixed point is unstable. Since we are searching for anisotropic solutions, this is the parameter space we want to consider: we require that $\rho>\frac{2}{\lambda}-\frac{\lambda}{2}(1-2 n)$.

Now, we look at two explicit examples to demonstrate that stable derivative anisotropic solutions can be found with small but non-zero anisotropy.. Consider first the case with $n=-1, \lambda=0.1$, and $\rho=20$, which has a fixed point at $(W, Y, Z)=(3.08249 \times$ $\left.10^{-6},-9.92559 \times 10^{-2}, \pm 5.26275 \times 10^{-3}\right)$. Linearisation around this point gives:

$$
\begin{aligned}
& \frac{d \delta W}{d \alpha}=-2.99504 \delta W-3.05955 \times 10^{-7} \delta Y \pm 3.50854 \times 10^{-3} \delta Z \\
& \frac{d \delta Y}{d \alpha}=-2.29684 \times 10^{-3} \delta W-3.08768 \delta Y \pm 0.87150 \delta Z \\
& \frac{d \delta Z}{d \alpha}=\mp 1.07688 \times 10^{-2} \delta W \mp 0.434252 \delta Y+9.26389 \times 10^{-2} \delta Z
\end{aligned}
$$




\section{ANISOTROPIC INFLATION}

where the change in signs is due to choosing either the positive or negative $Z$ solution. This system has eigenvalues $\left(-2.99504,-2.96384,-3.11922 \times 10^{-2}\right)$ whose real parts are all negative. This system has an average slow-roll parameter of $\epsilon=4.96279 \times 10^{-3}$ (from both 2.3.34 and $\epsilon_{-}$in 2.3.18) and anisotropy $\Sigma / H=3.08249 \times 10^{-6}$, which is however too large compared to current data [53, 70]. As a reference, from 2.3.9), we can also evaluate the constant of integration for the vector field defined in 2.2.10 in terms of $M_{\mathrm{pl}}, f_{0}$ and $\phi_{0} \sqrt{12}$. We can compare this solution with the non-derivative stable one $\lambda=0.1, \rho=50, n=0$. In that case, $\Sigma / H=4 \times 10^{-4}$ and thus we clearly see that the derivative coupling decreases the level of anisotropy.

In fact the condition that the anisotropy be small enough is very restrictive [53] - the anisotropy is not only required to be small but very small $\left(\sim \mathcal{O}\left(10^{-7}\right)\right.$ times smaller) relative to the the slow-roll parameter since $g_{*}=259200 \epsilon^{-1} \frac{\Sigma}{H}$ 2.1.4) (using that horizon-crossing takes place 60 e-folds before the end of inflation). However if we take some extreme values for the parameters it is possible to find a stable solution that admits an anisotropy small enough to avoid the bounds in [53, 70] 13 . As a second example we take $n=-100, \lambda=10^{-6}$, and $\rho=2 \times 10^{6}$. This has a fixed point at: $(W, Y, Z)=\left(2.78239 \times 10^{-26},-1.00000 \times 10^{-6}, \pm 5.00415 \times 10^{-13}\right)$. Linearisation of the $W, Y, Z$ equations around this point gives the equations:

$$
\begin{aligned}
& \frac{d \delta W}{d \alpha}=-3.00000 \delta W-2.78239 \times 10^{-32} \delta Y \pm 3.33610 \times 10^{-13} \delta Z \\
& \frac{d \delta Y}{d \alpha}=-2.00332 \times 10^{-16} \delta W-3.00000 \delta Y \pm 6.02500 \times 10^{-4} \delta Z \\
& \frac{d \delta Z}{d \alpha}=\mp 1.00083 \times 10^{-4} \delta W \mp 3.01250 \times 10^{-4} \delta Y+6.03001 \times 10^{-8} \delta Z
\end{aligned}
$$

where the change in signs is due to choosing either the positive or negative $Z$ solution, respectively. The eigenvalues for this set of equations are $(-3.00000,-3.00000,-2.00893 \times$ $\left.10^{-10}\right)$. The eigenvalues' real parts are all negative and hence this fixed point is stable. Therefore, this corresponds to a stable solution that produces anisotropy during inflation. Using (2.3.34), we find the slow-roll parameter to be $\epsilon=5.00000 \times 10^{-13}$ matching perfectly with the $\zeta_{-}$solution in 2.3 .15 , which gives the slow-roll parameter

\footnotetext{
${ }^{12}$ For example, for $\left(\phi_{0}, f_{0}\right)=(-1,1)$, we find $p_{A}= \pm 4.4 \times 10^{-7} M_{\mathrm{pl}}^{2}$. Inverting 2.3.28), we can also find the value of $\dot{v}$ in terms of all the parameters of the model, $\dot{v} \sim\left(\mathcal{R} V 2 f^{-2} e^{2 \alpha-4 \sigma}\right)^{1 / 2}$.

${ }^{13}$ The example shown here differs from that in the paper [51]. The example in the paper does not satisfy the constraints on anisotropy but this example, which has a different parameter set, does.
} 
(for $\lambda, \rho, n$ given above) as $\epsilon_{-}=5.00000 \times 10^{-13}$. The average anisotropy is given by $\Sigma / H=2.78239 \times 10^{-26}$ and in addition, $w$ is positive and real. This value of the anisotropy is consistent with observations because it predicts a value of $g_{*} \sim 10^{-8}$ (well below the bound obtained in [53] of $g_{*}=0.002 \pm 0.016$ ).

\subsection{More general solutions}

In the previous section we explored a suitable generalisation of the non-derivative anisotropic power-law inflation studied in [45] (the gauge kinetic function $f=f(\phi)$ did not depend on the inflaton velocity) where the gauge kinetic function has a monomial dependence on the inflaton's velocity. Our general equations, however, allow for an easy exploration of other interesting possibilities. One such possibility is the case of DBI inflation [46], where the inflaton can be identified with a D-brane position or a Wilson line. In any case, the vector field featuring on the inflationary D-brane may give rise to anisotropic solutions. In this model, the scalar action and gauge kinetic function are given by [64],

$$
P(\phi, X)=\frac{2 X \gamma}{\gamma+1}+V(\phi), \quad f(\phi, X)=\gamma^{1 / 2}, \quad \gamma=\frac{1}{\sqrt{1+2 h X}}
$$

where $h(\phi)$ is a function of the scalar field only (the warp factor associated with the 10dimensional geometry where the brane is moving). We see that in the non-relativistic case, when $\gamma \rightarrow 1$, the scalar field is canonically normalised and the vector field decouples from the inflaton. It is not difficult to check that power-law solutions with $h^{\prime} / h=$ const. $V^{\prime} / V=\lambda=$ const. cannot be found since the constraints $\epsilon \ll 1, \zeta \gg 1$, and $w>0$ 2.3.13 cannot be simultaneously satisfied. The same happens when considering a canonically normalised inflaton 2.3.1 coupled disformally to the vector via 2.4.1. This is consistent with the results of [74] where a detailed analysis is shown.

However, motivated by the DBI anisotropic solutions found in [47, we can modify slightly this Ansatz. Consider a DBI inflaton, with $P$ given as in 2.4.1), and a monomial, derivative coupling, $f$, as in (2.3.4). This could correspond to a model where the inflaton and the vector live on different D-branes. In [47] the authors found power-law anisotropic solutions with $h^{\prime} / h=$ const. which implies that $\gamma=\gamma_{0}=$ const. Let us see this in some detail. 


\subsubsection{DBI inflation with monomial, derivative coupling solutions}

Considering $h^{\prime} / h=$ const. implies an exponential form for $h$, which we take as:

$$
h(\phi)=\frac{h_{0}}{M_{\mathrm{pl}}^{4}} e^{\frac{\Lambda}{M_{\mathrm{pl}}} \phi} .
$$

Taking also an exponential form for the inflaton potential as before, 2.3.5, and powerlaw solutions for the scale factors and inflaton as in the canonically-normalised case (2.3.6), we obtain the conditions:

$$
\lambda \xi=-2, \quad \rho \xi+2 \zeta+2 \beta+2 n=1, \quad \Lambda=-\lambda .
$$

The requirement that $\Lambda=-\lambda$ is akin to setting $\gamma=\gamma_{0}=$ const. In terms of $h_{0}, \lambda$, and $\phi_{0} ; \gamma_{0}$ becomes:

$$
\gamma_{0}=\left(1-4 \frac{h_{0}}{\lambda^{2}} e^{-\lambda \phi_{0}}\right)^{-\frac{1}{2}}
$$

By applying the exact same procedure of balancing the amplitudes as we used for the canonically-normalised case 2.3.8 -2.3 .15 , we obtain two analogous solutions that satisfy all of the system's equations:

$$
\zeta_{+}=\frac{D+\sqrt{E}}{72 n \lambda}, \quad \zeta_{-}=\frac{D-\sqrt{E}}{72 n \lambda}
$$

where

$$
D=6 \lambda+18 n \lambda-36 n^{2} \lambda+12 \rho+36 n \rho,
$$

and

$$
E=D^{2}-144 n \lambda\left(\frac{8}{\gamma_{0} \lambda}+\lambda-2 n \lambda+8 \rho-12 n \rho+12 \frac{\rho^{2}}{\lambda}\right)
$$

Anisotropic inflationary solutions can now be found for suitable choices of the parameters, as long as they satisfy the constraints that $u, w$ (defined as before (2.3.9) must be real and positive, and, of course, $\epsilon=1 / \zeta \ll 1$. As a concrete example, a stable solution can be found by for $\lambda=0.01, \rho=300, n=-2, \gamma_{0}=1.5$. It has anisotropy $\Sigma / H=1.32254 \times 10^{-10}$ and slow-roll parameter $\epsilon=3.33306 \times 10^{-5}$.

We now consider the stability of this anisotropic DBI solution. We define dimen- 
sionless variables analogous to the canonically-normalised case:

$$
W=\frac{\dot{\sigma}}{\dot{\alpha}}, \quad Y=\frac{\gamma_{0}}{M_{\mathrm{pl}}} \frac{\dot{\phi}}{\dot{\alpha}}, \quad Z=\frac{f(\phi, X) e^{-\alpha+2 \sigma}}{M_{\mathrm{pl}}} \frac{\dot{v}}{\dot{\alpha}}
$$

The Hamiltonian constraint 2.2 .13$)$ for this system becomes:

$$
-\frac{V}{M_{\mathrm{pl}}^{2} \dot{\alpha}^{2}}=3\left(W^{2}-1\right)+\frac{Y^{2}}{1+\gamma_{0}}+\frac{Z^{2}}{2}(1-4 n),
$$

and the slow-roll parameter:

$$
\epsilon=3 W^{2}+\frac{Y^{2}}{2 \gamma_{0}}+\frac{Z^{2}}{3}(1-3 n) .
$$

The equations of motion in terms of (2.4.8) become:

$$
\begin{gathered}
\frac{d W}{d \alpha}=\frac{1}{3} Z^{2}(W+1-3 n W)+W\left(3\left(W^{2}-1\right)+\frac{Y^{2}}{2 \gamma_{0}}\right), \\
\frac{d Y}{d \alpha}=F(Y, Z) Y\left\{3 \gamma_{0} Y^{4}+4 \gamma_{0} n Z^{2}\left[-3 W(3 W(4 n-1)+4)+Z^{2}(n(12 n-7)+1)-3\right]+\right. \\
\quad 2 Y^{2}\left[Z^{2}\left(\gamma_{0}^{2}-3 n\left(\gamma_{0}^{2}+4 n-1\right)\right)+9 \gamma_{0}^{2} W^{2}-9\right]+3 \lambda Y\left(-4 n Z^{2}+6 W^{2}+Z^{2}-6\right) \\
\left.\quad+6 \rho Y Z^{2}(1-4 n)+3 \gamma_{0} \lambda Y^{3}\right\} \\
\frac{d Z}{d \alpha}= \\
\quad F(Y, Z) Z\left\{48 \gamma_{0} n^{3} Z^{4}+2 n\left[2 Z^{2} \gamma_{0}\left(-6-6 W+9 W^{2}+Z^{2}\right)+3 Y^{2}\left(-6+Z^{2}-\gamma_{0}^{2} Z^{2}\right)\right.\right. \\
\left.\quad+3 \lambda Y\left(-6+6 W^{2}+Z^{2}\right)+3 \lambda \gamma_{0} Y^{3}\right]-4 n^{2} Z^{2}\left[\gamma_{0}\left(-18+36 W^{2}+7 Z^{2}\right)+6 Y(Y+\lambda)\right] \\
\left.\quad+\gamma_{0} Y^{2}\left[2 \gamma_{0}\left(-6-6 W+9 W^{2}+Z^{2}\right)+3 Y(Y-2 \rho)\right]\right\},
\end{gathered}
$$

where

$$
F(Y, Z)=\frac{1}{6 \gamma_{0}\left(2 n Z^{2}(1-4 n)+\gamma_{0} Y^{2}\right)} .
$$




\section{ANISOTROPIC INFLATION}

This system reduces to the canonically-normalised case 2.3.35-2.3.37) when $\gamma_{0} \rightarrow 1$. This system permits stable, anisotropic solutions. As an example, a stable solution can be found by taking $\lambda=0.01, \rho=300, n=-2, \gamma_{0}=1.5$. With these parameters, we find a fixed point at $(W, Y, Z)=\left(1.32254 \times 10^{-10},-9.99917 \times 10^{-3}, \pm 3.45004 \times 10^{-5}\right)$. Linearisation of equations 2.4.11-2.4.13 around this fixed point yields:

$$
\begin{aligned}
& \frac{d \delta W}{d \alpha}=-2.99997 \delta W-8.81622 \times 10^{-13} \delta Y \pm 2.30003 \times 10^{-5} \delta Z \\
& \frac{d \delta Y}{d \alpha}=-1.2701 \times 10^{-6} \delta W-1.33371 \delta Y \pm 6.44229 \times 10^{-2} \delta Z \\
& \frac{d \delta Z}{d \alpha}=\mp 6.90183 \times 10^{-5} \delta W \mp 2.53082 \times 10^{-2} \delta Y+8.89147 \times 10^{-4} \delta Z .
\end{aligned}
$$

This fixed point has eigenvalues $\left(-2.99997,-1.33249,-3.33631 \times 10^{-4}\right)$ and is therefore stable. However, it has anisotropy $\Sigma / H=1.32254 \times 10^{-10}$ and slow-roll parameter $\epsilon=3.33306 \times 10^{-5}$, making it incompatible with data since this gives $g_{*}=1.03$ with horizon-crossing taken to be 60 e-folds before the end of inflation.

\subsection{Discussion}

We have studied anisotropic inflationary solutions where the inflaton couples to a vector field derivatively. That is, the gauge kinetic function depends both on the inflaton and its derivative, $f(\phi, X)$, with $2 X=(\partial \phi)^{2}$. This coupling is motivated by D-brane inflationary models, where the D-brane features a vector on its world-volume, and couples derivatively to the brane's position (or a Wilson line), the inflaton. Moreover, such couplings parametrise generic inflaton-matter couplings, which may be relevant in studies of the inflationary universe as a cosmological collider [67]. On the other hand, they also appear in the EFT of anisotropic inflation [68, 69].

We started by presenting a general set-up, which allows for the study of a wide range of models. We studied first an immediate generalisation of the power-law anisotropic model studied in [45, where the gauge kinetic function is a monomial in $X$, 2.3.4, while exponential in the inflaton. We found that there are no stable inflationary solutions for a purely shift symmetric coupling (that is $f_{\phi}=0$ ). However, stable derivative anisotropic solutions arise for a large range of parameters. Interestingly, compared to the non-derivative $(n=0)$ case of [45], the derivative anisotropic solutions have a lower level of anisotropy, although it still proves difficult to find solutions that can satisfy the 
constraints on the degree of anisotropy imposed by bounds on $g_{*}$ [53. We presented two illustrative examples, one of which satisfies this bound on the anisotropy, but requires very large values for $\rho, \lambda$ and $|n|$. We found that the value of the anisotropy depends mildly on the power $n$ in 2.3.4, which needs to be negative. We also found that the DBI generalisation of the power-law solutions in [45] can also be extended to the derivative case. That is, derivative anisotropic DBI solutions exists, where the gauge kinetic function is a monomial in $X$ (see (2.3.4)). This example could correspond to a DBI inflationary model where the inflaton and the vector field live on different D-branes. On the other hand, in the case where the inflaton and vector live on the same brane, the gauge kinetic function is dictated by the model and given by (2.4.1). In this case however, the requirements of inflation, small anisotropy and a positive vector energy density $(w>0)$ are not compatible and thus there are no solutions.

As we discussed in section 2.3, it is easy to understand the decrease in the anisotropy (that occurs with an increase in $|n|$ ) by looking at its final value, which is given by the ratio of the energy density of the vector field to that of the scalar field and is given by (2.3.30). The energy density of the vector field is reduced by the derivative coupling and thus there is a reduction in the anisotropy. An obvious follow-up to this work is to look at how the derivative coupling affects the induced anisotropy in the power spectra. 
2. ANISOTROPIC INFLATION 


\section{Chapter 3}

\section{Enhancement of the primordial gravitational wave spectrum}

In this chapter, we will discuss a popular mechanism for enhancing the gravitational wave spectrum of an inflationary model by the coupling of either the inflaton itself or a spectator axion field to a gauge field. This coupling can source tensor perturbations to the gauge field which can act, in turn, as a source for the metric tensor perturbations, effectively amplifying the tensor-to-scalar ratio.

\subsection{Motivations}

Next generation observations of the CMB such as by CMB-S4 [41, 75] will constrain the tensor-to-scalar ratio down to $r \sim \mathcal{O}\left(10^{-3}\right)$. A detection of $r$ at such a high value is of particular interest to low-energy models of inflation that predict an unobservably low value of $r$. As we will see in chapter 4, an example of this is Kähler moduli inflation whose tensor-to-scalar ratio is very low: the prototypical model predicts $r<10^{-10}$ [49] and examples with slightly higher energy scales predict at most $r \lesssim 10^{-6}[76]$. With this in mind, would a positive detection of $B$-mode polarisation in the CMB (at $r \gtrsim 10^{-3}$ ) rule out Kähler moduli inflation or is there a secondary mechanism that can act as a source for the gravitational wave spectrum and enhance it to a large value without the need for a high energy scale of inflation (see chapter 1)? This question is the main concern of this thesis. 


\section{ENHANCEMENT OF THE PRIMORDIAL GRAVITATIONAL WAVE SPECTRUM}

In this chapter we will review an enhancement mechanism which sources gravitational waves indirectly from a coupling between an axion field and a gauge field. This review will start with the mechanism described in [2] which, although not the first model to have successfully enhanced the gravity wave sector through the coupling of an axion to the gauge field, was the first model to do so with a non-constant velocity for the axion and with the evolution of the axion being subject to a potential [2]. We will then move on to refinements of the model that will prove useful in our quest to apply this mechanism to Kähler moduli inflation, particularly the model put forward in 3 .

\subsection{Basic model}

Our starting point is the model of [2, 48] which has an action given by

$$
\begin{aligned}
S=\int d^{4} x \sqrt{-g}\left\{\frac{M_{\mathrm{pl}}^{2}}{2} R-\frac{1}{2} \partial_{\mu} \phi \partial^{\mu} \phi-\frac{1}{2} \partial_{\mu} \sigma \partial^{\mu} \sigma-V(\phi)-U(\sigma)\right. \\
\left.-\frac{1}{4} F_{\mu \nu} F^{\mu \nu}-\alpha \frac{\sigma}{4 f} F_{\mu \nu} \tilde{F}^{\mu \nu}\right\}
\end{aligned}
$$

where $g$ is the spacetime metric determinant; $\{\mu, \nu, \ldots\}$ are spacetime indices; $R$ is the Ricci scalar; $\phi$ is explicitly the inflaton and is driven by the potential, $V(\phi) ; \sigma$ is a pseudo-scalar (axion) spectator field ${ }^{1}$ with its own separate potential, $U(\sigma) ; F_{\mu \nu}=$ $\partial_{\mu} A_{\nu}-\partial_{\nu} A_{\mu}$ is the field strength tensor of a vector field, $A_{\mu} ; \tilde{F}^{\mu \nu}=\frac{\epsilon^{\mu \nu \alpha \beta}}{2 \sqrt{-g}} F_{\alpha \beta}$ is the dual field strength of $A_{\mu}$ (with $\epsilon^{\mu \nu \rho \sigma}$ the Levi-Civita symbol with $\epsilon^{1234}=1$ ); and $\alpha$ and $f$ are constants (with $f$ the axion decay constant). Notice that due to the presence of the $1 / \sqrt{-g}$ in the definition of the dual field strength tensor, $\tilde{F}$, the term $\sqrt{-g} \alpha \frac{\sigma}{4 f} F \tilde{F}$ has no dependence on the metric and will therefore make no contribution to the energymomentum tensor. Also, it is important to understand that the term $F \tilde{F}$ cannot exist in the action on its own - it must be coupled to another field. This is because

$$
\begin{aligned}
S & \supset \int d^{4} x \sqrt{-g} F \tilde{F}=2 \int d^{4} x \epsilon^{\mu \nu \rho \sigma} \partial_{\mu} A_{\nu} \partial_{\rho} A_{\sigma} \\
& =-2 \int d^{4} x \epsilon^{\mu \nu \rho \sigma}\left(\partial_{\rho} \partial_{\mu} A_{\nu}\right) A_{\sigma}=0
\end{aligned}
$$

\footnotetext{
${ }^{1}$ 'Spectator' meaning it does not affect the background inflationary evolution.
} 
where we have integrated by parts and used the antisymmetry of $\epsilon$ and the symmetry of partial derivatives. Without going into a lot of detai $2^{2}$, the main goal of this section will be to argue why this coupling between the axion, $\sigma$ and the vector field introduces an extra source term for the primordial gravitational waves.

To start with it is important to understand that during inflation a vector field will be diluted (in particular its energy density will become negligible) because of the rapid expansion of the universe very quickly without some source that is continually producing it. We can see this by considering quickly the action for a vector field without a coupling like the one above

$$
S_{\text {vector }}=\int d^{4} x \sqrt{-g}\left\{-\frac{1}{4} F_{\mu \nu} F^{\mu \nu}\right\} .
$$

This of course gives equations of motion for the vector field

$$
\nabla^{\mu} F_{\mu \nu}=0
$$

which, under the Lorenz gauge $\left(A_{0}=0, \partial_{i} A_{i}=0\right)$ in a flat FRW spacetime $\left(d s^{2}=\right.$ $\left.-d t^{2}+a(t)^{2} \delta_{i j} d x^{i} d x^{j}\right)$ becomes

$$
\ddot{A}_{i}+H \dot{A}_{i}-\frac{1}{a^{2}} \nabla^{2} A_{i}=0
$$

or in conformal time

$$
A_{i}^{\prime \prime}-\nabla^{2} A_{i}=0
$$

where $H=\frac{\dot{a}}{a}$ is the Hubble parameter and primes indicate derivatives with respect to conformal time. Promoting $A_{i}$ to an operator, it can be defined through its mode functions as

$$
\begin{aligned}
& \hat{A}_{i}(\tau, \vec{x})=\int \frac{d^{3} k}{(2 \pi)^{3 / 2}} e^{i \vec{k} \cdot \vec{x}} \hat{A}_{i}(\tau, \vec{k}) \\
& =\sum_{\lambda= \pm} \int \frac{d^{3} k}{(2 \pi)^{3 / 2}}\left\{\epsilon_{i}^{\lambda}(\vec{k}) A_{\lambda}(\tau, \vec{k}) \hat{a}(\vec{k}) e^{i \vec{k} \cdot \vec{x}}+\epsilon_{i}^{\lambda}(\vec{k})^{*} A_{\lambda}(\tau, \vec{k})^{*} \hat{a}^{\dagger}(\vec{k}) e^{-i \vec{k} \cdot \vec{x}}\right\}
\end{aligned}
$$

where $\lambda$ denotes the helicity of the mode, $\hat{a}_{\lambda}$ are creation operators that satisfy

\footnotetext{
${ }^{2}$ Since the specific model relevant to this thesis is [3] which will be discussed in the next section.
} 


\section{ENHANCEMENT OF THE PRIMORDIAL GRAVITATIONAL WAVE SPECTRUM}

$\left[\hat{a}_{\lambda}(\vec{k}), \hat{a}_{\lambda^{\prime}}^{\dagger}\left(\vec{k}^{\prime}\right)\right]=(2 \pi)^{3} \delta_{\lambda \lambda^{\prime}} \delta^{3}\left(k-k^{\prime}\right)$ and $\epsilon_{i}^{\lambda}$ are polarisation tensors that satisfy

$$
\vec{k} \cdot \vec{\epsilon}^{ \pm}=0, \quad i \vec{k} \times \vec{\epsilon}^{ \pm}= \pm k \vec{\epsilon}^{ \pm}, \quad \vec{\epsilon}^{ \pm} \cdot \vec{\epsilon}^{ \pm}=0, \quad \vec{\epsilon}^{ \pm} \cdot \vec{\epsilon}^{\mp}=1, \quad\left(\vec{\epsilon}^{ \pm}\right)^{*}=\vec{\epsilon}^{\mp},
$$

as well as

$$
\vec{\epsilon}^{ \pm}(-\vec{p})=\vec{\epsilon}^{\mp}(\vec{p})
$$

With these conditions, it is easy to show that the equation of motion for the mode functions satisfies

$$
A_{ \pm}^{\prime \prime}+k^{2} A_{ \pm}=0
$$

which clearly has an oscillatory solution $\left(A(k, \tau) \sim e^{ \pm i k \tau}\right.$ with $\tau$ conformal time) and no growing mode. In an FRW spacetime, the electric, $E$, and magnetic, $B$, fields of $\hat{A}$ are given by

$$
\hat{E}_{i}=-\frac{1}{a^{2}} \hat{A}^{\prime}, \quad \hat{B}_{i}=\frac{1}{a^{2}} \epsilon_{i j k} \partial_{j} \hat{A}_{k}
$$

and therefore (with $A$ not growing), $E$ and $B$ quickly decrease as $a$ grows exponentially. The energy density in the vector field is

$$
\rho_{A}=\left\langle\frac{E^{2}+B^{2}}{2}\right\rangle
$$

and therefore the energy density of a (massless) vector field with no coupling to a secondary field scales as $a^{-4}$. This is why we say that vector fields are quickly diluted during inflation due to the exponentially fast increase in the scale factor, $a$.

A massless vector field therefore requires a coupling to some secondary field to be supported during inflation. This is why the coupling to the axion, $\sigma$, in (3.2.1) is required - under the right conditions for $\sigma$, this coupling can support the vector field for a sizeable time during inflation. With a vector field active, there exists a source for the gravitational waves as we will show later in this section. The equation of motion for the vector field including the coupling $\sqrt{-g} \alpha \frac{\sigma}{4 f} F \tilde{F}$ becomes

$$
A_{i}^{\prime \prime}-\nabla^{2} A_{i}-\frac{\alpha \sigma^{\prime}}{f}(\nabla \times \vec{A})_{i}=0
$$

where the axion has been taken to be homogeneous, $\sigma=\sigma(\tau)$ and, once again, we have

used the Lorenz gauge. Taking the same form for $\hat{A}_{i}$ as an operator as in 3.2.7), we 
arrive at the mode equation

$$
A_{ \pm}^{\prime \prime}+\left(k^{2} \mp k \frac{\alpha \sigma^{\prime}}{f}\right) A_{ \pm}=0
$$

The axion is taken to have potential

$$
U(\sigma)=\frac{\Lambda^{4}}{2}\left[\cos \left(\frac{\sigma}{f}\right)+1\right] .
$$

Ignoring the backreaction of the vector on the axion, it has equation of motion (in cosmic time)

$$
\ddot{\sigma}+3 H \dot{\sigma}-\frac{\Lambda^{4}}{2 f} \sin \left(\frac{\sigma}{f}\right)=0
$$

with an analytic solution

$$
\sigma=2 f \tan ^{-1}\left(e^{\delta H\left(t-t_{*}\right)}\right)
$$

where $\delta:=\frac{\Lambda^{4}}{6 H^{2} f^{2}}$ and $t_{*}$ refers to the time at which the axion goes to its minimum: $\sigma=\frac{\pi}{2} f$. Most interestingly for our purposes, its derivative has the form

$$
\dot{\sigma}=\frac{f H \delta}{\cosh \left(\delta H\left(t-t_{*}\right)\right)} .
$$

Defining $\xi:=\frac{\alpha \dot{\sigma}}{2 H f}$,

$$
\xi=\frac{\xi_{*}}{\cosh \left(\delta H\left(t-t_{*}\right)\right)}=\frac{2 \xi_{*}}{\left(\frac{a}{a_{*}}\right)^{\delta}+\left(\frac{a_{*}}{a}\right)^{\delta}}
$$

with $\xi_{*}=\alpha \delta / 2$ and $a_{*}$ the respective values of $\xi$ and $a$ at $t=t_{*}, \sigma^{\prime}$ can be written

$$
\sigma^{\prime}=\frac{2 a H f \xi}{\alpha}=\frac{4 a H f \xi_{*}}{\alpha\left[\left(\frac{a}{a_{*}}\right)^{\delta}+\left(\frac{a_{*}}{a}\right)^{\delta}\right]}=-\frac{4 \xi_{*} f}{\alpha \tau\left[\left(\frac{\tau_{*}}{\tau}\right)^{\delta}+\left(\frac{\tau}{\tau_{*}}\right)^{\delta}\right]}
$$

where we have used $a=-\frac{1}{H \tau}$ and $\tau_{*}$ is the value of $\tau$ at which the axion goes to its minimum. The vector equation 3.2 .14 becomes

$$
A_{ \pm}^{\prime \prime}+\left(k^{2} \pm \frac{4 k \xi_{*}}{\tau\left[\left(\frac{\tau_{*}}{\tau}\right)^{\delta}+\left(\frac{\tau}{\tau_{*}}\right)^{\delta}\right]}\right) A_{ \pm}=0 .
$$




\section{ENHANCEMENT OF THE PRIMORDIAL GRAVITATIONAL WAVE SPECTRUM}

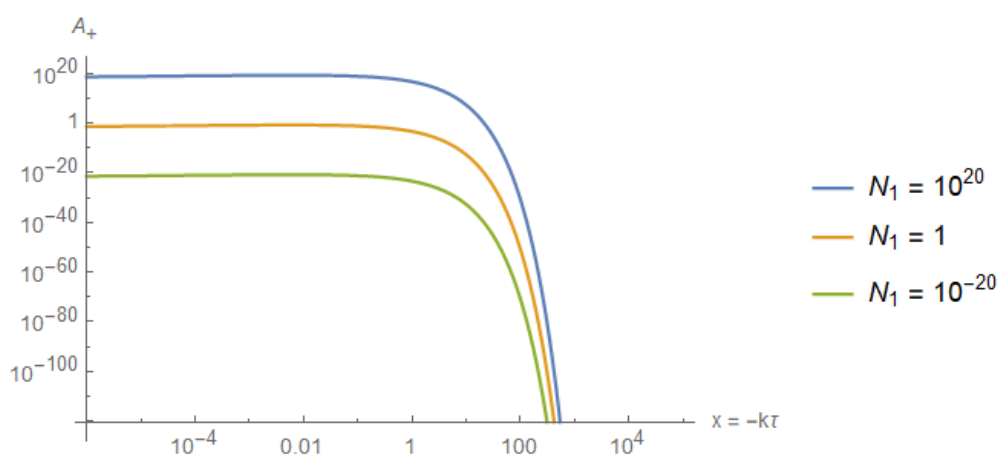

Figure 3.1: Plots (on a log-log scale) of $A+$ against $x=-k \tau=k / a H$ with different normalisation factors. We have used values from [2] of $\delta=0.2$ and $\xi_{*}=5$ and chosen the minimisation point of the axion, $x_{*}=-k \tau_{*}=1$, to be horizon-crossing. $A_{+}$is greatly enhanced as inflation proceeds.

With $\xi_{*}>0$ chosen to be positive, only the positive helicity modes, $A_{+}$, are amplified ${ }^{3}$ and the $A_{-}$modes can be ignored. An approximate analytic solution to this (see [2] Appendix A) is given by

$$
A_{+}(\tau, k) \simeq N_{1}\left(-\frac{\tau}{\xi(\tau)}\right)^{1 / 4} \exp \left(-\frac{4 \xi_{*}^{1 / 2}}{1+\delta}\left(\frac{\tau}{\tau_{*}}\right)^{\delta / 2} \sqrt{-k \tau}\right)
$$

and

$$
A_{+}^{\prime} \simeq N_{2}\left(\frac{\xi(\tau)}{-2 \tau}\right)^{1 / 4} \exp \left(-\frac{4 \xi_{*}^{1 / 2}}{1+\delta}\left(\frac{\tau}{\tau_{*}}\right)^{\delta / 2} \sqrt{-k \tau}\right)
$$

where $N_{1}$ and $N_{2}$ are normalisation factors that depend on $k, \xi_{*}, x_{*}, \delta$, the details of which are not important for our purposes - the key point is that $A_{+}$is indeed enhanced. To demonstrate this, the solution $(3.2 .22)$ is plotted in FIG. 3.1 for a range of normalisations ${ }^{4}, A_{+}$is greatly enhanced as inflation proceeds.

The electric and magnetic fields, $\hat{E}_{i}=-\frac{1}{a^{2}} \hat{A}_{i}^{\prime}$ and $\hat{B}_{i}=\frac{1}{a^{2}} \epsilon_{i j k} \partial_{j} \hat{A}_{k}$, can be written

$$
\begin{aligned}
& \hat{E}_{i}(\tau, \vec{x})=\int \frac{d^{3} k}{(2 \pi)^{3 / 2}} e^{i \vec{k} \cdot \vec{x}} \epsilon_{i}^{+}(\hat{k}) E_{+}(\tau, k)\left[\hat{a}_{+}(\vec{k})+\hat{a}_{+}^{\dagger}(-\vec{k})\right], \\
& \hat{B}_{i}(\tau, \vec{x})=\int \frac{d^{3} k}{(2 \pi)^{3 / 2}} e^{i \vec{k} \cdot \vec{x}} \epsilon_{i}^{+}(\hat{k}) B_{+}(\tau, k)\left[\hat{a}_{+}(\vec{k})+\hat{a}_{+}^{\dagger}(-\vec{k})\right]
\end{aligned}
$$

\footnotetext{
${ }^{3}$ Remember that conformal time is negative during inflation, therefore with $\xi_{*}$ positive, the term $4 k \xi_{*} /\left(\tau\left[\left(\tau_{*} / \tau\right)^{\delta}+\left(\tau / \tau_{*}\right)^{\delta}\right]\right)$ is negative.

${ }^{4}$ Strictly speaking, the normalisations in the plot do not correspond to $N$ as stated in 3.2 .22 - due to changing coordinates to $x=-k \tau$.
} 

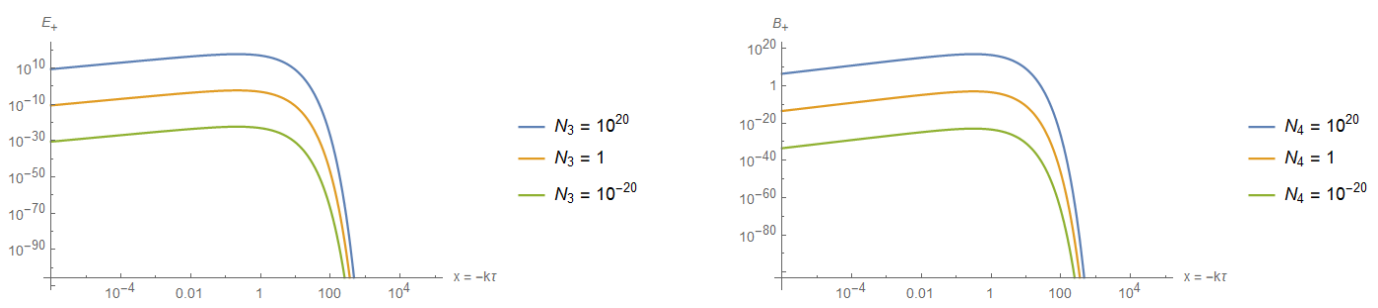

Figure 3.2: Plots (on a log-log scale) of $E+$ (left) and $B_{+}$(right) against $x=-k \tau=k / a H$ with different normalisation factors. We have used values from [2] of $\delta=0.2$ and $\xi_{*}=5$ and chosen the minimisation point of the axion, $x_{*}=-k \tau_{*}=1$, to be horizon-crossing. Both are greatly enhanced as inflation proceeds despite the accelerated expansion of the universe.

with

$$
\begin{aligned}
& E_{+}(\tau, k)=-N_{3} \tau^{2}\left(\frac{\xi(\tau)}{-2 \tau}\right)^{1 / 4} \exp \left(-\frac{4 \xi_{*}^{1 / 2}}{1+\delta}\left(\frac{\tau}{\tau_{*}}\right)^{\delta / 2} \sqrt{-k \tau}\right) \\
& B_{+}(\tau, k)=N_{4} \tau^{2}\left(\frac{-\tau}{8 \xi(\tau)}\right)^{1 / 4} \exp \left(-\frac{4 \xi_{*}^{1 / 2}}{1+\delta}\left(\frac{\tau}{\tau_{*}}\right)^{\delta / 2} \sqrt{-k \tau}\right) .
\end{aligned}
$$

In FIG. 3.2 , we plot $E_{+}$and $B_{+}$to show that they are both enhanced up until about horizon-crossing where the axion reaches its minimum. As we shall see, $E_{i}$ and $B_{i}$ appear in the source term for the gravitational tensor perturbations.

In order to determine the primordial gravitational wave spectrum, we must find the action to second order in tensor perturbations. Defining the perturbed metric tensor in conformal time through

$$
d s^{2}=a^{2}\left[-d \tau^{2}+\left(\delta_{i j}+h_{i j}(\tau, \vec{x})\right) d x^{i} d x^{j}\right]
$$

where the tensor perturbation to the metric is transverse, $\partial_{i} h_{i j}=0$, and traceless $h_{i i}=$ 0 . We are interested only in contributions with a coupling to $h_{i j}$ in the action - this automatically rules out the $\alpha \sqrt{-g} \frac{\sigma}{4 f} F \tilde{F}$ term since this contains no metric coupling. The Ricci scalar and metric determinant to second order are (see standard lecture notes e.g. [77])

$$
\sqrt{-g}=a^{4}-\frac{a^{4}}{4} h_{i j} h_{i j}
$$




\section{ENHANCEMENT OF THE PRIMORDIAL GRAVITATIONAL WAVE SPECTRUM}

$$
R=6 \frac{a^{\prime \prime}}{a^{3}}-\frac{1}{a^{2}}\left(h_{i j} h_{i j}^{\prime \prime}+\frac{1}{4} \partial_{i} h_{j k} \partial_{i} h_{j k}+\frac{3}{4} h_{i j}^{\prime} h_{i j}^{\prime}+3 \frac{a^{\prime}}{a} h_{i j} h_{i j}^{\prime}\right)
$$

where we are summing over lowered indices to emphasise that the metric is not involved in the summation. Combining these and considering only perturbations up to second order, we get the contribution to the action from the Einstein-Hilbert term

$$
\begin{aligned}
& \left(\frac{M_{\mathrm{pl}}^{2}}{2} \int d \tau d^{3} x \sqrt{-g} R\right)_{h_{\mu \nu}} \\
& =\frac{M_{\mathrm{pl}}^{2}}{2} \int d \tau d^{3} x\left\{-\frac{3}{2} a a^{\prime \prime} h_{i j} h_{i j}-a^{2} h_{i j} h_{i j}^{\prime \prime}-\frac{a^{2}}{4} \partial_{i} h_{j k} \partial_{i} h_{j k}-\frac{3 a^{2}}{4} h_{i j}^{\prime} h_{i j}^{\prime}-3 a^{\prime} a h_{i j} h_{i j}^{\prime}\right\} \\
& =\frac{M_{\mathrm{pl}}^{2}}{2} \int d \tau d^{3} x\left\{-\frac{3}{2} a a^{\prime \prime} h_{i j} h_{i j}-\frac{a^{2}}{4} \partial_{i} h_{j k} \partial_{i} h_{j k}+\frac{a^{2}}{4} h_{i j}^{\prime} h_{i j}^{\prime}-a^{\prime} a h_{i j} h_{i j}^{\prime}\right\} \\
& =\frac{M_{\mathrm{pl}}^{2}}{2} \int d \tau d^{3} x\left\{\frac{1}{2}\left(\left(a^{\prime}\right)^{2}-2 a a^{\prime \prime}\right) h_{i j} h_{i j}-\frac{a^{2}}{4} \partial_{i} h_{j k} \partial_{i} h_{j k}+\frac{a^{2}}{4} h_{i j}^{\prime} h_{i j}^{\prime}\right\}
\end{aligned}
$$

where we have repeatedly integrated by parts. An additional contribution that perfectly cancels the first term above comes from the minimal coupling of gravity to the scalar fields:

$$
\begin{aligned}
& \left(\int d \tau d^{3} x\left\{\sqrt{-g} \mathcal{L}_{\phi}\right\}\right)_{h_{\mu \nu}} \\
& =-\int d \tau d^{3} x \frac{a^{4}}{4} h_{i j} h_{i j}\left\{\frac{1}{2 a^{2}}\left(\phi^{\prime}\right)^{2}+\frac{1}{2 a^{2}}\left(\sigma^{\prime}\right)^{2}-V(\phi)-U(\sigma)\right\} \\
& =-\int d \tau d^{3} x \frac{a^{4}}{4} h_{i j} h_{i j} p=-\frac{M_{\mathrm{pl}}^{2}}{4} \int d \tau d^{3} x h_{i j} h_{i j}\left(\left(a^{\prime}\right)^{2}-2 a a^{\prime \prime}\right)
\end{aligned}
$$

where we have used the Friedmann equations in conformal time

$$
\begin{aligned}
& 3 M_{\mathrm{pl}}^{2}\left(a^{\prime}\right)^{2}=a^{4} \rho \\
& 6 M_{\mathrm{pl}}^{2}\left(\left(a^{\prime}\right)^{2}-a^{\prime \prime} a\right)=a^{4}(\rho+3 p)
\end{aligned}
$$

with $\rho=\frac{1}{2 a^{2}}\left(\phi^{\prime}\right)^{2}+\frac{1}{2 a^{2}}\left(\sigma^{\prime}\right)^{2}+V(\phi)+U(\sigma)$ and $p=\frac{1}{2 a^{2}}\left(\phi^{\prime}\right)^{2}+\frac{1}{2 a^{2}}\left(\sigma^{\prime}\right)^{2}-V(\phi)-U(\sigma)$ the energy density and pressure of the inflationary universe, respectively 5 . All that is

\footnotetext{
${ }^{5}$ As a condition the energy density in the vector field is taken to be much lower than that of inflaton to ensure the background inflationary evolution is not affected. In fact, we could easily ignore the contribution of the axion to $\rho$ and $p$ as well as this is also vastly sub-dominant with respect to the inflationary potential, $V$.
} 
left is therefore to find the perturbation to the term

$$
-\frac{1}{4} \sqrt{-g} g^{\mu \rho} g^{\nu \sigma} F_{\mu \nu} F_{\rho \sigma}
$$

The lowest order contribution is actually linear in $h_{i j}$ :

$$
\begin{aligned}
& \left(-\frac{1}{4} \sqrt{-g} g^{\mu \rho} g^{\nu \sigma} F_{\mu \nu} F_{\rho \sigma}\right)_{h_{\mu \nu}} \\
& =-\frac{1}{2} h_{i j}\left(A_{i}^{\prime} A_{j}^{\prime}+\epsilon_{i k l} \epsilon_{j m n} \partial_{k} A_{l} \partial_{m} A_{n}\right) \\
& =-\frac{a^{4}}{2} h_{i j}\left(E_{i} E_{j}+B_{i} B_{j}\right)
\end{aligned}
$$

and higher order terms in $h_{i j}$ are exponentially suppressed relative to the linear contribution to the source term for the tensor power spectrum. This means our perturbed action becomes

$$
S_{h_{\mu \nu}}=\int d \tau d^{3} x\left\{\frac{M_{\mathrm{pl}}^{2} a^{2}}{8}\left(\hat{h}_{i j}^{\prime} \hat{h}_{i j}^{\prime}-\partial_{i} \hat{h}_{j k} \partial_{i} \hat{h}_{j k}\right)-\frac{a^{4}}{2} \hat{h}_{i j}\left(\hat{E}_{i} \hat{E}_{j}+\hat{B}_{i} \hat{B}_{j}\right)\right\} .
$$

This leads to an equation of motion for $h_{i j}$ :

$$
\hat{h}_{i j}^{\prime \prime}+2 \frac{a^{\prime}}{a} \hat{h}_{i j}^{\prime}-\nabla^{2} \hat{h}_{i j}=-\frac{2 a^{2}}{M_{\mathrm{pl}}^{2}}\left(\hat{E}_{i} \hat{E}_{j}+\hat{B}_{i} \hat{B}_{j}\right)
$$

where it is clear that the right-hand side is a source term for the gravitational waves if the vector field is active during inflation.

\subsection{Using an isotropic gauge field}

Our attention now turns to a related model described in [3], where instead of a $U(1)$ vector, the axion is coupled to an $S U(2)$ gauge field. This model can be seen as an extension to chromo-natural inflation $[78,81]^{6}$ where the axion and gauge sector are now spectator fields, and there is a scalar field that acts as the inflaton.

\footnotetext{
${ }^{6}$ Chromo-natural inflation is a theory in which the inflaton is an axion field that enters slow-roll due to its coupling to an $S U(2)$ gauge field. This coupling leads to a large tensor-to-scalar ratio. In pure chromo-natural inflation, it is impossible to satisfy the bounds on $r$ and $n_{s}$ concurrently [81.
} 


\section{ENHANCEMENT OF THE PRIMORDIAL GRAVITATIONAL WAVE SPECTRUM}

\subsubsection{Background evolution}

The action of [3] takes the form

$$
\begin{aligned}
S=\int d^{4} x \sqrt{-g}\{ & \frac{M_{\mathrm{pl}}^{2}}{2} R-\frac{1}{2} \partial_{\mu} \phi \partial^{\mu} \phi-V(\phi)-\frac{1}{2} \partial_{\mu} \chi \partial^{\mu} \chi-U(\chi) \\
& \left.-\frac{1}{4} F_{\mu \nu}^{A} F^{A \mu \nu}+\frac{\lambda \chi}{4 f} F_{\mu \nu}^{A} \tilde{F}^{A \mu \nu}\right\}
\end{aligned}
$$

where again $\phi$ is the scalar field inflaton, driven by the potential $V(\phi) ; \chi$ is a spectator axion field, driven by the potential $U(\chi) ; F_{\mu \nu}^{A}=\partial_{\mu} A_{\nu}^{A}-\partial_{\nu} A_{\mu}^{A}-\mathrm{g} \epsilon^{A B C} A_{\mu}^{B} A_{\nu}^{C}$ is the field strength tensor of the $S U(2)$ gauge field, $A_{\mu}^{A}$, with $A, B, C \ldots$ the gauge indices and $\mathrm{g}$ the gauge coupling; and $\tilde{F}^{A \mu \nu}=\frac{1}{2 \sqrt{-g}} \epsilon^{\mu \nu \rho \sigma} F_{\rho \sigma}^{A}$ is the dual field strength. An explicit isotropic form for the gauge field, which greatly simplifies the background equations of motion, is assumed immediately:

$$
A_{0}^{A}=0, \quad A_{i}^{A}=\delta_{i}^{A} a(t) Q(t)
$$

where $Q$ is a scalar field and $a(t)$ is the scale factor of the universe. With this form for $A_{\mu}^{A}$, a few identities will prove useful

$$
F_{\mu \nu}^{A} F^{A \mu \nu}=6 \mathrm{~g}^{2} Q^{4}-6(\dot{Q}+H Q)^{2}
$$

and

$$
\tilde{F}^{A \mu \nu} F_{\mu \nu}^{A}=-12 \mathrm{~g} Q^{2}(\dot{Q}+H Q) .
$$

The background equations of motion for this system are then

$$
\begin{gathered}
\ddot{\phi}+3 H \dot{\phi}+\frac{d V}{d \phi}=0, \\
\ddot{\chi}+3 H \dot{\chi}+\frac{d U}{d \chi}=-\frac{3 \mathrm{~g} \lambda}{f} Q^{2}(\dot{Q}+H Q), \\
\ddot{Q}+3 H \dot{Q}+\left(\dot{H}+2 H^{2}\right) Q+2 \mathrm{~g}^{2} Q^{3}=\frac{\mathrm{g} \lambda}{f} \dot{\chi} Q^{2}, \\
3 M_{\mathrm{pl}}^{2} H^{2}=\frac{\dot{\phi}^{2}}{2}+V(\phi)+\frac{\dot{\chi}^{2}}{2}+U(\chi)+\frac{3}{2}(\dot{Q}+H Q)^{2}+\frac{3}{2} \mathrm{~g}^{2} Q^{4},
\end{gathered}
$$


and

$$
-2 M_{\mathrm{pl}}^{2} \dot{H}=\dot{\phi}^{2}+\dot{\chi}^{2}+2(\dot{Q}+H Q)^{2}+2 \mathrm{~g}^{2} Q^{4} .
$$

It will prove useful to define the slow-roll parameter in terms of components

$$
\epsilon=-\frac{\dot{H}}{H^{2}}=\epsilon_{\phi}+\epsilon_{\chi}+\epsilon_{E}+\epsilon_{B}
$$

with

$$
\epsilon_{\phi}:=\frac{\dot{\phi}^{2}}{2 M_{\mathrm{pl}}^{2} H^{2}}, \quad \epsilon_{\chi}:=\frac{\dot{\chi}^{2}}{2 M_{\mathrm{pl}}^{2} H^{2}}, \quad \epsilon_{E}:=\frac{(\dot{Q}+H Q)^{2}}{M_{\mathrm{pl}}^{2} H^{2}}, \quad \epsilon_{B}:=\frac{\mathrm{g}^{2} Q^{4}}{M_{\mathrm{pl}}^{2} H^{2}}
$$

where, using electromagnetic notation, $E$ and $B$ refer to the "electric" and "magnetic" parts of the gauge field, respectively. In [3], a specific inflationary model is not assumed, and instead they require only that the energy density in the inflaton field is much greater than the energy densities of the axion and the gauge field, $\rho_{\phi} \gg \rho_{\chi}, \rho_{Q}$. This ensures that $3 M_{\mathrm{pl}}^{2} H^{2} \sim V(\phi)$ and that the basic inflationary predictions of the model, including $n_{s}$ are negligibly affected by the presence of the spectator axion and gauge fields. As in the model presented in the previous section, the gauge field, $Q$, is supported by the rolling of the axion, $\chi$, and a rough estimate for its value can be found by considering (3.3.6) in the extreme slow-roll limit. The equations become

$$
\frac{d U}{d \chi} \approx-\frac{3 \mathrm{~g} \lambda}{f} H Q^{3} \Longrightarrow Q \approx\left(-\frac{f}{3 \mathrm{~g} \lambda H} \frac{d U}{d \chi}\right)^{1 / 3}
$$

and with this form for $Q$, the contribution from the potential to the equation of motion for $\chi$ is almost perfectly cancelled by the contribution of the gauge field - this allows the axion to slow roll. We will present a toy model where an inflationary potential of the form 7

$$
V(\phi)=\Lambda^{4}\left(1-m_{1} \phi e^{-m_{2} \phi}\right)
$$

is taken to demonstrate the key features of the enhancement mechanism. The potential for the axion is (as in [3])

$$
U(\chi)=\mu^{4}\left(1+\cos \left(\frac{\chi}{f}\right)\right) .
$$

\footnotetext{
${ }^{7}$ This is the same potential that was shown in the introduction Figure 1.2
} 


\section{ENHANCEMENT OF THE PRIMORDIAL GRAVITATIONAL WAVE SPECTRUM}

We use the parameters

$$
\mathrm{g}=1.11 \times 10^{-2}, \quad \mu=2.10 \times 10^{15} \mathrm{GeV}, \quad f=4.00 \times 10^{16} \mathrm{GeV}, \quad \lambda=280
$$

and

$$
\Lambda=8.80 \times 10^{15} \mathrm{GeV}, \quad m_{1}=8.91 \times 10^{-19} \mathrm{GeV}^{-1}, \quad m_{2}=3.29 \times 10^{-19} \mathrm{GeV}^{-1},
$$

as well as initial conditions

$$
\phi_{i n}=8.87 M_{\mathrm{pl}}, \quad \chi_{i n}=0.1 \pi f, \quad Q_{i n}=\left(-\left.\frac{f}{3 \mathrm{~g} \lambda \sqrt{V\left(\phi_{i n}\right) / 3}} \frac{d U}{d \chi}\right|_{\chi=\chi_{i n}}\right)^{1 / 3} M_{\mathrm{pl}}
$$

where we have used 3.3 .12 as an initial condition for $Q$, taken $H \sim \sqrt{V / 3 M_{\mathrm{pl}}^{2}}$ and converted to Planck units for convenience. We define the effective mass of the gauge field as

$$
\xi_{Q}:=\frac{\mathrm{g} Q}{H}
$$

and note for now that, in general, the higher the value of this at horizon-crossing, the greater the amplification to the gravitational wave spectrum. We define also

$$
\xi_{\chi}:=\frac{\lambda}{2 f H} \dot{\chi}
$$

and note that in the slow-roll limit 3.3 .6 becomes

$$
2 H^{2} Q+2 \mathrm{~g}^{2} Q^{3} \approx \frac{\mathrm{g} \lambda}{f} \dot{\chi} Q^{2} \Longrightarrow \frac{\lambda}{2 f H} \dot{\chi} \approx \frac{\mathrm{g} Q}{H}+\frac{H}{\mathrm{~g} Q} \Longrightarrow \xi_{\chi} \approx \xi_{Q}+\xi_{Q}{ }^{-1}
$$

With $\xi_{Q}>1, \xi_{\chi} \sim \xi_{Q}$ suggesting that the faster the axion is evolving after it has entered its slow-roll phase, the larger $\xi_{Q}$ will be. Plots of the evolutions of the (background) fields and their energy densities are shown in FIG. 3.3 3.6. The scalar field, $\phi$, completely dominates the energy density of the universe and therefore the basic inflationary predictions of this toy model are unaffected by the presence of the spectator fields. The axion rolls to its minimum and while it is rolling supports the gauge field through the coupling $\frac{\mathrm{g} \lambda}{f} \dot{\chi} Q^{2}$ that appears in the equation of motion for $Q$. The axion rolls at a speed that is roughly given by $\xi_{\chi}=\xi_{Q}+\xi_{Q}{ }^{-1}$. 

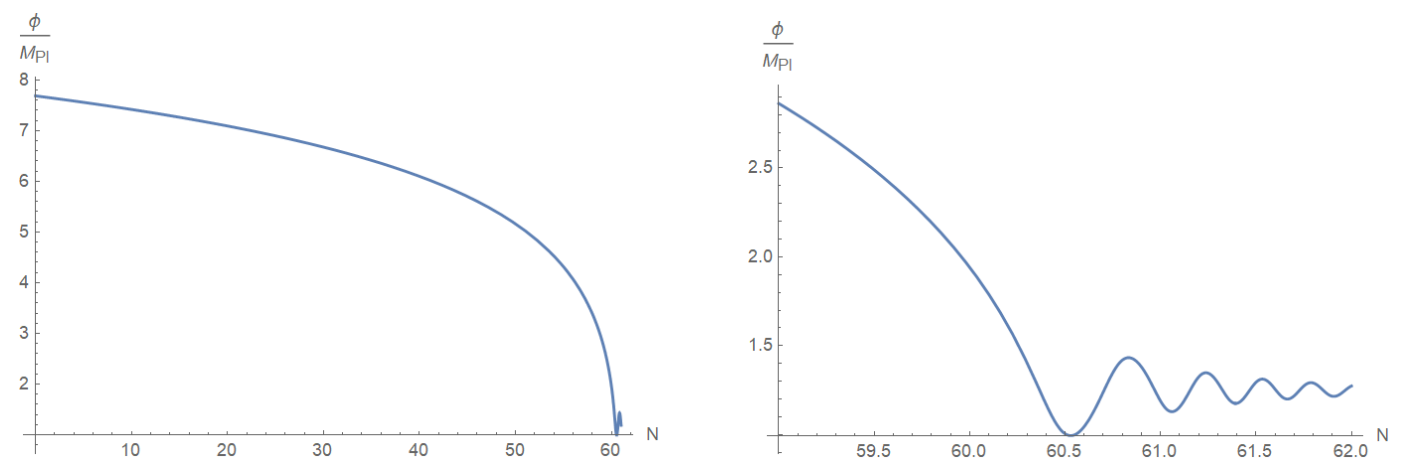

Figure 3.3: Plots of the evolution of the scalar inflaton, $\phi$, in units of $M_{\mathrm{pl}}$ during the last 60 e-folds and last few e-folds of inflation in the left and right figure, respectively.
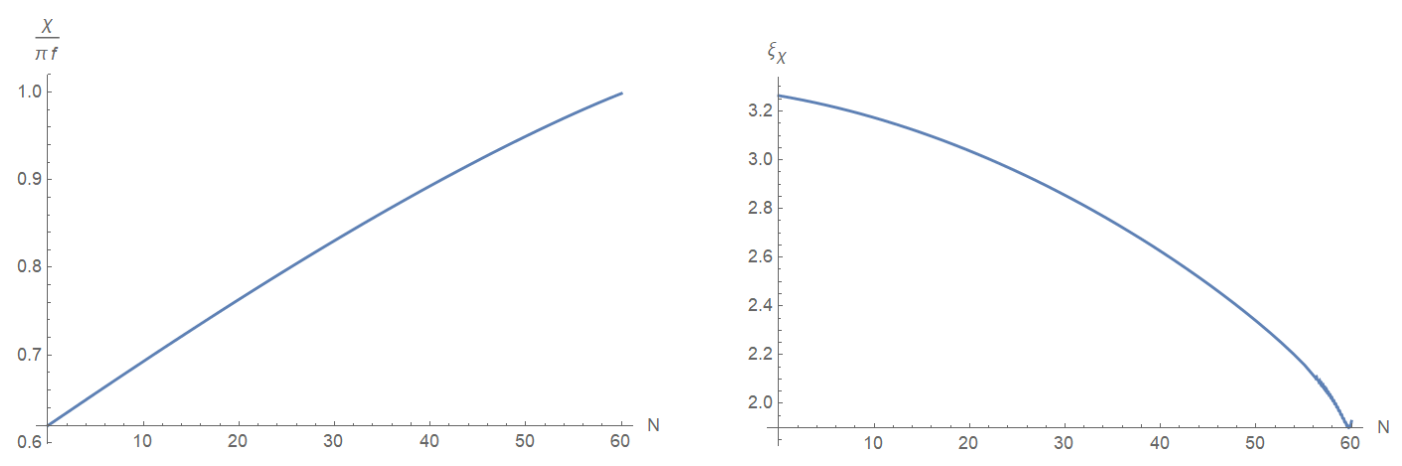

Figure 3.4: Plots of the evolution of the spectator axion (left figure) and $\xi_{\chi}=\frac{\lambda}{2 H f} \dot{\chi}$ (right figure) during the last 60 e-folds of inflation.
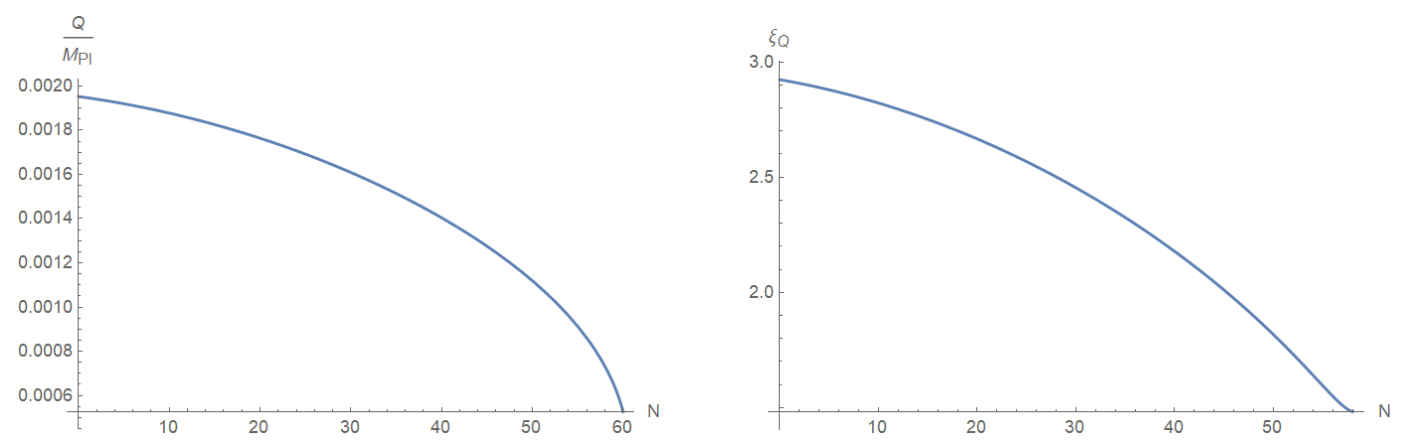

Figure 3.5: Plots of the evolution of the gauge field, $Q$ (left figure), and its effective mass, $\xi_{Q}=\frac{\mathrm{g} Q}{H}$ (right figure), during the last 60 e-folds of inflation. 


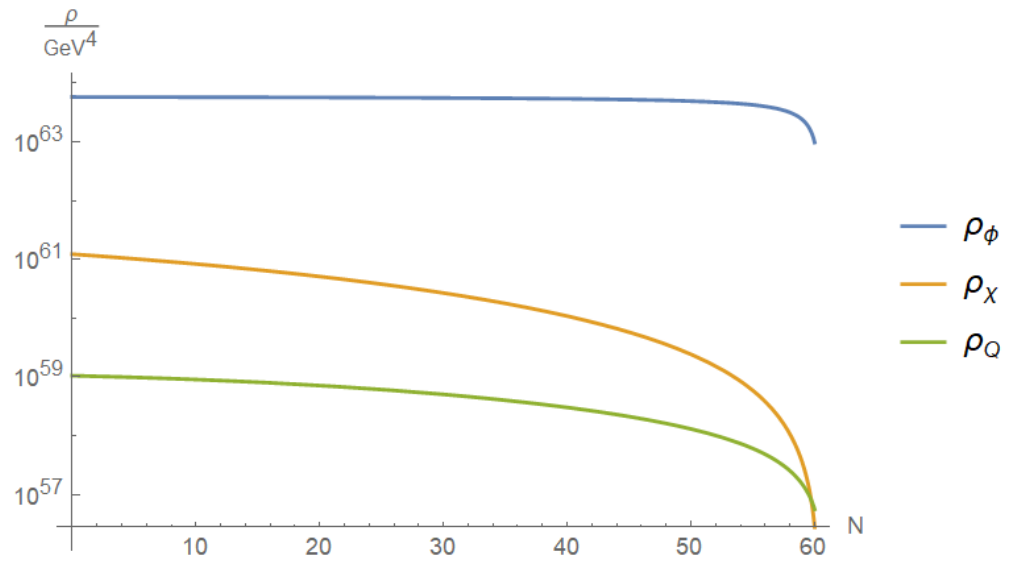

Figure 3.6: Plot (on a log scale) of the energy densities of the inflaton (blue), $\rho_{\phi}=\frac{1}{2} \dot{\phi}^{2}+$ $V$, the axion (orange), $\rho_{\chi}=\frac{1}{2} \dot{\chi}^{2}+U$, and the gauge field (green), $\rho_{Q}=\frac{3}{2}(\dot{Q}+H Q)^{2}+$ $\frac{3}{2} \mathrm{~g}^{2} Q^{4}$. The energy densities of the axion and the gauge field are vastly sub-dominant to the energy density in $\phi$, which allows $\phi$ to cause inflation.

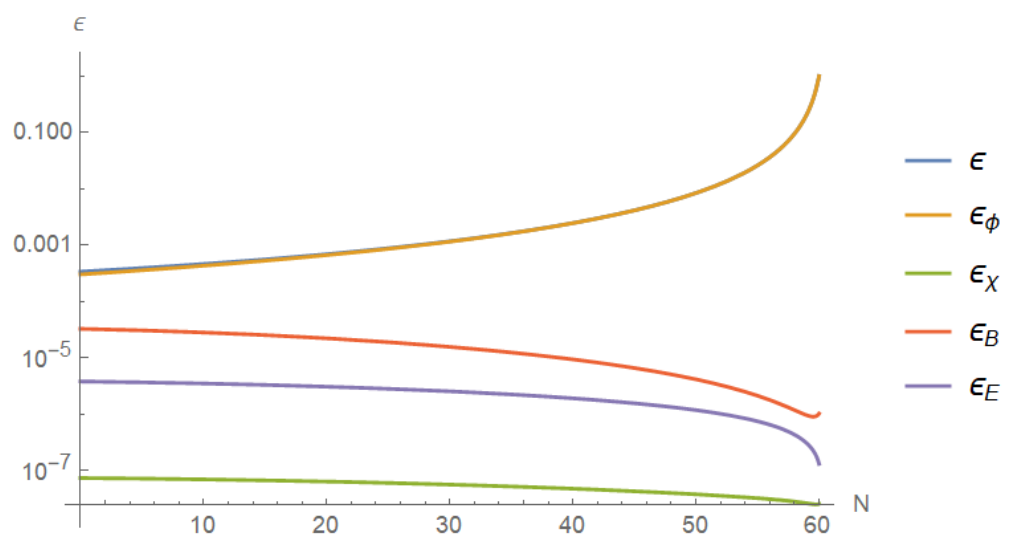

Figure 3.7: Plot (on a log scale) of $\epsilon=-\frac{\dot{H}}{H^{2}}$ (blue), and its components: $\epsilon_{\phi}=\frac{\dot{\phi}^{2}}{2 M_{\mathrm{pl}}^{2} H^{2}}$ (orange), $\epsilon_{\chi}=\frac{\dot{\chi}^{2}}{2 M_{\mathrm{pl}}^{2} H^{2}}$ (green), $\epsilon_{B}=\frac{\mathrm{g}^{2} Q^{4}}{M_{\mathrm{pl}}^{2} H^{2}}$ (red), and $\epsilon_{E}=\frac{(\dot{Q}+H Q)^{2}}{M_{\mathrm{pl}}^{2} H^{2}}$ during the last 60 e-folds of inflation. 
For reference, the basic inflationary predictions of this model are (taking horizoncrossing to be 60 e-folds before the end of inflation)

$$
n_{s}=0.966, \quad r_{b}=16 \epsilon=4.82 \times 10^{-3}
$$

where $r_{b}$ is the background (non-amplified) tensor-to-scalar ratio, and $n_{s}=1-2 \epsilon-\eta$ is the scalar spectral index.

\subsubsection{Tensor perturbations}

In order to evaluate the amplification of the gravitational wave spectrum, we must consider the tensor perturbations of the action (3.3.1) to second order. We set up the perturbations to the gauge field exactly as in [3, 80]

$$
\begin{aligned}
& A_{0}^{A}=a(t) \delta_{i}^{A}\left(Y_{i}+\partial_{i} Y\right) \\
& A_{i}^{A}=a(t)\left\{(Q+\delta Q) \delta_{i}^{A}+\partial_{i}\left(M_{j}+\partial_{j} M\right) \delta_{j}^{A}+\epsilon_{i}^{A C} \delta_{j}^{C}\left(W_{j}+\partial_{j} W\right)+\delta_{j}^{A} T_{i j}\right\}
\end{aligned}
$$

where $Y_{i}, M_{i}$ and $W_{i}$ are vector perturbations that are safe to neglect since they have been shown to decay rapidly on super-horizon scales [80]; $Y, \delta Q, M$, and $W$ are scalar perturbations; and $T_{i j}$ is a transverse, traceless tensor perturbation. In addition to this, the $S U(2)$ gauge freedom allows us to set $W=0$.

Following [3] and without loss of generality, we take the wavevector of the tensor modes to be along the $z$-axis, $\vec{k}=k_{z} \hat{z}$. The gauge field becomes

$$
\begin{aligned}
& A_{\mu}^{1}=a(t)\left(0, Q+\delta Q+T_{+}, T_{\times}, 0\right), \\
& A_{\mu}^{2}=a(t)\left(0, T_{\times}, Q+\delta Q-T_{+}, 0\right), \\
& A_{\mu}^{3}=a(t)\left(\partial_{z} Y, 0,0, Q+\delta Q+\partial_{z}^{2} M\right)
\end{aligned}
$$

where the tensor perturbations have been chosen to be of circular and cross polarisation with directionality along the $z$-axis:

$$
T_{i j}=\left(\begin{array}{ccc}
T_{+} & T_{\times} & 0 \\
T_{\times} & -T_{+} & 0 \\
0 & 0 & 0
\end{array}\right) .
$$




\section{ENHANCEMENT OF THE PRIMORDIAL GRAVITATIONAL WAVE SPECTRUM}

Equivalently to this, the perturbed metric can be written as (ignoring scalar perturbations to the metric) 8

$$
g_{\mu \nu}=a^{2}(\tau)\left(\begin{array}{cccc}
-1 & 0 & 0 & 0 \\
0 & 1+h_{+} & h_{\times} & 0 \\
0 & h_{\times} & 1-h_{+} & 0 \\
0 & 0 & 0 & 1
\end{array}\right)
$$

with inverse (to second order)

$$
g^{\mu \nu}=a^{2}(\tau)\left(\begin{array}{cccc}
-1 & 0 & 0 & 0 \\
0 & 1-h_{+}+h_{\times}^{2}+h_{+}^{2} & -h_{\times} & 0 \\
0 & -h_{\times} & 1-h_{+}+h_{\times}^{2}+h_{+}^{2} & 0 \\
0 & 0 & 0 & 1
\end{array}\right)
$$

where we have moved to conformal time, $\tau$, and the metric satisfies $g_{\mu \rho} g^{\rho \nu}=\delta_{\mu}^{\nu}+\mathcal{O}\left(h^{3}\right)$ up to second order in perturbations. We note that to second order $T_{+, \times}=T_{+, \times}(\tau, z)$ and $h_{+, \times}=h_{+, \times}(\tau, z)$ are the polarisations of the gauge and gravitational wave tensor. The system can be simplified by defining left- and right-moving modes for the tensors

$$
\psi_{L, R}:=\frac{a(\tau) M_{\mathrm{pl}}}{2}\left(h_{+} \pm i h_{\times}\right), \quad t_{L, R}:=a(\tau)\left(T_{+} \pm i T_{\times}\right) .
$$

Explicitly we wish to find the action

$$
\begin{array}{r}
S=\int d^{4} x\left\{\frac{M_{\mathrm{pl}}^{2}}{2} \sqrt{-g} R-\frac{1}{2} \sqrt{-g} g^{\mu \nu} \partial_{\mu} \phi \partial_{\nu} \phi-\sqrt{g} V-\frac{1}{2} \sqrt{g} g^{\mu \nu} \partial_{\mu} \chi \partial_{\nu} \chi\right. \\
\left.-\sqrt{-g} U-\frac{1}{4} \sqrt{-g} g^{\mu \rho} g^{\nu \sigma} F_{\mu \nu}^{a} F_{\rho \sigma}^{a}+\frac{\lambda}{8 f} \chi \epsilon^{\mu \nu \rho \sigma} F_{\mu \nu}^{a} F_{\rho \sigma}^{a}\right\}
\end{array}
$$

to second order. The full second order action is in Appendix B and leads to the following equations of motion for the tensor modes

$\partial_{x}^{2} \psi_{R, L}+\psi_{R, L}\left[1-\frac{1}{x^{2}}\left(2+\epsilon_{E}-3 \epsilon_{B}-4 \epsilon_{\phi}-4 \epsilon_{\chi}\right)\right]=\frac{2 \sqrt{\epsilon_{E}}}{M_{\mathrm{pl}} x} \partial_{x} t_{R, L}+\frac{2 \sqrt{\epsilon_{B}}}{x^{2}} t_{R, L}\left(\xi_{Q} \mp x\right)$

\footnotetext{
${ }^{8}$ The scalar perturbations to the metric have a negligible effect on the scalar equations of motion and we demonstrate this explicitly in chapter 5 when we apply this mechanism to Kähler moduli inflation.
} 
and

$$
\begin{aligned}
& \partial_{x}^{2} t_{R, L}+t_{R, L}\left[1 \mp \frac{2}{x}\left(\xi_{\chi}+\xi_{Q}\right)+\frac{2}{x^{2}} \xi_{\chi} \xi_{Q}\right] \\
& =-\frac{2 \sqrt{\epsilon_{E}}}{M_{\mathrm{pl}} x} \partial_{x} \psi_{R, L}+\psi_{R, L}\left[\frac{2}{x^{2}} \sqrt{\epsilon_{E}}+\frac{2}{x^{2}} \sqrt{\epsilon_{B}}\left(\xi_{Q} \mp x\right)\right]
\end{aligned}
$$

where we have changed variables to $x=-k \tau=k / a H$.

These are the full equations that will be used for numerical calculations, but first it will prove useful to study a slow-roll expansion of these equations to get a feel for what is going on. Ignoring anything higher order in slow-roll parameters than $\sqrt{\epsilon_{i}}$ and ignoring the RHS of equation of motion for $t_{R, L}$, which has a negligible effect on the evolution of $t_{R, L}$, we arrive at the much simpler equations of motion:

$$
\partial_{x}^{2} \psi_{R, L}+\psi_{R, L}\left(1-\frac{2}{x^{2}}\right)=\frac{2 \sqrt{\epsilon_{E}}}{x} \partial_{x} t_{R, L}+\frac{2 \sqrt{\epsilon_{B}}}{x^{2}}\left(\xi_{Q} \mp x\right) t_{R, L}
$$

and

$$
\partial_{x}^{2} t_{R, L}=-t_{R, L}\left[1 \mp \frac{2}{x}\left(\xi_{\chi}+\xi_{Q}\right)+\frac{2}{x^{2}} \xi_{\chi} \xi_{Q}\right]
$$

Examining first the equation of motion for the tensor perturbations of the gauge field (3.3.32), we see that well before horizon-crossing when $x \gg 1, \partial_{x}^{2} t_{R, L} \sim-t_{R, L}$ : both right- and left-moving modes are not growing. However as $x$ becomes smaller but still larger than 1 , there reaches a point where the dominant term inside the bracket on the RHS of 3.3 .32$)$ is $\mp \frac{2}{x}\left(\xi_{\chi}+\xi_{Q}\right)$ if $\xi_{\chi} \sim \xi_{Q}$ is large enough so that $\frac{2}{x}\left(\xi_{\chi}+\xi_{Q}\right)>1$ while $\frac{1}{x} \gg \frac{1}{x^{2}}$. We see immediately that in this region where $\mp \frac{2}{x}\left(\xi_{\chi}+\xi_{Q}\right)$ is dominant, that the right-moving tensor mode, $t_{R}$, will become a growing mode. Around horizoncrossing $x \sim 1$, the term $\frac{2}{x^{2}} \xi_{\chi} \xi_{Q}$ becomes the dominant term and in this region both $t_{R, L}$ are not growing modes and remain as such for $x<1$. Therefore, we see that $t_{L}$ will never become a growing mode and is of no interest, whereas it is possible that $t_{R}$ can become a growing mode for a short period of time (known as a transient instability) just before horizon-crossing $x \gtrsim 1$. Examining now the equation of motion for the gravitational tensor perturbations, (3.3.31), we see that well before horizon-crossing, $x \gg 1$, the RHS of the equation is heavily suppressed and therefore there is no source term for the modes. However as $x$ approaches 1 , the terms on the RHS can become

\footnotetext{
${ }^{9}$ In practice this is not difficult to achieve and a value of $\xi_{Q} \gtrsim 2$ will usually suffice.
} 


\section{ENHANCEMENT OF THE PRIMORDIAL GRAVITATIONAL WAVE SPECTRUM}

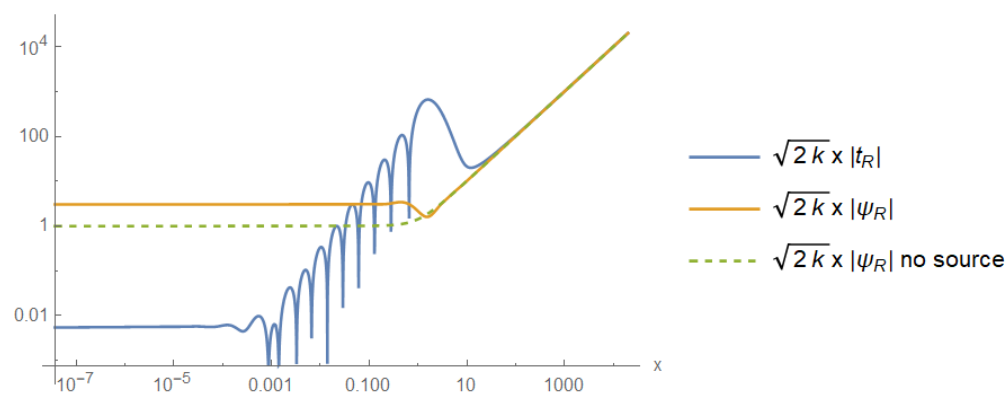

Figure 3.8: Plot of $\sqrt{2 k} x\left|\psi_{R}\right|$ and $\sqrt{2 k} x\left|t_{R}\right|$ against $x=k / a H$ with horizon-crossing, $x=1$, taken to be 60 e-folds before the end of inflation for the background solution shown in FIGs 3.3 3.7. The right-helicity mode of the gravitational wave tensor receives a modest enhancement. For reference the non-sourced solution for $\psi_{R}$ is also plotted (dashed line).

important if the gauge field mode is large enough, and since $t_{R}$ is a growing mode in this region, we see that the right-helicity mode of the gravity wave tensor, $\psi_{R}$, receives a source term and can be amplified if $t_{R}$ is large enough.

Solving equations (3.3.29)-3.3.30 numerically with the background system shown above (FIGs 3.3 3.7) leads to the solution for $\psi_{R}$ and $t_{R}$ shown in FIG. 3.8. We use standard Bunch-Davies initial conditions

$$
t_{R}\left(x_{i n}\right)=\psi_{R}\left(x_{i n}\right)=\frac{1}{\sqrt{2 k}}, \quad t_{R}^{\prime}\left(x_{i n}\right)=\psi_{R}^{\prime}\left(x_{i n}\right)=\frac{i}{\sqrt{2 k}}
$$

where $x_{i n}$ is some relatively large number numerically approximating infinity, we take to be $x_{i n}=2 \times 10^{4}$. The right-helicity mode of the gravitational wave tensor, $\psi_{R}$, receives a modest enhancement (compared to the non-sourced, dashed-line solution) due to its coupling to $t_{R}$ which experiences a transient instability that temporarily increases its magnitude round $x \sim 1$.

The right/left-helicity tensor power spectrum is given by

$$
\mathcal{P}_{t}^{R, L}=\frac{H^{2}}{\pi^{2}}\left|\sqrt{2 k} x \psi_{R, L}\right|^{2}
$$

where the non-sourced solution freezes out $\left|\sqrt{2 k} x \psi_{R, L}\right|^{2} \rightarrow 1$, and the full tensor power spectrum is given by $\mathcal{P}_{t}=\mathcal{P}_{t}^{R}+\mathcal{P}_{t}^{L}$ leading to $\mathcal{P}_{t}=\frac{2 H^{2}}{\pi^{2}}$ in the standard pure inflationary scenario. The sourced solution leads to $r=\frac{\mathcal{P}_{t}}{\mathcal{P}_{s}}=0.0417$, compared to the non-sourced solution which gives $r=\frac{\mathcal{P}_{t}}{\mathcal{P}_{s}}=0.00482$, so we see an enhancement of roughly an order of magnitude. Given that the vast majority of the tensor spectrum is provided by the 
right-helicity mode, we see that this spectrum is highly chiral.

\subsubsection{Scalar perturbations}

We will now discuss the scalar perturbations to this system: in particular we are interested to see whether the introduction of the gauge field and axion has a nonnegligible effect on the scalar power spectrum, $\mathcal{P}_{s}$.

We define the scalar perturbations as in [3] and, for simplicity, ignore scalar perturbations to the metric, which contribute negligibly ${ }^{10}$ [80. Considering the scalar part of 3.3 .22$)$ gives

$$
\begin{aligned}
& \Phi=(\phi(\tau)+\delta \phi(\tau, z), \chi(\tau)+\delta \chi(\tau, z)) \\
& A_{\mu}^{1}=a(\tau)(0, Q(\tau)+\delta Q(\tau, z), 0,0) \\
& A_{\mu}^{2}=a(\tau)(0,0, Q(\tau)+\delta Q(\tau, z), 0) \\
& A_{\mu}^{3}=a(\tau)\left(\partial_{z} Y(\tau, z), 0,0, Q(\tau)+\delta Q(\tau, z)+\partial_{z}^{2} M(\tau, z)\right) .
\end{aligned}
$$

where the again the wavevector is directed along the $z$-axis, $\vec{k}=k_{z} \hat{z}$. and we use a flat FRW metric in conformal time $d s^{2}=a(\tau)^{2} \eta_{\mu \nu} d x^{\mu} d x^{\nu}$. It is also convenient to redefine the perturbations in comoving form:

$$
\delta \phi=\frac{\Delta_{\phi}}{a}, \quad \delta \chi=\frac{\Delta_{\chi}}{a}, \quad \delta Q=\frac{\Delta_{1}}{\sqrt{2} a}, \quad M=\frac{a \mathrm{~g} Q \Delta_{1}+\sqrt{k^{2}+2 a^{2} \mathrm{~g}^{2} Q^{2}} \Delta_{2}}{\sqrt{2} \mathrm{~g} a^{2} k^{2} Q} .
$$

The equations for the scalar perturbations are found from the second order action shown in Appendix B where we also include the equations of motion for $\Delta_{\phi}, \Delta_{\chi}, \Delta_{1}, \Delta_{2}$. Evolving these equations with standard Bunch-Davies initial conditions, $\Delta_{i}\left(x_{i n}\right)=$ $1 / \sqrt{2 k}$ and $\Delta_{i}^{\prime}\left(x_{i n}\right)=i / \sqrt{2 k}$ with $i=\phi, \chi, 1,2$ and again we choose $x_{i n}=20000$. The evolution of the scalar perturbations is plotted in FIG. 3.9. In the case where the inflationary perturbation, $\Delta_{\phi}$ is massless (corresponding to a perfectly flat potential), after horizon-crossing, $\sqrt{2 k} x \Delta_{\phi} \rightarrow 1, x<1$. As can be seen, the gauge and axion perturbations are sub-dominant in comparison to the inflaton perturbation, $\Delta_{\phi}$.

\footnotetext{
${ }^{10}$ When we consider this mechanism applied to KMI (see chapter 5), we will explicitly demonstrate that the scalar perturbations of the metric (which are non-dynamical) make no difference to the evolution of the dynamical scalar perturbations.
} 


\section{ENHANCEMENT OF THE PRIMORDIAL GRAVITATIONAL WAVE SPECTRUM}

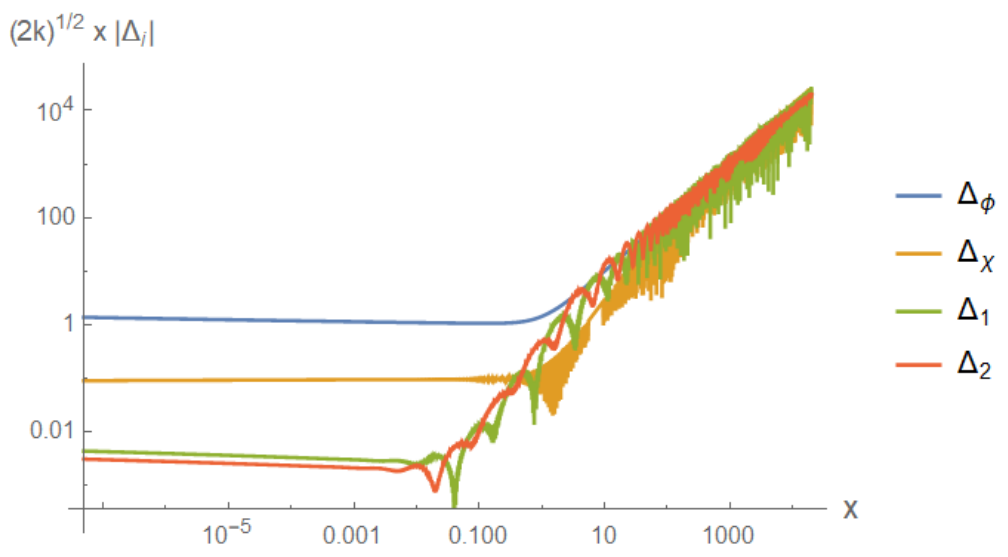

Figure 3.9: Plot of $\sqrt{2 k} x\left|\Delta_{\phi}\right|, \sqrt{2 k} x\left|\Delta_{\chi}\right|, \sqrt{2 k} x\left|\Delta_{1}\right|$ and $\sqrt{2 k} x\left|\Delta_{2}\right|$ against $x=k / a H$ with horizon-crossing, $x=1$, taken to be 60 e-folds before the end of inflation for the background solution shown in FIGs 3.33 .7

\subsubsection{Backreaction}

We have shown that the scalar perturbations from the gauge field are sub-dominant relative to that of the inflaton. However, the (right-helicity) tensor perturbation, $t_{R}$, is by necessity large and we need to check whether this tensor perturbation contributes significantly to the background equations of motion. If there were a large contribution from the tensor perturbation to the background equation of the motion, our assumption that, at the background level, we can take the gauge field in the isotropic configuration

$$
A_{i}^{A}=\delta_{i}^{A} a(t) Q(t)
$$

would be inconsistent. We follow [3] and in order to estimate this backreaction, we will take advantage of an analytic solution for the tensor fluctuation to the gauge field, $t_{R}$. The equation of motion for $t_{R}$ is given by (3.3.32), which has an analytic solution (assuming $\xi_{Q}$ and $\xi_{\chi}$ are constant) given by

$$
t_{R}(x)=\frac{1}{\sqrt{2 k}} i^{\beta} W_{\beta, \alpha}(-2 i x)
$$

where $W_{a, b}(z)$ is the Whittaker function, $\alpha:=-i \sqrt{2 \xi_{Q} \xi_{\chi}-\frac{1}{4}}$ and $\beta:=-i\left(\xi_{Q}+\xi_{\chi}\right)$. Of course this solution is merely a mode function, and we start by promoting $t_{R}$ and its conjugate $t_{L}$ to quantum operators. Starting with the definitions for $T_{+}, T_{\times}$, we arrive at the following forms for $\hat{t}_{R}$ and $\hat{t}_{L}$ : 


$$
\begin{aligned}
& \hat{t}_{R}(z)=\int \frac{d^{3} k}{(2 \pi)^{3}}\left\{t_{R}(k) \hat{a}_{R}(k) e^{i \vec{k} \cdot \vec{z}}+t_{L}^{*}(k) \hat{a}_{L}^{\dagger}(k) e^{-i \vec{k} \cdot \vec{z}}\right\} \\
& \hat{t}_{L}(z)=\int \frac{d^{3} k}{(2 \pi)^{3}}\left\{t_{L}(k) \hat{a}_{L}(k) e^{i \vec{k} \cdot \vec{z}}+t_{R}^{*}(k) \hat{a}_{R}^{\dagger}(k) e^{-i \vec{k} \cdot \vec{z}}\right\}
\end{aligned}
$$

where $\hat{t}_{R}=\hat{t}_{L}^{\dagger}$ but $t_{R} \neq t_{L}^{*}$. The creation and annihilation operators satisfy:

$$
\begin{aligned}
& {\left[\hat{a}_{R}(p), \hat{a}_{R}^{\dagger}(q)\right]=(2 \pi)^{3} \delta^{3}(\vec{p}-\vec{q})} \\
& {\left[\hat{a}_{L}(p), \hat{a}_{L}^{\dagger}(q)\right]=(2 \pi)^{3} \delta^{3}(\vec{p}-\vec{q})}
\end{aligned}
$$

and all other combinations are zero. The mode function $t_{R}$ and its conjugate $t_{R}^{*}$ are given by (3.3.38) and $t_{L}, t_{L}^{*}$ are assumed to be negligible over the relevant region. The following integrals prove useful:

$$
\begin{aligned}
\left\langle 0\left|\hat{t}_{R} \hat{t}_{L}\right| 0\right\rangle & =\int \frac{d^{3} k}{(2 \pi)^{3}}\left|t_{R}\right|^{2} \\
\left\langle 0\left|\hat{t}_{L} \hat{t}_{R}\right| 0\right\rangle=\int \frac{d^{3} k}{(2 \pi)^{3}}\left|t_{L}\right|^{2} \sim 0 & \\
\left\langle 0\left|\hat{t}_{R} \partial_{t} \hat{t}_{L}\right| 0\right\rangle & =\int \frac{d^{3} k}{(2 \pi)^{3}} t_{R}\left(\partial_{t} t_{R}^{*}\right) \\
\left\langle 0\left|\hat{t}_{L} \partial_{t} \hat{t}_{R}\right| 0\right\rangle & =\int \frac{d^{3} k}{(2 \pi)^{3}} t_{L}\left(\partial_{t} t_{L}^{*}\right) \sim 0 \\
\left\langle 0\left|\partial_{t} \hat{t}_{L} \hat{t}_{R}\right| 0\right\rangle & =\int \frac{d^{3} k}{(2 \pi)^{3}}\left(\partial_{t} t_{L}\right) t_{L}^{*} \sim 0 \\
\left\langle 0\left|\partial_{t} \hat{t}_{R} \hat{t}_{L}\right| 0\right\rangle & =\int \frac{d^{3} k}{(2 \pi)^{3}}\left(\partial_{t} t_{R}\right) t_{R}^{*} \\
\left\langle 0\left|\hat{t}_{R} \partial_{z} \hat{t}_{L}\right| 0\right\rangle & =\int \frac{d^{3} k}{(2 \pi)^{3}}(-i k)\left|t_{R}\right|^{2} \\
\left\langle 0\left|\hat{t}_{L} \partial_{z} \hat{t}_{R}\right| 0\right\rangle & =\int \frac{d^{3} k}{(2 \pi)^{3}}(-i k)\left|t_{L}\right|^{2} \sim 0 \\
\left\langle 0\left|\partial_{z} \hat{t}_{R} \hat{t}_{L}\right| 0\right\rangle & =\int \frac{d^{3} k}{(2 \pi)^{3}} i k\left|t_{R}\right|^{2}
\end{aligned}
$$




$$
\begin{aligned}
& \left\langle 0\left|\partial_{z} \hat{t}_{L} \hat{t}_{R}\right| 0\right\rangle=\int \frac{d^{3} k}{(2 \pi)^{3}} i k\left|t_{L}\right|^{2} \sim 0 \\
& \left\langle 0\left|\partial_{t} \hat{t}_{R} \partial_{t} \hat{t}_{L}\right| 0\right\rangle=\int \frac{d^{3} k}{(2 \pi)^{3}}\left|\partial_{t} t_{R}\right|^{2} \\
& \left\langle 0\left|\partial_{t} \hat{t}_{L} \partial_{t} \hat{t}_{R}\right| 0\right\rangle=\int \frac{d^{3} k}{(2 \pi)^{3}}\left|\partial_{t} t_{L}\right|^{2} \sim 0 \\
& \left\langle 0\left|\partial_{z} \hat{t}_{R} \partial_{z} \hat{t}_{L}\right| 0\right\rangle=\int \frac{d^{3} k}{(2 \pi)^{3}} k^{2}\left|t_{R}\right|^{2} \\
& \left\langle 0\left|\partial_{z} \hat{t}_{L} \partial_{z} \hat{t}_{R}\right| 0\right\rangle=\int \frac{d^{3} k}{(2 \pi)^{3}} k^{2}\left|t_{L}\right|^{2} \sim 0
\end{aligned}
$$

With these in hand, we can find the equation of motion for the gauge field, $Q$, including the backreaction of the tensor perturbations. 3.3.7 becomes

$$
\ddot{Q}+3 H \dot{Q}+\left(\dot{H}+2 H^{2}\right) Q+2 \mathrm{~g}^{2} Q^{3}-\frac{\mathrm{g} \lambda}{f} \dot{\chi} Q^{2}+\frac{\mathrm{g}}{3 a^{2}} \int \frac{d^{3} k}{(2 \pi)^{3}} \frac{k}{a}\left|t_{R}\right|^{2}+\frac{\mathrm{g} \xi_{\chi} H}{3 a^{2}} \int \frac{d^{3} k}{(2 \pi)^{3}}\left|t_{R}\right|^{2}=0 .
$$

The backreaction contribution can be estimated by defining

$$
\mathcal{T}_{B R}^{Q}:=\frac{\mathrm{g}}{3 a^{2}} \int \frac{d^{3} k}{(2 \pi)^{3}} \frac{k}{a}\left|t_{R}\right|^{2}+\frac{\mathrm{g} \xi_{\chi} H}{3 a^{2}} \int \frac{d^{3} k}{(2 \pi)^{3}}\left|t_{R}\right|^{2} \simeq \frac{\mathrm{g} H^{3}}{12 \pi^{2}}\left(\xi_{\chi} \beta_{1}\left(\xi_{Q}\right)+\beta_{2}\left(\xi_{Q}\right)\right)
$$

where

$$
\begin{aligned}
& \beta_{1}\left(\xi_{Q}\right)=\int_{0}^{x_{\max }} d x x\left|i^{\beta} W_{\beta, \alpha}(-2 i x)\right|^{2}, \\
& \beta_{2}\left(\xi_{Q}\right)=\int_{0}^{x_{\max }} d x x^{2}\left|i^{\beta} W_{\beta, \alpha}(-2 i x)\right|^{2},
\end{aligned}
$$

we have used the analytic solution for $t_{R}, 3.3 .38$, and used the cut-off described in [3], $x_{\max } \equiv \xi_{Q}+\xi_{\chi}+\sqrt{\xi_{Q}^{2}+\xi_{\chi}^{2}}$ which encompasses the main region for which $t_{R}$ is enhanced by the transient instability near $x=1$. As can be seen in FIG. 3.10, the backreaction term, $\mathcal{T}_{B R}^{Q}$ (red), on the equation of motion for $Q$ is sub-dominant relative to the leading terms in that equation, $2 \mathrm{~g} H \xi_{\chi} Q^{2}$ (blue), $2 \mathrm{~g}^{2} Q^{3}$ (orange), and $2 H^{2} Q$. The backreaction is under control and our assumption that we can take

$$
A_{i}^{A}=\delta_{i}^{A} a(t) Q(t)
$$




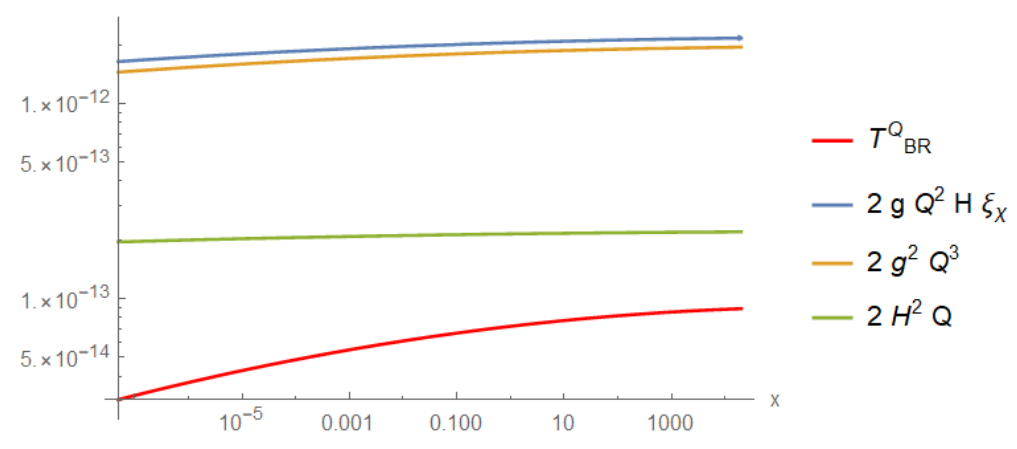

Figure 3.10: The backreaction, $\mathcal{T}_{B R}^{Q}$, (red), along with the dominant contributions of 3.3.7): $2 \mathrm{gH} \xi_{\chi} Q^{2}$ (blue), $2 \mathrm{~g}^{2} Q^{3}$ (orange), and $2 H^{2} Q$ plotted against $x=\frac{k}{a H}$ for the background solution shown in FIGs 3.3 3.7 and horizon-crossing, $x=1$, chosen to be 60 e-folds before the end of inflation.

is well justified.

\subsection{Summary}

In this chapter, we have presented two models where a gauge field can be supported during inflation by its coupling to an axion through the Chern-Simons-like term $\chi F \tilde{F}$. First, we presented the general features of the model put forward in [2, 48]. We showed why a vector field needs a source during inflation for it not to dilute rapidly due to the exponential expansion of the universe; and showed how, if the vector field were supported successfully by this coupling to the axion, it can contribute a source term to the primordial gravitational wave equation of motion.

Secondly, we considered a concrete example of the model put forward in [3], where instead of a vector field, an $S U(2)$ gauge field is coupled to an axion. We demonstrated how this coupling leads to the gauge field being sustained during inflation and how the gauge field can successfully act as a source for the primordial gravitational wave spectrum and enhance the tensor-to-scalar ratio, leading to a highly chiral tensor spectrum. We also showed that the scalar perturbations are under control and that the backreaction of the tensor perturbation to the gauge field does not contribute significantly to the background equations of motion. 
3. ENHANCEMENT OF THE PRIMORDIAL GRAVITATIONAL WAVE SPECTRUM 


\section{Chapter 4}

\section{Introduction to Kähler moduli inflation}

In this chapter, we will discuss the basics of two models of inflation whose inflatons are Kähler moduli. First, we will introduce the form of the potentials of both Kähler moduli inflation [49] and fibre inflation [76]. We will then look at some explicit examples of these two models. In chapter 5, we will attempt to apply the mechanism discussed in chapter 3 that leads to an enhancement of the inflationary tensor spectrum to Kähler moduli inflation and fibre inflation. With this in mind, we will be concerned with examples of Kähler and fibre inflation that are most applicable to successfully admitting this enhancement mechanism. This is of particular interest in Kähler moduli inflation, because, as we will see, it generally predicts a low value of the tensor-to-scalar ratio, and it is important to see if this value can be enhanced to observable $\left(r \gtrsim 10^{-3}\right)$ values, thus providing a lifeline to Kähler moduli inflation if inflationary tensor modes are ever detected. Kähler moduli inflation also naturally contains, in its particle content, axions with a non-perturbative superpotential term $W \supset e^{-a_{a}\left(\tau_{a}+i b_{a}\right)}$ (with $\tau_{a}$ Kähler moduli and $b_{a}$ their axionic superpartners), and therefore we will be interested in seeing if these native axions can be included in the inflationary evolution without disturbing the evolutions of the other field:1.

In general, Kähler moduli inflation can have many fields, which makes numerical simulation (particularly at the order of perturbations) more difficult. Therefore, much

\footnotetext{
${ }^{1}$ In the phenomenological example presented in chapter 3 the axion is included in an ad-hoc manner, whereas in Kähler inflation, axions appear naturally in the inflationary action, but are usually set to their minima for ease of calculation.
} 


\section{INTRODUCTION TO KÄHLER MODULI INFLATION}

of this chapter will be concerned with demonstrating that some of these fields can be set to their minima and removed from the equations of motion without affecting the evolutions of the other (important) fields and without significantly affecting the inflationary predictions of the model, thus allowing us to use a simplified model when applying the enhancement mechanism. The parameters in the examples in this chapter are taken from [76] for Kähler moduli inflation and [50] for fibre inflation.

In this chapter, we will not include the coupling between the Kähler moduli and the gauge field. However, all the work we do in this section is with a view to applying the enhancement mechanism of chapter 3 to Kähler moduli inflation. The interested reader is directed to Appendix A for some basics on Kähler moduli and Calabi-Yau compactification where a quick review is given of these concepts, but any familiarity with these concepts should not be required to understand the remainder of this thesis.

\subsection{Basics of Kähler moduli inflation}

The starting point for building an inflationary model from a high-energy theory is to derive the (low-energy) Lagrangian for an $\mathcal{N}=1$ four-dimensional supergravity theory. The bosonic fields of a general four-dimensional $\mathcal{N}=1$ supergravity theory are the spacetime metric, $g_{\mu \nu}$, gauge potentials, $A_{\mu}^{A}$, and complex scalar fields, $\Phi^{a}$. The lowenergy interactions of the scalar fields are determined by the superpotential, $W\left(\Phi^{a}\right)$, which is a holomorphic function of $\Phi^{a}$; the Kähler potential, $K\left(\Phi^{a}, \bar{\Phi}^{\bar{a}}\right)$, which is a real, analytic function of the scalars; and the gauge kinetic function, $\mathbf{f}\left(\Phi^{a}\right)$, which is a holomorphic function of $\Phi^{a}$ and provides the coupling between the scalars and gauge fields. Considering a theory without gauge fields, the scalar field Lagrangian is:

$$
\mathcal{L}_{\Phi}=-K_{a \bar{b}} \partial_{\mu} \Phi^{a} \partial^{\mu} \bar{\Phi}^{\bar{b}}-V
$$

where $K_{a \bar{b}}:=\partial_{a} \partial_{\bar{b}} K$ is the Kähler metrid ${ }^{2}$, and the potential is given by

$$
V\left(\Phi^{a}, \bar{\Phi}^{\bar{b}}\right)=e^{K / M_{\mathrm{pl}}^{2}}\left[K^{a \bar{b}} D_{a} W \overline{D_{b} W}-\frac{3}{M_{\mathrm{pl}}^{2}}|W|^{2}\right]
$$

where $K^{a \bar{b}}$ is the inverse Kähler metric and $D_{a} W:=\partial_{a} W+\frac{1}{M_{\mathrm{pl}}^{2}}\left(\partial_{a} K\right) W$.

\footnotetext{
${ }^{2}$ With $\partial_{a}=\partial / \partial \Phi^{a}$ and $\partial_{\bar{a}}=\partial / \partial \bar{\Phi}^{\bar{a}}$.
} 
Kähler moduli inflation [49] is an inflationary model derived from type IIB string theory. Compactification of a ten-dimensional string theory onto a (six-dimensional) Calabi-Yau manifold ${ }^{3}$ leads to an abundance of moduli (including the Kähler moduli, complex structure moduli and the axio-dilaton) that may appear as scalar fields in the four-dimensional cosmological action. These moduli are in fact at first glance problematic - they are massless and couple to Standard Model matter particles and lead to effects that have not been observed in nature. Therefore an important step in the compactification process is moduli stabilisation, the process by which a potential is found for the moduli that makes them massive enough so that their effect on the Standard Model fields at low energy is negligible. This process of moduli stabilisation leads to a scalar potential that, in the case of type IIB flux compactifications, can cause inflation through one or more Kähler moduli and stabilises all other moduli (in particular the axio-dilaton, all complex structure moduli, and the remaining Kähler moduli). The topic of moduli stabilisation and the derivations that lead to the superpotential and Kähler potential for Kähler moduli inflation from type IIB string theory are beyond the scope of this thesis. The interested reader is directed to 82 for a discussion of moduli stabilisation in type IIB string theory, Chapter 14 in 83 for a general discussion of moduli stabilisation via flux compactification, and [84, 85] for the explicit flux compactification mechanism that is used in the moduli stabilisation of Kähler modulus inflation and in particular justifies the forms of the volume, Kähler potential and superpotential used below.

The general idea however is as follows: a compactification from ten-dimensional type IIB string theory is made onto a Calabi-Yau three-fold (referring to the three complex dimensions of the CY), $\mathcal{M}$, with the addition of two non-trivial but sourceless 3-form fluxes, $F_{3}, H_{3}$ (hence the name flux compactification), being included in the 10-D action. These fluxes generate a superpotential for the Calabi-Yau moduli

$$
W_{\text {tree }}=\int_{\mathcal{M}} G_{3} \wedge \Omega
$$

\footnotetext{
${ }^{3}$ Calabi-Yau manifolds are complex manifolds that are often used as compactification manifolds due to some useful properties. Calabi-Yaus have structures determined by what are known as moduli - for example the Kähler moduli are related to volume of the manifold. See Appendix A if interested, but the important point from our perspective is that these moduli contain no four-dimensional spacetime index and can therefore appear as scalars in the effective four-dimensional cosmological action.
} 


\section{INTRODUCTION TO KÄHLER MODULI INFLATION}

where $G_{3}=F_{3}+i S H_{3}$ with $S=e^{-\phi}+i C_{0}$ the axio-dilaton of type IIB string theory and $\Omega$ is $\mathcal{M}$ 's $(3,0)$-form which as may be seen in Appendix A is related to $\mathcal{N}$ 's complex structure moduli, $u_{\alpha}$, and $\Omega$ should be viewed as being implicitly dependent on $u_{\alpha}$. The Kähler potential for the theory takes the form

$$
\frac{K}{M_{\mathrm{pl}}^{2}}=-2 \ln \mathcal{V}-\ln (S+\bar{S})-\ln \left(-i \int_{\mathcal{M}} \Omega \wedge \Omega\right)
$$

where $\mathcal{V}$ is the volume of the Calabi-Yau, $\mathcal{M}$, and should be viewed as being implicitly dependent on the Kähler moduli. The form for the superpotential, $W_{\text {tree }}$, stabilises both the axio-dilaton and the complex structure moduli by minimising it with $D_{a} W_{\text {tree }}=0$. The Kähler moduli do not appear in this superpotential so at this stage they are still massless. The Kähler potential can be rewritten after stabilising the complex structure moduli and axio-dilaton

$$
K=K_{c s}-\ln \left(\frac{2}{g_{s}}\right)+K_{T}
$$

where $K_{c s}=-\ln \left(\left\langle-i \int_{\mathcal{M}} \Omega \wedge \Omega\right\rangle\right)$ and $\ln \left(\frac{2}{g_{s}}\right)$ (with $g_{s}=e^{\langle\phi\rangle}$ the string coupling) are constant contributions since the relevant fields have been stabilised, and $K_{T}=K_{0}=$ $-2 \ln \mathcal{V}$. The superpotential for this system is constant: $W=W_{0}:=\left\langle W_{\text {tree }}\right\rangle$. Define the multiplet $T^{a}=\tau_{a}+i b_{d}$ where $\tau_{a}$ is a 4-cycle volume called a Kähler modulus and $b_{a}$ is its axionic partner: a component of a 4 -form, $C_{4}$, integrated on this cycle: $b_{a}=\int_{\tau_{a}} C_{4}$. A Kähler potential, $K$, is no-scale if it satisfies $K^{a \bar{b}} \partial_{a} K \partial_{\bar{b}} K=3$. Then with a constant superpotential, $W=W_{0}$ :

$$
V=e^{K / M_{\mathrm{pl}}^{2}}\left(K^{a \bar{b}} D_{a} W \overline{D_{\bar{b}} W}-\frac{3}{M_{\mathrm{pl}}^{2}}|W|^{2}\right)=0
$$

since $D_{a} W_{0}=0$ and $\partial_{a} K=\partial_{a} K_{0}$. With a no-scale Kähler potential, the Kähler moduli are therefore exactly massless at this order. The volume in Kähler inflation is chosen so that $K_{0}=-2 \ln (\mathcal{V})$ is no-scale.

\subsubsection{Kähler modulus inflation potential}

The superpotential receives no contributions from $\alpha^{\prime}$ and $g_{s}$ expansions. But a potential for the Kähler moduli can be generated by including non-perturbative corrections to

\footnotetext{
${ }^{4}$ We will refer to both $\tau_{a}$ and $T^{a}$ as Kähler moduli.
} 
the superpotential. In particular if a gauge field, $A^{A}$, with gauge group $S U(N)$, is included in the low-energy effective action, it is coupled to the scalar fields through the gauge kinetic function, $f_{A}$, and contributes to the Lagrangian

$$
\mathcal{L} \supset-\frac{\operatorname{Re}\left(f_{A}\right)}{4} F_{\mu \nu}^{A} F^{A \mu \nu}+\frac{\operatorname{Im}\left(f_{A}\right)}{4} F_{\mu \nu}^{A} \tilde{F}^{A \mu \nu}
$$

where $F^{A}=d A^{A}-A^{A} \wedge A^{A}, \tilde{F}^{A \mu \nu}=\frac{\epsilon^{\mu \nu \rho \sigma}}{2 \sqrt{-g}} F_{\rho \sigma}^{A}$ with $g$ the metric determinant, and $f_{A}$ is a holomorphic function of the scalar fields, in particular for our example, $T^{a}$. This coupling to an $S U(N)$ gauge field generates a contribution to the superpotential:

$$
W=\frac{g_{s}^{3 / 2} M_{\mathrm{pl}}^{3}}{\sqrt{4 \pi}}\left(W_{0}+\sum_{a} A_{a} e^{-a_{a} T^{a}}\right)
$$

where $A_{a}$ depend on the complex structure moduli so are constants after flux compactification stabilises these moduli, $a_{a}=2 \pi / N$, and we have now included the normalisation term for the superpotential as given in [85]. The Kähler potential does receive perturbative corrections, and to first order in $\alpha^{\prime}, K_{T}$ becomes

$$
K_{T}=K_{0}-2 \ln \left(1+\frac{\hat{\xi}}{2 \mathcal{V}}\right)=-2 \ln \left(\mathcal{V}+\frac{\hat{\xi}}{2}\right)
$$

where $\hat{\xi}:=\xi / g_{s}^{3 / 2}, \xi=-\frac{\chi(\mathcal{N}) \zeta(3)}{2(2 \pi)^{3}}, \chi(\mathcal{M})$ is called the Euler number of $\mathcal{M}$ that depends on the number of moduli in $\mathcal{M}$, and $\zeta$ is the Riemann zeta function with $\zeta(3) \sim 1.2$.

We are now interested to see what potential is generated by $4.1 .8-4.1 .9$ through 4.1.2). To do this, we first need a form for the volume in terms of the Kähler moduli. In this thesis, two models of Kähler moduli inflation are studied; namely, the eponymous Kähler moduli inflation [49] with a detailed analysis in [76], and fibre inflation [50]. We start with Kähler moduli inflation, a model in which the volume of the Calabi-Yau, $\mathcal{N}$, is taken to have the "Swiss cheese" form

$$
\mathcal{V}=\alpha\left(\tau_{1}^{3 / 2}-\sum_{a=2}^{n} \lambda_{a} \tau_{a}^{3 / 2}\right)
$$

where $\alpha$ and $\lambda_{a}$ are model-dependent constants, and it is assumed that $\tau_{1} \gg \tau_{a}, a>1$ so that the $\tau_{1}$ modulus forms the block of the "Swiss cheese" and the remaining $\tau_{a}$ form holes. The full Kähler potential written in terms of the multiplet Kähler moduli 


\section{INTRODUCTION TO KÄHLER MODULI INFLATION}

becomes

$$
\frac{K}{M_{\mathrm{pl}}^{2}}=\frac{K_{c s}}{M_{\mathrm{pl}}^{2}}-\ln \left(\frac{2}{g_{s}}\right)-2 \ln \left[\frac{\alpha}{2 \sqrt{2}}\left(\left(T^{1}+\bar{T}^{1}\right)^{3 / 2}-\sum_{a=2}^{n} \lambda_{a}\left(T^{a}+\bar{T}^{a}\right)^{3 / 2}\right)+\frac{\hat{\xi}}{2}\right] .
$$

In fact for the sake of simplicity, ignoring the constant contributions to the scalar potential $K_{c s}-\ln \left(\frac{2}{g_{s}}\right)$ which we will take to be an overall normalisation factor of the potential (through $\left.e^{K / M_{\mathrm{pl}}^{2}}\right) 5^{5}$ we write

$$
K=K_{T}=K_{0}-2 \ln \left(1+\frac{\hat{\xi}}{2 \mathcal{V}}\right)
$$

with $K_{0}=-2 \ln \mathcal{V}$ and we have set $M_{\mathrm{pl}}=1$. With this form for the volume, we have the following relations

$$
\begin{aligned}
& \partial_{1} K=\partial_{\overline{1}} K=-\frac{3 \alpha \sqrt{\tau_{1}}}{2 \mathcal{V}+\hat{\xi}}=-\frac{3 \alpha^{2 / 3}\left(\mathcal{V}+\alpha \sum_{a=2}^{n} \lambda_{a} \tau_{a}\right)^{1 / 3}}{2 \mathcal{V}+\hat{\xi}}, \\
& \partial_{a} K=\partial_{\bar{a}} K=\frac{3 \alpha \lambda_{a} \sqrt{\tau_{a}}}{2 \mathcal{V}+\hat{\xi}}, \quad a>1
\end{aligned}
$$

which leads to a Kähler metric, $K_{a \bar{b}}=\partial_{a} \partial_{\bar{b}} K$, of the form

$$
\begin{gathered}
K_{1 \overline{1}}=\frac{3 \alpha^{4 / 3}\left(4 \mathcal{V}-\hat{\xi}+6 \alpha \sum_{a=2}^{n} \lambda_{a} \tau_{a}^{3 / 2}\right)}{4(2 \mathcal{V}+\hat{\xi})^{2}\left(\mathcal{V}+\alpha \sum_{a=2}^{n} \lambda_{a} \tau_{a}^{3 / 2}\right)^{1 / 3}}, K_{1 \bar{b}}=-\frac{9 \alpha^{5 / 3} \lambda_{b} \sqrt{\tau_{b}}\left(\mathcal{V}+\alpha \sum_{c=2}^{n} \lambda_{c} \tau_{c}^{3 / 2}\right)^{1 / 3}}{2(2 \mathcal{V}+\hat{\xi})^{2}} \\
K_{a \bar{b}}=\frac{9 \alpha^{2} \lambda_{a} \lambda_{b} \sqrt{\tau_{a}} \sqrt{\tau_{b}}}{2(2 \mathcal{V}+\hat{\xi})^{2}}, \quad K_{a \bar{a}}=\frac{3 \alpha \lambda_{a}\left(2 \mathcal{V}+\hat{\xi}+6 \alpha \lambda_{a} \tau_{a}^{3 / 2}\right)}{4 \sqrt{\tau_{a}}(2 \mathcal{V}+\hat{\xi})^{2}}
\end{gathered}
$$

\footnotetext{
${ }^{5}$ Including the normalisation in front of $W$ as given in 4.1.8), we see our overall normalisation factor will become $\frac{g_{s}^{4}}{8 \pi} e^{K_{c s}}$. Furthermore in [85], it assumed that $e^{K_{c s}} \sim \mathcal{O}(1)$ so our normalisation is expected to be $\sim \frac{g_{s}^{4}}{8 \pi}$. In the examples of Kähler moduli inflation we will consider that are taken from [76], this whole normalisation term is absorbed into the parameters inside the potential. Meanwhile in the fibre inflation example we consider taken from [50], the power spectrum is explicitly normalised with $\frac{g_{s}^{4}}{8 \pi}$. From now on we will write the potential without this normalisation factor.
} 
and an inverse Kähler metric, $K^{a \bar{b}}$, of

$$
\begin{aligned}
K^{1 \overline{1}} & =\frac{4(2 \mathcal{V}+\hat{\xi})\left(\mathcal{V}+\alpha \sum_{a=2}^{n} \lambda_{a} \tau_{a}^{3 / 2}\right)^{1 / 3}\left(2 \mathcal{V}+\hat{\xi}+6 \alpha \sum_{a=2}^{n} \lambda_{a} \tau_{a}^{3 / 2}\right)}{3 \alpha^{4 / 3}(4 \mathcal{V}-\hat{\xi})} \\
K^{1 \bar{b}} & =\frac{8 \tau_{b}(2 \mathcal{V}+\hat{\xi})\left(\mathcal{V}+\alpha \sum_{c=2}^{n} \lambda_{c} \tau_{c}^{3 / 2}\right)^{2 / 3}}{\alpha^{2 / 3}(4 \mathcal{V}-\hat{\xi})}, \quad K^{a \bar{b}}=\frac{8(2 \mathcal{V}+\hat{\xi}) \tau_{a} \tau_{b}}{4 \mathcal{V}-\hat{\xi}} \\
K^{a \bar{a}} & =\frac{4(2 \mathcal{V}+\hat{\xi}) \sqrt{\tau_{a}}\left(4 \mathcal{V}-\hat{\xi}+6 \alpha \lambda_{a} \tau_{a}^{3 / 2}\right)}{3 \alpha \lambda_{a}(4 \mathcal{V}-\hat{\xi})}
\end{aligned}
$$

with $a, b>1$. This Kähler metric is almost no-scale with respect to $W_{0}$ in the sense that:

$$
K^{a \bar{b}} \partial_{a} K \partial_{\bar{b}} K=\frac{12 \mathcal{V}}{4 \mathcal{V}-\hat{\xi}} \simeq 3
$$

where the last step is valid when $\nu \gg \hat{\xi}$. The form for the Kähler metric is a little messy but will be greatly simplified once we apply the large volume approximation. The large volume approximation is an assumption of the moduli stabilisation regime for the Kähler moduli [85] - there is a minimum for the potential for the Kähler moduli at very large volume. Requiring therefore that $\mathcal{1} \gg 1$, we see immediately from the form of the volume that $\tau_{1}^{3 / 2} \gg \lambda_{a} \tau_{a}^{3 / 2}$ and for simplicity we take $\tau_{1} \gg \tau_{a}$ ] In the limit that $\mathcal{V} \gg 1$, the Kähler metric simplifies greatly

$$
\begin{aligned}
& K_{1 \overline{1}}=\frac{3 \alpha^{4 / 3}}{4 \mathcal{V}^{4 / 3}} \quad K_{1 \bar{b}}=-\frac{9 \alpha^{5 / 3} \lambda_{b} \sqrt{\tau_{b}}}{8 \mathcal{V}^{5 / 3}} \\
& K_{a \bar{b}}=\frac{9 \alpha^{2} \lambda_{a} \lambda_{b} \sqrt{\tau_{a}} \sqrt{\tau_{b}}}{8 \mathcal{V}^{2}}, \quad K_{a \bar{a}}=\frac{3 \alpha \lambda_{a}}{8 \mathcal{V} \sqrt{\tau_{a}}}
\end{aligned}
$$

and

$$
\begin{aligned}
K^{1 \overline{1}} & =\frac{4 \mathcal{V}^{4 / 3}}{3 \alpha^{4 / 3}}, & K^{1 \bar{b}} & =\frac{4 \tau_{b} \mathcal{V}^{2 / 3}}{\alpha^{2 / 3}}, \\
K^{a \bar{b}} & =4 \tau_{a} \tau_{b}, & K^{a \bar{a}} & =\frac{8 \sqrt{\tau_{a}} \mathcal{V}}{3 \alpha \lambda_{a}} .
\end{aligned}
$$

\footnotetext{
${ }^{6}$ In the examples we will consider later, $v$ takes values between $\sim 10^{4} l_{s}^{6}$ and $\sim 10^{6} l_{s}^{6}$, with $l_{s}$ the string length.

${ }^{7}$ In the explicit examples, $\lambda_{a}$ takes values between $\frac{1}{100}$ and 10 .
} 


\section{INTRODUCTION TO KÄHLER MODULI INFLATION}

In addition to this, we have

$$
\partial_{1} K=-\frac{3 \alpha^{2 / 3}}{2 \mathcal{V}^{2 / 3}}, \quad \partial_{a} K=\frac{3 \alpha \lambda_{a} \sqrt{\tau_{a}}}{2 \mathcal{V}}, \quad a>1 .
$$

We are now in a position to find the scalar potential for Kähler modulus inflation through 4.1.2). Considering first the contribution to the potential of terms independent of the Kähler moduli (i.e. terms that are proportional to $\left|W_{0}\right|^{2}$ ) and using the almost no-scale property of the full Kähler metric 4.1.16

$$
\begin{aligned}
V & \supset e^{K}\left(K^{a \bar{b}} D_{a} W_{0} \overline{D_{b} W_{0}}-3\left|W_{0}\right|^{2}\right) \\
& =\frac{1}{\mathcal{V}^{2}}\left(1+\frac{\hat{\xi}}{2 \mathcal{V}}\right)^{-2}\left(K^{a \bar{b}} \partial_{a} K \partial_{\bar{b}} K-3\right)\left|W_{0}\right|^{2} \\
& =\frac{1}{\mathcal{V}^{2}}\left(1+\frac{\hat{\xi}}{2 \mathcal{V}}\right)^{-2}\left(\frac{12 \mathcal{V}}{4 \mathcal{V}-\hat{\xi}}-3\right)\left|W_{0}\right|^{2} \\
& =\frac{1}{\mathcal{V}^{2}}\left(1+\frac{\hat{\xi}}{2 \mathcal{V}}\right)^{-2}\left(3\left(1-\frac{\hat{\xi}}{4 \mathcal{V}}\right)^{-1}-3\right)\left|W_{0}\right|^{2} \\
& \simeq \frac{1}{\mathcal{V}^{2}}\left(1-\frac{\hat{\xi}}{\mathcal{V}}\right)\left(3+\frac{3 \hat{\xi}}{4 \mathcal{V}}-3\right)\left|W_{0}\right|^{2} \\
& \simeq \frac{3 \hat{\xi}}{4 \mathcal{V}^{3}}\left|W_{0}\right|^{2}
\end{aligned}
$$

where we have explicitly used $\mathcal{V} \gg 18$ This term is the only contribution that the $\alpha^{\prime}$ correction to the Kähler potential, $\hat{\xi}$, makes to the scalar potential, $V$, in the large volume limit. The remaining potential is taken to be order $\mathcal{O}\left(1 / \mathcal{V}^{3}\right)$ in the large volume limit and it is assumed that $\mathcal{V} \sim e^{a_{a} \tau_{a}}$ where $a>1^{9}$ apart from an uplift term $V_{\text {uplift }}=\frac{\beta}{V^{2}}$ which is added to uplift the potential to a Minkowski minimum $\left(V_{\min }=0\right)$. Remembering $W=W_{0}+\sum_{a} A_{a} e^{-a_{a} T^{a}}$ and $e^{K_{0}}=\mathcal{V}^{-2}$, the remaining part of the po-

\footnotetext{
${ }^{8}$ In the examples we will consider, it is always the case that $\mathcal{V} \gg \hat{\xi}$.

${ }^{9}$ Terms that contain $e^{-a_{1} \tau_{1}}$ are ignored since this is effectively exponential in the volume and hence extremely small.
} 
tential is

$$
\begin{aligned}
V & \supset \frac{1}{\mathcal{V}^{2}}\left(K^{a \bar{b}} \partial_{a}\left(A_{a} e^{-a_{a} T^{a}}\right) \partial_{\bar{b}}\left(A_{b} e^{-a_{b} \bar{T}^{\bar{b}}}\right)+K^{a \bar{b}} \partial_{a}\left(A_{a} e^{-a_{a} T^{a}}\right) W_{0} \partial_{\bar{b}} K_{0}\right. \\
& +K^{a \bar{b}} \partial_{a}\left(A_{a} e^{-a_{a} T^{a}}\right) A_{b} e^{-a_{b} \bar{T}^{\bar{b}}} \partial_{\bar{b}} K_{0}+K^{a \bar{b}} W_{0} \partial_{a} K_{0} \partial_{\bar{b}}\left(A_{b} e^{-a_{b} \bar{T}^{\bar{b}}}\right) \\
& +K^{a \bar{b}} W_{0} \partial_{a} K_{0} A_{b} e^{-a_{b} \bar{T}^{\bar{b}}} \partial_{\bar{b}} K_{0}+K^{a \bar{b}} A_{a} e^{-a_{a} T^{a}} \partial_{a} K_{0} \partial_{\bar{b}}\left(A_{b} e^{-a_{b} \bar{T}^{\bar{b}}}\right) \\
& \left.+K^{a \bar{b}} A_{a} e^{-a_{a} T^{a}} \partial_{a} K_{0} W_{0} \partial_{\bar{b}} K_{0}+K^{a \bar{b}} A_{a} e^{-a_{a} T^{a}} \partial_{a} K_{0} A_{b} e^{-a_{a} \bar{T}^{\bar{b}}} \partial_{\bar{b}} K_{0}\right)
\end{aligned}
$$

where we have explicitly now assumed that $a_{a}, A_{a}$ and $W_{0}$ are all real. The relevant contributions are

$$
\begin{aligned}
& \frac{1}{\mathcal{V}^{2}} K^{a \bar{a}} \partial_{a}\left(A_{a} e^{-a_{a} T^{a}}\right) \partial_{\bar{a}}\left(A_{a} e^{-a_{a} \bar{T}^{\bar{a}}}\right)=\frac{1}{\mathcal{V}^{2}} \frac{8 \sqrt{\tau_{a}} \mathcal{V}}{3 \alpha \lambda_{a}}\left(A_{a} a_{a}\right)^{2} e^{-T^{a}-\bar{T}^{a}} \\
& =\frac{8 \sqrt{\tau_{a}}}{3 \alpha \lambda_{a} \mathcal{V}}\left(A_{a} a_{a}\right)^{2} e^{-2 a_{a} \tau_{a}} \\
& \frac{1}{\mathcal{V}^{2}} K^{a \overline{1}} \partial_{a}\left(A_{a} e^{-a_{a} T^{a}}\right) W_{0} \partial_{\overline{1}} K_{0}=\frac{1}{\mathcal{V}^{2}} \frac{4 \tau_{a} \mathcal{V}^{2 / 3}}{\alpha^{2 / 3}} a_{a} A_{a} e^{-a_{a} T^{a}} W_{0} \frac{2 \alpha^{2 / 3}}{2 \mathcal{V}^{2 / 3}} \\
& =\frac{6 \tau_{a} a_{a} A_{a} W_{0}}{\mathcal{V}^{2}} e^{-a_{a} \tau_{a}} e^{-i a_{a} b_{a}} \\
& \frac{1}{\mathcal{V}^{2}} K^{a \bar{a}} \partial_{a}\left(A_{a} e^{-a_{a} T^{a}}\right) W_{0} \partial_{a} K_{0}=-\frac{1}{\mathcal{V}^{2}} \frac{8 \sqrt{\tau_{a}} \mathcal{V}}{3 \alpha \lambda_{a}} a_{a} A_{a} W_{0} \frac{3 \alpha \lambda_{a} \sqrt{\tau_{a}}}{2 \mathcal{V}} e^{-a_{a} T^{a}} \\
& =-\frac{4 \tau_{a} a_{a} A_{a} W_{0}}{\mathcal{V}^{2}} e^{-a_{a} \tau_{a}} e^{-i a_{a} b_{a}}, \\
& \frac{1}{\mathcal{V}^{2}} K^{1 \bar{a}} W_{0} \partial_{1} K_{0} \partial_{\bar{a}}\left(A_{a} e^{-a_{a} \bar{T}_{\bar{a}}}\right)=\frac{1}{\mathcal{V}^{2}} \frac{4 \tau_{a} \mathcal{V}^{2 / 3}}{\alpha^{2 / 3}} W_{0} \frac{3 \alpha^{2 / 3}}{2 \mathcal{V}^{2 / 3}} a_{a} A_{a} e^{-a_{a} \bar{T}^{\bar{a}}} \\
& =\frac{6 \tau_{a} a_{a} A_{a} W_{0}}{\mathcal{V}^{2}} w^{-a_{a} \tau_{a}} e^{i a_{a} b_{a}}, \\
& \frac{1}{\mathcal{V}^{2}} K^{a \bar{a}} W_{0} \partial_{a} K_{0} \partial_{\bar{a}}\left(A_{a} e^{-a_{a} \bar{T}^{\bar{a}}}\right)=-\frac{1}{\mathcal{V}^{2}} \frac{8 \sqrt{\tau_{a}} \mathcal{V}}{3 \alpha \lambda_{a}} W_{0} \frac{3 \alpha \lambda_{a} \sqrt{\tau_{a}}}{2 \mathcal{V}} a_{a} A_{a} e^{-a_{a} \bar{T}^{\bar{a}}} \\
& =-\frac{4 \tau_{a} a_{a} A_{a} W_{0}}{\mathcal{V}^{2}} e^{-a_{a} \tau_{a}} e^{i a_{a} b_{a}}
\end{aligned}
$$

where we have ignored anything higher order than $\mathcal{O}\left(1 / \mathcal{V}^{3}\right)$ and anything proportional 


\section{INTRODUCTION TO KÄHLER MODULI INFLATION}

to $e^{-a_{1} \tau_{1}}-$ all the remaining terms are of cubic order in inverse volume. Putting the whole potential together gives

$$
\begin{aligned}
V= & \sum_{a=2}^{n} \frac{8 \sqrt{\tau_{a}}}{3 \alpha \lambda_{a} \mathcal{V}}\left(a_{a} A_{a}\right)^{2} e^{-2 a_{a} \tau_{a}} \\
& +\sum_{a=2}^{n} \frac{\tau_{a} a_{a} A_{a}}{\mathcal{V}^{2}} W_{0} e^{-a_{a} \tau_{a}}\left(6 e^{-i a_{a} b_{a}}-4 e^{-i a_{a} b_{a}}+6 e^{i a_{a} b_{a}}-4 e^{i a_{a} b_{a}}\right) \\
& +\frac{3 \hat{\xi}}{4 \mathcal{V}^{3}} W_{0}^{2}+V_{\text {uplift }}
\end{aligned}
$$

which leads to our large volume potential for Kähler moduli inflation

$$
V=\sum_{a=2}^{n} \frac{8 \sqrt{\tau_{a}}}{3 \alpha \lambda_{a} \mathcal{V}}\left(a_{a} A_{a}\right)^{2} e^{-2 a_{a} \tau_{a}}+\sum_{a=2}^{n} \frac{4 a_{a} A_{a} \tau_{a}}{\mathcal{V}^{2}} W_{0} e^{-a_{a} \tau_{a}} \cos \left(a_{a} b_{a}\right)+\frac{3 \hat{\xi}}{4 \mathcal{V}^{3}} W_{0}^{2}+\frac{\beta}{\mathcal{V}^{2}}
$$

where the (pseudo-) scalar fields are $\mathcal{V}$, the volume of the Calabi-Yau; $\tau_{a}$, 4-cycle Kähler moduli of the Calabi-Yau; and their axionic superpartners, $b_{a}$. Explicit inflationary examples of this model will be reviewed in the next section.

\subsubsection{Fibre inflation potential}

The potential for fibre inflation [50] is derived in the same way with some key differences to Kähler moduli inflation. The superpotential is of the same form

$$
W=W_{0}+\sum_{a} e^{-a_{a} T^{a}}
$$

A key difference is that the Kähler potential receives additional (string-loop) corrections. Without these string-loop corrections the Kähler potential has the same form as in Kähler moduli inflation 10

$$
K=-2 \ln \left(v+\frac{\hat{\xi}}{2}\right)
$$

\footnotetext{
${ }^{10}$ We have again removed the constant part $K_{c s}-\ln 2 / g_{s}$ of the Kähler potential and superpotential, as this contributes just an overall normalisation of the scalar potential.
} 
Another difference is the form of the volume, which, after taking the number of Kähler moduli to be 3 , has the form

$$
\mathcal{V}=\alpha\left(\sqrt{\tau_{1}} \tau_{2}-\gamma \tau_{3}^{3 / 2}\right)
$$

where it is important that $\tau_{1}, \tau_{2} \gg \tau_{3}$ and $\mathcal{V} \sim \mathcal{V}_{0}:=\alpha \sqrt{\tau_{1}} \tau_{2} \gg \alpha \gamma \tau_{3}^{3 / 2} \gg 1$. Since both $\tau_{1}$ and $\tau_{2}$ are very large, we can immediately see that their contribution to the superpotential will be negligible and we can write

$$
W=W_{0}+A_{3} e^{-a_{3} T_{3}}
$$

with $T^{3}=\tau_{3}+i b_{3}$ as before. Since, at this order, $K$ has the same form, we know that $\hat{\xi}$ contributes to the potential

$$
\frac{3 \hat{\xi}}{4 \mathcal{V}^{3}} W_{0}^{2}
$$

at large volume and with $K \simeq K_{0}=-2 \ln \mathcal{V}$ the remaining part of the Kähler potential. With this form for the volume, we get

$$
\partial_{a} K=\partial_{\bar{a}} K=\left(-\frac{\alpha \tau_{2}}{2 \sqrt{\tau_{1}} \mathcal{V}},-\frac{\alpha \sqrt{\tau_{1}}}{\mathcal{V}}, \frac{3 \alpha \gamma \sqrt{\tau_{3}}}{2 \mathcal{V}}\right)
$$

as well as

$$
K_{a \bar{b}}=\frac{1}{4 \tau_{2}^{2}}\left(\begin{array}{ccc}
\left(\frac{\tau_{2}}{\tau_{1}}\right)^{2} & \gamma\left(\frac{\tau_{3}}{\tau_{1}}\right)^{3 / 2} & -\frac{3 \gamma \sqrt{\tau_{3}} \tau_{2}}{2 \tau_{1}^{3 / 2}} \\
\gamma\left(\frac{\tau_{3}}{\tau_{2}}\right)^{3 / 2} & 2 & -\frac{3 \gamma \sqrt{\tau_{3}}}{\sqrt{\tau_{1}}} \\
-\frac{3 \gamma \sqrt{\tau_{3}} \tau_{2}}{2 \tau_{1}^{3 / 2}} & -\frac{3 \gamma \sqrt{\tau_{3}}}{\sqrt{\tau_{1}}} & \frac{3 \alpha \gamma \tau_{2}^{2}}{2 V \sqrt{\tau_{3}}}
\end{array}\right)
$$

and its inverse

$$
K^{a \bar{b}}=4\left(\begin{array}{ccc}
\tau_{1}^{2} & \gamma \sqrt{\tau_{1}} \tau_{3}^{3 / 2} & \tau_{1} \tau_{3} \\
\gamma \sqrt{\tau_{1}} \tau_{3}^{3 / 2} & \frac{\tau_{2}^{2}}{2} & \tau_{2} \tau_{3} \\
\tau_{1} \tau_{3} & \tau_{2} \tau_{3} & \frac{2 v_{\sqrt{\tau_{3}}}}{3 \alpha \gamma}
\end{array}\right)
$$

where in the Kähler metric, a large volume expansion has been applied: explicitly, all terms higher order in $\sqrt{\tau_{3} / \tau_{1,2}}$ than the lowest order in each matrix entry have been dropped, and $\mathcal{V}$ has been taken to be equal to $\mathcal{V}_{0}: \mathcal{V} \rightarrow \mathcal{V}_{0}:=\alpha \sqrt{\tau_{1}} \tau_{2}$. In completely 


\section{INTRODUCTION TO KÄHLER MODULI INFLATION}

the same way as was shown for Kähler moduli inflation, with this Kähler metric the contributions to the scalar potential can be calculated. Without the inclusion of the string-loop corrections, the potential for fibre inflation is

$$
V_{n g_{s}}=\frac{8 \sqrt{\tau_{3}}}{3 \alpha \lambda_{3} \mathcal{V}}\left(a_{3} A_{3}\right)^{2} e^{-2 a_{3} \tau_{3}}+\frac{4 a_{3} A_{3} \tau_{3}}{\mathcal{V}^{2}} W_{0} e^{-a_{3} \tau_{3}} \cos \left(a_{3} b_{3}\right)+\frac{3 \hat{\xi}}{4 \mathcal{V}^{3}} W_{0}^{2}
$$

where the subscript $n g_{s}$ stands for without string-loop corrections.

The string-loop corrections to the Kähler potential contribute (see [50, 86]) to the scalar potential

$$
\delta V_{g_{s}}=\frac{W_{0}^{2}}{\mathcal{V}^{2}}\left(\frac{A}{\tau_{1}^{2}}-\frac{B}{\mathcal{V} \sqrt{\tau_{1}}}+\frac{C \tau_{1}}{\mathcal{V}^{2}}\right)
$$

where $A, B$ and $C$ are constants that depend on $g_{s}$ that we treat as model-dependent parameters. This leads to an overall potential for fibre inflation of

$$
\begin{aligned}
V & =\frac{W_{0}^{2}}{\mathcal{V}^{2}}\left(\frac{A}{\tau_{1}^{2}}-\frac{B}{\mathcal{V} \sqrt{\tau_{1}}}+\frac{C \tau_{1}}{\mathcal{V}^{2}}\right)+\frac{8 \sqrt{\tau_{3}}}{3 \alpha \gamma \mathcal{V}}\left(a_{3} A_{3}\right)^{2} e^{-2 a_{3} \tau_{3}} \\
& +\frac{4 a_{3} A_{3} \tau_{3}}{\mathcal{V}^{2}} W_{0} e^{-a_{3} \tau_{3}} \cos \left(a_{3} b_{3}\right)+\frac{3 \hat{\xi}}{4 \mathcal{V}^{3}} W_{0}^{2}+\frac{\delta_{u p}}{\mathcal{V}^{4 / 3}}
\end{aligned}
$$

where we have included an uplift term that scales as $1 / \mathcal{V}^{4 / 3}$ in the volume to uplift this potential to Minkowski.

\subsection{Bog-standard Kähler moduli inflation}

We have seen that in Kähler moduli inflation [49, 76], the volume of the compactified space is given by

$$
\mathcal{V}=\alpha\left(\tau_{1}^{3 / 2}-\sum_{a=2}^{n} \lambda_{a} \tau_{a}^{3 / 2}\right)
$$

where $\tau_{a}$ are Kähler moduli with $\tau_{1}$ specifically a large modulus that controls the overall size of the volume, and the other moduli are necessarily smaller; and $\alpha$ and $\lambda_{a}$ are model-dependent constants. In Kähler inflation, it is standard to take $n=3$, because it is necessary that there be a stabiliser modulus (which must remain more or less constant) in addition to the inflaton (which of course has to be shifted away from 
its minimum). With three moduli, and in the large-volume limit, $\mathcal{V} \gg 1$, we have

$$
K_{a \bar{b}}=\left(\begin{array}{ccc}
\frac{3 \alpha^{4 / 3}}{4 \mathcal{V}^{4 / 3}} & -\frac{9 \alpha^{5 / 3} \lambda_{2} \sqrt{\tau_{2}}}{8 \mathcal{V}^{5 / 3}} & -\frac{9 \alpha^{5 / 3} \lambda_{3} \sqrt{\tau_{3}}}{8 \mathcal{V}^{5 / 3}} \\
-\frac{9 \alpha^{5 / 3} \lambda_{2} \sqrt{\tau_{2}}}{8 \mathcal{V}^{5 / 3}} & \frac{3 \alpha \lambda_{2}}{8 \mathcal{V} \sqrt{\tau_{2}}} & \frac{9 \alpha^{2} \lambda_{2} \lambda_{3} \sqrt{\tau_{2} \tau_{3}}}{8 \mathcal{V}^{2}} \\
-\frac{9 \alpha^{5 / 3} \lambda_{3} \sqrt{\tau_{3}}}{8 \mathcal{V}^{5 / 3}} & \frac{9 \alpha^{2} \lambda_{2} \lambda_{3} \sqrt{\tau_{2} \tau_{3}}}{8 \mathcal{V}^{2}} & \frac{3 \alpha \lambda_{3}}{8 \mathcal{V} \sqrt{\tau_{3}}}
\end{array}\right)
$$

where $K_{a \bar{b}}$ is the Kähler metric and we have taken only the highest order terms in $\mathcal{O}\left(\frac{1}{\mathcal{V}}\right)$ in each entry. The scalar potential is

$$
V=\sum_{a=2}^{3} \frac{8 \sqrt{\tau_{a}}}{3 \alpha \lambda_{a} \mathcal{V}}\left(a_{a} A_{a}\right)^{2} e^{-2 a_{a} \tau_{a}}+\sum_{a=2}^{3} \frac{4 a_{a} A_{a} \tau_{a}}{\mathcal{V}^{2}} W_{0} e^{-a_{a} \tau_{a}} \cos \left(a_{a} b_{a}\right)+\frac{3 \hat{\xi}}{4 \mathcal{V}^{3}} W_{0}^{2}+\frac{\beta}{\mathcal{V}^{2}}
$$

where all terms (bar the uplift term) scale like $\mathcal{O}\left(\frac{1}{\mathcal{V}^{3}}\right)$ since $a_{a} \tau_{a} \sim \ln \mathcal{V}, a>1$. The Kähler metric is the complex metric for the space spanned by the complex fields, $T^{a}=\tau_{a}+i b_{a}$. It is simpler to move to real space by making the association

$$
K_{a \bar{b}} \partial_{\mu} T^{a} \partial^{\mu} \bar{T}^{\bar{b}}=\frac{1}{2} \gamma_{a b} \partial_{\mu} \phi^{a} \partial^{\mu} \phi^{b}
$$

where $\phi^{a}=\left(\tau_{a}, b_{a}\right)$ are the real fields. It also proves convenient to use $\mathcal{V}$ as a field rather than $\tau_{1}$ since $\mathcal{V}$ naturally appears in the metric and the potential. The modulus, $\tau_{1}$, can of course always be found through 4.2.1. To change variables from $\tau_{1}$ to $\mathcal{V}$, use

$$
\tau_{1}=\alpha^{-2 / 3}\left(\mathcal{V}+\alpha \sum_{a=2}^{3} \lambda_{a} \tau_{a}^{3 / 2}\right)^{2 / 3} \simeq\left(\frac{\mathcal{V}}{\alpha}\right)^{2 / 3}
$$

then, for example, looking at the pure $T_{1}$ kinetic term:

$$
K_{1 \overline{1}} \partial_{\mu} T^{1} \partial^{\mu} \bar{T}^{\overline{1}}=\frac{3 \alpha^{4 / 3}}{4 \mathcal{V}^{4 / 3}} \partial_{\mu} \tau_{1} \partial^{\mu} \tau_{1}
$$

where we have ignored the contribution from the $b_{1}$ axion since the potential has no dependence on $b_{1}$ and therefore $b_{1}$ is effectively massless. Continuing

$$
\frac{3 \alpha^{4 / 3}}{4 \mathcal{V}^{4 / 3}} \partial_{\mu} \tau_{1} \partial^{\mu} \tau_{1} \simeq \frac{3 \alpha^{4 / 3}}{4 \mathcal{V}^{4 / 3}} \frac{2}{3 \alpha^{2 / 3} \mathcal{V}^{1 / 3}} \partial_{\mu} \mathcal{V} \frac{2}{3 \alpha^{2 / 3} \mathcal{V}^{1 / 3}} \partial^{\mu} \mathcal{V}=\frac{1}{3 \mathcal{V}^{2}} \partial_{\mu} \mathcal{V} \partial^{\mu} \mathcal{V}
$$

The system can therefore be set up as follows: we have fields $\phi^{a}=\left(\mathcal{V}, \tau_{2}, b_{2}, \tau_{3}, b_{3}\right)$ with 
real-space metric given by

$$
\gamma_{a b}=\left(\begin{array}{ccccc}
\frac{2}{3 \mathcal{V}^{2}} & -\frac{3 \alpha \lambda_{2} \sqrt{\tau_{2}}}{2 \mathcal{V}^{2}} & 0 & -\frac{3 \alpha \lambda_{3} \sqrt{\tau_{3}}}{2 \mathcal{V}^{2}} & 0 \\
-\frac{3 \alpha \lambda_{2} \sqrt{\tau_{2}}}{2 \mathcal{V}^{2}} & \frac{3 \alpha \lambda_{2}}{4 \mathcal{V} \sqrt{\tau_{2}}} & 0 & \frac{9 \alpha^{2} \lambda_{2} \lambda_{3} \sqrt{\tau_{2} \tau_{3}}}{4 \mathcal{V}^{2}} & 0 \\
0 & 0 & \frac{3 \alpha \lambda_{2}}{4 \mathcal{V} \sqrt{\tau_{2}}} & 0 & \frac{9 \alpha^{2} \lambda_{2} \lambda_{3} \sqrt{\tau_{2} \tau_{3}}}{4 \mathcal{V}^{2}} \\
-\frac{3 \alpha \lambda_{3} \sqrt{\tau_{3}}}{2 \mathcal{V}^{2}} & \frac{9 \alpha^{2} \lambda_{2} \lambda_{3} \sqrt{\tau_{2} \tau_{3}}}{4 \mathcal{V}^{2}} & 0 & \frac{3 \alpha \lambda_{3}}{4 \mathcal{V} \sqrt{\tau_{3}}} & 0 \\
0 & 0 & \frac{9 \alpha^{2} \lambda_{2} \lambda_{3} \sqrt{\tau_{2} \tau_{3}}}{4 \mathcal{V}^{2}} & 0 & \frac{3 \alpha \lambda_{3}}{4 \mathcal{V} \sqrt{\tau_{3}}}
\end{array}\right)
$$

and potential given in (4.2.3). The action describing this system is

$$
S=\int d^{4} x \sqrt{-g}\left\{\frac{M_{\mathrm{pl}}^{2}}{2} R-\frac{1}{2} \gamma_{a b} \partial_{\mu} \phi^{a} \partial^{\mu} \phi^{b}-V\left(\phi^{a}\right)\right\}
$$

with $g$ the determinant of the spacetime metric. At the background level, during inflation, $\phi^{a}=\phi^{a}(t)$, and therefore this action leads to equations of motion for the scalars

$$
\ddot{\phi}^{a}+3 H \dot{\phi}^{a}+\Gamma_{b c}^{a} \dot{\phi}^{b} \dot{\phi}^{c}+\gamma^{a b} \frac{\partial V}{\partial \phi^{b}}=0
$$

where $\Gamma_{b c}^{a}$ are the Christoffel symbols for metric, $\gamma_{a b}$. The Friedmann equations are

$$
3 M_{\mathrm{pl}}^{2} H^{2}=\frac{1}{2} \dot{\varphi}^{2}+V
$$

and

$$
\frac{\dot{H}}{H^{2}}=-\epsilon_{\varphi}
$$

where we have defined $\dot{\varphi}^{2}=\gamma_{a b} \dot{\phi}^{a} \dot{\phi}^{b}$ and $\epsilon_{\varphi}=\frac{\dot{\varphi}^{2}}{2 H^{2} M_{\mathrm{pl}}^{2}}$.

We consider first the parameter set given in example 1 in [76], a prototypical Kähler moduli inflation scenario. It has parameters

$$
\begin{gathered}
\xi=24, \quad \alpha=1, \quad \lambda_{2}=\frac{1}{100}, \quad \lambda_{3}=1, \quad a_{2}=20 \pi, \quad a_{3}=\frac{\pi}{2}, \\
A_{2}=\frac{1}{300}, \quad A_{3}=\frac{1}{300}, \quad \beta=1.984 \times 10^{-6}, \quad W_{0}=2 .
\end{gathered}
$$

These parameters are chosen to be consistent with the necessary constraint on the 
power spectrum, $H^{2} /\left(8 M_{\mathrm{pl}}^{2} \pi^{2} \epsilon_{\varphi}\right) \sim 2 \times 10^{-9}$, and $\beta$ is chosen so that the potential can be uplifted to as close a value of $\langle V\rangle=0$ as possible at the minimum of the potential. To be more explicit, with these parameters, $\tau_{2}$ is the inflaton 11 , and $\tau_{3}$ is a stabiliser, which is indispensable in its role of ensuring that the volume is stable. To emphasise why $\tau_{3}$ is a stabiliser, and cannot be the inflaton, we look at local minima of the potential. First, consider the full global minimum where the potential is minimised and all fields are at their respective minima. The axions are of course minimised at

$$
\left\langle b_{a}\right\rangle=\frac{\pi}{a_{a}}
$$

and the moduli are minimised at

$$
\langle\mathcal{V}\rangle=6.6010 \times 10^{6}, \quad\left\langle\tau_{2}\right\rangle=0.30205, \quad\left\langle\tau_{3}\right\rangle=5.8861
$$

which gives

$$
\left\langle\tau_{1}\right\rangle=3.5189 \times 10^{4}
$$

We are free to shift $\tau_{2}$ and $b_{2}$ considerably without affecting the volume's stability. If we choose

$$
\tau_{2}^{i n}=0.802, \quad b_{2}^{i n}=0.1 \frac{\pi}{a_{2}}
$$

there is still a local minimum at

$$
\mathcal{V}^{\text {in }}=6.6166 \times 10^{6}, \quad \tau_{3}^{\text {in }}=5.8875
$$

meaning we can safely shift $\tau_{2}$ and $b_{2}$ well away from their minima without ruining the stability of the system. The same is not true for $\tau_{3}$. Putting all fields to the global minimum apart from $\tau_{3}$ and the volume, we can get a feel for why changing $\tau_{3}$ 's position is crucial to the stability of the system. As can be seen in FIGs 4.1 4.2, the minimum for the volume is highly sensitive to both $\tau_{3}$ and $b_{3}$ - both cannot be perturbed far from their minima without destroying the global minimum, leading to the minimum being at infinite volume. The reason for this sensitivity is that with the parameters above 4.2 .13 the largest proportion of the potential is made up by the $\tau_{3}$ terms, and

\footnotetext{
${ }^{11}$ In most of the literature, the axions are set to their minima. But if we include the axion, $b_{2}$, in the evolution, we will see that to some extent, this is also an inflaton.
} 


\section{INTRODUCTION TO KÄHLER MODULI INFLATION}

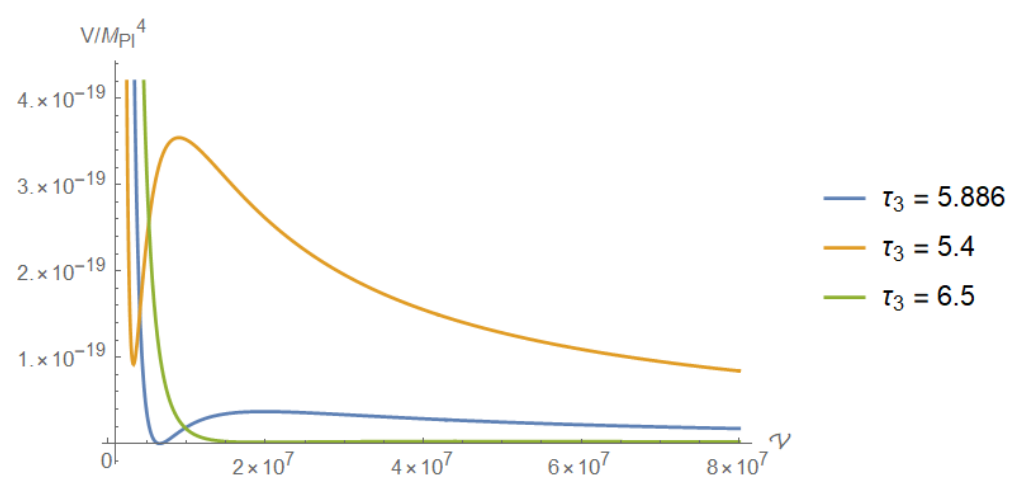

Figure 4.1: A plot of the potential, $V$, near its minimum with respect to the volume, $\mathcal{V}$, for three different values of $\tau_{3}$ with all other fields set to their values at the global minimum. Blue corresponding to the global minimum; and orange and green corresponding to values of $\tau_{3}$ just below and above its value at the global minimum, respectively. The minimum for the volume is highly sensitive to changes in $\tau_{3}$, and shifting it away from the global minimum destroys this minimum for the volume, which then has a minimum at $\mathcal{V} \rightarrow \infty$.

in particular, the term

$$
\frac{4 W_{0} a_{3} A_{3} \tau_{3}}{\mathcal{V}^{2}} e^{-a_{3} \tau_{3}} \cos \left(a_{3} b_{3}\right)
$$

is the most important term for the stabilisation of the potential since this term is negative when $b_{3}$ is near its minimum. The potential and the volume of this system are effectively fine-tuned to the values of $\tau_{3}$ and $b_{3}$. Therefore, immediately we can see why $\tau_{3}$ is not a viable inflaton candidate. Moreover, since we are interested in an axion that can evolve for many e-folds during inflation, $b_{3}$ is clearly not a suitable candidate for our interest in coupling the axion to a gauge field. This is a general feature of these models, the potential requires a stabilising modulus, which is chosen to be $\tau_{3}$, and therefore both $\tau_{3}$ and $b_{3}$ cannot be shifted far from their minima.

However, both $\tau_{2}$ and $b_{2}$ can be shifted away from the minimum of the potential without destroying this minimum and without greatly affecting the volume. The potential experienced by the inflaton, $\tau_{2}$, is shown in FIG. 4.3 . We evolve the system with initial conditions given by (4.2.17)-(4.2.18) and plot the evolution of the fields in FIGs 4.4 4.7. The inflaton modulus, $\tau_{2}$, and its axionic superpartner, $b_{2}$, roll slowly and thus cause inflation. Their evolutionary histories are linked, and they reach their minima at roughly the same time. The other three fields: the stabilisers, $\tau_{3}$ and $b_{3}$; and the volume modulus, $\mathcal{V}$, are all extremely stable during inflation. 


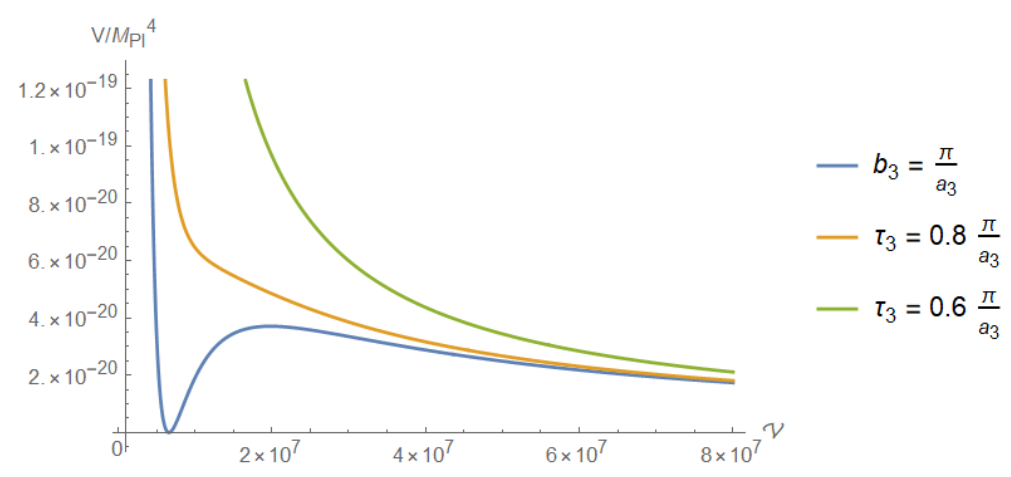

Figure 4.2: A plot of the potential, $V$, near its minimum with respect to the volume, $\mathcal{V}$, for three different values of $b_{3}$ with all other fields set to their values at the global minimum. Blue corresponding to the global minimum; and orange and green corresponding to values of $b_{3}$ shifted slightly away from the minimum. The minimum for the volume is highly sensitive to changes in $b_{3}$ and shifting it away from the global minimum destroys this minimum for the volume, which then has a minimum at $\mathcal{V} \rightarrow \infty$.

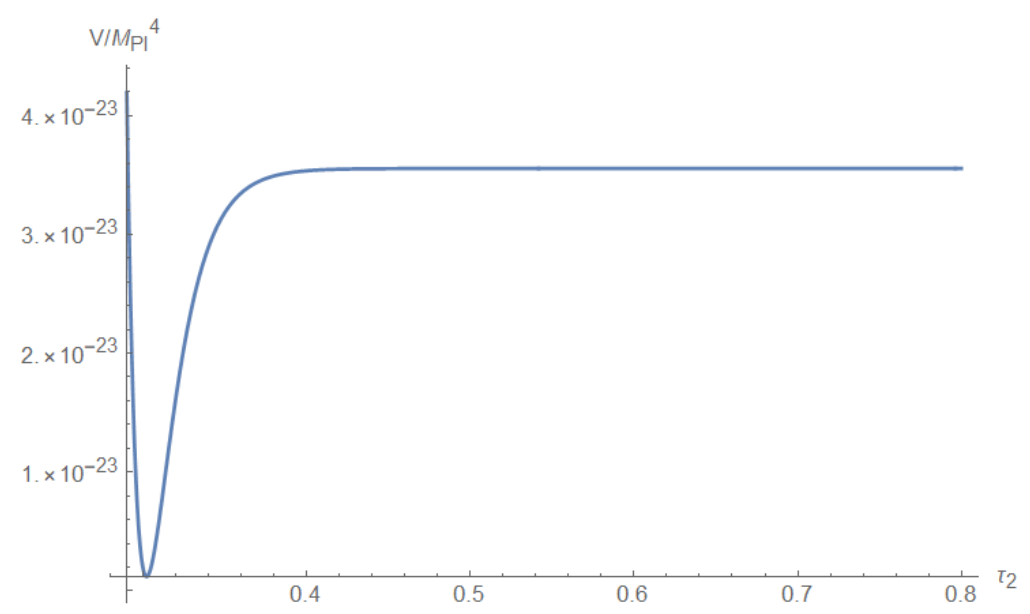

Figure 4.3: A plot of the potential, $V$, against the inflaton, $\tau_{2}$, with all other fields set to their values at the global minimum. Starting inflation with $\tau_{2}^{i n}=0.802$, the inflaton first rolls slowly down the flat part of the potential before exiting slow-roll as it nears the minimum and picks up speed. 

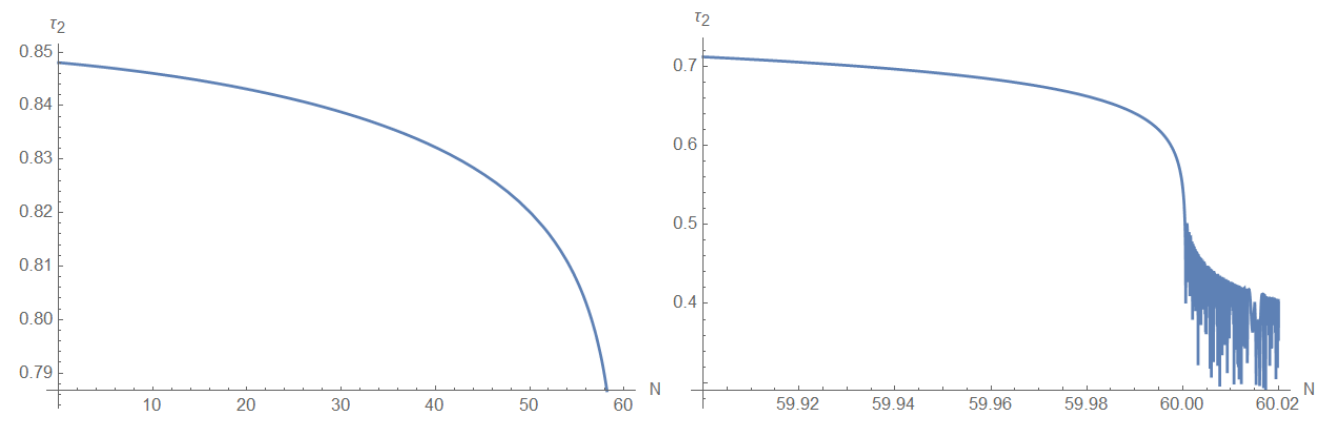

Figure 4.4: The evolution of the inflaton modulus, $\tau_{2}$, during the last 60 e-folds of inflation (left figure) and at the end of inflation when it decays (right figure) for the parameters given in 4.2.13.
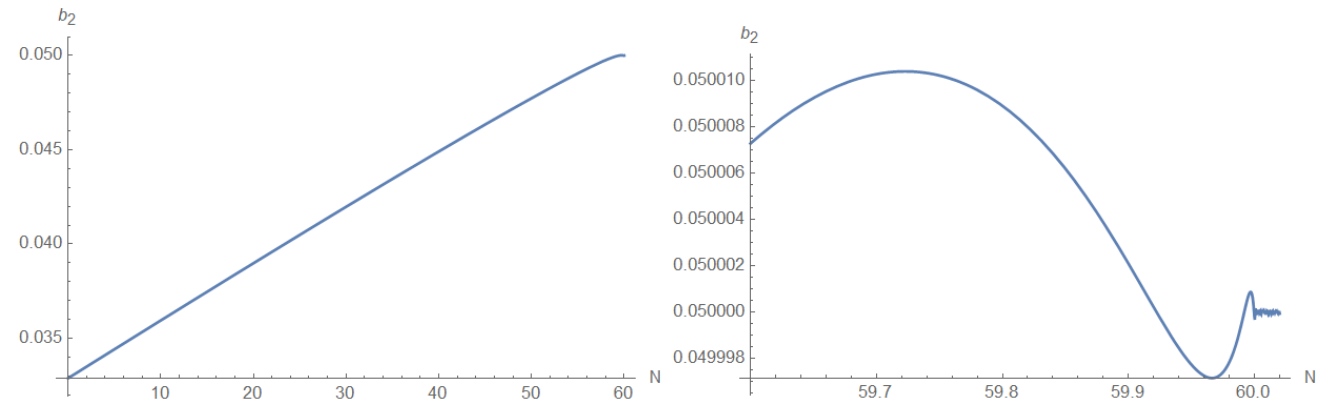

Figure 4.5: The evolution of the axionic partner to the inflaton modulus, $b_{2}$, during the last 60 e-folds of inflation (left figure) and at the end of inflation when it decays (right figure) for the parameters given in 4.2 .13 .
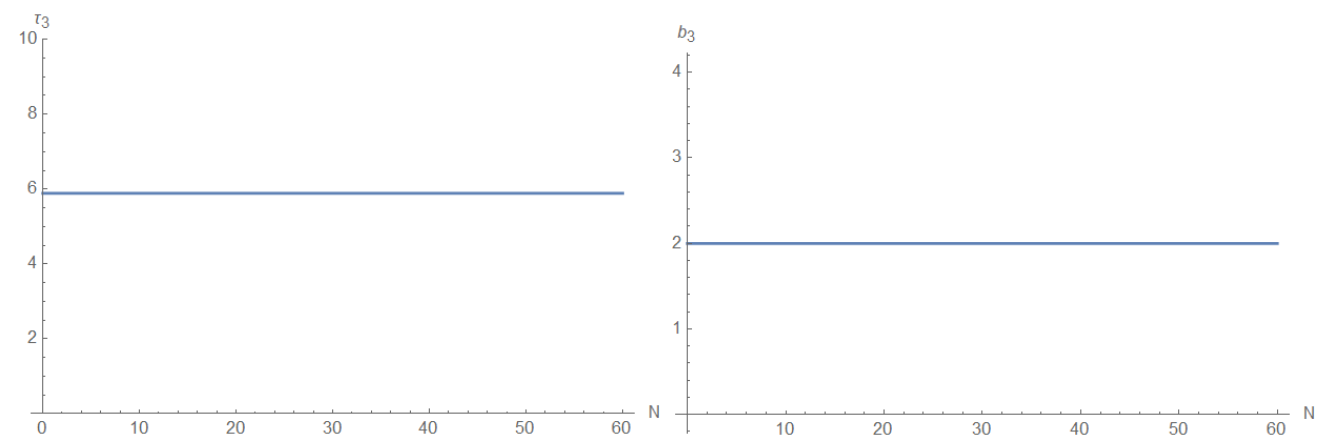

Figure 4.6: The evolution of the stabiliser modulus, $\tau_{3}$, and its axionic partner, $b_{3}$, during the last 60 e-folds of inflation for the parameters given in (4.2.13). Both fields are extremely stable during inflation. 


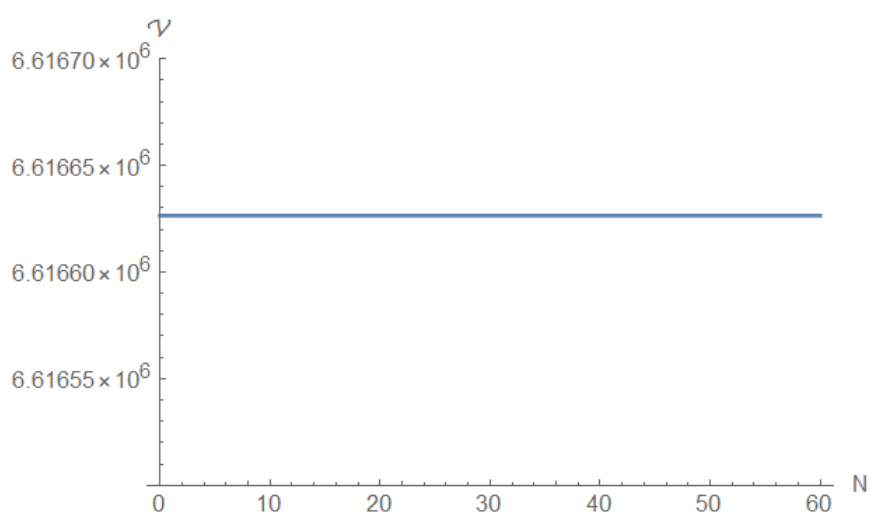

Figure 4.7: The evolution of the volume modulus, $\mathcal{V}$, during the last 60 e-folds of inflation for the parameters given in 4.2.13). $\mathcal{V}$ is extremely stable during inflation despite the relatively large change in $\tau_{2}$.

The inflationary predictions of this example are

$\epsilon=9.35 \times 10^{-17}, \quad n_{s}=0.971, \quad r=1.50 \times 10^{-15}, \quad V^{1 / 4}=5.89 \times 10^{12} \mathrm{GeV}$

where $n_{s}=1-2 \epsilon-\eta$ is the scalar spectral index (with $\eta=\frac{d \ln \epsilon}{d N}$ ), and $r=\frac{\mathcal{P}_{t}}{\mathcal{P}_{s}}=16 \epsilon$ is the tensor-to-scalar ratio with the scalar power spectrum normalised $\sqrt{12}$ to $\mathcal{P}_{s} \sim 2 \times 10^{-9}$. The tensor-to-scalar ratio, $r$, is extremely small in this example. This is because of the extremely low energy scale of inflation, which, in turn, is partly because of the large volume, $\mathcal{V}$, which appears (to various powers) in the denominator of all terms in the potential.

Concerning our interest of applying the mechanism described in chapter 3 to potentially enhance the gravitational spectrum of Kähler moduli inflation to observable values $\left(r \sim 10^{-3}\right)$, we see that with a tensor-to-scalar ratio of $r \sim 10^{-15}$, we would need an enhancement of order $10^{12}$ in the tensor power spectrum. To make things easier, we will therefore consider a different example put forward in [76] (see example 4), where the volume modulus, although still large, is a couple of orders of magnitude greater than in example 1. This, and a suitable choice of parameters, leads to a much larger inflationary energy scale, and consequently, a greater tensor-to-scalar ratio.

\footnotetext{
${ }^{12}$ Recall from 4.1 that the scalar potential actually contains a normalisation factor $\frac{g_{s}^{4}}{8 \pi} e^{K_{c s}}$ where $e^{K_{c s}}$ is assumed to be $\mathcal{O}(1)$. In the examples of Kähler moduli inflation we consider here (taken from [76]), this normalisation has been absorbed into the parameters, $W_{0}, A_{2}, \ldots$, etc.
} 


\subsection{Kähler moduli inflation with a higher energy scale (example 1)}

The parameters of example 4 in [76] are

$$
\begin{gathered}
\xi=\frac{1}{2}, \quad \alpha=\frac{1}{9 \sqrt{2}}, \quad \lambda_{2}=10, \quad \lambda_{3}=1, \quad a_{2}=\frac{2 \pi}{30}, \quad a_{3}=\frac{2 \pi}{3} \\
A_{2}=\frac{1}{1.7 \times 10^{6}}, \quad A_{3}=\frac{1}{425}, \quad \beta=6.947 \times 10^{-5}, \quad W_{0}=\frac{40}{17} .
\end{gathered}
$$

This potential has a global minimum at

$$
\langle\mathcal{V}\rangle=10142, \quad\left\langle\tau_{2}\right\rangle=4.7752, \quad\left\langle\tau_{3}\right\rangle=2.6511, \quad\left\langle b_{a}\right\rangle=\frac{\pi}{a_{a}}
$$

which gives

$$
\left\langle\tau_{1}\right\rangle=2555.69
$$

As before $\tau_{3}$ is the stabiliser and therefore it and $b_{3}$ cannot be shifted from their minima. In fact, since if $b_{3}$ is put to its minimum, it stays there, we will remove $b_{3}$ from the equations of motion and focus on a four-field model, $\phi^{a}=\left(\mathcal{V}, \tau_{2}, b_{2}, \tau_{3}\right)$. As before, $\tau_{2}$ is the inflaton modulus and shifting it as well as its axion, $b_{2}$, away from their minima does not destabilise the system. Taking

$$
\tau_{2}^{i n}=79.1, \quad b_{2}^{i n}=0.7 \frac{\pi}{a_{2}}
$$

shifts the minima for $\mathcal{V}, \tau_{3}$ and $\tau_{1}$ to

$$
V^{i n}=10972, \quad \tau_{3}^{i n}=2.6846, \quad \tau_{1}^{i n}=2644.39
$$

Unlike the previous example, the volume modulus is shifted by a non-negligible amount with these parameters:

$$
\frac{\Delta \mathcal{V}}{\langle\mathcal{V}\rangle}=\frac{10972-10142}{10142}=8.18 \%
$$

and therefore it is not trivial that this modulus can be safely set to its minimum without affecting the inflationary predictions. As mentioned in the introduction to this chapter, we would like to minimise the number of fields we evolve to ease the numerics when we 
attempt to apply the enhancement mechanism of chapter 3 to Kähler moduli inflation. With this in mind, we will show that it can be assumed that both $\mathcal{V}$ and $\tau_{3}$ are at the global minimum and, although this has a small impact on the fields' evolutions (in particular we will have to slightly shift the initial value for $\tau_{2}$ to recover the same results), it drastically simplifies the numerics and leaves the important inflationary predictions of the model intact.

We first evolve the system with all four fields active, $\phi^{a}=\left(\mathcal{V}, \tau_{2}, b_{2}, \tau_{3}\right)$, and with the full metric (to first order in each entry in $1 / \mathcal{V}$ ). This system has real-space metric given by

$$
\gamma_{a b}=\left(\begin{array}{cccc}
\frac{2}{3 \mathcal{V}^{2}} & -\frac{3 \alpha \lambda_{2} \sqrt{\tau_{2}}}{2 \mathcal{V}^{2}} & 0 & -\frac{3 \alpha \lambda_{3} \sqrt{\tau_{3}}}{2 \mathcal{V}^{2}} \\
-\frac{3 \alpha \lambda_{2} \sqrt{\tau_{2}}}{2 \mathcal{V}^{2}} & \frac{3 \alpha \lambda_{2}}{4 \mathcal{V} \sqrt{\tau_{2}}} & 0 & \frac{9 \alpha^{2} \lambda_{2} \lambda_{3} \sqrt{\tau_{2} \tau_{3}}}{4 \mathcal{V}^{2}} \\
0 & 0 & \frac{3 \alpha \lambda_{2}}{4 \mathcal{V} \sqrt{\tau_{2}}} & 0 \\
-\frac{3 \alpha \lambda_{3} \sqrt{\tau_{3}}}{2 \mathcal{V}^{2}} & \frac{9 \alpha^{2} \lambda_{2} \lambda_{3} \sqrt{\tau_{2} \tau_{3}}}{4 \mathcal{V}^{2}} & 0 & \frac{3 \alpha \lambda_{3}}{4 \mathcal{V} \sqrt{\tau_{3}}}
\end{array}\right) .
$$

The equations of motion $4.2 .10-4.2 .12$ and potential 4.2 .3 are of course the same as before. The evolutions of the fields for this higher-energy system are plotted in FIGs 4.84 .11 . As in the previous example, the evolutions of the inflaton, $\tau_{2}$, and its axionic partner, $b_{2}$, are linked and both decay at the same time. The volume modulus (FIG. 4.10 is stable for the majority of inflation while the inflaton is slowly rolling, but decays quickly in line with the decay of the inflaton and axion as the system settles into the global minimum of the potential, 4.3.2.

The inflationary predictions of this model are (50 e-folds before the end of inflation)

$$
\epsilon=4.14 \times 10^{-8}, \quad n_{s}=0.967, \quad r=6.62 \times 10^{-7}, \quad V^{1 / 4}=8.96 \times 10^{14} \mathrm{GeV}
$$

for power spectrum normalised to $\mathcal{P}_{s} \sim 2 \times 10^{-9}$. The much greater scale of inflation ( $V$ is 8 orders of magnitude larger), naturally leads to a much larger tensor-to-scalar ratio. Concerning our interest in enhancing the tensor spectrum to observable values $\left(r \sim 10^{-3}\right)$, we now require an enhancement of roughly 4 orders of magnitude. 


\section{INTRODUCTION TO KÄHLER MODULI INFLATION}
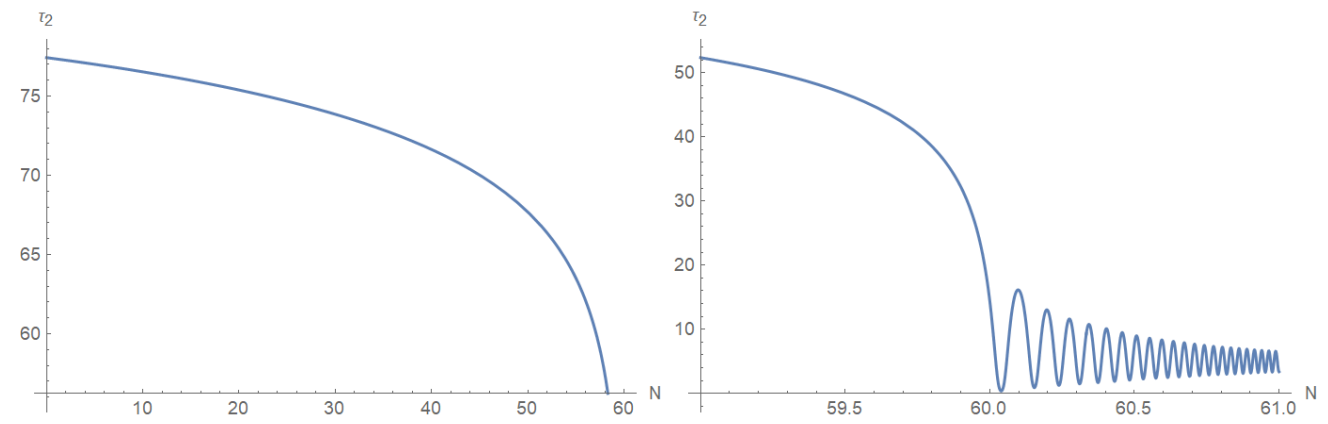

Figure 4.8: The evolution of the inflaton modulus, $\tau_{2}$, during the last 60 e-folds of inflation (left figure) and at the end of inflation when it decays (right figure) for the parameters given in 4.3.1 (example 1).
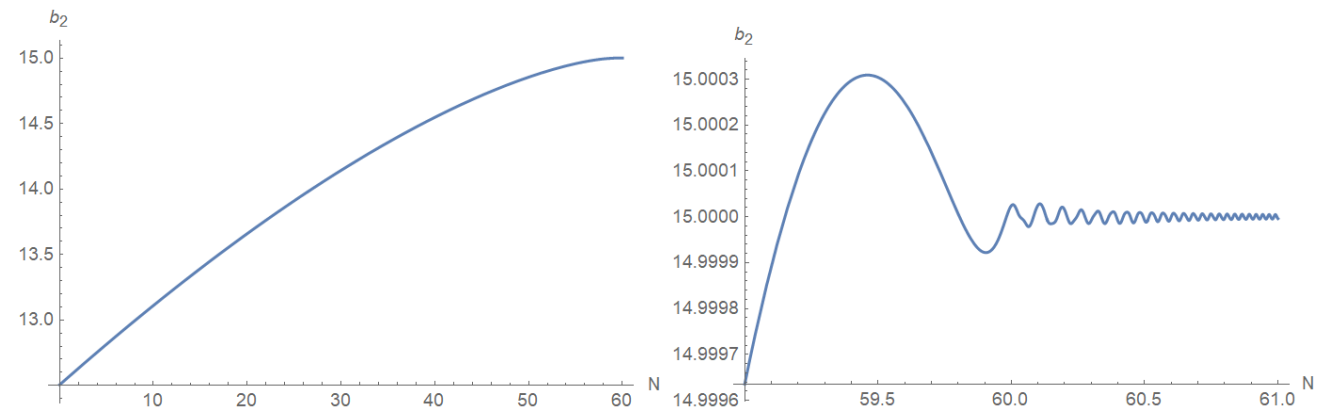

Figure 4.9: The evolution of the axionic partner to the inflaton modulus, $b_{2}$, during the last 60 e-folds of inflation (left figure) and at the end of inflation when it decays (right figure) for the parameters given in 4.3.1 (example 1).
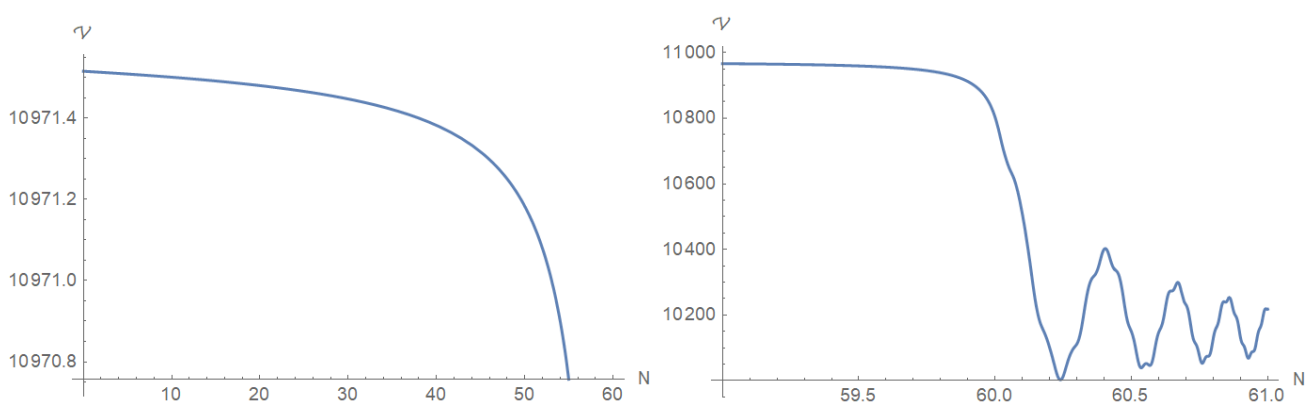

Figure 4.10: The evolution of volume modulus, $\mathcal{V}$, during the last 60 e-folds of inflation (left figure), and at the end of inflation when it decays (right figure) for the parameters given in 4.3.1 (example 1). The volume modulus is stable while the inflaton, $\tau_{2}$, is slowly rolling but decays quickly in line with the decay of the inflaton. 

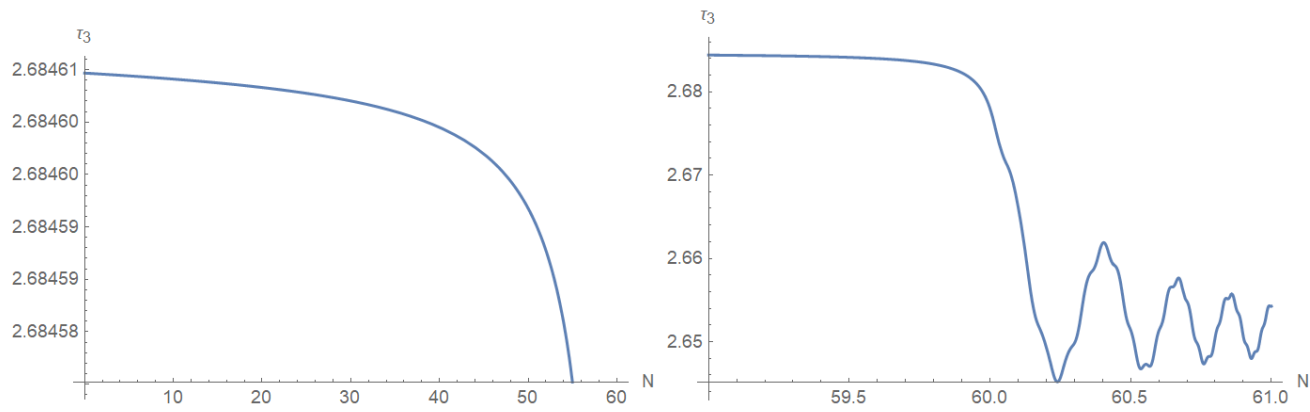

Figure 4.11: The evolution of the stabilising modulus, $\tau_{3}$, during the last 60 e-folds of inflation (left figure) and at the end of inflation when it decays (right figure) for the parameters given in 4.3.1) (example 1).

\section{Removing $\mathcal{V}$ and $\tau_{3}$ from the evolution}

We will now consider the same system but with $\tau_{3}$ and $\mathcal{V}$ set to their values at the global minimum

$$
\langle\mathcal{V}\rangle=10142, \quad\left\langle\tau_{3}\right\rangle=2.651
$$

to demonstrate that these two fields can be safely excluded from the numerical evolution without significantly impacting the inflationary predictions of the model. We now have a two-field model with $\phi^{a}=\left(\tau_{2}, b_{2}\right)$ and metric

$$
\gamma_{a b}=\left(\begin{array}{cc}
\frac{3 \alpha \lambda_{2}}{4 \mathcal{V} \sqrt{\tau_{2}}} & 0 \\
0 & \frac{3 \alpha \lambda_{2}}{4 \mathcal{V} \sqrt{\tau_{2}}}
\end{array}\right) .
$$

We use initial conditions with $\tau_{2}$ slightly shifted from the previous example (which allows us to recover the same value of $n_{s}$ )

$$
\tau_{2}^{i n}=78.5, \quad b_{2}^{i n}=0.7 \frac{\pi}{a_{2}} .
$$

The evolution during the last 60 e-folds of inflation of the two fields proceeds as expected and is plotted in FIGs 4.12 4.13. Despite the fact that the volume modulus does evolve non-negligibly during inflation (see FIG. 4.10), excluding it and the stabiliser from the evolution has only a very small effect on the evolution of the inflaton, $\tau_{2}$, and its axionic partner, $b_{2}$ (compare with FIGs 4.84 .9 ).

The inflationary predictions of this model are now (50 e-folds before the end of 

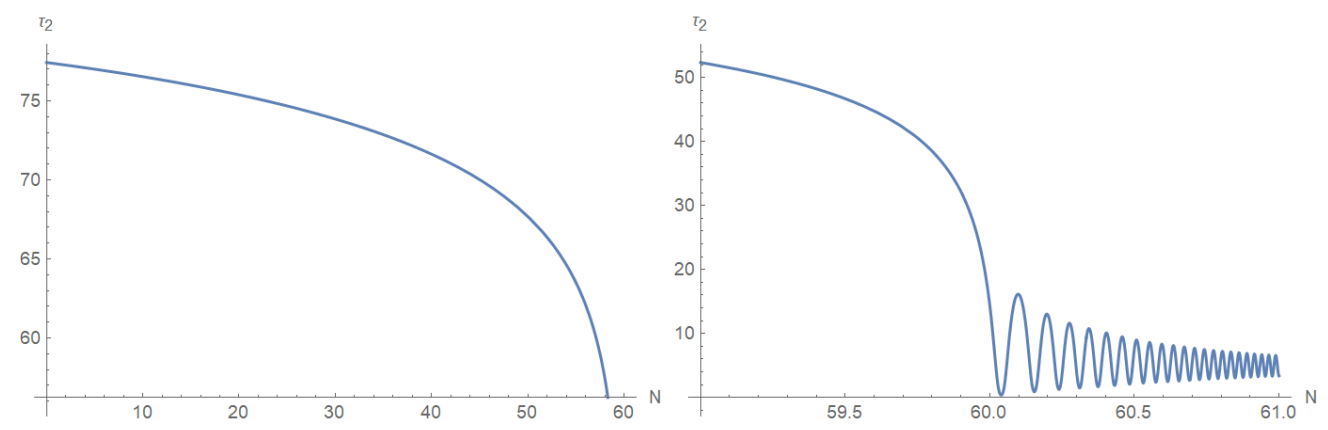

Figure 4.12: The evolution of the inflaton modulus, $\tau_{2}$, during the last 60 e-folds of inflation (left figure) and at the end of inflation when it decays (right figure) for the parameters given in 4.3.1 with the volume modulus, $\mathcal{V}$, and stabiliser, $\tau_{3}$, excluded from the evolution. The evolution proceeds almost identically to that of FIG. 4.8 for which $\mathcal{V}$ and $\tau_{3}$ were included in the evolution.
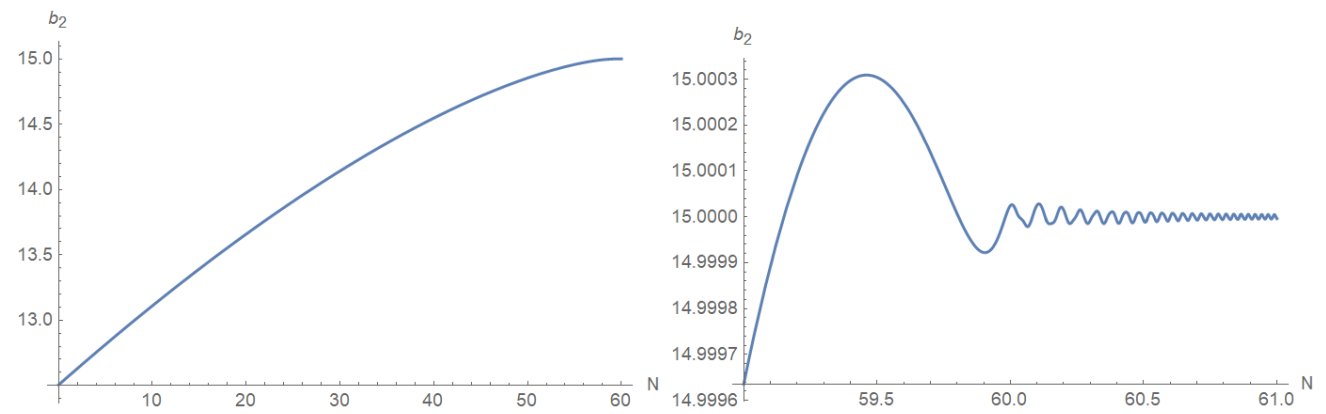

Figure 4.13: The evolution of the axionic partner to the inflaton modulus, $b_{2}$, during the last 60 e-folds of inflation (left figure) and at the end of inflation when it decays (right figure) for the parameters given in 4.3.1 with the volume modulus, $\mathcal{V}$, and stabiliser, $\tau_{3}$, excluded from the evolution. The evolution proceeds almost identically to that of FIG. 4.8 for which $\mathcal{V}$ and $\tau_{3}$ were included in the evolution. 
inflation)

$$
\epsilon=4.88 \times 10^{-8}, \quad n_{s}=0.967, \quad r=7.80 \times 10^{-7}, \quad V^{1 / 4}=9.13 \times 10^{14} \mathrm{GeV}
$$

for power spectrum normalised to $\mathcal{P}_{s} \sim 2 \times 10^{-9}$. The scalar spectral index is exactly the same as it was in the full evolution, however the potential, slow-roll parameter, and consequently the tensor-to-scalar ratio are all slightly greater in value. These differences are small however, and the generic inflationary predictions of the model are the same as they were in the full evolution. In particular, we still require an enhancement factor of roughly $10^{4}$ if we wish to have a detectable tensor spectrum.

The model shown in this section is very promising - it contains an axion that will couple naturally to a gauge field through the $b_{2} F \tilde{F}$ term in the action; with this axion active during inflation, the inflationary predictions of the model satisfy observational constraints; the tensor-to-scalar ratio is much greater than that of standard Kähler moduli inflation, and will therefore be much easier to enhance to observable values; and, we can reduce it to a two-field model without affecting its predictions significantly, greatly simplifying the numerics, which will become involved when we consider the perturbations of this system coupled to a gauge field in chapter 5. In the next section, we will consider a slight variation to this model, which, in practice, proves much more readily applicable to admit a large enhancement of the gravitational wave spectrum through the mechanism shown in chapter 3 .

\subsection{Including an extra modulus (example 2)}

Although the model shown in the previous section looks very promising, in practice it proves difficult to implement a successful enhancement of the gravitational wave spectrum without incurring an excessive backreaction of the gauge field tensor perturbation on the background equations of motion. This difficulty is in a large part because the axion, whose evolution can sustain the gauge field, is linked inextricably to the inflaton. This will be discussed in more detail in chapter 5. In the model discussed in chapter 3 , the axion is a spectator field, with no direct coupling to the inflaton. Using this as a guide, we pose the question in this section of whether it is possible to include an extra modulus, $T_{4}=\tau_{4}+i b_{4}$, that is neither responsible for inflation $\left(T_{2}\right)$ nor responsible 


\section{INTRODUCTION TO KÄHLER MODULI INFLATION}

for stabilising the potential $\left(T_{3}\right)$. It is easy to include an extra (small) modulus in the volume

$$
\mathcal{V}=\alpha\left(\tau_{1}^{3 / 2}-\sum_{a=2}^{4} \lambda_{a} \tau_{a}^{3 / 2}\right)
$$

and the potential (see 4.1) becomes

$$
V=\sum_{a=2}^{4} \frac{8 \sqrt{\tau_{a}}}{3 \alpha \lambda_{a} \mathcal{V}}\left(a_{a} A_{a}\right)^{2} e^{-2 a_{a} \tau_{a}}+\sum_{a=2}^{4} \frac{4 a_{a} A_{a} \tau_{a}}{\mathcal{V}^{2}} W_{0} e^{-a_{a} \tau_{a}} \cos \left(a_{a} b_{a}\right)+\frac{3 \hat{\xi}}{4 \mathcal{V}^{3}} W_{0}^{2}+\frac{\beta}{\mathcal{V}^{2}} .
$$

Likewise, the Kähler metric generalises easily

$$
K_{a \bar{b}}=\left(\begin{array}{cccc}
\frac{3 \alpha^{4 / 3}}{4 \mathcal{V}^{4 / 3}} & -\frac{9 \alpha^{5 / 3} \lambda_{2} \sqrt{\tau_{2}}}{8 \mathcal{V}^{5 / 3}} & -\frac{9 \alpha^{5 / 3} \lambda_{3} \sqrt{\tau_{3}}}{8 \mathcal{V}^{5 / 3}} & -\frac{9 \alpha^{5 / 3} \lambda_{4} \sqrt{\tau_{4}}}{8 \mathcal{V}^{5 / 3}} \\
-\frac{9 \alpha^{5 / 3} \lambda_{2} \sqrt{\tau_{2}}}{8 \mathcal{V}^{5 / 3}} & \frac{3 \alpha \lambda_{2}}{8 \mathcal{V} \sqrt{\tau_{2}}} & \frac{9 \alpha^{2} \lambda_{2} \lambda_{3} \sqrt{\tau_{2} \tau_{3}}}{8 \mathcal{V}^{2}} & \frac{9 \alpha^{2} \lambda_{2} \lambda_{4} \sqrt{\tau_{2} \tau_{4}}}{8 \mathcal{V}^{2}} \\
-\frac{9 \alpha^{5 / 3} \lambda_{3} \sqrt{\tau_{3}}}{8 \mathcal{V}^{5 / 3}} & \frac{9 \alpha^{2} \lambda_{2} \lambda_{3} \sqrt{\tau_{2} \tau_{3}}}{8 \mathcal{V}^{2}} & \frac{3 \alpha \lambda_{3}}{8 \mathcal{V} \sqrt{\tau_{3}}} & \frac{9 \alpha^{2} \lambda_{3} \lambda_{4} \sqrt{\tau_{3} \tau_{4}}}{8 \mathcal{V}^{2}} \\
-\frac{9 \alpha^{5 / 3} \lambda_{4} \sqrt{\tau_{4}}}{8 \mathcal{V}^{5 / 3}} & \frac{9 \alpha^{2} \lambda_{2} \lambda_{4} \sqrt{\tau_{2} \tau_{4}}}{8 \mathcal{V}^{2}} & \frac{9 \alpha^{2} \lambda_{3} \lambda_{4} \sqrt{\tau_{3} \tau_{4}}}{8 \mathcal{V}^{2}} & \frac{3 \lambda_{4}}{8 \mathcal{V} \sqrt{\tau_{4}}}
\end{array}\right)
$$

Our goal in this section is to show that this is consistent in that the model still predicts acceptable inflationary observables in $n_{s}, r$ and that, like in the previous section, we can safely set the volume modulus, $\mathcal{V}$, and the stabiliser, $\tau_{3}$, to their minima. Since the plan is to use the $b_{4}$ axion as the field coupled to the gauge field, we can set $b_{2}=\pi / a_{2}$ along with $b_{3}=\pi / a_{3}$. This leaves us with a 5 -field system, $\phi^{a}=\left(\mathcal{V}, \tau_{2}, \tau_{3}, \tau_{4}, b_{4}\right)$ with real-space metric given by

$$
\gamma_{a b}=\left(\begin{array}{ccccc}
\frac{2}{3 \mathcal{V}^{2}} & -\frac{3 \alpha \lambda_{2} \sqrt{\tau_{2}}}{2 \mathcal{V}^{2}} & -\frac{3 \alpha \lambda_{3} \sqrt{\tau_{3}}}{2 \mathcal{V}^{2}} & -\frac{3 \alpha \lambda_{4} \sqrt{\tau_{4}}}{2 \mathcal{V}^{2}} & 0 \\
-\frac{3 \alpha \lambda_{2} \sqrt{\tau_{2}}}{2 \mathcal{V}^{2}} & \frac{3 \alpha \lambda_{2}}{4 \mathcal{V} \sqrt{\tau_{2}}} & \frac{9 \alpha^{2} \lambda_{2} \lambda_{3} \sqrt{\tau_{2} \tau_{3}}}{4 \mathcal{V}^{2}} & \frac{9 \alpha^{2} \lambda_{2} \lambda_{4} \sqrt{\tau_{2} \tau_{4}}}{4 \mathcal{V}^{2}} & 0 \\
-\frac{3 \alpha \lambda_{3} \sqrt{\tau_{3}}}{2 \mathcal{V}^{2}} & \frac{9 \alpha^{2} \lambda_{2} \lambda_{3} \sqrt{\tau_{2} \tau_{3}}}{4 \mathcal{V}^{2}} & \frac{3 \alpha \lambda_{3}}{4 \mathcal{V} \sqrt{\tau_{3}}} & \frac{9 \alpha^{2} \lambda_{3} \lambda_{4} \sqrt{\tau_{3} \tau_{4}}}{4 \mathcal{V}^{2}} & 0 \\
-\frac{3 \alpha \lambda_{4} \sqrt{\tau_{4}}}{2 \mathcal{V}^{2}} & \frac{9 \alpha^{2} \lambda_{2} \lambda_{4} \sqrt{\tau_{2} \tau_{4}}}{4 \mathcal{V}^{2}} & \frac{9 \alpha^{2} \lambda_{3} \lambda_{4} \sqrt{\tau_{3} \tau_{4}}}{4 \mathcal{V}^{2}} & \frac{3 \alpha \lambda_{4}}{4 \mathcal{V} \sqrt{\tau_{4}}} & 0 \\
0 & 0 & 0 & 0 & \frac{3 \alpha \lambda_{4}}{4 \mathcal{V} \sqrt{\tau_{4}}}
\end{array}\right) .
$$


We use the same parameters as in the previous example (4.3.1) with the addition of

$$
\lambda_{4}=0.1, \quad a_{4}=\frac{2 \pi}{70}, \quad A_{4}=2 \times 10^{-9} .
$$

$A_{4}$ is chosen so that the contribution to the potential 4.4.2) from the $T_{4}$ terms is small; $\lambda_{4} \ll \lambda_{2}$ is chosen so that the contribution from the fields $\tau_{4}$ and $b_{4}$ to $\epsilon_{\varphi}=\frac{\dot{\varphi}^{2}}{2 H^{2} M_{\mathrm{pl}}^{2}}$ is small; and $a_{4}$ is chosen phenomenologically to help with the enhancement mechanism (see chapter 5). The minimum of this potential is found at

$$
\langle\mathcal{V}\rangle=10140, \quad\left\langle\tau_{2}\right\rangle=4.7752, \quad\left\langle\tau_{3}\right\rangle=2.6510, \quad\left\langle\tau_{4}\right\rangle=11.141, \quad\left\langle b_{a}\right\rangle=\frac{\pi}{a_{a}}
$$

and shifting both the inflaton and $b_{4}$ axion to their initial conditions of

$$
\tau_{2}^{i n}=80.5, \quad b_{4}^{i n}=0.59 \frac{\pi}{a_{4}}
$$

gives us the initial conditions for the other fields at the local minimum of the potential

$$
\mathcal{V}^{i n}=10971, \quad \tau_{3}^{i n}=2.6846, \quad \tau_{4}^{i n}=11.141
$$

The equations of motion are still given by 4.2.10 - 4.2.12). The evolutions of the fields for this 5-field model is shown in FIGs 4.14 4.17. The fields $\tau_{2}, \tau_{3}$ and $\mathcal{V}$ behave in much the same way they did in the previous, 3 moduli, example (see FIGs 4.8 4.11). The evolution of $\tau_{4}$ and, more importantly, the axion, $b_{4}$, (FIG. 4.15) are particularly uninteresting in this example: the axion moves to its minimum immediately (before the last 60 e-folds of inflation). However, as mentioned in chapter 3 , an axion can enter slow-roll through its coupling to a gauge field (the two fields, in essence, support one another), and this is exactly what we will see when we couple this system to a gauge field in chapter 5. Without the gauge-coupling, however, the axion does nothing of interest. The important thing is that the inflaton still slows rolls and causes inflation in a way compatible with observations after the introduction of an extra modulus, $T_{4}$.

The inflationary predictions of this example are (60 e-folds before the end of inflation)

$$
\epsilon=2.40 \times 10^{-8}, \quad n_{s}=0.965, \quad r=3.84 \times 10^{-7}, \quad V^{1 / 4}=8.14 \times 10^{14} \mathrm{GeV} .
$$




\section{INTRODUCTION TO KÄHLER MODULI INFLATION}
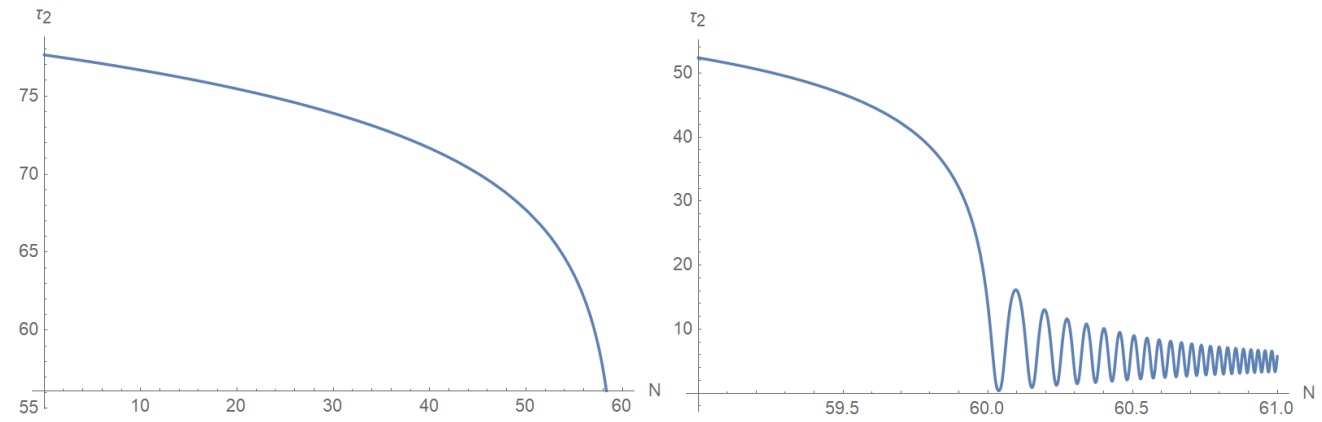

Figure 4.14: The evolution of the inflaton modulus, $\tau_{2}$, during the last 60 e-folds of inflation (left figure) and at the end of inflation when it decays (right figure) for the extra modulus example with the parameters given in 4.3.1) and 4.4.5).
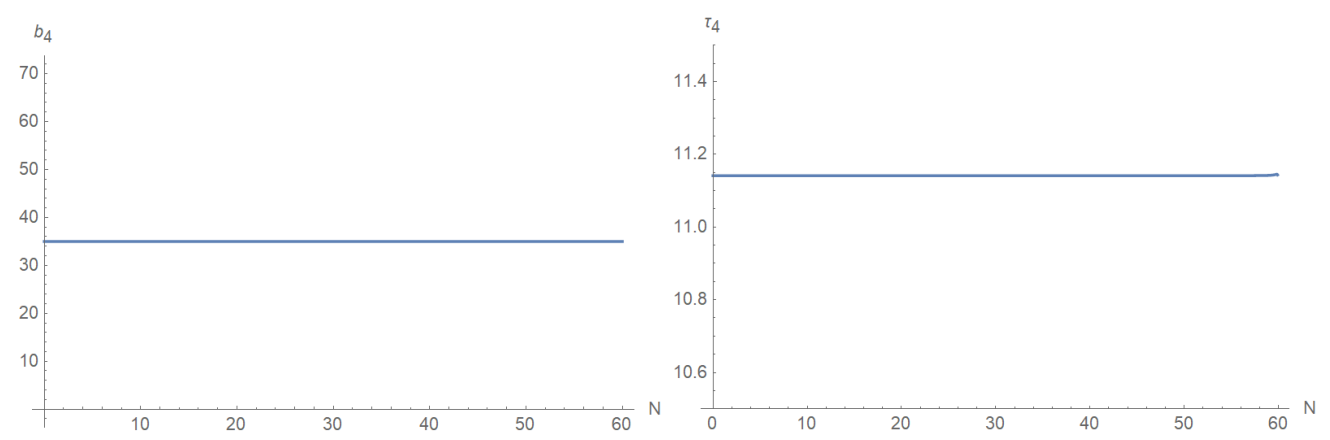

Figure 4.15: The evolution of the axion, $b_{4}$ (left figure), and its scalar superpartner, the $\tau_{4}$ modulus, during the last 60 e-folds of inflation for the extra modulus example with the parameters given in 4.3.1) and 4.4.5. Because $b_{4}$ has no gauge field to support it, it rapidly falls to its minimum and does not evolve.
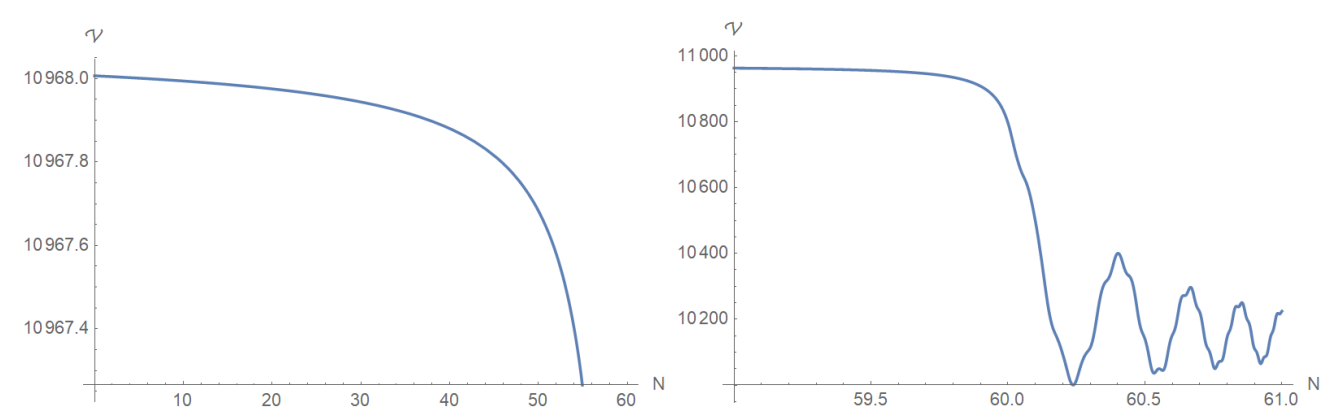

Figure 4.16: The evolution of volume modulus, $\mathcal{V}$, during the last 60 e-folds of inflation (left figure), and at the end of inflation when it decays (right figure) for the extra modulus example with the parameters given in (4.3.1) and 4.4.5). The volume modulus is stable while the inflaton, $\tau_{2}$, is slowly rolling but decays quickly in line with the decay of the inflaton. 

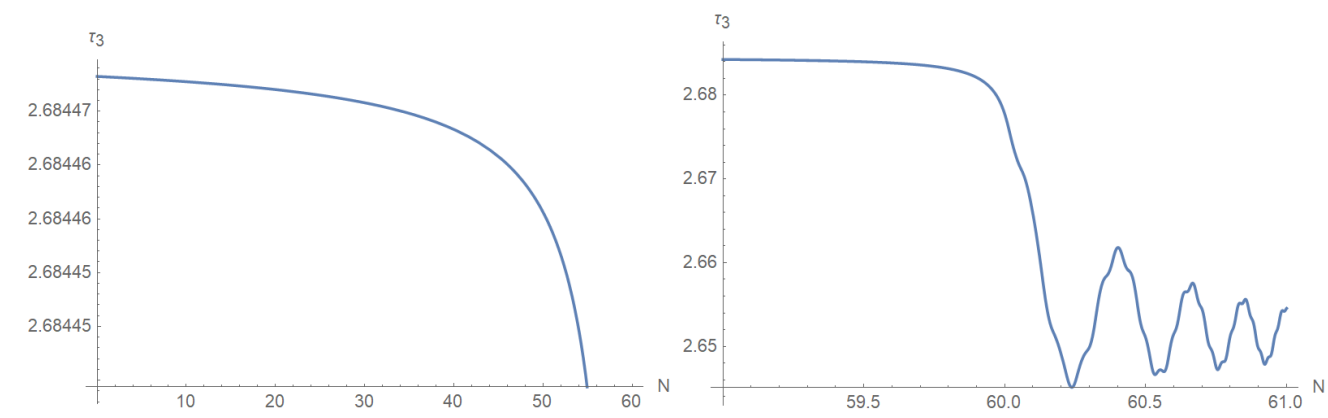

Figure 4.17: The evolution of the stabilising modulus, $\tau_{3}$, during the last 60 e-folds of inflation (left figure) and at the end of inflation when it decays (right figure) for the extra modulus example with the parameters given in 4.3.1) and 4.4.5.

In comparison to the 3-moduli case, where we took horizon-crossing to be 50 e-folds before the end of inflation, in this case $\eta=d(\ln \epsilon) / d N$ is larger earlier in inflation, meaning $n_{s}=1-2 \epsilon-\eta$ reaches an observable value earlier (in general $\eta$ will increase as inflation continues), hence why we measure 60 e-folds prior to the end of inflation rather than 50. Because this point is further from inflation's end $(\epsilon \rightarrow 1), \epsilon$ is slightly smaller than the 3-moduli example, and therefore so is $r$. This effect is not due to the additional modulus since both $\tau_{4}$ and $b_{4}$ are effectively stationary. Rather, it is because in this example we have $b_{2}$ set to its minimum - this changes the evolution of $\tau_{2}$, the inflaton, because it feels a stronger pull from the potential to its minimum. We note that both [49, 76] leave the axions at their minima so this is actually a more standard Kähler inflation scenario. But it is interesting that taking $b_{2}$ away from its minimum leads to a difference in the evolution. Despite this, in itself, this seems a perfectly viable multi-field model of inflation.

\section{Removing $\nu$ and $\tau_{3}$ from the evolution}

As already mentioned repeatedly, we very much would like to simplify our models as much as possible before attempting to include a gauge field as we do in chapter 5 . Therefore, we will consider this example again but with both $\mathcal{V}$ and $\tau_{3}$ at their minima as we did with the previous example. In addition to this we will also ignore the offdiagonal terms in the metric between $\tau_{2}$ and $\tau_{4}$. This massively simplifies the numerics, and we will show that, by slightly shifting the initial condition for $\tau_{2}$, we can arrive at a very similar evolution for the fields, and very similar inflationary predictions. 


\section{INTRODUCTION TO KÄHLER MODULI INFLATION}
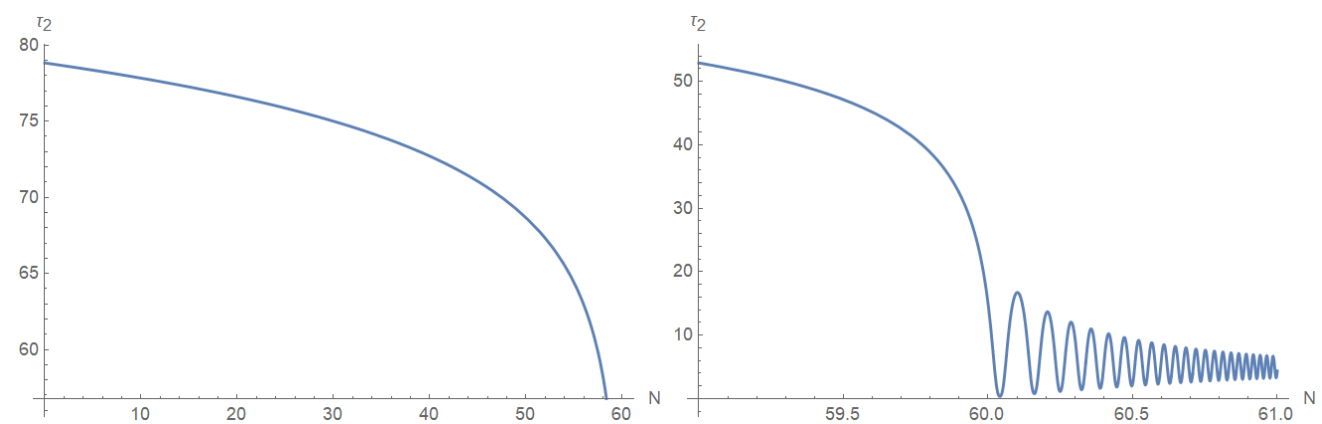

Figure 4.18: The evolution of the inflaton modulus, $\tau_{2}$, during the last 60 e-folds of inflation (left figure) and at the end of inflation when it decays (right figure) for the extra modulus example with the parameters given in 4.3.1 and 4.4.5 with the volume modulus, $\mathcal{V}$, and stabiliser, $\tau_{3}$, excluded from the evolution. The evolution proceeds almost identically to that of FIG. 4.14 for which $\mathcal{V}$ and $\tau_{3}$ were included in the evolution.

Setting $\mathcal{V}=\langle\mathcal{V}\rangle=10140$ and $\tau_{3}=\left\langle\tau_{3}\right\rangle=2.651$ to their values at the global minimum, and ignoring the off-diagonal terms leaves the three-dimensional real fieldspace metric as

$$
\gamma_{a b}=\left(\begin{array}{ccc}
\frac{3 \alpha \lambda_{2}}{4 \mathcal{V} \sqrt{\tau_{2}}} & 0 & 0 \\
0 & \frac{3 \alpha \lambda_{4}}{4 \mathcal{V} \sqrt{\tau_{4}}} & 0 \\
0 & 0 & \frac{3 \alpha \lambda_{4}}{4 \mathcal{V} \sqrt{\tau_{4}}}
\end{array}\right)
$$

with real fields $\phi^{a}=\left(\tau_{2}, \tau_{4}, b_{4}\right)$. We take

$$
\tau_{2}^{i n}=80, \quad b_{4}^{i n}=0.59 \frac{\pi}{a_{4}}, \quad \tau_{4}^{i n}=11.141
$$

The evolutions of the fields are plotted in FIGs 4.18 4.19. The inflationary predictions of this model are (60 e-folds before the end of inflation)

$$
\epsilon=2.71 \times 10^{-8}, \quad n_{s}=0.965, \quad r=4.34 \times 10^{-7}, \quad V^{1 / 4}=8.29 \times 10^{14} \mathrm{GeV} .
$$

As before, simplifying the system leads to a slight increase in the scale of the potential 60 e-folds before the end of inflation, leading to a slightly greater value for the tensorto-scalar ratio. However this system is well-approximated by this simplified example.

In this section, we have shown that we can safely introduce an extra (spectator) modulus to the system whilst satisfying observational constraints. This model is very promising with regards to our aim of enhancing its gravitational wave spectrum through the tensor modes of a gauge field supported by its coupling to an axion. 

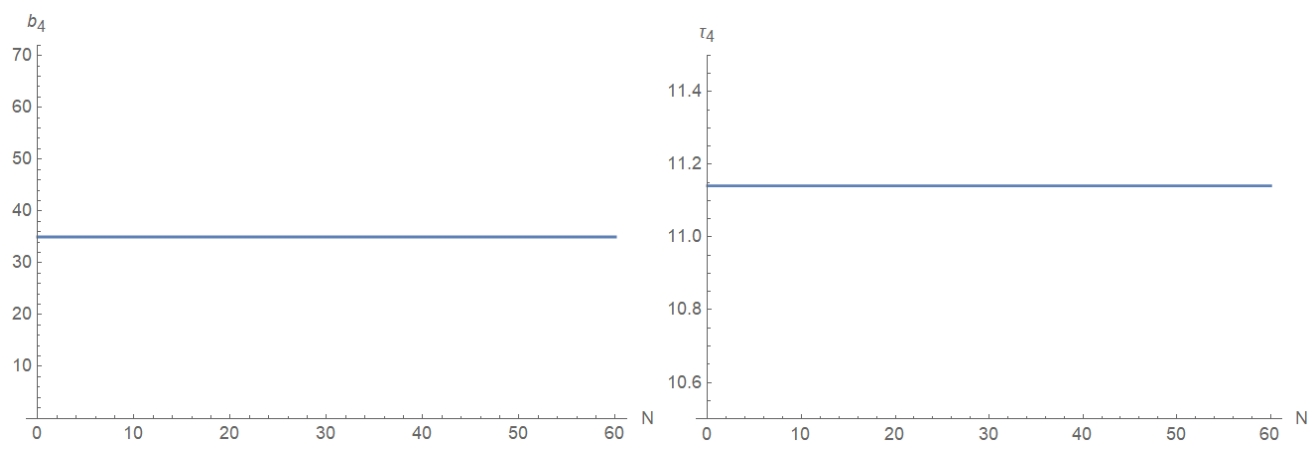

Figure 4.19: The evolution of the axion, $b_{4}$ (left figure), and its scalar superpartner, the $\tau_{4}$ modulus, during the last 60 e-folds of inflation for the extra modulus example with the parameters given in 4.3.1 and 4.4.5 with the volume modulus, $\mathcal{V}$, and stabiliser, $\tau_{3}$, excluded from the evolution. The evolution proceeds almost identically to that of FIG. 4.8 for which $\mathcal{V}$ and $\tau_{3}$ were included in the evolution. Because $b_{4}$ has no gauge field to support it, it rapidly falls to its minimum and does not evolve.

\subsection{Fibre inflation}

In relation to the mechanism of enhancing the tensor spectrum of an inflationary model, fibre inflation [50] is - at first glance - a less interesting model since it already predicts an observable tensor-to-scalar ratio. However, as we saw in chapter 3 , the tensor spectrum produced after applying the enhancement mechanism is highly chiral - giving models that use this enhancement mechanism a potentially observable (see chapter 5, 87]) and distinguishable feature, differentiating themselves from vanilla models of inflation. For this reason, in chapter 5, we will attempt to enhance the spectrum of fibre inflation in the manner described in chapter $3{ }^{13}$.

The first thing to note is that the potential in the standard case of fibre inflation

$$
\begin{aligned}
V= & \frac{W_{0}^{2}}{\mathcal{V}^{2}}\left(\frac{A}{\tau_{1}^{2}}-\frac{B}{\mathcal{V} \sqrt{\tau_{1}}}+\frac{C \tau_{1}}{\mathcal{V}^{2}}\right)+\frac{8 \sqrt{\tau_{3}}}{3 \alpha \lambda_{3} \mathcal{V}}\left(a_{3} A_{3}\right)^{2} e^{-2 a_{3} \tau_{3}} \\
& +\frac{4 a_{3} A_{3} \tau_{3}}{\mathcal{V}^{2}} W_{0} e^{-a_{3} \tau_{3}} \cos \left(a_{3} b_{3}\right)+\frac{3 \hat{\xi}}{4 \mathcal{V}^{3}} W_{0}^{2}+\frac{\delta_{u p}}{\mathcal{V}^{4 / 3}}
\end{aligned}
$$

contains no suitable axion. This is because like Kähler moduli inflation, $\tau_{3}$ is a stabiliser meaning that it and $b_{3}$ cannot be shifted far away from their minima without ruining

\footnotetext{
${ }^{13}$ We note that the original example of this enhancement mechanism in [3] was not applied to a model that initially predicted an unobservable tensor-to-scalar ratio, and was concerned merely with a modest enhancement to an already relatively large tensor spectrum.
} 


\section{INTRODUCTION TO KÄHLER MODULI INFLATION}

the stability of the potential. The reason there is no axion partner to $\tau_{1}$ nor $\tau_{2}$ is due to the form of the volume and superpotential in fibre inflation

$$
\mathcal{V}=\alpha\left(\sqrt{\tau_{1}} \tau_{2}-\gamma \tau_{3}^{3 / 2}\right)
$$

where by necessity, $\tau_{1}$ and $\tau_{2}$ are large moduli: $\tau_{1}, \tau_{2} \gg \tau_{3}$. This means that the superpotential (which is the same as in Kähler moduli inflation)

$$
W=W_{0}+\sum_{a=1}^{3} A_{a} e^{-a_{a} T^{a}} \simeq W_{0}+A_{3} e^{-a_{3} T_{3}}
$$

where the $T_{1}$ and $T_{2}$ terms are exponentially suppressed. The fact that the superpotential takes this form without the negligible contribution from the $T_{1}$ and $T_{2}$ fields leads to the potential above 4.5.1). Recall that the potential terms for $\tau_{1}$ and $\tau_{2}$ arrive from string-loop corrections to the Kähler potential. Although of course we can include the superpotential terms for $T_{1}$ and $T_{2}$ in the potential, in practice these are so small as to be irrelevant and the axions, $b_{1}$ and $b_{2}$ remain approximately massless. Therefore, our only hope of having an axion that can evolve during inflation is to include, as we did for Kähler moduli inflation in the section above, an extra modulus, $T_{4}=\tau_{4}+i b_{4}$, that has similar properties to $T_{3}$ but is not responsible for stabilising the potential. Consider

$$
\mathcal{V}=\alpha\left(\sqrt{\tau_{1}} \tau_{2}-\gamma_{3} \tau_{3}^{3 / 2}-\gamma_{4} \tau_{4}^{3 / 2}\right)
$$

where $T_{4}$ is chosen so that $\tau_{4} \sim \tau_{3} \ll \tau_{1}, \tau_{2}$. Now the superpotential becomes

$$
W \simeq W_{0}+A_{3} e^{-a_{3} \tau_{3}}+A_{4} e^{-a_{4} \tau_{4}} .
$$

The Kähler potential becomes

$$
K=-2 \ln \left(\mathcal{V}+\frac{\hat{\xi}}{2}\right) \simeq K_{0}=-2 \ln \mathcal{V}
$$

working in the large volume limit. Explicitly taking $\mathcal{V} \simeq \mathcal{V}_{0}=\alpha \sqrt{\tau_{1}} \tau_{2}$ and performing an expansion in $\sqrt{\tau_{3,4} / \tau_{1,2}}$ and keeping only the highest order term in each entry gives

$$
\partial_{a} K=\partial_{\bar{a}} K=\left(-\frac{1}{2 \tau_{1}},-\frac{1}{\tau_{2}}, \frac{3 \gamma_{3} \sqrt{\tau_{3}}}{2 \sqrt{\tau_{1}} \tau_{2}}, \frac{3 \gamma_{4} \sqrt{\tau_{4}}}{2 \sqrt{\tau_{1}} \tau_{2}}\right)
$$


The Kähler metric is

$$
K_{a \bar{b}}=\left(\begin{array}{cccc}
\frac{1}{4 \tau_{1}^{2}} & \frac{\gamma_{3} \tau_{3}^{3 / 2}+\gamma_{4} \tau_{4}^{3 / 2}}{4 \tau_{1}^{3 / 2} \tau_{2}^{2}} & -\frac{3 \gamma_{3} \sqrt{\tau_{3}}}{8 \tau_{1}^{3 / 2} \tau_{2}} & -\frac{3 \gamma_{4} \sqrt{\tau_{4}}}{8 \tau_{1}^{3 / 2} \tau_{2}} \\
\frac{\gamma_{3} \tau_{3}^{3 / 2}+\gamma_{4} \tau_{4}^{3 / 2}}{4 \tau_{1}^{3 / 2} \tau_{2}^{2}} & \frac{1}{2 \tau_{2}^{2}} & -\frac{3 \gamma_{3} \sqrt{\tau_{3}}}{4 \sqrt{\tau_{1}} \tau_{2}^{2}} & -\frac{3 \gamma_{4} \sqrt{\tau_{4}}}{4 \sqrt{\tau_{1}} \tau_{2}^{2}} \\
-\frac{3 \gamma_{3} \sqrt{\tau_{3}}}{8 \tau_{1}^{3 / 2} \tau_{2}} & -\frac{3 \gamma_{3} \sqrt{\tau_{3}}}{4 \sqrt{\tau_{1} \tau_{2}^{2}}} & \frac{3 \gamma_{3}}{8 \sqrt{\tau_{1} \tau_{2} \sqrt{\tau_{3}}}} & \frac{9 \gamma_{3} \gamma_{4} \sqrt{\tau_{3} \tau_{4}}}{8 \tau_{1} \tau_{2}^{2}} \\
-\frac{3 \gamma_{4} \sqrt{\tau_{4}}}{8 \tau_{1}^{3 / 2} \tau_{2}} & -\frac{3 \gamma_{4} \sqrt{\tau_{4}}}{4 \sqrt{\tau_{1}} \tau_{2}^{2}} & \frac{9 \gamma_{3} \gamma_{4} \sqrt{\tau_{3} \tau_{4}}}{8 \tau_{1} \tau_{2}^{2}} & \frac{3 \gamma_{4}}{8 \sqrt{\tau_{1}} \tau_{2} \sqrt{\tau_{4}}}
\end{array}\right)
$$

and its inverse is

$$
K^{a \bar{b}}=4\left(\begin{array}{cccc}
\tau_{1}^{2} & \gamma_{3} \sqrt{\tau_{1} \tau_{3}^{3}}+\gamma_{4} \sqrt{\tau_{1} \tau_{4}^{3}} & \tau_{1} \tau_{3} & \tau_{1} \tau_{4} \\
\gamma_{3} \sqrt{\tau_{1} \tau_{3}^{3}}+\gamma_{4} \sqrt{\tau_{1} \tau_{4}^{3}} & \frac{1}{2} \tau_{2}^{2} & \tau_{2} \tau_{3} & \tau_{2} \tau_{4} \\
\tau_{1} \tau_{3} & \tau_{2} \tau_{3} & \frac{2 \tau_{2} \sqrt{\tau_{1} \tau_{3}}}{3 \gamma_{3}} & \tau_{3} \tau_{4} \\
4 \tau_{1} \tau_{4} & \tau_{2} \tau_{4} & \tau_{3} \tau_{4} & \frac{2 \tau_{2} \sqrt{\tau_{1} \tau_{4}}}{3 \gamma_{4}}
\end{array}\right)
$$

where we have explicitly left everything in terms of the fields $\tau_{1,2,3,4}$. The $T_{4}$ modulus contributes equivalent terms to those of $T_{3}$ to the Kähler potential due to the symmetry of the $\tau_{3}$ and $\tau_{4}$ terms in the volume (4.5.4). Equivalently, because the superpotential 4.5.5 has symmetric terms in $T_{3}$ and $T_{4}$, we know that the potential 4.5.1) receives the additional termi 14

$$
V_{T_{4}}=\frac{8 \sqrt{\tau_{4}}}{3 \alpha \lambda_{3} \mathcal{V}}\left(a_{4} A_{4}\right)^{2} e^{-2 a_{4} \tau_{4}}+\frac{4 a_{4} A_{4} \tau_{4}}{\mathcal{V}^{2}} W_{0} e^{-a_{4} \tau_{4}} \cos \left(a_{4} b_{4}\right)
$$

and gives us the full potential (setting $b_{3}$ to its minimum)

$$
\begin{aligned}
V= & \left(\frac{A}{\tau_{1}^{2}}-\frac{B}{\mathcal{V} \sqrt{\tau_{1}}}+\frac{C \tau_{1}}{\mathcal{V}^{2}}\right) \frac{W_{0}^{2}}{\mathcal{V}^{2}}+\frac{8 a_{3}^{2} A_{3}^{2} \sqrt{\tau_{3}}}{3 \alpha \gamma \mathcal{V}} e^{-2 a_{3} \tau_{3}}-\frac{4 W_{0} a_{3} A_{3} \sqrt{\tau_{3}}}{\mathcal{V}^{2}} e^{-a_{3} \tau_{3}} \\
& +\frac{3 \hat{\xi} W_{0}^{2}}{4 \mathcal{V}^{3}}+\frac{8 \sqrt{\tau_{4}}}{3 \alpha \lambda_{3} \mathcal{V}}\left(a_{4} A_{4}\right)^{2} e^{-2 a_{4} \tau_{4}}+\frac{4 a_{4} A_{4} \tau_{4}}{\mathcal{V}^{2}} W_{0} e^{-a_{4} \tau_{4}} \cos \left(a_{4} b_{4}\right)+\frac{\delta_{u p}}{\mathcal{V}^{4 / 3}}
\end{aligned}
$$

\footnotetext{
${ }^{14}$ Cross terms in the potential involving both $\tau_{3}$ and $\tau_{4}$ are higher order in $\mathcal{O}\left(\frac{1}{v}\right)$ (see the derivation of the potential of Kähler moduli inflation in 4.1 which has the same form for the superpotential) and are therefore suppressed.
} 


\section{INTRODUCTION TO KÄHLER MODULI INFLATION}

Following [50], we wish to remove $\tau_{3}$ from the equations of motion to simplify the numerics. This is achieved by finding the minimum of $\tau_{3}$ in terms of $\mathcal{V}$. Solving $\partial V / \partial \tau_{3}=0$ gives

$$
\mathcal{V}=\frac{3 \alpha \gamma_{3} W_{0}}{a_{3} A_{3}} \sqrt{\tau_{3}}\left(\frac{1-a_{3} \tau_{3}}{1-4 a_{3} \tau_{3}}\right) e^{a_{3} \tau_{3}} \simeq \frac{3 \alpha \gamma_{3} W_{0}}{a_{3} A_{3}} \sqrt{\tau_{3}} e^{a_{3} \tau_{3}}
$$

where we have assumed $a_{3} \tau_{3} \gg 1$. This gives an approximate solution for $\tau_{3}$ of

$$
a_{3} \tau_{3} \simeq \ln \left(\frac{2 a_{3} A_{3}}{3 \alpha \gamma_{3} W_{0}}\right)
$$

and substituting this into the potential (4.5.11) leads to a four-field potential

$$
\begin{aligned}
V=\{ & \left.-\frac{3 \alpha \gamma_{3}}{2 a_{3}^{3 / 2}}\left(\ln \left(\frac{2 a_{3} A_{3}}{3 \alpha \gamma_{3} W_{0}} \mathcal{V}\right)\right)^{3 / 2}+\frac{3 \hat{\xi}}{4}\right\} \frac{W_{0}^{2}}{\mathcal{V}^{3}}+\left(\frac{A}{\tau_{1}^{2}}-\frac{B}{\mathcal{V} \sqrt{\tau_{1}}}+\frac{C \tau_{1}}{\mathcal{V}^{2}}\right) \frac{W_{0}^{2}}{\mathcal{V}^{2}} \\
& +\frac{8 a_{4}^{2} A_{4}^{2} \sqrt{\tau_{4}}}{3 \alpha \gamma_{4} \mathcal{V}} e^{-2 a_{4} \tau_{4}}+\frac{4 W_{0} a_{4} A_{4} \sqrt{\tau_{4}}}{\mathcal{V}^{2}} \cos \left(a_{4} b_{4}\right) e^{-a_{4} \tau_{4}}+\frac{\delta_{u p}}{\mathcal{V}^{4 / 3}}
\end{aligned}
$$

This is the exact same potential as in [50] (equation 3.60) with the addition of the two terms for $T_{4}$. As we did for Kähler inflation, we will use the volume modulus, $\mathcal{V}$, as a field since this is already in the potential. We therefore consider a four-field system, $\phi^{a}=\left(\tau_{1}, \mathcal{V}, \tau_{4}, b_{4}\right)$, and we note at this point that both $\tau_{1}$ and $\mathcal{V}$ can be considered as inflatons ${ }^{15}$ Again we use $K_{a \bar{b}} \partial_{\mu} T^{a} \partial^{\mu} \bar{T}^{\bar{b}}=\frac{1}{2} \gamma_{a b} \partial_{\mu} \phi^{a} \partial^{\mu} \phi^{b}$ to find the real-space field metric, $\gamma_{a b}$. We change variables from $\tau_{2}$ to $\mathcal{V} \simeq \mathcal{V}_{0}=\alpha \sqrt{\tau_{1}} \tau_{2}$ by

$$
\partial_{\mu} T_{2}=\partial_{\mu} \tau_{2}=\frac{1}{\alpha \sqrt{\tau_{1}}}\left(\partial_{\mu} \mathcal{\nu}-\frac{\mathcal{V}}{2 \tau_{1}} \partial_{\mu} \tau_{1}\right)
$$

where we are assuming the axions $b_{1}$ and $b_{2}$ are stationary. With this the real-space

\footnotetext{
${ }^{15}$ In the sense that they both have a non-negligible contribution to the number of e-folds that pass as the fields roll to their minima.
} 
metric can be found:

$$
\gamma_{a b}=\left(\begin{array}{cccc}
\frac{3}{4 \tau_{1}^{2}} & -\frac{1}{2 \tau_{1} \mathcal{V}} & 0 & 0 \\
-\frac{1}{2 \tau_{1} \mathcal{V}} & \frac{1}{\mathcal{V}^{2}} & -\frac{3 \alpha \gamma_{4} \sqrt{\tau_{4}}}{2 \mathcal{V}^{2}} & 0 \\
0 & -\frac{3 \alpha \gamma_{4} \sqrt{\tau_{4}}}{2 \mathcal{V}^{2}} & \frac{3 \alpha \gamma_{4}}{4 \mathcal{V} \sqrt{\tau_{4}}} & 0 \\
0 & 0 & 0 & \frac{3 \alpha \gamma_{4}}{4 \mathcal{V} \sqrt{\tau_{4}}}
\end{array}\right)
$$

with fields $\phi^{a}=\left(\tau_{1}, \mathcal{V}, \tau_{4}, b_{4}\right)$. The equations of motion and constraint equation of course take the same general form as in Kähler moduli inflation

$$
\begin{gathered}
\ddot{\phi}^{a}+3 H \dot{\phi}^{a}+\Gamma_{b c}^{a} \dot{\phi}^{b} \dot{\phi}^{c}+\gamma^{a b} \frac{\partial V}{\partial \phi^{b}}=0, \\
3 M_{\mathrm{pl}}^{2} H^{2}=\frac{1}{2} \dot{\varphi}^{2}+V
\end{gathered}
$$

and

$$
\frac{\dot{H}}{H^{2}}=-\epsilon_{\varphi}
$$

with $\Gamma_{b c}^{a}$ the Christoffel symbols, $\dot{\varphi}^{2}=\gamma_{a b} \dot{\phi}^{a} \dot{\phi}^{b}$ and $\epsilon_{\varphi}=\frac{\dot{\varphi}^{2}}{2 H^{2} M_{\mathrm{pl}}^{2}}$.

As an example we use the parameter set "SV2" in [50]:

$$
\begin{aligned}
& \hat{\xi}=\frac{\xi}{g_{s}^{3 / 2}}=4.59, \quad A=2.9 \times 10^{-3}, \quad B=0.93, \quad C=4.3 \times 10^{-5} \\
& W_{0}=100, \quad a_{3}=\frac{\pi}{4}, \quad A_{3}=1, \quad \alpha=0.1543, \quad \gamma_{3}=3.055, \quad \delta_{u p}=0.082
\end{aligned}
$$

and we note that there is an additional normalisation factor in front of the potential that comes from $e^{K_{c s}-\ln \left(\frac{2}{g_{s}}\right)}$, and a constant term in front of the superpotential, $\frac{g_{s}^{3}}{4 \pi}$. This normalisation is fixed so as to achieve the correct power spectrum 16 . In this example, the normalisation factor is $\frac{g_{s}^{4}}{8 \pi}=3.22 \times 10^{-4}$ with $g_{s}=0.3$. We introduce parameters for $T_{4}$

$$
a_{4}=\frac{2 \pi}{100}, \quad A_{4}=10^{-4}, \quad \gamma_{4}=0.1
$$

which are chosen so that $T_{4}$ has little effect on the inflationary evolution but so that

\footnotetext{
${ }^{16}$ As mentioned, in the Kähler moduli inflation example, this normalisation was assumed to be absorbed into the parameters, which were chosen so as to give the correct normalisation.
} 


\section{INTRODUCTION TO KÄHLER MODULI INFLATION}

$b_{4}$ is able to sustain a gauge field (see chapter 5 for this system coupled to a gauge field). Here we evolve the system without this coupling to demonstrate explicitly first the key predictions of fibre inflation, but also to show that the introduction of this fourth modulus does not ruin the inflationary predictions of the model. We find the global minimum of 4.5 .11 to be at

$$
\left\langle\tau_{1}\right\rangle=6.7637, \quad\langle\mathcal{V}\rangle=1408.1, \quad\left\langle\tau_{4}\right\rangle=15.849, \quad\left\langle b_{4}\right\rangle=\frac{\pi}{a_{4}}
$$

Shifting the principal inflaton, $\tau_{1}$, as well as $b_{4}$ (which we would like to evolve) away from their minima:

$$
\tau_{1}^{i n}=5000, \quad b_{4}^{i n}=0.5 \frac{\pi}{a_{4}}
$$

gives a local minimum for the remaining fields at

$$
\mathcal{V}^{i n}=1831.8, \quad \tau_{4}^{i n}=15.929
$$

Using these values as initial conditions, we can evolve the system. The evolutions of the fields are plotted in FIGs 4.204 .22 . The evolutions of $\tau_{1}$ and $\mathcal{V}$, the fields responsible for inflation proceed as expected (the same as in [50]) despite the introduction of the fourth modulus. As in the last example of Kähler moduli inflation above, the evolution of $\tau_{4}$ and, more importantly, $b_{4}$, is trivial. Without a gauge field to support $b_{4}$, it immediately goes to its minimum and remains there for the duration of inflation. In chapter 5, we will show that the introduction of a gauge field coupled to the axion, allows it to roll slowly for a sizeable duration of inflation. The inflationary predictions of this model are as follows (taken 50 e-folds before the end of inflation)

$$
\epsilon=3.70 \times 10^{-4}, \quad n_{s}=0.965, \quad r=5.39 \times 10^{-3}, \quad V^{1 / 4}=9.04 \times 10^{15} \mathrm{GeV} .
$$

As mentioned, fibre inflation predicts a considerably larger value for the tensor-to-scalar ratio (it is of observable value $r>10^{-3}$ ) when compared to Kähler moduli inflation - this is because the potential is of higher energy, which is likely due to the smaller value of the volume, $\mathcal{V} \sim \mathcal{O}\left(10^{3}\right)$. The introduction of the axion (and its spectator modulus) did not have a significant impact on the evolutions of the two inflationary fields, $\tau_{1}$ and $\mathcal{V}$. Although this model already predicts an observably high tensor-toscalar ratio, it is still a viable candidate for the enhancement mechanism described in 

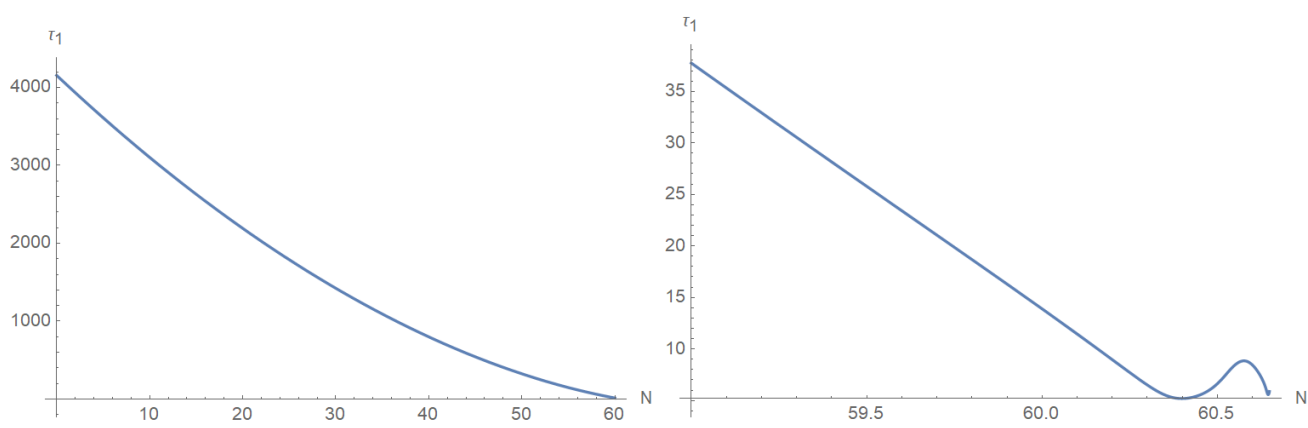

Figure 4.20: The evolution of the principal inflaton modulus, $\tau_{1}$, during the last 60 e-folds of inflation (left figure) and at the end of inflation when it decays (right figure) for fibre inflation with an additional, spectator, modulus, $T_{4}$, and parameters given in 4.5.20 4.5.21.
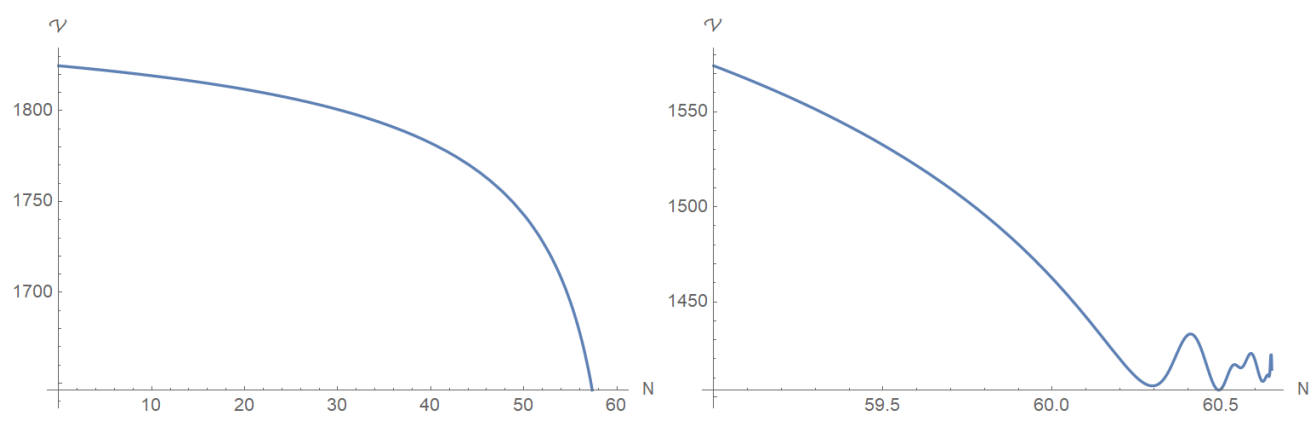

Figure 4.21: The evolution of the volume modulus, $\mathcal{V}$, during the last 60 e-folds of inflation (left figure) and at the end of inflation when it decays (right figure) for fibre inflation with an additional, spectator, modulus, $T_{4}$, and parameters given in 4.5.20 4.5.21.
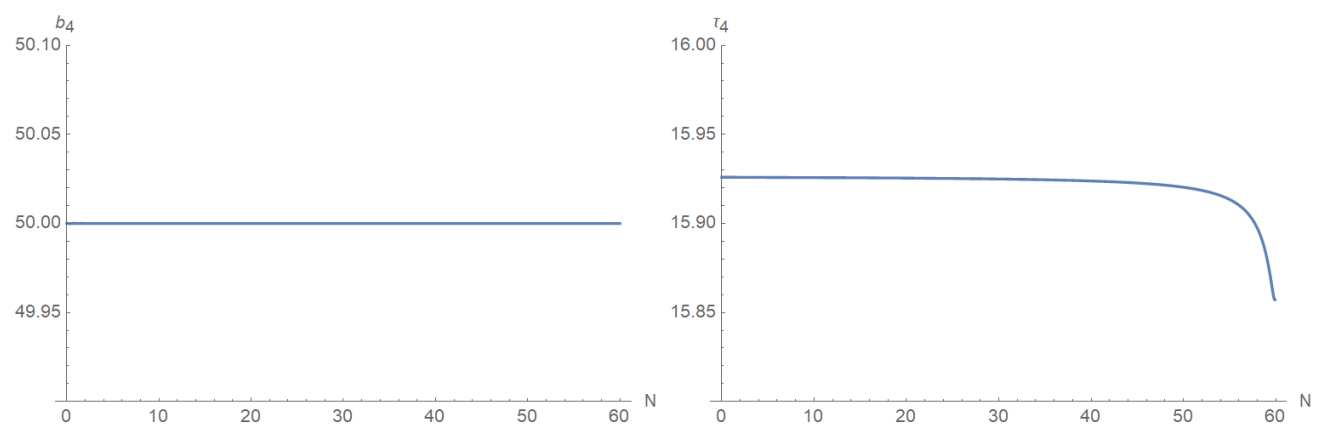

Figure 4.22: The evolutions of the axion, $b_{4}$ (left figure), and its superpartner modulus, $\tau_{4}$ (right figure), during the last 60 e-folds for fibre inflation with an additional, spectator, modulus, $T_{4}$, and parameters given in 4.5.20 -4.5.21). Because there is no gauge field to act to slow it, the axion immediately goes to its minimum and stays there for the duration of inflation. 


\section{INTRODUCTION TO KÄHLER MODULI INFLATION}

chapter 3 . In the next chapter we will apply this mechanism to a slightly modified version of this example of fibre inflation (whose inflationary sector is the same) by adding a further spectator axion. This does not modify the inflationary predictions of the model meaning the results of this section are still valid but helps to justify the necessarily large Chern-Simons-like coupling required to enhance the gravity sector.

\subsection{Summary}

In this chapter, we have considered three examples of Kähler moduli inflation and examined their general features: first, in section 4.2, we looked at a "bog-standard" low-energy example, where the tensor-to-scalar ratio is exceptionally small $\left(r \sim 10^{-15}\right)$. Since we are interested in enhancing the tensor-to-scalar ratio of Kähler inflation to observable values, we decided to a look at an example with higher energy, in section 4.3 this example predicts a considerably larger background tensor-to-scalar ratio, $r \sim 10^{-6}$, and naturally contains an axion that could be coupled to a gauge field. In addition to this we also considered a model, in section 4.4 with the same parameters, but with the addition of an extra modulus to act as the field coupled to the gauge field. We showed that this extra modulus, although following a trivial evolution without the presence of a gauge field, has no ill effect on the inflationary predictions of the model. We also importantly demonstrated that these last two models can be greatly simplified by setting both the volume modulus and the stabiliser modulus to their values at the global minimum and discarding them from the evolution whilst maintaining the generic features and inflationary predictions of the model. This will greatly simplify the numerics when we couple this system to a gauge field, which can be tricky, especially when we examine the perturbations of the system.

Finally, in section 4.5, we considered a model of fibre inflation to which we have added an extra modulus to act as a spectator and couple to a gauge field. This system satisfies observational constraints, and, being fibre inflation, predicts an observable value of the tensor-to-scalar ratio $\left(r \sim 10^{-3}\right)$. The inclusion of the extra modulus did not negatively impact the evolutions of the other fields, $\tau_{1}$ and $\mathcal{V}$, and inflation proceeds as expected when compared to the case without the extra modulus (see [50], section 3.4.2). 


\section{Chapter 5}

\section{Primordial Gravitational Waves in String Inflation}

This chapter includes much of a paper published in Journal of Cosmology and Astroparticle Physics written with Ivonne Zavala and Gianmassimo Tasinato titled On chromo-natural inflation in string theory [88]. We include examples of Kähler and fibre inflation where a Kähler modulus, $T$, is coupled to a gauge field and show how this coupling can lead to a large enhancement of the tensor-to-scalar ratio; large enough, in fact, to lift the tensor sector to a potentially observable value for $r$ despite the low energy scale of Kähler modulus inflation. As well as the example that is included in the paper, we discuss some additional examples. In particular, in this chapter we will first consider some phenomenological examples, before discussing the example that is included in the paper.

\subsection{Introduction}

Vanilla, single field, models of inflation are of course in good agreement with the most recent data. However ongoing and future experiments will allow us to take a step forward in testing the inflationary paradigm. Particularly interesting are the PGWs produced by inflation, which lead to a distinctive $B$-mode pattern in the CMB polarisation [89, 90], that is being searched for by a wide range of ground-based, balloon and satellite experiments 39 44]. Current bounds on the tensor-to-scalar ratio $r$ from 


\section{PRIMORDIAL GRAVITATIONAL WAVES IN STRING INFLATION}

Planck and BICEP/Keck restrict $r<0.07$ [37], but future experiments are likely to be able to reach a sensitivity of order $\Delta r \simeq 10^{-3}$.

In theories beyond the standard model of particle physics and cosmology, other fields besides the inflaton may be present during inflation and can have interesting consequences. Their dynamics can participate to the inflationary mechanism at the level of background or fluctuation evolution, and can leave imprints on the properties of tensor modes, for example by amplifying their spectrum. This implies that even models of inflation that normally predict a small value of $r$ can see the primordial tensor spectrum amplified by couplings with additional fields.

Perhaps the most studied example is the case with several scalar fields giving rise to a multi(scalar) field inflationary scenario (see e.g. 91] for a review and references therein). However, spin one particles have also been considered in various scenarios (see e.g. [58] for a review and references therein). Non-abelian gauge fields have attracted a lot of attention in cosmology recently [78, 79, 92, 93. Interestingly, in these models the spin-2 sector of gauge field fluctuations can provide a source for primordial gravitational waves as shown in chapter 3 , thus potentially enhancing the amplitude of the gravitational wave spectrum of low-energy models of inflation, up to values observable with future CMB polarisation experiments. Moreover, the PGW spectrum turns out to be chiral (only the right-helicity mode of the gauge field and hence the gravity mode gets enhanced), making it potentially distinguishable from a vanilla inflation scenario. These scenarios are consequently very interesting for their potential to generate distinctive observables that can be probed by the next generation of CMB polarisation experiments.

In particular, an $S U(2)$ gauge field $A_{\mu}^{a}$ is used in the chromo-natural inflation (CNI) model proposed in [78, 79]. The original motivation of this model was to relax the requirement of a super-Planckian decay constant $f$ in natural inflation 2 , by using the Chern-Simons (CS) coupling $\chi F_{\mu \nu}^{A} \tilde{F}^{A \mu \nu}$ of the axionic inflaton field $\chi$, to the gauge field with field strength $F_{\mu \nu}^{A}$. This coupling effectively provides a friction term on the axion dynamics, allowing inflation to occur in a steeper potential with $f<M_{\mathrm{pl}}$. It has however been demonstrated that in this original form the CNI model is not

\footnotetext{
${ }^{1}$ Also, amplification of tensor modes by spectator scalar fields has been discussed in e.g. [94 96, and the prospects for direct detection with future gravitational-wave experiments were recently discussed in 97 .

${ }^{2}$ See 98 for a review of natural inflation.
} 
observationally viable [81, 99] as it cannot satisfy constraints on the scalar spectral index. An interesting proposal to alleviate this problem is to introduce a separate inflationary sector, while the gauge-axion sector acts only as a spectator [3, 100] (this is the second model shown in chapter 3): this scenario is dubbed spectator CNI (SCNI). A common feature of both these proposals, which shall constitute an important point for our investigation, is a large axion-gauge CS coupling (where the axion, gauge field - and inflaton in SCNI - are canonically normalised):

$$
\mathcal{L} \supset \frac{\lambda}{4 f} \chi F_{\mu \nu}^{A} \tilde{F}^{A \mu \nu}
$$

(where $F=d A-g A \wedge A, g$ being the gauge coupling). Successful chromo-natural inflationary proposals that enhance tensor modes can be constructed, but must take into account that an enhancement of primordial tensor modes generally implies a very large enhancement of the tensor fluctuations of the gauge field. One needs to avoid excessive backreaction of the gauge field fluctuations to the background - as emphasised for example in [3, 101 103] - that would spoil the background inflationary dynamics. This condition typically requires very small values for the gauge coupling $\mathrm{g}$.

These facts indicate that it is not simple to design theories with the correct properties to realise satisfactory (S)CNI models, and one has to carefully balance between different requirements on the quantities involved. Here we will explore the possibility of embedding SCNI in models of Kähler moduli inflation and look for a consistent SCNI model, accompanied by a relatively large amplitude of the gravitational wave spectrum.

\section{Brief review of attempts so far to embed CNI in supergravity and string theory}

Before discussing our model, we review two ideas that have been proposed so far in this direction.

The first interesting possibility is to embed CNI in supergravity, as proposed in [104]. In supergravity, the coupling between the axion (and saxion) to the gauge sector is dictated by the gauge kinetic function, $f_{A}$, as:

$$
\mathcal{L} \supset-\frac{\operatorname{Re}\left(f_{A}\right)}{4} F_{\mu \nu}^{A} F^{A \mu \nu}+\frac{\operatorname{Im}\left(f_{A}\right)}{4} F_{\mu \nu}^{A} \tilde{F}^{A \mu \nu},
$$




\section{PRIMORDIAL GRAVITATIONAL WAVES IN STRING INFLATION}

where $f_{A}$ is a holomorphic function of the superfields, including the axion that acts as the inflaton in CNI. The proposal to account for a large CS coupling was to introduce two canonically normalised (super)fields, $\Phi_{j}=\alpha_{j}+i \phi_{j}, j=1,2$ and choose the gauge kinetic function as $f_{A}=1+i c \frac{\Phi_{1}}{\Phi_{2}}$ with $c$ a number of order one. The inflationary trajectory occurs for $\alpha_{i}=0$, such that, $\operatorname{Re}\left(f_{A}\right)=1$, while $\operatorname{Im}\left(f_{A}\right)=\phi_{1} / \phi_{2}$. The superpotential is chosen such that $\phi_{2}$ is heavier than the Hubble scale and it is thus kept fixed at its minimum at a suitable value of $\left\langle\phi_{2}\right\rangle$ during inflation 4 , As we have mentioned before, CNI has been shown to be observationally unviable, however, one could in principle add to this set-up an inflationary sector as in [3], while keeping the axion-gauge sector as spectators. However, the viability of such a model will need to be carefully scrutinised when adding more fields; also the backreaction of the gauge field tensor fluctuations on the background evolution will be hard to control without introducing new parameters (i.e. superfields) 5 [3, 101, 102]. We will discuss this aspect in detail.

The second possibility is the work of [4] to embed SCNI in a string theory set-up. In [4] a simplified string model is considered using gaugino condensation on magnetised D7-branes in type IIB CY orientifold compactifications, and the axion associated to the 2-form potential $C_{2}$ present in the compactification (this was used in [105, 106] to realise natural inflation in string theory). It is argued that this set-up could in principle be embedded in a large volume scenario (LV) for moduli stabilisation [84, 85], with the inflationary sector being a model of Kähler inflation [49]. Although an explicit string model was not presented they consider as possible values for the relevant parameters: $\lambda=50, f=10^{-12} M_{\mathrm{pl}}$, a gauge coupling $g_{A}=0.7$, and an axion scale 6 $\mu \sim 5.89 \times 10^{-8} M_{\mathrm{pl}}$, that is, $\mu \gg f$, which may be theoretically inconsistent due to unitarity constraints as discussed in [107]. Importantly, the backreaction of the gauge field tensor fluctuations on the background was not considered in [4]. It has been

\footnotetext{
${ }^{3}$ In [104], $f_{A}=1+c \frac{\Phi_{1}}{\Phi_{2}}$ with the norm $|c|$ a number of order one; $c$ needs to be purely imaginary in order for the axion-gauge coupling to be non-zero along the inflationary trajectory, which is given by $\operatorname{Re} \Phi_{i}=\alpha_{i}=0$.

${ }^{4}$ The Kähler potential and superpotential for this model are given by $K=\frac{1}{2}\left(\Phi_{1}+\bar{\Phi}_{1}\right)^{2}+\frac{1}{2}\left(\Phi_{2}+\right.$ $\left.\bar{\Phi}_{2}\right)^{2}+S \bar{S}$ and $W=S\left[i \sqrt{2} \Lambda \sinh \left(i \Phi_{1} / \sqrt{2} \Phi_{2}\right)+\left(\Lambda_{2}^{2}+\Phi_{2}^{2}\right)\right]$, where $\Phi_{i}=\alpha_{i}+i \phi_{i}$ and $\alpha_{i}=S=0$ during inflation, while $\Lambda_{2}=\left\langle\phi_{2}\right\rangle$ sets the large coupling required.

${ }^{5}$ As we will discuss, the gauge coupling needs to be sufficiently small to guarantee control on the backreaction. This could be introduced in supergravity by adding a third superfield, $\Phi_{3}$ to the configuration of [104], in addition to the inflationary sector, such that $\left\langle\Phi_{3}\right\rangle$ sets the required small coupling.

${ }^{6}$ Assuming an effective potential for the axion of the form $V=\mu^{4}(1-\cos (\chi / f))$.
} 
argued in the literature that backreaction imposes strong constraints on the parameters [3, 101, 102]. As we shall discuss in detail, keeping under control the backreaction of gauge fluctuations requires an additional parameter, which needs to be sufficiently small.

\section{What we do in this work}

In light of these results, we consider in detail the requirements for an explicit realisation of the SCNI scenario in a string theory set-up, including for the first time a careful estimate of backreaction issues for gauge fluctuations. As we will see, and already pointed out in [4] (and [104] in supergravity), string theory naturally includes the field content of SCNI, albeit in a more intricate form. Given the phenomenologically appealing features of this scenario, and the forthcoming experimental opportunities associated with CMB polarisation, it is of paramount importance to investigate the possibility of realising this model in a theoretically consistent theory, keeping backreaction under control.

As a concrete set-up, we adopt the LV scenario [84, 85] in type IIB CY orientifold compactifications. Within this framework, we consider Kähler inflation, where the tensor-to-scalar ratio is too small to be observationally relevant, namely $r<10^{-6}$ [49, [76], and include one of the gaugino condensation sectors involved in moduli stabilisation as the spectator sector to explore realisations of SCNI [3, 101], which will be capable of enhancing the amplitude of primordial tensor modes to observable levels.

We organise our discussion as follows:

- In section 5.2, we discuss the dynamics of gauge fields in general multi-field inflationary scenarios that arise in typical supergravity and string theory models. In particular, the metric in the scalar manifold is generically curved, the scalar potential is non-separable, and the coupling of the scalars to gauge fields is nontrivial. Within this general set-up, we discuss the background evolution and requirements for successful inflation in this more general configuration. Once specialised on the case of spectator chromo-natural inflation, we show how the background evolution leads to conditions on the parameters in 5.1.1.

- In section 5.3 we then analyse gauge fields in Kähler inflation and the embedding of SCNI in this framework, introducing the relevant parameters discussed above 


\section{PRIMORDIAL GRAVITATIONAL WAVES IN STRING INFLATION}

and presenting the full background evolution, setting up the system phenomenologically with two parameters $c_{1}$ and $c_{2}$. We attempt to apply the enhancement mechanism discussed in chapter 3 to the examples considered in chapter 4.

- In sections 5.4 5.6 we focus on a detailed analysis of the tensor, scalar perturbations for the models of section 5.3 , including a careful estimate of the backreaction of the gauge fluctuations on the background equations of motion. We show that the amplitude of primordial tensor modes can be enhanced by a factor of order $10^{4}$ with respect to the typical values met in Kähler inflation set-up. In order to avoid excessive backreaction from gauge fluctuations, we need to reduce the value of the effective gauge coupling for the gauge group under consideration, and this implies we need to choose a large value $N \sim 10^{6}$ for the degree of the gauge group $S U(N)$. We find that the simplest example from chapter 4 where the axion coupled to the gauge field is the superpartner of the inflaton is unable to satisfy constraints on the backreaction, whereas the four-field model provides a viable model.

- In section 5.7, we consider the slightly more complex scenario discussed in [88] where the superpotential of Kähler moduli inflation is modified in order to justify the large coupling between the axion and the gauge field. We show that the conditions to obtain successful slow-roll inflation can be satisfied by turning on large values for the magnetic flux on the D7 branes.

- In section 5.8 we consider the feasibility of this model.

- In section 5.9, we apply this same model to fibre inflation and demonstrate that one can achieve a highly chiral spectrum for fibre inflation.

\subsection{Gauge fields in general multi-field inflation}

We start by introducing the general set-up that arises when considering $S U(N)$ gauge fields coupled to an axion in a general multi-field inflationary system, and then study in detail the requirements for successful inflationary dynamics. Our aim is to determine general conditions that a successful model of slow-roll inflation has to satisfy in the 
general set-up we study. Moreover, when specialising to the case spectator chromonatural inflation, we show that our formulae require large Chern-Simons couplings between the axion and gauge fields (as introduced in eqn. (5.1.1)).

The action describing the system we are interested in consists of multiple scalar fields interacting with a gauge field. It reads

$S=\int \mathrm{d}^{4} x \sqrt{-g}\left[\frac{M_{\mathrm{pl}}^{2}}{2} R-\frac{\gamma_{a b}\left(\phi^{c}\right)}{2} \partial_{\mu} \phi^{a} \partial^{\mu} \phi^{b}-V\left(\phi^{a}\right)-\frac{f\left(\phi^{a}\right)}{4} F_{\mu \nu}^{A} F^{A \mu \nu}+\frac{h\left(\phi^{a}\right)}{4} F_{\mu \nu}^{A} \tilde{F}^{A \mu \nu}\right]$,

where $\gamma_{a b}\left(\phi^{c}\right)$ is the metric of the scalar manifold spanned by the scalar fields $\phi^{a}$, and $V\left(\phi^{c}\right)$ is the scalar potential, which is generally not separable; that is, the scalar fields generically interact via the kinetic and/or potential terms. The scalar sector with $a=1, \ldots n$ scalars, contains the axion as well as the inflaton(s), which may be constituted by one or more fields, non-trivially coupled to one another. The coupling of the scalars (inflaton(s) and axion(s)) to the gauge sector is dictated by the functions $f\left(\phi^{a}\right), h\left(\phi^{a}\right)$, which generically depend on the scalar fields ${ }^{8}$

The gauge group is in general $S U(N)$ and the gauge field strength, $F_{\mu \nu}^{A}$, is given by

$$
F_{\mu \nu}^{A}=\partial_{\mu} A_{\nu}^{A}-\partial_{\nu} A_{\mu}^{A}-f^{A B C} A_{\mu}^{B} A_{\nu}^{C},
$$

where $f^{A B C}$ are the structure functions of $S U(N)$, and the dual, $\tilde{F}^{A \mu \nu}$, is defined as $\tilde{F}^{A \mu \nu}=\epsilon^{\mu \nu \alpha \beta} F_{\alpha \beta}^{A} /(2 \sqrt{-g})$ with $g$ the metric determinant. Let us stress that at this stage the gauge field is not canonically normalised. Thus, there is no gauge coupling, $g_{A}$, appearing in the definition of $F^{A}$, nor in the action. The gauge coupling is fielddependent and it is given by $g_{A}^{2}=1 / f\left(\phi^{a}\right)$ once the scalar field $\phi^{a}$, coupled to the gauge field, has been stabilised. However, while the scalar is evolving, we can define an instantaneous gauge coupling at a fixed time, $t_{0}$ as, $g_{A, 0}^{2}=1 / f\left(\phi^{a}\left(t_{0}\right)\right)$ as will be the case in our string theory set-up.

We now discuss the cosmological background evolution and slow-roll dynamics of the system. Specifically we are interested in the case where two scalar fields couple to

\footnotetext{
${ }^{7}$ In [100], a generalisation of CNI was presented where the canonically normalised inflaton - driven by a dilaton - and the axion, are both coupled to a canonically normalised $S U(2)$ gauge field. In our notation, they had a flat scalar metric, $\gamma_{a b}=\delta_{a b}, f\left(\phi^{a}\right)$ a function of the inflaton and $h\left(\phi^{a}\right)=\frac{\lambda}{f} \sigma$, where $\sigma$ denotes the axion.

${ }^{8}$ As can be inferred from the introduction, and as we will discuss below, they are related to the real and imaginary parts of the gauge kinetic function.
} 


\section{PRIMORDIAL GRAVITATIONAL WAVES IN STRING INFLATION}

the gauge field via the functions $f\left(\phi^{a}\right)$ and $h\left(\phi^{a}\right)$, plus an additional scalar field, not coupled to the gauge field, which acts as the inflaton. That is, the spectator sector consists of two scalars coupled to the gauge field. This system generalises the chromonatural inflation models discussed in [3, 78].

\subsubsection{Background evolution and slow-roll inflation}

We consider a homogeneous and isotropic flat FRW metric $d s^{2}=-d t^{2}+a(t)^{2} d x^{i} d x_{i}$, where $a(t)$ is the scale factor. To treat the $S U(N)$ case, we proceed as follows. First, homogeneity and isotropy of the gauge field energy density, and hence the background can be maintained by splitting the $S U(N)$ gauge group into $\mathcal{N}=[N / 2](N / 2 \bmod 2)$ disjoint sub-groups of $S U(2)$ [108, 109]. In each sub-group, the gauge field equals a different scalar, which can be assumed to be locked into the following isotropic configuration,

$$
A_{0(n)}^{A}=0, \quad A_{i(n)}^{A}=a(t) Q_{n}(t) \delta_{i}^{A}, \quad n=1, \ldots, \mathcal{N}
$$

Notice that now within each sub-group, $f^{A B C}=\epsilon^{A B C}$. In the background configuration 5.2 .3 , the field strength tensor components within each sub-group are,

$$
\begin{aligned}
F_{0 i(n)}^{A} & =-a E_{i(n)}^{A}=a(t)\left[H Q_{(n)}(t)+\dot{Q}_{(n)}(t)\right] \delta_{i}^{A}, \\
F_{i j(n)}^{A} & =a^{2} \epsilon_{i j k} B_{k(n)}^{A}=-\epsilon_{i j}^{A}\left[a(t) Q_{(n)}(t)\right]^{2}
\end{aligned}
$$

where $H=\dot{a} / a$ is the Hubble parameter.

For $\mathcal{N}>1$, it is difficult to solve the full background evolution. However, a simplification occurs if one assumes that each $S U(2)$ sub-group has a common field strength 9 , $F^{A}$. In this case, the system and equations of motion are equivalent to those of the single $S U(2)$ cas ${ }^{10}$ by replacing $A_{i}^{A} \rightarrow A_{i}^{A} / \sqrt{\mathcal{N}}$, that is,

$$
Q \rightarrow \frac{Q}{\sqrt{\mathcal{N}}}
$$

and defining an effective gauge coupling, $\mathrm{g}$, as

$$
g:=\frac{1}{\sqrt{\mathcal{N}}}
$$

\footnotetext{
${ }^{9}$ This can be realised by initialising the system with a common initial condition. See also the discussion in [108.

${ }^{10}$ In this way, the system becomes equivalent to that studied in chapter 3
} 
which is now equivalent to having $F=d A-\mathrm{g} A \wedge A$, without introducing this coupling into the function $h(\phi)$. Let us stress again that this is not the standard gauge coupling, which is still given by $g_{A}^{2}=1 / f\left(\phi^{a}\right)$ defined above and at this stage is field dependent. From (5.2.7) we already see that a small (effective) gauge coupling g can be achieved for a large gauge group, $N$. Keeping this in mind, we focus on the single $S U(2)$ case with an effective gauge coupling given by (5.2.7), to investigate the background evolution of the system.

In this case, the equations of motion for the metric are given by

$$
\begin{aligned}
3 M_{\mathrm{pl}}^{2} H^{2} & =\rho_{\varphi}+\rho_{Y M}, \\
2 M_{\mathrm{pl}}^{2} \dot{H} & =-\dot{\varphi}^{2}-2 f\left(\phi^{a}\right)\left[(H Q+\dot{Q})^{2}+\mathrm{g}^{2} Q^{4}\right],
\end{aligned}
$$

where in $(5.2 .8)$ the energy densities are

$$
\rho_{\varphi}=\frac{1}{2} \dot{\varphi}^{2}+V\left(\phi^{a}\right), \quad \rho_{Y M}=\frac{3}{2} f\left[(H Q+\dot{Q})^{2}+\mathrm{g}^{2} Q^{4}\right]
$$

and we have defined:

$$
\dot{\varphi}^{2}:=\gamma_{a b} \dot{\phi}^{a} \dot{\phi}^{b}
$$

The scalar equations of motion are given by

$\ddot{\phi}^{a}+3 H \dot{\phi}^{a}+\Gamma_{b c}^{a} \dot{\phi}^{b} \dot{\phi}^{c}+\gamma^{a b} V_{, b}=-3 \mathrm{~g} \gamma^{a b} h_{, b} Q^{2}(H Q+\dot{Q})+\frac{3}{2} \gamma^{a b} f_{, b}\left((H Q+\dot{Q})^{2}-\mathrm{g}^{2} Q^{4}\right)$,

where $X_{, a}$ denotes a derivative with respect to the field $\phi^{a}$. Note that the RHS of this equation will be zero for any scalar field that is not coupled to the gauge field. Note as well that these equations are valid also if the inflationary sector (decoupled from the gauge field) includes several scalars. In this case, there may be further interesting phenomenology due to possible large turns (see e.g. [110 116]). We leave exploration of this possibility for future work.

Finally, the gauge field equation is given by

$$
\ddot{Q}+3 H \dot{Q}+Q\left(\dot{H}+2 H^{2}\right)+2 \mathrm{~g}^{2} Q^{3}=\mathrm{g} Q^{2} \dot{\phi}^{a} \frac{h_{, a}}{f}-\dot{\phi}^{a} \frac{f_{, a}}{f}(Q H+\dot{Q})
$$

where on the RHS only the scalar fields coupled to the gauge field will appear. 


\section{PRIMORDIAL GRAVITATIONAL WAVES IN STRING INFLATION}

The energy-momentum conservation equation, $\nabla_{\mu} T^{\mu \nu}=0$, where $T^{\mu \nu}$ is the total energy momentum tensor including the scalars and gauge field, further gives $(\nu=0)$ :

$$
\begin{aligned}
& \dot{\rho}_{\varphi}+3 H\left(\rho_{\varphi}+p_{\varphi}\right)=\dot{\phi}^{a} Q_{a Y M}, \\
& \dot{\rho}_{Y M}+4 H \rho_{Y M}=-\dot{\phi}^{a} Q_{a Y M},
\end{aligned}
$$

with $p_{\varphi}=\frac{1}{2} \dot{\varphi}^{2}-V\left(\phi^{a}\right)$ and

$$
Q_{a Y M}:=-3 \mathrm{~g} h_{, a} Q^{2}(H Q+\dot{Q})+\frac{3}{2} f_{, a}\left[(H Q+\dot{Q})^{2}-\mathrm{g}^{2} Q^{4}\right] .
$$

Equations (5.2.14 show in a neat way the non-trivial interplay between the scalar and gauge field dynamics in a cosmological setting.

\subsubsection{Slow-roll Dynamics}

To study inflation, we define the first slow-roll parameter in the usual way as

$$
\epsilon:=-\frac{\dot{H}}{H^{2}}=\epsilon_{\varphi}+\epsilon_{E}+\epsilon_{B}
$$

where we introduced the slow-roll parameters for the scalars and the gauge field 11 .

$$
\epsilon_{\varphi}:=\frac{\dot{\varphi}^{2}}{2 H^{2} M_{\mathrm{pl}}^{2}}, \quad \epsilon_{E}:=\frac{f(H Q+\dot{Q})^{2}}{H^{2} M_{\mathrm{pl}}^{2}}, \quad \epsilon_{B}:=\frac{f \mathrm{~g}^{2} Q^{4}}{H^{2} M_{\mathrm{pl}}^{2}}
$$

During inflation, $\epsilon=\epsilon_{\varphi}+\epsilon_{E}+\epsilon_{B} \ll 1$, and the energy density must be dominated by the scalar potential, $3 M_{\mathrm{pl}}^{2} H^{2} \sim V$. Note that there may be different hierarchies among the individual slow-roll parameters, but the overall $\epsilon \ll 1$ has to be small.

We now define a second small slow-roll parameter as follows:

$$
\eta:=\frac{\dot{\epsilon}}{H \epsilon}=2 \epsilon_{H}-2 \frac{\epsilon_{\varphi}}{\epsilon_{H}} \delta_{\varphi}+\xi_{f}\left(\epsilon_{B}+\epsilon_{E}\right)+2 \frac{\epsilon_{E}}{\epsilon_{H}} \delta_{E}+4 \frac{\epsilon_{B}}{\epsilon_{H}} \delta_{B} \ll 1 .
$$

where we introduce the quantities

$$
\delta_{\varphi}:=-\frac{\ddot{\varphi}}{H \dot{\varphi}}, \quad \delta_{E}:=\frac{\frac{d}{d t}(\dot{Q}+H Q)}{H(\dot{Q}+H Q)}, \quad \delta_{B}:=\frac{\dot{Q}}{H Q},
$$

\footnotetext{
${ }^{11}$ Notice in comparison to the case of chapter 3, $\epsilon_{E, B}$ have an additional factor of $f\left(\phi^{a}\right)$. This is because of the non-canonical nature of the $f F^{2}$ term in the action.
} 
which we take to be small during inflation (to ensure that $\eta$ is small barring cancellations), as well as the small parameter

$$
\xi_{f}:=M_{\mathrm{pl}} \frac{f_{a} \dot{\phi}^{a}}{2 f \dot{\varphi}} \sqrt{2 \epsilon_{\varphi}}=\frac{f_{a} \dot{\phi}^{a}}{2 f H} .
$$

Notice that the slow-roll conditions defined above do not involve the coupling between the axion and the gauge field, $h$. Therefore, this coupling can be large during inflation.

Dropping all terms with time derivatives except on the RHS of the equation for $Q$, 5.2.13), which transfers part of the scalar sector kinetic energy to the background gauge field, we get the relation

$$
2 H^{2} Q+2 \mathrm{~g}^{2} Q^{3} \simeq \mathrm{g} Q^{2} \frac{h_{a} \dot{\phi}^{a}}{f}-H Q \frac{f_{a} \dot{\phi}^{a}}{f}
$$

Introducing the parameters

$$
\xi_{h}:=M_{\mathrm{pl}} \frac{h_{a} \dot{\phi}^{a}}{2 f \dot{\varphi}} \sqrt{2 \epsilon_{\varphi}}=\frac{h_{a} \dot{\phi}^{a}}{2 f H}, \quad \xi_{Q}:=\frac{\mathrm{g} Q}{H},
$$

eq. (5.2.21) becomes

$$
1+\xi_{Q}^{2} \simeq \xi_{h} \xi_{Q}-\xi_{f}
$$

In fact in every scenario we consider (5.2.21) is dominated by $2 \mathrm{~g}^{2} Q^{3}$ and $\mathrm{g} Q^{2} \frac{h_{a} \dot{\phi}^{a}}{f}$ and we get the very approximate solution

$$
2 \mathrm{~g}^{2} Q^{3} \sim \mathrm{g} Q^{2} \frac{h_{a} \dot{\phi}^{a}}{f} \Longrightarrow \xi_{Q} \sim \xi_{h}
$$

Physically, the parameters $\xi_{f}$ and $\xi_{h}$ represent a dimensionless (i.e. scaled by $\frac{1}{H}$ ) measure of the speeds of the fields coupled to the gauge field through $f$ and $h$, respectively, scaled by $\frac{1}{f}$. Meanwhile, the parameter $\xi_{Q}$ can be seen to be roughly equal to the square root of the ratio of the magnetic and electric parts of the energy density of the gauge field (see 5.2 .10$)$ ) when the system is in slow-roll:

$$
\sqrt{\frac{\rho_{B}}{\rho_{E}}}=\frac{\mathrm{g} Q^{2}}{H Q+\dot{Q}} \simeq \frac{\mathrm{g} Q}{H}=\xi_{Q}
$$

where in the second step we have assumed slow-roll evolution for $Q$ : $\dot{Q} \ll H Q$. As we 


\section{PRIMORDIAL GRAVITATIONAL WAVES IN STRING INFLATION}

saw, the slow-roll conditions imply that $\xi_{f} \ll 1$, while no condition is required for $\xi_{Q}, \xi_{h}$, which can be significant, and given (5.2.23), we have, $\xi_{h} \gtrsim \xi_{Q}$. Stability analysis in CNI has shown that scalar perturbations are stable for $\xi_{Q}>\sqrt{2}$ and therefore $\xi_{h} \gtrsim \xi_{Q}>\sqrt{2}$ [78 80, 117]. Then $\xi_{h}>1$, which implies

$$
\frac{M_{\mathrm{pl}}}{f} \frac{h_{a} \dot{\phi}^{a}}{2 \dot{\varphi}}>\frac{1}{\sqrt{2 \epsilon_{\varphi}}}
$$

This is the first important constraint on the background field evolution that any successful model of inflation described by action (5.2.1) should satisfy. Note that this relation depends on the ratio between the gauge field coupling to the scalars via $h_{a} / f$ and has non-trivial implications for their values as we now discuss.

\subsubsection{Large Chern-Simons couplings are needed in spectator chromo- natural inflation}

Let us consider the condition in eq. 5.2.26 in the known cases of CNI and SCNI. In those examples, $h\left(\phi^{a}\right)=\lambda \chi / f$ (see eq. 5.1.1) in the Introduction) where $\chi$ is the axion, while $f\left(\phi^{a}\right)=1$. Then, condition (5.2.26) reduces to [117]

$$
\frac{M_{\mathrm{pl}}}{f} \frac{\lambda}{\sqrt{2}}>\frac{1}{\sqrt{\epsilon_{\chi}}},
$$

where $\epsilon_{\chi}$ is the slow-roll parameter associated to the axion field alone: $\epsilon_{\chi}=\frac{\dot{\chi}^{2}}{2 M_{\mathrm{pl}}^{2} H^{2}}$ (that is, the inflaton does not enter in this relation in the case of SCNI). Therefore it is clear that in CNI, where the axion is also the inflaton, the larger the combination $\lambda M_{\mathrm{pl}} / f$ is, the smaller $\epsilon_{\chi}$ will be. In other words, to avoid an excessively large coupling $\lambda M_{\mathrm{pl}} / f, \epsilon_{\chi}$ will be as large as possible (see e.g. [117] where $M_{\mathrm{pl}} \lambda / f \sim 10$ ). On the other hand, in order to ensure a maximal enhancement of the tensor fluctuations from the gauge field, the parameter $\xi_{Q}$ needs to be as large as possible, while keeping the backreaction of the gauge tensor perturbations on the background equations of motion under control [3, 101]. We will see this in detail in the string theory realisation in sections 5.3 and 5.4 .

In the extended version of chromo-natural inflation [3], the axion and gauge fields act as spectators and therefore the requirement of a large axion coupling from the 
condition (5.2.27) can be relaxed by consistently maximising the value of $\epsilon_{\chi}$, while having $\epsilon_{\phi}<\epsilon_{\chi} \ll 1$, where $\phi$ is the inflaton. An extreme example of this situation with $\epsilon_{\phi} \sim 10^{-73}, H_{\text {inf }} \sim 10^{-40} M_{\mathrm{pl}}$ and $\epsilon_{\chi} \sim 10^{-6}$ (with $\lambda / f \sim 10^{4} M_{\mathrm{pl}}^{-1}, \xi_{Q} \sim 44$, $g \sim 10^{-36}$ ) was discussed in 101$]^{12}$

In the rich multi-field model we consider in the next section, there is not a neat distinction between each scalar's dynamics, and therefore, $\xi_{h}$ is dictated by the evolution of all scalar fields, as seen in 5.2.22. This means that this large coupling requirement could be mildly relaxed due to the multiple field evolution 13 . Moreover, as we already mentioned, we are also aiming to maximise the tensor fluctuations' enhancement, that is largely determined by the magnitude of $\xi_{Q}$, while keeping the backreaction under control.

\subsection{Examples of (spectator) chromo-natural Kähler infla- tion}

In this section we will put forward two examples of of Kähler moduli inflation coupled to an $S U(2)$ gauge field. The two inflationary examples are given in chapter 4 as example 1 and 2. In example 1 , the inflaton itself, $\tau_{2}$, as well as its axionic partner $b_{2}$ is coupled to the gauge field. In example 2 , an additional spectator modulus $T_{4}=\tau_{4}+i b_{4}$ is coupled to the gauge field while the inflaton remains as $\tau_{2}$. The second example is SCNI. Example 1, although capable of producing a large enhancement to the PGW spectrum of Kähler inflation, cannot do so without incurring excessive backreaction by the tensor fluctuations to the gauge field, which we will discuss in detail in 5.5. Example 2 has more freedom in choosing parameters in its potential because the field coupled to the gauge field does not need to support inflation. Example 2 permits a large enhancement with controlled backreaction, but controlling the backreaction requires $g \ll 1$. These examples are phenomenological in the sense that we do not motivate or justify the necessary large and small parameters. In particular we require the coupling between the axion and the gauge field to be large and as stated $\mathrm{g} \ll 1$. In 5.6 we will introduce a tweak to example 2 that complicates the model slightly but gives some motivation to the magnitude of these couplings.

\footnotetext{
${ }^{12}$ See however [103] for restrictions on the model in [101].

${ }^{13}$ As all the scalar fields interact non-trivially via the kinetic and potential terms, non-linearities may restrict further the parameter space along the lines discussed in [103.
} 


\section{PRIMORDIAL GRAVITATIONAL WAVES IN STRING INFLATION}

The four dimensional effective action arising in models of inflation in string theory such as Kähler moduli inflation is given by (after moving into real space, see chapter 4)

$S=\int d^{4} x \sqrt{-g}\left[\frac{M_{\mathrm{pl}}^{2}}{2} R-\frac{1}{2} \gamma_{a b} \partial_{\mu} \phi^{a} \partial^{\mu} \phi^{b}-V\left(\phi^{a}\right)-\frac{\operatorname{Re}\left(f^{a}\right)}{4} F_{\mu \nu}^{A} F^{A \mu \nu}+\frac{\operatorname{Im}\left(f^{a}\right)}{4} F_{\mu \nu}^{A} \tilde{F}^{A \mu \nu}\right]$,

where the Planck scale is given by $M_{\mathrm{pl}}^{2}=2 V_{6}^{0} /\left((2 \pi)^{7} \alpha^{\prime 4} g_{s}^{2}\right)$, with $V_{6}^{0}$ the dimensionful six-dimensional volume vev, $(2 \pi)^{2} \alpha^{\prime}=\ell_{s}^{2}$ the string scale and $g_{s}=e^{\langle\phi\rangle}$ the string coupling given in terms of the dilaton $\phi$ vev. The $S U(N)$ gauge field (that, as has been shown, is equivalent to one $S U(2)$ gauge field of field strength $F_{\mu \nu}^{A}=\partial_{\mu} A_{\nu}^{A}-\partial_{\nu} A_{\mu}^{A}-$ $\mathrm{g} \epsilon^{A B C} A_{\mu}^{B} A_{\nu}^{C}$ with $\left.\mathrm{g}=\frac{1}{\sqrt{N / 2}}\right)$, gives rise to gaugino condensation on the D7-branes, which generates a potential for the Kähler moduli $T^{a}=\tau_{a}+i b_{a}$. The dual is defined as $\tilde{F}^{A \mu \nu}=\epsilon^{\mu \nu \alpha \beta} F_{\alpha \beta}^{A} /(2 \sqrt{-g})$ with $A=1,2,3$ the gauge index. The other moduli present, namely the axio-dilaton and complex structure (and deformation moduli of D7-branes) are assumed to be already stabilised at the perturbative level by internal fluxes at a higher scale and integrated out consistently (as mentioned in chapter 4).

This action could of course be written in terms of the Kähler metric and complex scalar fields $T^{a}=\tau_{a}+i b_{a}$. The moduli $\tau_{a}$ are the (Einstein frame) volumes of the 4-cycles $\Sigma_{a}$ wrapped by the D7-branes in units of $\ell_{s}$; while the imaginary parts, $b_{a}$, are axions that are the components of the $C_{4}$-forms along these cycles $b_{a}=\alpha^{\prime-2} \int_{\Sigma_{a}} C_{4}$. This action has been written in terms of the real fields $\phi^{a}=\left(\tau_{a}, b_{a}\right)$. To see how we arrive at $\gamma_{a b}$ for the different examples, see chapter 4. The coupling between the scalars (Kähler moduli) and the gauge field is given by the holomorphic gauge kinetic function, $f^{a}\left(T^{a}\right)$.

Comparing (5.3.1) to the general multi-field action 5.2.1) we introduced in section 5.2 . we have a non-trivial scalar metric $\left(\gamma_{a b}\right)$ mixing non-trivially the scalar fields; the scalar potential is in general a non-separable, non-trivial function of all the scalar fields, and there is a non-trivial well-defined coupling between the scalar fields and the gauge field. We saw in the previous section how to deal with the general $S U(N)$ group in terms of an effective single $S U(2)$ group by introducing an effective gauge coupling (5.2.7), $\mathrm{g}=1 / \sqrt{N / 2}$. 
Let us now discuss the gauge field coupling to the scalars in (5.3.1) and (5.2.1). In the original LV Kähler inflation scenario, the gauge kinetic function is given by [85]:

$$
f^{a}=\frac{T^{a}}{2 \pi},
$$

where $T^{a}=\tau_{a}+i b_{a}$ is the Kähler modulus coupled to the gauge field we want to use to realise SCNI. Therefore, we can immediately identify the functions $f$ and $h$ in 5.2.1 with the real and imaginary parts of $f^{a}$ respectively. As we discussed in the previous section, a successful background evolution implies the condition 5.2.26, which depends on the ratio $h_{, a} / f$. For $f$ given by (5.3.2) this ratio gives $h_{, a} / f \sim 1 / \tau_{a}$, and the constraint (5.2.26), becomes

$$
M_{\mathrm{pl}} \frac{\dot{b}_{a}}{\tau_{a} \dot{\varphi}} \gtrsim \frac{1}{\sqrt{2 \epsilon_{\varphi}}},
$$

or more simply

$$
\frac{\dot{b}_{a}}{\tau_{a} H}=\frac{1}{\tau_{a}} \frac{d b_{a}}{d N} \gtrsim 1
$$

where $\dot{\varphi}$ and $\epsilon_{\varphi}$ are defined in (5.2.11) and (5.2.17) respectively, and $N=\ln a$ is the e-folding number. Although it is in principle possible that the cosmological evolution satisfies this condition, we did not find any example where this could be satisfied for a non-negligible time during inflation ${ }^{14}$. Therefore, it is clear that we need the real and imaginary parts of the gauge kinetic function $f^{a}$ to have different coefficients, which is not possible in the original Kähler inflation model. We therefore propose for example 1 and 2 (see below) that the coupling functions $f$ and $h$ are given purely phenomenologically as

$$
f\left(\phi^{a}\right)=\frac{c_{1}}{2 \pi} \tau_{a}, \quad h\left(\phi^{a}\right)=\frac{c_{2}}{2 \pi} b_{a}
$$

where $a$ corresponds to the modulus coupled to the gauge field $T^{a}=\tau_{a}+i b_{a}$. We see that (5.3.3) now becomes

$$
\frac{c_{2}}{c_{1} \tau_{a}} \frac{d b_{a}}{d N} \gtrsim 1
$$

and we therefore require the hierarchy $c_{2} \gg c_{1}$.

\footnotetext{
${ }^{14}$ Recall that in the slow-roll solution described in chapter 3 the axion is actually slowed down by its coupling to the gauge field, making this condition even more difficult to achieve.
} 


\section{PRIMORDIAL GRAVITATIONAL WAVES IN STRING INFLATION}

\subsubsection{Background evolution of example 1}

We first consider a model with three Kähler moduli, whose basic inflationary predictions are shown in chapter 4. The scalar potential is derived using the Kähler potential, $K$, and superpotential, $W$, using, as standard

$$
V\left(\phi^{a}, \bar{\phi}^{b}\right)=e^{K / M_{\mathrm{pl}}^{2}}\left[K^{a \bar{b}} D_{a} W \overline{D_{b} W}-\frac{3}{M_{\mathrm{pl}}^{2}}|W|^{2}\right] .
$$

The derivation for the potential of Kähler moduli inflation is given in chapter 4 . In the three-field case, it has potential

$$
V_{1}=\frac{8\left(a_{2} A_{2}\right)^{2} e^{-2 a_{2} \tau_{2}} \sqrt{\tau_{2}}}{3 \alpha \lambda_{2} \mathcal{V}}+\frac{4 W_{0} a_{2} A_{2} e^{-a_{2} \tau_{2}} \cos \left(a_{2} b_{2}\right) \tau_{2}}{\mathcal{V}^{2}}+\frac{3 \hat{\xi} W_{0}}{4 \mathcal{V}^{3}}+\frac{\beta}{\mathcal{V}^{2}}+V_{3},
$$

where the term proportional to $\beta$ is the uplifting piece, taken to be of the form $V_{\text {uplift }}=$ $\beta / \mathcal{V}^{2}$ as in [49] and $V_{3}$ comes from the stabilisation of the $T_{3}$ modulus and it is given by:

$$
V_{3}=\frac{8\left(a_{3} A_{3}\right)^{2} e^{-2 a_{3}\left\langle\tau_{3}\right\rangle} \sqrt{\left\langle\tau_{3}\right\rangle}}{3 \alpha \lambda_{3} \mathcal{V}}-\frac{4 W_{0} a_{3} A_{3} e^{-a_{3}\left\langle\tau_{3}\right\rangle}\left\langle\tau_{3}\right\rangle}{\mathcal{V}^{2}}
$$

with $b_{3}$ set to its minimum, $\left\langle b_{3}\right\rangle=\pi / a_{3}$. As we show in chapter 4 , the small cycle $\tau_{3}$ acts as a stabiliser for the potential at large volume $\mathcal{V}$ so cannot be shifted far from its minimum. However $\tau_{2}$ and $b_{2}$ can be displaced away from their minima leading to an effective two-field inflationary model as shown in [76. The $T_{3}$ modulus stays at its minimum with $\left\langle b_{3}\right\rangle=\pi / a_{3}$ during the cosmological evolution in this case.

We can now use our general discussion in section 5.3 to study the scalar field dynamics in the presence of the gauge field. Our scalar fields are given by

$$
\phi^{a}=\left(\tau_{2}, b_{2}\right)
$$

and we have simplified field-space metric (with $\mathcal{V}$ and $\tau_{3}$ set to their minima (see chapter 4p) given by

$$
\gamma_{a b}=\left(\frac{3 \alpha \lambda_{2}}{4 \mathcal{V} \sqrt{\tau_{2}}}\right) \delta_{a b}
$$

Moreover, the coupling functions $f, h$ in 5.2 .1 can now be easily identified. We have

$$
f\left(\phi^{a}\right)=\frac{c_{1}}{2 \pi} \tau_{2}, \quad h\left(\phi^{a}\right)=\frac{c_{2}}{2 \pi} b_{2},
$$


where the phenomenological constants $c_{1}, c_{2}$ allow for some freedom in the parameter space.

\section{Parameters}

We use parameters as in example 1 in chapter 4 (this is example 4 in [76]):

$$
\begin{aligned}
& \hat{\xi}=1 / 2, \quad \alpha=\frac{1}{9 \sqrt{2}}, \quad \lambda_{2}=10, \quad \lambda_{3}=1, \quad a_{2}=\frac{2 \pi}{30}, \quad a_{3}=\frac{2 \pi}{3}, \\
& A_{2}=\frac{1}{1.7 \times 10^{6}}, \quad A_{3}=\frac{1}{425}, \quad \beta=6.9468131457 \times 10^{-5}, \quad W_{0}=\frac{40}{17} .
\end{aligned}
$$

The global minimum of the potential at zero cosmological constant is found to be at:

$$
\left\langle\tau_{1}\right\rangle=2555.69, \quad\left\langle\tau_{2}\right\rangle=4.77519, \quad\left\langle\tau_{3}\right\rangle=2.65111, \quad \rightarrow \quad\langle\mathcal{V}\rangle=10142.4
$$

while the axions' minima lie at $b_{a}=\pi / a_{a}$. This is a relatively small volume example for Kähler modulus inflation, however it is still consistent with the large volume approximation. We are interested in the cosmological evolution when moving $T_{2}$ away from its minimum while $T_{1}, T_{3}$ are consistently kept at their minima (see chapter 4 ).

The scalar sector is coupled to the gauge sector through the gauge kinetic functions $f$ and $h$. We are searching for solutions which yield a sizeable period of inflation in which the isotropic gauge field, $Q$, is supported. This support is given by its effective coupling to the axion, $\xi_{h}=\frac{c_{2} \dot{b_{2}}}{2 c_{1} H \tau_{2}}$. As we shall see, the effective mass of the gauge field goes $\xi_{h} \simeq \xi_{Q}+\xi_{Q}^{-1}$ so the larger $\xi_{h}$ the larger $\xi_{Q}$; and as we shall also see in section 5.4 the larger $\xi_{Q}$ the larger the enhancement to the primordial gravitational wave spectrum. $c_{1}$ and $c_{2}$ are therefore chosen so that there is $\xi_{h}$ and consequently $\xi_{Q}$ are large enough to get a considerable amplification. We take:

$$
c_{1}=1, \quad c_{2}=7000
$$

and we take the gauge coupling, g, which we will see is an extremely important factor in determining how large the backreaction of the gauge field perturbation on the 

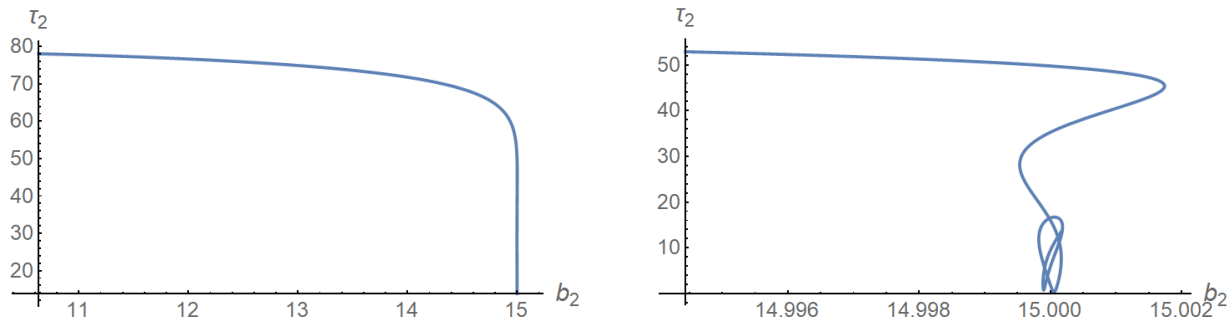

Figure 5.1: Parametric plot of the cosmological evolution of $\tau_{2}$ and $b_{2}$, during the last 60 e-folds on the left and the last e-fold of inflation in example 1.
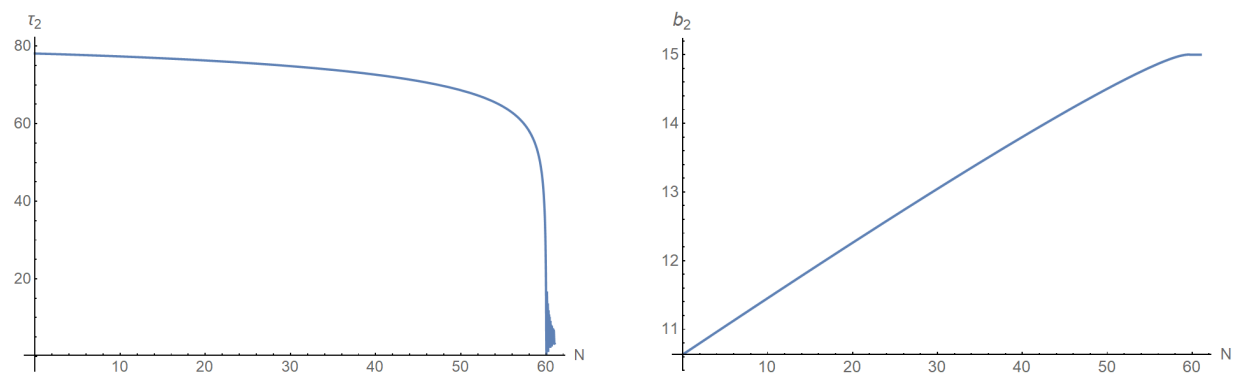

Figure 5.2: Cosmological evolution of $\tau_{2}$ and $b_{2}$, during the last $61 \mathrm{e}$-folds in example 1 . Inflation ends at $N=60$.

background equations of motion is in section 5.6 , to be

$$
\mathrm{g}=\frac{1}{10}
$$

This system is evolved under the equations of motion given by $(5.2 .8)-(5.2 .9)$ and 5.2 .12 -5.2.13) and the background evolution is plotted in FIGs 5.1 5.8,

Interestingly, as can be seen in FIG 5.8, the contribution from the gauge field,
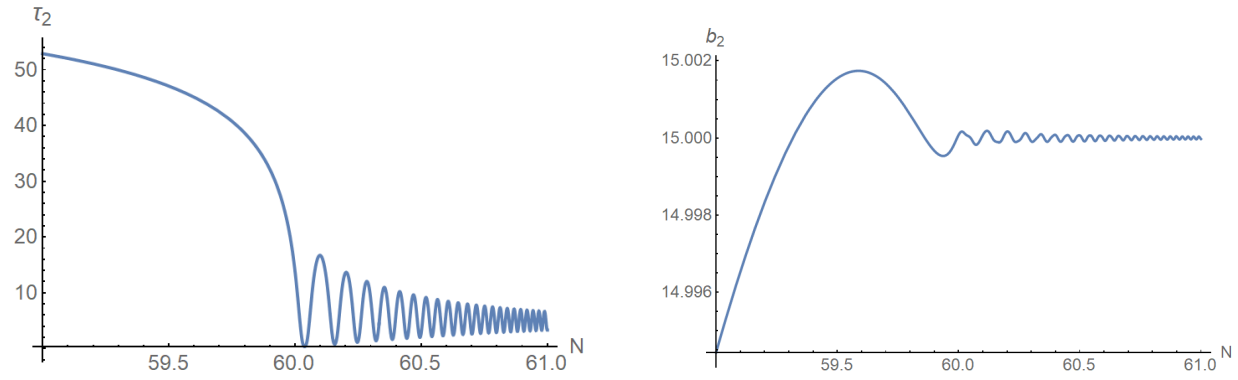

Figure 5.3: Cosmological evolution of $\tau_{2}$ and $b_{2}$, during the last e-fold in example 1 . Inflation ends at $N=60$. 

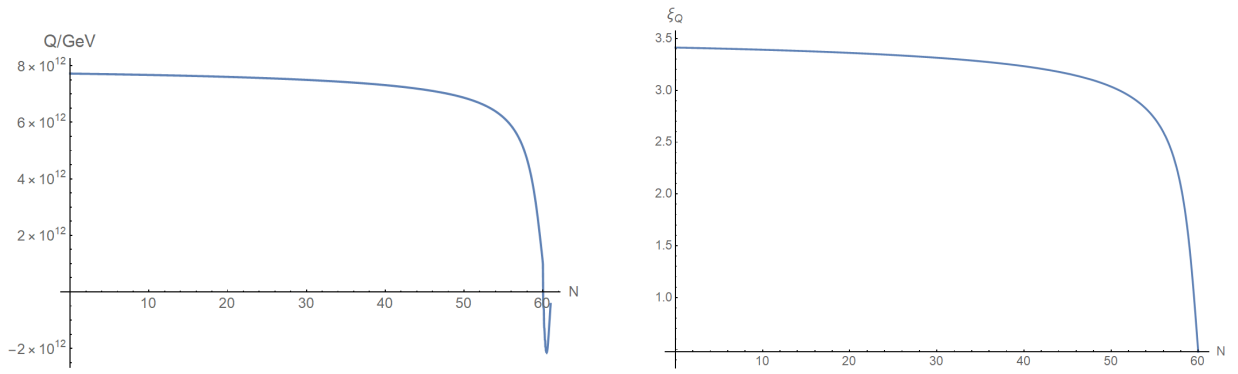

Figure 5.4: Cosmological evolution of the gauge field $Q$ (left figure) and $\xi_{Q}=\frac{g Q}{H}$ (right figure) during the last 60 e-folds of inflation in example 1 . The gauge field is supported by its coupling to the axion determined by $\xi_{h}$.
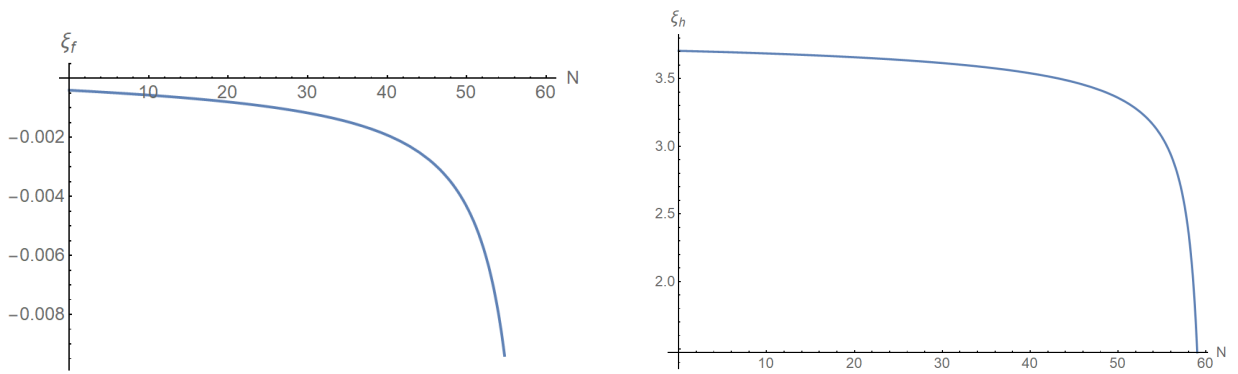

Figure 5.5: Cosmological evolution of the effective coupling terms $\xi_{f}=\frac{\dot{\tau}_{2}}{2 \mathrm{H} \tau_{2}}$ (left figure) and $\xi_{h}=\frac{c_{2} \dot{b}_{2}}{2 c_{1} H \tau_{2}}$ (right figure) during the last 60 e-folds of inflation in example 1 .
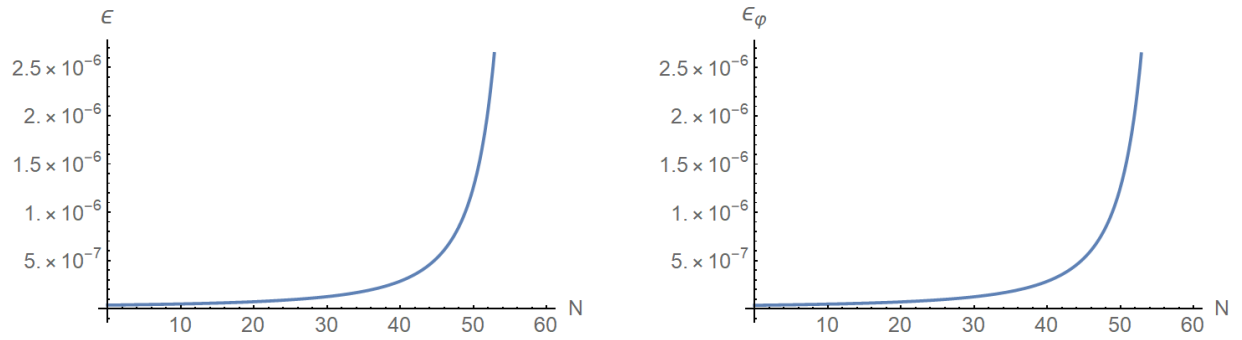

Figure 5.6: The evolution of the slow-roll parameter, $\epsilon=-\frac{\dot{H}}{H^{2}}$ (left figure), and the proportion of $\epsilon$ made up by $\epsilon_{\varphi}=\frac{1}{2 H^{2}} \gamma_{a b} \dot{\phi}^{a} \dot{\phi}^{b}$ (right figure) during the last 60 e-folds of inflation in example 1. $\epsilon_{\varphi}$ dominates over the other components of the slow-roll parameter. 

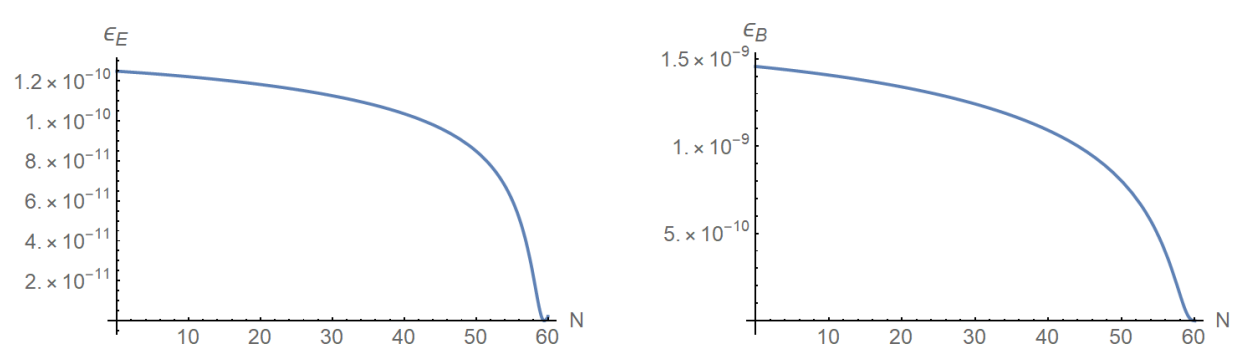

Figure 5.7: The evolution of the electric and magnetic components of the slow roll parameter, $\epsilon_{E}=\frac{c_{1}}{2 \pi} \tau_{2} \frac{(\dot{Q}+H Q)^{2}}{H^{2} M_{\mathrm{pl}}^{2}}$ (left figure) and $\epsilon_{B}=\frac{c_{1}}{2 \pi} \tau_{2} \frac{\mathrm{g}^{2} Q^{4}}{H^{2} M_{\mathrm{pl}}^{2}}$ (right figure) respectively during the last 60 e-folds of inflation in example 1 . They contribute only a small amount to the overall slow-roll parameter, FIG. 5.6

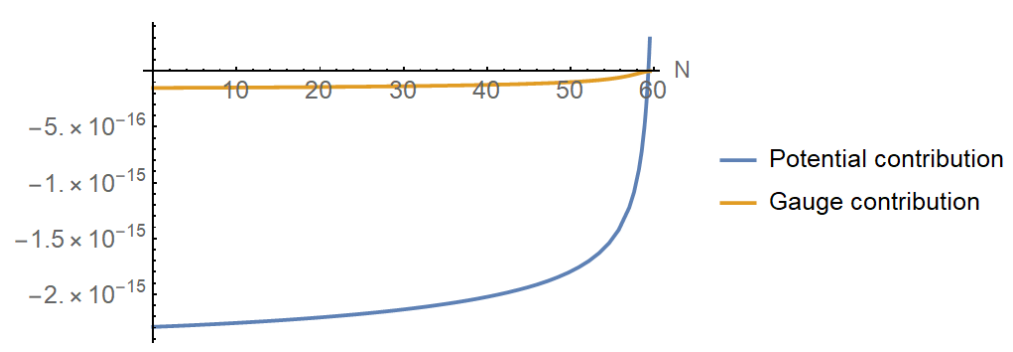

Figure 5.8: A comparison of the contributions to $b_{2}$ 's equation of motion (5.2.12) from the term provided by the potential, $\frac{4 \mathcal{V} \sqrt{\tau_{2}}}{3 \alpha \lambda_{2}} \frac{d V}{d b_{2}}$; and the term provided by the gauge field, $-3 \mathrm{~g} \frac{c_{2}}{2 \pi} \frac{4 \mathcal{V} \sqrt{\tau_{2}}}{3 \alpha \lambda_{2}}(H Q+\dot{Q})$ for example 1. Unlike the examples shown in [3] and [4], this is not a "slow-roll solution" whereby the axion, $b_{2}$ is slowed by the backreaction of the gauge field, $Q$. In this example $b_{2}$ 's evolution is unaffected by the presence of $Q$ but $Q$ is supported nonetheless. 
$-3 \mathrm{~g} \frac{c_{2}}{2 \pi} \frac{4 \mathcal{V} \sqrt{\tau_{2}}}{3 \alpha \lambda_{2}}(H Q+\dot{Q})$, to $b_{2}$ 's equation of motion 5.2 .12 is vastly sub-dominant compared to the contribution from the potential, $\frac{4 \mathcal{V} \sqrt{\tau}}{3 \alpha \lambda_{2}} \frac{d V}{d b_{2}}$. This suggests that example 1 is not a "slow-roll solution" (as described in [3] and [4] and the situation in chapter 3) whereby the axion, $b_{2}$, is slowed by the backreaction of the gauge field, $Q$ - in the slowroll case, $Q$ settles to a value that enables the contribution from the gauge field to almost perfectly cancel the contribution from the potential to the axion's equation of motion. In this example, $b_{2}$ 's evolution is unaffected by the presence of $Q$ but $Q$ is supported nonetheless. This is in part necessary since the evolutionary histories of the inflaton, $\tau_{2}$, and $b_{2}$ are intertwined (see FIG 5.1 5.2 - if $b_{2}$ 's evolution were slowed considerably by the presence of $Q$, this would have a large effect on $\tau_{2}$ and consequently the basic inflationary predictions of Kähler inflation. This model makes the following inflationary predictions, 50 e-folds before the end of inflation:

$$
\begin{aligned}
& \epsilon_{\varphi}=4.75 \times 10^{-8}, \quad n_{s}=0.966, \quad r_{b}=7.61 \times 10^{-7}, \\
& V_{\text {inf }}^{1 / 4}=9.77 \times 10^{14} \mathrm{GeV}, \quad \Delta \varphi=0.194 M_{\mathrm{pl}}
\end{aligned}
$$

where $\Delta \phi=\int_{N_{*}}^{N_{e}} \sqrt{2 \epsilon_{\varphi}} d N$ with $N_{e}$ the end of inflation and $N_{*}=N_{e}-50, n_{s}$ is the scalar spectral index, and $r=\mathcal{P}_{T} / \mathcal{P}_{S}=16 \epsilon_{\varphi}$ is the non-sourced estimate for the tensor-to-scalar ratio.

Although this model seems promising as a candidate to have a large enhancement of the gravitational wave sector induced by the gauge field as it supports $Q$ for a long period during inflation, it turns out to be ineffective. The backreaction (we will derive in detail an estimate for the backreaction in section 5.6 induced by the tensor perturbation to the gauge field, $t_{R}$, responsible for the enhancement to the gravity wave sector, is too large. The principle concern of this backreaction is onto the equation of motion for $Q, 55.2 .13$. We require that the backreaction on this equation, $\mathcal{T}_{B R}^{Q}$, be much smaller than the largest term in (5.2.13), namely $2 \mathrm{Hg} Q^{2} \xi_{h}$ (see (5.2.24)). As is discussed in [3], $\mathcal{T}_{B R}^{Q}$ is dependent on the size of $\xi_{Q}$ and $g$ and we show this in FIG. 5.9 . Since our background inflationary system is of a lower energy scale than the example provided in [3], we naturally require a larger enhancement to the gravity wave sector to uplift $r$ to observable values. This requires a larger value of $\xi_{Q}$ and hence leads to larger backreaction. On top of this, the largest term in (5.2.13) is considerably smaller due to the lower energy scale - one can write $2 H \mathrm{~g} Q^{2} \xi_{h}=2 H^{3} \frac{\xi_{Q}^{2} \xi_{h}}{g}$ and we see that a 


\section{PRIMORDIAL GRAVITATIONAL WAVES IN STRING INFLATION}

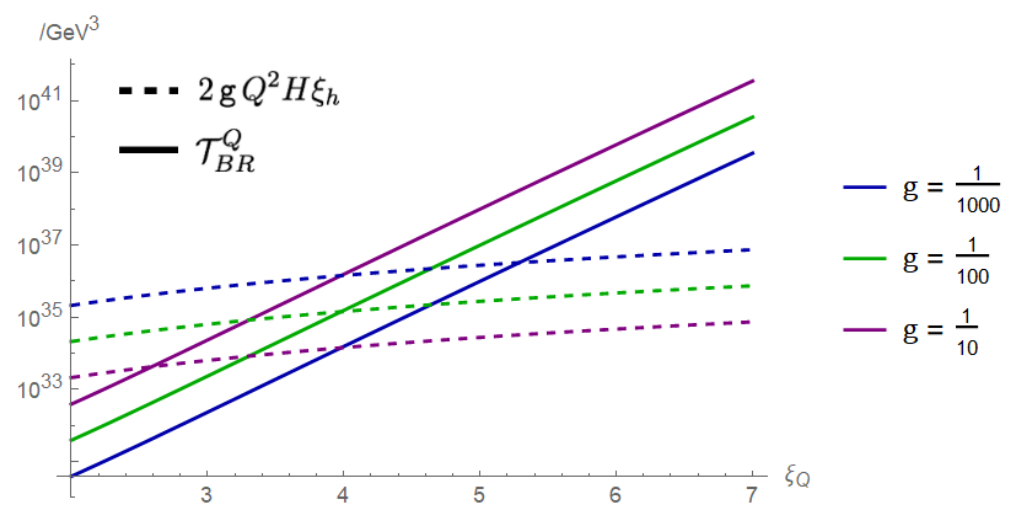

Figure 5.9: A quick comparison of the backreaction, $\mathcal{T}_{B R}^{Q}$, on (5.2.13) (solid lines), and the largest term in 5.2 .13$), 2 H \mathrm{gQ} Q^{2} \xi_{h}$ (dashed lines) against $\xi_{Q}$ for different values of $\mathrm{g}$. Reducing $g$ decreases the backreaction and increases the largest term in 5.2.13).

lower value of $V \propto H^{2}$ reduces the size of this term, consequently meaning we require even smaller backreaction on this equation. As we can see in FIG. 5.9, this effect can be mitigated however by choosing a smaller value for $g$ and as is seen in [101, having a low energy scale does not necessarily ruin the efficacy of this model. Unfortunately, we were unable to find a working example where the evolution of $b_{2}$ could support a good value of $\xi_{Q}$ with low enough $g$ for the backreaction to be under control without spoiling the generic inflationary predictions of Kähler inflation caused by $\tau_{2}$. In fact in the example shown here the value of $\xi_{Q} \sim 3.5$ is not large enough to generate a substantial enhancement to the PGW spectrum despite the fact we have $g=\frac{1}{10}$ at a relatively large value and the backreaction is already too large. In practice we were unable to even get close to a working model with this two scalar field model. This is in a large part due to the interaction between $\tau_{2}$ and $b_{2}$; as can be seen in FIG 5.1 5.2 , their evolutionary histories are tied. The fact that the field responsible for inflation, $\tau_{2}$, is directly (through the coupling $f$ ) and indirectly (through $b_{2}$ ) coupled to the evolution of the gauge field, $Q$, is in fact quite restrictive.

This is the reason we modify this model by introducing a fourth Kähler modulus, $T_{4}$, to act as an inflationary spectator and instead choose this modulus to be coupled to the gauge field, while allowing the inflaton, $\tau_{2}$, to be free. This modified model with an extra Kähler modulus is example 2 in chapter 4 and we now couple it to a gauge field. 


\subsubsection{Background evolution of example 2}

We now consider example 2 , the case where an extra modulus, $T_{4}=\tau_{4}+i b_{4}$, is included in the system. We choose this modulus to be coupled to the gauge field through the gauge kinetic functions, $f$ and $h$, while $\tau_{2}$ remains the inflaton. For simplicity, $b_{2}$ is taken to be at its minimum. The inflationary potential can again be found from the superpotential and Kähler potential as shown in chapter 4.

$$
V_{2}=V_{1}+\frac{8\left(a_{4} A_{4}\right)^{2} e^{-2 a_{4} \tau_{4}} \sqrt{\tau_{4}}}{3 \alpha \lambda_{4} \mathcal{V}}+\frac{4 W_{0} a_{4} A_{4} e^{-a_{4} \tau_{4}} \cos \left(a_{4} b_{4}\right) \tau_{4}}{\mathcal{V}^{2}}
$$

where $V_{1}$ is the potential for example 1 (5.3.7). The advantage of this system, where we effectively have spectator fields, $\tau_{4}$ and $b_{4}$, coupled to the gauge sector, is that there is now a lot more freedom in choosing the parameters and initial conditions for the axion and gauge field because we do not have to worry about inflationary predictions. We do however require that both the kinetic and potential energy densities in the spectator fields are less than those of the inflaton. This ensures that the inflationary predictions of Kähler inflation are not affected and that $\tau_{4}$ and $b_{4}$ are true spectator fields.

\section{Parameters}

We choose parameters to be

$$
\begin{aligned}
& \hat{\xi}=\frac{1}{2}, \quad \alpha=\frac{1}{9 \sqrt{2}}, \quad \lambda_{2}=10, \quad \lambda_{3}=1, \quad \lambda_{4}=0.1, \\
& a_{2}=\frac{2 \pi}{30}, \quad a_{3}=\frac{2 \pi}{3}, \quad a_{4}=\frac{2 \pi}{70}, \quad A_{2}=\frac{1}{1.7 \times 10^{6}}, \quad A_{3}=\frac{1}{425} \\
& A_{4}=2 \times 10^{-9}, \quad \beta=6.94681 \times 10^{-5}, \quad W_{0}=\frac{40}{17} .
\end{aligned}
$$

The parameters are the same as in 5.3.12 with the addition of $\lambda_{4}$, chosen to be much smaller than $\lambda_{2}$ so that $\tau_{4}$ and $b_{4}$ have lower kinetic energy and therefore have a negligible contribution to $\epsilon_{\varphi}$; and $a_{4}$ and $A_{4}$ are chosen to be much smaller than $a_{2}$ and $A_{2}$, respectively, so that the energy density of the universe is dominated completely by the inflationary sector. 


\section{PRIMORDIAL GRAVITATIONAL WAVES IN STRING INFLATION}

The global minimum of this potential is found at:

$$
\left\langle\tau_{1}\right\rangle=2555.35, \quad\left\langle\tau_{2}\right\rangle=4.77519, \quad\left\langle\tau_{3}\right\rangle=2.65102, \quad\left\langle\tau_{4}\right\rangle=11.141, \quad\langle\mathcal{V}\rangle \rightarrow 10140.1
$$

with $b_{a}=\pi / a_{a}$. Displacement of $\tau_{1}, \tau_{4}$ and $b_{4}$ away from this minimum leads to a small shift in the values of $\tau_{3}$ and $\mathcal{V}$ at the new local minimum. For numerical simplicity, we set $\tau_{3}$ and $\mathcal{V}$, as well as the $b_{2}$ and $b_{3}$ axions, to their minima. We are now considering a three-field system of $\left(\tau_{2}, \tau_{4}, b_{4}\right)$ with real field space metric

$$
\gamma_{a b}=\frac{3 \alpha}{4 \mathcal{V}}\left(\begin{array}{ccc}
\frac{\lambda_{2}}{\sqrt{\tau_{2}}} & 0 & 0 \\
0 & \frac{\lambda_{4}}{\sqrt{\tau_{4}}} & 0 \\
0 & 0 & \frac{\lambda_{4}}{\sqrt{\tau_{4}}}
\end{array}\right) .
$$

This system is coupled to a gauge field exactly as in example 1, however, this time it is not the inflaton, $\tau_{2}$, that is coupled to the gauge field. Instead, the two spectator fields, $\tau_{4}$ and $b_{4}$ are coupled to it through the terms in the action:

$$
\mathcal{L} \supset-\frac{f\left(\phi^{a}\right)}{4} F_{\mu \nu}^{A} F^{A \mu \nu}+\frac{h\left(\phi^{a}\right)}{4} F_{\mu \nu}^{A} \tilde{F}^{A \mu \nu}
$$

where

$$
f\left(\phi^{a}\right)=\frac{c_{1}}{2 \pi} \tau_{4}, \quad h\left(\phi^{a}\right)=\frac{c_{2}}{2 \pi} b_{4} .
$$

The equations of motion are as before 5.2.12 5.2.13). The initial conditions for $\tau_{4}$, $b_{4}$, and $Q$; as well as the values of $c_{1}, c_{2}$, and $g$ are chosen phenomenologically to lead to a large (observable) enhancement of the gravitational wave spectrum (see section 5.4 without leading to excessive backreaction from the gauge tensor perturbation (see section 5.6). With this in mind, the parameters are chosen to be

$$
c_{1}=1, \quad c_{2}=450, \quad \mathrm{~g}=\frac{1}{2000} .
$$

Although smaller than in example 1 , again the value of $c_{2} \gg 1$. This seems to be a general requirement of these models. The initial conditions are taken as:

$$
\tau_{2}=80.17, \quad \tau_{4}=12.6, \quad b_{4}=0.59 \frac{\pi}{a_{4}}, \quad Q=8 \times 10^{-4} M_{\mathrm{pl}}
$$

The modulus $\tau_{4}$ is taken to start near its minimum for simplicity, and as can be 

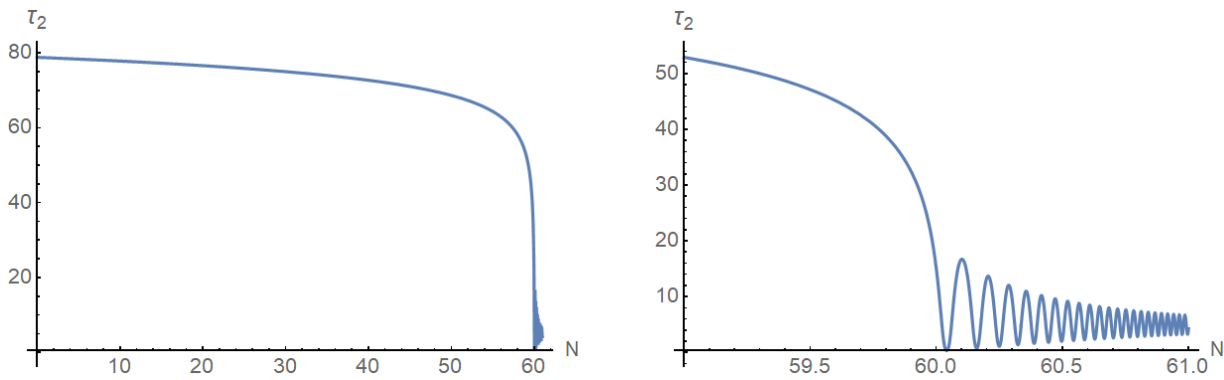

Figure 5.10: The evolution of the inflaton, $\tau_{2}$ during the last 60 e-folds of inflation (left) and during the last few e-folds (right) for example 2.

seen in FIG. 5.11, it remains roughly constant. The important spectator field is of course the axion, $b_{4}$, which moves slowly towards its minimum as can be seen in FIG. 5.11. Contrary to the situation described in example 1 above, in this example, the axion's $\left(b_{4}\right)$ evolution is slowed considerably by its coupling to the gauge field, $Q$, which backreacts on $b_{4}$ through the term on the RHS of $(5.2 .12),-3 \mathrm{~g} \gamma^{a b} h_{, b} Q^{2}(H Q+\dot{Q})=$ $3 \mathrm{~g} \frac{c_{2}}{2 \pi} \frac{4 \mathcal{V} \sqrt{\tau_{4}}}{3 \alpha \lambda_{4}} Q^{2}(H Q+\dot{Q})$. This term almost completely cancels with the potential term $\gamma^{a b} V_{, b}=\frac{4 \mathcal{V} \sqrt{\tau_{4}}}{3 \alpha \lambda_{4}} \frac{d V}{d b_{4}}$ as can be seen in FIG. 5.12. This "slow-roll solution" is the situation described and expected in chromo-natural inflation [78] and more generally in models with a spectator axion coupled to a gauge field $([3$, , [4] $)$ where the gauge field, $Q$, has an attractor solution such that it forces the axion to roll slowly. This slow evolution of the axion leads to the gauge field, $Q$, being sustained for a large period of time during inflation as is shown in FIG. 5.13. The evolution of the inflaton, $\tau_{2}$, as well as plots of $\xi_{f}, \xi_{h}$ and the slow-parameters, $\epsilon, \epsilon_{\varphi}, \epsilon_{B}, \epsilon_{E}$ are shown in FIG. 5.10 5.17. Notice also that $\epsilon_{B} \sim \epsilon>\epsilon_{\varphi}$ in the early stages of inflation. This is a similar situation to that described in [101] and is acceptable as long as the scalar perturbations of the gauge field are very small relative to the inflationary perturbation and therefore the scalar power spectrum can be taken as $\frac{H^{2}}{8 \pi \epsilon_{\varphi}}$, which we will discuss in section 5.5 .

We turn now to the inflationary predictions of this model. Let us start discussing the scalar spectral index: $n_{s}=1-2 \epsilon-\eta_{\varphi}$ with $\eta_{\varphi}=d\left(\ln \epsilon_{\varphi}\right) / d N$. This form for $n_{s}$ arises under the assumption that the power spectrum is well-approximated by $\mathcal{P}_{s}=\frac{H^{2}}{8 \pi^{2} \epsilon_{\varphi}}$ instead of $\mathcal{P}_{s}=\frac{H^{2}}{8 \pi^{2} \epsilon}$. Since in the model described, $\epsilon \sim \epsilon_{B} \gg \epsilon_{\varphi}$ for much of inflation, this a very important distinction. This assumption is well-justified if the scalar power spectrum receives a negligible contribution from the gauge field perturbations on super- 

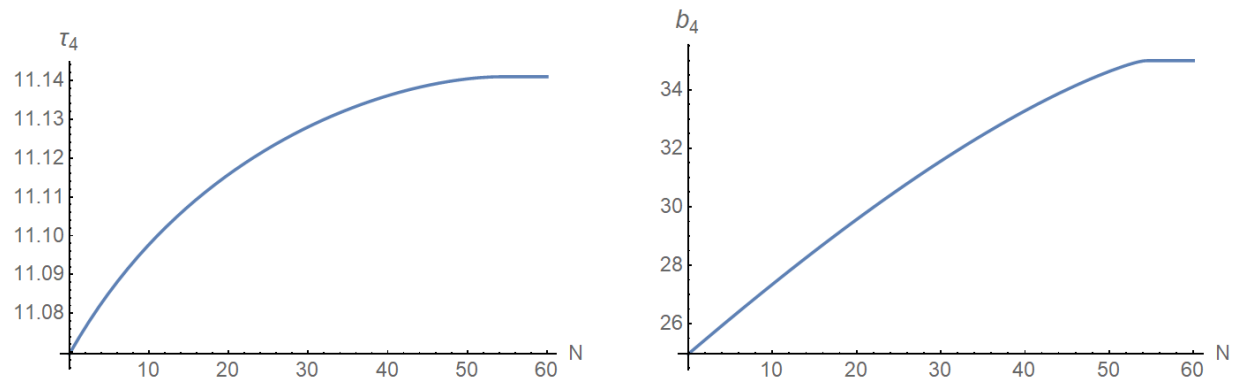

Figure 5.11: The evolution of the secondary modulus, $\tau_{4}$ and its axion partner $b_{4}$ in example 2. Both fields decay well before the end of inflation.
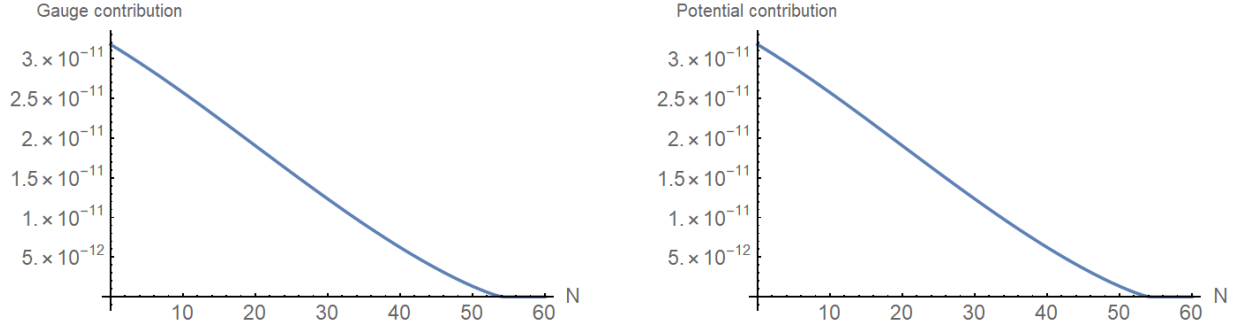

Figure 5.12: The contributions of (left) the term $3 \mathrm{~g} \frac{c_{2}}{2 \pi} \frac{4 \mathcal{V} \sqrt{\tau_{4}}}{3 \alpha \lambda_{4}}(H Q+\dot{Q})$ provided by the gauge field, $Q$; and (right) the term $\frac{4 \mathcal{V} \sqrt{\tau_{4}}}{3 \alpha} \frac{d V}{d b_{1}}$ provided by the potential, to the equation of motion for $b_{4}$ given by the form in $(5.2 .12)$ in example 2. The contribution from the gauge field almost exactly cancels the contribution from the potential leading to a slow-roll evolution for $b_{4}$.
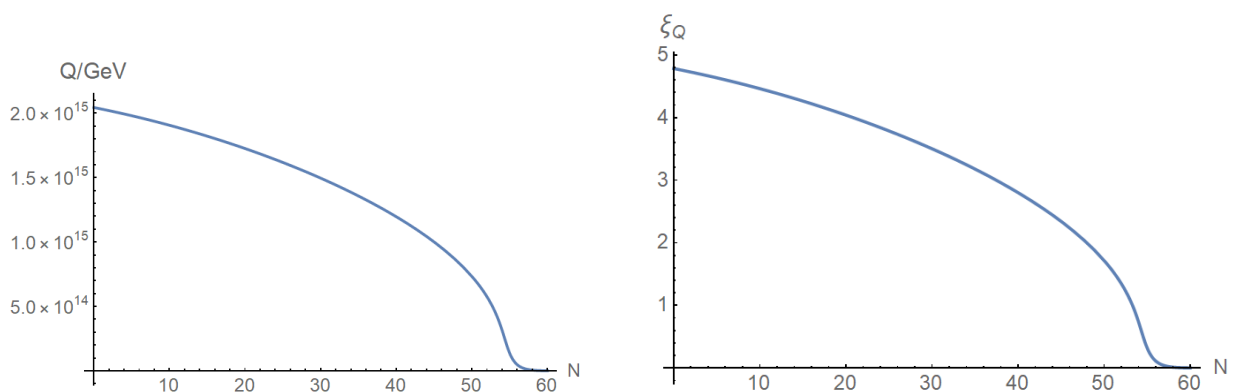

Figure 5.13: The evolution of the gauge field, $Q$ (left) and $\xi_{Q}=\frac{\mathrm{g} Q}{H}$ (right) during the last 60 e-folds of inflation in example 2. The evolution of $Q$ is tied to the evolution of $b_{4}$. 

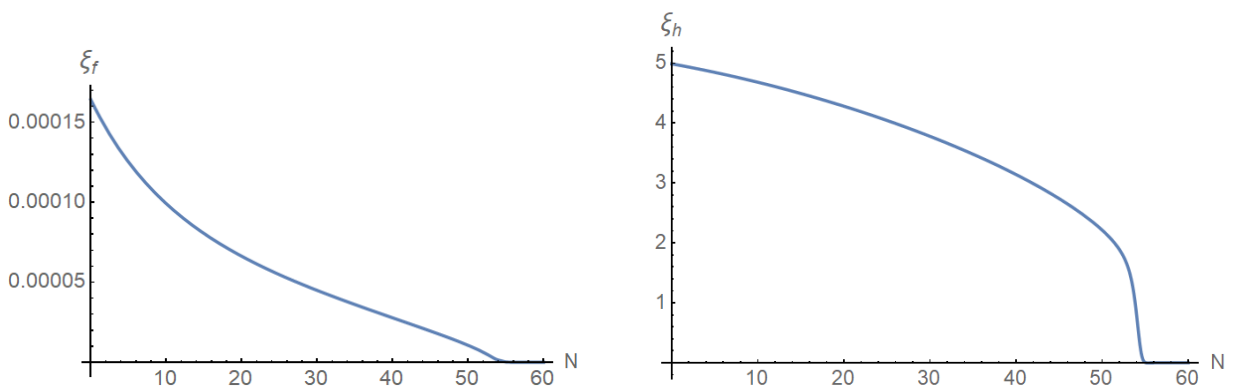

Figure 5.14: The evolution of the effective coupling constants of $\tau_{4}$ and $b_{4}$ to the gauge field, $\xi_{f}$ (left) and $\xi_{h}$ (right) during the last 60 e-folds in example 2.
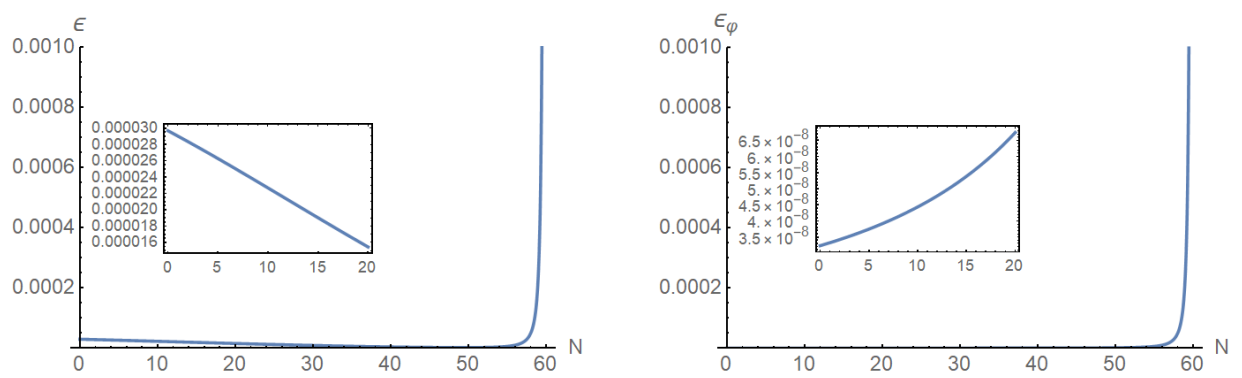

Figure 5.15: The evolution of the slow-roll parameter, $\epsilon$ (left), and the proportion of $\epsilon$ made up by $\epsilon_{\varphi}$, during the last 60 e-folds of inflation in example 2 .
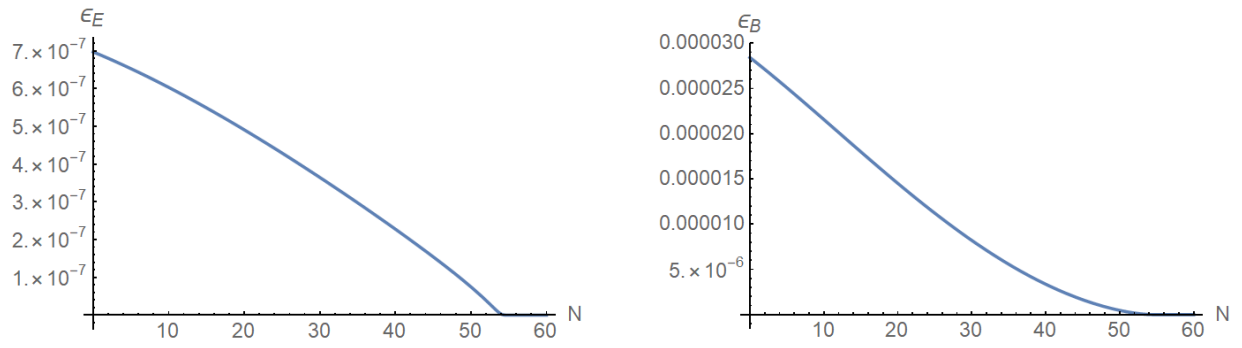

Figure 5.16: The evolution of the electric and magnetic components of the slow roll parameter, $\epsilon_{E}$ (left figure) and $\epsilon_{B}$ (right figure) respectively during the last 60 e-folds of inflation in example 2. 


\section{PRIMORDIAL GRAVITATIONAL WAVES IN STRING INFLATION}

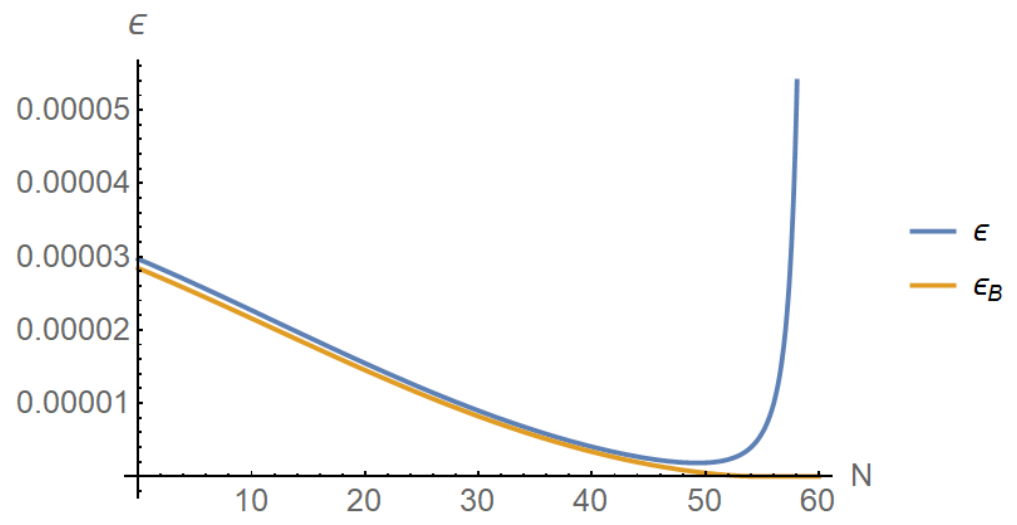

Figure 5.17: Comparing $\epsilon_{B}$ with the overall slow-roll parameter, $\epsilon . \epsilon_{B}$ provides the largest contribution to $\epsilon$ for the majority of the last 60 e-folds of inflation.

horizon scales [101] (see 5.5) and is therefore the same power spectrum that would arise in this model if the gauge fields were ignored, i.e., the one approximated by

$$
\mathcal{P}_{s}=\frac{H^{2}}{8 \pi^{2} \epsilon_{\varphi}} .
$$

The value of the scalar spectral index can then be determined following the usual procedure:

$$
n_{s}-1=\frac{d \ln \mathcal{P}_{s}}{d \ln k}
$$

where

$$
\mathcal{P}_{s}=\frac{H^{2}}{8 \pi^{2} \epsilon_{\varphi}}, \quad \ln k=N+\ln H
$$

because $x=k / a H=1$ at horizon-crossing. Using the chain rule

$$
\frac{d \ln \mathcal{P}_{s}}{d \ln k}=\frac{d \ln \mathcal{P}_{s}}{d N} \frac{d N}{d \ln k}
$$

and

$$
d \ln \mathcal{P}_{s}=d \ln \left(\frac{H^{2}}{8 \pi^{2} \epsilon_{\varphi}}\right)=2 d \ln H-d \ln \epsilon_{\varphi} \Longrightarrow \frac{d \ln \mathcal{P}_{s}}{d N}=2 \frac{d \ln H}{d N}-\frac{d \epsilon_{\varphi}}{d N}
$$

but

$$
\epsilon=-\frac{1}{H} \frac{d H}{d N}=-\frac{d \ln H}{d N} \Longrightarrow \frac{d \ln \mathcal{P}_{s}}{d N}=-2 \epsilon-\eta_{\varphi}
$$


where we have explicitly defined $\eta_{\varphi}=\frac{d \ln \epsilon_{\varphi}}{d N}$. Also

$$
\begin{gathered}
\frac{d N}{d \ln k}=\left(\frac{d \ln k}{d N}\right)^{-1}=\left(1+\frac{d \ln H}{d N}\right)^{-1} \approx 1+\epsilon \\
\Longrightarrow n_{s}-1=\frac{d \ln \mathcal{P}_{s}}{d \ln k}=\frac{d \ln \mathcal{P}_{s}}{d N} \frac{d N}{d \ln k}=\left(-2 \epsilon-\eta_{\varphi}\right)(1+\epsilon) \approx-2 \epsilon-\eta_{\varphi}
\end{gathered}
$$

leads us to

$$
n_{s}=1-2 \epsilon-\eta_{\varphi}
$$

The expression for the non-sourced tensor-to-scalar ratio $r_{b}$ is equivalently

$$
r_{b}=\frac{\mathcal{P}_{t}}{\mathcal{P}_{s}}=16 \epsilon_{\varphi}
$$

This model makes the following inflationary predictions, 50 e-folds before the end of inflation:

$$
\begin{aligned}
& \epsilon_{\varphi}=4.44 \times 10^{-8}, \quad n_{s}=0.967, \quad r_{b}=7.10 \times 10^{-7} \\
& V_{\text {inf }}^{1 / 4}=9.49 \times 10^{14} \mathrm{GeV}, \quad \Delta \varphi=0.189 M_{\mathrm{pl}}
\end{aligned}
$$

where $\Delta \phi=\int_{N_{*}}^{N_{e}} \sqrt{2 \epsilon_{\varphi}} d N$ with $N_{e}$ the end of inflation.

\subsection{Perturbations}

We now consider perturbations to this system. We are particularly interested in the tensor sector as our hope is that the gauge field, supported during inflation by the evolution of the axion - which can act as a source term for the tensor perturbations - provides a large amplification to the gravitational wave spectrum of Kähler modulus inflation. In the next few sections we will discuss the following aspects that must be simultaneously satisfied

- Amplification of primordial tensor fluctuations: we show that the primordial spectrum of tensor fluctuations of Kähler inflation can be amplified by a factor of order $10^{3}$. The enhanced spectrum reaches values that can be probed by the future generation of CMB polarisation experiments [39 44]. The spectrum produced will 


\section{PRIMORDIAL GRAVITATIONAL WAVES IN STRING INFLATION}

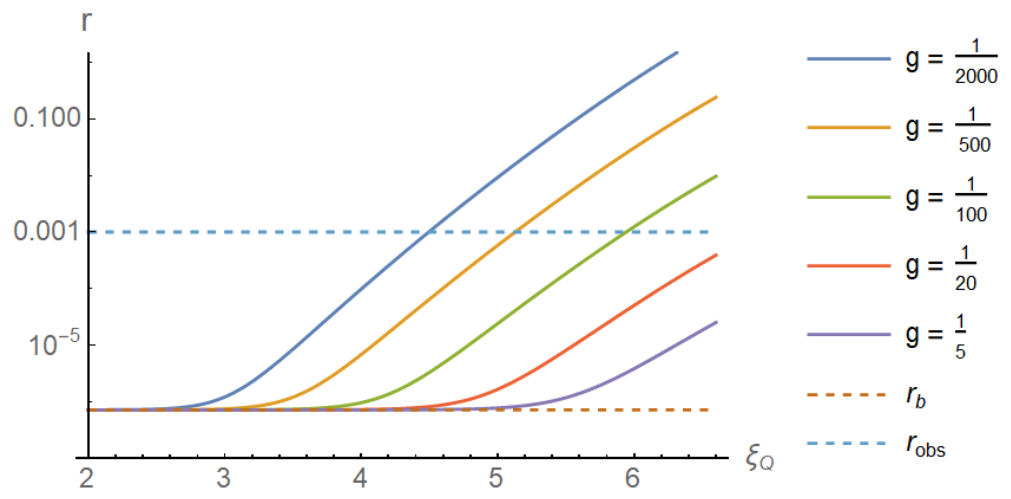

Figure 5.18: Analytic estimate of the sourced tensor-to-scalar ratio, $r$, against $\xi_{Q}=\frac{\mathrm{gQ}}{H}$ for different values of $\mathrm{g}$. The dashed lines correspond to the (non-sourced) background tensor-to-scalar ratio for our example $r_{b}=7.10 \times 10^{-7}$ (orange) and the observational cut-off $r_{o b s} \gtrsim 10^{-3}$ (blue).

be almost completely chiral. However, we shall explain that the tensor chirality is likely not observable for the predicted value of the parameters of our Kähler moduli inflation model.

- Backreaction of gauge fluctuations: In section 5.6 we perform a careful estimate of the backreaction of fluctuations. We show that the backreaction of a potentially large amplitude of gauge field fluctuations can be made sub-dominant in the evolution equations for background quantities. As anticipated, a small backreaction requires a small effective gauge coupling, which is why in example 2, $\mathrm{g}=\frac{1}{2000}$ was chosen. We were unable to find a parameter set for example 1 where the value of $g$ could be very low and the gauge field could be sustained at a large enough value for sizeable enhancement.

Before discussing the perturbations in detail, we show in figures 5.185 .19 (repeating a very similar plot as FIG. 5.9 for emphasis of this point) why the system is heavily constrained, and in particular why we require a very low value for g. In figure 5.18 we plot the analytical estimate (see below) of the sourced tensor-to-scalar ratio as a function of the parameter $\xi_{Q}=\mathrm{g} Q / H$ for different values of the effective gauge coupling, g. The enhancement is exponentially sensitive to the value of $\xi_{Q}$. In figure 5.19 we show an estimate of the backreaction (see 5.6) of the gauge field tensor perturbations on the equation of motion for $Q \sqrt{5.2 .13}$ as a function of $\xi_{Q}$ for different values of g. In order to ensure that our approach of choosing the gauge field to be in the 


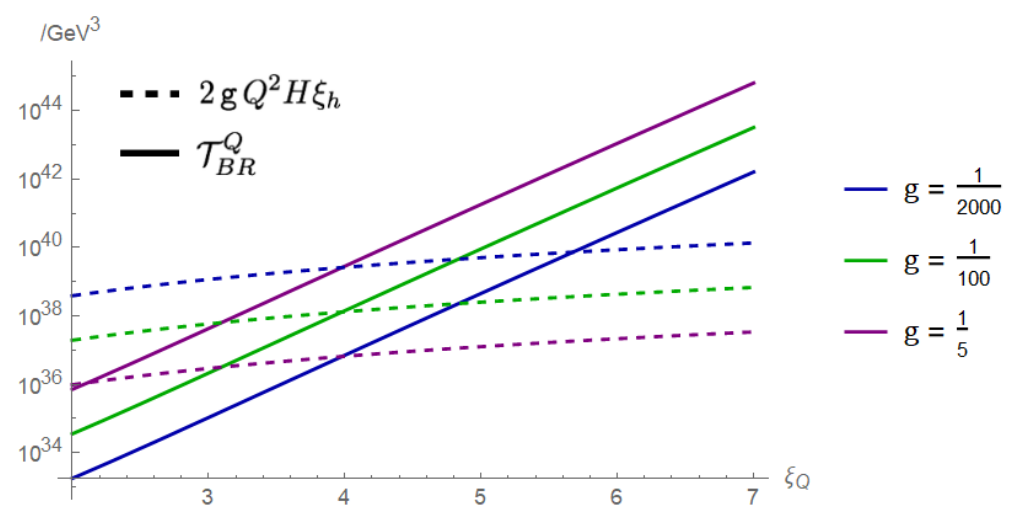

Figure 5.19: The backreaction of the tensor fluctuations on the equation of motion for $Q, \mathcal{T}_{B R}^{Q}$ (solid lines) along with the largest term in the equation of motion for $Q, 2 \mathrm{~g} Q^{2} H \xi_{h}$ (dashed lines) plotted against $\xi_{Q}$ for different values of $\mathrm{g}$. Increasing $\xi_{Q}$ increases $\mathcal{T}_{B R}^{Q}$ much faster than $2 \mathrm{~g} Q^{2} H \xi_{h}$, but decreasing g reduces $\mathcal{T}_{B R}^{Q}$ whilst increasing $2 \mathrm{~g} Q^{2} H \xi_{h}$.

isotropic configuration, $A_{i}^{A}=\delta_{i}^{A} a Q$, is consistent, we require that the backreaction $\mathcal{T}_{B R}^{Q}$, be considerably smaller than the largest term in 5.2 .13$)$, namely $2 \mathrm{~g} Q^{2} H \xi_{h}$. The backreaction also increases exponentially with $\xi_{Q}$. However, the lower $\mathrm{g}$, the smaller the backreaction. Since we require a large enhancement to uplift the tensor spectrum of Kähler moduli inflation, we need a relatively large value for $\xi_{Q} \gtrsim 4$ (in [3], $\xi_{Q} \lesssim 3.5$ ) and consequently a very small value for $g$.

\subsubsection{Set-up}

Following [80], the perturbations can be decomposed as (equivalently to chapter 3):

$$
\begin{aligned}
& \phi^{a}=\phi^{a}(t)+\delta \phi^{a}\left(t, x^{i}\right) \\
& A_{0}^{A}=a(t)\left(Y_{A}\left(t, x^{i}\right)+\partial_{A} Y\left(t, x^{i}\right)\right) \\
& \begin{array}{r}
A_{i}^{A}=a(t)\left[\left(Q(t)+\delta Q\left(t, x^{i}\right)\right) \delta_{A i}+\partial_{i}\left(M_{A}\left(t, x^{i}\right)+\partial_{A} M\left(t, x^{i}\right)\right)\right. \\
\left.\quad+\epsilon_{i A C}\left(U_{C}\left(t, x^{i}\right)+\partial_{C} U\left(t, x^{i}\right)\right)+t_{i A}\left(t, x^{i}\right)\right]
\end{array} \\
& \begin{aligned}
g_{00}=-a^{2}(t)\left(1-2 \phi\left(t, x^{i}\right)\right) \\
g_{0 i}=a^{2}(t)\left(B_{i}\left(t, x^{i}\right)+\partial_{i} B\left(t, x^{i}\right)\right) \\
g_{i j}=a^{2}(t)\left[\left(1+2 \psi\left(t, x^{i}\right)\right) \delta_{i j}+2 \partial_{i} \partial_{j} E\left(t, x^{i}\right)+\partial_{i} E_{j}\left(t, x^{i}\right)+\partial_{j} E_{i}\left(t, x^{i}\right)+h_{i j}\left(t, x^{i}\right)\right]
\end{aligned}
\end{aligned}
$$




\section{PRIMORDIAL GRAVITATIONAL WAVES IN STRING INFLATION}

Here, again, $A=1,2,3$ is the $\mathrm{SU}(2)$ index and $i=1,2,3$ is the spatial index. The tensor modes are the perturbations $t_{i A}$ and $h_{i j}$, on which we impose the transverse and traceless gauge: $\partial_{i} h_{i j}=\partial_{i} t_{i A}=t_{i A}=t_{i i}=h_{i i}=0$ which leaves us with 4 tensor perturbations. The vector modes are $Y_{A}, M_{A}, U_{C}, B_{i}, E_{i}$, which are also chosen to be transverse leaving 10 vector perturbations. The scalar modes contribute another 10 (example 1) or 11 (example 2) perturbations. However, the $S U(2)$ gauge freedom allows us to set $U=U_{i}=0$, immediately removing a scalar and vector perturbation. However, vector perturbations have been shown to quickly decay on super-horizon scales [3, 80] and so these are ignored.

\section{Tensors}

First we focus on the tensor perturbations ${ }^{15}$. This is the same set-up as in chapter 3. Taking our momentum vector along the $z$-axis, $k=k_{z}$ and including only the remaining tensor perturbations, we can write our gauge fields and metric as:

$$
\begin{aligned}
& A_{\mu}^{1}=a\left(0, Q+T_{+}, T_{\times}, 0\right) \\
& A_{\mu}^{2}=a\left(0, T_{\times}, Q-T_{+}, 0\right), \\
& A_{\mu}^{3}=a(0,0,0, Q)
\end{aligned}
$$

and

$$
g_{\mu \nu}=a^{2}\left(\begin{array}{cccc}
-1 & 0 & 0 & 0 \\
0 & 1+h_{+} & h_{\times} & 0 \\
0 & h_{\times} & 1-h_{+} & 0 \\
0 & 0 & 0 & 1
\end{array}\right)
$$

where $T_{+}, T_{\times}$and $h_{+}, h_{\times}$are the transverse tensor perturbations of the gauge fields and metric, respectively. To quadratic order the metric inverse is:

$$
g^{\mu \nu}=a^{-2}\left(\begin{array}{cccc}
-1 & 0 & 0 & 0 \\
0 & 1-h_{+}+h_{+}^{2}+h_{\times}^{2} & -h_{\times} & 0 \\
0 & -h_{\times} & 1+h_{+}+h_{+}^{2}+h_{\times}^{2} & 0 \\
0 & 0 & 0 & 1
\end{array}\right) .
$$

\footnotetext{
${ }^{15}$ We will use a different formalism for the metric when working with the scalars for convenience.
} 


\subsubsection{Analytic approximation}

After finding the tensor action to 2nd order in perturbations, the linearised equations of motion for the tensor modes to leading order in slow-roll can be found and these are given below. We use notation standard to the literature (as in [3]), whereby we split the tensor modes into left and right-moving modes: $\psi_{L, R}=\left(a M_{p} / 2\right)\left(h_{+} \pm i h_{\times}\right)$and $t_{L, R}=a\left(T_{+} \pm i T_{\times}\right)$. By applying a first order slow-roll expansion ${ }^{16}$, we arrive at the equations of motion 17 :

$$
\begin{aligned}
& \partial_{x}^{2} \psi_{R, L}+\left(1-\frac{2}{x^{2}}\right) \psi_{R, L}=\frac{2 \sqrt{f \epsilon_{E}}}{x} \partial_{x} t_{R, L}+\frac{2 \sqrt{f \epsilon_{B}}}{x^{2}}\left(\xi_{Q} \mp x\right) t_{R} \\
& \partial_{x}^{2} t_{R, L}+\left[1+\frac{2}{x^{2}}\left(\xi_{Q} \xi_{h} \mp x\left(\xi_{Q}+\xi_{h}\right)\right)\right] t_{R, L}-\frac{2 \xi_{f}}{x} \partial_{x} t_{R, L} \\
& =-\frac{2 \sqrt{\epsilon_{E} / f}}{x} \partial_{x} \psi_{R, L}+\frac{2}{x^{2}}\left[\left(\xi_{Q} \mp x\right) \sqrt{\epsilon_{B} / f}+\left(1+2 \xi_{f}\right) \sqrt{\epsilon_{E} / f}\right] \psi_{R, L}
\end{aligned}
$$

where, to first order in slow-roll:

$$
\sqrt{\epsilon_{E}}=\frac{\left.\sqrt{f}\left(Q-x \partial_{x} Q\right)\right)}{M_{\mathrm{pl}}} \sim \frac{\sqrt{f} Q}{M_{\mathrm{pl}}}, \quad \sqrt{\epsilon_{B}}=\frac{\sqrt{f} \mathrm{~g} Q^{2}}{H M_{\mathrm{pl}}}
$$

and recall that

$$
\xi_{Q}=\frac{\mathrm{gQ}}{H}, \quad \xi_{h}=\frac{c_{2} \dot{b_{a}}}{2 f H}, \quad \xi_{f}=\frac{c_{1} \dot{\tau_{a}}}{2 f H}
$$

where $a=2,4$ corresponding to example 1 and 2 above, respectively. Before discussing our numerical results, we can make some progress analytically as in [3]. First, we note that only the right-helicity mode can be enhanced - the relevant term in (5.4.6) is given by $\mp \frac{2}{x}\left(\xi_{Q}+\xi_{h}\right) t_{R, L}$, which can only cause an enhancement in the right-helicity mode, $t_{R}$ due to the minus sign 18 For this reason, we will only consider the right-helicity mode, $t_{R}$, in our discussion as this is the one relevant to enhancing the gravitational wave spectrum. Proceeding to find an analytic approximation, we start by solving

\footnotetext{
${ }^{16}$ Here by, first order, we mean objects proportional to $\sqrt{\epsilon_{i}}, i=\phi, E, B$. The full equations are in Appendix C

${ }^{17}$ These equations of motion are completely equivalent to those in chapter 3 and $[3$. (set $f=1$ ).

${ }^{18}$ Due to the $1 / x$ factor, and $\xi_{Q}, \xi_{h}>1$, this term dominates over the other two terms proportional to $t_{R, L}$ when $x \sim \mathcal{O}(1)$. When $x \gg 1$, the term $t_{R, L}$ dominates, $t_{R, L}$ does not grow; and then when $x \ll 1$, the term $\frac{2}{x^{2}} \xi_{q} \xi_{h} t_{R, L}$ dominates and the solution once again stops growing.
} 


\section{PRIMORDIAL GRAVITATIONAL WAVES IN STRING INFLATION}

the right-helicity homogeneous equation of (5.4.6) with $\xi_{f}, \xi_{h}$ and $\xi_{Q}$ assumed to be constant 19 . This gives solutions

$$
t_{R}=\frac{1}{\sqrt{2 k}} i^{\beta} x^{\xi_{f}} W_{\beta, \alpha}(-2 i x)
$$

where $W_{k, m}(z)$ is the Whittaker function, $\beta=-i\left(\xi_{Q}+\xi_{h}\right)$ and $\alpha=\frac{1}{2} \sqrt{-8 \xi_{Q} \xi_{h}+\left(1+2 \xi_{f}\right)^{2}}$. The solution is normalised by the early-time solution, $t_{R} \rightarrow(2 k)^{-1 / 2}(2 x)^{\beta} e^{i x} x^{\xi_{f}}$, $x \rightarrow \infty$, which is the equivalent initial condition to the one given in [3] ( $\operatorname{set} \xi_{f}=0$ ).

We then wish to find the Green function for (5.4.5) for which we use the following formula

$$
G\left(x, x^{\prime}\right)=\frac{\nu_{1}(x) \nu_{2}\left(x^{\prime}\right)-\nu_{1}\left(x^{\prime}\right) \nu_{2}(x)}{\nu_{1}^{\prime}\left(x^{\prime}\right) \nu_{2}\left(x^{\prime}\right)-\nu_{1}\left(x^{\prime}\right) \nu_{2}^{\prime}\left(x^{\prime}\right)}
$$

where $\nu_{1}$ and $\nu_{2}$ are the homogeneous solutions to the equation one wishes to solve. In our case, 5.4.5 has homogeneous solutions:

$$
\nu_{1}(x)=e^{i x}\left(1+\frac{i}{x}\right)
$$

and

$$
\nu_{2}(x)=e^{-i x}\left(1-\frac{i}{x}\right)
$$

With this, our Green's function is

$$
G\left(x, x^{\prime}\right)=\frac{\left(x^{\prime}-x\right) \cos \left(x^{\prime}-x\right)-\left(1+x x^{\prime}\right) \sin \left(x^{\prime}-x\right)}{x x^{\prime}} .
$$

We now need to perform the integral

$$
\int d x^{\prime}\left\{\frac{2 \sqrt{f \epsilon_{E}}}{x^{\prime}} \partial_{x^{\prime}} t_{R, L}+\frac{2 \sqrt{f \epsilon_{B}}}{\left(x^{\prime}\right)^{2}}\left(\xi_{Q}-x^{\prime}\right)\right\} G\left(x, x^{\prime}\right) .
$$

An analytic estimate for this integral can be found by first performing an indefinite integral over $x^{\prime}$, then by taking two limits: first $x^{\prime} \rightarrow \infty$ (the dominant contribution to this integral will be in the sub-horizon limit before the gauge field decays). Then in

\footnotetext{
${ }^{19}$ Of course in reality, as can be seen in e.g. FIGs 5.135 .14 these are not constants and this is a limiting factor on the accuracy of this analytic approximation. Despite this, it proves to be a good order of magnitude estimate of the enhancement.
} 
the super-horizon limit

$$
\lim _{x \rightarrow 0} \psi_{R}=\frac{1}{\sqrt{2 k x}}\left\{\mathcal{F}_{E}^{R} \sqrt{f \epsilon_{E}}+\mathcal{F}_{B}^{R} \sqrt{f \epsilon_{B}}\right\}
$$

The sourced tensor power spectrum is given by:

$$
\mathcal{P}_{t}^{s}=\mathcal{P}_{t}^{R, s}+\mathcal{P}_{t}^{L, s}
$$

where

$$
\mathcal{P}_{t}^{L, s}=0, \quad \mathcal{P}_{t}^{R, s}(k)=\frac{H^{2}}{\pi^{2} M_{\mathrm{pl}}^{2}}\left|\sqrt{2 k} x \lim _{x \rightarrow 0} \psi_{R}\right|^{2}=f \frac{\epsilon_{B} H^{2}}{\pi^{2} M_{\mathrm{pl}}^{2}} \mathcal{F}_{R}^{2}
$$

and $\mathcal{F}_{R}^{2}:=\left|\mathcal{F}_{B}^{R}+\sqrt{\epsilon_{E} / \epsilon_{B}} \mathcal{F}_{E}^{R}\right|^{2}$ is a measure of the amplification. $\mathcal{F}_{E}^{R}$ and $\mathcal{F}_{B}^{R}$ are given by

$$
\begin{aligned}
& \mathcal{F}_{E}^{R}= \\
& -\frac{i 2^{4-\xi_{f}}}{256} e^{\frac{1}{2} i \pi\left(\beta+\xi_{f}\right)} \Gamma\left(-\alpha+\xi_{f}-\frac{3}{2}\right) \Gamma\left(\alpha+\xi_{f}-\frac{3}{2}\right)\left\{\frac{\Gamma\left(-\beta-\xi_{f}\right)}{\Gamma\left(-\alpha-\beta+\frac{1}{2}\right) \Gamma\left(\alpha-\beta+\frac{1}{2}\right)}\right. \\
& {\left[-16 \alpha^{4}+8 \alpha^{2}\left(8 \beta+4 \xi_{f}^{2}+5\right)-8\left((8 \beta+3) \xi_{f}^{2}+8 \beta \xi_{f}+2 \beta(8 \beta+1)+2 \xi_{f}^{4}\right)+32 \xi_{f}-9\right]} \\
& +\frac{1}{\Gamma\left(-\beta+\xi_{f}+1\right)}\left[16 \alpha^{4}+8 \alpha^{2}\left(8 \beta-4 \xi_{f}^{2}-5\right)+128 \beta^{2}-16 \beta\left(2 \xi_{f}+1\right)^{2}\right. \\
& \left.\left.+\left(1-2 \xi_{f}\right)^{2}\left(4 \xi_{f}\left(\xi_{f}+1\right)+9\right)\right]\right\},
\end{aligned}
$$

$$
\begin{aligned}
& \mathcal{F}_{B}^{R}= \\
& \frac{2^{4-\xi_{f}}}{256} e^{\frac{1}{2} i \pi\left(\beta+\xi_{f}\right)} \Gamma\left(-\alpha+\xi_{f}-\frac{3}{2}\right) \Gamma\left(\alpha+\xi_{f}-\frac{3}{2}\right)\left\{\frac{\Gamma\left(-\beta-\xi_{f}\right)}{\Gamma\left(-\alpha-\beta+\frac{1}{2}\right) \Gamma\left(\alpha-\beta+\frac{1}{2}\right)}\right. \\
& {\left[8 i \xi_{Q}\left(\beta+\xi_{f}\right)\left(1-4 \alpha^{2}+8 \beta+4\left(\xi_{f}-1\right) \xi_{f}\right)\right.} \\
& \left.-\left(4 \alpha^{2}-4\left(\xi_{f}-3\right) \xi_{f}-9\right)\left(4 \alpha^{2}-8 \beta-4 \xi_{f}\left(\xi_{f}+1\right)-1\right)\right] \\
& -\frac{1}{\Gamma\left(-\beta+\xi_{f}+1\right)}\left[8 i \xi_{Q}\left(\beta-\xi_{f}\right)\left(4 \alpha^{2}+8 \beta-4\left(\xi_{f}-1\right) \xi_{f}-1\right)\right. \\
& \left.\left.+\left(4 \alpha^{2}-4\left(\xi_{f}-3\right) \xi_{f}-9\right)\left(4 \alpha^{2}+8 \beta-4 \xi_{f}\left(\xi_{f}+1\right)-1\right)\right]\right\} .
\end{aligned}
$$




\section{PRIMORDIAL GRAVITATIONAL WAVES IN STRING INFLATION}
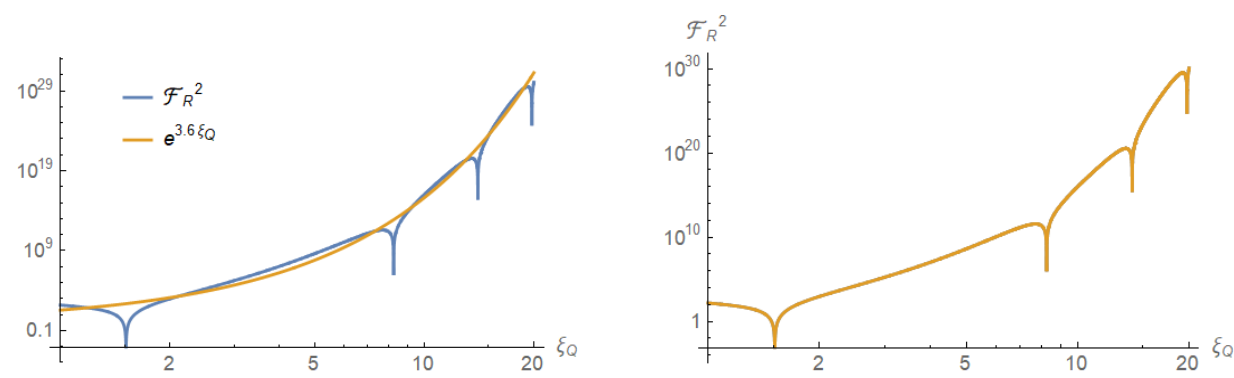

Figure 5.20: The amplification factor, $\mathcal{F}^{2}$, of the tensor power spectrum for right-helicity modes in our analytic estimate plotted against the effective mass of the gauge field, $\xi_{Q}$. The left figure uses $\xi_{f}=9.91 \times 10^{-5}$ and shows the function $e^{3.6 \xi_{Q}}$ which gives an idea of how the enhancement scales with $\xi_{Q}$. The right figure shows two cases, $\xi_{f}=9.91 \times 10^{-5}$ and $\xi_{f}=0$ demonstrating that $\xi_{f}$ is too small to have any effect on the tensor perturbations. This plot is therefore completely equivalent to that given in 3 .

As was mentioned in section 5.3, example 1 leads to too great backreaction from $t_{R}$ on the background equations of motion meaning it is inconsistent. For this reason, we focus on example 2 where an extra modulus, $T_{4}$ has been introduced and is coupled to the gauge field. Using the slow-roll approximation, $\xi_{h}=\xi_{Q}+\xi_{Q}^{-1}+\xi_{f} / \xi_{Q}$ and taking $\xi_{f}=9.91 \times 10^{-5}$, the value it takes 50 e-folds before the end of inflation in example 2 above, we plot the amplification factor $\mathcal{F}^{2}$ for right mode in FIG. 5.20 and we compare the amplification factor for $\xi_{f}=9.91 \times 10^{-5}$ and $\xi_{f}=0$. There is negligible difference, suggesting that this coupling is too small to affect the tensor spectrum.

We define the total tensor spectrum as $r=r_{b}+r_{e n h}$ where $r_{b}$ is the background inflationary tensor spectrum $r_{b}=\left(2 H^{2} / \pi^{2}\right) / \mathcal{P}_{s}$ and $r_{e n h}=\mathcal{P}_{t}^{s} / \mathcal{P}_{s}$ with $\mathcal{P}_{s}=2 \times 10^{-9}$ where $\mathcal{P}_{t}^{s}$ is calculated through (5.4.16). For example 2, 50 e-folds before the end of inflation we have $r_{b}=7.10 \times 10^{-7}$ and $r_{e n h}=8.57 \times 10^{-4}$ leading to an overall estimate for the tensor-to-scalar ratio of $r=8.58 \times 10^{-4}$.

\subsubsection{Numerical results}

We will now discuss the full numerical solution for the tensor perturbations for example 2 . We consider the full equations of motion without employing the slow-roll approximation and normalise our solutions in the Bunch-Davies form $t_{R}\left(x_{i n}\right)=\psi_{R}\left(x_{i n}\right)=1 / \sqrt{2 k}$, $t_{R}^{\prime}\left(x_{i n}\right)=\psi_{R}^{\prime}\left(x_{i n}\right)=i / \sqrt{2 k}$ where $x_{i n}$ should be some relatively large number that we take to be $x_{i n}=2 \times 10^{4}$, numerically approximating infinity, and ${ }^{20} k=k_{*}=$

\footnotetext{
${ }^{20}$ This is default pivot scale for Planck [37.
} 
$0.05 \mathrm{Mpc}^{-1}$. The evolutions of $t_{R}$ and $\psi_{R}$ are plotted in FIG 5.21. With no enhancement to the gravity sector, $|\sqrt{2 k} x \psi| \rightarrow 1$ at super-horizon scales, $x<1$, but as we can see $\psi_{R}$ freezes out at super-horizon scales with an enhanced value due to a transient instability experienced by $t_{R}$ just before horizon-crossing even as $t_{R}$ decays. The freeze-out value of $\frac{1}{2}\left|\sqrt{2 k} x \psi_{R}\right|^{2}$ (the factor of a half arrives because only one of the two helicities is amplified) is the amplification factor for the tensor power spectrum. Evaluating the tensor-to-scalar ratio, $r=\mathcal{P}_{t} / \mathcal{P}_{s}$ with this freeze-out value leads to a larger enhancement than that predicted by our analytic estimate above, giving an enhancement of $r_{b}=7.10 \times 10^{-7} \longrightarrow r=1.27 \times 10^{-3}$, an amplification of $\sim 1791$ for example 2. The value of $r=1.27 \times 10^{-3}$ is large enough to be potentially observable at next generation detectors such as CMB-S4 [41] and many others [39, 40, 42, 44]. We define the tensor power spectrum a: 21

$$
\mathcal{P}_{t}=\frac{H^{2}}{\pi^{2} M_{P l}^{2}}\left|\sqrt{2 k} x \psi_{R}\right|^{2}
$$

and evaluate this with the freeze-out value $(x \ll 1)$ for $\left|\sqrt{2 k} x \psi_{R}\right|^{2}$, with horizoncrossing, $x=1$, taken to be 50 e-folds before the end of inflation.

It is interesting to stress that the resulting tensor spectrum is fully chiral, since only the right-helicity tensor modes get amplified. On the other hand, the resulting tensor-to-scalar ratio is at least one order of magnitude too small for its chirality to be detected by cross-correlating $T, E$ and $B$ spectra with future CMB experiments - see e.g. [87] for a detailed analysis.

\subsection{Scalar perturbations}

We will now discuss the scalar perturbations in this system. As mentioned, we will use a different formalism for the metric than the one used for the tensors. This will prove to be consistent as we will show that the scalar metric perturbations have no impact at linear order, and can therefore be set to zero. Our starting point is the ADM formalism as described for generalised multi-field inflation in $\left[118{ }^{22}\right.$. In the ADM formalism, the

\footnotetext{
${ }^{21}$ This is not the same as $(5.4 .16)$, which accounted only for the sourced contribution to the tensor power spectrum. The following is defined through the full numerical solution and is therefore the full (right-helicity) tensor power spectrum. Since this is so much larger than the (non-enhanced) left-helicity power spectrum, we can safely neglect the contribution from the left-helicity mode.

${ }^{22}$ This is simply a multi-field generalisation of the formalism discussed in chapter 1 .
} 


\section{PRIMORDIAL GRAVITATIONAL WAVES IN STRING INFLATION}

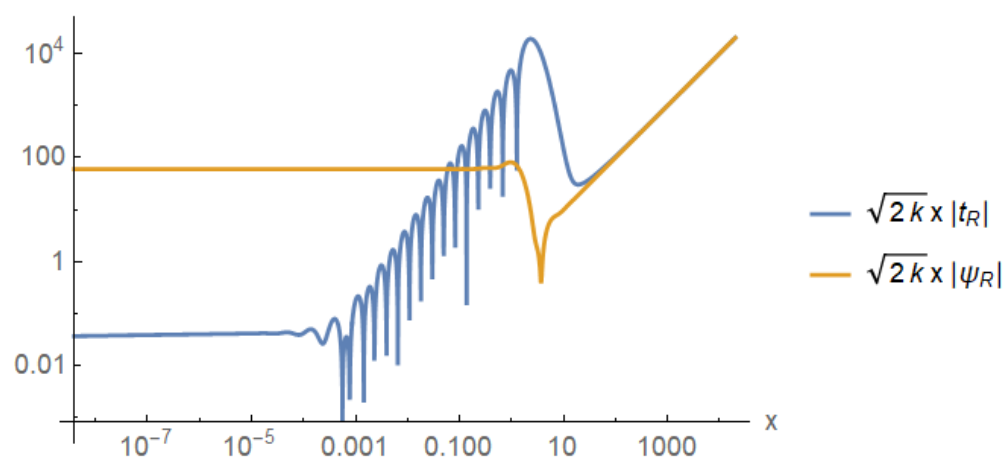

Figure 5.21: The evolution of the (right-helicity) tensor modes for the gauge field, $t_{R}$, and the gravity sector, $\psi_{R}$ for example 2 plotted against $x=k / a H$.

metric is taken to be:

$$
d s^{2}=-N^{2} d t^{2}+h_{i j}\left(d x^{i}+N^{i} d t\right)\left(d x^{j}+N^{j} d t\right)
$$

where $N$ and $N^{i}$ are the lapse and shift functions, respectively. With this choice, our full action, (5.2.1), can be written in the form:

$$
S=\frac{1}{2} \int d t d^{3} x \sqrt{h} N\left(R^{(3)}+2 P\right)+\frac{1}{2} \int d t d^{3} x \frac{\sqrt{h}}{N}\left(E_{i j} E^{i j}-E^{2}\right),
$$

where $R^{(3)}$ is the Ricci scalar calculated with the spatial metric, $h_{i j}$, whose determinant is $h=\operatorname{det}\left(h_{i j}\right) . E_{i j}$ is the symmetric extrinsic curvature tensor given by:

$$
E_{i j}=\frac{1}{2} \cdot \dot{h_{i j}}-\frac{1}{2} \nabla_{j}^{(3)} N_{i}-\frac{1}{2} \nabla_{i}^{(3)} N_{j}
$$

and $E$ is its trace, $E=h^{i j} E_{i j}$. Finally, $P$, is the matter sector and for our system is:

$$
P=X-V-\frac{f\left(\phi^{a}\right)}{4} F_{\mu \nu}^{A} F^{A \mu \nu}+\frac{h\left(\phi^{a}\right)}{4} \tilde{F}_{\mu \nu}^{A} F^{A \mu \nu}
$$

where $X$ is the kinetic term and can be decomposed as:

$$
X=-\frac{1}{2} \gamma_{a b} \partial_{\mu} \phi^{a} \partial^{\mu} \phi^{b}=\frac{1}{2 N^{2}} \gamma_{a b} v^{a} v^{b}-\frac{\gamma_{a b}}{2} h^{i j} \partial_{i} \phi^{a} \partial_{j} \phi^{b}
$$


with $v^{a}=\dot{\phi}^{a}-N^{j} \partial_{j} \phi^{a}$. Variation with respect to the lapse, $N$, leads to the energy constraint:

$$
\begin{aligned}
& R^{(3)}-2 V-\gamma_{a b} h^{i j} \partial_{i} \phi^{a} \partial_{j} \phi^{b}-\frac{1}{N^{2}}\left\{E_{i j} E^{i j}-E^{2}+\gamma_{a b} v^{a} v^{b}\right. \\
& \left.+f\left(h^{i j} F_{0 i}^{A} F_{0 j}^{A}-h^{j k} N^{i} F_{0 j}^{A} F_{i k}^{A}+h^{i j} N^{k} N^{l} F_{i k}^{A} F_{j l}^{A}+h^{i j} N^{k} F_{0 i}^{A} F_{j k}^{A}\right)\right\}=0
\end{aligned}
$$

and variation with respect to the shift, $N^{i}$, leads the momentum constraint:

$$
\nabla_{j}^{(3)}\left(\frac{1}{N}\left(E_{i}^{j}-E \delta_{i}^{j}\right)\right) N=\gamma_{a b} v^{a} \partial_{i} \phi^{b}+f h^{j k}\left(N^{l} F_{k l}^{A} F_{i j}^{A}+F_{0 j}^{A} F_{i k}^{A}\right) .
$$

We now wish to linearise the system in scalar perturbations. We choose the spatially flat gauge to set $h_{i j}=a(t)^{2} \delta_{i j}$ and define the scalar perturbed lapse and shift function as:

$$
N=1+\alpha, \quad N_{i}=\partial_{i} \beta
$$

where $\alpha$ and $\beta$ are linear perturbations. As defined previously, we decompose our fields in the following way (considering now only scalar perturbations):

$$
\begin{aligned}
& \phi^{a}=\phi^{a}(t)+\delta \phi^{a}\left(t, x^{i}\right) \\
& A_{0}^{A}=a(t) \partial_{A} Y\left(t, x^{i}\right) \\
& A_{i}^{A}=a(t)\left[\left(Q(t)+\delta Q\left(t, x^{i}\right)\right) \delta_{A i}+\partial_{i} \partial_{A} M\left(t, x^{i}\right)\right] .
\end{aligned}
$$

In example $2, \delta \phi^{a}=\left\{\delta \tau_{2}, \delta \tau_{4}, \delta b_{4}\right\}$. Including $\alpha$ and $\beta$, we therefore have 8 remaining perturbations. However, the perturbations $Y, \alpha, \beta$ are all non-dynamical (no time derivatives of, e.g., $Y$, appear in its equation of motion). The equations of motion for these three perturbations can therefore be used as constraints. From the energy constraint (5.5.6), we get the following equation for $\beta$ :

$$
\begin{aligned}
4 H \partial^{2} \beta+12 a^{2} H^{2} \alpha= & 2 a H \sqrt{f \epsilon_{E}} \partial^{2} Y-6 a^{2} H \sqrt{f \epsilon_{E}}(H \delta Q+\dot{\delta Q})-2 a^{2} V_{, a} \delta \phi^{a} \\
& -2 a^{2} H \sqrt{f \epsilon_{E}} \partial^{2}(H M+\dot{M})-2 a^{2} \gamma_{a b} \dot{\phi}^{a} \mathcal{D}_{t} \delta \phi^{b}
\end{aligned}
$$

and from the momentum constraint (5.5.7), we get the $\alpha$ equation:

$$
\alpha=\frac{1}{2 H}\left(\gamma_{a b} \dot{\phi}^{a} \delta \phi^{b}+2 H \sqrt{f \epsilon_{E}} \delta Q+2 H \xi_{Q} \sqrt{f \epsilon_{B}} Y\right)
$$




\section{PRIMORDIAL GRAVITATIONAL WAVES IN STRING INFLATION}

where $\mathcal{D}_{t} \delta \phi^{a}=\delta \dot{\phi}^{a}+\Gamma_{b c}^{a} \dot{\phi}^{b} \delta \phi^{c}, \partial^{2}=\partial_{i} \partial^{i}$, and we have performed a systematic slowroll expansion up to first order in $\sqrt{\epsilon}$ as was described for the tensor perturbations. By expanding the action up to second order in the scalar perturbations, and substituting the values for $\alpha$ and $\beta$ found from (5.5.10) and (5.5.11), we find equations for the remaining perturbations, $Y, \delta \phi^{a}, \delta Q, M$. The constraint equation for $Y$ can then be found after moving into momentum space and choosing the wave-vector to be purely on the $z$-axis, $k=k_{z} . Y$ can be found from this equation then substituted into the Fourierspace equations of motion for $\delta \phi^{a}, \delta Q, M$. Whether we set the metric perturbations $\alpha$ and $\beta$ to zero or not, the algebra is extremely involved, and we will therefore only summarise our results here. Before attempting to numerically solve the equations for $\delta \phi^{a}, \delta Q, M$, we change to $x=k / a H$ coordinates, and by redefining the fields as in $([3,80])$

$$
\begin{array}{ll}
\delta \tau_{2}=\frac{\Delta_{\tau_{2}}}{a}, & \delta \tau_{4}=\frac{\Delta_{\tau_{4}}}{a}, \quad \delta b_{4}=\frac{\Delta_{b_{4}}}{a} \\
\delta Q=\frac{\Delta_{1}}{\sqrt{2} a}, & M=\frac{a \mathrm{~g} Q \Delta_{1}+\sqrt{k^{2}+2 a^{2} \mathrm{~g}^{2} Q^{2}} \Delta_{2}}{\sqrt{2} \mathrm{~g} a^{2} k^{2} Q}
\end{array}
$$

we can remove $a$ and $k$ from the equations of motion, using $k=x a H$. The initial conditions for the scalar field perturbations have the multi-field inflation form [26]:

$$
\Delta^{a}\left(x_{i n}\right)=\frac{-x H \frac{d \phi^{a}}{d x}}{\sqrt{2 k} \dot{\varphi}}, \quad \frac{d \Delta^{a}}{d x}\left(x_{i n}\right)=\frac{-i x H \frac{d \phi^{a}}{d x}}{\sqrt{2 k} \dot{\varphi}}
$$

where $\Delta^{a}=\left(\Delta_{\tau_{2}}, \Delta_{\tau_{4}}, \Delta_{b_{4}}\right)$ and $\dot{\varphi}^{2}=\gamma_{a b} \dot{\phi^{a}} \dot{\phi^{b}}=x^{2} H^{2} \gamma_{a b} \frac{d \phi^{a}}{d x} \frac{d \phi^{b}}{d x}$ while the scalar gauge field perturbations have the standard Bunch-Davies initial conditions:

$$
\Delta_{i}\left(x_{i n}\right)=\frac{1}{\sqrt{2 k}}, \quad \frac{d \Delta_{i}}{d x}\left(x_{i n}\right)=\frac{i}{\sqrt{2 k}}
$$

where $\Delta_{i}=\left(\Delta_{1}, \Delta_{2}\right), k=k_{*}=0.05 \mathrm{Mpc}^{-1}$ is the pivot scale and as before $x_{i n}=2 \times 10^{4}$.

We first demonstrate that the inclusion of the scalar metric perturbations, $\alpha, \beta$, in the equations of motion for scalar perturbations 55.5 .12 has no effect on the evolution as these contribute negligibly. The complexity of the equations including the contribution from the metric perturbations is so high that a full evolution of the system is beyond the scope of this work. Instead, we show that over a shorter evolution ${ }^{23}$, there is

\footnotetext{
${ }^{23}$ The smaller the final value of $x$, the closer to the end of inflation.
} 

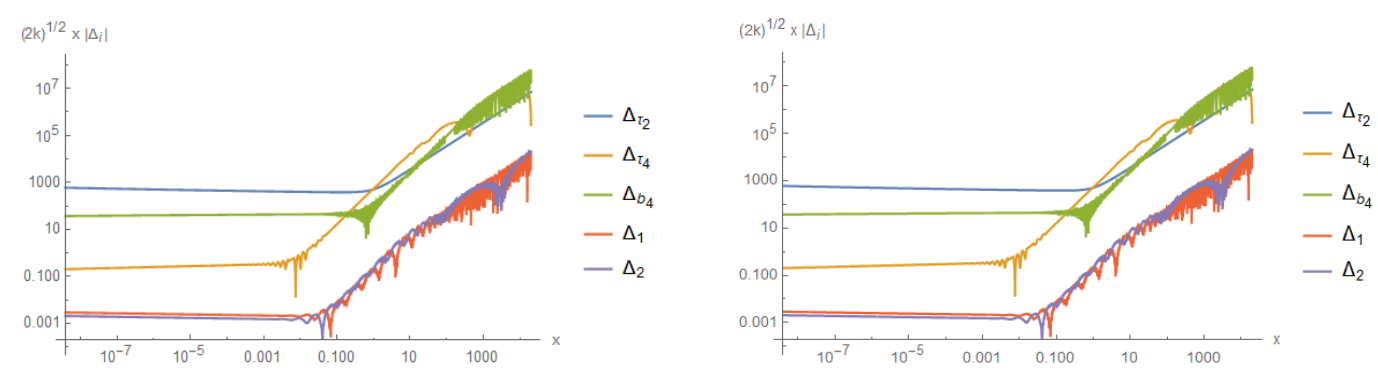

Figure 5.22: The evolution of the scalar perturbations defined in 5.5 .12 with (left figure) and without (right figure) the inclusion of the metric perturbations $\alpha, \beta$ found through (5.5.11) and (5.5.10) for example 2 plotted against $x=k / a H$. The metric perturbations have a negligible effect on the evolution and can safely be set to zero.

no difference in the system's evolution with or without the inclusion of the metric perturbations. We then do a full evolution without the metric perturbations to include the decays of all the fields. As can be seen in FIG. 5.22, the metric perturbations have a negligible effect on the evolution of the scalar perturbations and can therefore be set to zero. We include the scalar perturbation equations for example 2 in Appendix C.

In order to see that the perturbations are well behaved, we define the (tangential) multi-field scalar perturbation

$$
\delta_{s}=\frac{\gamma_{a b} \dot{\phi}^{a} \delta \phi^{b}}{\dot{\varphi}}=\frac{\Delta_{s}}{a}=\frac{\gamma_{a b} \dot{\phi}^{a} \Delta^{b}}{a \dot{\varphi}}
$$

with $\Delta^{a}=\left(\Delta_{\tau_{2}}, \Delta_{\tau_{4}}, \Delta_{b_{4}}\right)$. In the standard multi-field inflation case, the combination $\left|\sqrt{2 k} x \Delta_{s}\right|$ should be $\mathcal{O}(1) \sqrt{24}$ after horizon-crossing, $x<1$ before the background fields decay and lead to a decay in the scalar perturbations. In FIG. 5.23, $\Delta_{s}$ is plotted with the gauge perturbations, $\Delta_{1}, \Delta_{2}$. As can be seen, $\Delta_{s}$ freezes out $(x<1)$ with $\left|\sqrt{2 k} x \Delta_{s}\right| \sim \mathcal{O}(1) \gg\left|\sqrt{2 k} x \Delta_{1}\right|,\left|\sqrt{2 k} x \Delta_{2}\right|$ suggesting the gauge field has negligible effect on the scalar power spectrum. The full evolution including the decays of all the perturbations is shown in FIG. 5.24.

We have shown that the metric scalar perturbations contribute negligibly and that the scalar perturbations to the gauge field are very small relative to the tangential inflationary perturbation. Our assumption is that the scalar power spectrum can be

\footnotetext{
${ }^{24}$ In the perfectly massless case, when the potential is perfectly flat, $\left|\sqrt{2 k} x \Delta_{s}\right|=1$ after horizoncrossing. Therefore a value close to 1 is expected during slow-roll inflation. When the slow-roll approximation breaks down, as happens when the inflaton nears its minimum, the perturbation will grow before decaying as the background inflaton settles to its minimum (see 5.24).
} 


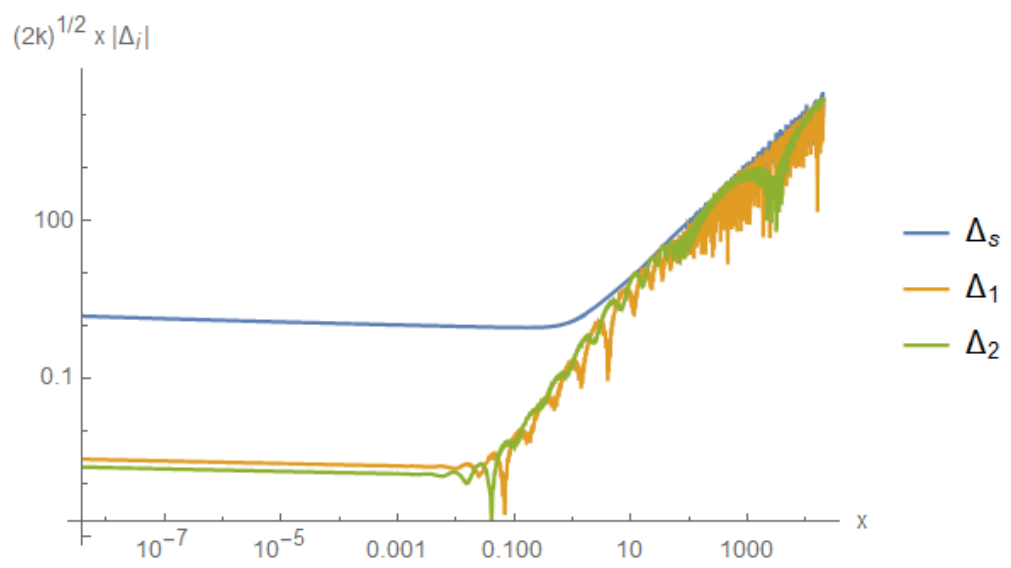

Figure 5.23: The evolution of the scalar perturbations defined in (5.5.12) and (5.5.15) plotted against $x=k / a H$. After horizon-crossing, $x<1$, the scalar perturbation associated with the scalar fields, $\Delta_{s}$ is consistently much larger than the scalars associated with the gauge field, $\Delta_{1}, \Delta_{2}$.
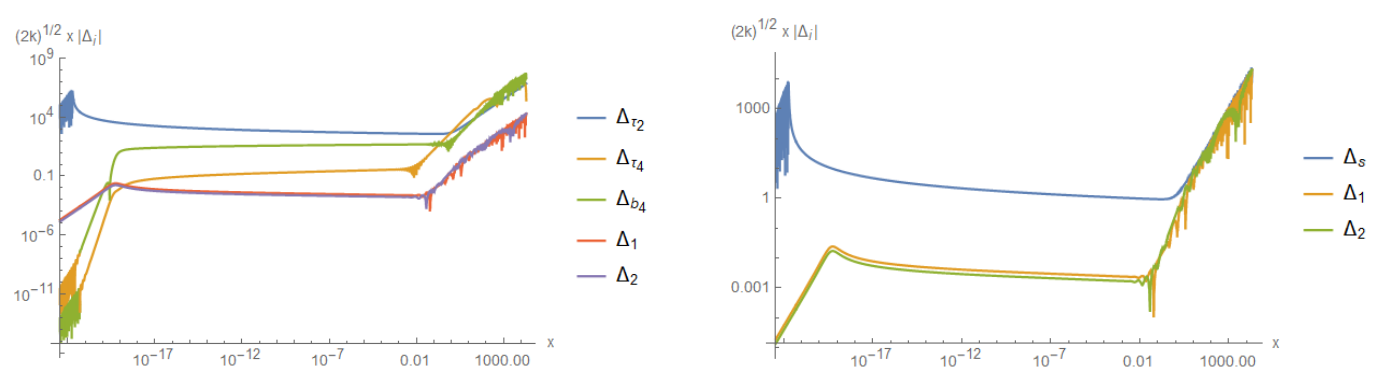

Figure 5.24: The full evolution of the scalar perturbations defined in (5.5.12) and (5.5.15) plotted against $x=k / a H$ including the decays of all the perturbations which take place concurrently with the decays of the corresponding background fields. 
split into a contribution from the gauge field and the standard inflationary part. With the gauge field perturbations sub-dominant, this means that the power spectrum will be well-approximated by $\mathcal{P}_{s}=\frac{H^{2}}{8 \pi^{2} \epsilon_{\varphi}}$ and that therefore the inflationary predictions of both $\mathcal{P}_{s}$ and $n_{s}$ are not spoiled by the presence of the spectator gauge field.

\subsection{Backreaction}

In order to verify the consistency of this model, particularly the assumption that at the background level, the gauge field, $A_{i}^{A}$, can be taken in the isotropic form, $A_{i}^{A}=$ $a(t) \delta_{i}^{A} Q(t)$, we must check whether the tensor perturbation to the gauge field, which is, by necessity, large when $x \sim 1$, does not produce a large backreaction on the background equations of motion. In order to estimate this backreaction, we will take advantage of our analytic solution for the tensor fluctuation to the gauge field, $t_{R}$, given in 5.4.9). Of course this solution is merely a mode function, and we start by promoting $t_{R}$ and its conjugate $t_{L}$ to quantum operators. Starting with the definitions for $T_{+}, T_{\times}$, we arrive at the following forms for $\hat{t}_{R}$ and $\hat{t}_{L}{ }^{25}$.

$$
\begin{aligned}
& \hat{t}_{R}(z)=\int \frac{d^{3} k}{(2 \pi)^{3}}\left\{t_{R}(k) \hat{a}_{R}(k) e^{i \vec{k} \cdot \vec{z}}+t_{L}^{*}(k) \hat{a}_{L}^{\dagger}(k) e^{-i \vec{k} \cdot \vec{z}}\right\} \\
& \hat{t}_{L}(z)=\int \frac{d^{3} k}{(2 \pi)^{3}}\left\{t_{L}(k) \hat{a}_{L}(k) e^{i \vec{k} \cdot \vec{z}}+t_{R}^{*}(k) \hat{a}_{R}^{\dagger}(k) e^{-i \vec{k} \cdot \vec{z}}\right\}
\end{aligned}
$$

where $\hat{t}_{R}=\hat{t}_{L}^{\dagger}$ but $t_{R} \neq t_{L}^{*}$. The creation and annihilation operators satisfy:

$$
\begin{aligned}
& {\left[\hat{a}_{R}(p), \hat{a}_{R}^{\dagger}(q)\right]=(2 \pi)^{3} \delta^{3}(\vec{p}-\vec{q})} \\
& {\left[\hat{a}_{L}(p), \hat{a}_{L}^{\dagger}(q)\right]=(2 \pi)^{3} \delta^{3}(\vec{p}-\vec{q})}
\end{aligned}
$$

and all other combinations are zero. The mode function $t_{R}$ and its conjugate $t_{R}^{*}$ are given by 5.4 .9 and $t_{L}, t_{L}^{*}$ are assumed to be negligible over the relevant region. The

\footnotetext{
${ }^{25}$ This is completely the same as discussed in chapter 3
} 


\section{PRIMORDIAL GRAVITATIONAL WAVES IN STRING INFLATION}

following integrals prove useful:

$$
\begin{aligned}
& \left\langle 0\left|\hat{t}_{R} \hat{t}_{L}\right| 0\right\rangle=\int \frac{d^{3} k}{(2 \pi)^{3}}\left|t_{R}\right|^{2} \\
& \left\langle 0\left|\hat{t}_{L} \hat{t}_{R}\right| 0\right\rangle=\int \frac{d^{3} k}{(2 \pi)^{3}}\left|t_{L}\right|^{2} \sim 0 \\
& \left\langle 0\left|\hat{t}_{R} \partial_{t} \hat{t}_{L}\right| 0\right\rangle=\int \frac{d^{3} k}{(2 \pi)^{3}} t_{R}\left(\partial_{t} t_{R}^{*}\right) \\
& \left\langle 0\left|\hat{t}_{L} \partial_{t} \hat{t}_{R}\right| 0\right\rangle=\int \frac{d^{3} k}{(2 \pi)^{3}} t_{L}\left(\partial_{t} t_{L}^{*}\right) \sim 0 \\
& \left\langle 0\left|\partial_{t} \hat{t}_{L} \hat{t}_{R}\right| 0\right\rangle=\int \frac{d^{3} k}{(2 \pi)^{3}}\left(\partial_{t} t_{L}\right) t_{L}^{*} \sim 0 \\
& \left\langle 0\left|\partial_{t} \hat{t}_{R} \hat{t}_{L}\right| 0\right\rangle=\int \frac{d^{3} k}{(2 \pi)^{3}}\left(\partial_{t} t_{R}\right) t_{R}^{*} \\
& \left\langle 0\left|\hat{t}_{R} \partial_{z} \hat{t}_{L}\right| 0\right\rangle=\int \frac{d^{3} k}{(2 \pi)^{3}}(-i k)\left|t_{R}\right|^{2} \\
& \left\langle 0\left|\hat{t}_{L} \partial_{z} \hat{t}_{R}\right| 0\right\rangle=\int \frac{d^{3} k}{(2 \pi)^{3}}(-i k)\left|t_{L}\right|^{2} \sim 0 \\
& \left\langle 0\left|\partial_{z} \hat{t}_{R} \hat{t}_{L}\right| 0\right\rangle=\int \frac{d^{3} k}{(2 \pi)^{3}} i k\left|t_{R}\right|^{2} \\
& \left\langle 0\left|\partial_{z} \hat{t}_{L} \hat{t}_{R}\right| 0\right\rangle=\int \frac{d^{3} k}{(2 \pi)^{3}} i k\left|t_{L}\right|^{2} \sim 0 \\
& \left\langle 0\left|\partial_{t} \hat{t}_{R} \partial_{t} \hat{t}_{L}\right| 0\right\rangle=\int \frac{d^{3} k}{(2 \pi)^{3}}\left|\partial_{t} t_{R}\right|^{2} \\
& \left\langle 0\left|\partial_{t} \hat{t}_{L} \partial_{t} \hat{t}_{R}\right| 0\right\rangle=\int \frac{d^{3} k}{(2 \pi)^{3}}\left|\partial_{t} t_{L}\right|^{2} \sim 0 \\
& \left\langle 0\left|\partial_{z} \hat{t}_{R} \partial_{z} \hat{t}_{L} \partial_{z} \hat{t}_{R}\right| 0\right\rangle=\int \frac{d^{3} k}{(2 \pi)^{3}} k^{2}\left|t_{R}\right|^{2} \\
& \left\langle 0 \frac{d^{3} k}{(2 \pi)^{3}} k^{2}\left|t_{L}\right|^{2} \sim 0\right.
\end{aligned}
$$

With these in hand, we can find equation of motion for $Q$, (5.2.13), including the backreaction from $t_{R}$ :

$$
\begin{aligned}
& \ddot{Q}+3 H \dot{Q}+Q\left(\dot{H}+2 H^{2}\right)+2 \mathrm{~g}^{2} Q^{3}-2 \mathrm{~g} Q^{2} H \xi_{h}+2 H \xi_{f}(Q H+\dot{Q}) \\
& +\frac{\mathrm{g}}{3 a^{2}} \int \frac{d^{3} k}{(2 \pi)^{3}} \frac{k}{a}\left|t_{R}\right|^{2}+\frac{\mathrm{g} \xi_{h} H}{3 a^{2}} \int \frac{d^{3} k}{(2 \pi)^{3}}\left|t_{R}\right|^{2}=0
\end{aligned}
$$




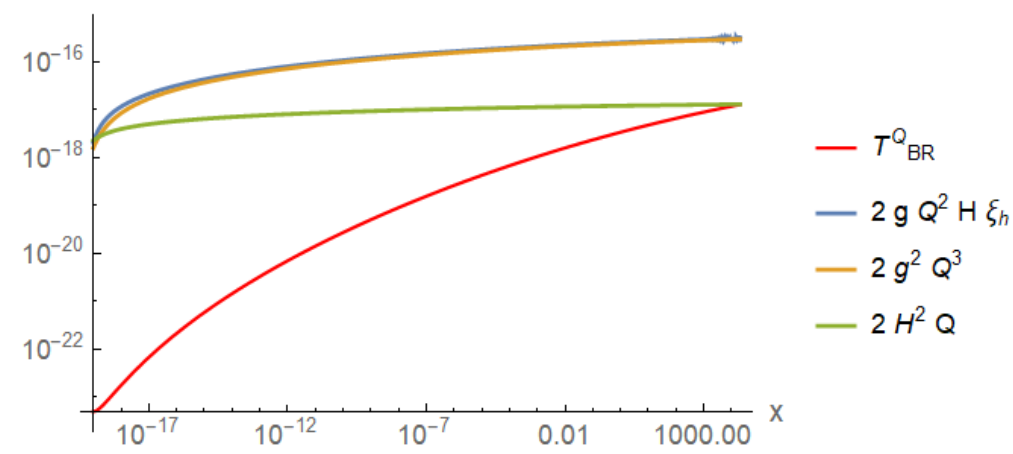

Figure 5.25: The evolution of the leading terms in the equation of motion for $Q(5.6 .4$, $2 \mathrm{~g} Q^{2} H \xi_{h}$ (blue), $2 \mathrm{~g}^{2} Q^{3}$ (orange), $2 H^{2} Q$ (green) and the backreaction induced by the gauge tensor perturbation, $\mathcal{T}_{B R}^{Q}$ (red) in example 2 plotted against $x=k / a H$.

which is in fact identical to the equivalent expression in chapter 3 and [3] - the difference is accounted for in the definition of $\xi_{h}$. To make an estimate of the additional terms' magnitudes in terms of the effective mass of the gauge field, $\xi_{Q}$, we again follow [3] by defining:

$$
\mathcal{T}_{B R}^{Q}:=\frac{\mathrm{g} \xi_{h} H}{3 a^{2}} \int \frac{d^{3} k}{(2 \pi)^{3}}\left|t_{R}\right|^{2}+\frac{\mathrm{g}}{3 a^{2}} \int \frac{d^{3} k}{(2 \pi)^{3}} \frac{k}{a}\left|t_{R}\right|^{2} \simeq \frac{\mathrm{g} H^{3}}{12 \pi^{2}}\left(\xi_{h} \beta_{1}\left(\xi_{Q}\right)+\beta_{2}\left(\xi_{Q}\right)\right)
$$

where

$$
\begin{aligned}
& \beta_{1}\left(\xi_{Q}\right)=\int_{0}^{x_{\max }} d x x\left|i^{\beta} x^{\xi_{f}} W_{\beta, \alpha}(-2 i x)\right|^{2}, \\
& \beta_{2}\left(\xi_{Q}\right)=\int_{0}^{x_{\max }} d x x^{2}\left|i^{\beta} x^{\xi_{f}} W_{\beta, \alpha}(-2 i x)\right|^{2}
\end{aligned}
$$

where we have used the analytic solution for $t_{R}$ given in (5.4.9); used the same cut-off described in [3], $x_{\max }:=\xi_{Q}+\xi_{h}+\sqrt{\xi_{Q}^{2}+\xi_{h}^{2}}$ which encompasses the main region for which $t_{R}$ is enhanced by the transient instability near $x=1$; and $\beta=-i\left(\xi_{Q}+\xi_{h}\right)$ and $\alpha=\frac{1}{2} \sqrt{-8 \xi_{Q} \xi_{h}+\left(1+2 \xi_{f}\right)^{2}}$. FIG. 5.25 shows the evolution of the backreaction term, $\mathcal{T}_{B R}^{Q}$, plotted with the leading contributions to the equation of motion for $Q$ for example 2. $\mathcal{T}_{B R}^{Q}$ is indeed small relative to the largest contribution given by $2 \mathrm{~g} Q^{2} H \xi_{h}$. As mentioned in section 5.3 , in example 1 the backreaction of $t_{R}$ is too large and this is shown in FIG. 5.26 ,

We also want to check that the contribution to the energy density is low. The contribution to the energy density from the gauge tensor perturbation is found to be 


\section{PRIMORDIAL GRAVITATIONAL WAVES IN STRING INFLATION}

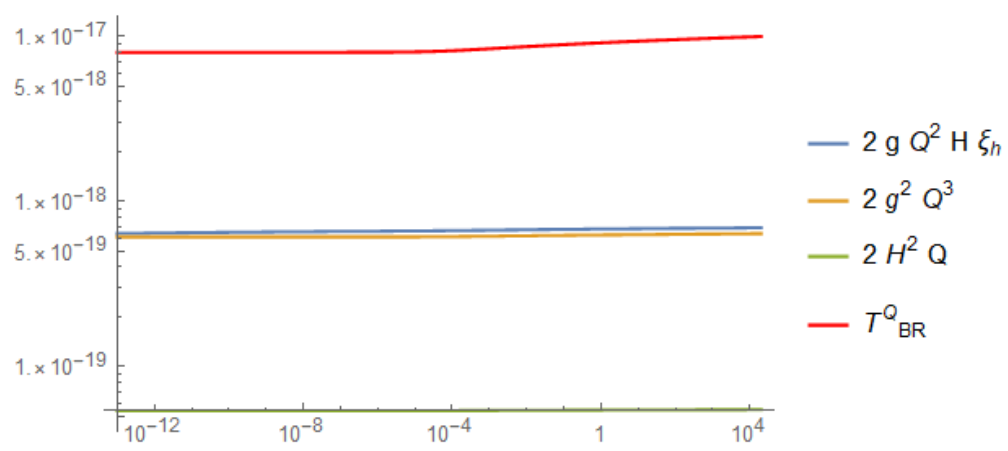

Figure 5.26: The evolution of the leading terms in the equation of motion for $Q$ (5.6.4), $2 \mathrm{~g} Q^{2} H \xi_{h}$ (blue), $2 \mathrm{~g}^{2} Q^{3}$ (orange), $2 H^{2} Q$ (green) and the backreaction induced by the gauge tensor perturbation, $\mathcal{T}_{B R}^{Q}$ (red) in example 1 plotted against $x=k / a H$.

(exactly analogously to [3] )

$$
\rho_{t_{R}}=\frac{1}{a^{4}} \int \frac{d^{3} k}{(2 \pi)^{3}}\left\{\frac{1}{2}\left|\partial_{\eta} t_{R}\right|^{2}+\left(\frac{k}{2}+a g Q\right) k\left|t_{R}\right|^{2}\right\} \simeq f \frac{H^{4}}{8 \pi^{2}} J_{t_{R}}
$$

where $\eta$ is conformal time and

$$
\mathcal{J}_{t_{R}}=\int_{0}^{x_{\max }} d x x^{3}\left\{\left|i^{\beta} \partial_{x}\left(x^{\xi_{f}} W_{\beta, \alpha}(-2 i x)\right)\right|^{2}+\left(1+2 \frac{\xi_{Q}}{x}\right)\left|i^{\beta} x^{\xi_{f}} W_{\beta, \alpha}(-2 i x)\right|^{2}\right\} \text {. }
$$

Using this expression, in FIG. 5.27, the evolution of $\rho_{t_{R}}$ is plotted alongside $\rho_{\varphi} \simeq V$ and $\rho_{Q}=\frac{3}{2} f\left[(H Q+\dot{Q})^{2}+\mathrm{g}^{2} Q^{4}\right]$. It is a sub-dominant contribution throughout.

Example 2 is therefore a working phenomenological model which is capable of producing a large enhancement to the gravity wave spectrum without excessive backreaction and without a large contribution to the scalar power spectrum from the scalar perturbations to the gauge field. This model requires without justification that $c_{2} \gg 1$ and $\mathrm{g} \ll 1$. The coupling between the gauge field and the axion must be large (see (5.2.27) and since in Kähler inflation we expect that $c_{1}=c_{2}=1$ [119], there is no theoretical justification for this, and we treat $c_{1}$ and $c_{2}$ as purely phenomenological. In addition to this, since $\mathrm{g}=\frac{1}{\sqrt{\mathcal{N}}} \ll 1$ (with $\mathcal{N}=[N / 2]$ ) 5.2.7), we see that immediately that with $\mathrm{g}=\frac{1}{2000}$, we require an extremely large value for the degree of the gauge group, $S U(N)$ with $N=8 \times 10^{6}$.

In the next section we consider a very similar but slightly more involved model in which the necessary large coupling between the gauge field and axion can be given some 


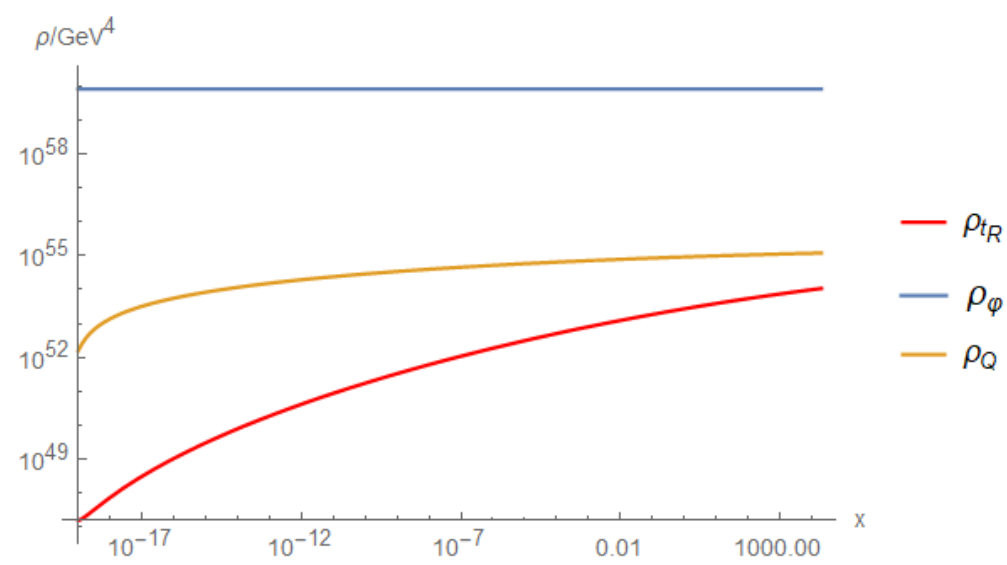

Figure 5.27: The evolution of the energy densities in $\phi^{a}, \rho_{\varphi} \simeq V$ (blue); in $Q, \rho_{Q}=$ $\frac{3}{2} f\left[(H Q+\dot{Q})^{2}+\mathrm{g}^{2} Q^{4}\right]$ (orange); and in $t_{R}, 5.6 .8$ (green), in example 2 plotted against $x=k / a H$.

theoretical grounding. We will also consider more specifically what the requirement of small $g$ means for the gauge group degree.

\subsection{Justifying the large Chern-Simons coupling (example} 3)

The action is still given by (5.3.1) and as in example 2, we use an extra modulus, $T_{4}=\tau_{4}+i b_{4}$, as the spectator scalar field coupled to the gauge field - the key difference here is that instead of $b_{4}$ being coupled to the gauge field through the gauge kinetic coupling, we introduce a further $C_{2}$ axion that allows for a difference between $f$, the coupling to $F^{2}$ and $h$, the coupling to $F \tilde{F}$.

The spectator sector arises from a multiply-wrapped magnetised D7-brane stack along a 4 -cycle, $\Sigma_{4}$, parametrised by the fourth Kähler modulus $\tau_{4}$. In this case, the gauge kinetic function becomes [4, 105, 119, 121,26

$$
f_{4}=n\left(T_{4}+\kappa_{b c}^{A} G^{b} \mathbf{f}^{c}+\frac{\kappa_{b c}^{A} f^{b} f^{c}}{2 g_{s}}\right)
$$

where $n$ is the wrapping number, $\kappa_{b c}^{A}$ are the intersection numbers between the 2-cycles of the Calabi-Yau, $\mathrm{f}^{c}$ is the D7-brane magnetic flux and $G^{a}$ contains the 2 -form axions

\footnotetext{
${ }^{26}$ In example 2 , we used $f_{4}=\frac{c_{1}}{2 \pi} \tau_{4}+i \frac{c_{2}}{2 \pi} \tau_{4}$.
} 


\section{PRIMORDIAL GRAVITATIONAL WAVES IN STRING INFLATION}

and is given by ${ }^{27} G^{a}=\frac{1}{g_{s}} \mathrm{~b}^{a}+i \mathrm{c}^{a}$, where $\mathrm{b}^{a}$ and $\mathrm{c}^{a}$ are the axions descending from the $B_{2}$ and $C_{2}$ forms present in the theory. The Kähler coordinate $T_{4}$ is also shifted by $G$ as $T_{4} \rightarrow T_{4}-\frac{g_{s}}{4} \kappa_{b c}^{A} G^{b}(G+\bar{G})^{c}$ [119 121]. The magnetisation of the D7-branes also contributes to the D-term for the D7-brane gauge theory [120, 121]. In general this can receive contributions from matter fields living on the D7-branes. Here we assume that these have been stabilised at a high scale together with the complex structure moduli and the axiodilaton. Furthermore, we assume that the D-terms also contribute to the stabilisation of the $B_{2}$ axion at $\mathrm{b}=0$ similarly to [4, 105].

Finally, for a successful stabilisation of $\tau_{4}$ consistent with the SCNI scenario we plan to construct, we include as before a second stack of unmagnetised D7-branes, wrapping the same cycle, $\Sigma_{4}$, as the spectator brane, which gives rise to a second non-perturbative contribution for the modulus $\tau_{4}$. That is, the superpotential includes two terms for the spectator sector given by

$$
W_{n p}^{(s)}=A_{4} e^{-a_{4} T_{4}}+A e^{-a f_{4}},
$$

where

$$
f_{4}=n\left(T_{4}+i m b\right)
$$

with $T_{4}=\tau_{4}+i b_{4}$ and we have denoted the magnetic brane flux with $m$ and renamed the $C_{2}$ axion as $\ell^{28}$. As before, the 4 -cycles, $\Sigma_{2}$ and $\Sigma_{3}$, with volumes given by $\tau_{2}$ and $\tau_{3}$, respectively, are wrapped by stacks of unmagnetised D7-branes that give rise to gaugino condensation and introduce the standard Kähler inflation superpotential terms $W \supset e^{-a_{2} T_{2}}$ and $W \supset e^{-a_{3} T_{3}}$. The set-up of the 4-cycles and their respective brane wrappings is summarised in FIG. 5.28 ,

Let us summarise the parameter freedom we have and compare to the phenomenological DFF model [3]. The spectator sector's action takes the following form 29 .

$$
\mathcal{L} \supset-\frac{f\left(\tau_{4}\right)}{4} F_{\mu \nu}^{A} F^{A \mu \nu}+\frac{h(b)}{4} F_{\mu \nu}^{A} \tilde{F}^{A \mu \nu},
$$

\footnotetext{
${ }^{27}$ Where we have assumed that the axiodilaton imaginary part has been fixed to zero.

${ }^{28} \mathrm{We}$ emphasise that this is not $b_{4}$ which is still included in $T_{4}=\tau_{4}+i b_{4}$.

${ }^{29}$ We have ignored here the shift in $\operatorname{Im} f_{4}$ due to $b_{4}$, which will be stabilised during the cosmological evolution. We do this because the required value of the magnetic flux $m \gg\left\langle b_{4}\right\rangle$ and thus will not change the results.
} 


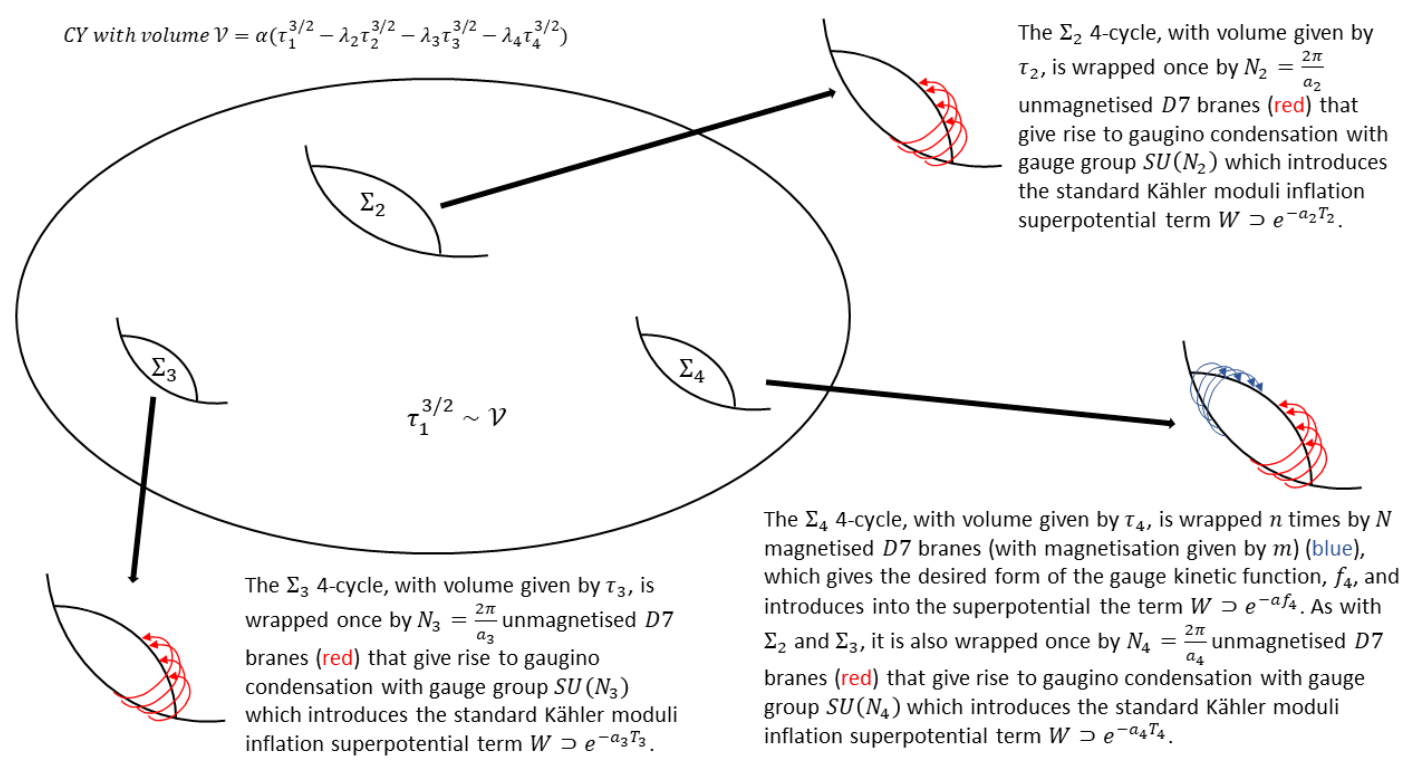

Figure 5.28: A schematic of the set-up used in this section.

where $f\left(\tau_{4}\right)=\tau_{4}, h(b)=m b$, we have absorbed the wrapping number $n$ into the gauge field $A^{A}$, whose field strength is now given by $F_{2}=d A-\mathrm{g} A \wedge A$ with our gauge coupling now redefined as $\mathrm{g}=1 / \sqrt{n N / 2}$ for convenience, and as we have discussed, both $\tau_{4}$ and $b$ are dynamical during inflation. We therefore see that the key differences between this and the previous examples are $\frac{c_{1}}{2 \pi} \rightarrow 1, \frac{c_{2}}{2 \pi} \rightarrow m$, and $\mathrm{g}=\frac{1}{\sqrt{N / 2}} \rightarrow \mathrm{g}=\frac{1}{\sqrt{n N / 2}}$. Another difference is that the scalar potential is changed by the introduction of the second term in (5.7.2), however the potential for $b$ will still take the form (see below)

$$
V(b) \propto g\left(\tau_{4}\right) \cos (n a m b),
$$

where $g\left(\tau_{4}\right)$ is a function of the spectator saxion, $a=2 \pi / N$.

\section{Scalar potential}

The scalar potential is exactly the same as the four-field potential used in example 2 (5.3.17) whose large-volume derivation was given in chapter 4, starting with (5.3.6) with the addition of the terms dubbed $V_{n}$, i.e. our potential is

$$
V_{3}=V_{2}+V_{n}
$$




\section{PRIMORDIAL GRAVITATIONAL WAVES IN STRING INFLATION}

where

$$
\begin{aligned}
V_{n}= & \frac{8 \tilde{a}^{2} \tilde{A}^{2} \sqrt{\tau_{4}}}{3 \alpha \lambda_{4} \mathcal{V}} e^{-\frac{2 \tilde{a}}{m} \tau_{4}}+\frac{16 \tilde{a} \tilde{A} a_{4} A_{4} \sqrt{\tau_{4}}}{3 \alpha \lambda_{4} \mathcal{V}} e^{-\left(a_{4}+\frac{\tilde{a}}{m}\right) \tau_{4}} \cos \left[a_{4} b_{4}-\tilde{a}\left(b+\frac{b_{4}}{m}\right)\right] \\
& +\frac{4 \tilde{a} \tilde{A} W_{0} \tau_{4}}{\mathcal{V}^{2}} e^{-\frac{\tilde{a}}{m} \tau_{4}} \cos \left[\tilde{a}\left(b+\frac{b_{4}}{m}\right)\right] .
\end{aligned}
$$

where we have defined

$$
\tilde{a}:=\text { anm }, \quad \tilde{A}:=\frac{A}{m}
$$

for convenience. These terms were found in the exact same manner as for the original potential (see chapter 4 ) whereby terms higher order than $\mathcal{O}\left((1 / \mathcal{V})^{3}\right)$ in $1 / \mathcal{V}$ with $\mathcal{V} \sim$ $e^{-a_{a} \tau_{a}}$ are discarded. The now four-field system can be described by real-space metric [88, 119, 120]:

$$
\gamma_{a b}=\left(\begin{array}{cccc}
\frac{3 \alpha \lambda_{2}}{4 \sqrt{\tau_{2}} \mathcal{V}} & 0 & 0 & 0 \\
0 & \frac{3 \alpha \lambda_{4}}{4 \sqrt{\tau_{4}} \mathcal{V}} & 0 & 0 \\
0 & 0 & \frac{3 \alpha \lambda_{4}}{4 \sqrt{\tau_{4}} \mathcal{V}} & 0 \\
0 & 0 & 0 & \frac{2 g_{s} \sqrt{\tau_{4}}}{\sqrt{\zeta} \mathcal{V}}
\end{array}\right) .
$$

where $g_{s}$ is the string-coupling and $\zeta$ is a model-dependent constant. The majority of this section will in fact assume a three-field model as we will show that if the $b_{4}$ axion is started at its minimum $b_{4}=\frac{\pi}{a_{4}}$, it only moves negligibly and has no effect on the overall system. The new terms in the potential (5.7.7) with $b_{4}$ at its minimum simplify a little to become

$$
\begin{aligned}
& V_{n} \rightarrow \frac{8 \tilde{a}^{2} \tilde{A}^{2} \sqrt{\tau_{4}}}{3 \alpha \lambda_{4} \mathcal{V}} e^{-\frac{2 \tilde{a}}{m} \tau_{4}}-\frac{16 \tilde{a} \tilde{A} a_{4} A_{4} \sqrt{\tau_{4}}}{3 \alpha \lambda_{4} \mathcal{V}} e^{-\left(a_{4}+\frac{\tilde{a}}{m}\right) \tau_{4}} \cos \left[\tilde{a}\left(b+\frac{b_{4}}{m}\right)\right] \\
& +\frac{4 \tilde{a} \tilde{A} W_{0} \tau_{4}}{\mathcal{V}^{2}} e^{-\frac{\tilde{a}}{m} \tau_{4}} \cos \left[\tilde{a}\left(b+\frac{\pi}{a_{4} M}\right)\right] .
\end{aligned}
$$

With this system $\tau_{4}$ and $b$, coupled to the gauge sector act fully as spectator fields. We require that both the kinetic and potential energy densities in the spectator fields are less than those of the inflaton. This ensures that the inflationary predictions of Kähler inflation driven by $\tau_{2}$ are not affected and thus $\tau_{4}, b$ and the gauge field, are true spectator fields.

Considering our discussion in section 5.2, and the clear need for large parameter to uplift the large coupling between the axion and the gauge field, we now see that this can be achieved by a large magnetic flux, $m$. We also need the gauge field to 
be sustained for a sufficiently long time, and we can parametrise this now with a "decay constant". Sustaining the gauge field for a sizeable period requires a specific value for the decay constant of the axion. Note that since the scalar fields are not canonically normalised, the naive decay constant read from the scalar potential 5.7.5) $f_{c}=1 /(m n a)$ is not correct. However we can identify an instantaneous decay constant as $f_{c}=M_{\mathrm{pl}} \sqrt{\gamma_{b b}\left(\tau_{4}\right)} /(m n a)$ and as we will see, this is of order $f_{c} \sim 10^{-3} M_{\mathrm{pl}}$. We see that now we can constrain our parameters $(n, m, N)$. At this stage, we have two constraints for the three parameters in order to have a successful inflationary evolution with a gauge field that can be sustained for long enough to enhance the tensor spectrum. However, on top of a successful background evolution, one needs to make sure that the backreaction of the tensor gauge perturbations are under control [3, 101,103], which will require $\mathrm{g} \ll 1$, thus introducing a third constraint, fixing the three parameters we have available. We choose $m$, the D7-brane flux, $n$, the D7-brane wrap number and $N$, the condensing group, to ensure a successful background evolution, large enhancement of tensor perturbations and good control on the backreaction.

\subsubsection{Background evolution and cosmological parameters}

We consider the following set of parameters for the Kähler moduli 30 ,

$$
\begin{aligned}
& \hat{\xi}=\frac{1}{2}, \quad \alpha=\frac{1}{9 \sqrt{2}}, \quad \lambda_{2}=10, \quad \lambda_{3}=1, \quad \lambda_{4}=0.01 \\
& a_{2}=\frac{2 \pi}{30}, \quad a_{3}=\frac{2 \pi}{3}, \quad a_{4}=\frac{2 \pi}{50}, \quad \tilde{a}=40 a_{4}, \quad g_{s}=0.1 \\
& A_{2}=\frac{1}{1.7 \times 10^{6}}, \quad A_{3}=\frac{1}{425}, \quad A_{4}=4.2 \times 10^{-9}, \quad \tilde{A}=0.0034 A_{4}, \\
& m=10000, \quad \zeta=5, \quad \beta=6.94681 \times 10^{-5}, \quad W_{0}=\frac{40}{17} .
\end{aligned}
$$

As in example $2, \lambda_{4}$ is chosen to be much smaller than $\lambda_{2}$ so that $\tau_{4}$ has lower kinetic energy, and therefore a negligible contribution to $\epsilon_{\varphi}$. We require that the energy density of the universe receives only a small contribution from the spectator part so that

\footnotetext{
${ }^{30}$ As in example 1 and 2, we are still using the parameters from example 4 in [76] as a base for the inflationary sector.
} 


\section{PRIMORDIAL GRAVITATIONAL WAVES IN STRING INFLATION}

inflation can proceed as expected with the important inflationary terms involving $\tau_{2}$. With this in mind, $A_{4}, a_{4}, \tilde{A}, \tilde{a}$ are chosen to be small relative to $A_{2}, a_{2}$. In addition to this, we would like the stabilisation of $\tau_{4}$ and $b_{4}$ to be dictated by the terms

$$
V \supset \frac{8\left(a_{4} A_{4}\right)^{2} e^{-2 a_{4} \tau_{4}} \sqrt{\tau_{4}}}{3 \alpha \lambda_{4} \mathcal{V}}+\frac{4 W_{0} a_{4} A_{4} e^{-a_{4} \tau_{4}} \cos \left(a_{4} b_{4}\right) \tau_{4}}{\mathcal{V}^{2}},
$$

which is why $\tilde{A}$ is taken to be small relative to $A_{4}$. Ensuring the terms involving $b$ are much smaller than the terms in (5.7.12) ensures that the $b_{4}$ axion will be minimised at $\left\langle b_{4}\right\rangle=\frac{\pi}{a_{4}}$, which in turn ensures that $\tau_{4}$ is stabilised at a much smaller value than $\tau_{1} 31$. We are then left with some freedom in choosing $a$ and $m$, which are chosen phenomenologically to lead to a successful enhancement of the PGW spectrum.

The global minimum of the potential for this set of parameters is found to be at:

$\tau_{1}=2554.50, \quad \tau_{2}=4.77523, \quad \tau_{3}=2.65081, \quad \tau_{4}=14.8743, \quad \nu \rightarrow 10135.3$,

while the axions' minima of course lie at $b^{a}=\pi / a^{a}$. We are now in a position to evolve the system under equations of motion (5.2.8)-(5.2.13). The initial conditions are taken as:

$$
\tau_{2}=80.17, \quad \tau_{4}=10, \quad b=0.4 \frac{\pi}{a}, \quad Q=8 \times 10^{-4} M_{\mathrm{pl}} .
$$

As in example $2, \mathrm{~g}=\frac{1}{2000}$. The rest of this analysis is exactly equivalent to example 2 with of course the key difference being the axion coupled to the gauge field is $b$ not $b_{4}$. We therefore quickly go through the background evolution, perturbations and backreaction.

The background evolution of this system is plotted in FIGs 5.29 5.37. The evolution proceeds very similarly to that of example $2-b$ evolves slowly (see FIG. 5.30) because of its coupling to $Q$, which cancels the potential term in the equation of motion for $b$ (see FIG. 5.31), while $\tau_{4}$ 's evolution is quite trivial: it is shifted only a small amount from its minimum while $b$ is away from its minimum, and decays as $b$ does. The "slow-roll" evolution of $b$ supports $Q$ (see FIG. 5.32) which roughly satisfies $\xi_{Q} \sim \xi_{h}$ (see FIG. 5.33 , left figure). Also in the the right figure of FIG. 5.33, we see that the evolution of $b_{4}$ takes it only a negligible distance away from its minimum - in fact it has a completely

\footnotetext{
${ }^{31}$ Recall $\tau_{4}$ must be a 'small' blow-up modulus, and a minimum at low values of $\tau_{4}$ is achieved through the terms in the potential of (5.7.12). The minimum can be ruined if the terms involving the $b$ axion are too large and $\tau_{4}$ can be destabilised to very large values, $\tau_{4} \gtrsim 10^{3}$.
} 

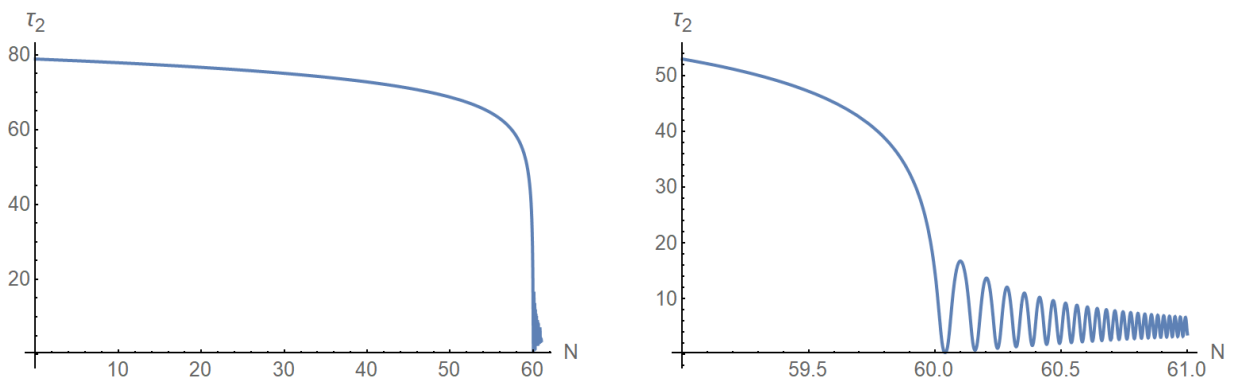

Figure 5.29: The evolution of the inflaton, $\tau_{2}$ during the last 60 e-folds of inflation (left) and during the last few e-folds (right) for example 3.
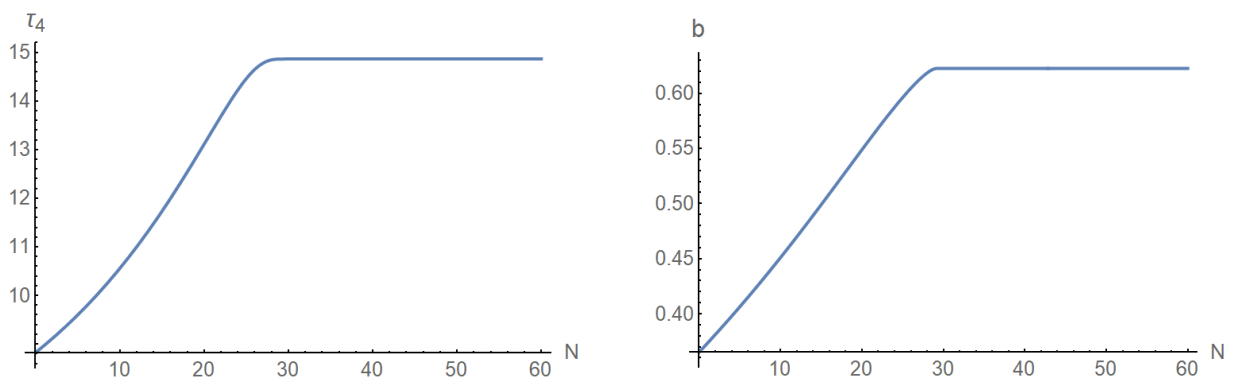

Figure 5.30: The evolution of the spectator modulus, $\tau_{4}$ and the axion $b$ during the last 60 e-folds of inflation for example 3. Both fields reach their minima well before the end of inflation.
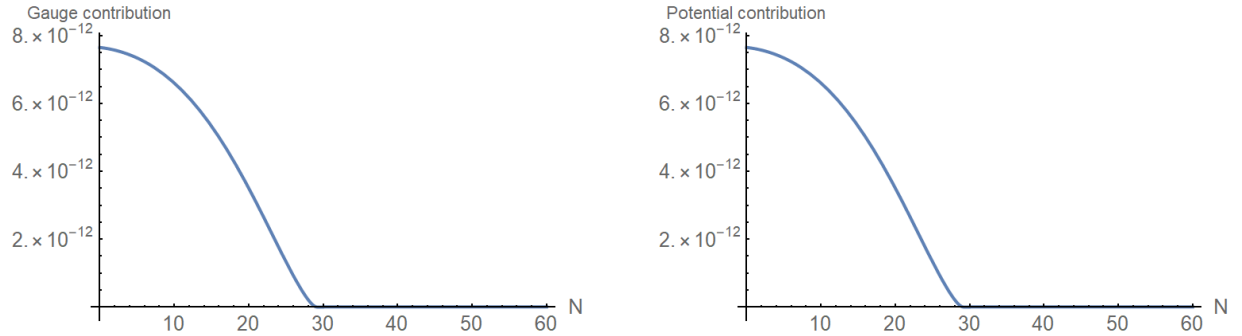

Figure 5.31: The contributions of (left) the term $-3 \mathrm{~g} m \frac{\sqrt{\zeta} \mathcal{V}}{2 g_{s} \sqrt{\tau_{4}}} Q^{2}(H Q+\dot{Q})$ provided by the gauge field, $Q$; and (right) the term $\frac{\sqrt{\zeta} \mathcal{V}}{2 g_{s} \sqrt{\tau_{4}}} \frac{\partial V}{\partial b}$ provided by the potential, to the equation of motion for $b$ given by the form in (5.2.12) for example 3. The contribution from the gauge field almost exactly cancels the contribution from the potential leading to a slow-roll evolution for $b$. 

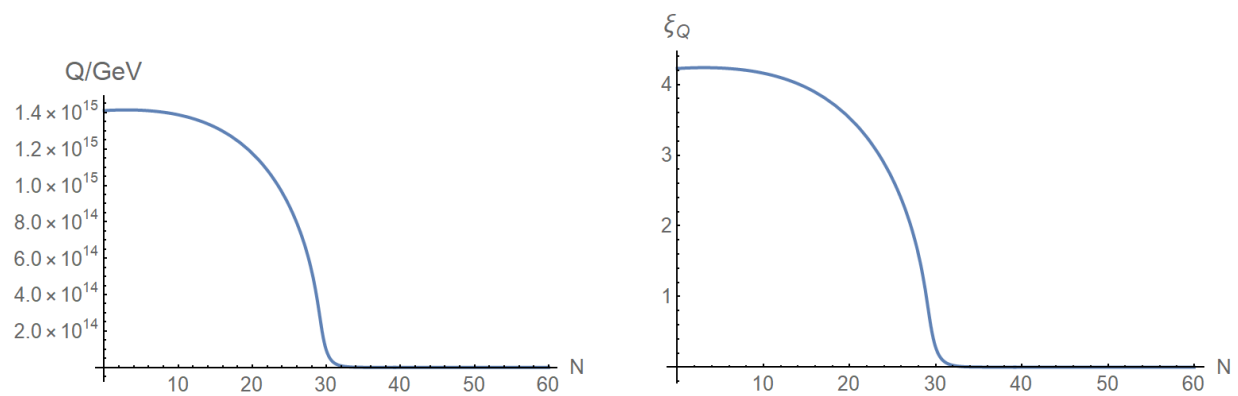

Figure 5.32: The evolution of the gauge field, $Q$ (left) and $\xi_{Q}=\frac{\mathrm{g} Q}{H}$ (right) during the last 60 e-folds of inflation for example 3. The evolution of $Q$ is tied to the evolution of $b$.
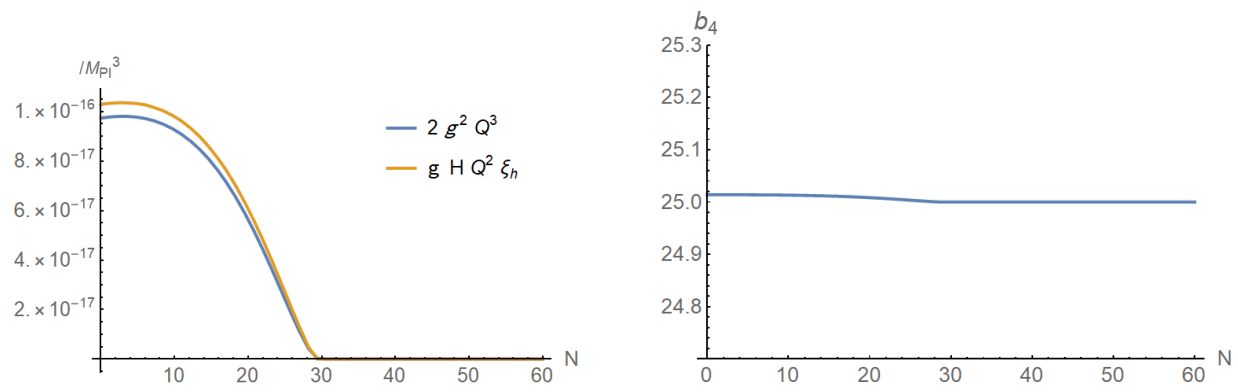

Figure 5.33: Left figure: The two dominant terms in the equation of motion for $Q$, 5.2 .13 , satisfy $2 \mathrm{~g}^{2} Q^{3} \sim \mathrm{g} H Q^{2} \xi_{h}$ for example 3 . The term introduced through the coupling to the axion almost cancels $2 \mathrm{~g}^{2} Q^{3}$, a term that sends $Q$ to zero. In this way the axiongauge coupling supports the gauge field. Right figure: The evolution of the $b_{4}$ modulus for example 3 - started at its minimum, it moves only negligibly during inflation, and can be safely ignored in the numerics.
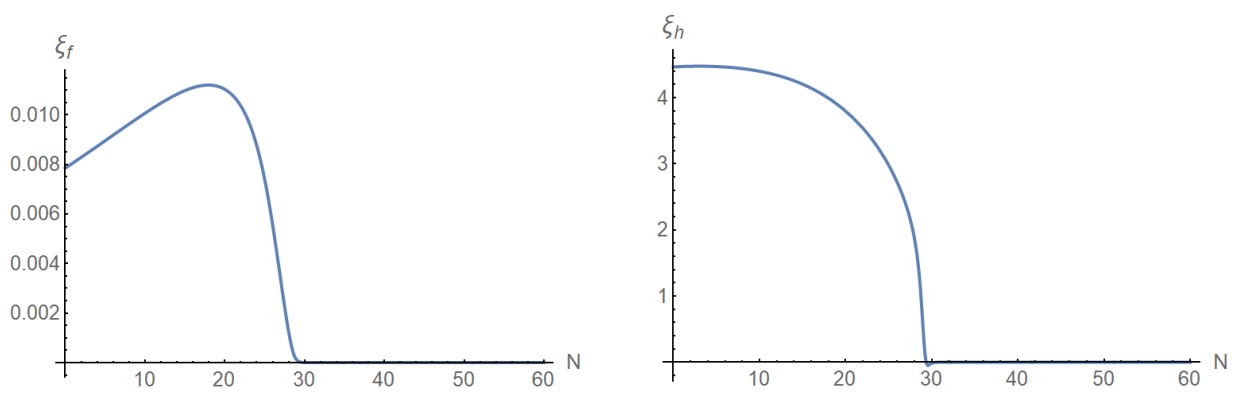

Figure 5.34: The evolution of the effective couplings of $\tau_{4}$ and $b$ to the gauge field, $\xi_{f}$ (left) and $\xi_{h}$ (right) during the last 60 e-folds of example 3. 

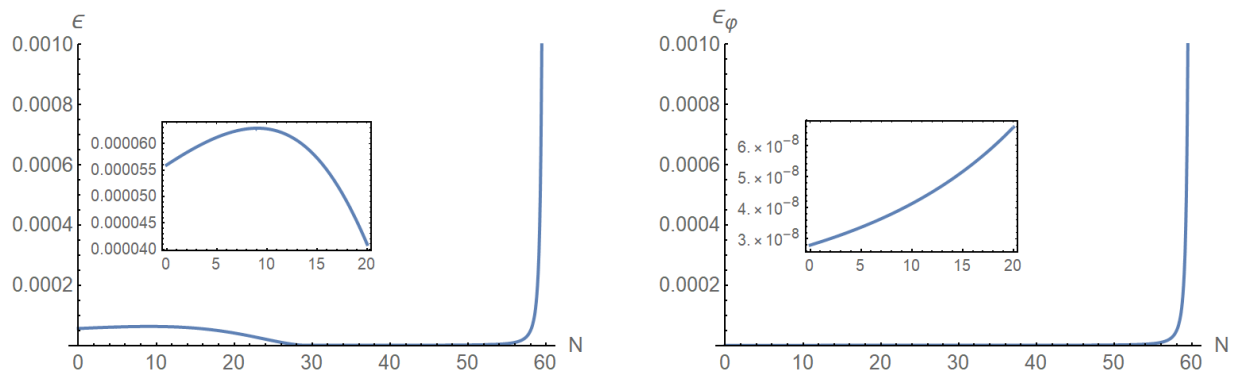

Figure 5.35: The evolution of the slow-roll parameter, $\epsilon$ (left), and the proportion of $\epsilon$ made up by $\epsilon_{\varphi}$, during the last 60 e-folds of inflation for example 3 .
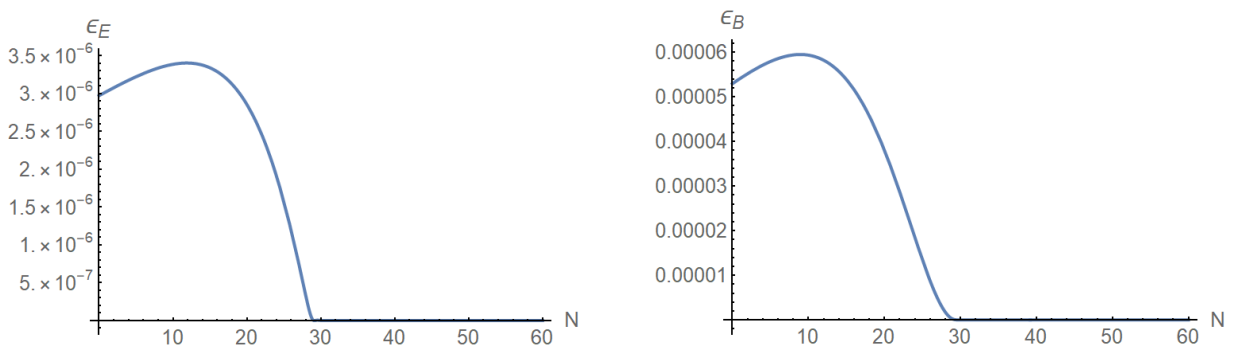

Figure 5.36: The evolution of the electric and magnetic components of the slow roll parameter, $\epsilon_{E}$ (left figure) and $\epsilon_{B}$ (right figure) respectively during the last 60 e-folds of inflation for example 3 .
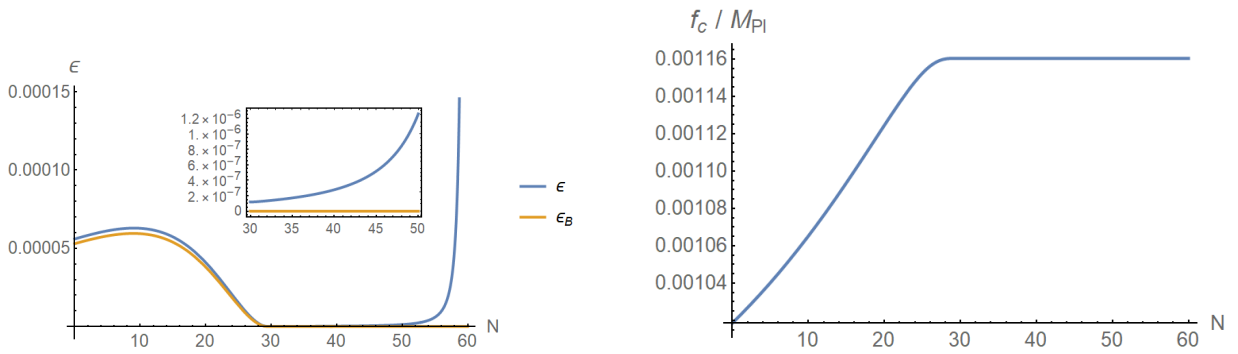

Figure 5.37: Left figure: Comparing $\epsilon_{B}$ with the overall slow-roll parameter, $\epsilon . \epsilon_{B}$ provides the largest contribution to $\epsilon$ for a substantial part of the last 60 e-folds of inflation in example 3. Right figure: Plot of the instantaneous decay constant, $f_{c}=\frac{\sqrt{\gamma_{b b}}}{a m n}$, during the last 60 e-folds of inflation in example 3. 


\section{PRIMORDIAL GRAVITATIONAL WAVES IN STRING INFLATION}

negligible effect on the overall evolution and it is safe to consider a three scalar field system of $\left(\tau_{2}, \tau_{4}, b\right)(+Q)$ from here on. Finally in the left figure of 5.37, we see that as in example 2, the slow-roll parameter $\epsilon \sim \epsilon_{B}$ is dominated by the gauge field for a large period of inflation. This of course requires us to assume again that the power spectrum can be well-approximated by $\mathcal{P}_{s}=\frac{H^{2}}{8 \pi^{2} \epsilon_{\varphi}}$. In the right figure of 5.37 , we see that the instantaneous decay constant of the axion is around $f_{c} \sim 10^{-3} M_{\mathrm{pl}}$.

The inflationary predictions of this model (assuming $\mathcal{P}_{s}=\frac{H^{2}}{8 \pi^{2} \epsilon_{\varphi}}, n_{s}=1-2 \epsilon-\eta_{\varphi}$, $\left.r=16 \epsilon_{\varphi}\right)$ are (now taken to be 60 e-folds before the end of inflation)

$$
\begin{aligned}
& \epsilon_{\varphi}=2.80 \times 10^{-8}, \quad n_{s}=0.964, \quad r_{b}=4.48 \times 10^{-7}, \\
& V_{\text {inf }}^{1 / 4}=8.39 \times 10^{14} \mathrm{GeV}, \quad \Delta \varphi=0.190 M_{\mathrm{pl}}
\end{aligned}
$$

where $\Delta \varphi=\int_{N_{*}}^{N_{e}} \sqrt{2 \epsilon_{\varphi}} d N$ with $N_{e}$ the end of inflation and $N_{*}=N_{e}-60$.

We see here at the background level, we have a working model where the large coupling between the axion and gauge field is accounted for by including very large magnetic flux, $m=10000$. We also require a small value for $g=\frac{1}{\sqrt{N n / 2}}=\frac{1}{2000}$, and constraints on the decay speed of the axion have us choose (to achieve the best enhancement) $\tilde{a}=a n m=\frac{8 \pi}{5}$, with $a=\frac{2 \pi}{N}$. We see in this way, we have 3 constraints and 3 unknowns and can solve to get a slightly smaller value for the gauge degree than in example 2, $N \sim 3 \times 10^{5}$ with the wrapping number at $n=25$. In FIG. 5.38, we plot g against $N$ for example 3 where $n=25$ and $m=10000$ have already been constrained. Requiring small $\mathrm{g}$ means we need very large gauge degree, $N$.

\subsubsection{Tensors}

The perturbations are found in the exact same way as for example 2 , and, in fact the analytical estimate for the enhancement to the tensor power spectrum is identical to that given in (5.4.16)-(5.4.18). 60 e-folds before the end of inflation, we have in example 3: $\xi_{f}=7.85 \times 10^{-3}$ and $\xi_{Q}=4.23$, which gives us the analytic estimate for the enhancement to $r$ of

$$
r_{b}=4.48 \times 10^{-7} \rightarrow r=2.86 \times 10^{-3} .
$$

The full equations of motion for the right-helicity tensor modes, $t_{R}$ and $\psi_{R}$ are 


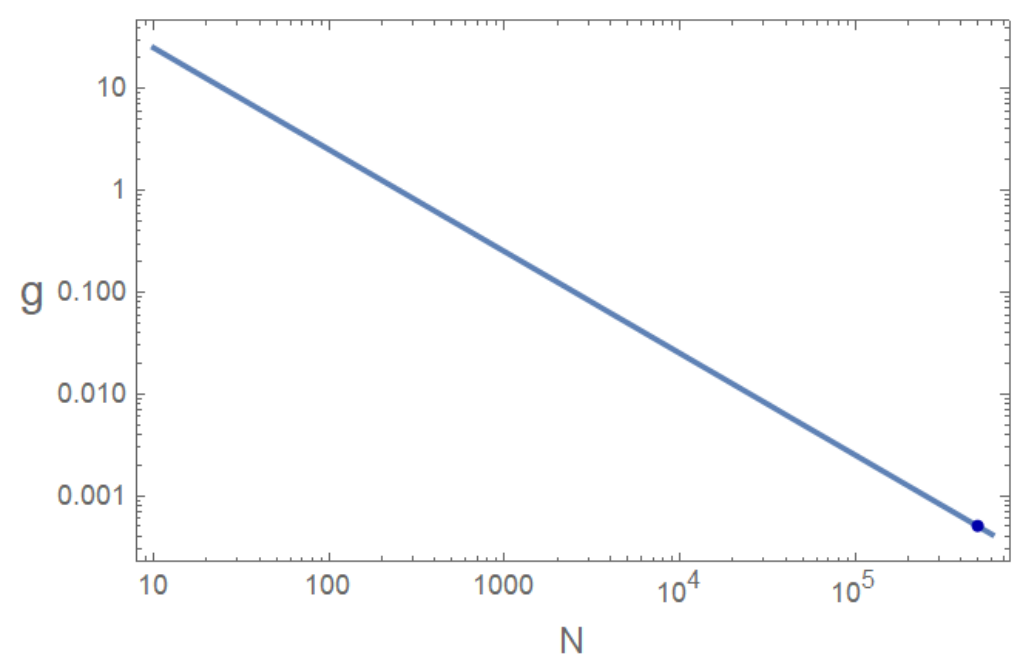

Figure 5.38: Plot of how the effective gauge coupling $g$ constrains the value of the gauge degree $N$ in example 3 . The example given $\mathrm{g}=\frac{1}{2000}$ is marked with a blue dot.

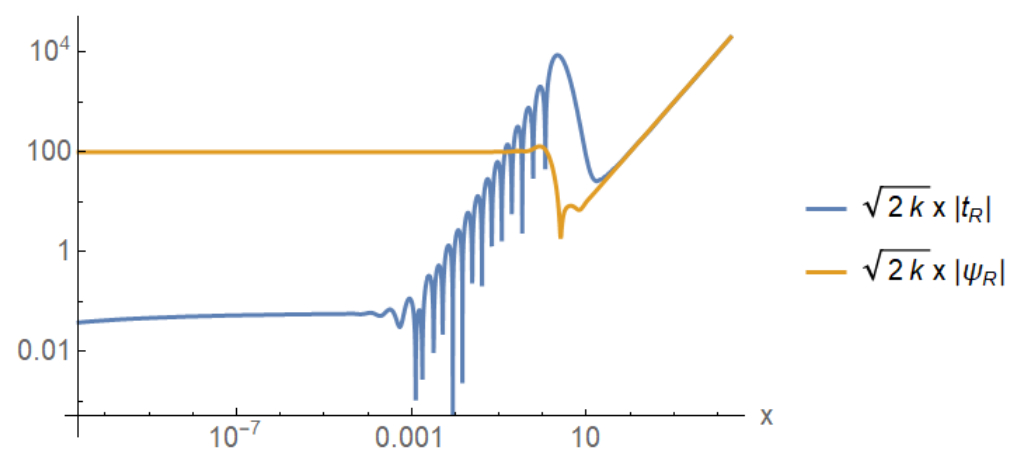

Figure 5.39: The evolution of the (right-helicity) tensor modes for the gauge field, $t_{R}$, and the gravity sector, $\psi_{R}$ for example 3 plotted against $x=k / a H$.

written in Appendix C, and their numerical evolution is plotted in FIG. 5.39 using the standard initial conditions of $\psi_{R}\left(x_{i n}\right)=t_{R}\left(x_{i n}\right)=\frac{1}{\sqrt{2 k}}$ with $k=k_{*}=0.05 \mathrm{Mpc}^{-1}$ the pivot scale, $x_{i n}=2 \times 10^{4}$ and horizon-crossing, $x=1$, is taken to be 60 e-folds before the end of inflation. As in example 2, we see a large enhancement to $\psi_{R}$, and using the freeze-out value $(x \ll 1)$, we get an enhancement of

$$
r_{b}=4.48 \times 10^{-7} \rightarrow r=2.29 \times 10^{-3},
$$

of a similar value to that predicted by the analytic estimate. This corresponds to an enhancement of $5 \times 10^{3}$. 


\section{PRIMORDIAL GRAVITATIONAL WAVES IN STRING INFLATION}

Comparing this to the example given in [3] where the freeze-out value of $\sqrt{2 k} x \psi_{R} \lesssim$ 10 (FIG. 4), we see that the amplification factor in our model is much larger. This is of course necessary because we wish to amplify the tensor-to-scalar ratio to observable values $r \gtrsim 10^{-3}$. In the example given in [3], $r_{b} \sim 10^{-3}$ whereas in our example, $r_{b} \lesssim 10^{-6}$ meaning we require a much greater enhancement. This does not come for free and the larger enhancement leads to larger backreaction (see section 5.6), which can be compensated for by reducing the value of $\mathrm{g}$, and in $[3], \mathrm{g}=1.11 \times 10^{-2}$, compared to our value of $\mathrm{g}=5 \times 10^{-4}$.

As in example 2, we are able to greatly amplify the tensor power spectrum which becomes almost completely right-handed. Although as in example 2, this chirality is unlikely to be detectable because $r$ is still about an order of magnitude too small [87].

\subsubsection{Scalars}

The set-up of the scalars proceeds again exactly as for example 2 - starting with the $\mathrm{ADM}$ formalism, the constraint equations for the metric perturbations, $\alpha$ and $\beta$ are given by $5.5 .10-5.5 .11{ }^{32}$. The remaining scalar perturbations are then given by

$$
\begin{aligned}
& \phi^{a}=\phi^{a}(t)+\delta \phi^{a}\left(t, x^{i}\right) \\
& A_{0}^{A}=a(t) \partial_{A} Y\left(t, x^{i}\right) \\
& A_{i}^{A}=a(t)\left[\left(Q(t)+\delta Q\left(t, x^{i}\right)\right) \delta_{A i}+\partial_{i} \partial_{A} M\left(t, x^{i}\right)\right] .
\end{aligned}
$$

where we are now explicitly assuming a three-field system $\phi^{a}=\left(\tau_{2}, \tau_{4}, b\right)$. Again after moving to momentum space and assuming the wave-vector is purely along the $z$-axis, $k=k_{z}$, the non-dynamical $Y$ can be solved for then substituted along with $\alpha$ and $\beta$ into the remaining equations of motion. Again it is simplest to work with $x=k / a H$, use

$$
\begin{array}{ll}
\delta \tau_{2}=\frac{\Delta_{\tau_{2}}}{a}, & \delta \tau_{4}=\frac{\Delta_{\tau_{4}}}{a}, \quad \delta b=\frac{\Delta_{b}}{a} \\
\delta Q=\frac{\Delta_{1}}{\sqrt{2} a}, & M=\frac{a \mathrm{~g} Q \Delta_{1}+\sqrt{k^{2}+2 a^{2} \mathrm{~g}^{2} Q^{2}} \Delta_{2}}{\sqrt{2} \mathrm{~g} a^{2} k^{2} Q}
\end{array}
$$

\footnotetext{
${ }^{32}$ These equations are written in terms of the functions $f$ and $h$, and the scalar metric $\gamma_{a b}$ so are quite general, and can be applied equally to examples 1,2 and 3 (in ex. $3, f=\tau_{4}, h=M b$ ).
} 

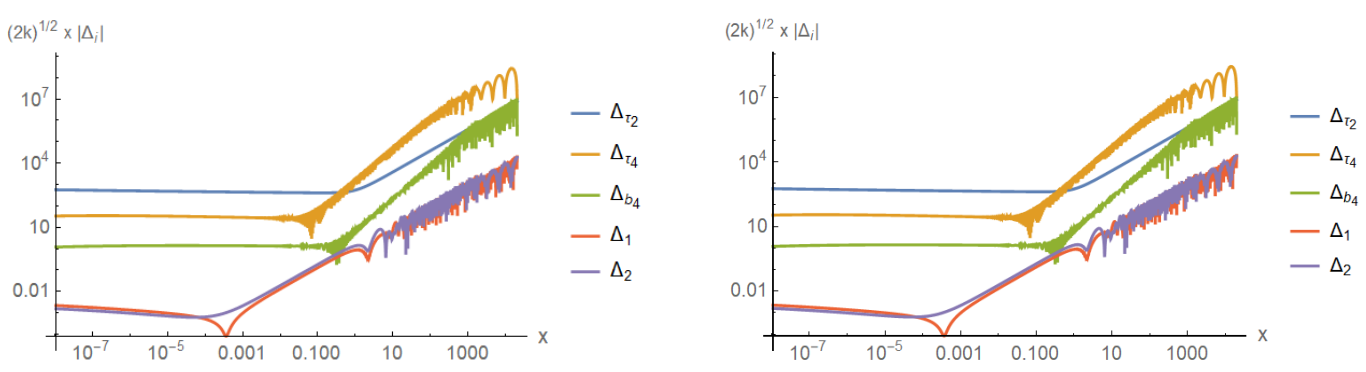

Figure 5.40: The evolution of the scalar perturbations defined in 5.7.19) with (left figure) and without (right figure) the inclusion of the metric perturbations $\alpha, \beta$ found through (5.5.10), (5.5.11) for the example 3 plotted against $x=k / a H$. As we can see, the metric perturbations have a negligible effect on the evolution and can safely be set to zero.

and remove $a$ and $k$ from the equations of motion, using $k=x a H$. The initial conditions for the scalar field perturbations have the multi-field inflation form [26]:

$$
\Delta^{a}\left(x_{i n}\right)=\frac{-x H \frac{d \phi^{a}}{d x}}{\sqrt{2 k} \dot{\varphi}}, \quad \frac{d \Delta^{a}}{d x}\left(x_{i n}\right)=\frac{-i x H \frac{d \phi^{a}}{d x}}{\sqrt{2 k \dot{\varphi}}}
$$

where $\Delta^{a}=\left(\Delta_{\tau_{2}}, \Delta_{\tau_{4}}, \Delta_{b}\right)$ while the scalar gauge field perturbations have the standard Bunch-Davies initial conditions:

$$
\Delta_{i}\left(x_{i n}\right)=\frac{1}{\sqrt{2 k}}, \quad \frac{d \Delta_{i}}{d x}\left(x_{i n}\right)=\frac{i}{\sqrt{2 k}}
$$

where $\Delta_{i}=\left(\Delta_{1}, \Delta_{2}\right), k=k_{*}=0.05 \mathrm{Mpc}^{-1}$ is the pivot scale and as before $x_{i n}=2 \times 10^{4}$. As we did for example 2, we first demonstrate graphically that the inclusion of the metric perturbations has no effect on the evolution of the dynamical perturbations as can be seen in FIG. 5.40 ,

The equations of motion (with the metric perturbations set to zero) for the five remaining scalars without the metric perturbations are included in Appendix C. We again define the (tangential) multi-field scalar perturbation

$$
\delta_{s}=\frac{\gamma_{a b} \dot{\phi}^{a} \delta \phi^{b}}{\dot{\varphi}}=\frac{\Delta_{s}}{a}=\frac{\gamma_{a b} \dot{\phi}^{a} \Delta^{b}}{a \dot{\varphi}}
$$

with $\Delta^{a}=\left(\Delta_{\tau_{2}}, \Delta_{\tau_{4}}, \Delta_{b}\right)$. We plot clearly its evolution during slow-roll in FIG. 5.41 . As in example 2, the gauge perturbations are vastly sub-dominant to the inflationary perturbation, and during slow-roll of the background fields, $\Delta_{s}$ freezes out $(x<1)$ with $\left|\sqrt{2 k} x \Delta_{s}\right| \sim \mathcal{O}(1)$ suggesting the gauge field has a negligible effect on the scalar 


\section{PRIMORDIAL GRAVITATIONAL WAVES IN STRING INFLATION}

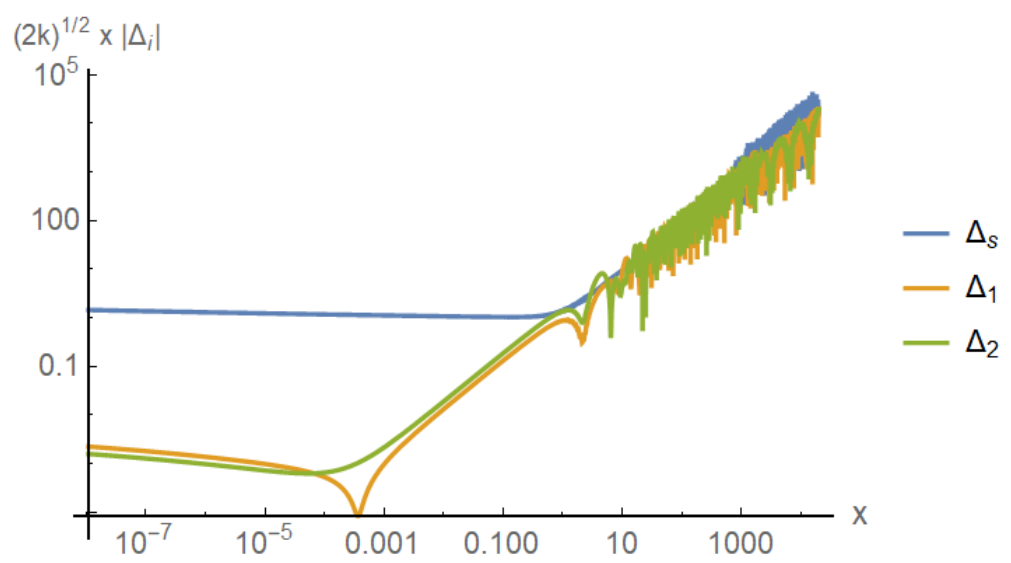

Figure 5.41: The evolution of the scalar perturbations defined in 5.7 .19 and 5.7 .22 for example 3 plotted against $x=k / a H$. After horizon-crossing, $x<1$, the scalar perturbation associated with the scalar fields, $\Delta_{s}$ is consistently much larger than the scalars associated with the gauge field, $\Delta_{1}, \Delta_{2}$.
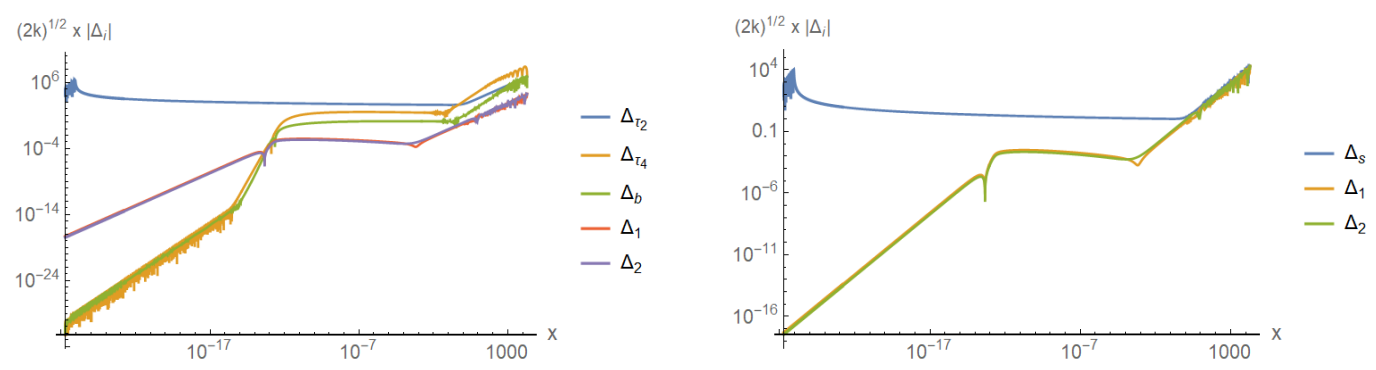

Figure 5.42: The full evolution of the scalar perturbations defined in $(5.7 .19)$ and $(5.7 .22)$ plotted against $x=k / a H$ for example 3 including the decays of all the perturbations which take place concurrently with the decays of the corresponding background fields.

power spectrum. In this way we seek to justify our assumption that the scalar power spectrum is well-approximated by $\frac{H^{2}}{8 \pi^{2} \epsilon_{\varphi}}$.

Finally in FIG. 5.42, we plot the full evolution of all the perturbations including their decays, which occur concurrently to the decays of their background fields. We have shown that the metric scalar perturbations' contribution is negligible and that the scalar perturbations to the gauge field are very small relative to the tangential inflationary perturbation. Our assumption is that the scalar power spectrum can be split into a contribution from the gauge field and the standard inflationary part. With the gauge field perturbations sub-dominant, this means that the power spectrum is well-approximated by $\mathcal{P}_{s}=\frac{H^{2}}{8 \pi^{2} \epsilon_{\varphi}}$ and that therefore the inflationary predictions of both $\mathcal{P}_{s}$ and $n_{s}$ are not spoiled by the presence of the spectator gauge field. 


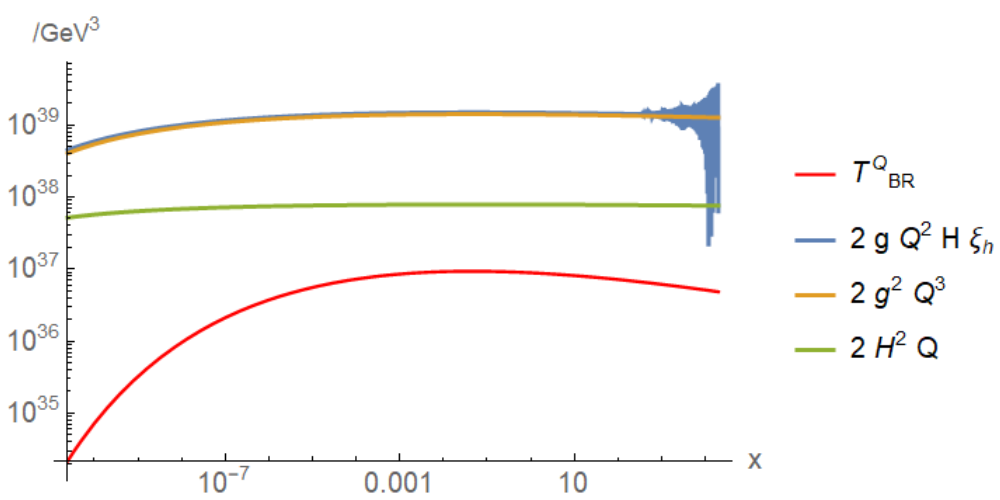

Figure 5.43: The evolution of the leading terms in the equation of motion for $Q(5.6 .4$, $2 \mathrm{~g} Q^{2} H \xi_{h}$ (blue), $2 \mathrm{~g}^{2} Q^{3}$ (orange), $2 H^{2} Q$ (green) and the backreaction induced by the gauge tensor perturbation, $\mathcal{T}_{B R}^{Q}$ (red) for example 3 plotted against $x=k / a H$.

\subsubsection{Backreaction}

Finally we demonstrate that the backreaction for example 3 is under control. The backreaction of the tensor perturbations onto the gauge field equation of motion, $\mathcal{T}_{B R}^{Q}$, as well as the energy density in the tensor modes, $\rho_{t_{R}}$, is given again by (5.6.4)-55.6.8) Since these equations are written in terms of $\xi_{h}$, they are quite general. It is shown in FIGs 5.435 .44 that the backreaction is well under control in this example.

We have therefore shown that if we can include an extremely large magnetisation of the branes $m=10^{4}$ in order to sustain the gauge field, while having a gauge field of extremely large degree $\left(N \sim 10^{5}\right)$, it is possible to greatly enhance the spectrum of Kähler moduli inflation without incurring excessive backreaction. The viability of this model is discussed in the next section.

\subsection{Discussion of viability of example 3}

As we have discussed, designing field theory models with the correct properties to realise satisfactory (S)CNI seems rather challenging [107, 122]. Supergravity and string theory models of inflation offer a natural framework where one can study possible embeddings of SCNI that may overcome some of these challenges. A first look at the supergravity embedding of CNI was taken in [104], while in string theory, a proposal to embed SCNI was first made in [4]. 


\section{PRIMORDIAL GRAVITATIONAL WAVES IN STRING INFLATION}

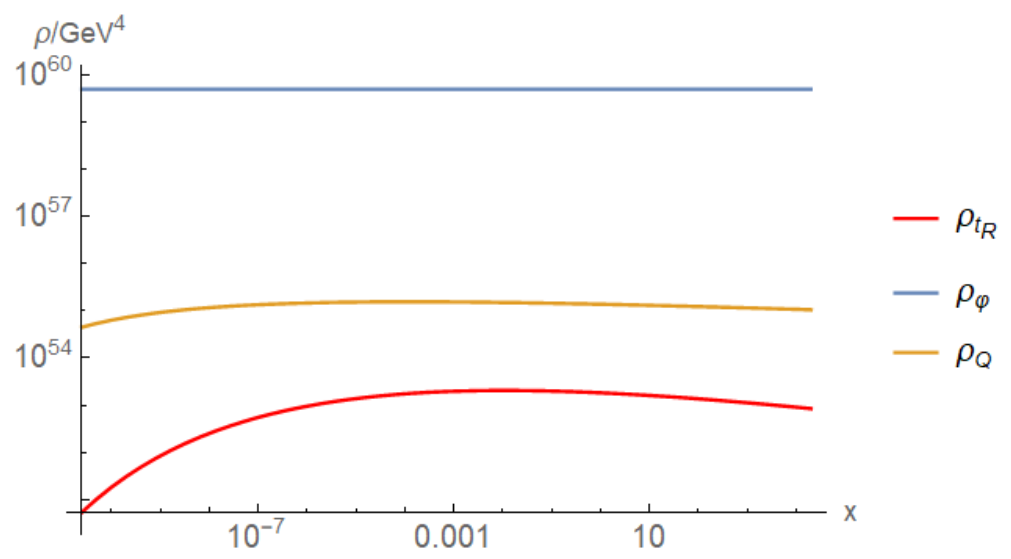

Figure 5.44: The evolution of the energy densities in $\phi^{a}, \rho_{\varphi} \simeq V$ (blue); in $Q, \rho_{Q}=$ $\frac{3}{2} f\left[(H Q+\dot{Q})^{2}+\mathrm{g}^{2} Q^{4}\right]$ (orange); and in $t_{R}, 5.6 .8$ (red), for the example 3 plotted against $x=k / a H$.

In example 1 and 2, we attempted to use Kähler inflation in its original form, but without introducing a large phenomenological constant, $c_{2}$, this cannot be used to realise SCNI. Thus, similarly to [4], for example 3, we introduced as a spectator sector magnetised D7-branes, multiply wrapping four cycles, parametrised by a Kähler modulus $T_{4}$ and a $C_{2}$ axion, $b$, coupled to the non-abelian gauge fields living on the magnetised multiply-wrapped D7-branes. The parameters available in this set-up are the magnetic field on the spectator D7-branes, $m$, its wrapping number, $n$, and the degree of the gauge group, $N$. From the cosmological point of view, we were interested in realising successfully three specific goals: successful inflation, a sustained gauge field to successfully source gravitational waves at the observable level, $r \sim 10^{-3}$, and a controllable backreaction from the tensor gauge fluctuations. We therefore fixed the parameters $(m, N, n)$ in order to realise these goals.

We were then left with a general multi-field system described from the field theory point of view by the action (5.2.1), where the field space metric $\gamma_{a b}$ is given by 535.7 .9 , the scalar potential is given by (5.7.6), the couplings $f\left(\phi^{a}\right), h\left(\phi^{a}\right)$ are given by $f=\tau_{4}$, $h=m b$, the gauge field $F=d A-\mathrm{g} A \wedge A$ and we defined the "effective gauge coupling" as $\mathrm{g}=1 / \sqrt{n N / 2}$

A successful period of inflation and large enough enhancement of the gravitational wave spectrum impose two conditions on the parameters, which can be fixed by two of

\footnotetext{
${ }^{33}$ Where we are working in the large volume limit.
} 
the three parameters in the model, namely $m$ and $n$. However, keeping the backreaction under control imposes a third condition, which then fixes the third parameter, $N$.

We show in FIG. 5.18 the enhancement of $r$ for different values of $g$ as a function of the parameter $\xi_{Q}$, while in FIG. 5.19, we see the backreaction estimate for three values of $\mathrm{g}$ as a function of $\xi_{Q}$. As we have discussed, for a fixed value of the effective coupling, the larger $\xi_{Q}$, the larger the enhancement, but also the backreaction (see also FIGs 5.25 and 5.43). We show the dependence of $\mathrm{g}$ as a function of $N$ in figure $5.38-$ low g requires very high $N$.

Since the spectator axion is not canonically normalised, and its kinetic term depends on $\tau_{4}$, a dynamical field, we cannot define a decay "constant" in the usual way. However, we can define an "instantaneous decay constant", as in [116, which we show in FIG. 5.37 in Planck units and turns out to be sub-Planckian as expected, $f_{c} \sim 10^{-3} M_{\mathrm{pl}}$. On the other hand, because the host inflationary model is Kähler inflation, the field excursion is sub-Planckian as well, $\Delta \phi \sim 0.2 M_{\mathrm{pl}}$ and the large-potential bound on $r$ is avoided, since the enhanced tensor spectrum gives $r=2.29 \times 10^{-3}$. The actual gauge kinetic coupling is field dependent and set by $\tau_{4}$. At the minimum of $\tau_{4}$, this is given by $g^{2}=1 /\left\langle\tau_{4}\right\rangle \sim \frac{1}{15}$, and remember that it is displaced only a small distance from its minimum, so an instantaneous gauge coupling defined at time $t$ differs little from its value at the minimum.

An important feature of the model from the cosmological point of view, is that it realises a very mild version of the proposal in [101] to enhance the gravitational wave spectrum. Indeed, the model requires a specific hierarchy in the slow-roll parameters, namely $\epsilon \sim \epsilon_{B} \gg \epsilon_{\varphi}$ in order to ensure such a large enhancement. To see why this is we plot in FIG. 5.45 how $\epsilon_{B}$ is affected by reducing the value of $\mathrm{g}$. Despite the fact that, naively, it seems $\epsilon_{B}=f \frac{\mathrm{g}^{2} Q^{4}}{M_{\mathrm{pl}}^{2} H^{2}} \propto \mathrm{g}^{2}$, in fact if we plot it against $\xi_{Q}$, we see that $\epsilon_{B}=f \frac{H^{2} \xi_{Q}^{4}}{\mathrm{~g}^{2} M_{\mathrm{pl}}^{2}}$. This fact has the important consequence that if we wish to have a model that produces a relatively large value of $\xi_{Q}\left(e . g\right.$. in our case $\left.\xi_{Q} \sim 4\right)$ so that we can get a large enough enhancement to the tensor-to-scalar (see FIG. 5.18) to achieve $r \sim 10^{-3}$, whilst having a small value of $\mathrm{g}$ so as not to produce too large a backreaction (see FIG. 5.19), it is unavoidable that $\epsilon \sim \epsilon_{B}>\epsilon_{\varphi}$. This point is further emphasised in FIG. 5.46 where we plot the enhancement factor against the ratio of $\epsilon_{B}$ to $\epsilon_{\varphi}$ for two values of $g$. In [3] (and equivalent chapter 3), their inflationary model already predicts a background tensor-to-scalar ratio of $r_{b} \sim 10^{-3}$; they have a much larger value of $\epsilon_{\varphi}$ than 


\section{PRIMORDIAL GRAVITATIONAL WAVES IN STRING INFLATION}

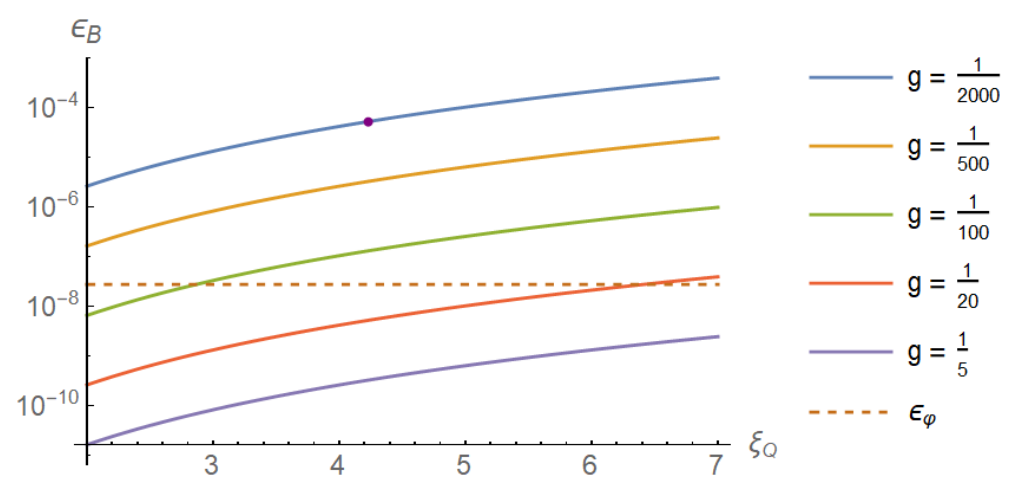

Figure 5.45: The value of $\epsilon_{B}=f \frac{\mathrm{g}^{2} Q^{4}}{M_{\mathrm{pl}}^{2} H^{2}}=f \frac{H^{2} \xi_{Q}^{4}}{\mathrm{~g}^{2} M_{\mathrm{pl}}^{2}}$ plotted against $\xi_{Q}=\frac{\mathrm{g} Q}{H}$. If one wishes to achieve the same value of $\xi_{Q}$ after reducing $g$ to mitigate the backreaction, $\epsilon_{B}$ will be larger. The dashed line shows the value of $\epsilon_{\varphi} 60$ e-folds before the end of inflation for example 3. Similarly the dot corresponds to the values for $\xi_{Q}$ and $\epsilon_{B}$ in example 360 e-folds before the end of inflation.

in our example; and their model predicts a considerably smaller enhancement factor $\frac{r_{s}+r_{b}}{r_{b}} \sim 20$ (compared to $\frac{r_{s}+r_{b}}{r_{b}} \sim 5000$ in example 3). To achieve this enhancement, they only require $\xi_{Q} \sim 3.4$ compared to our $\xi_{Q} \sim 4.2$ and as discussed the backreaction scales exponentially in $\xi_{Q}$. For these reasons, in [3], they are able to choose a relatively large value for $\mathrm{g}=1.11 \times 10^{-2}$ compared to our $\mathrm{g}=\frac{1}{2000}$, and therefore they are able to satisfy $\epsilon \sim \epsilon_{\varphi} \gg \epsilon_{B}$. In this regard our example has more in common with [101] where they demonstrate that one can achieve exceptionally large enhancements whilst controlling the backreaction if $\mathrm{g}$ is taken to be small enough and it is allowed that $\epsilon \sim \epsilon_{B}$. In the specific example in [101], they achieve an enhancement of $\frac{r_{s}+r_{b}}{r_{b}} \sim 10^{68}$ and have $\epsilon \sim \epsilon_{B} \sim 10^{-2}$.

The fact that we have $\epsilon \sim \epsilon_{B}$ may be seen as a problem. However as in [101, we assume that scalar power spectrum, $\mathcal{P}_{s}$, is dominated by the scalar field perturbations and the gauge field perturbations therefore contribute negligibly to $\mathcal{P}_{s}$. This is a crucial assumption of this work and is of course also valid for example 2 as well as example 3. This in turn allows us to assume that the scalar power spectrum is well approximated by $\mathcal{P}_{s}=\frac{H^{2}}{8 \pi^{2} \epsilon_{\varphi}}$ and consequently that $n_{s}=1-2 \epsilon-\eta_{\varphi}$. This of course ensures that the important inflationary predictions of Kähler inflation are not spoiled. In FIGs 5.40 5.42 , we show that the scalar field perturbations are much larger in magnitude than the scalar perturbations of the gauge field.

Comparing to the phenomenological models in the literature, we have seen that a 

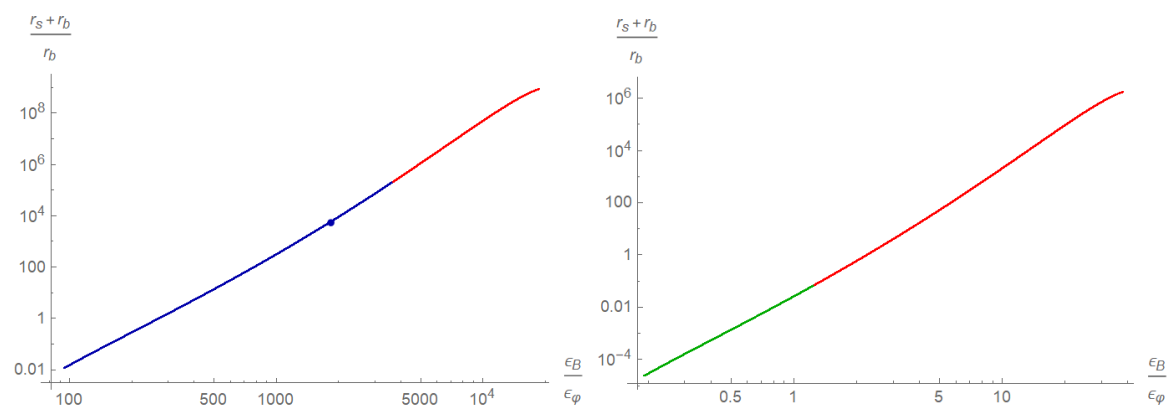

Figure 5.46: Two plots showing how the enhancement factor, $\frac{r_{s}+r_{b}}{r_{b}}$, varies relative to the ratio of $\frac{\epsilon_{B}}{\epsilon_{\varphi}}$ where $\epsilon_{\varphi}=2.80 \times 10^{-8}$, the value it takes 60 e-folds before the end of inflation in example 3. The left plot corresponds to $g=\frac{1}{2000}$, the value of $g$ used in example 3 and the right plot shows $g=\frac{1}{100}$. The dot corresponds to the values in example 3 . The red part of the lines corresponds to parameter space where the backreaction is too large. It can be seen immediately that with $\mathrm{g}=\frac{1}{100}$, it is impossible to get a substantial enhancement without incurring excessive backreaction. Because of the low value of $\epsilon_{\varphi}$ in Kähler inflation, in order to get a large enhancement with controlled backreaction, we require g small, which leads to a large value of $\epsilon_{B}$ relative to $\epsilon_{\varphi}$.

relatively simple string theory construction has enough parameters to account for the cosmological constraints required to realise our goals. At least one of these parameters can be easily incorporated in field theory models, namely rather than working with $S U(2)$, one can work with $S U(N)$, introducing an effective coupling that can be made small, at the price of a large degree. However it is not clear whether there are field theory equivalents of the magnetic flux and the D7-brane wrapping number.

Let us now discuss in more detail the values of the model parameters $(m, N, n)$. As we saw, for a successful evolution we require a large magnetic flux $m=10^{4}$. Requiring that the gauge field is sustained for enough e-folds to enhance the tensor spectrum then requires that the instantaneous decay constant $f_{c} \sim 10^{-3} M_{\mathrm{pl}}$, which fixes $n=25$. Finally control of the backreaction requires a specific value for the effective decay constant $\mathrm{g} \sim \frac{1}{2000}$, which fixes $N \sim 3 \times 10^{5}$. The winding number is of a similar order to the values used in e.g. [105]. The magnetic flux on the other hand is much larger, and may backreact on the geometry. Moreover, the gauge group degree required is very large $N \sim 10^{5}$. Since $N$ is basically the number of D7-branes, such a large number of them may backreact on the full geometry. In any case, it is not clear that such a large number for $N$ can be realised in any realistic large volume compactification. So we see this as the biggest challenge of the construction. Remember however, that we have 


\section{PRIMORDIAL GRAVITATIONAL WAVES IN STRING INFLATION}

required three very specific cosmological objectives and the large value of $N$ is needed to realise these.

Finally we note that although the tensor spectrum that is produced is fully chiral, this chirality is unlikely to be detectable by cross-correlating $T, E$ and $B$ spectra with future CMB experiments because overall the tensor-to-scalar ratio is about one order of magnitude too small [87]. This leads us nicely into our next section in which we apply this enhancement mechanism to fibre inflation, a model that already predicts an observable tensor-to-scalar ratio, $r \sim 5 \times 10^{-3}$.

\subsection{Fibre inflation (example 4)}

In chapter 4, we considered an example of fibre inflation with an additional spectator modulus, $T_{4}$, and we saw that fibre inflation is capable of producing an observable tensor-to-scalar ratio without the need for an enhancement - it has a high energy potential ( $V$ is about 4 orders of magnitude larger in fibre inflation than Kähler modulus inflation). Although we could work with this model straight away and consider a more phenomenological scenario equivalent to example 2 above for Kähler moduli inflation, we modify the spectator sector as in example 3, coupling again to an $S U(N)$ gauge field. Applying this mechanism to fibre inflation is potentially interesting because, with a small enhancement to the right-handed part of the tensor spectrum, the chirality of this model could potentially be observable (we will see that we can enhance the spectrum up to $r \sim 10^{-2}$ very easily), giving it a distinguishable feature. Because the superpotential terms for $T_{4}$ are assumed to be the same as in example 3 , i.e.

$$
W_{n p}^{(s)}=A_{4} e^{-a_{4} T_{4}}+A e^{-a f_{4}},
$$

and because the $\tau_{4}$ modulus is manifestly chosen to be small relative to $\tau_{1}$ and $\tau_{2}$ so it does not receive a correction from the Kähler potential, we can see that the spectator part of the potential will be the same as it was in example 3 for Kähler moduli inflation. 
The whole potential is as follows

$$
\begin{aligned}
V=\{ & \left.-\frac{3 \alpha \gamma_{3}}{2 a_{3}^{3 / 2}}\left(\ln \left(\frac{2 a_{3} A_{3}}{3 \alpha \gamma_{3} W_{0}} \mathcal{V}\right)\right)^{3 / 2}+\frac{3 \hat{\xi}}{4}\right\} \frac{W_{0}^{2}}{\mathcal{V}^{3}}+\left(\frac{\mathcal{A}}{\tau_{1}^{2}}-\frac{B}{\mathcal{V} \sqrt{\tau_{1}}}+\frac{C \tau_{1}}{\mathcal{V}^{2}}\right) \frac{W_{0}^{2}}{\mathcal{V}^{2}} \\
& +\frac{8 a_{4}^{2} A_{4}^{2} \sqrt{\tau_{4}}}{3 \alpha \gamma_{4} \mathcal{V}} e^{-2 a_{4} \tau_{4}}+\frac{4 W_{0} a_{4} A_{4} \tau_{4}}{\mathcal{V}^{2}} \cos \left(a_{4} b_{4}\right) e^{-a_{4} \tau_{4}} \\
& \frac{8 \tilde{a}^{2} \tilde{A}^{2} \sqrt{\tau_{4}}}{3 \alpha \gamma_{4} \mathcal{V}} e^{-\frac{2 \tilde{a}}{m} \tau_{4}}+\frac{16 \tilde{a} \tilde{A} a_{4} A_{4} \sqrt{\tau_{4}}}{3 \alpha \gamma_{4} \mathcal{V}} e^{-\left(a_{4}+\frac{\tilde{a}}{m}\right) \tau_{4}} \cos \left[a_{4} b_{4}-\tilde{a}\left(b+\frac{b_{4}}{m}\right)\right] \\
& +\frac{4 \tilde{a} \tilde{A} W_{0} \tau_{4}}{\mathcal{V}^{2}} e^{-\frac{\tilde{a}}{m} \tau_{4}} \cos \left[\tilde{a}\left(b+\frac{b_{4}}{m}\right)\right]+\frac{\delta_{u p}}{\mathcal{V}^{4 / 3}}
\end{aligned}
$$

where the first line corresponds to the inflationary part of the potential after substituting for $\tau_{3}$ (which is a stabiliser) as in [50] (equation 3.60) (see chapter 4 for details) with $\mathcal{A}, B, C, a_{3}, A_{3}$ model-dependent constants; $\frac{\delta_{u p}}{v^{4 / 3}}$ is an uplift term used in fibre inflation; and the remaining terms are entirely equivalent to the spectator part of example 3 (replace $\gamma_{4} \rightarrow \lambda_{4}$ ) recalling that we have made the replacement $\tilde{a}=$ anm and $\tilde{A}=\frac{A}{m}$ with $a=\frac{2 \pi}{N}, N$ the degree of the gauge group to which the spectator sector is coupled, $n$ the wrapping number, and $m$ the magnetisation of the branes. In fibre inflation both $\mathcal{V}$ and $\tau_{1}$ contribute to inflation, and here we have added as spectators $\tau_{4}, b_{4}, b$. We will show that, as in example 3 , the $b_{4}$ axion can be safely set to its minimum as it only moves negligibly during inflation. We therefore start with a five-scalar field system $\left(\tau_{1}, \mathcal{V}, \tau_{4}, b_{4}, b\right)$ coupled to the gauge field, $Q$, in the usual way through $f=\tau_{4}$ and $h=M b$. The scalar manifold metric is given by the four-field metric used in chapter 4 with the addition of the term for the $b$ axion $[88,119,120$,

$$
\gamma_{a b}=\left(\begin{array}{ccccc}
\frac{3}{4 \tau_{1}^{2}} & -\frac{1}{2 \tau_{1} \mathcal{V}} & 0 & 0 & 0 \\
-\frac{1}{2 \tau_{1} \mathcal{V}} & \frac{1}{\mathcal{V}^{2}} & -\frac{3 \alpha \gamma_{4} \sqrt{\tau_{4}}}{2 \mathcal{V}^{2}} & 0 & 0 \\
0 & -\frac{3 \alpha \gamma_{4} \sqrt{\tau_{4}}}{2 \mathcal{V}^{2}} & \frac{3 \alpha \gamma_{4}}{4 \mathcal{V} \sqrt{\tau_{4}}} & 0 & 0 \\
0 & 0 & 0 & \frac{3 \alpha \gamma_{4}}{4 \mathcal{V} \sqrt{\tau_{4}}} & 0 \\
0 & 0 & 0 & 0 & \frac{2 g_{s} \sqrt{\tau_{4}}}{\sqrt{\zeta} \mathcal{V}}
\end{array}\right) .
$$




\section{PRIMORDIAL GRAVITATIONAL WAVES IN STRING INFLATION}

\subsubsection{Background evolution}

The background evolution of this system can now be described by (5.2.8)-(5.2.13). We use as parameters:

$$
\begin{aligned}
& \hat{\xi}=\frac{\xi}{g_{s}^{3 / 2}}=4.59, \quad \mathcal{A}=2.9 \times 10^{-3}, \quad B=0.93, \quad C=4.3 \times 10^{-5}, \\
& W_{0}=100, \quad a_{3}=\frac{\pi}{4}, \quad A_{3}=1, \quad \alpha=0.1543, \quad \gamma_{3}=3.055, \\
& \delta_{u p}=0.082, \quad g_{s}=0.3, \quad a_{4}=\frac{2 \pi}{70}, \quad A_{4}=1.5 \times 10^{-4}, \quad \gamma_{4}=0.1 \\
& \tilde{a}=7 a_{4}, \quad \tilde{A}=10^{-2} A_{4}, \quad m=500, \quad \zeta=5, \quad \mathrm{~g}=\frac{1}{50}
\end{aligned}
$$

where the inflationary parameters are taken from example set "SV2" in [50]. As in example 3 , the parameters $a_{4}, A_{4}$ are chosen so that the terms involving the modulus $T_{4}$ are small relative to the inflationary terms and do not spoil the minimum for the $\tau_{1}$ and $\mathcal{V}$, the inflatons. On top of this, $\tilde{A}$ is taken to be small relative to $A_{4}$ so that the minimum for $\tau_{4}$ and $b_{4}$ is dictated by the terms not involving the $C_{2}$ axion as was discussed for example 3. Finally $\tilde{a}$ (as well $A_{4}, a_{4}, \tilde{A}$ ) and $\mathrm{g}$ are chosen to lead to a phenomenologically interesting example where the tensor power spectrum can be enhanced. Notice immediately that $g$ is much larger than in the Kähler moduli inflation examples - this is because we now only require a small enhancement (and hence small $\left.\xi_{Q}\right)$, which means the backreaction is not as difficult to control.

The minimum of this potential is found at

$$
\left\langle\tau_{1}\right\rangle=6.7570, \quad\langle\mathcal{V}\rangle=1408.2, \quad\left\langle\tau_{4}\right\rangle=13.741
$$

We take $\tau_{1}^{i n}=5000$ and $b^{i n}=\frac{0.001 \pi}{a}$ as chosen initial conditions and this leaves $\mathcal{V}$ and $\tau_{4}$ at the new minimum

$$
\mathcal{V}^{\text {in }}=1830, \quad \tau_{4}^{i n}=9.46,
$$

which we use as their initial conditions. The evolution of this system is plotted in FIGs 5.475 .55 . Inflation proceeds normally due to the slow evolution of $\tau_{1}$ and $\mathcal{V}$ plotted in FIGs 5.47 and 5.48 . As in example 2 and 3 , the evolution of the $\tau_{4}$ modulus is trivial (FIG. 5.49) - it goes to its minimum as $b$ does. The axion $b$ (FIG. 5.49) is supported by its coupling to the gauge field - the potential term in its equation of motion is 

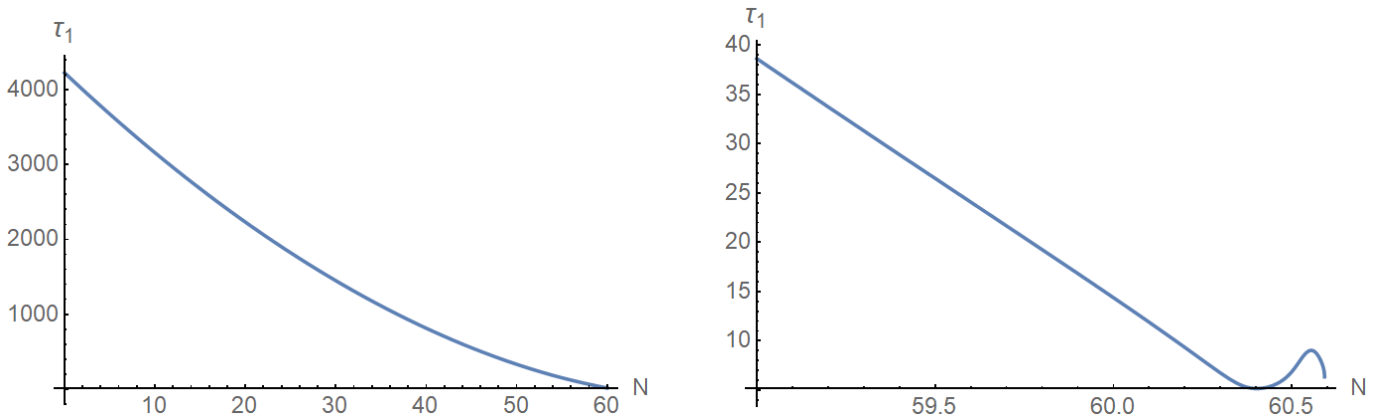

Figure 5.47: The evolution of the inflaton field, $\tau_{1}$, during the last 60 e-folds (left figure) and its decay as inflation ends (right figure) for example 4 plotted against $N$ the e-fold number.
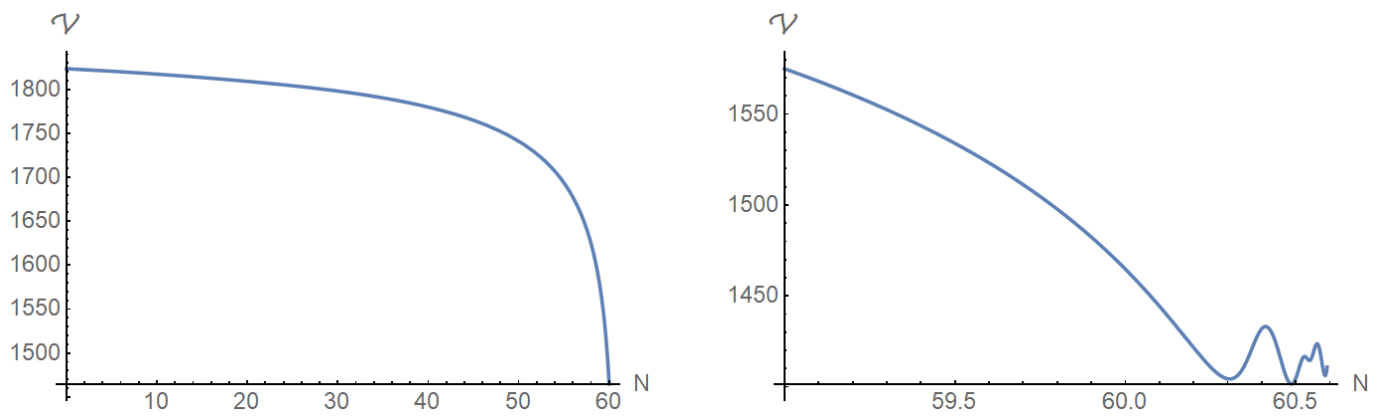

Figure 5.48: The evolution of the volume modulus, $\mathcal{V}$, during the last 60 e-folds (left figure) and its decay as inflation ends (right figure) for example 4 plotted against $N$ the e-fold number.

almost perfectly by the term from the gauge field (FIG. 5.52). In this way, this is another example of the slow-roll solution described before. As the axion is active for many e-folds during inflation, it supports $Q$ (see FIG. 5.50) and notice that the value of $\xi_{Q} \sim 2.5$ is much smaller than in the Kähler moduli case. In 5.53, we see that the gauge field roughly satisfies $\xi_{Q} \sim \xi_{h}$ and that the $b_{4}$ axion only moves negligibly during inflation and can therefore safely be set to its minimum and removed from the evolution as expected. Unlike examples 2 and 3 , we see that in this case, $\epsilon \sim \epsilon_{\varphi}$ - the slow-roll parameter is not dominated by the magnetic part of the gauge field in this example. This is because of the much larger value of $\mathrm{g}$ used here (see FIG. 5.45 in the previous section). 

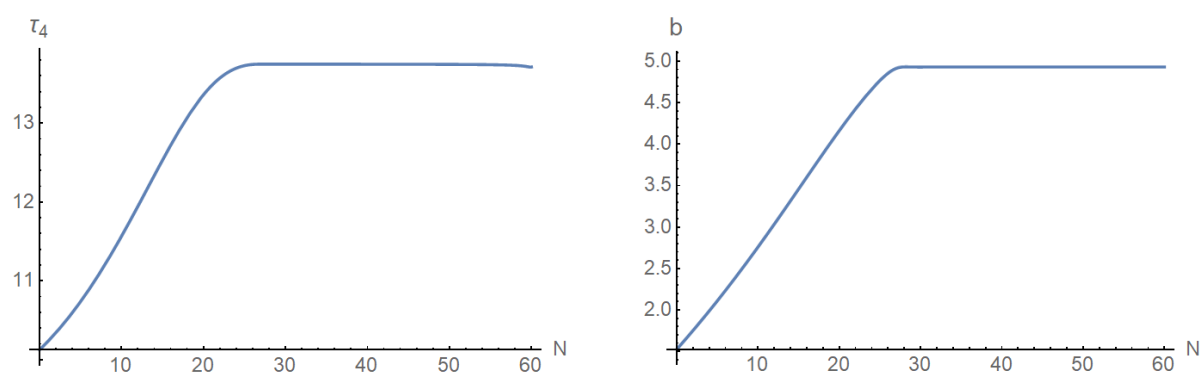

Figure 5.49: The evolution of the spectator fields, $\tau_{4}$ (left figure) and $b$ (right figure), during the last 60 e-folds of inflation for example 4 plotted against $N$ the e-fold number.
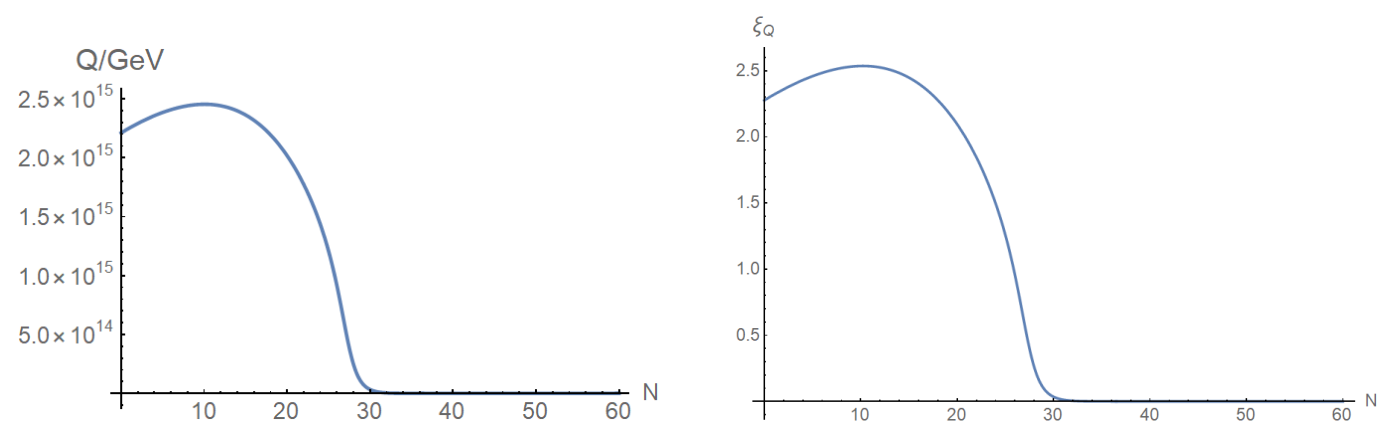

Figure 5.50: The evolution of the gauge field, $Q$ (left figure), and its effective mass, $\xi_{Q}$ (right figure), during the last 60 e-folds of inflation for example 4 plotted against $N$ the e-fold number.
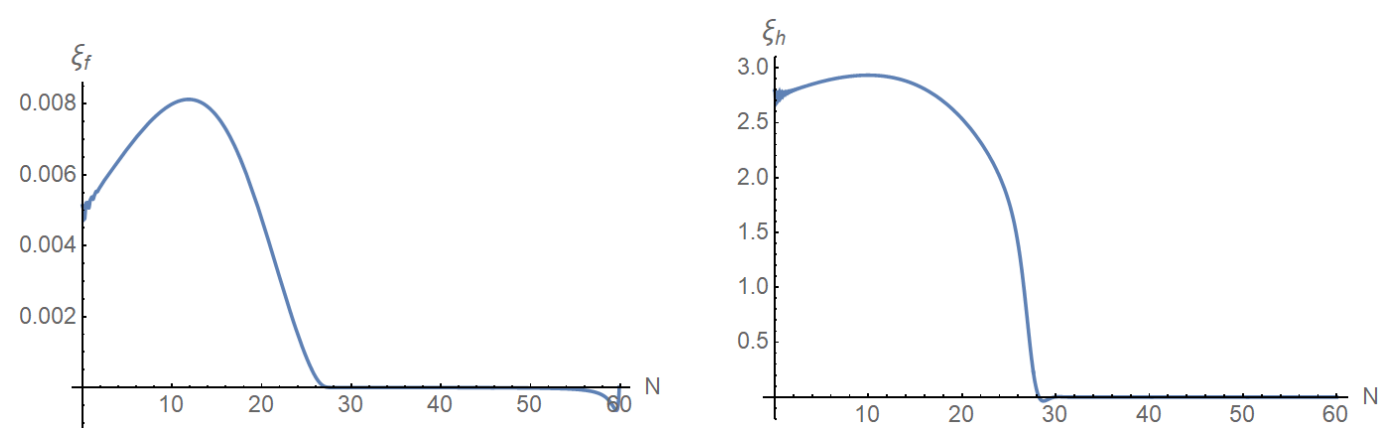

Figure 5.51: The evolution of the effective couplings from the spectator fields to the gauge field, $\xi_{f}=\frac{\dot{f}}{2 H f}$ (left figure) and $\xi_{h}=\frac{\dot{h}}{2 H f}$ (right figure), during the last 60 e-folds of inflation for example 4 plotted against $N$ the e-fold number. 

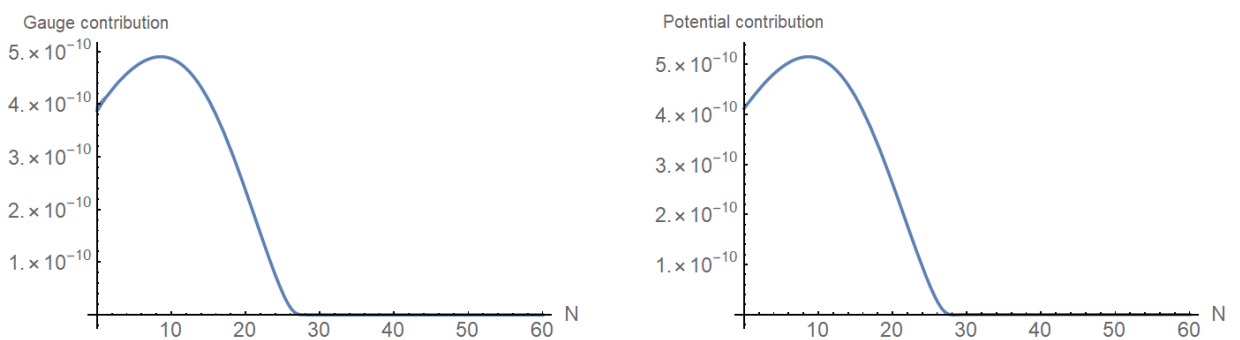

Figure 5.52: The contributions of (left) the term $-3 \mathrm{~g} m \frac{\sqrt{\zeta} \mathcal{V}}{2 g_{s} \sqrt{\tau_{4}}} Q^{2}(H Q+\dot{Q})$ provided by the gauge field, $Q$; and (right) the term $\frac{\sqrt{\zeta} \mathcal{V}}{2 g_{s} \sqrt{\tau_{4}}} \frac{\partial V}{\partial b}$ provided by the potential, to the equation of motion for $b$ given by the form in $(5.2 .12)$ for example 4 . The contribution from the gauge field almost exactly cancels the contribution from the potential leading to a slow-roll evolution for $b$.
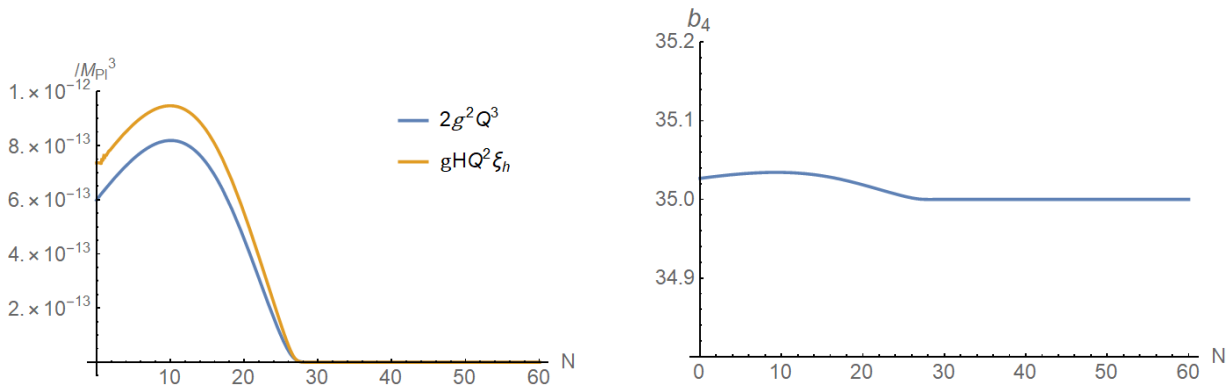

Figure 5.53: Left figure: The two dominant terms in the equation of motion for $Q$, (5.2.13), satisfy $2 \mathrm{~g}^{2} Q^{3} \sim \mathrm{g} H Q^{2} \xi_{h}$ for example 4 . The term introduced through the coupling to the axion almost cancels $2 \mathrm{~g}^{2} Q^{3}$, a term that sends $Q$ to zero. In this way the axiongauge coupling supports the gauge field. Right figure: The evolution of the $b_{4}$ modulus for example 4 - started at its minimum, it moves only negligibly during inflation, and can be safely ignored in the numerics.
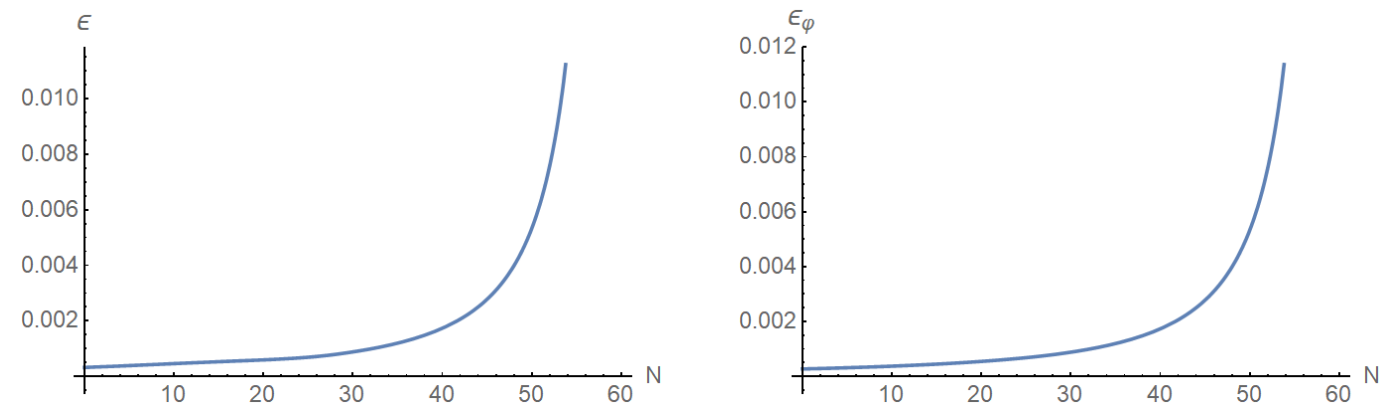

Figure 5.54: The evolution of the slow-roll parameter, $\epsilon=-\frac{\dot{H}}{H^{2}}$ (left figure) and its scalar component $\epsilon_{\varphi}=\gamma_{a b} \frac{\dot{\phi}^{a} \dot{\phi}^{b}}{2 H^{2}}$ (right figure), during the last 60 e-folds of inflation for example 4 plotted against $N$ the e-fold number. 


\section{PRIMORDIAL GRAVITATIONAL WAVES IN STRING INFLATION}
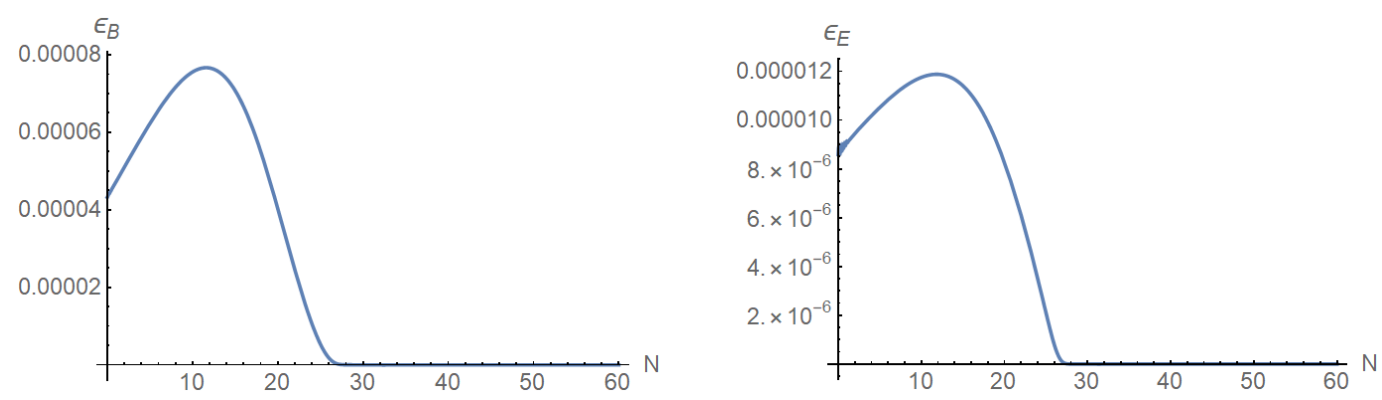

Figure 5.55: The evolution of the magnetic component of slow-roll parameter, $\epsilon_{B}=$ $f \frac{\mathrm{g}^{2} Q^{4}}{M_{\mathrm{pl}}^{2} H^{2}}$ (left figure) and the electric component of the slow-roll parameter $\epsilon_{E}=f \frac{(H Q+\dot{Q})^{2}}{M_{\mathrm{pl}}^{2} H^{2}}$ (right figure), during the last 60 e-folds of inflation for example 4 plotted against $N$ the e-fold number.

The inflationary predictions of this model (using as before $\mathcal{P}_{s}=\frac{H^{2}}{8 \pi^{2} \epsilon_{\varphi}}, n_{s}=1-$ $\left.2 \epsilon-\eta_{\varphi}, r=16 \epsilon_{\varphi}\right) 50$ e-folds before the end of inflation

$$
\begin{aligned}
& \epsilon_{\varphi}=3.73 \times 10^{-4}, \quad n_{s}=0.964, \quad r_{b}=5.97 \times 10^{-3}, \\
& V_{i n f}^{1 / 4}=9.03 \times 10^{15} \mathrm{GeV}, \quad \Delta \varphi=4.55 M_{\mathrm{pl}} .
\end{aligned}
$$

The much higher energy scale of fibre inflation (as well as much larger field displacement) allows for a much greater value of the tensor-to-scalar ratio. We wish to enhance this but must be careful that the enhancement does not take $r$ above the observational bound $r \lesssim 0.07$ set by Planck [37]. The parameters we use of $\tilde{a}=a m n=\frac{2 \pi}{10}$, with $a=\frac{2 \pi}{N}$ and $m=500$ as well as $\mathrm{g}=\frac{1}{\sqrt{N n / 2}}=\frac{1}{50}$. Satisfying these requirements simultaneously leaves us with a wrapping number of $n=1$ and a gauge group degree of $N=5000$.

\subsubsection{Tensors}

The tensor perturbations are found in exactly the same way as 5.4 and the analytic estimate is again identical to that found there. Using the analytic estimate, we get the sourced right-helicity tensor-to-scalar ratio as

$$
r^{s, R}=4.59 \times 10^{-2} .
$$




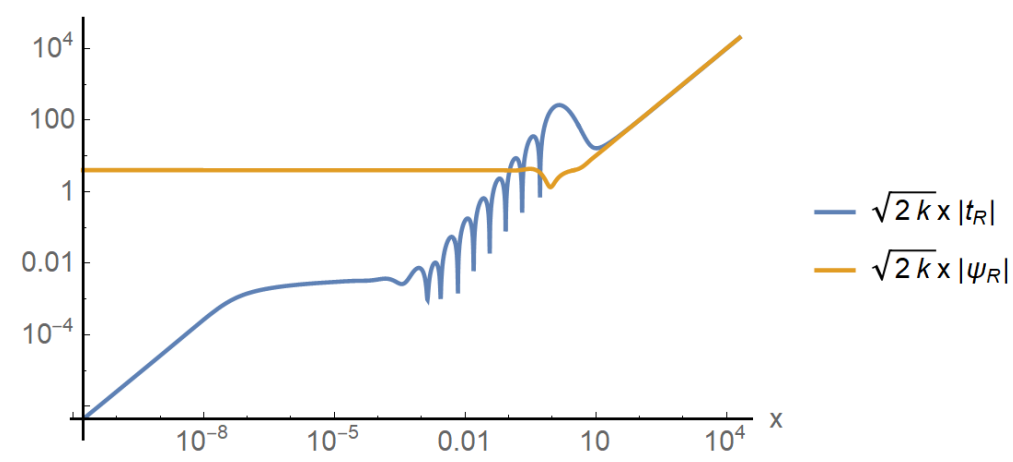

Figure 5.56: The evolution of the (right-helicity) tensor modes for the gauge field, $t_{R}$, and the gravity sector, $\psi_{R}$ for example 4 plotted against $x=k / a H$.

The non-sourced right and left-helicities both contribute $\frac{r_{b}}{2}=2.98 \times 10^{-3}$ meaning our total estimate for the tensor-to-scalar ratio is

$$
r=5.18 \times 10^{-2}
$$

corresponding to an enhancement of roughly an order of magnitude. This spectrum is highly chiral $-94.2 \%$ of it is from the right-helicity mode.

The full equations of motion for the tensors are written in Appendix C. We evolve these with the usual initial conditions $\psi_{R}\left(x_{i n}\right)=t_{R}\left(x_{i n}\right)=\frac{1}{\sqrt{2 k}}$ with $x_{i n}=2 \times 10^{4}$ and $k=k_{*}=0.05 \mathrm{Mpc}^{-1}$. The evolution is plotted in FIG. 5.56 .

We see the enhancement to the right-helicity mode of the gravity sector is considerably smaller than in our Kähler moduli inflation examples (see e.g. FIG. 5.39) with $\left|\sqrt{2 k} x \psi_{R}\right| \rightarrow \sim 4$. Since the enhancement is only to the right-helicity mode, we must add to this the background left-helicity contribution to the tensor spectrum to get the full tensor-to-scalar ratio, i.e. ${ }^{34}$

$$
\mathcal{P}_{t}=\mathcal{P}_{t}^{R}+\mathcal{P}_{t}^{L} \quad \text { with } \quad \mathcal{P}_{t}^{R}=\left.\left|\sqrt{2 k} x \psi_{R}\right|^{2} \frac{H^{2}}{\pi^{2}}\right|_{x<1}, \quad \mathcal{P}_{t}^{R}=\frac{H^{2}}{\pi^{2}}
$$

Using this method with the numerical solution and taking the freeze-out $(x<1)$ value of $\left|\sqrt{2 k} x \psi_{R}\right|^{2}=15.9$, we get

$$
r_{b}=5.97 \times 10^{-3} \rightarrow r=4.87 \times 10^{-2},
$$

\footnotetext{
${ }^{34}$ We did not need to be this careful when considering the power spectra for the Kähler moduli inflation examples because the left-helicity mode was completely negligible.
} 


\section{PRIMORDIAL GRAVITATIONAL WAVES IN STRING INFLATION}

an enhancement of $\sim 8$. The spectrum is highly chiral - approximately $94.1 \%$ of it comes from the right-helicity mode, $\psi_{R}$. Given that this spectrum is an order of magnitude larger than that predicted in example 2 and 3 , it is possible that this righthandedness could potentially be detectable by analysis of cross-correlation of CMB polarisations [87, giving this model a distinguishable feature.

\subsubsection{Scalars}

The scalar perturbations are again set up exactly as in example 2 and 3, except we now have one extra:

$$
\begin{aligned}
& \phi^{a}=\phi^{a}(t)+\delta \phi^{a}\left(t, x^{i}\right) \\
& A_{0}^{A}=a(t) \partial_{A} Y\left(t, x^{i}\right) \\
& A_{i}^{A}=a(t)\left[\left(Q(t)+\delta Q\left(t, x^{i}\right)\right) \delta_{A i}+\partial_{i} \partial_{A} M\left(t, x^{i}\right)\right] .
\end{aligned}
$$

with four-field system $\phi^{a}=\left(\tau_{1}, \mathcal{V}, \tau_{4}, b\right)$. We use again $x=k / a H$ and comoving perturbations are defined

$$
\begin{array}{ll}
\delta \tau_{1}=\frac{\Delta_{\tau_{1}}}{a}, & \delta \mathcal{V}=\frac{\Delta_{\mathcal{V}}}{a}, \quad \delta \tau_{4}=\frac{\Delta_{\tau_{4}}}{a}, \quad \delta b=\frac{\Delta_{b}}{a} \\
\delta Q=\frac{\Delta_{1}}{\sqrt{2} a}, & M=\frac{a \mathrm{~g} Q \Delta_{1}+\sqrt{k^{2}+2 a^{2} \mathrm{~g}^{2} Q^{2}} \Delta_{2}}{\sqrt{2} \mathrm{~g} a^{2} k^{2} Q} .
\end{array}
$$

Once again the initial conditions for the scalar field perturbations have the multi-field inflation form [26]:

$$
\Delta^{a}\left(x_{i n}\right)=\frac{-x H \frac{d \phi^{a}}{d x}}{\sqrt{2 k} \dot{\varphi}}, \quad \frac{d \Delta^{a}}{d x}\left(x_{i n}\right)=\frac{-i x H \frac{d \phi^{a}}{d x}}{\sqrt{2 k} \dot{\varphi}}
$$

where $\Delta^{a}=\left(\Delta_{\tau_{1}}, \Delta_{\mathcal{V}}, \Delta_{\tau_{4}}, \Delta_{b}\right)$ while the scalar gauge field perturbations have the standard Bunch-Davies initial conditions:

$$
\Delta_{i}\left(x_{i n}\right)=\frac{1}{\sqrt{2 k}}, \quad \frac{d \Delta_{i}}{d x}\left(x_{i n}\right)=\frac{i}{\sqrt{2 k}}
$$

where $\Delta_{i}=\left(\Delta_{1}, \Delta_{2}\right), k=k_{*}=0.05 \mathrm{Mpc}^{-1}$ is the pivot scale and as before $x_{i n}=2 \times 10^{4}$ with $x=1$ taken to be 50 e-folds before the end of inflation. 


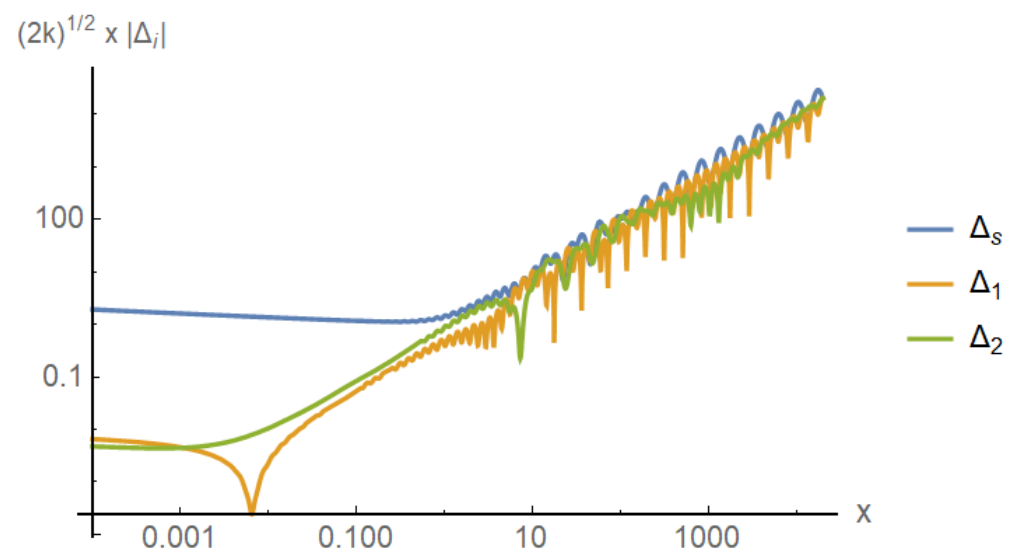

Figure 5.57: The evolution of the scalar perturbations defined in 5.9 .13$)$ and 5.9 .16 for example 4 plotted against $x=k / a H$. After horizon-crossing, $x<1$, the scalar perturbation associated with the scalar fields, $\Delta_{s}$ is consistently much larger than the scalars associated with the gauge field, $\Delta_{1}, \Delta_{2}$.
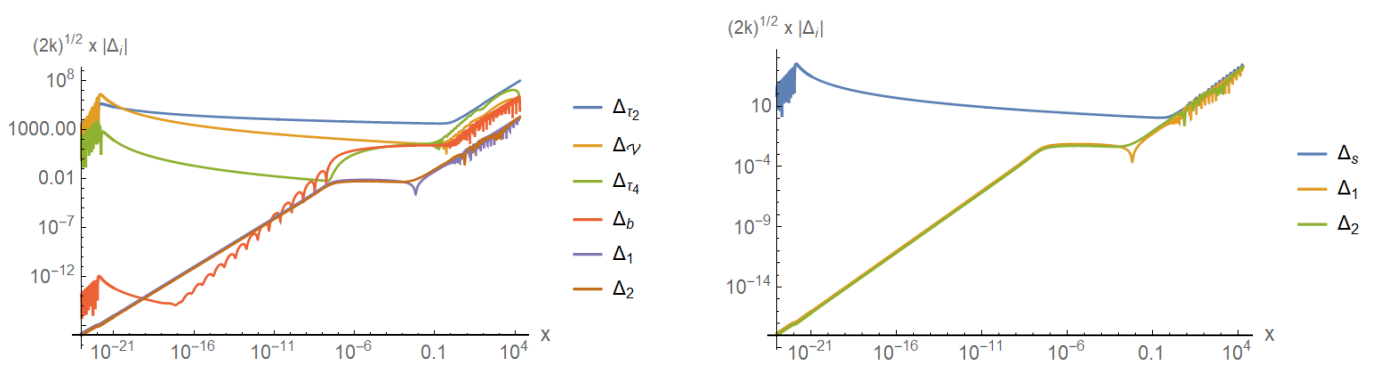

Figure 5.58: The full evolution of the scalar perturbations defined in (5.9.13) and (5.9.16) plotted against $x=k / a H$ for example 4 including the decays of all the perturbations which take place concurrently with the decays of the corresponding background fields.

The equations of motion for the six remaining scalars without the metric perturbations and after solving for $Y$ are included in Appendix C. Using the multi-field scalar perturbation

$$
\delta_{s}=\frac{\gamma_{a b} \dot{\phi}^{a} \delta \phi^{b}}{\dot{\varphi}}=\frac{\Delta_{s}}{a}=\frac{\gamma_{a b} \dot{\phi}^{a} \Delta^{b}}{a \dot{\varphi}}
$$

with $\Delta^{a}=\left(\Delta_{\tau_{1}}, \Delta_{\mathcal{V}}, \Delta_{\tau_{4}}, \Delta_{b}\right)$. We plot clearly its evolution during slow-roll in FIG. 5.57. As in examples 2 and 3, the gauge perturbations are vastly sub-dominant to the inflationary perturbation, and during slow-roll of the background fields, $\Delta_{s}$ freezes out $(x<1)$ with $\left|\sqrt{2 k} x \Delta_{s}\right| \sim \mathcal{O}(1)$ suggesting the gauge field has a negligible effect on the scalar power spectrum.

Finally in FIG. 5.58, we plot the full evolution of all the perturbations including 


\section{PRIMORDIAL GRAVITATIONAL WAVES IN STRING INFLATION}

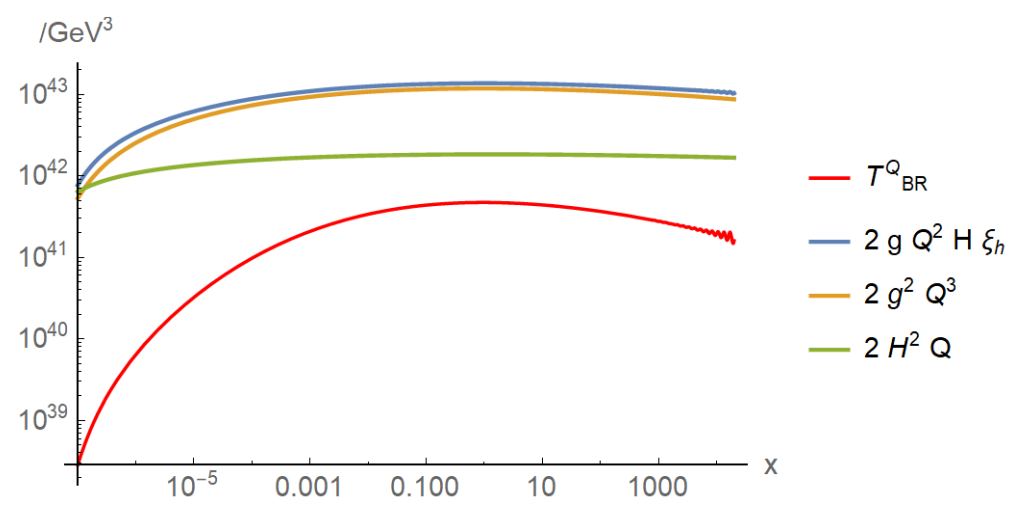

Figure 5.59: The evolution of the leading terms in the equation of motion for $Q$ (5.6.4), $2 \mathrm{~g} Q^{2} H \xi_{h}$ (blue), $2 \mathrm{~g}^{2} Q^{3}$ (orange), $2 H^{2} Q$ (green) and the backreaction induced by the gauge tensor perturbation, $\mathcal{T}_{B R}^{Q}$ (red) for example 4 plotted against $x=k / a H$.

their decays, which occur concurrently to the decays of their background fields. We have shown that the scalar perturbations to the gauge field are very small relative to the tangential inflationary perturbation.

\subsubsection{Backreaction}

The backreaction of the gauge tensor modes onto the equation of motion for $Q, \mathcal{T}_{B R}^{Q}$ is again given by (5.6.4) and is plotted against the leading terms in this equation in FIG. 5.59. The energy density contribution from the tensor modes of the gauge field, $\rho_{t_{R}}$ is shown in FIG. 5.60. Both contributions are vastly sub-dominant relative to the background terms, meaning our approach of choosing an isotropic background form for the gauge field is consistent.

We have therefore shown that it is possible to amplify by roughly an order of magnitude the tensor spectrum of fibre inflation while keeping the backreaction under control. Although fibre inflation naturally predicts an observable tensor-to-scalar ratio $r \sim 5 \times 10^{-3}$, this is interesting because it renders the tensor spectrum highly chiral giving it a distinguishable feature that may be detectable.

\subsection{Summary}

In this chapter we have applied the enhancement mechanism introduced in chapter 3 to various examples of Kähler moduli inflation and fibre inflation where a modulus is 


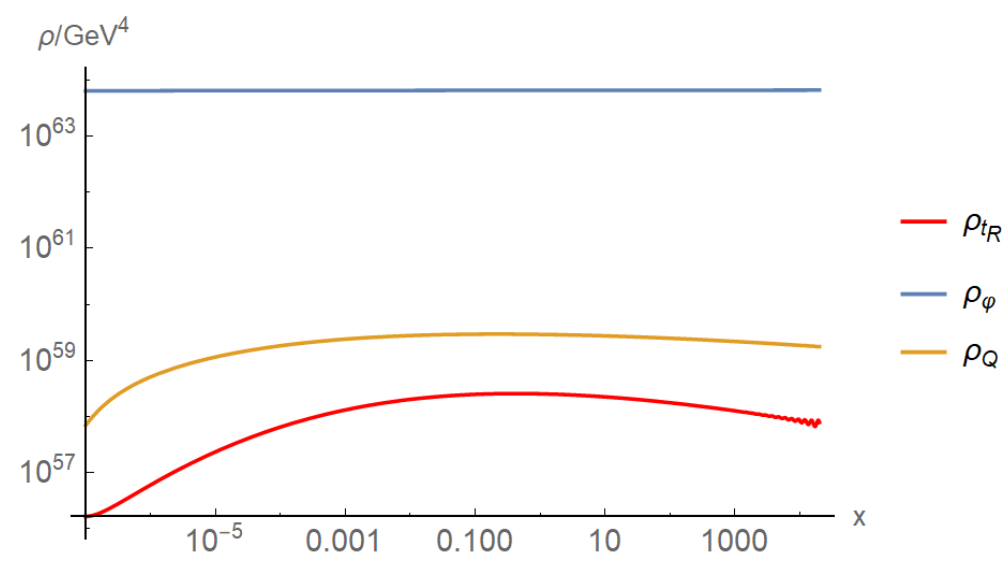

Figure 5.60: The evolution of the energy densities in $\phi^{a}, \rho_{\varphi} \simeq V$ (blue); in $Q, \rho_{Q}=$ $\frac{3}{2} f\left[(H Q+\dot{Q})^{2}+\mathrm{g}^{2} Q^{4}\right]$ (orange); and in $t_{R}, 5.6 .8$ (red), for the example 4 plotted against $x=k / a H$.

coupled to an $S U(N)$ gauge field that can be split into a set of $S U(2)$ gauge fields with common field strength. Redefinitions of the gauge field then allow us to treat this system as one $S U(2)$ field and take the same approach as in chapter 3 . First we applied the mechanism to example 4 of [76] where the axion coupled to the gauge field is the superpartner of the inflaton (and in fact itself is partially responsible for inflation). Due to constraints put on the axion due to its intrinsic role in inflation (in particular the inflaton cannot decay until its axionic partner has decayed), we were unable to find any working examples where the tensor spectrum could be greatly amplified without incurring excessive backreaction.

In order to get a working example, we required a bit more freedom for the fields coupled to the gauge field, and thus we introduced a spectator sector modulus, $T_{4}$, to be coupled to the $S U(N)$ gauge field while the inflationary sector remained unchanged. This extra freedom that comes from not needing the fields coupled to the gauge sector to also satisfy inflationary requirements allows us in particular to reduce the gauge coupling $\mathrm{g}$ that necessarily needs to be small to control the backreaction of the gauge tensor modes (see FIG. 5.19). With this model we are able to amplify the tensor spectrum of Kähler inflation by $\sim 10^{3}$ and uplift it to potentially observable values $\left(r \gtrsim 10^{-3}\right.$ [39 44]). The spectrum produced is fully chiral but this chirality is unlikely to be detectable because the tensor-to-scalar ratio is still roughly an order of magnitude too small [87]. Although this model is extremely positive, it requires the introduction 


\section{PRIMORDIAL GRAVITATIONAL WAVES IN STRING INFLATION}

of a large parameter to induce a large difference in the axion-gauge coupling, $h$, and the four-cycle-gauge coupling, $f$. We parametrise this be defining $f=\frac{c_{1}}{2 \pi} \tau_{4}$ and $h=\frac{c_{2}}{2 \pi} b_{4}$, and in this example we need $c_{2}=450, c_{1}=1$. There is unfortunately no theoretical justification for this and it is expected that $c_{1}=c_{2}=1$ [85]. For this reason we introduce another model, where the axion is not the direct superpartner to four-cycle, $\tau_{4}$, coupled to the gauge field, but is instead an additional axion that arises from a multiply-wrapped D7-brane stack along $\tau_{4}$.

This new model immediately allows for a theoretical justification for the difference between $f=\tau_{4}$ and $h=m b$ where $m$ is the magnetisation and $b$ is the new axion. In this way we can parametrise the necessary requirements of the models through physical means. Another important parameter is g, which now, after successive redefinitions, takes the form $\mathrm{g}=\frac{1}{\sqrt{N n / 2}}$ where $N$ is the gauge degree and $n$ is the wrapping number. Using this model we are able to get a working solution very similar to the previous example, increasing the tensor-to-scalar ratio to observable values, having a completely chiral spectrum - but at too low a tensor-to-scalar ratio for this to be detectable. This model requires that the magnetisation $m=10000$ and the gauge degree $N \sim 10^{5}$ be very large, which may be physically unreasonable. In addition to this, this example and the previous one both require that the slow-roll parameter be dominated by the magnetic component of the gauge field $\epsilon \sim \epsilon_{B}$ (see FIG. 5.45). This introduces the possibility that the scalar power spectrum of the inflationary model is spoiled - we assume therefore that if the gauge field perturbations are very small relative to those of the scalar sector (see FIG. 5.41 that we can assume that $\mathcal{P}_{s}=\frac{H^{2}}{8 \pi^{2} \epsilon_{\varphi}}$. This follows [101] and is the main assumption of this work.

Finally we applied this same model to fibre inflation, a model that already predicts an observable tensor-to-scalar ratio. We were able to uplift the tensor spectrum by about an order of magnitude making it largely chiral - a chirality that for this example may be detectable [87], making this an interesting and distinguishable feature of the model. 


\section{Chapter 6}

\section{Summary and discussions}

In this thesis we have considered two possible implications of including spectator gauge fields in the inflationary action. By "spectator" we mean a field not responsible for driving inflation, but a field that nonetheless can affect inflationary predictions. In chapter 2, we showed how a $U(1)$ gauge field - supported through its coupling to the inflaton - can sustain a global universal anisotropy under the right conditions; and in chapter 5, we showed how the tensor-to-scalar ratio of Kähler modulus models of inflation can be enhanced through a coupling of an axion and an $S U(N)$ gauge field. In this chapter, we review the main results; consider the feasibility of the models and their assumptions; and discuss possibilities for future work.

\subsection{Anisotropic inflation}

In chapter 2 (based on [51]), we consider an extension to the model put forward in [45], which was the first working example of a system capable of sustaining an anisotropy in the metric after a period of inflation. The system is set up with the anisotropic metric

$$
d s^{2}=-d t^{2}+e^{2 \alpha(t)}\left[e^{-4 \sigma(t)} d x^{2}+e^{2 \sigma(t)}\left(d y^{2}+d z^{2}\right)\right]
$$

with $\alpha \sim H t$ and $\sigma \sim \Sigma t$ (with the anisotropy characterised by $\frac{\Sigma}{H}$ ) the isotropic and anisotropic e-folding numbers, respectively; and this anisotropy can be sustained during inflation if a vector field, chosen to be directed along the direction of anisotropy

$$
A^{\mu}=(0, v(t), 0,0)
$$




\section{SUMMARY AND DISCUSSIONS}

is supported through a coupling to the inflaton, $\phi$, appearing in the action as $f^{2}(\phi) F^{2}$. The extension we consider is to generalise this coupling to include derivative terms of the inflaton, i.e. $f=f(\phi, X)$ with $2 X=-\dot{\phi}^{2}$. We then follow [45] exactly and choose a power-law form for the (single-field) inflationary potential and gauge kinetic function

$$
V(\phi)=V_{0} e^{\frac{\lambda}{M_{\mathrm{pl}}} \phi}, \quad f(\phi, X)=M_{\mathrm{pl}}^{4 n} f_{0} \frac{e^{\frac{\rho}{M_{\mathrm{pl}}} \phi}}{(-X)^{n}}
$$

and use as Ansätze that the isotropic and anisotropic e-folding numbers and the inflaton scale logarithmically with time. Using this set-up, we study analytically possible solutions. In particular, we perform a dynamical system analysis to search for stable (attractor) solutions that support a non-negligible anisotropy. We find no stable solutions when the gauge kinetic function is taken to depend only on the derivative term $(\rho=0) f=f(X)$; however, stable, anisotropic solutions exist when $\rho$ is non-zero. The anisotropy can be constrained through $g_{*}$, a parameter that measures the degree to which the scalar power spectrum fails to be isotropic through

$$
\mathcal{P}_{s}(\vec{k})=\mathcal{P}_{s}(k)\left(1+g_{*} \sin ^{2} \theta\right)
$$

with $\theta$ the angle between the mode function wave-vector and the anisotropic direction. For an anisotropic inflationary model that predicts a certain anisotropy, $\frac{\Sigma}{H}$, we can evaluate $g_{*}$ through

$$
g_{*}=24 I N_{e}^{2}
$$

where $N_{e}$ is the number of e-folds after the end of inflation, and $I=3 \epsilon^{-1} \frac{\Sigma}{H}$ [55]. In [53], they constrain $g_{*}=0.002 \pm 0.016^{1}$. This greatly constrains $\frac{\Sigma}{H}$ relative to $\epsilon$. Taking $N_{e}=60$, we get $g_{*}=259200 \epsilon^{-1} \frac{\Sigma}{H}$, which using the constraint tells us

$$
\frac{1}{\epsilon} \frac{\Sigma}{H} \lesssim 6.94 \times 10^{-8}
$$

for positive $\frac{\Sigma}{H}$. We provide one stable example that satisfies this constraint with $\epsilon=$ $5.00 \times 10^{-13}$ and $\frac{\Sigma}{H}=2.78 \times 10^{-26}$. Interestingly the derivative coupling parametrised by $n$ reduces the anisotropy as is shown in FIG. 2.2 while only inducing a small reduction in $\epsilon$. This helps to satisfy constraints on $g_{*}$. All the same as is shown in [70],

\footnotetext{
${ }^{1}$ This is a true intrinsic anisotropy - the foreground di-polar anisotropy (assumed to be our motion relative to the $\mathrm{CMB}$ ) has been removed.
} 
the anisotropic attractor solution described in [45] cannot satisfy the constraint on $g_{*}$ without a fine-tuning of the parameters - and the same seems to be the case here. If we examine the form for the anisotropy given in 2.3.19), we get

$$
\frac{\Sigma}{H}=\epsilon\left(\frac{1}{2}+\frac{\rho}{\lambda}-\epsilon^{-1}-n\right)
$$

The parameters used for 2.3.46 are $n=-100, \lambda=10^{-6}, \rho=2 \times 10^{6}, \epsilon^{-1}=2 \times 10^{12}$ we see that $\rho / \lambda$ and $\epsilon^{-1}$ roughly cancel - and in fact at high precision $\epsilon^{-1}$ cancels with $\frac{1}{2}+\frac{\rho}{\lambda}-n$ to give $\mathcal{O}\left(10^{-13}\right)$. This non-trivial cancellation provides the necessarilysmall value for the anisotropy, but requires some extreme values for the parameters - particularly $\rho$ and $\lambda$. The extremely small value of $\lambda$ means that the inflationary potential would require an extremely large (super-Planckian) excursion for the inflaton.

More generally of course the exponential function chosen for the inflationary potential is very much out of favour with constraints on $n_{s}$ and $r$ [37] and it would be interesting to see if an example of anisotropic inflation could be found with a more realistic potential and after performing a full numerical evolution of the inflationary period with the inflaton-vector field coupling. This is an obvious follow-up to this work. The work shown in this thesis focussed on analytic solutions and therefore may have missed some subtle problems that can arise when a full numerical evolution is attempted.

Finally the perturbations of this system were not considered - it would be interesting to see if the vector field has any effect on the scalar and tensor power spectrum besides the introduction of an anisotropy ${ }^{2}$.

\subsection{Gauge field-induced enhancement of the tensor power spectrum}

The other main topic of this thesis is concerned with the coupling of axions to gauge fields through the action term $\chi F \tilde{F}$ - under the right conditions the axion, $\chi$, can support the gauge field during inflation, and interestingly a gauge field supported during inflation introduces a source term for inflationary gravitational waves. In this way the

\footnotetext{
${ }^{2}$ The tensor spectrum is also expected to receive an anisotropy given by $g_{*}=6 I \epsilon N_{e}^{2}[55$ - slow-roll suppressed when compared to the anisotropy induced in the scalar power spectrum.
} 


\section{SUMMARY AND DISCUSSIONS}

tensor-to-scalar ratio of an inflationary model may be enhanced without increasing the energy scale of the inflationary potential.

In chapter 3, we introduce two models [2, 3] where an axion is coupled to a gauge field that introduces a source term for the tensor modes. We first however demonstrated why a vector field dilutes during inflation - demonstrating that - without a source term of its own, its energy density decreases with the expansion of the universe. We then showed how a vector field introduces a source term into the equation of motion for gravitational waves. In the second half of chapter 3 , we considered a specific example of the model introduced in [3, an extension of chromo-natural inflation where the inflaton and axion sectors are separate. The axion is coupled to an $S U(2)$ gauge field (through the term $\frac{\lambda \chi}{4 f}$ ) which is assumed (at the background level) to be locked into an isotropic configuration $A_{0}=0, A_{i}^{A}=a(t) Q(t) \delta_{i}^{A}$ with $A$ the gauge index. In [3], the authors leave the background inflationary model unspecified whereas here we chose, as a toy model, a potential of $V(\phi)=\Lambda^{4}\left(1-m_{1} \phi e^{-m_{2} \phi}\right)$. We showed how the axion and gauge field can interact in such a way that allows the axion to support the gauge field for a lengthy period of time during inflation if the coupling constant $\frac{\lambda}{f}$ is large enough. We then showed how the tensor perturbations of the gauge field can enhance the righthelicity mode of the gravity sector inducing a large increase in the tensor-to-scalar ratio and rendering the tensor spectrum chiral. We also demonstrated how we can estimate the backreaction of the tensor modes, and that this backreaction is under control in this specific example.

In chapter 4 we introduced Kähler moduli inflation [49] and fibre inflation [50]two models of inflation to which we wished to apply the enhancement mechanism of [3]. We derived the large-volume form for the potential in Kähler moduli inflation and introduced the potential of fibre inflation before studying several examples of Kähler moduli and fibre inflation numerically, all the time bearing in mind that our goal was to enhance the models' tensor spectra through an axion-gauge field coupling. We first show a prototypical example of Kähler inflation (based on the parameter set "example 1 " in [76]) with very large volume $\left(\mathcal{V} \sim 7 \times 10^{6}\right)$ and extremely low energy scale $\left(V^{1 / 4} \sim 6 \times 10^{12} \mathrm{GeV}\right)$ which consequently predicts a very low value for the tensorto-scalar ratio $\left(r \sim 10^{-15}\right)$ and since our goal was to enhance the tensor spectrum of Kähler moduli inflation to observable values $\left(r \sim 10^{-3}\right)$ we see we would have required an enhancement of $\mathcal{O}\left(10^{12}\right)$ with this parameter set. We therefore use as our basic 
inflationary model the parameter set "example 4" in [76] which leads to a much higher scale for the potential $-V^{1 / 4} \sim 8-9 \times 10^{14} \mathrm{GeV}$ and consequently the tensor-to-scalar ratio $r \lesssim 10^{-6}$. The field content of Kähler inflation models naturally allows for the inclusion of axions in the inflationary evolution, and we showed that we can evolve both the superpartner axion of the inflaton (dubbed example 1) and the additional modulus, $T_{4}=\tau_{4}+i b_{4}$, introduced to allow for a bit more freedom in the parameters (example 2 ), while satisfying standard constraints on inflation (particularly on $n_{s}$ ). Much of the chapter was concerned with the simplification of the models - in full Kähler inflation, there are many fields in the evolution including the volume, $\mathcal{V}$, and a stabiliser modulus, $\tau_{3}$ that only evolve a small amount and have little effect on the inflationary predictions of the model, and we demonstrated that they can be safely removed from the evolution, easing the numerics that become convoluted when we involve the gauge field. Finally we showed an example of fibre inflation (using parameter set "SV2" of [50] as a base) to which we add again an additional modulus, $T_{4}$.

Finally in chapter 5 (large parts based on [88]), we couple Kähler moduli inflation and fibre inflation to an $S U(N)$ gauge field. We showed that the $S U(N)$ gauge field can be split into $S U(2)$ sub-groups such that, if assumed to have common field strength, we can recover the system of chapter 3 with effective gauge coupling, $g=\frac{1}{\mathcal{N}}$ where $\mathcal{N}=[N / 2]$. The scalar sector was coupled to the gauge sector through

$$
\mathcal{L} \supset-\frac{f\left(\phi^{a}\right)}{4} F_{\mu \nu}^{A} F^{A \mu \nu}+\frac{h\left(\phi^{a}\right)}{4} \tilde{F}^{A \mu \nu} F_{\mu \nu}^{A}
$$

where $f$ is a function of the 4-cycle saxion $\tau_{a}$ and $h$ a function of its axion partner $b_{a}$. We first considered the case where the scalar sector coupled to the gauge field is taken from the intrinsic field content of Kähler moduli inflation. In order to support the gauge coupling we unfortunately required a hierarchy between the couplings of $f$ to $F^{2}$ and $h$ to $\tilde{F} F$ - we parametrised this by defining $f=\frac{c_{1}}{2 \pi} \tau_{a}$ and $h=\frac{c_{2}}{2 \pi} b_{a}$ where $T^{a}=\tau_{a}+i b_{a}$ is the modulus we choose to couple to the gauge field and left $c_{1}$ and $c_{2}$ as phenomenological constants. We note that however it is expected that $c_{1} \sim c_{2} \sim \mathcal{O}(1)$ [85] whereas we required $c_{1} \ll c_{2}$ in order for the axion to support the gauge field in every example we considered. In example 1 we couple the inflaton, $\tau_{2}$, and its axion, $b_{2}$, to the gauge field. We were unable to find a working example for this scenario - the principal problem being the backreaction of the tensor fluctuations to the gauge field, 


\section{SUMMARY AND DISCUSSIONS}

which was too large. As is plotted in FIGs 5.9 and 5.19, the backreaction onto the background equation of motion of the gauge field $(Q)$ scales exponentially with $\xi_{Q}$, and since we require a large enhancement of the gravity sector $\left(\sim 10^{4}\right)$ to uplift the tensor spectrum of Kähler moduli inflation to observable values, we require a larger value of $\xi_{Q}$ (see FIG. 5.20) than in e.g. [3] and the example we considered in chapter 3. This problem is compounded by the fact that the largest background terms in the equation of motion for $Q, \frac{2}{\mathrm{~g}} H^{3} \xi_{Q}^{3} \sim \frac{2}{\mathrm{~g}} H^{3} \xi_{Q}^{2} \xi_{h}$ (see (5.6.4)), are smaller in lower energy inflation due to the scaling $H^{3}$. This effect can be mitigated by reducing the gauge coupling $\mathrm{g}$, which not only reduces the backreaction (FIGs 5.9 and 5.19 but also increases the terms $\frac{2}{\mathrm{~g}} H^{3} \xi_{Q}^{3} \sim \frac{2}{\mathrm{~g}} H^{3} \xi_{Q}^{2} \xi_{h}$ and actually increases the enhancement to the tensor spectrum (see FIG. 5.18) for a given value of $\xi_{Q}$ meaning we do not require as large a value for $\xi_{Q}$ to achieve our goal of enhancing the tensor spectrum to observable values, and this further reduces the backreaction. For the set-up described in example 1 where the inflaton itself is coupled to the gauge field, we were unable to find a background solution which could sustain $\xi_{Q}$ at a large value (so as to lead to a large enhancement) and for which $g$ was small enough to mitigate the backreaction. In reality the set-up of example 1 was very restrictive - the axion's and inflaton's evolutions were intertwined due to the form of the scalar metric and the potential, in particular the term in $V$

$$
\frac{4 W_{0} a_{2} A_{2} e^{-a_{2} \tau_{2}} \cos \left(a_{2} b_{2}\right) \tau_{2}}{\mathcal{V}^{2}}
$$

is vital to the evolution of both $\tau_{2}$ and $b_{2}-$ while $b_{2}$ is well away from the minimum, this term is positive and $\tau_{2}$ continues in slow-roll until $b_{2}$ nears its minimum. In this way their evolutionary histories are tied and this is shown nicely in FIG. 5.1. The fact that $b_{2}$ is so important to the inflationary evolution means we do not have much freedom in choosing the parameters that govern its evolution $\left(a_{2}, A_{2}, \lambda_{2}\right)$, and this makes it very difficult to find solutions that support large $\xi_{Q}$, particularly when $g$ is reduced.

For this reason, in example 2, we introduce an additional modulus, $T_{4}=\tau_{4}+i b_{4}$, as a spectator sector that is coupled to the $S U(N)$ gauge field instead of the inflaton. This gives us considerably more freedom in choosing the parameters that dictate the evolution of the axion coupled to the gauge field $\left(a_{4}, A_{4}, \lambda_{4}\right)$. These parameters are constrained in that we require that the new terms $\left(T_{4}\right)$ introduced into the potential (5.3.17) are sub-dominant so we do not affect the inflationary evolution. This 
still leaves us with a lot more freedom however, and in this example we are able to reduce $g$ considerably and take $g=\frac{1}{2000}$. We showed, both through an analytic approximation and numerically, that this set-up can lead to a large enhancement of the gravitational wave spectrum, and enhance the tensor spectrum of Kähler moduli inflation from $r \sim 10^{-6} \rightarrow r \sim 10^{-3}$, uplifting the tensor-to-scalar ratio to a value that may be observable at next generation detectors [39 44]. Interestingly this spectrum is almost perfectly right-handed - a feature that would in theory give it a distinguishable observable. However, due to the relatively low value of the tensor-to-scalar ratio (even after the enhancement), this chirality is likely unobservable [87]. We then considered the scalar perturbations and demonstrated that: the metric scalar perturbations contribute negligibly and can be safely set to zero; the inflationary scalar perturbations are well behaved in that the tangential multi-field perturbation satisfies $\left|\Delta_{s} \sqrt{2 k} x\right| \sim 1$ after horizon-crossing when the background system is still in slow roll; and the scalar perturbations to the gauge field are much smaller than the inflationary perturbation after horizon-crossing. We then derived an analytic approximation of the backreaction of the tensor perturbations of the gauge field onto the equation of motion for $Q$, which we showed is sub-dominant in example 2 . In this way example 2 is a successful phenomenological model in that we see a large enhancement to the gravitational wave spectrum of Kähler moduli inflation, the scalar perturbations are under control, and the model is consistent in that the backreaction from the tensor perturbations of the gauge field is sub-dominant. However, this model requires without justification the hierarchy $c_{2} \gg c_{1}$ despite the fact the expected gauge kinetic function for Kähler moduli inflation would give $c_{2} \sim c_{1} \sim \mathcal{O}(1)$ [85].

With this in mind, in example 3 , we considered an extension to this set-up, whereby the superpotential (given in Kähler inflation as $W=W_{0}+\sum_{a} A_{a} e^{-a_{a} T^{a}}$ ) receives a correction of the form $A e^{-a f_{4}}$ (arising from the introduction of multiply-wrapped magnetised D7-branes) where $f_{4}=n\left(T_{4}+i m b\right)$ where $m$ is the magnetisation of the branes and $n$ is the wrapping number. Setting the $b_{4}$ axion to its minimum, this allowed us to generate a hierarchy between the axion-gauge coupling and the saxiongauge coupling. This set-up leads to $f=\tau_{4}$ and $h=m b$, and the (effective) gauge coupling constant was $\mathrm{g}=\frac{1}{\sqrt{n N / 2}}$ with $N$ the degree of the gauge group and $a=\frac{2 \pi}{N}$. The analysis and results for this model were almost identical to those of example 2 : 


\section{SUMMARY AND DISCUSSIONS}

we see a large enhancement of the tensor spectrum, lifting $r$ to observable values $3^{3}$ with $r \sim 10^{-7} \rightarrow r \sim 10^{-3}$, the inflationary scalar perturbations are dominant over those of the gauge field, and the backreaction of the tensor perturbations to the gauge field is under control. Achieving this however requires very large magnetisation $m=10000$, and very small $\mathrm{g}=\frac{1}{2000}$. Along with the required value for $a$, we get from this that $n=25$ and $N \sim 3 \times 10^{5}$. The large values of $m$ and $N$ (effectively the number of D7-branes) are likely unrealistic in any true compactification scenario and both the large magnetic flux and the large number of D7-branes would likely backreact on the geometry. Achieving these large values is certainly one of the biggest challenges presented by this model, and a full consideration of the compactification process would need to be considered in a further work.

Another potential issue with the set-ups of both example 2 and example 3 is that requiring that there be a large enhancement while maintaining control over the backreaction seems to guarantee that $\epsilon \sim \epsilon_{B} \gg \epsilon_{\varphi}$. This is particularly problematic when starting with a low value for $\epsilon_{\varphi}$ as is the case here where $\epsilon_{\varphi} \sim 10^{-8}$. This is plotted nicely in FIGs 5.455 .46 where it is shown that even with a gauge coupling as small as $g=\frac{1}{100}$, it is impossible to achieve a greater than $\mathcal{O}(1)$ enhancement to the tensorto-scalar ratio (with our value of $\epsilon_{\varphi} \sim 10^{-8}$ ) without incurring excessive backreaction. Thus one requires a lower value of $g$ to mitigate the backreaction and this leads to a larger value of $\epsilon_{B}$. We therefore take it as a necessity that $\epsilon \sim \epsilon_{B}$ and follow [101] by assuming that if the scalar perturbations to the gauge field are vastly sub-dominant relative to the inflationary perturbations, then we are free to take the power spectrum $\mathcal{P}_{s}=\frac{H^{2}}{8 \pi^{2} \epsilon_{\varphi}}$ and therefore $n_{s}=1-2 \epsilon-\eta_{\varphi}$. We see in FIGs 5.225 .24 for example 2 and FIGs 5.405 .42 for example 3 that this is indeed the case. However, if this assumption does not hold, then $\mathcal{P}_{s}=\frac{H^{2}}{8 \pi^{2} \epsilon}$ and $n_{s}=1-2 \epsilon-\eta$, which dramatically changes the inflationary predictions of the model - in particular, since $\epsilon \sim \epsilon_{B}$, this model becomes effectively equivalent (in its predictions of the scalar spectral index) to chromo-natural inflation and is therefore not observationally viable [81, 99]. We therefore see that this assumption is absolutely pivotal to the success of this model, and a thorough consideration of the viability of this assumption is required in a future work. Another assumption of this work is that the isocurvature perturbations are under

\footnotetext{
${ }^{3}$ As before the produced tensor spectrum is perfectly chiral, however this chirality is likely unobservable due to the low tensor-to-scalar ratio.
} 
control. Throughout this work we have neglected isocurvature perturbations, but given that this example is a multi-field model of inflation, a complete analysis would include a study of the isocurvature perturbations. This is left to a further work.

We note finally with regards to example 3 that a similar set-up was considered in [4] where they also include a $C_{2}$ axion coupled to a gauge field with Kähler moduli inflation. However they do not consider the backreaction, and achieve extremely large enhancements $\left(\frac{r}{r_{b}} \sim \mathcal{O}\left(10^{7}\right)\right.$ ) with (relatively large) $\mathrm{g}=\frac{1}{\sqrt{2}}$ and therefore this model is extremely likely to incur excessive backreaction from the tensor perturbations to the gauge field onto the equation of motion for $Q$ (see e.g. FIGs 5.19 and 5.46, as well as [101]) and is therefore inconsistent.

At the end of chapter 5 (example 4), we applied the same set-up of example 3 (whereby $f=\tau_{4}, h=m b$ and $\mathrm{g}=\frac{1}{\sqrt{n N / 2}}$ ) to fibre inflation (using parameter set "SV2" from [50]). This example of fibre inflation already predicts an observable tensor spectrum, $r \sim 5 \times 10^{-3}$ so we were searching only for a relatively small enhancement. This allowed us to relax somewhat the constraints on $m$ and $g$. In this example we had $m=500, \mathrm{~g}=\frac{1}{50}$, which gives a wrapping number of $n=1$ and a gauge degree of $N=5000$. With this set-up we were able to achieve an enhancement of about an order of magnitude and uplift the tensor-to-scalar ratio to $r \sim 5 \times 10^{-2}$, satisfying the current bound of $r \lesssim 0.07$ [37] but leading to a large chirality ( $\sim 94 \%$ of it is from the right-handed mode), and given the higher overall value of the tensor-to-scalar ratio in this example, this chirality is potentially detectable [87], giving this model a distinguishable feature. Unlike examples 2 and 3, this model does not require a very low value for $\mathrm{g}$ to satisfy constraints on the backreaction (since we do not require a very large enhancement) - this means, the hierarchy $\epsilon \sim \epsilon_{\varphi} \gg \epsilon_{B}$ is maintained and we do not need to make the assumption of examples 2 and 3 that the scalar power spectrum is approximated by $\mathcal{P}_{s}=\frac{H^{2}}{8 \pi^{2} \epsilon_{\varphi}}$ despite the fact that $\epsilon \sim \epsilon_{B} \gg \epsilon_{\varphi}$ in those examples. The values taken for the magnetic flux $m=500$ and the gauge degree $N=5000$ in this fibre inflation example are both roughly two orders of magnitude smaller than the Kähler moduli inflation case, however it is likely these are both still too large for a realistic compactification scenario. As was stated for example 3, a full consideration of the compactification process and a study of the isocurvature perturbations would need to be considered in a further work. 


\section{SUMMARY AND DISCUSSIONS}

\subsection{Final thoughts}

In this thesis we have considered two possible implications of including spectator gauge fields in the inflationary action. Gauge fields, of course, naturally dilute during the exponential expansion of space, however they can be supported through couplings to the inflaton (as in the anisotropic inflation part of this thesis) or to rolling axions (as in the enhancement part) that arise naturally in high energy theories such as string theory. In this way the two scenarios considered in this thesis are linked. The main difference between the two is that in the anisotropic inflation case, we have a massless vector field that is supported through its coupling to the inflaton; whereas in the enhancement scenario, we have an $S U(N)$ gauge field supported by a rolling axion, that is usually taken to be a spectator in its own right. We have demonstrated that it is possible to sustain a consistent anisotropy after inflation and that the tensor spectrum of a low energy inflationary model such as Kähler moduli inflation can be enhanced to potentially observable levels while rendering the tensor spectrum chiral. These two paradigms both require specific assumptions that may be impractical in a realistic effective field theory derived from string theory to achieve the exact goals set out in this thesis, but are interesting in their own rights as stand-alone models and certainly warrant further investigation. An interesting follow-up to this work would be the exploration of the possibility that these two models could be combined - starting with an anisotropic metric, is it possible to have a scenario that both enhances the gravity wave spectrum whilst also supporting an anisotropy? 


\section{Appendix A}

\section{Calabi-Yau compactification and the moduli space}

In this appendix, we discuss how compactifications onto Calabi-Yau manifolds lead to the introduction of scalar fields into the four-dimensional effective action. The majority of this review is inspired by chapters in [83] with help from [123 130].

\section{A.1 Maths}

For completeness we will briefly review some mathematical concepts that will prove useful in the section ahead. The expert reader will wish to skip to the next section, section A.2,

\section{A.1.1 Differential forms and boundaries}

A differential form, $C_{p}$, is a tensor of rank $[0, p]$ whose components $C_{m_{1} . . m_{p}}$ are completely antisymmetric. It is expanded in terms of its components as follows:

$$
C_{p}=\frac{1}{p !} C_{m_{1} . . m_{p}}(x) d x^{m_{1}} \wedge \ldots \wedge d x^{m_{p}}
$$

The exterior derivative maps $p$-forms to $(p+1)$-forms via

$$
d C_{p}=\frac{1}{p !} \partial_{m_{0}} C_{m_{1} . ., m_{p}}(x) d x^{m_{0}} \wedge d x^{m_{1}} \wedge \ldots \wedge d x^{m_{p}} .
$$




\section{A. CALABI-YAU COMPACTIFICATION AND THE MODULI SPACE}

and satisfies

$$
d\left(d C_{p}\right)=0 \forall C_{p} \Longrightarrow d^{2}=0 .
$$

Analogous to the exterior derivative is the "boundary operator", $\partial$. For some manifold, $\mathcal{M}$, consider an $n$-dimensional sub-manifold, $\mathcal{N}$. $\mathcal{N}$ is defined as a subset of $\mathcal{N}$ and has the structure of an $n$-dimensional manifold. The boundary of $\mathcal{N}$, denoted $\partial \mathcal{N}$ is $(n-1)$ dimensional. The operator, $\partial$, analogously to the exterior derivative, satisfies

$$
\partial(\partial \mathcal{N})=0 \Longrightarrow \partial^{2}=0
$$

In words: the boundary of a manifold can have no boundary.

\section{A.1.2 Cohomology}

A $p$-form, $A_{p}$, is closed if it satisfies $d A_{p}=0$. Furthermore, $A_{p}$ is exact if there exists some $B_{p-1}$ for which $A_{p}=d B_{p-1}$. By the basic properties of the exterior derivative $\left(d^{2}=0\right)$, it is trivial to see that any exact form is closed; the opposite is not always true however. In a topologically-trivial space, $\mathcal{R}^{d}$, any closed form is exact but this is not true generally. In fact, for some manifold, $\mathcal{M}$, the closed forms that are not exact provide a characterisation of the degree to which $\mathcal{M}$ fails to be $\mathcal{R}^{d}$.

A manifold, $\mathcal{M}$, can be defined by glueing together a set of coordinate patches, $U_{n}$. A closed $p$-form $A_{p}$ on $\mathcal{M}$ defines closed $p$-forms, $A_{p}^{n}$, on the coordinate patches $U_{n}$. Each patch, $U_{n}$, is locally homeomorphic to $\mathcal{R}^{d}$ and therefore the closed $p$-forms on $U_{n}$ are exact, and there exists some $B_{p}^{n}$ on $U_{n}$ for which $A_{p}^{n}=d B_{p-1}^{n}$. However, this does not necessarily imply that $B_{p-1}^{n}$ will patch up to a globally well-defined $B_{p-1}$ for which $A_{p}=d B_{p-1}$ on $\mathcal{M}$. Whether this is possible depends on the global structure of $\mathcal{M}$ and therefore the existence on $\mathcal{M}$ of closed forms that are not exact is a measure of the topological non-triviality of $\mathcal{M}$. For this reason, the topological non-triviality of $\mathcal{M}$ is determined by its cohomology groups. The $p$ th cohomology group of $\mathcal{M}, \mathcal{H}_{p}(\mathcal{M}, \mathcal{R})$ is defined as the quotient:

$$
\mathcal{H}_{p}(\mathcal{M}, \mathcal{R})=\frac{Z_{p}}{B_{p}}
$$

where $Z_{p}$ and $B_{p}$ are the set of closed and exact $p$-forms on $\mathcal{M}$, respectively. In other words, the $p$ th cohomology group contains the closed $p$-forms on $\mathcal{M}$ that are not exact. $\mathcal{H}_{p}(\mathcal{N}, \mathcal{R})$ have the structure of vector spaces with dimension (the number of non-exact 
closed $p$-forms on $\mathcal{M})$ given by the Betti numbers of $\mathcal{M}, b_{p}(\mathcal{M})$ :

$$
b_{p}(\mathcal{M})=\operatorname{dim}\left(\mathcal{H}_{p}(\mathcal{M}, \mathcal{R})\right)
$$

\section{A.1.3 Homology}

A set of sub-manifolds of dimension $p, \mathcal{N}_{p}^{k}$ of $\mathcal{M} \prod^{1}$, can be given the structure of a vector space by introducing linear combinations of $N_{p}^{k}$ called $p$-chains:

$$
a_{p}=c_{k} N_{p}^{k}
$$

where $c_{k}$ are real coefficients. A $p$-chain, $a_{p} \in \mathcal{M}$ with no boundary

$$
\partial a_{p}=0
$$

is called a $p$-cycle. A $p$-chain, $a_{p}$, that is the boundary of some $(p+1)$-chain, $b_{p+1}$, $a_{p}=\partial b_{p+1}$ is called trivial. Since the boundary of a sub-manifold has no boundary, $\partial\left(\partial a_{p}\right)=0$, all $p$-chains are $p$-cycles. Completely analogously to the case of $p$-forms, in $\mathcal{R}^{d}$ all $p$-cycles are trivial but this may not be true for some general manifold, $\mathcal{M}$, and the existence of $p$-cycles on $\mathcal{M}$ that are not trivial is a measure of the non-triviality of $\mathcal{M}$ for analogous reasons to those described in section A.1.2. The homology groups of $\mathcal{M}$ are the sets of non-trivial cycles on $\mathcal{M}$. The $p$ th homology group of $\mathcal{M}$ is defined as

$$
\mathcal{H}^{p}(\mathcal{M}, \mathcal{R})=\frac{Z^{p}}{B^{p}}
$$

where $Z^{p}$ and $B^{p}$ are the sets of $p$-cycles and trivial $p$-cycles, respectively. There exists a duality between the vector spaces of the cohomology groups, $\mathcal{H}_{p}$, and the homology groups, $\mathcal{H}^{p}$; and their product defines a linear map to the reals: $\mathcal{H}_{p} \times \mathcal{H}^{p} \rightarrow$ $\mathcal{R}$. This duality means that, equivalently to the case of the cohomology groups, the dimensionalities of the homology groups, $\mathcal{H}^{p}(\mathcal{M}, \mathcal{R})$, are given by the Betti numbers, $b_{p}(\mathcal{M})$.

\footnotetext{
${ }^{1}$ Here $p$ denotes the dimension of the sub-manifold and $k$ is just a label.
} 


\section{A. CALABI-YAU COMPACTIFICATION AND THE MODULI SPACE}

\section{A.2 Calabi-Yau compactifications}

In the context of cosmology, models derived from string theory are almost always done so via Calabi-Yau compactifications, the motivation for which is discussed here.

String theories are 10-dimensional so if we wish to use string theory in cosmology, we need to compactify our string theory space, $S_{10}$, to $4 \mathrm{D}$ :

$$
S_{10} \rightarrow \Sigma_{4} \times \mathcal{M}
$$

where $\Sigma_{4}$ is our cosmological spacetime and $\mathcal{M}$ is the compactified space of the remaining six dimensions. How can we perform this compactification? Progress can be made by posing the question: what features would we like the compactified space, $\mathcal{M}$, to have? Principally we would like the compactification process to preserve some supersymmetry $(S u S y)$ on $\mathcal{M}$. This is because $S u S y$ provides a good starting point to build particle physics models.

\section{A.2.1 Preserving supersymmetry on $\mathcal{M}$}

At each point on $\Sigma_{4} \times \mathcal{M}$, there exists a local set of supercharges that transforms under $S O(10)$. $\mathcal{M}$ is a curved manifold and therefore local supercharges at different points on $\mathcal{M}$ can be related by parallel transport with respect to the $S O(6)$ spin connection. A local supercharge, $\mathcal{S}$, at a point, $P$ on $\mathcal{M}$, parallel transported around some closed loop, $C$, will return to $P$ having been rotated by some element of $S O(6), R_{C}$, which will be in general non-trivial due to the curvature of $\mathcal{M}$. For this reason $\mathcal{S}$ is not a globally well-defined supercharge on $\mathcal{M}$ and therefore supersymmetry has been broken.

The condition that compactification onto $\mathcal{M}$ preserves some supersymmetry is therefore that there exist non-trivial 6-dimensional spinors $\xi\left(x^{m}\right)$ on $\mathcal{M}$ that are covariantly conserved

$$
\nabla_{\mathcal{M}} \xi\left(x^{m}\right)=0
$$

where $x^{m}$ are our coordinates on $\mathcal{M}$ and $\xi$ are Killing spinors. Whether there exist solutions to this equation can be determined by the holonomy groun $2^{2}$ of $\mathcal{M}$. The holonomy group of $\mathcal{M}$ is the set of rotations $R_{C}$ undergone by a spinor after parallel transport around a closed loop, $C$, for every closed loop on $\mathcal{M}$. The holonomy group

\footnotetext{
${ }^{2}$ Not to be confused with the homology groups defined in section A.1.3
} 
depends on the metric and spin connection of $\mathcal{M}$. In general a 6-dimensional manifold will have holonomy group of $S O(6)$ and therefore every spinor parallel transported around a closed loop on $\mathcal{M}$ will be rotated, i.e. there are no Killing spinors on $\mathcal{M}$ and therefore all supersymmetry has been broken by compactification. Therefore to preserve some supersymmetry, $\mathcal{M}$ must possess some special holonomy (not $S O(6)$ ), in particular, if $\mathcal{N}$ has holonomy group of $S U(3)$ some supersymmetry is conserved 3 .

\section{A.2.2 Calabi-Yau manifolds and their moduli}

If our compactified space, $\mathcal{M}$, has holonomy of $S U(3)$ some supersymmetry is conserved after compactification. Therefore if we know a manifold has $S U(3)$ holonomy it is a good candidate for compactification. Here a conjecture by Eugenio Calabi [131] (proved by Shing-Tung Yau [132]) proves extremely useful. Calabi's conjecture states that an $N$-dimensional complex manifold that is Kähler and admits a nowhere-vanishing holomorphic $N$-form will have an $S U(N)$ holonomy metric. A manifold that satisfies this is called a Calabi-Yau (CY) manifold. An $N$-dimensional complex manifold is a $2 N$-dimensional manifold endowed with a complex structure. Complex coordinates can be decomposed into holomorphic and anti-holomorphic coordinates:

$$
d z^{j}=d x^{j}+i d y^{j+N}
$$

and

$$
d \bar{z}^{\bar{j}}=d x^{j}-i d y^{j+N}
$$

where $j=\{1, \ldots, N\}, d z^{k}$ and $d \bar{z}^{k}$ are called holomorphic and anti-holomorphic coordinates, respectively; $x^{i}(i=\{1, \ldots, N\})$ and $y^{i}(i=\{N+1, \ldots, 2 N\})$ are two sets of real coordinates that together span the $2 N$ real coordinates of $\mathcal{M}$. From now on to avoid confusion, we will use "early" Latin letters $\{a, \bar{a}, b, \bar{b}, .$.$\} (running from \{1, \ldots, N\}$ ) to mark holomorphic and anti-holomorphic indices, and "middle" Latin letters $\{i, j, k .$. (running from $\{1, \ldots, 2 N\}$ ) to mark the $2 N$ real coordinates. A complex manifold is endowed with a complex structure that relates coordinates through

$$
I_{j}^{i} d x^{j}=-d y^{i+N}, \quad I_{j}^{i} d y^{j+N}=d x^{i}
$$

\footnotetext{
${ }^{3}$ The compactification leads to a reduction of the supersymmetry on the cosmological 4-dimensional space, $\Sigma_{4}$, as well.
} 


\section{A. CALABI-YAU COMPACTIFICATION AND THE MODULI SPACE}

which implies

$$
I_{b}^{a} d z^{b}=i d z^{a}, \quad I_{\bar{b}}^{\bar{a}} d \bar{z}^{\bar{b}}=-i d \bar{z}^{\bar{a}}
$$

where $I_{j}^{i}$ is the complex structure tensor of $\mathcal{M}$ whose fundamental property is

$$
I_{j}^{i} I_{k}^{j} X^{k}=-X^{i} \forall X \in \mathcal{M} \Longrightarrow I_{k}^{i} I_{j}^{k}=-\delta_{j}^{i}
$$

With these rules in place, tensors can be decomposed according to their holomorphic and anti-holomorphic indices, in particular, a $p$-form, $A_{p}$, can be decomposed on a complex manifold as

$$
A_{p}=A_{p, 0}+A_{p-1,1}+\cdots+A_{1, p-1}+A_{0, p}
$$

where $A_{m, n}$ is a form with $m$ holomorphic and $n$ anti-holomorphic indices with basis $d z^{a_{1}} \wedge \ldots \wedge d z^{a_{m}} \wedge d \bar{z}^{\bar{b}_{1}} \wedge \ldots \wedge d \bar{z}^{b_{n}}$. The complex structure tensor can be decomposed in terms of the complex coordinates as

$$
I=I_{b}^{a} \frac{\partial}{\partial z^{a}} \otimes d z^{b}+I_{\bar{b}}^{a} \frac{\partial}{\partial z^{a}} \otimes d \bar{z}^{\bar{b}}+I_{b}^{\bar{a}} \frac{\partial}{\partial \bar{z}^{\bar{a}}} \otimes d z^{b}+I_{\bar{b}}^{\bar{a}} \frac{\partial}{\partial \bar{z}^{\bar{a}}} \otimes d \bar{z}^{\bar{b}}
$$

where $\partial / \partial z^{a}$ and $\partial / \partial \bar{z}^{\bar{a}}$ are basis vectors dual to $\mathrm{A} .2 .3$ and $\mathrm{A} .2 .4$. It can be shown that (see e.g. [126]) that the mixed components of $I$ are zero, $I_{\bar{b}}^{a}=I_{b}^{\bar{a}}=0$. In fact the components of $I$ can be chosen to be

$$
I_{j}^{i}=\left(\begin{array}{cc}
I_{b}^{a} & I_{\bar{b}}^{a} \\
I_{b}^{\bar{a}} & I_{\bar{b}}^{\bar{a}}
\end{array}\right)=\left(\begin{array}{cc}
i \delta_{b}^{a} & 0 \\
0 & -i \delta_{\bar{b}}^{\bar{a}}
\end{array}\right) .
$$

A complex manifold, $\mathcal{M}$, is hermitian if it admits a metric that satisfies

$$
I_{j}^{i} X^{j} I_{l}^{k} Y^{l} g_{i k}=X^{i} Y^{j} g_{i j} \forall X, Y \in \mathcal{M} \Longrightarrow I_{i}^{k} I_{j}^{l} g_{k l}=g_{i j}
$$

This tells us that $g_{i j}$ must be a covariant $(1,1)$ type tensor, i.e. its only non-zero components are mixed holomorphic and anti-holomorphic since by A.2.10

$$
\begin{aligned}
I_{i}^{k} I_{j}^{l} g_{k l} & =\left(i \delta_{a}^{c}\right)\left(i \delta_{b}^{d}\right) g_{c d}+\left(i \delta_{a}^{c}\right)\left(-i \delta_{\bar{b}}^{\bar{d}}\right) g_{c \bar{d}}+\left(-i \delta_{\bar{a}}^{\bar{c}}\right)\left(i \delta_{b}^{d}\right) g_{\bar{c} d}+\left(-i \delta_{\bar{a}}^{\bar{c}}\right)\left(-i \delta_{\bar{b}}^{\bar{d}}\right) g_{\bar{c} \bar{d}} \\
& =-g_{a b}+g_{a \bar{b}}+g_{\bar{a} b}-g_{\bar{a} \bar{b}} \stackrel{!}{=} g_{i j}=g_{a b}+g_{a \bar{b}}+g_{\bar{a} b}+g_{\bar{a} \bar{b}} \\
& \Longrightarrow g_{a b}=g_{\bar{a} \bar{b}}=0
\end{aligned}
$$


Therefore $g_{i j}=g_{a \bar{b}}+g_{\bar{a} b}$ or in perhaps better notation

$$
g_{i j}=\left(\begin{array}{cc}
0 & g_{a \bar{b}} \\
g_{\bar{c} d} & 0
\end{array}\right) .
$$

The fundamental form of a hermitian manifold, $J$, is defined by lowering an index of the complex structure to make a two-form $J_{i j}=g_{i k} I_{j}^{k}$. Using A.2.10, we get

$$
J_{i j}=i g_{a \bar{b}}-i g_{\bar{a} b}
$$

which is antisymmetric since $g_{a \bar{b}}=g_{\bar{b} a}$. Expanded in terms of the basis, it becomes clear that $J$ is a $(1,1)$-form:

$$
J=i g_{a \bar{b}} d z^{a} \otimes d \bar{z}^{\bar{b}}-i g_{\bar{a} b} d \bar{z}^{\bar{a}} \otimes d z^{b}=i g_{a \bar{b}} d z^{a} \wedge d \bar{z}^{\bar{b}}
$$

where in the last step we have again used the symmetry of the metric and the fact that $d z^{a} \wedge d \bar{z}^{\bar{b}}=d z^{a} \otimes d \bar{z}^{\bar{b}}-d \bar{z}^{\bar{b}} \otimes d z^{a}$. A hermitian manifold is Kähler if $J$ is closed, $d J=0$, in which case $J$ is called the Kähler form. Therefore a manifold for which $J$ is closed and for which there may exist a $(N, 0)$-form that is nowhere vanishing is a CalabiYau manifold and thus admits an $S U(N)$ holonomy metric and will preserve some supersymmetry after compactification. In particular, in the case of compactification onto cosmological spacetimes where our compactified space, $\mathcal{M}$, is 6-dimensional, the holonomy group is $S U(3)$ and $\mathcal{M}$ is called a Calabi-Yau three-fold.

On a complex manifold, cohomology classes (see section A.1.2 are split by holomorphic and anti-holomorphic components. For example, the 3-form cohomology group of $\mathcal{M}$ becomes

$$
\mathcal{H}_{3}(\mathcal{M}, \mathcal{R})=\mathcal{H}_{3,0}(\mathcal{M}, \mathcal{R})+\mathcal{H}_{2,1}(\mathcal{M}, \mathcal{R})+\mathcal{H}_{1,2}(\mathcal{M}, \mathcal{R})+\mathcal{H}_{0,3}(\mathcal{M}, \mathcal{R})
$$

where $\mathcal{H}_{m, n}(\mathcal{M}, \mathcal{R})$ is the cohomology group for forms with $m$ holomorphic and $n$ antiholomorphic indices. The Hodge numbers, $h_{m, n}=\operatorname{dim}\left(\mathcal{H}_{m, n}(\mathcal{M}, \mathcal{R})\right)$, are the complex generalisation of the Betti numbers defined in section A.1.2. The Hodge numbers are used to define the Euler characteristic, the weighted sum of the Hodge numbers:

$$
\chi(\mathcal{M})=\sum_{m, n=0}^{3}(-1)^{m+n} h_{m, n}(\mathcal{M})=2\left[h_{1,1}-h_{1,2}\right] .
$$




\section{A. CALABI-YAU COMPACTIFICATION AND THE MODULI SPACE}

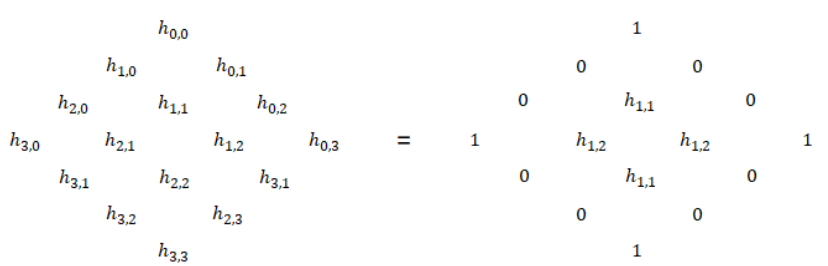

Figure A.1: Hodge diamond for a Calabi-Yau three-fold with $S U(3)$ holonomy.

Hodge numbers are represented in diamond diagrams. The Hodge diamond for $\mathcal{M}$, a Calabi-Yau three-fold with $S U(3)$ holonomy, is shown in Figure A.1. Notice that $h_{3,0}=1$, showing that $\mathcal{M}$ has a $(3,0)$-form as required by Calabi's conjecture, the second part of which is the existence a holomorphic $N$-form 4 i.e. an $(N, 0)$-form. We will refer to this holomorphic 3 -form as $\Omega$.

Let us consider some properties of the Kähler form, $J$, on a Calabi-Yau three-fold. Consider the integral over $\mathcal{M}$ :

$$
\int_{\mathcal{M}} J^{3}=\int_{\mathcal{M}} J \wedge J \wedge J=i^{3} \int_{\mathcal{M}} g_{a \bar{b}} g_{c \bar{d}} g_{e \bar{f}} d z^{a} \wedge d \bar{z}^{\bar{b}} \wedge d z^{c} \wedge d \bar{z}^{\bar{d}} \wedge d z^{e} \wedge d \bar{z}^{\bar{f}} .
$$

Expanding and using the antisymmetry of the wedge product this becomes

$$
\int_{\mathcal{M}} J^{3}=3 !(i) \int_{\mathcal{M}} \operatorname{det}\left(g_{a \bar{b}}\right) d z^{1} \wedge d \bar{z}^{1} \wedge d z^{2} \wedge d \bar{z}^{2} \wedge d z^{3} \wedge d \bar{z}^{3}
$$

which, converting back to real coordinates through $d z^{a} \wedge d \bar{z}^{\bar{a}}=-i d x^{a} \wedge d y^{a+3}+i d y^{a+3} \wedge$ $d x^{a}=-2 i d x^{a} \wedge d y^{a+3}$ and then permuting through the wedge products, becomes

$$
\int_{\mathcal{M}} J^{3}=6 i(-i)^{3} \int_{\mathcal{M}} 2^{3} \sqrt{\operatorname{det}\left(g_{i j}\right)} d x^{1} \wedge d x^{2} \wedge d x^{3} \wedge d y^{4} \wedge d y^{5} \wedge d y^{6}=6 \mathcal{V}_{\mathcal{M}}
$$

where $\mathcal{V}_{\mathcal{M}}$ is the volume of $\mathcal{M}$, the factor of $2^{3}$ in the volume element is conventional, and $\operatorname{det}\left(g_{i j}\right)=\operatorname{det}\left(g_{a \bar{b}}\right)^{2}$ because of the block form of $g_{i j}$ in A.2.13. This is an important result because it demonstrates that the Kähler form, $J$, cannot be exact and yet we know by definition that is it closed, $d J=0$. If $J$ were exact, there would exist some $A$ for which $J=d A$ then

$$
\int_{\mathcal{M}} J \wedge J \wedge J=\int_{\mathcal{M}} d A \wedge J \wedge J=-\int_{\mathcal{M}} A \wedge d(J \wedge J)=0 \Longrightarrow \mathcal{V}_{\mathcal{M}}=0
$$

\footnotetext{
${ }^{4}$ For the case of an $N$-dimensional complex manifold. In our example, $\mathcal{M}$ is a 3 -dimensional complex manifold.
} 
where we have assumed that $A, J$ disappear on the boundary of $\mathcal{M}$ and explicitly used that $d J=0$. Since the volume of our Calabi-Yau must be non-zero we know that $J$ cannot be exact. The Kähler form, $J$, is a closed, non-exact $(1,1)$-form and is therefore in the $\mathcal{H}_{1,1}$ cohomology group and can be expanded in a basis as such

$$
J=\sum_{\alpha=1}^{h_{1,1}} t_{\alpha} w_{\alpha}
$$

where $w_{\alpha}$ are $(1,1)$-forms that form a basis $\left\{w_{\alpha}\right\}$ of $\mathcal{H}_{1,1}$ (cohomology groups have the structure of vector spaces) and $t_{\alpha}$ are 2-cycle volumes that control the size of the even-dimensional cycle volumes in $\mathcal{M}$, through

$$
\begin{aligned}
& \mathcal{V}=\frac{1}{6} \int_{\mathcal{M}} J \wedge J \wedge J=\frac{1}{6} \kappa_{\alpha \beta \gamma} t^{\alpha} t^{\beta} t^{\gamma} \\
& \tau_{\alpha}=\partial_{t_{\alpha}} \nu=\frac{1}{2} \kappa_{\alpha \beta \gamma} t^{\beta} t^{\gamma}
\end{aligned}
$$

where $\kappa_{\alpha \beta \gamma}$ are constants and $\tau_{a}$ are 4 -cycle volumes that we will refer to as Kähler moduli. The key point to notice about these moduli is that they have no $4 \mathrm{D}$ spacetime index $(\mu, \nu, .$.$) and therefore from the point of view of our compactified 4 \mathrm{D}$ cosmological theory, they are scalars. In addition to this, these moduli can appear in the low-energy effective action of the cosmological spacetime. Since the value of $h_{1,1}$ can be very large depending on the Calabi-Yau that is chosen, we can have a large number of these moduli appearing in our $4 \mathrm{D}$ theory. Could one or more of these be suitable inflaton candidates? In addition to the Kähler moduli, a Calabi-Yau also contains complex structure moduli. The simplest way to define the complex structure moduli is by considering them as deformations to the metric $g_{i j} \rightarrow g_{i j}+\delta g_{i} \sqrt{5}$. We first define a $(2,1)$-form, $\mathcal{C}$, with help from the unique holomorphic 3 -form, $\Omega$ :

$$
\mathcal{C}=\Omega_{a b c} \delta g_{\bar{d}}^{c} d z^{a} \wedge d z^{b} \wedge d \bar{z}^{\bar{d}}
$$

\footnotetext{
${ }^{5}$ The Kähler moduli can in fact be defined similarly through $\delta g_{a \bar{b}}=\sum_{\alpha=1}^{h_{1,1}} b_{a \bar{b}} t_{\alpha}$ where $b_{\alpha}$ form a basis of $(1,1)$-forms.

${ }^{6}$ The relevance of the variation of the Kähler metric is that a CY manifold has a Ricci-flat metric: $R_{i j}(g)=0$. The condition that a variation of this metric should leave it Ricci-flat: $R_{i j}(g+\delta g)=0$ can be cast in terms of the Kähler and complex structure moduli. For more information, see [130].
} 


\section{A. CALABI-YAU COMPACTIFICATION AND THE MODULI SPACE}

which can be expanded in a basis of the $\mathcal{H}_{2,1}$ cohomology group:

$$
\mathcal{C}_{a b \bar{c}}=\Omega_{a b c} \delta g_{\bar{d}}^{c}=\sum_{\alpha=1}^{h_{1,2}} u_{\alpha} b_{a b \bar{c} \alpha}
$$

where $\left\{u_{\alpha}\right\}$ form a basis for $\mathcal{H}_{2,1}$ and $u_{\alpha}$ are 3 -cycle volumes called complex structure moduli. 


\section{Appendix B}

\section{Second order action for action with isotropic gauge field}

We are interested in perturbing to second order the action

$$
\begin{array}{r}
S=\int d^{4} x\left\{\frac{M_{\mathrm{pl}}^{2}}{2} \sqrt{-g} R-\frac{1}{2} \sqrt{-g} g^{\mu \nu} \partial_{\mu} \phi \partial_{\nu} \phi-\sqrt{g} V-\frac{1}{2} \sqrt{g} g^{\mu \nu} \partial_{\mu} \chi \partial_{\nu} \chi\right. \\
\left.-\sqrt{-g} U-\frac{1}{4} \sqrt{-g} g^{\mu \rho} g^{\nu \sigma} F_{\mu \nu}^{a} F_{\rho \sigma}^{a}+\frac{\lambda}{8 f} \chi \epsilon^{\mu \nu \rho \sigma} F_{\mu \nu}^{a} F_{\rho \sigma}^{a}\right\}
\end{array}
$$

with

$$
\begin{gathered}
g_{\mu \nu}=a^{2}\left(\begin{array}{cccc}
-1 & 0 & 0 & 0 \\
0 & 1+h_{+} & h_{\times} & 0 \\
0 & h_{\times} & 1-h_{+} & 0 \\
0 & 0 & 0 & 1
\end{array}\right), \\
g^{\mu \nu}=a^{2}\left(\begin{array}{cccc}
-1 & 0 & 0 & 0 \\
0 & 1-h_{+}+h_{\times}^{2}+h_{+}^{2} & -h_{\times} & 0 \\
0 & -h_{\times} & 1-h_{+}+h_{\times}^{2}+h_{+}^{2} & 0 \\
0 & 0 & 0 & 1
\end{array}\right), \\
A_{\mu}^{1}=a\left(0, Q+\delta Q+T_{+}, T_{\times}, 0\right), \\
A_{\mu}^{2}=a\left(0, T_{\times}, Q+\delta Q-T_{+}, 0\right), \\
A_{\mu}^{3}=a\left(\partial_{z} Y, 0,0, Q+\delta Q+\partial_{z}^{2} M\right)
\end{gathered}
$$




\section{B. SECOND ORDER ACTION FOR ACTION WITH ISOTROPIC GAUGE FIELD}

and after the redefinition of the tensor modes to left- and right-moving ones

$$
\psi_{L, R}:=\frac{a M_{\mathrm{pl}}}{2}\left(h_{+} \pm i h_{\times}\right), \quad t_{L, R}:=a\left(T_{+} \pm i T_{\times}\right)
$$

With this form for metric, square root of the determinant of the metric to second order is

$$
\sqrt{-g}=a^{4}-2 a^{2} \psi_{L} \psi_{R}
$$

and the Ricci scalar is

$$
\begin{aligned}
R= & \frac{8 a_{, \tau, \tau} \psi_{L} \psi_{R}}{a^{5}}+\frac{2 a^{\prime 2} \psi_{L} \psi_{R}}{a^{6}}+\frac{2 a_{, \tau} \psi_{L, \tau} \psi_{R}}{a^{5}}+\frac{2 a_{, \tau} \psi_{L} \psi_{R, \tau}}{a^{5}}+\frac{6 \psi_{L, z} \psi_{R, z}}{a^{4}} \\
& +\frac{4 \psi_{L, z, z} \psi_{R}}{a^{4}}+\frac{4 \psi_{L} \psi_{R, z, z}}{a^{4}}-\frac{6 \psi_{L, \tau} \psi_{R, \tau}}{a^{4}}-\frac{4 \psi_{L, \tau, \tau} \psi_{R}}{a^{4}}-\frac{4 \psi_{L} \psi_{R, \tau, \tau}}{a^{4}}+\frac{6 a_{, \tau, \tau}}{a^{3}} .
\end{aligned}
$$

With this, the second order action (B.0.1) can be found

$$
\begin{aligned}
& S_{2}=-9 \mathrm{~g}^{2} Q^{2} \delta \mathrm{Q}^{2} a^{4}+\mathrm{g}^{2} Q^{2} Y_{, z}^{2} a^{4}-\mathrm{g}^{2} Q^{2} M_{, z, z}^{2} a^{4}-\frac{1}{2} \delta \chi^{2} U_{, \tau, \tau}(\chi) a^{4}-\frac{1}{2} \delta \phi^{2} V_{, \tau, \tau}(\phi) a^{4} \\
& -6 \mathrm{~g}^{2} Q^{2} \delta \mathrm{Q} M_{, z, z} a^{4}+\frac{2 \mathrm{~g} \lambda Q \chi Y_{, z} \delta \mathrm{Q}_{, z} a^{3}}{f}+\frac{\mathrm{g} \lambda Q^{2} \delta \chi Y_{, z, z} a^{3}}{f}+\frac{2 \mathrm{~g} \lambda Q \delta \mathrm{Q} \chi Y_{, z, z} a^{3}}{f} \\
& -\frac{6 \mathrm{~g} \lambda Q \delta \mathrm{Q} \delta \chi Q_{, \tau} a^{3}}{f}-\frac{3 \mathrm{~g} \lambda \delta \mathrm{Q}^{2} \chi Q, \tau}{f}-\frac{2 \mathrm{~g} \lambda Q \delta \chi Q_{, \tau} M_{, z, z} a^{3}}{f} \\
& -\frac{2 \mathrm{~g} \lambda \delta \mathrm{Q} \chi Q_{, \tau} M_{, z, z} a^{3}}{f}-\frac{3 \mathrm{~g} \lambda Q^{2} \delta \chi \delta \mathrm{Q}_{, \tau} a^{3}}{f}-\frac{6 \mathrm{~g} \lambda Q \delta \mathrm{Q} \chi \delta \mathrm{Q}_{, \tau} a^{3}}{f} \\
& -\frac{2 \mathrm{~g} \lambda Q \chi M_{, z, z} \delta \mathrm{Q}_{, \tau} a^{3}}{f}-\frac{\mathrm{g} \lambda Q^{2} \delta \chi M_{, \tau, z, z} a^{3}}{f}-\frac{2 \mathrm{~g} \lambda Q \delta \mathrm{Q} \chi M_{, \tau, z, z} a^{3}}{f}-\delta \mathrm{Q}_{, z}^{2} a^{2} \\
& -\frac{1}{2} \delta \phi_{, z}^{2} a^{2}-\frac{1}{2} \delta \chi_{, z}^{2} a^{2}+\frac{1}{2} Y_{, z, z}^{2} a^{2}+\frac{3}{2} \delta \mathrm{Q}_{, \tau}^{2} a^{2}+\frac{1}{2} \delta \phi_{, \tau}^{2} a^{2}+\frac{1}{2} \delta \chi_{, \tau}^{2} a^{2}+\frac{1}{2} M_{, \tau, z, z}^{2} a^{2} \\
& +2 \mathrm{~g}^{2} Q^{3} t_{R} \psi_{L} a^{2}+2 \mathrm{~g}^{2} Q^{3} t_{L} \psi_{R} a^{2}-3 \mathrm{~g}^{2} Q^{4} \psi_{L} \psi_{R} a^{2}+2 U(\chi) \psi_{L} \psi_{R} a^{2} \\
& +2 V(\phi) \psi_{L} \psi_{R} a^{2}-Y_{, z, z} \delta \mathrm{Q}_{, \tau} a^{2}-Y_{, z, z} M_{, \tau, z, z} a^{2}+\delta \mathrm{Q}_{, \tau} M_{, \tau, z, z} a^{2}-\frac{9 \mathrm{~g} \lambda Q^{2} \delta \mathrm{Q} \delta \chi a_{, \tau} a^{2}}{f} \\
& -\frac{9 \mathrm{~g} \lambda Q \delta \mathrm{Q}^{2} \chi a_{, \tau} a^{2}}{f}-\frac{3 \mathrm{~g} \lambda Q^{2} \delta \chi a_{, \tau} M_{, z, z} a^{2}}{f}-\frac{6 \mathrm{~g} \lambda Q \delta \mathrm{Q} \chi a_{, \tau} M_{, z, z} a^{2}}{f} \\
& +\frac{\mathrm{g} \lambda \chi t_{L} t_{R} Q_{, \tau} a}{f}-i \mathrm{~g} Q t_{R} t_{L, z} a+2 \mathrm{~g} i Q^{2} \psi_{R} t_{L, z} a+\mathrm{g} i Q t_{L} t_{R, z} a \\
& -2 i \mathrm{~g} Q^{2} \psi_{L} t_{R, z} a-\delta \mathrm{Q} a_{, \tau} Y_{, z, z} a-a_{, \tau} M_{, z, z} Y_{, z, z} a+3 \delta \mathrm{Q} a_{, \tau} \delta \mathrm{Q}_{, \tau} a+a_{, \tau} M_{, z, z} \delta \mathrm{Q}_{, \tau} a
\end{aligned}
$$




$$
\begin{aligned}
& +\frac{\mathrm{g} \lambda Q \chi t_{R} t_{L, \tau} a}{f}+\frac{\mathrm{g} \lambda Q \chi t_{L} t_{R, \tau} a}{f}+\delta \mathrm{Q} a_{, \tau} M_{, \tau, z, z} a+a_{, \tau} M_{, z, z} M_{, \tau, z, z} a \\
& +\frac{3}{2} \delta \mathrm{Q}^{2} a^{\prime 2}+\frac{Q^{2} \psi_{L} \psi_{R} a^{\prime 2}}{a^{2}}+\frac{\psi_{L} \psi_{R} a^{\prime 2}}{a^{2}}+\psi_{L} \psi_{R} Q^{\prime 2}-\psi_{L} \psi_{R} \phi^{\prime 2} \\
& -\psi_{L} \psi_{R} \chi^{\prime 2}+\frac{1}{2} a^{\prime 2} M_{, z, z}^{2}+\frac{\mathrm{g} \lambda Q \chi t_{L} t_{R} a_{, \tau}}{f}-t_{L, z} t_{R, z}+3 \psi_{L, z} \psi_{R, z} \\
& +\delta \mathrm{Q} a^{\prime 2} M_{, z, z}+2 \psi_{R} \psi_{L, z, z}+2 \psi_{L} \psi_{R, z, z}-2 \psi_{R} Q_{, \tau} t_{L, \tau}-2 \psi_{L} Q_{, \tau} t_{R, \tau} \\
& +\frac{i \lambda \chi t_{L, z} t_{R, \tau}}{f}+t_{L, \tau} t_{R, \tau}-3 \psi_{L, \tau} \psi_{R, \tau}-2 \psi_{R} \psi_{L, \tau, \tau}-2 \psi_{L} \psi_{R, \tau, \tau} \\
& -\frac{i \lambda \chi t_{R, z} t_{L, \tau}}{f}+\frac{2 Q \psi_{L} \psi_{R} a_{, \tau} Q_{, \tau}}{a}-\frac{2 \psi_{L} \psi_{R} a_{, \tau, \tau}}{a}-\frac{2 Q \psi_{R} a_{, \tau} t_{L, \tau}}{a} \\
& -\frac{2 Q \psi_{L} a_{, \tau} t_{R, \tau}}{a}+\frac{\psi_{R} a_{, \tau} \psi_{L, \tau}}{a}+\frac{\psi_{L} a_{, \tau} \psi_{R, \tau}}{a}
\end{aligned}
$$

The perturbation, $Y$, is non-dynamical (its equation of motion contains no timederivatives of $Y$ ) and after moving into Fourier space, it can solved for explicitly in terms of the other fields. After substituting this into the other equations of motion, the scalar perturbation equations from this action become

$$
H^{2}\left(x^{2} \Delta_{\phi, x, x}+\Delta_{\phi}\left(x^{2}+\epsilon-2\right)\right)+\Delta_{\phi} V_{, \phi, \phi}=0,
$$




$$
\begin{aligned}
& \frac{x^{5} \lambda \Delta_{2} Q_{, x} H^{5}}{\sqrt{\frac{1}{2} x^{2} H^{2}+\mathrm{g}^{2} Q^{2}}}-\frac{x^{4} \lambda Q \Delta_{2} H^{5}}{\sqrt{\frac{1}{2} x^{2} H^{2}+\mathrm{g}^{2} Q^{2}}}+f x^{4} \Delta_{\chi} H^{4}-2 f x^{2} \Delta_{\chi} H^{4} \\
& +f x^{2} \epsilon \Delta_{\chi} H^{4}+f x^{4} \Delta_{\chi, x, x} H^{4}+\frac{2 \mathrm{~g} x^{2} \lambda Q^{2} \sqrt{x^{2} H^{2}+2 \mathrm{~g}^{2} Q^{2}} \Delta_{1} H^{3}}{\sqrt{\frac{1}{2} x^{2} H^{2}+\mathrm{g}^{2} Q^{2}}} \\
& +\frac{3 \mathrm{~g}^{2} x^{3} \lambda Q^{2} \Delta_{2} Q_{, x} H^{3}}{\sqrt{\frac{1}{2} x^{2} H^{2}+\mathrm{g}^{2} Q^{2}}}+\frac{\mathrm{g}^{2} x^{3} \lambda Q^{3} \Delta_{2, x} H^{3}}{\sqrt{\frac{1}{2} x^{2} H^{2}+\mathrm{g}^{2} Q^{2}}} \\
& -\frac{3 \mathrm{~g}^{2} x^{2} \lambda Q^{3} \Delta_{2} H^{3}}{\sqrt{\frac{1}{2} x^{2} H^{2}+\mathrm{g}^{2} Q^{2}}}-\frac{2 \mathrm{~g} x^{3} \lambda Q \sqrt{x^{2} H^{2}+2 \mathrm{~g}^{2} Q^{2}} \Delta_{1} Q_{, x} H^{3}}{\sqrt{\frac{1}{2} x^{2} H^{2}+\mathrm{g}^{2} Q^{2}}} \\
& -\frac{\mathrm{g} x^{3} \lambda Q^{2} \sqrt{x^{2} H^{2}+2 \mathrm{~g}^{2} Q^{2}} \Delta_{1, x} H^{3}}{\sqrt{\frac{1}{2} x^{2} H^{2}+\mathrm{g}^{2} Q^{2}}}+\frac{\mathrm{g}^{2} x^{2} \lambda^{2} Q^{4} \Delta_{\chi} H^{2}}{f} \\
& -4 f \mathrm{~g}^{2} Q^{2} \Delta_{\chi} H^{2}+2 f \mathrm{~g}^{2} x^{2} Q^{2} \Delta_{\chi} H^{2}+2 f \mathrm{~g}^{2} \epsilon Q^{2} \Delta_{\chi} H^{2} \\
& +f x^{2} \Delta_{\chi} U_{, \chi, \chi} H^{2}+2 f \mathrm{~g}^{2} x^{2} Q^{2} \Delta_{\chi, x, x} H^{2} \\
& +\frac{4 \mathrm{~g}^{3} \lambda Q^{4} \sqrt{x^{2} H^{2}+2 \mathrm{~g}^{2} Q^{2}} \Delta_{1} H}{\sqrt{\frac{1}{2} x^{2} H^{2}+\mathrm{g}^{2} Q^{2}}}+\frac{4 \mathrm{~g}^{4} x \lambda Q^{4} \Delta_{2} Q_{, x} H}{\sqrt{\frac{1}{2} x^{2} H^{2}+\mathrm{g}^{2} Q^{2}}} \\
& +\frac{2 \mathrm{~g}^{4} x \lambda Q^{5} \Delta_{2, x} H}{\sqrt{\frac{1}{2} x^{2} H^{2}+\mathrm{g}^{2} Q^{2}}}-\frac{4 \mathrm{~g}^{4} \lambda Q^{5} \Delta_{2} H}{\sqrt{\frac{1}{2} x^{2} H^{2}+\mathrm{g}^{2} Q^{2}}} \\
& -\frac{4 \mathrm{~g}^{3} x \lambda Q^{3} \sqrt{x^{2} H^{2}+2 \mathrm{~g}^{2} Q^{2}} \Delta_{1} Q_{, x} H}{\sqrt{\frac{1}{2} x^{2} H^{2}+\mathrm{g}^{2} Q^{2}}} \\
& -\frac{2 \mathrm{~g}^{3} x \lambda Q^{4} \sqrt{x^{2} H^{2}+2 \mathrm{~g}^{2} Q^{2}} \Delta_{1, x} H}{\sqrt{\frac{1}{2} x^{2} H^{2}+\mathrm{g}^{2} Q^{2}}}+2 f \mathrm{~g}^{2} Q^{2} \Delta_{\chi} U_{, \chi, \chi}=0,
\end{aligned}
$$




$$
\begin{aligned}
& -\frac{\sqrt{2} x^{7} \lambda \Delta_{2} \chi_{, x} H^{7}}{f \sqrt{x^{2} H^{2}+2 \mathrm{~g}^{2} Q^{2}}}+x^{6} \sqrt{2} \Delta_{1} H^{6}+\frac{2 \mathrm{~g} x^{4} Q \sqrt{2} \Delta_{2} H^{6}}{\sqrt{x^{2} H^{2}+2 \mathrm{~g}^{2} Q^{2}}}+\frac{\mathrm{g} x^{6} \sqrt{2} \Delta_{2} Q_{, x, x} H^{6}}{\sqrt{x^{2} H^{2}+2 \mathrm{~g}^{2} Q^{2}}} \\
& +x^{6} \sqrt{2} \Delta_{1, x, x} H^{6}-\frac{3 \sqrt{2} \mathrm{~g} x^{6} Q \Delta_{2} H^{6}}{\sqrt{x^{2} H^{2}+2 \mathrm{~g}^{2} Q^{2}}}-\frac{2 \sqrt{2} \mathrm{~g} x^{5} \Delta_{2} Q_{, x} H^{6}}{\sqrt{x^{2} H^{2}+2 \mathrm{~g}^{2} Q^{2}}} \\
& -\frac{\sqrt{2} \mathrm{~g} x^{6} Q \Delta_{2, x, x} H^{6}}{\sqrt{x^{2} H^{2}+2 \mathrm{~g}^{2} Q^{2}}}+\frac{4 \mathrm{~g} x^{4} \lambda Q^{2} \Delta_{\chi} H^{5}}{f}+\frac{x^{5} \sqrt{2 x^{2} H^{2}+4 \mathrm{~g}^{2} Q^{2}} \Delta_{1} H_{, x} H^{5}}{\sqrt{x^{2} H^{2}+2 \mathrm{~g}^{2} Q^{2}}} \\
& +\frac{2 \mathrm{~g} x^{5} \lambda Q^{2} \Delta_{\chi, x} H^{5}}{f}+\frac{2 \mathrm{~g} x^{5} \lambda Q \sqrt{2} \Delta_{1} \chi_{, x} H^{5}}{f}-\frac{2 \mathrm{~g} x^{5} \lambda Q \Delta_{\chi} Q_{, x} H^{5}}{f} \\
& -\frac{2 \sqrt{2} \mathrm{~g} x^{5} Q \Delta_{2} H_{, x} H^{5}}{\sqrt{x^{2} H^{2}+2 \mathrm{~g}^{2} Q^{2}}}-\frac{6 \sqrt{2} \mathrm{~g}^{2} x^{5} \lambda Q^{2} \Delta_{2} \chi_{, x} H^{5}}{f \sqrt{x^{2} H^{2}+2 \mathrm{~g}^{2} Q^{2}}}+10 \mathrm{~g}^{2} x^{4} Q^{2} \sqrt{2} \Delta_{1} H^{4} \\
& +\frac{8 \mathrm{~g}^{3} x^{3} Q^{2} \sqrt{2} \Delta_{2} Q_{, x} H^{4}}{\sqrt{x^{2} H^{2}+2 \mathrm{~g}^{2} Q^{2}}}+\frac{2 \mathrm{~g}^{3} x^{4} Q^{2} \sqrt{2} \Delta_{2} Q_{, x, x} H^{4}}{\sqrt{x^{2} H^{2}+2 \mathrm{~g}^{2} Q^{2}}}+4 \mathrm{~g}^{2} x^{4} Q^{2} \sqrt{2} \Delta_{1, x, x} H^{4} \\
& -\frac{6 \sqrt{2} \mathrm{~g}^{3} x^{4} Q \Delta_{2} Q_{, x}^{2} H^{4}}{\sqrt{x^{2} H^{2}+2 \mathrm{~g}^{2} Q^{2}}}-\frac{18 \sqrt{2} \mathrm{~g}^{3} x^{4} Q^{3} \Delta_{2} H^{4}}{\sqrt{x^{2} H^{2}+2 \mathrm{~g}^{2} Q^{2}}}-\frac{2 \sqrt{2} \mathrm{~g}^{3} x^{2} Q^{3} \Delta_{2} H^{4}}{\sqrt{x^{2} H^{2}+2 \mathrm{~g}^{2} Q^{2}}} \\
& -\frac{4 \sqrt{2} \mathrm{~g}^{3} x^{4} Q^{3} \Delta_{2, x, x} H^{4}}{\sqrt{x^{2} H^{2}+2 \mathrm{~g}^{2} Q^{2}}}+\frac{10 \mathrm{~g}^{3} x^{2} \lambda Q^{4} \Delta_{\chi} H^{3}}{f} \\
& +\frac{4 \mathrm{~g}^{2} x^{3} Q^{2} \sqrt{2 x^{2} H^{2}+4 \mathrm{~g}^{2} Q^{2}} \Delta_{1} H_{, x} H^{3}}{\sqrt{x^{2} H^{2}+2 \mathrm{~g}^{2} Q^{2}}}+\frac{10 \mathrm{~g}^{3} x^{3} \lambda Q^{4} \Delta_{\chi, x} H^{3}}{f} \\
& +\frac{8 \mathrm{~g}^{3} x^{3} \lambda Q^{3} \sqrt{2} \Delta_{1} \chi_{, x} H^{3}}{f}-\frac{6 \sqrt{2} \mathrm{~g}^{3} x^{3} Q^{3} \Delta_{2} H_{, x} H^{3}}{\sqrt{x^{2} H^{2}+2 \mathrm{~g}^{2} Q^{2}}} \\
& -\frac{12 \sqrt{2} \mathrm{~g}^{4} x^{3} \lambda Q^{4} \Delta_{2} \chi_{, x} H^{3}}{f \sqrt{x^{2} H^{2}+2 \mathrm{~g}^{2} Q^{2}}}+28 \mathrm{~g}^{4} x^{2} Q^{4} \sqrt{2} \Delta_{1} H^{2} \\
& +4 \mathrm{~g}^{4} x^{2} Q^{4} \sqrt{2} \Delta_{1, x, x} H^{2}-\frac{36 \sqrt{2} \mathrm{~g}^{5} x^{2} Q^{5} \Delta_{2} H^{2}}{\sqrt{x^{2} H^{2}+2 \mathrm{~g}^{2} Q^{2}}} \\
& -\frac{4 \sqrt{2} \mathrm{~g}^{5} x^{2} Q^{5} \Delta_{2, x, x} H^{2}}{\sqrt{x^{2} H^{2}+2 \mathrm{~g}^{2} Q^{2}}}+\frac{12 \mathrm{~g}^{5} \lambda Q^{6} \Delta_{\chi} H}{f} \\
& +\frac{4 \mathrm{~g}^{4} x Q^{4} \sqrt{2 x^{2} H^{2}+4 \mathrm{~g}^{2} Q^{2}} \Delta_{1} H_{, x} H}{\sqrt{x^{2} H^{2}+2 \mathrm{~g}^{2} Q^{2}}}+\frac{12 \mathrm{~g}^{5} x \lambda Q^{6} \Delta_{\chi, x} H}{f} \\
& +\frac{8 \mathrm{~g}^{5} x \lambda Q^{5} \sqrt{2} \Delta_{1} \chi_{, x} H}{f}-\frac{4 \sqrt{2} \mathrm{~g}^{5} x Q^{5} \Delta_{2} H_{, x} H}{\sqrt{x^{2} H^{2}+2 \mathrm{~g}^{2} Q^{2}}} \\
& -\frac{8 \sqrt{2} \mathrm{~g}^{6} x \lambda Q^{6} \Delta_{2} \chi_{, x} H}{f \sqrt{x^{2} H^{2}+2 \mathrm{~g}^{2} Q^{2}}}+24 \mathrm{~g}^{6} Q^{6} \sqrt{2} \Delta_{1}-\frac{24 \sqrt{2} \mathrm{~g}^{7} Q^{7} \Delta_{2}}{\sqrt{x^{2} H^{2}+2 \mathrm{~g}^{2} Q^{2}}}=0,
\end{aligned}
$$




$$
\begin{aligned}
& \frac{\mathrm{g} x^{7} Q \sqrt{2} \Delta_{2} H^{7}}{\sqrt{x^{2} H^{2}+2 \mathrm{~g}^{2} Q^{2}}}+\frac{2 \mathrm{~g} x^{6} \sqrt{2} \Delta_{2} Q_{, x} H^{7}}{\sqrt{x^{2} H^{2}+2 \mathrm{~g}^{2} Q^{2}}}+\frac{\mathrm{g} x^{7} Q \sqrt{2} \Delta_{2, x, x} H^{7}}{\sqrt{x^{2} H^{2}+2 \mathrm{~g}^{2} Q^{2}}} \\
& -\frac{2 \sqrt{2} \mathrm{~g} x^{5} Q \Delta_{2} H^{7}}{\sqrt{x^{2} H^{2}+2 \mathrm{~g}^{2} Q^{2}}}-\frac{\sqrt{2} \mathrm{~g} x^{7} \Delta_{2} Q_{, x, x} H^{7}}{\sqrt{x^{2} H^{2}+2 \mathrm{~g}^{2} Q^{2}}}+\frac{2 \mathrm{~g} x^{6} Q \sqrt{2} \Delta_{2} H_{, x} H^{6}}{\sqrt{x^{2} H^{2}+2 \mathrm{~g}^{2} Q^{2}}} \\
& +\frac{2 \mathrm{~g} x^{6} \lambda Q \Delta_{\chi} Q_{, x} H^{6}}{f}-\frac{2 \mathrm{~g} x^{5} \lambda Q^{2} \Delta_{\chi} H^{6}}{f}-\frac{\sqrt{2} \mathrm{~g} x^{6} \lambda Q \Delta_{1} \chi_{, x} H^{6}}{f} \\
& +\frac{6 \mathrm{~g}^{3} x^{5} Q \sqrt{2} \Delta_{2} Q_{, x}^{2} H^{5}}{\sqrt{x^{2} H^{2}+2 \mathrm{~g}^{2} Q^{2}}}-2 \sqrt{2} \mathrm{~g}^{2} x^{5} Q^{2} \Delta_{1} H^{5} \\
& +\frac{6 \mathrm{~g}^{3} x^{5} Q^{3} \sqrt{2} \Delta_{2} H^{5}}{\sqrt{x^{2} H^{2}+2 \mathrm{~g}^{2} Q^{2}}}+\frac{2 \mathrm{~g}^{3} x^{3} Q^{3} \sqrt{2} \Delta_{2} H^{5}}{\sqrt{x^{2} H^{2}+2 \mathrm{~g}^{2} Q^{2}}} \\
& +\frac{4 \mathrm{~g}^{3} x^{5} Q^{3} \sqrt{2} \Delta_{2, x, x} H^{5}}{\sqrt{x^{2} H^{2}+2 \mathrm{~g}^{2} Q^{2}}}-\frac{8 \sqrt{2} \mathrm{~g}^{3} x^{4} Q^{2} \Delta_{2} Q_{, x} H^{5}}{\sqrt{x^{2} H^{2}+2 \mathrm{~g}^{2} Q^{2}}} \\
& -\frac{2 \sqrt{2} \mathrm{~g}^{3} x^{5} Q^{2} \Delta_{2} Q_{, x, x} H^{5}}{\sqrt{x^{2} H^{2}+2 \mathrm{~g}^{2} Q^{2}}}+\frac{6 \mathrm{~g}^{3} x^{4} Q^{3} \sqrt{2} \Delta_{2} H_{, x} H^{4}}{\sqrt{x^{2} H^{2}+2 \mathrm{~g}^{2} Q^{2}}} \\
& -\frac{2 \mathrm{~g}^{3} x^{3} \lambda Q^{4} \Delta_{\chi} H^{4}}{f}-\frac{2 \mathrm{~g}^{3} x^{4} \lambda Q^{4} \Delta_{\chi, x} H^{4}}{f} \\
& -\frac{4 \sqrt{2} \mathrm{~g}^{3} x^{4} \lambda Q^{3} \Delta_{1} \chi_{, x} H^{4}}{f}-8 \sqrt{2} \mathrm{~g}^{4} x^{3} Q^{4} \Delta_{1} H^{3} \\
& +\frac{12 \mathrm{~g}^{5} x^{3} Q^{5} \sqrt{2} \Delta_{2} H^{3}}{\sqrt{x^{2} H^{2}+2 \mathrm{~g}^{2} Q^{2}}}+\frac{4 \mathrm{~g}^{5} x^{3} Q^{5} \sqrt{2} \Delta_{2, x, x} H^{3}}{\sqrt{x^{2} H^{2}+2 \mathrm{~g}^{2} Q^{2}}} \\
& +\frac{4 \mathrm{~g}^{5} x^{2} Q^{5} \sqrt{2} \Delta_{2} H_{, x} H^{2}}{\sqrt{x^{2} H^{2}+2 \mathrm{~g}^{2} Q^{2}}}-\frac{4 \mathrm{~g}^{5} x \lambda Q^{6} \Delta_{\chi} H^{2}}{f} \\
& -\frac{4 \mathrm{~g}^{5} x^{2} \lambda Q^{6} \Delta_{\chi_{, x}} H^{2}}{f}-\frac{4 \sqrt{2} \mathrm{~g}^{5} x^{2} \lambda Q^{5} \Delta_{1} \chi_{, x} H^{2}}{f} \\
& -8 \sqrt{2} \mathrm{~g}^{6} x Q^{6} \Delta_{1} H+\frac{8 \mathrm{~g}^{7} x Q^{7} \sqrt{2} \Delta_{2} H}{\sqrt{x^{2} H^{2}+2 \mathrm{~g}^{2} Q^{2}}}=0
\end{aligned}
$$

where the scalar perturbations are defined through

$$
\delta \phi=\frac{\Delta_{\phi}}{a}, \quad \delta \chi=\frac{\Delta_{\chi}}{a}, \quad \delta Q=\frac{\Delta_{1}}{\sqrt{2} a}, \quad M=\frac{a \mathrm{~g} Q \Delta_{1}+\sqrt{k^{2}+2 a^{2} \mathrm{~g}^{2} Q^{2}} \Delta_{2}}{\sqrt{2} \mathrm{~g} a^{2} k^{2} Q} .
$$




\section{Appendix C}

\section{Full perturbation equations for chapter 5}

In this appendix we write the full perturbation equations for chapter 5. We exclude the scalar equations including the metric perturbations as these are infeasibly long.

\section{C.1 Example 2}

Considering first example 2 in chapter 5, and looking at the tensor equations of motion for the right-helicity modes. Recalling that

$$
\psi_{L, R}:=\frac{a M_{\mathrm{pl}}}{2}\left(h_{+} \pm i h_{\times}\right), \quad t_{L, R}:=a\left(T_{+} \pm i T_{\times}\right)
$$

the full equations of motion for $t_{R}$ and $\psi_{R}$ are

$$
\begin{aligned}
2 \mathrm{~g}^{2} Q^{3} \psi_{R}= & -4 H^{2} Q \xi_{f} \psi_{R}+4 H^{2} x \xi_{f} Q_{, x} \psi_{R}-2 H^{2} x \xi_{f} t_{R, x}+2 \mathrm{~g} H Q \xi_{h} t_{R}+2 \mathrm{~g} H Q^{2} x \psi_{R} \\
& -2 \mathrm{~g} H Q x t_{R}-2 H^{2} x \xi_{h} t_{R}+2 H^{2} x Q_{, x} \psi_{R}-2 H^{2} x^{2} Q_{, x, x} \psi_{R}-2 H^{2} Q \psi_{R} \\
& +2 H^{2} Q \epsilon \psi_{R}-2 H^{2} x^{2} Q_{, x} \psi_{R, x}+2 H^{2} Q x \psi_{R, x}+H^{2} x^{2} t_{R}+H^{2} x^{2} t_{R, x, x}
\end{aligned}
$$




$$
\begin{aligned}
& -\frac{3 \alpha H^{2} c_{1}^{2} \lambda_{4} \xi_{h}^{2} \psi_{R} \sqrt{\tau_{2} \tau_{4}^{3}}}{\mathcal{V} c_{2}^{2} \sqrt{\tau_{2}}}-3 \mathrm{~g}^{2} Q^{4} c_{1} \tau_{4} \psi_{R}+2 \mathrm{~g}^{2} Q^{3} c_{1} \tau_{4} t_{R} \\
& -2 \mathrm{~g} H Q^{2} x c_{1} \tau_{4} t_{R}+H^{2} Q^{2} c_{1} \tau_{4} \psi_{R}+H^{2} x^{2} c_{1} \tau_{4} Q_{, x}^{2} \psi_{R} \\
& -2 H^{2} Q x c_{1} \tau_{4} Q_{, x} \psi_{R}-2 H^{2} x^{2} c_{1} \tau_{4} Q_{, x} t_{R, x}+2 H^{2} Q x c_{1} \tau_{4} t_{R, x} \\
& -\frac{3 \alpha H^{2} x^{2} \lambda_{2} \tau_{2, x}^{2} \psi_{R}}{4 \mathcal{V} \sqrt{\tau_{2}}}-\frac{3 \alpha H^{2} \lambda_{4} \xi_{f}^{2} \psi_{R} \sqrt{\tau_{2} \tau_{4}^{3}}}{\mathcal{V} \sqrt{\tau_{2}}}-H^{2} x^{2} \psi_{R} \\
& -4 H^{2} \psi_{R}+3 H^{2} \epsilon \psi_{R}-H^{2} x^{2} \psi_{R, x, x}+2 V \psi_{R}=0
\end{aligned}
$$

where, $x$ means derivatives with respect to $x=\frac{k}{a H}$.

The scalar perturbations for example 2 are split as

$$
\begin{array}{ll}
\delta \tau_{2}=\frac{\Delta_{\tau_{2}}}{a}, & \delta \tau_{4}=\frac{\Delta_{\tau_{4}}}{a}, \quad \delta b_{4}=\frac{\Delta_{b_{4}}}{a} \\
\delta Q=\frac{\Delta_{1}}{\sqrt{2} a}, & M=\frac{a \mathrm{~g} Q \Delta_{1}+\sqrt{k^{2}+2 a^{2} \mathrm{~g}^{2} Q^{2}} \Delta_{2}}{\sqrt{2} \mathrm{~g} a^{2} k^{2} Q}
\end{array}
$$

and their equations of motion (with the metric perturbations set to zero) are as follows

$$
\begin{aligned}
& \frac{32 \mathrm{~g}^{5} \sqrt{2} \sqrt{\tau_{4}} \sqrt{\left(2 \mathrm{~g}^{2} Q^{2}+H^{2} x^{2}\right) \tau_{4}} c_{1} c_{2} \Delta_{2} Q^{6}}{2 \mathrm{~g}^{2} Q^{2}+H^{2} x^{2}} \\
& -\frac{32 \mathrm{~g}^{4} \sqrt{4 \mathrm{~g}^{2} Q^{2}+2 H^{2} x^{2}} c_{1} c_{2} \Delta_{1} \sqrt{\tau_{4}} \sqrt{\left(2 \mathrm{~g}^{2} Q^{2}+H^{2} x^{2}\right) \tau_{4}} Q^{5}}{2 \mathrm{~g}^{2} Q^{2}+H^{2} x^{2}} \\
& +\frac{32 \mathrm{~g}^{3} H^{2} x^{2} \sqrt{2} \sqrt{\tau_{4}} \sqrt{\left(2 \mathrm{~g}^{2} Q^{2}+H^{2} x^{2}\right) \tau_{4}} c_{1} c_{2} \Delta_{2} Q^{4}}{2 \mathrm{~g}^{2} Q^{2}+H^{2} x^{2}} \\
& +\frac{16 \mathrm{~g}^{3} H^{2} x \sqrt{2} \sqrt{\tau_{4}} \sqrt{\left(2 \mathrm{~g}^{2} Q^{2}+H^{2} x^{2}\right) \tau_{4}} c_{1} c_{2} \Delta_{2, x} Q^{4}}{2 \mathrm{~g}^{2} Q^{2}+H^{2} x^{2}}+8 \mathrm{~g} H^{3} x^{2} c_{2}^{2} \Delta_{b_{4}} Q^{3} \\
& -\frac{16 \sqrt{2} \mathrm{~g}^{3} H^{2} x^{2} c_{1} c_{2} Q_{, x} \Delta_{2, x} \sqrt{\tau_{4}} \sqrt{\left(2 \mathrm{~g}^{2} Q^{2}+H^{2} x^{2}\right) \tau_{4}} Q^{3}}{2 \mathrm{~g}^{2} Q^{2}+H^{2} x^{2}} \\
& -\frac{16 \sqrt{2} \mathrm{~g}^{2} H^{2} x^{2} c_{1} c_{2} \Delta_{1} \sqrt{\tau_{4}} \sqrt{\left(2 \mathrm{~g}^{2} Q^{2}+H^{2} x^{2}\right) \tau_{4}} Q^{3}}{\sqrt{2 \mathrm{~g}^{2} Q^{2}+H^{2} x^{2}}} \\
& +\frac{18 \mathrm{~g}^{2} H^{2} \alpha \sqrt{\left(2 \mathrm{~g}^{2} Q^{2}+H^{2} x^{2}\right) \tau_{4}} c_{1}^{2} \Delta_{\tau_{4}} \lambda_{4} \xi_{h}^{2} Q_{2} \Delta_{1, x} \sqrt{\tau_{4}} \sqrt{\left(2 \mathrm{~g}^{2} Q^{2}+H^{2} x^{2}\right) \tau_{4}} Q^{3}}{\sqrt{2 \mathrm{~g}^{2} Q^{2}+H^{2} x^{2}} V_{c_{2}}}
\end{aligned}
$$




$$
\begin{aligned}
& +\frac{16 \mathrm{~g}^{2} H^{2} x^{2} \sqrt{2} \sqrt{\tau_{4}} \sqrt{\left(2 \mathrm{~g}^{2} Q^{2}+H^{2} x^{2}\right) \tau_{4}} c_{1} c_{2} Q_{, x} \Delta_{1, x} Q^{2}}{\sqrt{2 \mathrm{~g}^{2} Q^{2}+H^{2} x^{2}}} \\
& +\frac{8 \mathrm{~g} H^{4} x^{4} \sqrt{2} \sqrt{\tau_{4}} \sqrt{\left(2 \mathrm{~g}^{2} Q^{2}+H^{2} x^{2}\right) \tau_{4}} c_{1} c_{2} \Delta_{2} Q^{2}}{2 \mathrm{~g}^{2} Q^{2}+H^{2} x^{2}} \\
& +\frac{8 \mathrm{~g} H^{4} x^{2} \sqrt{2} \sqrt{\tau_{4}} \sqrt{\left(2 \mathrm{~g}^{2} Q^{2}+H^{2} x^{2}\right) \tau_{4}} c_{1} c_{2} \Delta_{2} Q^{2}}{2 \mathrm{~g}^{2} Q^{2}+H^{2} x^{2}} \\
& +\frac{8 \mathrm{~g} H^{4} x^{3} \sqrt{2} \sqrt{\tau_{4}} \sqrt{\left(2 \mathrm{~g}^{2} Q^{2}+H^{2} x^{2}\right) \tau_{4}} c_{1} c_{2} \Delta_{2, x} Q^{2}}{2 \mathrm{~g}^{2} Q^{2}+H^{2} x^{2}}-8 \mathrm{~g} H^{3} x^{3} c_{2}^{2} Q_{, x} \Delta_{b_{4}} Q^{2} \\
& -8 H^{4} x^{2} c_{1} c_{2} \Delta_{\tau_{4}} Q^{2}+\frac{24 \mathrm{~g}^{2} H^{2} \alpha \sqrt{\left(2 \mathrm{~g}^{2} Q^{2}+H^{2} x^{2}\right) \tau_{4}} c_{2} \Delta_{\tau_{4}} \lambda_{4} Q^{2}}{\sqrt{2 \mathrm{~g}^{2} Q^{2}+H^{2} x^{2}} \mathcal{V}} \\
& +\frac{12 \mathrm{~g}^{2} H^{2} \alpha \sqrt{\left(2 \mathrm{~g}^{2} Q^{2}+H^{2} x^{2}\right) \tau_{4}} c_{2} \Delta_{\tau_{4}} \lambda_{4} \xi_{f} Q^{2}}{\sqrt{2 \mathrm{~g}^{2} Q^{2}+H^{2} x^{2}} \mathcal{V}} \\
& +\frac{12 \mathrm{~g}^{2} H^{2} \alpha \sqrt{\left(2 \mathrm{~g}^{2} Q^{2}+H^{2} x^{2}\right) \tau_{4}} c_{1} \Delta_{b_{4}} \lambda_{4} \xi_{h} Q^{2}}{\sqrt{2 \mathrm{~g}^{2} Q^{2}+H^{2} x^{2}} \mathcal{V}} \\
& +\frac{12 \mathrm{~g}^{2} H^{2} x \alpha \sqrt{\left(2 \mathrm{~g}^{2} Q^{2}+H^{2} x^{2}\right) \tau_{4}} c_{1} \Delta_{b_{4}, x} \lambda_{4} \xi_{h} Q^{2}}{\sqrt{2 \mathrm{~g}^{2} Q^{2}+H^{2} x^{2}} \mathcal{V}}
\end{aligned}
$$

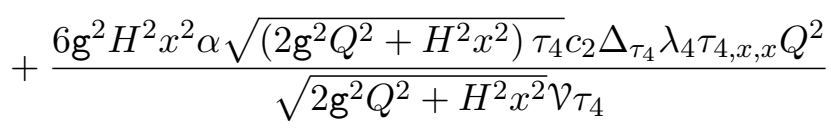




$$
\begin{aligned}
& -\frac{18 \mathrm{~g}^{2} H^{2} \alpha c_{2} \Delta_{\tau_{4}} \lambda_{4} \xi_{f}^{2} \sqrt{\left(2 \mathrm{~g}^{2} Q^{2}+H^{2} x^{2}\right) \tau_{4}} Q^{2}}{\sqrt{2 \mathrm{~g}^{2} Q^{2}+H^{2} x^{2}} \mathcal{V}} \\
& -\frac{12 \mathrm{~g}^{2} H^{2} x^{2} \alpha c_{2} \Delta_{\tau_{4}} \lambda_{4} \sqrt{\left(2 \mathrm{~g}^{2} Q^{2}+H^{2} x^{2}\right) \tau_{4}} Q^{2}}{\sqrt{2 \mathrm{~g}^{2} Q^{2}+H^{2} x^{2}} \mathcal{V}} \\
& -\frac{12 \mathrm{~g}^{2} H^{2} \alpha \epsilon c_{2} \Delta_{\tau_{4}} \lambda_{4} \sqrt{\left(2 \mathrm{~g}^{2} Q^{2}+H^{2} x^{2}\right) \tau_{4}} Q^{2}}{\sqrt{2 \mathrm{~g}^{2} Q^{2}+H^{2} x^{2}} \mathcal{V}} \\
& -\frac{12 \mathrm{~g}^{2} H^{2} x^{2} \alpha c_{2} \Delta_{\tau_{4}, x, x} \lambda_{4} \sqrt{\left(2 \mathrm{~g}^{2} Q^{2}+H^{2} x^{2}\right) \tau_{4}} Q^{2}}{\sqrt{2 \mathrm{~g}^{2} Q^{2}+H^{2} x^{2}} \mathcal{V}} \\
& -\frac{12 \mathrm{~g}^{2} H^{2} x \alpha c_{2} \Delta_{\tau_{4}, x} \lambda_{4} \xi_{f} \sqrt{\left(2 \mathrm{~g}^{2} Q^{2}+H^{2} x^{2}\right) \tau_{4}} Q^{2}}{\sqrt{2 \mathrm{~g}^{2} Q^{2}+H^{2} x^{2}} \mathcal{V}} \\
& -\frac{16 \mathrm{~g}^{2} c_{2} V_{, b_{4}, \tau_{4}} \Delta_{b_{4}} \sqrt{\tau_{4}} \sqrt{\left(2 \mathrm{~g}^{2} Q^{2}+H^{2} x^{2}\right) \tau_{4}} Q^{2}}{\sqrt{2 \mathrm{~g}^{2} Q^{2}+H^{2} x^{2}}} \\
& -\frac{16 \mathrm{~g}^{2} c_{2} V_{, \tau_{2}, \tau_{4}} \Delta_{\tau_{2}} \sqrt{\tau_{4}} \sqrt{\left(2 \mathrm{~g}^{2} Q^{2}+H^{2} x^{2}\right) \tau_{4}} Q^{2}}{\sqrt{2 \mathrm{~g}^{2} Q^{2}+H^{2} x^{2}}} \\
& -\frac{16 \mathrm{~g}^{2} c_{2} V_{, \tau_{4}, \tau_{4}} \Delta_{\tau_{4}} \sqrt{\tau_{4}} \sqrt{\left(2 \mathrm{~g}^{2} Q^{2}+H^{2} x^{2}\right) \tau_{4}} Q^{2}}{\sqrt{2 \mathrm{~g}^{2} Q^{2}+H^{2} x^{2}}} \\
& +16 H^{4} x^{3} c_{1} c_{2} Q_{, x} \Delta_{\tau_{4}} Q-8 \sqrt{2} H^{4} x^{3} c_{1} c_{2} \Delta_{1, x} \tau_{4} Q \\
& -\frac{16 \sqrt{2} \mathrm{~g} H^{4} x^{3} c_{1} c_{2} Q_{, x} \Delta_{2} \sqrt{\tau_{4}} \sqrt{\left(2 \mathrm{~g}^{2} Q^{2}+H^{2} x^{2}\right) \tau_{4}} Q}{2 \mathrm{~g}^{2} Q^{2}+H^{2} x^{2}} \\
& -\frac{8 \sqrt{2} \mathrm{~g} H^{4} x^{4} c_{1} c_{2} Q_{, x} \Delta_{2, x} \sqrt{\tau_{4}} \sqrt{\left(2 \mathrm{~g}^{2} Q^{2}+H^{2} x^{2}\right) \tau_{4}} Q}{2 \mathrm{~g}^{2} Q^{2}+H^{2} x^{2}} \\
& +\frac{9 H^{4} x^{2} \alpha \sqrt{\left(2 \mathrm{~g}^{2} Q^{2}+H^{2} x^{2}\right) \tau_{4}} c_{1}^{2} \Delta_{\tau_{4}} \lambda_{4} \xi_{h}^{2}}{\sqrt{2 \mathrm{~g}^{2} Q^{2}+H^{2} x^{2}} \mathcal{c}_{2}} \\
& +\frac{8 \mathrm{~g} H^{4} x^{4} \sqrt{2} \sqrt{\tau_{4}} \sqrt{\left(2 \mathrm{~g}^{2} Q^{2}+H^{2} x^{2}\right) \tau_{4}} c_{1} c_{2} Q_{, x}^{2} \Delta_{2}}{2 \mathrm{~g}^{2} Q^{2}+H^{2} x^{2}} \\
& -8 H^{4} x^{4} c_{1} c_{2} Q_{, x}^{2} \Delta_{\tau_{4}}+\frac{12 H^{4} x^{2} \alpha \sqrt{\left(2 \mathrm{~g}^{2} Q^{2}+H^{2} x^{2}\right) \tau_{4}} c_{2} \Delta_{\tau_{4}} \lambda_{4}}{\sqrt{2 \mathrm{~g}^{2} Q^{2}+H^{2} x^{2}} \mathcal{V}} \\
& +\frac{6 H^{4} x^{2} \alpha \sqrt{\left(2 \mathrm{~g}^{2} Q^{2}+H^{2} x^{2}\right) \tau_{4}} c_{2} \Delta_{\tau_{4}} \lambda_{4} \xi_{f}}{\sqrt{2 \mathrm{~g}^{2} Q^{2}+H^{2} x^{2}} \mathcal{V}} \\
& +\frac{6 H^{4} x^{2} \alpha \sqrt{\left(2 \mathrm{~g}^{2} Q^{2}+H^{2} x^{2}\right) \tau_{4}} c_{1} \Delta_{b_{4}} \lambda_{4} \xi_{h}}{\sqrt{2 \mathrm{~g}^{2} Q^{2}+H^{2} x^{2}} \mathcal{V}} \\
& +\frac{6 H^{4} x^{3} \alpha \sqrt{\left(2 \mathrm{~g}^{2} Q^{2}+H^{2} x^{2}\right) \tau_{4}} c_{1} \Delta_{b_{4}, x} \lambda_{4} \xi_{h}}{\sqrt{2 \mathrm{~g}^{2} Q^{2}+H^{2} x^{2}} \mathcal{V}} \\
& +8 H^{4} x^{4} \sqrt{2} c_{1} c_{2} Q_{, x} \Delta_{1, x} \tau_{4}
\end{aligned}
$$




$$
\begin{aligned}
& +\frac{3 H^{4} x^{4} \alpha \sqrt{\left(2 \mathrm{~g}^{2} Q^{2}+H^{2} x^{2}\right) \tau_{4}} c_{2} \Delta_{\tau_{4}} \lambda_{4} \tau_{4, x, x}}{\sqrt{2 \mathrm{~g}^{2} Q^{2}+H^{2} x^{2}} \nu \tau_{4}} \\
& -\frac{9 H^{4} x^{2} \alpha c_{2} \Delta_{\tau_{4}} \lambda_{4} \xi_{f}^{2} \sqrt{\left(2 \mathrm{~g}^{2} Q^{2}+H^{2} x^{2}\right) \tau_{4}}}{\sqrt{2 \mathrm{~g}^{2} Q^{2}+H^{2} x^{2}} \mathcal{V}} \\
& -\frac{6 H^{4} x^{4} \alpha c_{2} \Delta_{\tau_{4}} \lambda_{4} \sqrt{\left(2 \mathrm{~g}^{2} Q^{2}+H^{2} x^{2}\right) \tau_{4}}}{\sqrt{2 \mathrm{~g}^{2} Q^{2}+H^{2} x^{2}} \mathcal{V}} \\
& -\frac{6 H^{4} x^{2} \alpha \epsilon c_{2} \Delta_{\tau_{4}} \lambda_{4} \sqrt{\left(2 \mathrm{~g}^{2} Q^{2}+H^{2} x^{2}\right) \tau_{4}}}{\sqrt{2 \mathrm{~g}^{2} Q^{2}+H^{2} x^{2}} \mathcal{V}} \\
& -\frac{6 H^{4} x^{4} \alpha c_{2} \Delta_{\tau_{4}, x, x} \lambda_{4} \sqrt{\left(2 \mathrm{~g}^{2} Q^{2}+H^{2} x^{2}\right) \tau_{4}}}{\sqrt{2 \mathrm{~g}^{2} Q^{2}+H^{2} x^{2}} \mathcal{V}} \\
& -\frac{6 H^{4} x^{3} \alpha c_{2} \Delta_{\tau_{4}, x_{4}} \lambda_{4} \xi_{f} \sqrt{\left(2 \mathrm{~g}^{2} Q^{2}+H^{2} x^{2}\right) \tau_{4}}}{\sqrt{2 \mathrm{~g}^{2} Q^{2}+H^{2} x^{2}} \mathcal{V}} \\
& -\frac{8 H^{2} x^{2} c_{2} V_{, b_{4}, \tau_{4}} \Delta_{b_{4}} \sqrt{\tau_{4}} \sqrt{\left(2 \mathrm{~g}^{2} Q^{2}+H^{2} x^{2}\right) \tau_{4}}}{\sqrt{2 \mathrm{~g}^{2} Q^{2}+\tau_{4}} \Delta_{\tau_{2}} \sqrt{\tau_{4} x^{2}} \sqrt{\left(2 \mathrm{~g}^{2} Q^{2}+H^{2} x^{2}\right) \tau_{4}}} \\
& \sqrt{2 \mathrm{~g}^{2} Q^{2}+H^{2} x^{2}}
\end{aligned}
$$




$$
\begin{aligned}
& \frac{8 x^{5} \sqrt{2} \sqrt{\tau_{4}} \sqrt{\left(2 \mathrm{~g}^{2} Q^{2}+H^{2} x^{2}\right) \tau_{4}} c_{2} Q_{, x} \Delta_{2} H^{5}}{2 \mathrm{~g}^{2} Q^{2}+H^{2} x^{2}} \\
& -\frac{8 \sqrt{2} Q x^{4} c_{2} \Delta_{2} \sqrt{\tau_{4}} \sqrt{\left(2 \mathrm{~g}^{2} Q^{2}+H^{2} x^{2}\right) \tau_{4}} H^{5}}{2 \mathrm{~g}^{2} Q^{2}+H^{2} x^{2}} \\
& +\frac{6 x^{4} \alpha \sqrt{\left(2 \mathrm{~g}^{2} Q^{2}+H^{2} x^{2}\right) \tau_{4}} \Delta_{b_{4}} \lambda_{4} H^{4}}{\sqrt{2 \mathrm{~g}^{2} Q^{2}+H^{2} x^{2}} \mathcal{V}} \\
& +\frac{6 x^{2} \alpha \epsilon \sqrt{\left(2 \mathrm{~g}^{2} Q^{2}+H^{2} x^{2}\right) \tau_{4}} \Delta_{b_{4}} \lambda_{4} H^{4}}{\sqrt{2 \mathrm{~g}^{2} Q^{2}+H^{2} x^{2}} \mathcal{V}} \\
& +\frac{6 x^{4} \alpha \sqrt{\left(2 \mathrm{~g}^{2} Q^{2}+H^{2} x^{2}\right) \tau_{4}} \Delta_{b_{4}, x, x} \lambda_{4} H^{4}}{\sqrt{2 \mathrm{~g}^{2} Q^{2}+H^{2} x^{2}} \nu} \\
& +\frac{6 x^{2} \alpha \sqrt{\left(2 \mathrm{~g}^{2} Q^{2}+H^{2} x^{2}\right) \tau_{4}} \Delta_{b_{4}} \lambda_{4} \xi_{f} H^{4}}{\sqrt{2 \mathrm{~g}^{2} Q^{2}+H^{2} x^{2}} \mathcal{V}} \\
& +\frac{6 x^{3} \alpha \sqrt{\left(2 \mathrm{~g}^{2} Q^{2}+H^{2} x^{2}\right) \tau_{4}} \Delta_{b_{4}, x} \lambda_{4} \xi_{f} H^{4}}{\sqrt{2 \mathrm{~g}^{2} Q^{2}+H^{2} x^{2}} \mathcal{V}} \\
& +\frac{6 x^{3} \alpha \sqrt{\left(2 \mathrm{~g}^{2} Q^{2}+H^{2} x^{2}\right) \tau_{4}} c_{1} \Delta_{\tau_{4}, x} \lambda_{4} \xi_{h} H^{4}}{\sqrt{2 \mathrm{~g}^{2} Q^{2}+H^{2} x^{2}} \nu_{c_{2}}} \\
& +\frac{18 x^{2} \alpha \sqrt{\left(2 \mathrm{~g}^{2} Q^{2}+H^{2} x^{2}\right) \tau_{4}} c_{1} \Delta_{\tau_{4}} \lambda_{4} \xi_{f} \xi_{h} H^{4}}{\sqrt{2 \mathrm{~g}^{2} Q^{2}+H^{2} x^{2}} v c_{2}} \\
& -\frac{12 x^{2} \alpha \Delta_{b_{4}} \lambda_{4} \sqrt{\left(2 \mathrm{~g}^{2} Q^{2}+H^{2} x^{2}\right) \tau_{4}} H^{4}}{\sqrt{2 \mathrm{~g}^{2} Q^{2}+H^{2} x^{2}} \mathcal{V}} \\
& -\frac{6 x^{2} \alpha c_{1} \Delta_{\tau_{4}} \lambda_{4} \xi_{h} \sqrt{\left(2 \mathrm{~g}^{2} Q^{2}+H^{2} x^{2}\right) \tau_{4}} H^{4}}{\sqrt{2 \mathrm{~g}^{2} Q^{2}+H^{2} x^{2}} \mathcal{V}_{2}} \\
& -\frac{3 x^{4} \alpha b_{4, x, x} \Delta_{\tau_{4}} \lambda_{4} \sqrt{\left(2 \mathrm{~g}^{2} Q^{2}+H^{2} x^{2}\right) \tau_{4}} H^{4}}{\sqrt{2 \mathrm{~g}^{2} Q^{2}+H^{2} x^{2}} \mathcal{V} \tau_{4}} \\
& +\frac{16 \mathrm{~g} Q^{2} x^{2} \sqrt{4 \mathrm{~g}^{2} Q^{2}+2 H^{2} x^{2}} \sqrt{\tau_{4}} \sqrt{\left(2 \mathrm{~g}^{2} Q^{2}+H^{2} x^{2}\right) \tau_{4}} c_{2} \Delta_{1} H^{3}}{2 \mathrm{~g}^{2} Q^{2}+H^{2} x^{2}}
\end{aligned}
$$




$$
\begin{aligned}
& +\frac{24 \mathrm{~g}^{2} Q^{2} x^{3} \sqrt{2} \sqrt{\tau_{4}} \sqrt{\left(2 \mathrm{~g}^{2} Q^{2}+H^{2} x^{2}\right) \tau_{4}} c_{2} Q_{, x} \Delta_{2} H^{3}}{2 \mathrm{~g}^{2} Q^{2}+H^{2} x^{2}} \\
& +\frac{8 \mathrm{~g}^{2} Q^{3} x^{3} \sqrt{2} \sqrt{\tau_{4}} \sqrt{\left(2 \mathrm{~g}^{2} Q^{2}+H^{2} x^{2}\right) \tau_{4}} c_{2} \Delta_{2, x} H^{3}}{2 \mathrm{~g}^{2} Q^{2}+H^{2} x^{2}} \\
& -8 \mathrm{~g} Q^{3} x^{2} c_{2} \Delta_{\tau_{4}} H^{3}+8 \mathrm{~g} Q^{2} x^{3} c_{2} Q_{, x} \Delta_{\tau_{4}} H^{3} \\
& -\frac{16 \mathrm{~g} Q x^{3} \sqrt{4 \mathrm{~g}^{2} Q^{2}+2 H^{2} x^{2}} c_{2} Q_{, x} \Delta_{1} \sqrt{\tau_{4}} \sqrt{\left(2 \mathrm{~g}^{2} Q^{2}+H^{2} x^{2}\right) \tau_{4}} H^{3}}{2 \mathrm{~g}^{2} Q^{2}+H^{2} x^{2}} \\
& -\frac{8 \mathrm{~g} Q^{2} x^{3} \sqrt{4 \mathrm{~g}^{2} Q^{2}+2 H^{2} x^{2}} c_{2} \Delta_{1, x} \sqrt{\tau_{4}} \sqrt{\left(2 \mathrm{~g}^{2} Q^{2}+H^{2} x^{2}\right) \tau_{4}} H^{3}}{2 \mathrm{~g}^{2} Q^{2}+H^{2} x^{2}} \\
& -\frac{24 \sqrt{2} \mathrm{~g}^{2} Q^{3} x^{2} c_{2} \Delta_{2} \sqrt{\tau_{4}} \sqrt{\left(2 \mathrm{~g}^{2} Q^{2}+H^{2} x^{2}\right) \tau_{4}} H^{3}}{2 \mathrm{~g}^{2} Q^{2}+H^{2} x^{2}} \\
& +\frac{8 \mathrm{~g}^{2} Q^{4} x^{2} c_{2}^{2} \Delta_{b_{4}} H^{2}}{c_{1}}+\frac{8 x^{2} \sqrt{\tau_{4}} \sqrt{\left(2 \mathrm{~g}^{2} Q^{2}+H^{2} x^{2}\right) \tau_{4}} V_{, b_{4}, b_{4}} \Delta_{b_{4}} H^{2}}{\sqrt{2 \mathrm{~g}^{2} Q^{2}+H^{2} x^{2}}} \\
& +\frac{8 x^{2} \sqrt{\tau_{4}} \sqrt{\left(2 \mathrm{~g}^{2} Q^{2}+H^{2} x^{2}\right) \tau_{4}} V_{, \tau_{2}, b_{4}} \Delta_{\tau_{2}} H^{2}}{\sqrt{2 \mathrm{~g}^{2} Q^{2}+H^{2} x^{2}}} \\
& +\frac{8 x^{2} \sqrt{\tau_{4}} \sqrt{\left(2 \mathrm{~g}^{2} Q^{2}+H^{2} x^{2}\right) \tau_{4}} V_{, b_{4}, \tau_{4}} \Delta_{\tau_{4}} H^{2}}{\sqrt{2 \mathrm{~g}^{2} Q^{2}+H^{2} x^{2}}} \\
& +\frac{12 \mathrm{~g}^{2} Q^{2} x^{2} \alpha \sqrt{\left(2 \mathrm{~g}^{2} Q^{2}+H^{2} x^{2}\right) \tau_{4}} \Delta_{b_{4}} \lambda_{4} H^{2}}{\sqrt{2 \mathrm{~g}^{2} Q^{2}+H^{2} x^{2}} \mathcal{V}} \\
& +\frac{12 \mathrm{~g}^{2} Q^{2} \alpha \epsilon \sqrt{\left(2 \mathrm{~g}^{2} Q^{2}+H^{2} x^{2}\right) \tau_{4}} \Delta_{b_{4}} \lambda_{4} H^{2}}{\sqrt{2 \mathrm{~g}^{2} Q^{2}+H^{2} x^{2}} \mathcal{V}}
\end{aligned}
$$

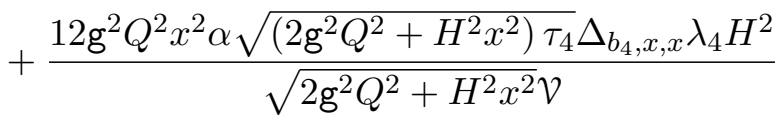

$$
\begin{aligned}
& +\frac{12 \mathrm{~g}^{2} Q^{2} \alpha \sqrt{\left(2 \mathrm{~g}^{2} Q^{2}+H^{2} x^{2}\right) \tau_{4}} \Delta_{b_{4}} \lambda_{4} \xi_{f} H^{2}}{\sqrt{2 \mathrm{~g}^{2} Q^{2}+H^{2} x^{2}} \mathcal{V}} \\
& +\frac{12 \mathrm{~g}^{2} Q^{2} x \alpha \sqrt{\left(2 \mathrm{~g}^{2} Q^{2}+H^{2} x^{2}\right) \tau_{4}} \Delta_{b_{4}, x} \lambda_{4} \xi_{f} H^{2}}{\sqrt{2 \mathrm{~g}^{2} Q^{2}+H^{2} x^{2}} \mathcal{V}} \\
& +\frac{12 \mathrm{~g}^{2} Q^{2} x \alpha \sqrt{\left(2 \mathrm{~g}^{2} Q^{2}+H^{2} x^{2}\right) \tau_{4}} c_{1} \Delta_{\tau_{4}, x} \lambda_{4} \xi_{h} H^{2}}{\sqrt{2 \mathrm{~g}^{2} Q^{2}+H^{2} x^{2}} v_{c_{2}}}
\end{aligned}
$$




$$
\begin{aligned}
& +\frac{36 \mathrm{~g}^{2} Q^{2} \alpha \sqrt{\left(2 \mathrm{~g}^{2} Q^{2}+H^{2} x^{2}\right) \tau_{4}} c_{1} \Delta_{\tau_{4}} \lambda_{4} \xi_{f} \xi_{h} H^{2}}{\sqrt{2 \mathrm{~g}^{2} Q^{2}+H^{2} x^{2}} v c_{2}} \\
& -\frac{24 \mathrm{~g}^{2} Q^{2} \alpha \Delta_{b_{4}} \lambda_{4} \sqrt{\left(2 \mathrm{~g}^{2} Q^{2}+H^{2} x^{2}\right) \tau_{4}} H^{2}}{\sqrt{2 \mathrm{~g}^{2} Q^{2}+H^{2} x^{2}} \mathcal{V}} \\
& -\frac{12 \mathrm{~g}^{2} Q^{2} \alpha c_{1} \Delta_{\tau_{4}} \lambda_{4} \xi_{h} \sqrt{\left(2 \mathrm{~g}^{2} Q^{2}+H^{2} x^{2}\right) \tau_{4}} H^{2}}{\sqrt{2 \mathrm{~g}^{2} Q^{2}+H^{2} x^{2}} c_{c_{2}}} \\
& -\frac{6 \mathrm{~g}^{2} Q^{2} x^{2} \alpha b_{4, x, x} \Delta_{\tau_{4}} \lambda_{4} \sqrt{\left(2 \mathrm{~g}^{2} Q^{2}+H^{2} x^{2}\right) \tau_{4}} H^{2}}{\sqrt{2 \mathrm{~g}^{2} Q^{2}+H^{2} x^{2}} \nu \tau_{4}} \\
& +\frac{32 \mathrm{~g}^{3} Q^{4} \sqrt{2} \sqrt{\tau_{4}} \sqrt{\left(2 \mathrm{~g}^{2} Q^{2}+H^{2} x^{2}\right) \tau_{4}} c_{2} \Delta_{1} H}{\sqrt{2 \mathrm{~g}^{2} Q^{2}+H^{2} x^{2}}} \\
& +\frac{32 \mathrm{~g}^{4} Q^{4} x \sqrt{2} \sqrt{\tau_{4}} \sqrt{\left(2 \mathrm{~g}^{2} Q^{2}+H^{2} x^{2}\right) \tau_{4}} c_{2} Q_{, x} \Delta_{2} H}{2 \mathrm{~g}^{2} Q^{2}+H^{2} x^{2}} \\
& +\frac{16 \mathrm{~g}^{4} Q^{5} x \sqrt{2} \sqrt{\tau_{4}} \sqrt{\left(2 \mathrm{~g}^{2} Q^{2}+H^{2} x^{2}\right) \tau_{4}} c_{2} \Delta_{2, x} H}{2 \mathrm{~g}^{2} Q^{2}+H^{2} x^{2}} \\
& -\frac{32 \sqrt{2} \mathrm{~g}^{4} Q^{5} c_{2} \Delta_{2} \sqrt{\tau_{4}} \sqrt{\left(2 \mathrm{~g}^{2} Q^{2}+H^{2} x^{2}\right) \tau_{4}} H}{2 \mathrm{~g}^{2} Q^{2}+H^{2} x^{2}} \\
& -\frac{32 \sqrt{2} \mathrm{~g}^{3} Q^{3} x c_{2} Q_{, x} \Delta_{1} \sqrt{\tau_{4}} \sqrt{\left(2 \mathrm{~g}^{2} Q^{2}+H^{2} x^{2}\right) \tau_{4}} H}{\sqrt{2 \mathrm{~g}^{2} Q^{2}+H^{2} x^{2}}} \\
& -\frac{16 \sqrt{2} \mathrm{~g}^{3} Q^{4} x c_{2} \Delta_{1, x} \sqrt{\tau_{4}} \sqrt{\left(2 \mathrm{~g}^{2} Q^{2}+H^{2} x^{2}\right) \tau_{4}} H}{\sqrt{2 \mathrm{~g}^{2} Q^{2}+H^{2} x^{2}}} \\
& +\frac{16 \mathrm{~g}^{2} Q^{2} \sqrt{\tau_{4}} \sqrt{\left(2 \mathrm{~g}^{2} Q^{2}+H^{2} x^{2}\right) \tau_{4}} V_{, b_{4}, b_{4}} \Delta_{b_{4}}}{\sqrt{2 \mathrm{~g}^{2} Q^{2}+H^{2} x^{2}}} \\
& +\frac{16 \mathrm{~g}^{2} Q^{2} \sqrt{\tau_{4}} \sqrt{\left(2 \mathrm{~g}^{2} Q^{2}+H^{2} x^{2}\right) \tau_{4}} V_{, \tau_{2}, b_{4}} \Delta_{\tau_{2}}}{\sqrt{2 \mathrm{~g}^{2} Q^{2}+H^{2} x^{2}}} \\
& +\frac{16 \mathrm{~g}^{2} Q^{2} \sqrt{\tau_{4}} \sqrt{\left(2 \mathrm{~g}^{2} Q^{2}+H^{2} x^{2}\right) \tau_{4}} V_{, b_{4}, \tau_{4}} \Delta_{\tau_{4}}}{\sqrt{2 \mathrm{~g}^{2} Q^{2}+H^{2} x^{2}}}=0 \\
& \begin{array}{c}
\frac{9 \alpha H^{2} x^{2} \lambda_{2} \tau_{2, x}^{2} \Delta_{\tau_{2}}}{\mathcal{V} \sqrt{\tau_{2}}}+\frac{12 \alpha H^{2} x \lambda_{2} \sqrt{\tau_{2}} \tau_{2, x} \Delta_{\tau_{2}}}{\mathcal{V}}-\frac{12 \alpha H^{2} x^{2} \lambda_{2} \sqrt{\tau_{2}} \tau_{2, x} \Delta_{\tau_{2, x}}}{\mathcal{V}} \\
-\frac{12 \alpha H^{2} x^{2} \lambda_{2} \sqrt{\tau_{2}} \tau_{2, x, x} \Delta_{\tau_{2}}}{\mathcal{V}}-\frac{48 \alpha H^{2} \lambda_{2} \tau_{2}^{3 / 2} \Delta_{\tau_{2}}}{\mathcal{V}}+\frac{24 \alpha H^{2} \epsilon \lambda_{2} \tau_{2}^{3 / 2} \Delta_{\tau_{2}}}{\mathcal{V}}
\end{array} \\
& +\frac{24 \alpha H^{2} x^{2} \lambda_{2} \tau_{2}^{3 / 2} \Delta_{\tau_{2}}}{\mathcal{V}}+\frac{24 \alpha H^{2} x^{2} \lambda_{2} \tau_{2}^{3 / 2} \Delta_{\tau_{2}, x, x}}{\mathcal{V}} \\
& +32 \tau_{2}^{2} \Delta_{b_{4}} V_{, \tau_{2}, b_{4}}+32 \tau_{2}^{2} \Delta_{\tau_{2}} V_{, \tau_{2}, \tau_{2}}+32 \tau_{2}^{2} V_{, \tau_{2}, \tau_{4}} \Delta_{\tau_{4}}=0,
\end{aligned}
$$




$$
\begin{aligned}
\Delta_{1, x, x}= & -\frac{\sqrt{2} \mathrm{~g} Q^{2} c_{2} \Delta_{b_{4}}}{H x^{2} c_{1} \tau_{4}}-\frac{\sqrt{2} \mathrm{~g} Q^{2} c_{2} \Delta_{b_{4}, x}}{H x c_{1} \tau_{4}}-\Delta_{1}-\frac{4 \mathrm{~g}^{2} Q^{2} \Delta_{1}}{H^{2} x^{2}}+\frac{2 \mathrm{~g} Q \Delta_{1} \xi_{h}}{H x^{2}}+\frac{2 \Delta_{1, x} \xi_{f}}{x} \\
& -\frac{4 \mathrm{~g}^{2} Q^{2} \Delta_{2} \xi_{h}}{H x^{2} \sqrt{2 \mathrm{~g}^{2} Q^{2}+H^{2} x^{2}}}-\frac{2 H \Delta_{2} \xi_{h}}{\sqrt{2 \mathrm{~g}^{2} Q^{2}+H^{2} x^{2}}}+\frac{2 \mathrm{~g} Q \Delta_{2}}{\sqrt{2 \mathrm{~g}^{2} Q^{2}+H^{2} x^{2}}} \\
& +\frac{4 \mathrm{~g}^{3} Q^{3} \Delta_{2}}{H^{2} x^{2} \sqrt{2 \mathrm{~g}^{2} Q^{2}+H^{2} x^{2}}}-\frac{2 \sqrt{2} \mathrm{~g}^{2} Q^{3} \Delta_{\tau_{4}}}{H^{2} x^{2} \tau_{4}}+\frac{\sqrt{2} Q_{, x} \Delta_{\tau_{4}}}{x \tau_{4}}-\frac{\sqrt{2} Q_{\tau_{4}}}{x^{2} \tau_{4}} \\
& +\frac{\sqrt{2} Q \epsilon \Delta_{\tau_{4}}}{x^{2} \tau_{4}}-\frac{\sqrt{2} Q_{, x, x} \Delta_{\tau_{4}}}{\tau_{4}}-\frac{\sqrt{2} Q_{, x} \Delta_{\tau_{4}, x}}{\tau_{4}}+\frac{\sqrt{2} Q \Delta_{\tau_{4}, x}}{x \tau_{4}}
\end{aligned}
$$

$$
\begin{aligned}
& \Delta_{2, x, x}=\frac{8 \mathrm{~g}^{5} \sqrt{2 \mathrm{~g}^{2} Q^{2}+H^{2} x^{2}} c_{1} \Delta_{\tau_{4}} Q^{7}}{H^{6} Q \sqrt{2} c_{1} \tau_{4} x^{6}+4 \mathrm{~g}^{2} H^{4} Q^{3} \sqrt{2} c_{1} \tau_{4} x^{4}+4 \mathrm{~g}^{4} H^{2} Q^{5} \sqrt{2} c_{1} \tau_{4} x^{2}} \\
& -\frac{8 \sqrt{2} \mathrm{~g}^{6} c_{1} \Delta_{2} \tau_{4} Q^{7}}{H^{6} Q \sqrt{2} c_{1} \tau_{4} x^{6}+4 \mathrm{~g}^{2} H^{4} Q^{3} \sqrt{2} c_{1} \tau_{4} x^{4}+4 \mathrm{~g}^{4} H^{2} Q^{5} \sqrt{2} c_{1} \tau_{4} x^{2}} \\
& +\frac{4 \mathrm{~g}^{4} H \sqrt{2 \mathrm{~g}^{2} Q^{2}+H^{2} x^{2}} c_{2} \Delta_{b_{4}} Q^{6}}{H^{6} Q \sqrt{2} c_{1} \tau_{4} x^{6}+4 \mathrm{~g}^{2} H^{4} Q^{3} \sqrt{2} c_{1} \tau_{4} x^{4}+4 \mathrm{~g}^{4} H^{2} Q^{5} \sqrt{2} c_{1} \tau_{4} x^{2}} \\
& +\frac{4 \mathrm{~g}^{4} H x \sqrt{2 \mathrm{~g}^{2} Q^{2}+H^{2} x^{2}} c_{2} \Delta_{b_{4}, x} Q^{6}}{H^{6} Q \sqrt{2} c_{1} \tau_{4} x^{6}+4 \mathrm{~g}^{2} H^{4} Q^{3} \sqrt{2} c_{1} \tau_{4} x^{4}+4 \mathrm{~g}^{4} H^{2} Q^{5} \sqrt{2} c_{1} \tau_{4} x^{2}} \\
& +\frac{8 \mathrm{~g}^{5} \sqrt{4 \mathrm{~g}^{2} Q^{2}+2 H^{2} x^{2}} c_{1} \Delta_{1} \tau_{4} Q^{6}}{H^{6} Q \sqrt{2} c_{1} \tau_{4} x^{6}+4 \mathrm{~g}^{2} H^{4} Q^{3} \sqrt{2} c_{1} \tau_{4} x^{4}+4 \mathrm{~g}^{4} H^{2} Q^{5} \sqrt{2} c_{1} \tau_{4} x^{2}} \\
& -\frac{4 \mathrm{~g}^{3} H^{2} \sqrt{2 \mathrm{~g}^{2} Q^{2}+H^{2} x^{2}} \epsilon c_{1} \Delta_{\tau_{4}} Q^{5}}{H^{6} Q \sqrt{2} c_{1} \tau_{4} x^{6}+4 \mathrm{~g}^{2} H^{4} Q^{3} \sqrt{2} c_{1} \tau_{4} x^{4}+4 \mathrm{~g}^{4} H^{2} Q^{5} \sqrt{2} c_{1} \tau_{4} x^{2}} \\
& +\frac{4 \mathrm{~g}^{3} H^{2} \sqrt{2 \mathrm{~g}^{2} Q^{2}+H^{2} x^{2}} c_{1} \Delta_{\tau_{4}} Q^{5}}{H^{6} Q \sqrt{2} c_{1} \tau_{4} x^{6}+4 \mathrm{~g}^{2} H^{4} Q^{3} \sqrt{2} c_{1} \tau_{4} x^{4}+4 \mathrm{~g}^{4} H^{2} Q^{5} \sqrt{2} c_{1} \tau_{4} x^{2}} \\
& +\frac{8 \mathrm{~g}^{3} H^{2} x^{2} \sqrt{2 \mathrm{~g}^{2} Q^{2}+H^{2} x^{2}} c_{1} \Delta_{\tau_{4}} Q^{5}}{H^{6} Q \sqrt{2} c_{1} \tau_{4} x^{6}+4 \mathrm{~g}^{2} H^{4} Q^{3} \sqrt{2} c_{1} \tau_{4} x^{4}+4 \mathrm{~g}^{4} H^{2} Q^{5} \sqrt{2} c_{1} \tau_{4} x^{2}} \\
& -\frac{4 \mathrm{~g}^{3} H^{2} x \sqrt{2 \mathrm{~g}^{2} Q^{2}+H^{2} x^{2}} c_{1} \Delta_{\tau_{4}, x} Q^{5}}{H^{6} Q \sqrt{2} c_{1} \tau_{4} x^{6}+4 \mathrm{~g}^{2} H^{4} Q^{3} \sqrt{2} c_{1} \tau_{4} x^{4}+4 \mathrm{~g}^{4} H^{2} Q^{5} \sqrt{2} c_{1} \tau_{4} x^{2}}
\end{aligned}
$$




$$
\begin{aligned}
& -\frac{12 \sqrt{2} \mathrm{~g}^{4} H^{2} x^{2} c_{1} \Delta_{2} \tau_{4} Q^{5}}{H^{6} Q \sqrt{2} c_{1} \tau_{4} x^{6}+4 \mathrm{~g}^{2} H^{4} Q^{3} \sqrt{2} c_{1} \tau_{4} x^{4}+4 \mathrm{~g}^{4} H^{2} Q^{5} \sqrt{2} c_{1} \tau_{4} x^{2}} \\
& +\frac{8 \mathrm{~g}^{4} H^{2} x \sqrt{2} c_{1} \Delta_{2, x} \xi_{f} \tau_{4} Q^{5}}{H^{6} Q \sqrt{2} c_{1} \tau_{4} x^{6}+4 \mathrm{~g}^{2} H^{4} Q^{3} \sqrt{2} c_{1} \tau_{4} x^{4}+4 \mathrm{~g}^{4} H^{2} Q^{5} \sqrt{2} c_{1} \tau_{4} x^{2}} \\
& -\frac{8 \sqrt{2} \mathrm{~g}^{4} H \sqrt{2 \mathrm{~g}^{2} Q^{2}+H^{2} x^{2}} c_{1} \Delta_{1} \xi_{h} \tau_{4} Q^{5}}{H^{6} Q \sqrt{2} c_{1} \tau_{4} x^{6}+4 \mathrm{~g}^{2} H^{4} Q^{3} \sqrt{2} c_{1} \tau_{4} x^{4}+4 \mathrm{~g}^{4} H^{2} Q^{5} \sqrt{2} c_{1} \tau_{4} x^{2}} \\
& +\frac{2 \mathrm{~g}^{2} H^{3} x^{2} \sqrt{2 \mathrm{~g}^{2} Q^{2}+H^{2} x^{2}} c_{2} \Delta_{b_{4}} Q^{4}}{H^{6} Q \sqrt{2} c_{1} \tau_{4} x^{6}+4 \mathrm{~g}^{2} H^{4} Q^{3} \sqrt{2} c_{1} \tau_{4} x^{4}+4 \mathrm{~g}^{4} H^{2} Q^{5} \sqrt{2} c_{1} \tau_{4} x^{2}} \\
& +\frac{2 \mathrm{~g}^{2} H^{3} x^{3} \sqrt{2 \mathrm{~g}^{2} Q^{2}+H^{2} x^{2}} c_{2} \Delta_{b_{4}, x} Q^{4}}{H^{6} Q \sqrt{2} c_{1} \tau_{4} x^{6}+4 \mathrm{~g}^{2} H^{4} Q^{3} \sqrt{2} c_{1} \tau_{4} x^{4}+4 \mathrm{~g}^{4} H^{2} Q^{5} \sqrt{2} c_{1} \tau_{4} x^{2}} \\
& -\frac{4 \mathrm{~g}^{3} H^{2} x \sqrt{2 \mathrm{~g}^{2} Q^{2}+H^{2} x^{2}} c_{1} Q_{, x} \Delta_{\tau_{4}} Q^{4}}{H^{6} Q \sqrt{2} c_{1} \tau_{4} x^{6}+4 \mathrm{~g}^{2} H^{4} Q^{3} \sqrt{2} c_{1} \tau_{4} x^{4}+4 \mathrm{~g}^{4} H^{2} Q^{5} \sqrt{2} c_{1} \tau_{4} x^{2}} \\
& +\frac{4 \mathrm{~g}^{3} H^{2} x^{2} \sqrt{2 \mathrm{~g}^{2} Q^{2}+H^{2} x^{2}} c_{1} Q_{, x, x} \Delta_{\tau_{4}} Q^{4}}{H^{6} Q \sqrt{2} c_{1} \tau_{4} x^{6}+4 \mathrm{~g}^{2} H^{4} Q^{3} \sqrt{2} c_{1} \tau_{4} x^{4}+4 \mathrm{~g}^{4} H^{2} Q^{5} \sqrt{2} c_{1} \tau_{4} x^{2}} \\
& +\frac{4 \mathrm{~g}^{3} H^{2} x^{2} \sqrt{2 \mathrm{~g}^{2} Q^{2}+H^{2} x^{2}} c_{1} Q_{, x} \Delta_{\tau_{4}, x} Q^{4}}{H^{6} Q \sqrt{2} c_{1} \tau_{4} x^{6}+4 \mathrm{~g}^{2} H^{4} Q^{3} \sqrt{2} c_{1} \tau_{4} x^{4}+4 \mathrm{~g}^{4} H^{2} Q^{5} \sqrt{2} c_{1} \tau_{4} x^{2}} \\
& +\frac{8 \mathrm{~g}^{3} H^{2} x^{2} \sqrt{2} \sqrt{2 \mathrm{~g}^{2} Q^{2}+H^{2} x^{2}} c_{1} \Delta_{1} \tau_{4} Q^{4}}{H^{6} Q \sqrt{2} c_{1} \tau_{4} x^{6}+4 \mathrm{~g}^{2} H^{4} Q^{3} \sqrt{2} c_{1} \tau_{4} x^{4}+4 \mathrm{~g}^{4} H^{2} Q^{5} \sqrt{2} c_{1} \tau_{4} x^{2}} \\
& -\frac{2 \mathrm{~g} H^{4} x^{2} \sqrt{2 \mathrm{~g}^{2} Q^{2}+H^{2} x^{2}} \epsilon c_{1} \Delta_{\tau_{4}} Q^{3}}{H^{6} Q \sqrt{2} c_{1} \tau_{4} x^{6}+4 \mathrm{~g}^{2} H^{4} Q^{3} \sqrt{2} c_{1} \tau_{4} x^{4}+4 \mathrm{~g}^{4} H^{2} Q^{5} \sqrt{2} c_{1} \tau_{4} x^{2}} \\
& +\frac{2 \mathrm{~g} H^{4} x^{4} \sqrt{2 \mathrm{~g}^{2} Q^{2}+H^{2} x^{2}} c_{1} \Delta_{\tau_{4}} Q^{3}}{H^{6} Q \sqrt{2} c_{1} \tau_{4} x^{6}+4 \mathrm{~g}^{2} H^{4} Q^{3} \sqrt{2} c_{1} \tau_{4} x^{4}+4 \mathrm{~g}^{4} H^{2} Q^{5} \sqrt{2} c_{1} \tau_{4} x^{2}} \\
& +\frac{6 \mathrm{~g} H^{4} x^{2} \sqrt{2 \mathrm{~g}^{2} Q^{2}+H^{2} x^{2}} c_{1} \Delta_{\tau_{4}} Q^{3}}{H^{6} Q \sqrt{2} c_{1} \tau_{4} x^{6}+4 \mathrm{~g}^{2} H^{4} Q^{3} \sqrt{2} c_{1} \tau_{4} x^{4}+4 \mathrm{~g}^{4} H^{2} Q^{5} \sqrt{2} c_{1} \tau_{4} x^{2}}
\end{aligned}
$$




$$
\begin{aligned}
& 2 \mathrm{~g} H^{4} x^{3} \sqrt{2 \mathrm{~g}^{2} Q^{2}+H^{2} x^{2}} c_{1} \Delta_{\tau_{4}, x} Q^{3} \\
& H^{6} Q \sqrt{2} c_{1} \tau_{4} x^{6}+4 \mathrm{~g}^{2} H^{4} Q^{3} \sqrt{2} c_{1} \tau_{4} x^{4}+4 \mathrm{~g}^{4} H^{2} Q^{5} \sqrt{2} c_{1} \tau_{4} x^{2} \\
& -\frac{6 \sqrt{2} \mathrm{~g}^{2} H^{4} x^{4} c_{1} \Delta_{2} \tau_{4} Q^{3}}{H^{6} Q \sqrt{2} c_{1} \tau_{4} x^{6}+4 \mathrm{~g}^{2} H^{4} Q^{3} \sqrt{2} c_{1} \tau_{4} x^{4}+4 \mathrm{~g}^{4} H^{2} Q^{5} \sqrt{2} c_{1} \tau_{4} x^{2}} \\
& -\frac{2 \sqrt{2} \mathrm{~g}^{2} H^{4} x^{2} c_{1} \Delta_{2} \tau_{4} Q^{3}}{H^{6} Q \sqrt{2} c_{1} \tau_{4} x^{6}+4 \mathrm{~g}^{2} H^{4} Q^{3} \sqrt{2} c_{1} \tau_{4} x^{4}+4 \mathrm{~g}^{4} H^{2} Q^{5} \sqrt{2} c_{1} \tau_{4} x^{2}} \\
& -\frac{2 \sqrt{2} \mathrm{~g}^{2} H^{4} x^{2} \epsilon c_{1} \Delta_{2} \tau_{4} Q^{3}}{H^{6} Q \sqrt{2} c_{1} \tau_{4} x^{6}+4 \mathrm{~g}^{2} H^{4} Q^{3} \sqrt{2} c_{1} \tau_{4} x^{4}+4 \mathrm{~g}^{4} H^{2} Q^{5} \sqrt{2} c_{1} \tau_{4} x^{2}} \\
& +\frac{4 \mathrm{~g}^{2} H^{4} x^{2} \sqrt{2} c_{1} \Delta_{2} \xi_{f} \tau_{4} Q^{3}}{H^{6} Q \sqrt{2} c_{1} \tau_{4} x^{6}+4 \mathrm{~g}^{2} H^{4} Q^{3} \sqrt{2} c_{1} \tau_{4} x^{4}+4 \mathrm{~g}^{4} H^{2} Q^{5} \sqrt{2} c_{1} \tau_{4} x^{2}} \\
& +\frac{8 \mathrm{~g}^{2} H^{4} x^{3} \sqrt{2} c_{1} \Delta_{2, x} \xi_{f} \tau_{4} Q^{3}}{H^{6} Q \sqrt{2} c_{1} \tau_{4} x^{6}+4 \mathrm{~g}^{2} H^{4} Q^{3} \sqrt{2} c_{1} \tau_{4} x^{4}+4 \mathrm{~g}^{4} H^{2} Q^{5} \sqrt{2} c_{1} \tau_{4} x^{2}} \\
& -\frac{8 \sqrt{2} \mathrm{~g}^{2} H^{3} x^{2} \sqrt{2 \mathrm{~g}^{2} Q^{2}+H^{2} x^{2}} c_{1} \Delta_{1} \xi_{h} \tau_{4} Q^{3}}{H^{6} Q \sqrt{2} c_{1} \tau_{4} x^{6}+4 \mathrm{~g}^{2} H^{4} Q^{3} \sqrt{2} c_{1} \tau_{4} x^{4}+4 \mathrm{~g}^{4} H^{2} Q^{5} \sqrt{2} c_{1} \tau_{4} x^{2}} \\
& +\frac{2 H^{5} x^{4} \sqrt{2 \mathrm{~g}^{2} Q^{2}+H^{2} x^{2}} c_{2} \Delta_{b_{4}} Q^{2}}{H^{6} Q \sqrt{2} c_{1} \tau_{4} x^{6}+4 \mathrm{~g}^{2} H^{4} Q^{3} \sqrt{2} c_{1} \tau_{4} x^{4}+4 \mathrm{~g}^{4} H^{2} Q^{5} \sqrt{2} c_{1} \tau_{4} x^{2}} \\
& -\frac{10 \mathrm{~g} H^{4} x^{3} \sqrt{2 \mathrm{~g}^{2} Q^{2}+H^{2} x^{2}} c_{1} Q_{, x} \Delta_{\tau_{4}} Q^{2}}{H^{6} Q \sqrt{2} c_{1} \tau_{4} x^{6}+4 \mathrm{~g}^{2} H^{4} Q^{3} \sqrt{2} c_{1} \tau_{4} x^{4}+4 \mathrm{~g}^{4} H^{2} Q^{5} \sqrt{2} c_{1} \tau_{4} x^{2}} \\
& +\frac{2 \mathrm{~g} H^{4} x^{4} \sqrt{2 \mathrm{~g}^{2} Q^{2}+H^{2} x^{2}} c_{1} Q_{, x, x} \Delta_{\tau_{4}} Q^{2}}{H^{6} Q \sqrt{2} c_{1} \tau_{4} x^{6}+4 \mathrm{~g}^{2} H^{4} Q^{3} \sqrt{2} c_{1} \tau_{4} x^{4}+4 \mathrm{~g}^{4} H^{2} Q^{5} \sqrt{2} c_{1} \tau_{4} x^{2}} \\
& +\frac{2 \mathrm{~g} H^{4} x^{4} \sqrt{2 \mathrm{~g}^{2} Q^{2}+H^{2} x^{2}} c_{1} Q_{, x} \Delta_{\tau_{4}, x} Q^{2}}{H^{6} Q \sqrt{2} c_{1} \tau_{4} x^{6}+4 \mathrm{~g}^{2} H^{4} Q^{3} \sqrt{2} c_{1} \tau_{4} x^{4}+4 \mathrm{~g}^{4} H^{2} Q^{5} \sqrt{2} c_{1} \tau_{4} x^{2}} \\
& +\frac{2 \mathrm{~g} H^{4} x^{4} \sqrt{4 \mathrm{~g}^{2} Q^{2}+2 H^{2} x^{2}} c_{1} \Delta_{1} \tau_{4} Q^{2}}{H^{6} Q \sqrt{2} c_{1} \tau_{4} x^{6}+4 \mathrm{~g}^{2} H^{4} Q^{3} \sqrt{2} c_{1} \tau_{4} x^{4}+4 \mathrm{~g}^{4} H^{2} Q^{5} \sqrt{2} c_{1} \tau_{4} x^{2}} \\
& +\frac{8 \mathrm{~g}^{2} H^{4} x^{3} \sqrt{2} c_{1} Q_{, x} \Delta_{2} \tau_{4} Q^{2}}{H^{6} Q \sqrt{2} c_{1} \tau_{4} x^{6}+4 \mathrm{~g}^{2} H^{4} Q^{3} \sqrt{2} c_{1} \tau_{4} x^{4}+4 \mathrm{~g}^{4} H^{2} Q^{5} \sqrt{2} c_{1} \tau_{4} x^{2}}
\end{aligned}
$$




$$
\begin{aligned}
& +\frac{2 \mathrm{~g}^{2} H^{4} x^{4} \sqrt{2} c_{1} Q_{, x, x} \Delta_{2} \tau_{4} Q^{2}}{H^{6} Q \sqrt{2} c_{1} \tau_{4} x^{6}+4 \mathrm{~g}^{2} H^{4} Q^{3} \sqrt{2} c_{1} \tau_{4} x^{4}+4 \mathrm{~g}^{4} H^{2} Q^{5} \sqrt{2} c_{1} \tau_{4} x^{2}} \\
& -\frac{4 \sqrt{2} \mathrm{~g}^{2} H^{4} x^{3} c_{1} Q_{, x} \Delta_{2} \xi_{f} \tau_{4} Q^{2}}{H^{6} Q \sqrt{2} c_{1} \tau_{4} x^{6}+4 \mathrm{~g}^{2} H^{4} Q^{3} \sqrt{2} c_{1} \tau_{4} x^{4}+4 \mathrm{~g}^{4} H^{2} Q^{5} \sqrt{2} c_{1} \tau_{4} x^{2}} \\
& -\frac{2 H^{5} x^{5} \sqrt{2 \mathrm{~g}^{2} Q^{2}+H^{2} x^{2}} c_{2} Q_{, x} \Delta_{b_{4}} Q}{H^{6} Q \sqrt{2} c_{1} \tau_{4} x^{6}+4 \mathrm{~g}^{2} H^{4} Q^{3} \sqrt{2} c_{1} \tau_{4} x^{4}+4 \mathrm{~g}^{4} H^{2} Q^{5} \sqrt{2} c_{1} \tau_{4} x^{2}} \\
& +\frac{4 \mathrm{~g} H^{4} x^{4} \sqrt{2 \mathrm{~g}^{2} Q^{2}+H^{2} x^{2}} c_{1} Q_{, x}^{2} \Delta_{\tau_{4}} Q}{H^{6} Q \sqrt{2} c_{1} \tau_{4} x^{6}+4 \mathrm{~g}^{2} H^{4} Q^{3} \sqrt{2} c_{1} \tau_{4} x^{4}+4 \mathrm{~g}^{4} H^{2} Q^{5} \sqrt{2} c_{1} \tau_{4} x^{2}} \\
& -\frac{6 \sqrt{2} \mathrm{~g}^{2} H^{4} x^{4} c_{1} Q_{, x}^{2} \Delta_{2} \tau_{4} Q}{H^{6} Q \sqrt{2} c_{1} \tau_{4} x^{6}+4 \mathrm{~g}^{2} H^{4} Q^{3} \sqrt{2} c_{1} \tau_{4} x^{4}+4 \mathrm{~g}^{4} H^{2} Q^{5} \sqrt{2} c_{1} \tau_{4} x^{2}} \\
& -\frac{\sqrt{2} H^{6} x^{6} c_{1} \Delta_{2} \tau_{4} Q}{H^{6} Q \sqrt{2} c_{1} \tau_{4} x^{6}+4 \mathrm{~g}^{2} H^{4} Q^{3} \sqrt{2} c_{1} \tau_{4} x^{4}+4 \mathrm{~g}^{4} H^{2} Q^{5} \sqrt{2} c_{1} \tau_{4} x^{2}} \\
& -\frac{\sqrt{2} H^{6} x^{4} \epsilon c_{1} \Delta_{2} \tau_{4} Q}{H^{6} Q \sqrt{2} c_{1} \tau_{4} x^{6}+4 \mathrm{~g}^{2} H^{4} Q^{3} \sqrt{2} c_{1} \tau_{4} x^{4}+4 \mathrm{~g}^{4} H^{2} Q^{5} \sqrt{2} c_{1} \tau_{4} x^{2}} \\
& +\frac{2 H^{6} x^{4} \sqrt{2} c_{1} \Delta_{2} \tau_{4} Q}{H^{6} Q \sqrt{2} c_{1} \tau_{4} x^{6}+4 \mathrm{~g}^{2} H^{4} Q^{3} \sqrt{2} c_{1} \tau_{4} x^{4}+4 \mathrm{~g}^{4} H^{2} Q^{5} \sqrt{2} c_{1} \tau_{4} x^{2}} \\
& +\frac{2 H^{6} x^{4} \sqrt{2} c_{1} \Delta_{2} \xi_{f} \tau_{4} Q}{H^{6} Q \sqrt{2} c_{1} \tau_{4} x^{6}+4 \mathrm{~g}^{2} H^{4} Q^{3} \sqrt{2} c_{1} \tau_{4} x^{4}+4 \mathrm{~g}^{4} H^{2} Q^{5} \sqrt{2} c_{1} \tau_{4} x^{2}} \\
& +\frac{2 H^{6} x^{5} \sqrt{2} c_{1} \Delta_{2, x} \xi_{f} \tau_{4} Q}{H^{6} Q \sqrt{2} c_{1} \tau_{4} x^{6}+4 \mathrm{~g}^{2} H^{4} Q^{3} \sqrt{2} c_{1} \tau_{4} x^{4}+4 \mathrm{~g}^{4} H^{2} Q^{5} \sqrt{2} c_{1} \tau_{4} x^{2}} \\
& -\frac{2 \sqrt{2} H^{5} x^{4} \sqrt{2 \mathrm{~g}^{2} Q^{2}+H^{2} x^{2}} c_{1} \Delta_{1} \xi_{h} \tau_{4} Q}{H^{6} Q \sqrt{2} c_{1} \tau_{4} x^{6}+4 \mathrm{~g}^{2} H^{4} Q^{3} \sqrt{2} c_{1} \tau_{4} x^{4}+4 \mathrm{~g}^{4} H^{2} Q^{5} \sqrt{2} c_{1} \tau_{4} x^{2}} \\
& -\frac{2 \sqrt{2} H^{6} x^{5} c_{1} Q_{, x} \Delta_{2} \tau_{4}}{H^{6} Q \sqrt{2} c_{1} \tau_{4} x^{6}+4 \mathrm{~g}^{2} H^{4} Q^{3} \sqrt{2} c_{1} \tau_{4} x^{4}+4 \mathrm{~g}^{4} H^{2} Q^{5} \sqrt{2} c_{1} \tau_{4} x^{2}} \\
& +\frac{H^{6} x^{6} \sqrt{2} c_{1} Q_{, x, x} \Delta_{2} \tau_{4}}{H^{6} Q \sqrt{2} c_{1} \tau_{4} x^{6}+4 \mathrm{~g}^{2} H^{4} Q^{3} \sqrt{2} c_{1} \tau_{4} x^{4}+4 \mathrm{~g}^{4} H^{2} Q^{5} \sqrt{2} c_{1} \tau_{4} x^{2}} \\
& -\frac{2 \sqrt{2} H^{6} x^{5} c_{1} Q_{, x} \Delta_{2} \xi_{f} \tau_{4}}{H^{6} Q \sqrt{2} c_{1} \tau_{4} x^{6}+4 \mathrm{~g}^{2} H^{4} Q^{3} \sqrt{2} c_{1} \tau_{4} x^{4}+4 \mathrm{~g}^{4} H^{2} Q^{5} \sqrt{2} c_{1} \tau_{4} x^{2}} .
\end{aligned}
$$

\section{C.2 Example 3}

The e.o.m for $t_{R}$ and $\psi_{R}$ are now given by, respectively

$$
\begin{aligned}
& -4 H^{2} Q \xi_{f} \psi_{R}+4 H^{2} x \xi_{f} Q_{, x} \psi_{R}-2 H^{2} x \xi_{f} t_{R, x}-2 \mathrm{~g}^{2} Q^{3} \psi_{R}+2 \mathrm{~g} H Q \xi_{h} t_{R} \\
& \quad+2 \mathrm{~g} H Q^{2} x \psi_{R}-2 \mathrm{~g} H Q x t_{R}-2 H^{2} x \xi_{h} t_{R}+2 H^{2} x Q, x \psi_{R}-2 H^{2} x^{2} Q_{, x, x} \psi_{R} \\
& -2 H^{2} Q \psi_{R}+2 H^{2} Q \epsilon \psi_{R}-2 H^{2} x^{2} Q_{, x} \psi_{R, x}+2 H^{2} Q x \psi_{R, x}+H^{2} x^{2} t_{R} \\
& \quad+H^{2} x^{2} t_{R, x, x}=0
\end{aligned}
$$


and

$$
\begin{aligned}
- & \frac{3 \alpha H^{2} x^{2} \lambda_{2} \tau_{2, x}^{2} \psi_{R}}{4 \mathcal{V} \sqrt{\tau_{2}}}-\frac{3 \alpha H^{2} \lambda_{4} \tau_{4}^{3 / 2} \xi_{f}^{2} \psi_{R}}{\mathcal{V}}-3 \mathrm{~g}^{2} Q^{4} \tau_{4} \psi_{R}+2 \mathrm{~g}^{2} Q^{3} \tau_{4} t_{R} \\
& -\frac{8 \sqrt{2} H^{2} \tau_{4}^{5 / 2} g_{s} \xi_{h}^{2} \psi_{R}}{\sqrt{\zeta} m^{2} \mathcal{V}}-2 \mathrm{~g} H Q^{2} x \tau_{4} t_{R}+H^{2} Q^{2} \tau_{4} \psi_{R}+H^{2} x^{2} \tau_{4} Q_{, x}^{2} \psi_{R} \\
& -2 H^{2} Q x \tau_{4} Q_{, x} \psi_{R}-2 H^{2} x^{2} \tau_{4} Q_{, x} t_{R, x}+2 H^{2} Q x \tau_{4} t_{R, x} \\
- & H^{2} x^{2} \psi_{R}-4 H^{2} \psi_{R}+3 H^{2} \epsilon \psi_{R}-H^{2} x^{2} \psi_{R, x, x}+2 V \psi_{R}=0 .
\end{aligned}
$$

The scalar metric perturbations are defined through

$$
\begin{array}{ll}
\delta \tau_{2}=\frac{\Delta_{\tau_{2}}}{a}, & \delta \tau_{4}=\frac{\Delta_{\tau_{4}}}{a}, \quad \delta b=\frac{\Delta_{b}}{a} \\
\delta Q=\frac{\Delta_{1}}{\sqrt{2} a}, & M=\frac{a \mathrm{~g} Q \Delta_{1}+\sqrt{k^{2}+2 a^{2} \mathrm{~g}^{2} Q^{2}} \Delta_{2}}{\sqrt{2} \mathrm{~g} a^{2} k^{2} Q} .
\end{array}
$$

Their equations of motion are given by

$$
\begin{aligned}
& \frac{32 \mathrm{~g}^{5} m \sqrt{2} \sqrt{\tau_{4}} \sqrt{\left(2 \mathrm{~g}^{2} Q^{2}+H^{2} x^{2}\right) \tau_{4}} \Delta_{2} Q^{6}}{2 \mathrm{~g}^{2} Q^{2}+H^{2} x^{2}} \\
& -\frac{32 \mathrm{~g}^{4} m \sqrt{4 \mathrm{~g}^{2} Q^{2}+2 H^{2} x^{2}} \Delta_{1} \sqrt{\tau_{4}} \sqrt{\left(2 \mathrm{~g}^{2} Q^{2}+H^{2} x^{2}\right) \tau_{4}} Q^{5}}{2 \mathrm{~g}^{2} Q^{2}+H^{2} x^{2}} \\
& +\frac{32 \mathrm{~g}^{3} H^{2} m x^{2} \sqrt{2} \sqrt{\tau_{4}} \sqrt{\left(2 \mathrm{~g}^{2} Q^{2}+H^{2} x^{2}\right) \tau_{4}} \Delta_{2} Q^{4}}{2 \mathrm{~g}^{2} Q^{2}+H^{2} x^{2}} \\
& +\frac{16 \mathrm{~g}^{3} H^{2} m x \sqrt{2} \sqrt{\tau_{4}} \sqrt{\left(2 \mathrm{~g}^{2} Q^{2}+H^{2} x^{2}\right) \tau_{4}} \Delta_{2, x} Q^{4}}{2 \mathrm{~g}^{2} Q^{2}+H^{2} x^{2}}+8 \mathrm{~g} H^{3} m^{2} x^{2} \Delta_{b} Q^{3} \\
& -\frac{16 \sqrt{2} \mathrm{~g}^{3} H^{2} m x^{2} Q_{, x} \Delta_{2, x} \sqrt{\tau_{4}} \sqrt{\left(2 \mathrm{~g}^{2} Q^{2}+H^{2} x^{2}\right) \tau_{4}} Q^{3}}{2 \mathrm{~g}^{2} Q^{2}+H^{2} x^{2}} \\
& -\frac{16 \sqrt{2} \mathrm{~g}^{2} H^{2} m x^{2} \Delta_{1} \sqrt{\tau_{4}} \sqrt{\left(2 \mathrm{~g}^{2} Q^{2}+H^{2} x^{2}\right) \tau_{4}} Q^{3}}{\sqrt{2 \mathrm{~g}^{2} Q^{2}+H^{2} x^{2}}} \\
& -\frac{16 \sqrt{2} \mathrm{~g}^{2} H^{2} m x \Delta_{1, x} \sqrt{\tau_{4}} \sqrt{\left(2 \mathrm{~g}^{2} Q^{2}+H^{2} x^{2}\right) \tau_{4}} Q^{3}}{\sqrt{2 \mathrm{~g}^{2} Q^{2}+H^{2} x^{2}}} \\
& +\frac{8 \mathrm{~g} H^{4} m x^{4} \sqrt{2} \sqrt{\tau_{4}} \sqrt{\left(2 \mathrm{~g}^{2} Q^{2}+H^{2} x^{2}\right) \tau_{4}} \Delta_{2} Q^{2}}{2 \mathrm{~g}^{2} Q^{2}+H^{2} x^{2}} \\
& +\frac{8 \mathrm{~g} H^{4} m x^{2} \sqrt{2} \sqrt{\tau_{4}} \sqrt{\left(2 \mathrm{~g}^{2} Q^{2}+H^{2} x^{2}\right) \tau_{4}} \Delta_{2} Q^{2}}{2 \mathrm{~g}^{2} Q^{2}+H^{2} x^{2}} \\
& +\frac{8 \mathrm{~g} H^{4} m x^{3} \sqrt{2} \sqrt{\tau_{4}} \sqrt{\left(2 \mathrm{~g}^{2} Q^{2}+H^{2} x^{2}\right) \tau_{4}} \Delta_{2, x} Q^{2}}{2 \mathrm{~g}^{2} Q^{2}+H^{2} x^{2}}-8 \mathrm{~g} H^{3} m^{2} x^{3} Q_{, x} \Delta_{b} Q^{2} \\
& -8 H^{4} m x^{2} \Delta_{\tau_{4}} Q^{2}+\frac{24 \mathrm{~g}^{2} H^{2} m \alpha \sqrt{\left(2 \mathrm{~g}^{2} Q^{2}+H^{2} x^{2}\right) \tau_{4}} \Delta_{\tau_{4}} \lambda_{4} Q^{2}}{\sqrt{2 \mathrm{~g}^{2} Q^{2}+H^{2} x^{2}} \mathcal{V}}
\end{aligned}
$$




$$
\begin{aligned}
& +\frac{12 \mathrm{~g}^{2} H^{2} m \alpha \sqrt{\left(2 \mathrm{~g}^{2} Q^{2}+H^{2} x^{2}\right) \tau_{4}} \Delta_{\tau_{4}} \lambda_{4} \xi_{f} Q^{2}}{\sqrt{2 \mathrm{~g}^{2} Q^{2}+H^{2} x^{2}} \mathcal{V}} \\
& +16 \mathrm{~g}^{2} H^{2} m x^{2} \sqrt{2} Q_{, x} \Delta_{1, x} \tau_{4} Q^{2} \\
& +\frac{6 \mathrm{~g}^{2} H^{2} m x^{2} \alpha \sqrt{\left(2 \mathrm{~g}^{2} Q^{2}+H^{2} x^{2}\right) \tau_{4}} \Delta_{\tau_{4}} \lambda_{4} \tau_{4, x, x} Q^{2}}{\sqrt{2 \mathrm{~g}^{2} Q^{2}+H^{2} x^{2}} v \tau_{4}} \\
& -\frac{32 \sqrt{2} \mathrm{~g}^{2} H^{2} g_{s} \Delta_{b} \xi_{h} \sqrt{\tau_{4}} \sqrt{\left(2 \mathrm{~g}^{2} Q^{2}+H^{2} x^{2}\right) \tau_{4}} \sqrt{\frac{\left(2 \mathrm{~g}^{2} Q^{2}+H^{2} x^{2}\right) \tau_{4}}{\zeta}} Q^{2}}{\left(2 \mathrm{~g}^{2} Q^{2}+H^{2} x^{2}\right) \mathcal{V}} \\
& -\frac{32 \sqrt{2} \mathrm{~g}^{2} H^{2} x g_{s} \Delta_{b, x} \xi_{h} \sqrt{\tau_{4}} \sqrt{\left(2 \mathrm{~g}^{2} Q^{2}+H^{2} x^{2}\right) \tau_{4}} \sqrt{\frac{\left(2 \mathrm{~g}^{2} Q^{2}+H^{2} x^{2}\right) \tau_{4}}{\zeta}} Q^{2}}{\left(2 \mathrm{~g}^{2} Q^{2}+H^{2} x^{2}\right) \mathcal{V}} \\
& -\frac{16 \sqrt{2} \mathrm{~g}^{2} H^{2} g_{s} \Delta_{\tau_{4}} \xi_{h}^{2} \sqrt{\tau_{4}} \sqrt{\left(2 \mathrm{~g}^{2} Q^{2}+H^{2} x^{2}\right) \tau_{4}} \sqrt{\frac{\left(2 \mathrm{~g}^{2} Q^{2}+H^{2} x^{2}\right) \tau_{4}}{\zeta}} Q^{2}}{m\left(2 \mathrm{~g}^{2} Q^{2}+H^{2} x^{2}\right) \mathcal{V}} \\
& -\frac{18 \mathrm{~g}^{2} H^{2} m \alpha \Delta_{\tau_{4}} \lambda_{4} \xi_{f}^{2} \sqrt{\left(2 \mathrm{~g}^{2} Q^{2}+H^{2} x^{2}\right) \tau_{4}} Q^{2}}{\sqrt{2 \mathrm{~g}^{2} Q^{2}+H^{2} x^{2}} \mathcal{V}} \\
& -\frac{12 \mathrm{~g}^{2} H^{2} m x^{2} \alpha \Delta_{\tau_{4}} \lambda_{4} \sqrt{\left(2 \mathrm{~g}^{2} Q^{2}+H^{2} x^{2}\right) \tau_{4}} Q^{2}}{\sqrt{2 \mathrm{~g}^{2} Q^{2}+H^{2} x^{2}} \mathcal{V}} \\
& -\frac{12 \mathrm{~g}^{2} H^{2} m \alpha \epsilon \Delta_{\tau_{4}} \lambda_{4} \sqrt{\left(2 \mathrm{~g}^{2} Q^{2}+H^{2} x^{2}\right) \tau_{4}} Q^{2}}{\sqrt{2 \mathrm{~g}^{2} Q^{2}+H^{2} x^{2}} \mathcal{V}} \\
& -\frac{12 \mathrm{~g}^{2} H^{2} m x^{2} \alpha \Delta_{\tau_{4}, x, x} \lambda_{4} \sqrt{\left(2 \mathrm{~g}^{2} Q^{2}+H^{2} x^{2}\right) \tau_{4}} Q^{2}}{\sqrt{2 \mathrm{~g}^{2} Q^{2}+H^{2} x^{2}} \mathcal{V}} \\
& -\frac{12 \mathrm{~g}^{2} H^{2} m x \alpha \Delta_{\tau_{4}, x} \lambda_{4} \xi_{f} \sqrt{\left(2 \mathrm{~g}^{2} Q^{2}+H^{2} x^{2}\right) \tau_{4}} Q^{2}}{\sqrt{2 \mathrm{~g}^{2} Q^{2}+H^{2} x^{2}} \mathcal{V}} \\
& -\frac{16 \mathrm{~g}^{2} m V_{, b, \tau_{4}} \Delta_{b} \sqrt{\tau_{4}} \sqrt{\left(2 \mathrm{~g}^{2} Q^{2}+H^{2} x^{2}\right) \tau_{4}} Q^{2}}{\sqrt{2 \mathrm{~g}^{2} Q^{2}+H^{2} x^{2}}} \\
& -\frac{16 \mathrm{~g}^{2} m V_{, \tau_{2}, \tau_{4}} \Delta_{\tau_{2}} \sqrt{\tau_{4}} \sqrt{\left(2 \mathrm{~g}^{2} Q^{2}+H^{2} x^{2}\right) \tau_{4}} Q^{2}}{\sqrt{2 \mathrm{~g}^{2} Q^{2}+H^{2} x^{2}}} \\
& -\frac{16 \mathrm{~g}^{2} m V_{, \tau_{4}, \tau_{4}} \Delta_{\tau_{4}} \sqrt{\tau_{4}} \sqrt{\left(2 \mathrm{~g}^{2} Q^{2}+H^{2} x^{2}\right) \tau_{4}} Q^{2}}{\sqrt{2 \mathrm{~g}^{2} Q^{2}+H^{2} x^{2}}}+16 H^{4} m x^{3} Q_{, x} \Delta_{\tau_{4}} Q \\
& -8 \sqrt{2} H^{4} m x^{3} \Delta_{1, x} \tau_{4} Q-\frac{16 \sqrt{2} \mathrm{~g} H^{4} m x^{3} Q_{, x} \Delta_{2} \sqrt{\tau_{4}} \sqrt{\left(2 \mathrm{~g}^{2} Q^{2}+H^{2} x^{2}\right) \tau_{4}} Q}{2 \mathrm{~g}^{2} Q^{2}+H^{2} x^{2}} \\
& -\frac{8 \sqrt{2} \mathrm{~g} H^{4} m x^{4} Q_{, x} \Delta_{2, x} \sqrt{\tau_{4}} \sqrt{\left(2 \mathrm{~g}^{2} Q^{2}+H^{2} x^{2}\right) \tau_{4}} Q}{2 \mathrm{~g}^{2} Q^{2}+H^{2} x^{2}} \\
& +\frac{8 \mathrm{~g} H^{4} m x^{4} \sqrt{2} \sqrt{\tau_{4}} \sqrt{\left(2 \mathrm{~g}^{2} Q^{2}+H^{2} x^{2}\right) \tau_{4}} Q_{, x}^{2} \Delta_{2}}{2 \mathrm{~g}^{2} Q^{2}+H^{2} x^{2}}-8 H^{4} m x^{4} Q_{, x}^{2} \Delta_{\tau_{4}}
\end{aligned}
$$




$$
\begin{aligned}
& +\frac{12 H^{4} m x^{2} \alpha \sqrt{\left(2 \mathrm{~g}^{2} Q^{2}+H^{2} x^{2}\right) \tau_{4}} \Delta_{\tau_{4}} \lambda_{4}}{\sqrt{2 \mathrm{~g}^{2} Q^{2}+H^{2} x^{2}} \nu} \\
& +\frac{6 H^{4} m x^{2} \alpha \sqrt{\left(2 \mathrm{~g}^{2} Q^{2}+H^{2} x^{2}\right) \tau_{4}} \Delta_{\tau_{4}} \lambda_{4} \xi_{f}}{\sqrt{2 \mathrm{~g}^{2} Q^{2}+H^{2} x^{2}} \mathcal{V}} \\
& +8 H^{4} m x^{4} \sqrt{2} Q_{, x} \Delta_{1, x} \tau_{4} \\
& +\frac{3 H^{4} m x^{4} \alpha \sqrt{\left(2 \mathrm{~g}^{2} Q^{2}+H^{2} x^{2}\right) \tau_{4}} \Delta_{\tau_{4}} \lambda_{4} \tau_{4, x, x}}{\sqrt{2 \mathrm{~g}^{2} Q^{2}+H^{2} x^{2}} \nu \tau_{4}} \\
& -\frac{16 \sqrt{2} H^{4} x^{2} g_{s} \Delta_{b} \xi_{h} \sqrt{\tau_{4}} \sqrt{\left(2 \mathrm{~g}^{2} Q^{2}+H^{2} x^{2}\right) \tau_{4}} \sqrt{\frac{\left(2 \mathrm{~g}^{2} Q^{2}+H^{2} x^{2}\right) \tau_{4}}{\zeta}}}{\left(2 \mathrm{~g}^{2} Q^{2}+H^{2} x^{2}\right) \mathcal{V}} \\
& -\frac{16 \sqrt{2} H^{4} x^{3} g_{s} \Delta_{b, x} \xi_{h} \sqrt{\tau_{4}} \sqrt{\left(2 \mathrm{~g}^{2} Q^{2}+H^{2} x^{2}\right) \tau_{4}} \sqrt{\frac{\left(2 \mathrm{~g}^{2} Q^{2}+H^{2} x^{2}\right) \tau_{4}}{\zeta}}}{\left(2 \mathrm{~g}^{2} Q^{2}+H^{2} x^{2}\right) \mathcal{V}} \\
& -\frac{8 \sqrt{2} H^{4} x^{2} g_{s} \Delta_{\tau_{4}} \xi_{h}^{2} \sqrt{\tau_{4}} \sqrt{\left(2 \mathrm{~g}^{2} Q^{2}+H^{2} x^{2}\right) \tau_{4}} \sqrt{\frac{\left(2 \mathrm{~g}^{2} Q^{2}+H^{2} x^{2}\right) \tau_{4}}{\zeta}}}{m\left(2 \mathrm{~g}^{2} Q^{2}+H^{2} x^{2}\right) \mathcal{V}} \\
& -\frac{9 H^{4} m x^{2} \alpha \Delta_{\tau_{4}} \lambda_{4} \xi_{f}^{2} \sqrt{\left(2 \mathrm{~g}^{2} Q^{2}+H^{2} x^{2}\right) \tau_{4}}}{\sqrt{2 \mathrm{~g}^{2} Q^{2}+H^{2} x^{2}} \mathcal{V}} \\
& -\frac{6 H^{4} m x^{4} \alpha \Delta_{\tau_{4}} \lambda_{4} \sqrt{\left(2 \mathrm{~g}^{2} Q^{2}+H^{2} x^{2}\right) \tau_{4}}}{\sqrt{2 \mathrm{~g}^{2} Q^{2}+H^{2} x^{2}} \mathcal{V}} \\
& -\frac{6 H^{4} m x^{2} \alpha \epsilon \Delta_{\tau_{4}} \lambda_{4} \sqrt{\left(2 \mathrm{~g}^{2} Q^{2}+H^{2} x^{2}\right) \tau_{4}}}{\sqrt{2 \mathrm{~g}^{2} Q^{2}+H^{2} x^{2}} \mathcal{V}} \\
& -\frac{6 H^{4} m x^{4} \alpha \Delta_{\tau_{4}, x, x} \lambda_{4} \sqrt{\left(2 \mathrm{~g}^{2} Q^{2}+H^{2} x^{2}\right) \tau_{4}}}{\sqrt{2 \mathrm{~g}^{2} Q^{2}+H^{2} x^{2}} \mathcal{V}} \\
& -\frac{6 H^{4} m x^{3} \alpha \Delta_{\tau_{4}, x} \lambda_{4} \xi_{f} \sqrt{\left(2 \mathrm{~g}^{2} Q^{2}+H^{2} x^{2}\right) \tau_{4}}}{\sqrt{2 \mathrm{~g}^{2} Q^{2}+H^{2} x^{2}} \mathcal{V}} \\
& -\frac{8 H^{2} m x^{2} V_{, b, \tau_{4}} \Delta_{b} \sqrt{\tau_{4}} \sqrt{\left(2 \mathrm{~g}^{2} Q^{2}+H^{2} x^{2}\right) \tau_{4}}}{\sqrt{2 \mathrm{~g}^{2} Q^{2}+H^{2} x^{2}}} \\
& -\frac{8 H^{2} m x^{2} V_{, \tau_{2}, \tau_{4}} \Delta_{\tau_{2}} \sqrt{\tau_{4}} \sqrt{\left(2 \mathrm{~g}^{2} Q^{2}+H^{2} x^{2}\right) \tau_{4}}}{\sqrt{2 \mathrm{~g}^{2} Q^{2}+H^{2} x^{2}}} \\
& -\frac{8 H^{2} m x^{2} V_{, \tau_{4}, \tau_{4}} \Delta_{\tau_{4}} \sqrt{\tau_{4}} \sqrt{\left(2 \mathrm{~g}^{2} Q^{2}+H^{2} x^{2}\right) \tau_{4}}}{\sqrt{2 \mathrm{~g}^{2} Q^{2}+H^{2} x^{2}}}=0
\end{aligned}
$$




$$
\begin{aligned}
& \frac{m Q x^{4} \zeta \sqrt{2} \sqrt{\frac{\tau_{4}}{\zeta}} \sqrt{\frac{\left(2 \mathrm{~g}^{2} Q^{2}+H^{2} x^{2}\right) \tau_{4}}{\zeta}} \Delta_{2} H^{5}}{2 \mathrm{~g}^{2} Q^{2}+H^{2} x^{2}} \\
& -\frac{\sqrt{2} m x^{5} \zeta Q_{, x} \Delta_{2} \sqrt{\frac{\tau_{4}}{\zeta}} \sqrt{\frac{\left(2 \mathrm{~g}^{2} Q^{2}+H^{2} x^{2}\right) \tau_{4}}{\zeta}} H^{5}}{2 \mathrm{~g}^{2} Q^{2}+H^{2} x^{2}} \\
& +\frac{4 x^{2} \sqrt{4 \mathrm{~g}^{2} Q^{2}+2 H^{2} x^{2}} \sqrt{\frac{\left(2 \mathrm{~g}^{2} Q^{2}+H^{2} x^{2}\right) \tau_{4}}{\zeta}} g_{s} \Delta_{b} \tau_{4} H^{4}}{\left(2 \mathrm{~g}^{2} Q^{2}+H^{2} x^{2}\right) \mathcal{V}} \\
& +\frac{2 x^{2} \sqrt{4 \mathrm{~g}^{2} Q^{2}+2 H^{2} x^{2}} \sqrt{\frac{\left(2 \mathrm{~g}^{2} Q^{2}+H^{2} x^{2}\right) \tau_{4}}{\zeta}} g_{s} \Delta_{b} \xi_{f} \tau_{4} H^{4}}{\left(2 \mathrm{~g}^{2} Q^{2}+H^{2} x^{2}\right) \mathcal{V}} \\
& +\frac{2 x^{3} \sqrt{4 \mathrm{~g}^{2} Q^{2}+2 H^{2} x^{2}} \sqrt{\frac{\left(2 \mathrm{~g}^{2} Q^{2}+H^{2} x^{2}\right) \tau_{4}}{\zeta}} g_{s} \Delta_{b, x} \xi_{f} \tau_{4} H^{4}}{\left(2 \mathrm{~g}^{2} Q^{2}+H^{2} x^{2}\right) \mathcal{V}} \\
& +\frac{2 x^{3} \sqrt{4 \mathrm{~g}^{2} Q^{2}+2 H^{2} x^{2}} \sqrt{\frac{\left(2 \mathrm{~g}^{2} Q^{2}+H^{2} x^{2}\right) \tau_{4}}{\zeta}} g_{s} \Delta_{\tau_{4}, x} \xi_{h} \tau_{4} H^{4}}{m\left(2 \mathrm{~g}^{2} Q^{2}+H^{2} x^{2}\right) \mathcal{V}} \\
& +\frac{2 x^{2} \sqrt{4 \mathrm{~g}^{2} Q^{2}+2 H^{2} x^{2}} \sqrt{\frac{\left(2 \mathrm{~g}^{2} Q^{2}+H^{2} x^{2}\right) \tau_{4}}{\zeta}} g_{s} \Delta_{\tau_{4}} \xi_{f} \xi_{h} \tau_{4} H^{4}}{m\left(2 \mathrm{~g}^{2} Q^{2}+H^{2} x^{2}\right) \mathcal{V}} \\
& -\frac{x^{4} \sqrt{4 \mathrm{~g}^{2} Q^{2}+2 H^{2} x^{2}} b_{, x, x} g_{s} \Delta_{\tau_{4}} \sqrt{\frac{\left(2 \mathrm{~g}^{2} Q^{2}+H^{2} x^{2}\right) \tau_{4}}{\zeta}} H^{4}}{\left(2 \mathrm{~g}^{2} Q^{2}+H^{2} x^{2}\right) \mathcal{V}} \\
& -\frac{2 x^{2} \sqrt{4 \mathrm{~g}^{2} Q^{2}+2 H^{2} x^{2}} \epsilon g_{s} \Delta_{b} \tau_{4} \sqrt{\frac{\left(2 \mathrm{~g}^{2} Q^{2}+H^{2} x^{2}\right) \tau_{4}}{\zeta}} H^{4}}{\left(2 \mathrm{~g}^{2} Q^{2}+H^{2} x^{2}\right) \mathcal{V}} \\
& -\frac{2 x^{4} \sqrt{4 \mathrm{~g}^{2} Q^{2}+2 H^{2} x^{2}} g_{s} \Delta_{b} \tau_{4} \sqrt{\frac{\left(2 \mathrm{~g}^{2} Q^{2}+H^{2} x^{2}\right) \tau_{4}}{\zeta}} H^{4}}{\left(2 \mathrm{~g}^{2} Q^{2}+H^{2} x^{2}\right) \mathcal{V}} \\
& -\frac{2 x^{4} \sqrt{4 \mathrm{~g}^{2} Q^{2}+2 H^{2} x^{2}} g_{s} \Delta_{b, x, x} \tau_{4} \sqrt{\frac{\left(2 \mathrm{~g}^{2} Q^{2}+H^{2} x^{2}\right) \tau_{4}}{\zeta}} H^{4}}{\left(2 \mathrm{~g}^{2} Q^{2}+H^{2} x^{2}\right) \mathcal{V}} \\
& -\frac{2 x^{2} \sqrt{4 \mathrm{~g}^{2} Q^{2}+2 H^{2} x^{2}} g_{s} \Delta_{\tau_{4}} \xi_{h} \tau_{4} \sqrt{\frac{\left(2 \mathrm{~g}^{2} Q^{2}+H^{2} x^{2}\right) \tau_{4}}{\zeta}} H^{4}}{m\left(2 \mathrm{~g}^{2} Q^{2}+H^{2} x^{2}\right) \mathcal{V}} \\
& +\frac{3 \mathrm{~g}^{2} m Q^{3} x^{2} \zeta \sqrt{2} \sqrt{\frac{\tau_{4}}{\zeta}} \sqrt{\frac{\left(2 \mathrm{~g}^{2} Q^{2}+H^{2} x^{2}\right) \tau_{4}}{\zeta}} \Delta_{2} H^{3}}{2 \mathrm{~g}^{2} Q^{2}+H^{2} x^{2}}+\mathrm{g} m Q^{3} x^{2} \Delta_{\tau_{4}} H^{3} \\
& -\mathrm{g} m Q^{2} x^{3} Q_{, x} \Delta_{\tau_{4}} H^{3}-2 \sqrt{2} \mathrm{~g} m Q^{2} x^{2} \Delta_{1} \tau_{4} H^{3}+2 \mathrm{~g} m Q x^{3} \sqrt{2} Q_{, x} \Delta_{1} \tau_{4} H^{3} \\
& +\mathrm{g} m Q^{2} x^{3} \sqrt{2} \Delta_{1, x} \tau_{4} H^{3}-\frac{3 \sqrt{2} \mathrm{~g}^{2} m Q^{2} x^{3} \zeta Q_{, x} \Delta_{2} \sqrt{\frac{\tau_{4}}{\zeta}} \sqrt{\frac{\left(2 \mathrm{~g}^{2} Q^{2}+H^{2} x^{2}\right) \tau_{4}}{\zeta}} H^{3}}{2 \mathrm{~g}^{2} Q^{2}+H^{2} x^{2}} \\
& -\frac{\sqrt{2} \mathrm{~g}^{2} m Q^{3} x^{3} \zeta \Delta_{2, x} \sqrt{\frac{\tau_{4}}{\zeta}} \sqrt{\frac{\left(2 \mathrm{~g}^{2} Q^{2}+H^{2} x^{2}\right) \tau_{4}}{\zeta}} H^{3}}{2 \mathrm{~g}^{2} Q^{2}+H^{2} x^{2}}
\end{aligned}
$$




$$
\begin{aligned}
& -\mathrm{g}^{2} m^{2} Q^{4} x^{2} \Delta_{b} H^{2} \\
& +\frac{8 \mathrm{~g}^{2} Q^{2} \sqrt{4 \mathrm{~g}^{2} Q^{2}+2 H^{2} x^{2}} \sqrt{\frac{\left(2 \mathrm{~g}^{2} Q^{2}+H^{2} x^{2}\right) \tau_{4}}{\zeta}} g_{s} \Delta_{b} \tau_{4} H^{2}}{\left(2 \mathrm{~g}^{2} Q^{2}+H^{2} x^{2}\right) \mathcal{V}} \\
& -x^{2} V_{, b, b} \Delta_{b} \tau_{4} H^{2}-x^{2} V_{, \tau_{2}, b} \Delta_{\tau_{2}} \tau_{4} H^{2}-x^{2} V_{, b, \tau_{4}} \Delta_{\tau_{4}} \tau_{4} H^{2} \\
& +\frac{4 \mathrm{~g}^{2} Q^{2} \sqrt{4 \mathrm{~g}^{2} Q^{2}+2 H^{2} x^{2}} \sqrt{\frac{\left(2 \mathrm{~g}^{2} Q^{2}+H^{2} x^{2}\right) \tau_{4}}{\zeta}} g_{s} \Delta_{b} \xi_{f} \tau_{4} H^{2}}{\left(2 \mathrm{~g}^{2} Q^{2}+H^{2} x^{2}\right) \mathcal{V}} \\
& +\frac{4 \mathrm{~g}^{2} Q^{2} x \sqrt{4 \mathrm{~g}^{2} Q^{2}+2 H^{2} x^{2}} \sqrt{\frac{\left(2 \mathrm{~g}^{2} Q^{2}+H^{2} x^{2}\right) \tau_{4}}{\zeta}} g_{s} \Delta_{b, x} \xi_{f} \tau_{4} H^{2}}{\left(2 \mathrm{~g}^{2} Q^{2}+H^{2} x^{2}\right) \mathcal{V}} \\
& +\frac{4 \mathrm{~g}^{2} Q^{2} x \sqrt{4 \mathrm{~g}^{2} Q^{2}+2 H^{2} x^{2}} \sqrt{\frac{\left(2 \mathrm{~g}^{2} Q^{2}+H^{2} x^{2}\right) \tau_{4}}{\zeta}} g_{s} \Delta_{\tau_{4}, x} \xi_{h} \tau_{4} H^{2}}{m\left(2 \mathrm{~g}^{2} Q^{2}+H^{2} x^{2}\right) \mathcal{V}} \\
& +\frac{4 \mathrm{~g}^{2} Q^{2} \sqrt{4 \mathrm{~g}^{2} Q^{2}+2 H^{2} x^{2}} \sqrt{\frac{\left(2 \mathrm{~g}^{2} Q^{2}+H^{2} x^{2}\right) \tau_{4}}{\zeta}} g_{s} \Delta_{\tau_{4}} \xi_{f} \xi_{h} \tau_{4} H^{2}}{m\left(2 \mathrm{~g}^{2} Q^{2}+H^{2} x^{2}\right) \mathcal{V}} \\
& -\frac{2 \mathrm{~g}^{2} Q^{2} x^{2} \sqrt{4 \mathrm{~g}^{2} Q^{2}+2 H^{2} x^{2}} b_{, x, x} g_{s} \Delta_{\tau_{4}} \sqrt{\frac{\left(2 \mathrm{~g}^{2} Q^{2}+H^{2} x^{2}\right) \tau_{4}}{\zeta}} H^{2}}{\left(2 \mathrm{~g}^{2} Q^{2}+H^{2} x^{2}\right) \mathcal{V}} \\
& -\frac{4 \mathrm{~g}^{2} Q^{2} \sqrt{4 \mathrm{~g}^{2} Q^{2}+2 H^{2} x^{2}} \epsilon g_{s} \Delta_{b} \tau_{4} \sqrt{\frac{\left(2 \mathrm{~g}^{2} Q^{2}+H^{2} x^{2}\right) \tau_{4}}{\zeta}} H^{2}}{\left(2 \mathrm{~g}^{2} Q^{2}+H^{2} x^{2}\right) \mathcal{V}} \\
& -\frac{4 \mathrm{~g}^{2} Q^{2} x^{2} \sqrt{4 \mathrm{~g}^{2} Q^{2}+2 H^{2} x^{2}} g_{s} \Delta_{b} \tau_{4} \sqrt{\frac{\left(2 \mathrm{~g}^{2} Q^{2}+H^{2} x^{2}\right) \tau_{4}}{\zeta}} H^{2}}{\left(2 \mathrm{~g}^{2} Q^{2}+H^{2} x^{2}\right) \mathcal{V}} \\
& -\frac{4 \mathrm{~g}^{2} Q^{2} x^{2} \sqrt{4 \mathrm{~g}^{2} Q^{2}+2 H^{2} x^{2}} g_{s} \Delta_{b, x, x} \tau_{4} \sqrt{\frac{\left(2 \mathrm{~g}^{2} Q^{2}+H^{2} x^{2}\right) \tau_{4}}{\zeta}} H^{2}}{\left(2 \mathrm{~g}^{2} Q^{2}+H^{2} x^{2}\right) \mathcal{V}} \\
& -\frac{4 \mathrm{~g}^{2} Q^{2} \sqrt{4 \mathrm{~g}^{2} Q^{2}+2 H^{2} x^{2}} g_{s} \Delta_{\tau_{4}} \xi_{h} \tau_{4} \sqrt{\frac{\left(2 \mathrm{~g}^{2} Q^{2}+H^{2} x^{2}\right) \tau_{4}}{\zeta}} H^{2}}{m\left(2 \mathrm{~g}^{2} Q^{2}+H^{2} x^{2}\right) \mathcal{V}} \\
& +\frac{4 \mathrm{~g}^{4} m Q^{5} \zeta \sqrt{2} \sqrt{\frac{\tau_{4}}{\zeta}} \sqrt{\frac{\left(2 \mathrm{~g}^{2} Q^{2}+H^{2} x^{2}\right) \tau_{4}}{\zeta}} \Delta_{2} H}{2 \mathrm{~g}^{2} Q^{2}+H^{2} x^{2}} \\
& -4 \sqrt{2} \mathrm{~g}^{3} m Q^{4} \Delta_{1} \tau_{4} H+4 \mathrm{~g}^{3} m Q^{3} x \sqrt{2} Q_{, x} \Delta_{1} \tau_{4} H \\
& +2 \mathrm{~g}^{3} m Q^{4} x \sqrt{2} \Delta_{1, x} \tau_{4} H \\
& -\frac{4 \sqrt{2} \mathrm{~g}^{4} m Q^{4} x \zeta Q_{, x} \Delta_{2} \sqrt{\frac{\tau_{4}}{\zeta}} \sqrt{\frac{\left(2 \mathrm{~g}^{2} Q^{2}+H^{2} x^{2}\right) \tau_{4}}{\zeta}} H}{2 \mathrm{~g}^{2} Q^{2}+H^{2} x^{2}} \\
& -\frac{2 \sqrt{2} \mathrm{~g}^{4} m Q^{5} x \zeta \Delta_{2, x} \sqrt{\frac{\tau_{4}}{\zeta}} \sqrt{\frac{\left(2 \mathrm{~g}^{2} Q^{2}+H^{2} x^{2}\right) \tau_{4}}{\zeta}} H}{2 \mathrm{~g}^{2} Q^{2}+H^{2} x^{2}} \\
& -2 \mathrm{~g}^{2} Q^{2} V_{, b, b} \Delta_{b} \tau_{4}-2 \mathrm{~g}^{2} Q^{2} V_{, \tau_{2}, b} \Delta_{\tau_{2}} \tau_{4} \\
& -2 \mathrm{~g}^{2} Q^{2} V_{, b, \tau_{4}} \Delta_{\tau_{4}} \tau_{4}=0 \text {, }
\end{aligned}
$$




$$
\begin{aligned}
& \frac{9 \alpha H^{2} x^{2} \lambda_{2} \tau_{2, x}^{2} \Delta_{\tau_{2}}}{\mathcal{V} \sqrt{\tau_{2}}}+\frac{12 \alpha H^{2} x \lambda_{2} \sqrt{\tau_{2}} \tau_{2, x} \Delta_{\tau_{2}}}{\mathcal{V}}-\frac{12 \alpha H^{2} x^{2} \lambda_{2} \sqrt{\tau_{2}} \tau_{2, x} \Delta_{\tau_{2, x}}}{\mathcal{V}} \\
& -\frac{12 \alpha H^{2} x^{2} \lambda_{2} \sqrt{\tau_{2}} \tau_{2, x, x} \Delta_{\tau_{2}}}{\mathcal{V}}+32 \tau_{2}^{2} \Delta_{b} V_{, \tau_{2}, b}-\frac{48 \alpha H^{2} \lambda_{2} \tau_{2}^{3 / 2} \Delta_{\tau_{2}}}{\mathcal{V}} \\
& +\frac{24 \alpha H^{2} \epsilon \lambda_{2} \tau_{2}^{3 / 2} \Delta_{\tau_{2}}}{\mathcal{V}}+\frac{24 \alpha H^{2} x^{2} \lambda_{2} \tau_{2}^{3 / 2} \Delta_{\tau_{2}}}{\mathcal{V}} \\
& +\frac{24 \alpha H^{2} x^{2} \lambda_{2} \tau_{2}^{3 / 2} \Delta_{\tau_{2}, x, x}}{\mathcal{V}}+32 \tau_{2}^{2} \Delta_{\tau_{2}} V_{, \tau_{2}, \tau_{2}}+32 \tau_{2}^{2} V_{, \tau_{2}, \tau_{4}} \Delta_{\tau_{4}}=0 \\
& \Delta_{1, x, x}=-\Delta_{1}-\frac{4 \mathrm{~g}^{2} Q^{2} \Delta_{1}}{H^{2} x^{2}}+\frac{2 \mathrm{~g} Q \Delta_{1} \xi_{h}}{H x^{2}}+\frac{2 \Delta_{1, x} \xi_{f}}{x}-\frac{4 \mathrm{~g}^{2} Q^{2} \Delta_{2} \xi_{h}}{H x^{2} \sqrt{2 \mathrm{~g}^{2} Q^{2}+H^{2} x^{2}}} \\
& -\frac{2 H \Delta_{2} \xi_{h}}{\sqrt{2 \mathrm{~g}^{2} Q^{2}+H^{2} x^{2}}}+\frac{2 \mathrm{~g} Q \Delta_{2}}{\sqrt{2 \mathrm{~g}^{2} Q^{2}+H^{2} x^{2}}}+\frac{4 \mathrm{~g}^{3} Q^{3} \Delta_{2}}{H^{2} x^{2} \sqrt{2 \mathrm{~g}^{2} Q^{2}+H^{2} x^{2}}} \\
& -\frac{\sqrt{2} \mathrm{~g} m Q^{2} \Delta_{b}}{H x^{2} \tau_{4}}-\frac{\sqrt{2} \mathrm{~g} m Q^{2} \Delta_{b, x}}{H x \tau_{4}}-\frac{2 \sqrt{2} \mathrm{~g}^{2} Q^{3} \Delta_{\tau_{4}}}{H^{2} x^{2} \tau_{4}}+\frac{\sqrt{2} Q_{{ }_{x}} \Delta_{\tau_{4}}}{x \tau_{4}} \\
& -\frac{\sqrt{2} Q \Delta_{\tau_{4}}}{x^{2} \tau_{4}}+\frac{\sqrt{2} Q \epsilon \Delta_{\tau_{4}}}{x^{2} \tau_{4}}-\frac{\sqrt{2} Q_{, x, x} \Delta_{\tau_{4}}}{\tau_{4}}-\frac{\sqrt{2} Q_{, x} \Delta_{\tau_{4}, x}}{\tau_{4}}+\frac{\sqrt{2} Q \Delta_{\tau_{4}, x}}{x \tau_{4}},
\end{aligned}
$$

and

$$
\begin{aligned}
\Delta_{2, x, x}= & \frac{8 \mathrm{~g}^{5} \sqrt{2 \mathrm{~g}^{2} Q^{2}+H^{2} x^{2}} \Delta_{\tau_{4}} Q^{7}}{H^{6} Q \sqrt{2} \tau_{4} x^{6}+4 \mathrm{~g}^{2} H^{4} Q^{3} \sqrt{2} \tau_{4} x^{4}+4 \mathrm{~g}^{4} H^{2} Q^{5} \sqrt{2} \tau_{4} x^{2}} \\
& -\frac{8 \sqrt{2} \mathrm{~g}^{6} \Delta_{2} \tau_{4} Q^{7}}{H^{6} Q \sqrt{2} \tau_{4} x^{6}+4 \mathrm{~g}^{2} H^{4} Q^{3} \sqrt{2} \tau_{4} x^{4}+4 \mathrm{~g}^{4} H^{2} Q^{5} \sqrt{2} \tau_{4} x^{2}} \\
& +\frac{4 \mathrm{~g}^{4} H m \sqrt{2 \mathrm{~g}^{2} Q^{2}+H^{2} x^{2}} \Delta_{b} Q^{6}}{H^{6} Q \sqrt{2} \tau_{4} x^{6}+4 \mathrm{~g}^{2} H^{4} Q^{3} \sqrt{2} \tau_{4} x^{4}+4 \mathrm{~g}^{4} H^{2} Q^{5} \sqrt{2} \tau_{4} x^{2}} \\
& +\frac{4 \mathrm{~g}^{4} H m x \sqrt{2 \mathrm{~g}^{2} Q^{2}+H^{2} x^{2}} \Delta_{b, x} Q^{6}}{H^{6} Q \sqrt{2} \tau_{4} x^{6}+4 \mathrm{~g}^{2} H^{4} Q^{3} \sqrt{2} \tau_{4} x^{4}+4 \mathrm{~g}^{4} H^{2} Q^{5} \sqrt{2} \tau_{4} x^{2}} \\
& +\frac{8 \mathrm{~g}^{5} \sqrt{4 \mathrm{~g}^{2} Q^{2}+2 H^{2} x^{2}} \Delta_{1} \tau_{4} Q^{6}}{H^{6} Q \sqrt{2} \tau_{4} x^{6}+4 \mathrm{~g}^{2} H^{4} Q^{3} \sqrt{2} \tau_{4} x^{4}+4 \mathrm{~g}^{4} H^{2} Q^{5} \sqrt{2} \tau_{4} x^{2}} \\
& -\frac{4 \mathrm{~g}^{3} H^{2} \sqrt{2 \mathrm{~g}^{2} Q^{2}+H^{2} x^{2}} \epsilon \Delta_{\tau_{4}} Q^{5}}{H^{6} Q \sqrt{2} \tau_{4} x^{6}+4 \mathrm{~g}^{2} H^{4} Q^{3} \sqrt{2} \tau_{4} x^{4}+4 \mathrm{~g}^{4} H^{2} Q^{5} \sqrt{2} \tau_{4} x^{2}}
\end{aligned}
$$




$$
\begin{aligned}
& +\frac{4 \mathrm{~g}^{3} H^{2} \sqrt{2 \mathrm{~g}^{2} Q^{2}+H^{2} x^{2}} \Delta_{\tau_{4}} Q^{5}}{H^{6} Q \sqrt{2} \tau_{4} x^{6}+4 \mathrm{~g}^{2} H^{4} Q^{3} \sqrt{2} \tau_{4} x^{4}+4 \mathrm{~g}^{4} H^{2} Q^{5} \sqrt{2} \tau_{4} x^{2}} \\
& +\frac{8 \mathrm{~g}^{3} H^{2} x^{2} \sqrt{2 \mathrm{~g}^{2} Q^{2}+H^{2} x^{2}} \Delta_{\tau_{4}} Q^{5}}{H^{6} Q \sqrt{2} \tau_{4} x^{6}+4 \mathrm{~g}^{2} H^{4} Q^{3} \sqrt{2} \tau_{4} x^{4}+4 \mathrm{~g}^{4} H^{2} Q^{5} \sqrt{2} \tau_{4} x^{2}} \\
& -\frac{4 \mathrm{~g}^{3} H^{2} x \sqrt{2 \mathrm{~g}^{2} Q^{2}+H^{2} x^{2}} \Delta_{\tau_{4}, x} Q^{5}}{H^{6} Q \sqrt{2} \tau_{4} x^{6}+4 \mathrm{~g}^{2} H^{4} Q^{3} \sqrt{2} \tau_{4} x^{4}+4 \mathrm{~g}^{4} H^{2} Q^{5} \sqrt{2} \tau_{4} x^{2}} \\
& -\frac{12 \sqrt{2} \mathrm{~g}^{4} H^{2} x^{2} \Delta_{2} \tau_{4} Q^{5}}{H^{6} Q \sqrt{2} \tau_{4} x^{6}+4 \mathrm{~g}^{2} H^{4} Q^{3} \sqrt{2} \tau_{4} x^{4}+4 \mathrm{~g}^{4} H^{2} Q^{5} \sqrt{2} \tau_{4} x^{2}} \\
& +\frac{8 \mathrm{~g}^{4} H^{2} x \sqrt{2} \Delta_{2, x} \xi_{f} \tau_{4} Q^{5}}{H^{6} Q \sqrt{2} \tau_{4} x^{6}+4 \mathrm{~g}^{2} H^{4} Q^{3} \sqrt{2} \tau_{4} x^{4}+4 \mathrm{~g}^{4} H^{2} Q^{5} \sqrt{2} \tau_{4} x^{2}} \\
& -\frac{8 \sqrt{2} \mathrm{~g}^{4} H \sqrt{2 \mathrm{~g}^{2} Q^{2}+H^{2} x^{2}} \Delta_{1} \xi_{h} \tau_{4} Q^{5}}{H^{6} Q \sqrt{2} \tau_{4} x^{6}+4 \mathrm{~g}^{2} H^{4} Q^{3} \sqrt{2} \tau_{4} x^{4}+4 \mathrm{~g}^{4} H^{2} Q^{5} \sqrt{2} \tau_{4} x^{2}} \\
& +\frac{2 \mathrm{~g}^{2} H^{3} m x^{2} \sqrt{2 \mathrm{~g}^{2} Q^{2}+H^{2} x^{2}} \Delta_{b} Q^{4}}{H^{6} Q \sqrt{2} \tau_{4} x^{6}+4 \mathrm{~g}^{2} H^{4} Q^{3} \sqrt{2} \tau_{4} x^{4}+4 \mathrm{~g}^{4} H^{2} Q^{5} \sqrt{2} \tau_{4} x^{2}} \\
& +\frac{2 \mathrm{~g}^{2} H^{3} m x^{3} \sqrt{2 \mathrm{~g}^{2} Q^{2}+H^{2} x^{2}} \Delta_{b, x} Q^{4}}{H^{6} Q \sqrt{2} \tau_{4} x^{6}+4 \mathrm{~g}^{2} H^{4} Q^{3} \sqrt{2} \tau_{4} x^{4}+4 \mathrm{~g}^{4} H^{2} Q^{5} \sqrt{2} \tau_{4} x^{2}} \\
& -\frac{4 \mathrm{~g}^{3} H^{2} x \sqrt{2 \mathrm{~g}^{2} Q^{2}+H^{2} x^{2}} Q_{, x} \Delta_{\tau_{4}} Q^{4}}{H^{6} Q \sqrt{2} \tau_{4} x^{6}+4 \mathrm{~g}^{2} H^{4} Q^{3} \sqrt{2} \tau_{4} x^{4}+4 \mathrm{~g}^{4} H^{2} Q^{5} \sqrt{2} \tau_{4} x^{2}} \\
& +\frac{4 \mathrm{~g}^{3} H^{2} x^{2} \sqrt{2 \mathrm{~g}^{2} Q^{2}+H^{2} x^{2}} Q_{, x, x} \Delta_{\tau_{4}} Q^{4}}{H^{6} Q \sqrt{2} \tau_{4} x^{6}+4 \mathrm{~g}^{2} H^{4} Q^{3} \sqrt{2} \tau_{4} x^{4}+4 \mathrm{~g}^{4} H^{2} Q^{5} \sqrt{2} \tau_{4} x^{2}}
\end{aligned}
$$




$$
\begin{aligned}
& +\frac{4 \mathrm{~g}^{3} H^{2} x^{2} \sqrt{2 \mathrm{~g}^{2} Q^{2}+H^{2} x^{2}} Q_{, x} \Delta_{\tau_{4}, x} Q^{4}}{H^{6} Q \sqrt{2} \tau_{4} x^{6}+4 \mathrm{~g}^{2} H^{4} Q^{3} \sqrt{2} \tau_{4} x^{4}+4 \mathrm{~g}^{4} H^{2} Q^{5} \sqrt{2} \tau_{4} x^{2}} \\
& +\frac{8 \mathrm{~g}^{3} H^{2} x^{2} \sqrt{2} \sqrt{2 \mathrm{~g}^{2} Q^{2}+H^{2} x^{2}} \Delta_{1} \tau_{4} Q^{4}}{H^{6} Q \sqrt{2} \tau_{4} x^{6}+4 \mathrm{~g}^{2} H^{4} Q^{3} \sqrt{2} \tau_{4} x^{4}+4 \mathrm{~g}^{4} H^{2} Q^{5} \sqrt{2} \tau_{4} x^{2}} \\
& -\frac{2 \mathrm{~g} H^{4} x^{2} \sqrt{2 \mathrm{~g}^{2} Q^{2}+H^{2} x^{2}} \epsilon \Delta_{\tau_{4}} Q^{3}}{H^{6} Q \sqrt{2} \tau_{4} x^{6}+4 \mathrm{~g}^{2} H^{4} Q^{3} \sqrt{2} \tau_{4} x^{4}+4 \mathrm{~g}^{4} H^{2} Q^{5} \sqrt{2} \tau_{4} x^{2}} \\
& +\frac{2 \mathrm{~g} H^{4} x^{4} \sqrt{2 \mathrm{~g}^{2} Q^{2}+H^{2} x^{2}} \Delta_{\tau_{4}} Q^{3}}{H^{6} Q \sqrt{2} \tau_{4} x^{6}+4 \mathrm{~g}^{2} H^{4} Q^{3} \sqrt{2} \tau_{4} x^{4}+4 \mathrm{~g}^{4} H^{2} Q^{5} \sqrt{2} \tau_{4} x^{2}} \\
& +\frac{6 \mathrm{~g} H^{4} x^{2} \sqrt{2 \mathrm{~g}^{2} Q^{2}+H^{2} x^{2}} \Delta_{\tau_{4}} Q^{3}}{H^{6} Q \sqrt{2} \tau_{4} x^{6}+4 \mathrm{~g}^{2} H^{4} Q^{3} \sqrt{2} \tau_{4} x^{4}+4 \mathrm{~g}^{4} H^{2} Q^{5} \sqrt{2} \tau_{4} x^{2}} \\
& -\frac{2 \mathrm{~g} H^{4} x^{3} \sqrt{2 \mathrm{~g}^{2} Q^{2}+H^{2} x^{2}} \Delta_{\tau_{4}, x} Q^{3}}{H^{6} Q \sqrt{2} \tau_{4} x^{6}+4 \mathrm{~g}^{2} H^{4} Q^{3} \sqrt{2} \tau_{4} x^{4}+4 \mathrm{~g}^{4} H^{2} Q^{5} \sqrt{2} \tau_{4} x^{2}} \\
& -\frac{6 \sqrt{2} \mathrm{~g}^{2} H^{4} x^{4} \Delta_{2} \tau_{4} Q^{3}}{H^{6} Q \sqrt{2} \tau_{4} x^{6}+4 \mathrm{~g}^{2} H^{4} Q^{3} \sqrt{2} \tau_{4} x^{4}+4 \mathrm{~g}^{4} H^{2} Q^{5} \sqrt{2} \tau_{4} x^{2}} \\
& -\frac{2 \sqrt{2} \mathrm{~g}^{2} H^{4} x^{2} \Delta_{2} \tau_{4} Q^{3}}{H^{6} Q \sqrt{2} \tau_{4} x^{6}+4 \mathrm{~g}^{2} H^{4} Q^{3} \sqrt{2} \tau_{4} x^{4}+4 \mathrm{~g}^{4} H^{2} Q^{5} \sqrt{2} \tau_{4} x^{2}} \\
& -\frac{2 \sqrt{2} \mathrm{~g}^{2} H^{4} x^{2} \epsilon \Delta_{2} \tau_{4} Q^{3}}{H^{6} Q \sqrt{2} \tau_{4} x^{6}+4 \mathrm{~g}^{2} H^{4} Q^{3} \sqrt{2} \tau_{4} x^{4}+4 \mathrm{~g}^{4} H^{2} Q^{5} \sqrt{2} \tau_{4} x^{2}} \\
& +\frac{4 \mathrm{~g}^{2} H^{4} x^{2} \sqrt{2} \Delta_{2} \xi_{f} \tau_{4} Q^{3}}{H^{6} Q \sqrt{2} \tau_{4} x^{6}+4 \mathrm{~g}^{2} H^{4} Q^{3} \sqrt{2} \tau_{4} x^{4}+4 \mathrm{~g}^{4} H^{2} Q^{5} \sqrt{2} \tau_{4} x^{2}} \\
& +\frac{8 \mathrm{~g}^{2} H^{4} x^{3} \sqrt{2} \Delta_{2, x} \xi_{f} \tau_{4} Q^{3}}{H^{6} Q \sqrt{2} \tau_{4} x^{6}+4 \mathrm{~g}^{2} H^{4} Q^{3} \sqrt{2} \tau_{4} x^{4}+4 \mathrm{~g}^{4} H^{2} Q^{5} \sqrt{2} \tau_{4} x^{2}} \\
& -\frac{8 \sqrt{2} \mathrm{~g}^{2} H^{3} x^{2} \sqrt{2 \mathrm{~g}^{2} Q^{2}+H^{2} x^{2}} \Delta_{1} \xi_{h} \tau_{4} Q^{3}}{H^{6} Q \sqrt{2} \tau_{4} x^{6}+4 \mathrm{~g}^{2} H^{4} Q^{3} \sqrt{2} \tau_{4} x^{4}+4 \mathrm{~g}^{4} H^{2} Q^{5} \sqrt{2} \tau_{4} x^{2}} \\
& +\frac{2 H^{5} m x^{4} \sqrt{2 \mathrm{~g}^{2} Q^{2}+H^{2} x^{2}} \Delta_{b} Q^{2}}{H^{6} Q \sqrt{2} \tau_{4} x^{6}+4 \mathrm{~g}^{2} H^{4} Q^{3} \sqrt{2} \tau_{4} x^{4}+4 \mathrm{~g}^{4} H^{2} Q^{5} \sqrt{2} \tau_{4} x^{2}} \\
& -\frac{10 \mathrm{~g} H^{4} x^{3} \sqrt{2 \mathrm{~g}^{2} Q^{2}+H^{2} x^{2}} Q_{, x} \Delta_{\tau_{4}} Q^{2}}{H^{6} Q \sqrt{2} \tau_{4} x^{6}+4 \mathrm{~g}^{2} H^{4} Q^{3} \sqrt{2} \tau_{4} x^{4}+4 \mathrm{~g}^{4} H^{2} Q^{5} \sqrt{2} \tau_{4} x^{2}} \\
& +\frac{2 \mathrm{~g} H^{4} x^{4} \sqrt{2 \mathrm{~g}^{2} Q^{2}+H^{2} x^{2}} Q_{, x, x} \Delta_{\tau_{4}} Q^{2}}{H^{6} Q \sqrt{2} \tau_{4} x^{6}+4 \mathrm{~g}^{2} H^{4} Q^{3} \sqrt{2} \tau_{4} x^{4}+4 \mathrm{~g}^{4} H^{2} Q^{5} \sqrt{2} \tau_{4} x^{2}}
\end{aligned}
$$




$$
\begin{aligned}
& +\frac{2 \mathrm{~g} H^{4} x^{4} \sqrt{2 \mathrm{~g}^{2} Q^{2}+H^{2} x^{2}} Q_{, x} \Delta_{\tau_{4}, x} Q^{2}}{H^{6} Q \sqrt{2} \tau_{4} x^{6}+4 \mathrm{~g}^{2} H^{4} Q^{3} \sqrt{2} \tau_{4} x^{4}+4 \mathrm{~g}^{4} H^{2} Q^{5} \sqrt{2} \tau_{4} x^{2}} \\
& +\frac{2 \mathrm{~g} H^{4} x^{4} \sqrt{4 \mathrm{~g}^{2} Q^{2}+2 H^{2} x^{2}} \Delta_{1} \tau_{4} Q^{2}}{H^{6} Q \sqrt{2} \tau_{4} x^{6}+4 \mathrm{~g}^{2} H^{4} Q^{3} \sqrt{2} \tau_{4} x^{4}+4 \mathrm{~g}^{4} H^{2} Q^{5} \sqrt{2} \tau_{4} x^{2}} \\
& +\frac{8 \mathrm{~g}^{2} H^{4} x^{3} \sqrt{2} Q_{, x} \Delta_{2} \tau_{4} Q^{2}}{H^{6} Q \sqrt{2} \tau_{4} x^{6}+4 \mathrm{~g}^{2} H^{4} Q^{3} \sqrt{2} \tau_{4} x^{4}+4 \mathrm{~g}^{4} H^{2} Q^{5} \sqrt{2} \tau_{4} x^{2}} \\
& +\frac{2 \mathrm{~g}^{2} H^{4} x^{4} \sqrt{2} Q_{, x, x} \Delta_{2} \tau_{4} Q^{2}}{H^{6} Q \sqrt{2} \tau_{4} x^{6}+4 \mathrm{~g}^{2} H^{4} Q^{3} \sqrt{2} \tau_{4} x^{4}+4 \mathrm{~g}^{4} H^{2} Q^{5} \sqrt{2} \tau_{4} x^{2}} \\
& -\frac{4 \sqrt{2} \mathrm{~g}^{2} H^{4} x^{3} Q_{, x} \Delta_{2} \xi_{f} \tau_{4} Q^{2}}{H^{6} Q \sqrt{2} \tau_{4} x^{6}+4 \mathrm{~g}^{2} H^{4} Q^{3} \sqrt{2} \tau_{4} x^{4}+4 \mathrm{~g}^{4} H^{2} Q^{5} \sqrt{2} \tau_{4} x^{2}} \\
& -\frac{2 H^{5} m x^{5} \sqrt{2 \mathrm{~g}^{2} Q^{2}+H^{2} x^{2}} Q_{, x} \Delta_{b} Q}{H^{6} Q \sqrt{2} \tau_{4} x^{6}+4 \mathrm{~g}^{2} H^{4} Q^{3} \sqrt{2} \tau_{4} x^{4}+4 \mathrm{~g}^{4} H^{2} Q^{5} \sqrt{2} \tau_{4} x^{2}} \\
& +\frac{4 \mathrm{~g} H^{4} x^{4} \sqrt{2 \mathrm{~g}^{2} Q^{2}+H^{2} x^{2}} Q_{, x}^{2} \Delta_{\tau_{4}} Q}{H^{6} Q \sqrt{2} \tau_{4} x^{6}+4 \mathrm{~g}^{2} H^{4} Q^{3} \sqrt{2} \tau_{4} x^{4}+4 \mathrm{~g}^{4} H^{2} Q^{5} \sqrt{2} \tau_{4} x^{2}} \\
& -\frac{\sqrt{2} H^{6} x^{6} \Delta_{2} \tau_{4} Q}{H^{6} Q \sqrt{2} \tau_{4} x^{6}+4 \mathrm{~g}^{2} H^{4} Q^{3} \sqrt{2} \tau_{4} x^{4}+4 \mathrm{~g}^{4} H^{2} Q^{5} \sqrt{2} \tau_{4} x^{2}} \\
& -\frac{6 \sqrt{2} \mathrm{~g}^{2} H^{4} x^{4} Q_{, x}^{2} \Delta_{2} \tau_{4} Q}{H^{6} Q \sqrt{2} \tau_{4} x^{6}+4 \mathrm{~g}^{2} H^{4} Q^{3} \sqrt{2} \tau_{4} x^{4}+4 \mathrm{~g}^{4} H^{2} Q^{5} \sqrt{2} \tau_{4} x^{2}} \\
& -\frac{\sqrt{2} H^{6} x^{4} \epsilon \Delta_{2} \tau_{4} Q}{H^{6} Q \sqrt{2} \tau_{4} x^{6}+4 \mathrm{~g}^{2} H^{4} Q^{3} \sqrt{2} \tau_{4} x^{4}+4 \mathrm{~g}^{4} H^{2} Q^{5} \sqrt{2} \tau_{4} x^{2}} \\
& +\frac{2 H^{6} x^{4} \sqrt{2} \Delta_{2} \tau_{4} Q}{H^{6} Q \sqrt{2} \tau_{4} x^{6}+4 \mathrm{~g}^{2} H^{4} Q^{3} \sqrt{2} \tau_{4} x^{4}+4 \mathrm{~g}^{4} H^{2} Q^{5} \sqrt{2} \tau_{4} x^{2}} \\
& +\frac{2 H^{6} x^{4} \sqrt{2} \Delta_{2} \xi_{f} \tau_{4} Q}{H^{6} Q \sqrt{2} \tau_{4} x^{6}+4 \mathrm{~g}^{2} H^{4} Q^{3} \sqrt{2} \tau_{4} x^{4}+4 \mathrm{~g}^{4} H^{2} Q^{5} \sqrt{2} \tau_{4} x^{2}} \\
& +\frac{2 H^{6} x^{5} \sqrt{2} \Delta_{2, x} \xi_{f} \tau_{4} Q}{H^{6} Q \sqrt{2} \tau_{4} x^{6}+4 \mathrm{~g}^{2} H^{4} Q^{3} \sqrt{2} \tau_{4} x^{4}+4 \mathrm{~g}^{4} H^{2} Q^{5} \sqrt{2} \tau_{4} x^{2}} \\
& -\frac{2 \sqrt{2} H^{5} x^{4} \sqrt{2 \mathrm{~g}^{2} Q^{2}+H^{2} x^{2}} \Delta_{1} \xi_{h} \tau_{4} Q}{H^{6} Q \sqrt{2} \tau_{4} x^{6}+4 \mathrm{~g}^{2} H^{4} Q^{3} \sqrt{2} \tau_{4} x^{4}+4 \mathrm{~g}^{4} H^{2} Q^{5} \sqrt{2} \tau_{4} x^{2}} \\
& -\frac{2 \sqrt{2} H^{6} x^{5} Q_{, x} \Delta_{2} \tau_{4}}{H^{6} Q \sqrt{2} \tau_{4} x^{6}+4 \mathrm{~g}^{2} H^{4} Q^{3} \sqrt{2} \tau_{4} x^{4}+4 \mathrm{~g}^{4} H^{2} Q^{5} \sqrt{2} \tau_{4} x^{2}} \\
& +\frac{H^{6} x^{6} \sqrt{2} Q_{, x, x} \Delta_{2} \tau_{4}}{H^{6} Q \sqrt{2} \tau_{4} x^{6}+4 \mathrm{~g}^{2} H^{4} Q^{3} \sqrt{2} \tau_{4} x^{4}+4 \mathrm{~g}^{4} H^{2} Q^{5} \sqrt{2} \tau_{4} x^{2}} \\
& -\frac{2 \sqrt{2} H^{6} x^{5} Q_{, x} \Delta_{2} \xi_{f} \tau_{4}}{H^{6} Q \sqrt{2} \tau_{4} x^{6}+4 \mathrm{~g}^{2} H^{4} Q^{3} \sqrt{2} \tau_{4} x^{4}+4 \mathrm{~g}^{4} H^{2} Q^{5} \sqrt{2} \tau_{4} x^{2}} .
\end{aligned}
$$




\section{C.3 Example 4}

The tensor perturbation equations for the fibre inflation example are

$$
\begin{aligned}
t_{R, x, x}= & -\frac{4 \xi_{f} Q_{, x} \psi_{R}}{x}+\frac{4 Q \xi_{f} \psi_{R}}{x^{2}}+\frac{2 \xi_{f} t_{R, x}}{x}+\frac{2 \mathrm{~g}^{2} Q^{3} \psi_{R}}{H^{2} x^{2}}-\frac{2 \mathrm{~g} Q \xi_{h} t_{R}}{H x^{2}}-\frac{2 \mathrm{~g} Q^{2} \psi_{R}}{H x} \\
& +\frac{2 \mathrm{~g} Q t_{R}}{H x}+\frac{2 \xi_{h} t_{R}}{x}-\frac{2 Q_{, x} \psi_{R}}{x}+\frac{2 Q \psi_{R}}{x^{2}}+2 Q_{, x, x} \psi_{R}+2 Q_{, x} \psi_{R, x}-\frac{2 Q \psi_{R, x}}{x}-t_{R},
\end{aligned}
$$

and

$$
\begin{aligned}
\psi_{R, x, x}= & -\frac{3 \tau_{1, x}^{2} \psi_{R}}{8 \tau_{1}^{2}}+\frac{\tau_{1, x} \psi_{R} \mathcal{V}_{, x}}{2 \mathcal{V} \tau_{1}}-\frac{3 \alpha \gamma_{4} \sqrt{\tau_{4}^{3}} \xi_{f} \psi_{R} \mathcal{V}_{, x}}{\mathcal{V}^{2} x}-\frac{3 \alpha \gamma_{4} \sqrt{\tau_{4}^{3}} \xi_{f}^{2} \psi_{R}}{2 \mathcal{V} x^{2}}-\frac{3 \mathrm{~g}^{2} Q^{4} \tau_{4} \psi_{R}}{H^{2} x^{2}} \\
& +\frac{2 \mathrm{~g}^{2} Q^{3} \tau_{4} t_{R}}{H^{2} x^{2}}-\frac{8 \sqrt{2} \tau_{4}^{5 / 2} g_{s} \xi_{h}^{2} \psi_{R}}{\sqrt{\zeta} m^{2} \mathcal{V} x^{2}}-\frac{2 \mathrm{~g} Q^{2} \tau_{4} t_{R}}{H x}+\frac{Q^{2} \tau_{4} \psi_{R}}{x^{2}}+\tau_{4} Q_{, x}^{2} \psi_{R} \\
& -\frac{2 Q \tau_{4} Q_{, x} \psi_{R}}{x}-2 \tau_{4} Q_{, x} t_{R, x}+\frac{2 Q \tau_{4} t_{R, x}}{x}+\frac{2 V \psi_{R}}{H^{2} x^{2}}-\frac{\psi_{R} \mathcal{V}_{, x}^{2}}{2 \mathcal{V}^{2}}-\frac{4 \psi_{R}}{x^{2}}-\psi_{R} .
\end{aligned}
$$


The scalar perturbation equations are

$$
\begin{aligned}
& \frac{64 \mathrm{~g}^{2} H^{2} Q^{2} \mathcal{V} \sqrt{2} \sqrt{\frac{\left(2 \mathrm{~g}^{2} Q^{2}+H^{2} x^{2}\right) \tau_{4}}{\zeta}} \sqrt{\left(2 \mathrm{~g}^{2} Q^{2}+H^{2} x^{2}\right) \tau_{4}} g_{s} \Delta_{\nu} \xi_{h}^{2} \tau_{4}^{3 / 2}}{m\left(2 \mathrm{~g}^{2} Q^{2}+H^{2} x^{2}\right)} \\
& +\frac{32 H^{4} x^{2} \mathcal{V} \sqrt{2} \sqrt{\frac{\left(2 \mathrm{~g}^{2} Q^{2}+H^{2} x^{2}\right) \tau_{4}}{\zeta}} \sqrt{\left(2 \mathrm{~g}^{2} Q^{2}+H^{2} x^{2}\right) \tau_{4}} g_{s} \Delta_{\mathcal{V}} \xi_{h}^{2} \tau_{4}^{3 / 2}}{m\left(2 \mathrm{~g}^{2} Q^{2}+H^{2} x^{2}\right)} \\
& +\frac{12 \mathrm{~g}^{2} H^{2} m Q^{2} \nu \alpha \sqrt{\left(2 \mathrm{~g}^{2} Q^{2}+H^{2} x^{2}\right) \tau_{4}} \gamma_{4} \Delta_{\mathcal{}} \xi_{f}^{2} \tau_{4}}{\sqrt{2 \mathrm{~g}^{2} Q^{2}+H^{2} x^{2}}} \\
& +\frac{6 H^{4} m x^{2} \mathcal{V} \alpha \sqrt{\left(2 \mathrm{~g}^{2} Q^{2}+H^{2} x^{2}\right) \tau_{4}} \gamma_{4} \Delta_{\nu} \xi_{f}^{2} \tau_{4}}{\sqrt{2 \mathrm{~g}^{2} Q^{2}+H^{2} x^{2}}} \\
& +\frac{48 \mathrm{~g}^{2} H^{2} m Q^{2} \nu \alpha \sqrt{\left(2 \mathrm{~g}^{2} Q^{2}+H^{2} x^{2}\right) \tau_{4}} \gamma_{4} \Delta_{\mathcal{V}} \tau_{4}}{\sqrt{2 \mathrm{~g}^{2} Q^{2}+H^{2} x^{2}}} \\
& +\frac{24 H^{4} m x^{2} \nu \alpha \sqrt{\left(2 \mathrm{~g}^{2} Q^{2}+H^{2} x^{2}\right) \tau_{4}} \gamma_{4} \Delta_{\mathcal{V}} \tau_{4}}{\sqrt{2 \mathrm{~g}^{2} Q^{2}+H^{2} x^{2}}} \\
& +\frac{24 H^{4} m x^{4} \alpha \sqrt{\left(2 \mathrm{~g}^{2} Q^{2}+H^{2} x^{2}\right) \tau_{4}} \nu_{, x, x} \gamma_{4} \Delta_{\mathcal{V}} \tau_{4}}{\sqrt{2 \mathrm{~g}^{2} Q^{2}+H^{2} x^{2}}} \\
& +\frac{48 \mathrm{~g}^{2} H^{2} m Q^{2} x^{2} \alpha \sqrt{\left(2 \mathrm{~g}^{2} Q^{2}+H^{2} x^{2}\right) \tau_{4}} \mathcal{V}_{, x, x} \gamma_{4} \Delta_{\mathcal{V}} \tau_{4}}{\sqrt{2 \mathrm{~g}^{2} Q^{2}+H^{2} x^{2}}} \\
& +\frac{48 H^{4} m x^{4} \alpha \sqrt{\left(2 \mathrm{~g}^{2} Q^{2}+H^{2} x^{2}\right) \tau_{4}} \mathcal{V}_{, x} \gamma_{4} \Delta_{\mathcal{V}, x} \tau_{4}}{\sqrt{2 \mathrm{~g}^{2} Q^{2}+H^{2} x^{2}}} \\
& +\frac{96 \mathrm{~g}^{2} H^{2} m Q^{2} x^{2} \alpha \sqrt{\left(2 \mathrm{~g}^{2} Q^{2}+H^{2} x^{2}\right) \tau_{4}} \mathcal{V}_{, x} \gamma_{4} \Delta_{\mathcal{V}, x} \tau_{4}}{\sqrt{2 \mathrm{~g}^{2} Q^{2}+H^{2} x^{2}}} \\
& +\frac{12 H^{4} m x^{3} \nu \alpha \sqrt{\left(2 \mathrm{~g}^{2} Q^{2}+H^{2} x^{2}\right) \tau_{4}} \gamma_{4} \Delta_{\mathcal{V}, x} \xi_{f} \tau_{4}}{\sqrt{2 \mathrm{~g}^{2} Q^{2}+H^{2} x^{2}}} \\
& +\frac{24 \mathrm{~g}^{2} H^{2} m Q^{2} x \mathcal{V} \alpha \sqrt{\left(2 \mathrm{~g}^{2} Q^{2}+H^{2} x^{2}\right) \tau_{4}} \gamma_{4} \Delta_{\mathcal{V}, x} \xi_{f} \tau_{4}}{\sqrt{2 \mathrm{~g}^{2} Q^{2}+H^{2} x^{2}}} \\
& -\frac{72 H^{4} m x^{4} \alpha \mathcal{V}_{, x}^{2} \gamma_{4} \Delta_{\mathcal{V}} \sqrt{\left(2 \mathrm{~g}^{2} Q^{2}+H^{2} x^{2}\right) \tau_{4}} \tau_{4}}{\sqrt{2 \mathrm{~g}^{2} Q^{2}+H^{2} x^{2}} \mathcal{V}} \\
& -\frac{144 \mathrm{~g}^{2} H^{2} m Q^{2} x^{2} \alpha \mathcal{V}_{, x}^{2} \gamma_{4} \Delta_{\nu} \sqrt{\left(2 \mathrm{~g}^{2} Q^{2}+H^{2} x^{2}\right) \tau_{4}} \tau_{4}}{\sqrt{2 \mathrm{~g}^{2} Q^{2}+H^{2} x^{2}} \nu} \\
& -\frac{12 H^{4} m x^{4} \mathcal{V} \alpha \gamma_{4} \Delta_{\mathcal{V}} \sqrt{\left(2 \mathrm{~g}^{2} Q^{2}+H^{2} x^{2}\right) \tau_{4}} \tau_{4}}{\sqrt{2 \mathrm{~g}^{2} Q^{2}+H^{2} x^{2}}} \\
& -\frac{24 \mathrm{~g}^{2} H^{2} m Q^{2} x^{2} \mathcal{V} \alpha \gamma_{4} \Delta_{\nu} \sqrt{\left(2 \mathrm{~g}^{2} Q^{2}+H^{2} x^{2}\right) \tau_{4}} \tau_{4}}{\sqrt{2 \mathrm{~g}^{2} Q^{2}+H^{2} x^{2}}}
\end{aligned}
$$




$$
\begin{aligned}
& -\frac{24 \mathrm{~g}^{2} H^{2} m Q^{2} \mathcal{V} \alpha \epsilon \gamma_{4} \Delta_{\nu} \sqrt{\left(2 \mathrm{~g}^{2} Q^{2}+H^{2} x^{2}\right) \tau_{4}} \tau_{4}}{\sqrt{2 \mathrm{~g}^{2} Q^{2}+H^{2} x^{2}}} \\
& -\frac{12 H^{4} m x^{2} \mathcal{V} \alpha \epsilon \gamma_{4} \Delta_{v} \sqrt{\left(2 \mathrm{~g}^{2} Q^{2}+H^{2} x^{2}\right) \tau_{4}} \tau_{4}}{\sqrt{2 \mathrm{~g}^{2} Q^{2}+H^{2} x^{2}}}
\end{aligned}
$$

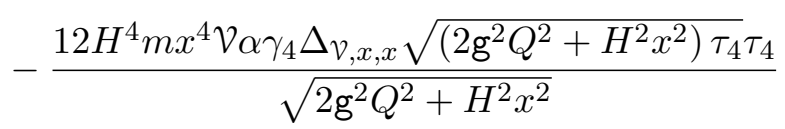

$$
\begin{aligned}
& -\frac{24 \mathrm{~g}^{2} H^{2} m Q^{2} x^{2} \mathcal{V} \alpha \gamma_{4} \Delta_{\mathcal{V}, x, x} \sqrt{\left(2 \mathrm{~g}^{2} Q^{2}+H^{2} x^{2}\right) \tau_{4}} \tau_{4}}{\sqrt{2 \mathrm{~g}^{2} Q^{2}+H^{2} x^{2}}} \\
& -\frac{24 \mathrm{~g}^{2} H^{2} m Q^{2} \nu \alpha \gamma_{4} \Delta_{\nu} \xi_{f} \sqrt{\left(2 \mathrm{~g}^{2} Q^{2}+H^{2} x^{2}\right) \tau_{4}} \tau_{4}}{\sqrt{2 \mathrm{~g}^{2} Q^{2}+H^{2} x^{2}}} \\
& -\frac{12 H^{4} m x^{2} \mathcal{V} \alpha \gamma_{4} \Delta_{v} \xi_{f} \sqrt{\left(2 \mathrm{~g}^{2} Q^{2}+H^{2} x^{2}\right) \tau_{4}} \tau_{4}}{\sqrt{2 \mathrm{~g}^{2} Q^{2}+H^{2} x^{2}}} \\
& -\frac{24 H^{4} m x^{3} \alpha \mathcal{V}_{, x} \gamma_{4} \Delta_{\nu} \xi_{f} \sqrt{\left(2 \mathrm{~g}^{2} Q^{2}+H^{2} x^{2}\right) \tau_{4}} \tau_{4}}{\sqrt{2 \mathrm{~g}^{2} Q^{2}+H^{2} x^{2}}} \\
& -\frac{48 \mathrm{~g}^{2} H^{2} m Q^{2} x \alpha \mathcal{V}_{, x} \gamma_{4} \Delta_{\nu} \xi_{f} \sqrt{\left(2 \mathrm{~g}^{2} Q^{2}+H^{2} x^{2}\right) \tau_{4}} \tau_{4}}{\sqrt{2 \mathrm{~g}^{2} Q^{2}+H^{2} x^{2}}} \\
& +\frac{18 \mathrm{~g}^{2} H^{2} m Q^{2} \mathcal{V}^{2} \alpha \sqrt{\left(2 \mathrm{~g}^{2} Q^{2}+H^{2} x^{2}\right) \tau_{4}} \gamma_{4} \Delta_{\tau_{4}} \xi_{f}^{2}}{\sqrt{2 \mathrm{~g}^{2} Q^{2}+H^{2} x^{2}}} \\
& +\frac{9 H^{4} m x^{2} \mathcal{V}^{2} \alpha \sqrt{\left(2 \mathrm{~g}^{2} Q^{2}+H^{2} x^{2}\right) \tau_{4}} \gamma_{4} \Delta_{\tau_{4}} \xi_{f}^{2}}{\sqrt{2 \mathrm{~g}^{2} Q^{2}+H^{2} x^{2}}} \\
& +\frac{32 \mathrm{~g}^{2} H^{2} Q^{2} \mathcal{V}^{2} \sqrt{2} \sqrt{\frac{\left(2 \mathrm{~g}^{2} Q^{2}+H^{2} x^{2}\right) \tau_{4}}{\zeta}} \sqrt{\tau_{4}} \sqrt{\left(2 \mathrm{~g}^{2} Q^{2}+H^{2} x^{2}\right) \tau_{4}} g_{s} \Delta_{\tau_{4}} \xi_{h}^{2}}{m\left(2 \mathrm{~g}^{2} Q^{2}+H^{2} x^{2}\right)} \\
& +\frac{16 H^{4} x^{2} \mathcal{V}^{2} \sqrt{2} \sqrt{\frac{\left(2 \mathrm{~g}^{2} Q^{2}+H^{2} x^{2}\right) \tau_{4}}{\zeta}} \sqrt{\tau_{4}} \sqrt{\left(2 \mathrm{~g}^{2} Q^{2}+H^{2} x^{2}\right) \tau_{4}} g_{s} \Delta_{\tau_{4}} \xi_{h}^{2}}{m\left(2 \mathrm{~g}^{2} Q^{2}+H^{2} x^{2}\right)} \\
& +\frac{64 \mathrm{~g}^{4} m Q^{5} \mathcal{V}^{3} \sqrt{4 \mathrm{~g}^{2} Q^{2}+2 H^{2} x^{2}} \sqrt{\tau_{4}} \sqrt{\left(2 \mathrm{~g}^{2} Q^{2}+H^{2} x^{2}\right) \tau_{4}} \Delta_{1}}{2 \mathrm{~g}^{2} Q^{2}+H^{2} x^{2}}
\end{aligned}
$$




$$
\begin{aligned}
& +\frac{32 \mathrm{~g}^{2} H^{2} m Q^{3} x^{2} \mathcal{V}^{3} \sqrt{2} \sqrt{\tau_{4}} \sqrt{\left(2 \mathrm{~g}^{2} Q^{2}+H^{2} x^{2}\right) \tau_{4}} \Delta_{1}}{\sqrt{2 \mathrm{~g}^{2} Q^{2}+H^{2} x^{2}}} \\
& +\frac{16 H^{4} m Q x^{3} \nu^{3} \sqrt{2} \sqrt{\tau_{4}} \sqrt{\left(2 \mathrm{~g}^{2} Q^{2}+H^{2} x^{2}\right) \tau_{4}} \Delta_{1, x}}{\sqrt{2 \mathrm{~g}^{2} Q^{2}+H^{2} x^{2}}} \\
& +\frac{32 \mathrm{~g}^{2} H^{2} m Q^{3} x \mathcal{V}^{3} \sqrt{2} \sqrt{\tau_{4}} \sqrt{\left(2 \mathrm{~g}^{2} Q^{2}+H^{2} x^{2}\right) \tau_{4}} \Delta_{1, x}}{\sqrt{2 \mathrm{~g}^{2} Q^{2}+H^{2} x^{2}}} \\
& +\frac{32 \mathrm{~g} H^{4} m Q x^{3} \mathcal{V}^{3} \sqrt{2} \sqrt{\tau_{4}} \sqrt{\left(2 \mathrm{~g}^{2} Q^{2}+H^{2} x^{2}\right) \tau_{4}} Q_{, x} \Delta_{2}}{2 \mathrm{~g}^{2} Q^{2}+H^{2} x^{2}} \\
& +\frac{16 \mathrm{~g} H^{4} m Q x^{4} \mathcal{V}^{3} \sqrt{2} \sqrt{\tau_{4}} \sqrt{\left(2 \mathrm{~g}^{2} Q^{2}+H^{2} x^{2}\right) \tau_{4}} Q_{, x} \Delta_{2, x}}{2 \mathrm{~g}^{2} Q^{2}+H^{2} x^{2}} \\
& +\frac{32 \mathrm{~g}^{3} H^{2} m Q^{3} x^{2} \mathcal{V}^{3} \sqrt{2} \sqrt{\tau_{4}} \sqrt{\left(2 \mathrm{~g}^{2} Q^{2}+H^{2} x^{2}\right) \tau_{4}} Q_{, x} \Delta_{2, x}}{2 \mathrm{~g}^{2} Q^{2}+H^{2} x^{2}} \\
& -16 \mathrm{~g} H^{3} m^{2} Q^{3} x^{2} \nu^{3} \Delta_{b}+16 \mathrm{~g} H^{3} m^{2} Q^{2} x^{3} \mathcal{V}^{3} Q_{, x} \Delta_{b} \\
& +\frac{32 \mathrm{~g}^{2} m Q^{2} V^{3} \sqrt{\tau_{4}} \sqrt{\left(2 \mathrm{~g}^{2} Q^{2}+H^{2} x^{2}\right) \tau_{4}} V_{, b, \tau_{4}} \Delta_{b}}{\sqrt{2 \mathrm{~g}^{2} Q^{2}+H^{2} x^{2}}} \\
& +\frac{16 H^{2} m x^{2} \mathcal{V}^{3} \sqrt{\tau_{4}} \sqrt{\left(2 \mathrm{~g}^{2} Q^{2}+H^{2} x^{2}\right) \tau_{4}} V_{, b, \tau_{4}} \Delta_{b}}{\sqrt{2 \mathrm{~g}^{2} Q^{2}+H^{2} x^{2}}} \\
& +\frac{32 \mathrm{~g}^{2} m Q^{2} \mathcal{V}^{3} \sqrt{\tau_{4}} \sqrt{\left(2 \mathrm{~g}^{2} Q^{2}+H^{2} x^{2}\right) \tau_{4}} V_{\tau_{1}, \tau_{4}} \Delta_{\tau_{1}}}{\sqrt{2 \mathrm{~g}^{2} Q^{2}+H^{2} x^{2}}} \\
& +\frac{16 H^{2} m x^{2} V^{3} \sqrt{\tau_{4}} \sqrt{\left(2 \mathrm{~g}^{2} Q^{2}+H^{2} x^{2}\right) \tau_{4}} V_{, \tau_{1}, \tau_{4}} \Delta_{\tau_{1}}}{\sqrt{2 \mathrm{~g}^{2} Q^{2}+H^{2} x^{2}}} \\
& +16 H^{4} m Q^{2} x^{2} \mathcal{V}^{3} \Delta_{\tau_{4}}+16 H^{4} m x^{4} \mathcal{V}^{3} Q_{, x}^{2} \Delta_{\tau_{4}} \\
& -32 H^{4} m Q x^{3} \nu^{3} Q_{, x} \Delta_{\tau_{4}} \\
& +\frac{32 \mathrm{~g}^{2} m Q^{2} \nu^{3} \sqrt{\tau_{4}} \sqrt{\left(2 \mathrm{~g}^{2} Q^{2}+H^{2} x^{2}\right) \tau_{4}} V_{, \tau_{4}, \tau_{4}} \Delta_{\tau_{4}}}{\sqrt{2 \mathrm{~g}^{2} Q^{2}+H^{2} x^{2}}} \\
& +\frac{16 H^{2} m x^{2} \mathcal{V}^{3} \sqrt{\tau_{4}} \sqrt{\left(2 \mathrm{~g}^{2} Q^{2}+H^{2} x^{2}\right) \tau_{4}} V_{, \tau_{4}, \tau_{4}} \Delta_{\tau_{4}}}{\sqrt{2 \mathrm{~g}^{2} Q^{2}+H^{2} x^{2}}} \\
& +\frac{12 H^{4} m x^{4} \alpha \sqrt{\left(2 \mathrm{~g}^{2} Q^{2}+H^{2} x^{2}\right) \tau_{4}} \mathcal{V}_{, x}^{2} \gamma_{4} \Delta_{\tau_{4}}}{\sqrt{2 \mathrm{~g}^{2} Q^{2}+H^{2} x^{2}}} \\
& +\frac{24 \mathrm{~g}^{2} H^{2} m Q^{2} x^{2} \alpha \sqrt{\left(2 \mathrm{~g}^{2} Q^{2}+H^{2} x^{2}\right) \tau_{4}} \mathcal{V}_{, x}^{2} \gamma_{4} \Delta_{\tau_{4}}}{\sqrt{2 \mathrm{~g}^{2} Q^{2}+H^{2} x^{2}}} \\
& +\frac{6 H^{4} m x^{4} \mathcal{V}^{2} \alpha \sqrt{\left(2 \mathrm{~g}^{2} Q^{2}+H^{2} x^{2}\right) \tau_{4}} \gamma_{4} \Delta_{\tau_{4}}}{\sqrt{2 \mathrm{~g}^{2} Q^{2}+H^{2} x^{2}}} \\
& +\frac{12 \mathrm{~g}^{2} H^{2} m Q^{2} x^{2} \mathcal{V}^{2} \alpha \sqrt{\left(2 \mathrm{~g}^{2} Q^{2}+H^{2} x^{2}\right) \tau_{4}} \gamma_{4} \Delta_{\tau_{4}}}{\sqrt{2 \mathrm{~g}^{2} Q^{2}+H^{2} x^{2}}} \\
& +\frac{12 \mathrm{~g}^{2} H^{2} m Q^{2} \nu^{2} \alpha \epsilon \sqrt{\left(2 \mathrm{~g}^{2} Q^{2}+H^{2} x^{2}\right) \tau_{4}} \gamma_{4} \Delta_{\tau_{4}}}{\sqrt{2 \mathrm{~g}^{2} Q^{2}+H^{2} x^{2}}}
\end{aligned}
$$




$$
\begin{aligned}
& +\frac{6 H^{4} m x^{2} \mathcal{V}^{2} \alpha \epsilon \sqrt{\left(2 \mathrm{~g}^{2} Q^{2}+H^{2} x^{2}\right) \tau_{4}} \gamma_{4} \Delta_{\tau_{4}}}{\sqrt{2 \mathrm{~g}^{2} Q^{2}+H^{2} x^{2}}} \\
& +\frac{6 H^{4} m x^{3} \mathcal{V} \alpha \sqrt{\left(2 \mathrm{~g}^{2} Q^{2}+H^{2} x^{2}\right) \tau_{4}} \nu_{, x} \gamma_{4} \Delta_{\tau_{4}}}{\sqrt{2 \mathrm{~g}^{2} Q^{2}+H^{2} x^{2}}} \\
& +\frac{12 \mathrm{~g}^{2} H^{2} m Q^{2} x \mathcal{V} \alpha \sqrt{\left(2 \mathrm{~g}^{2} Q^{2}+H^{2} x^{2}\right) \tau_{4}} \mathcal{V}_{, x} \gamma_{4} \Delta_{\tau_{4}}}{\sqrt{2 \mathrm{~g}^{2} Q^{2}+H^{2} x^{2}}} \\
& +\frac{6 H^{4} m x^{4} \mathcal{V}^{2} \alpha \sqrt{\left(2 \mathrm{~g}^{2} Q^{2}+H^{2} x^{2}\right) \tau_{4}} \gamma_{4} \Delta_{\tau_{4}, x, x}}{\sqrt{2 \mathrm{~g}^{2} Q^{2}+H^{2} x^{2}}} \\
& +\frac{12 \mathrm{~g}^{2} H^{2} m Q^{2} x^{2} \mathcal{V}^{2} \alpha \sqrt{\left(2 \mathrm{~g}^{2} Q^{2}+H^{2} x^{2}\right) \tau_{4}} \gamma_{4} \Delta_{\tau_{4}, x, x}}{\sqrt{2 \mathrm{~g}^{2} Q^{2}+H^{2} x^{2}}} \\
& +\frac{32 \mathrm{~g}^{2} m Q^{2} \mathcal{V}^{3} \sqrt{\tau_{4}} \sqrt{\left(2 \mathrm{~g}^{2} Q^{2}+H^{2} x^{2}\right) \tau_{4}} V_{, \nu, \tau_{4}} \Delta_{\mathcal{V}}}{\sqrt{2 \mathrm{~g}^{2} Q^{2}+H^{2} x^{2}}} \\
& +\frac{16 H^{2} m x^{2} \nu^{3} \sqrt{\tau_{4}} \sqrt{\left(2 \mathrm{~g}^{2} Q^{2}+H^{2} x^{2}\right) \tau_{4}} V_{, \nu, \tau_{4}} \Delta_{\mathcal{V}}}{\sqrt{2 \mathrm{~g}^{2} Q^{2}+H^{2} x^{2}}} \\
& +\frac{6 H^{4} m x^{3} \nu^{2} \alpha \sqrt{\left(2 \mathrm{~g}^{2} Q^{2}+H^{2} x^{2}\right) \tau_{4}} \gamma_{4} \Delta_{\tau_{4}, x} \xi_{f}}{\sqrt{2 \mathrm{~g}^{2} Q^{2}+H^{2} x^{2}}} \\
& +\frac{12 \mathrm{~g}^{2} H^{2} m Q^{2} x \mathcal{V}^{2} \alpha \sqrt{\left(2 \mathrm{~g}^{2} Q^{2}+H^{2} x^{2}\right) \tau_{4}} \gamma_{4} \Delta_{\tau_{4}, x} \xi_{f}}{\sqrt{2 \mathrm{~g}^{2} Q^{2}+H^{2} x^{2}}} \\
& +\frac{64 \mathrm{~g}^{2} H^{2} Q^{2} \mathcal{V}^{2} \sqrt{2} \sqrt{\frac{\left(2 \mathrm{~g}^{2} Q^{2}+H^{2} x^{2}\right) \tau_{4}}{\zeta}} \sqrt{\tau_{4}} \sqrt{\left(2 \mathrm{~g}^{2} Q^{2}+H^{2} x^{2}\right) \tau_{4}} g_{s} \Delta_{b} \xi_{h}}{2 \mathrm{~g}^{2} Q^{2}+H^{2} x^{2}} \\
& +\frac{32 H^{4} x^{2} V^{2} \sqrt{2} \sqrt{\frac{\left(2 \mathrm{~g}^{2} Q^{2}+H^{2} x^{2}\right) \tau_{4}}{\zeta}} \sqrt{\tau_{4}} \sqrt{\left(2 \mathrm{~g}^{2} Q^{2}+H^{2} x^{2}\right) \tau_{4}} g_{s} \Delta_{b} \xi_{h}}{2 \mathrm{~g}^{2} Q^{2}+H^{2} x^{2}} \\
& +\frac{32 H^{4} x^{3} V^{2} \sqrt{2} \sqrt{\frac{\left(2 \mathrm{~g}^{2} Q^{2}+H^{2} x^{2}\right) \tau_{4}}{\zeta}} \sqrt{\tau_{4}} \sqrt{\left(2 \mathrm{~g}^{2} Q^{2}+H^{2} x^{2}\right) \tau_{4}} g_{s} \Delta_{b, x} \xi_{h}}{2 \mathrm{~g}^{2} Q^{2}+H^{2} x^{2}} \\
& +\frac{64 \mathrm{~g}^{2} H^{2} Q^{2} x \mathcal{V}^{2} \sqrt{2} \sqrt{\frac{\left(2 \mathrm{~g}^{2} Q^{2}+H^{2} x^{2}\right) \tau_{4}}{\zeta}} \sqrt{\tau_{4}} \sqrt{\left(2 \mathrm{~g}^{2} Q^{2}+H^{2} x^{2}\right) \tau_{4}} g_{s} \Delta_{b, x} \xi_{h}}{2 \mathrm{~g}^{2} Q^{2}+H^{2} x^{2}} \\
& -\frac{16 H^{4} m x^{4} \sqrt{4 \mathrm{~g}^{2} Q^{2}+2 H^{2} x^{2}} \mathcal{V}^{3} Q_{, x} \Delta_{1, x} \sqrt{\tau_{4}} \sqrt{\left(2 \mathrm{~g}^{2} Q^{2}+H^{2} x^{2}\right) \tau_{4}}}{2 \mathrm{~g}^{2} Q^{2}+H^{2} x^{2}} \\
& -\frac{32 \mathrm{~g}^{2} H^{2} m Q^{2} x^{2} \sqrt{4 \mathrm{~g}^{2} Q^{2}+2 H^{2} x^{2}} \nu^{3} Q_{, x} \Delta_{1, x} \sqrt{\tau_{4}} \sqrt{\left(2 \mathrm{~g}^{2} Q^{2}+H^{2} x^{2}\right) \tau_{4}}}{2 \mathrm{~g}^{2} Q^{2}+H^{2} x^{2}} \\
& -\frac{64 \sqrt{2} \mathrm{~g}^{5} m Q^{6} \nu^{3} \Delta_{2} \sqrt{\tau_{4}} \sqrt{\left(2 \mathrm{~g}^{2} Q^{2}+H^{2} x^{2}\right) \tau_{4}}}{2 \mathrm{~g}^{2} Q^{2}+H^{2} x^{2}} \\
& -\frac{16 \sqrt{2} \mathrm{~g} H^{4} m Q^{2} x^{4} \mathcal{V}^{3} \Delta_{2} \sqrt{\tau_{4}} \sqrt{\left(2 \mathrm{~g}^{2} Q^{2}+H^{2} x^{2}\right) \tau_{4}}}{2 \mathrm{~g}^{2} Q^{2}+H^{2} x^{2}}
\end{aligned}
$$




$$
\begin{aligned}
& -\frac{64 \sqrt{2} \mathrm{~g}^{3} H^{2} m Q^{4} x^{2} \mathcal{V}^{3} \Delta_{2} \sqrt{\tau_{4}} \sqrt{\left(2 \mathrm{~g}^{2} Q^{2}+H^{2} x^{2}\right) \tau_{4}}}{2 \mathrm{~g}^{2} Q^{2}+H^{2} x^{2}} \\
& -\frac{16 \sqrt{2} \mathrm{~g} H^{4} m Q^{2} x^{2} \nu^{3} \Delta_{2} \sqrt{\tau_{4}} \sqrt{\left(2 \mathrm{~g}^{2} Q^{2}+H^{2} x^{2}\right) \tau_{4}}}{2 \mathrm{~g}^{2} Q^{2}+H^{2} x^{2}} \\
& -\frac{16 \sqrt{2} \mathrm{~g} H^{4} m x^{4} \mathcal{V}^{3} Q_{, x}^{2} \Delta_{2} \sqrt{\tau_{4}} \sqrt{\left(2 \mathrm{~g}^{2} Q^{2}+H^{2} x^{2}\right) \tau_{4}}}{2 \mathrm{~g}^{2} Q^{2}+H^{2} x^{2}} \\
& -\frac{16 \sqrt{2} \mathrm{~g} H^{4} m Q^{2} x^{3} \mathcal{V}^{3} \Delta_{2, x} \sqrt{\tau_{4}} \sqrt{\left(2 \mathrm{~g}^{2} Q^{2}+H^{2} x^{2}\right) \tau_{4}}}{2 \mathrm{~g}^{2} Q^{2}+H^{2} x^{2}} \\
& -\frac{32 \sqrt{2} \mathrm{~g}^{3} H^{2} m Q^{4} x \mathcal{V}^{3} \Delta_{2, x} \sqrt{\tau_{4}} \sqrt{\left(2 \mathrm{~g}^{2} Q^{2}+H^{2} x^{2}\right) \tau_{4}}}{2 \mathrm{~g}^{2} Q^{2}+H^{2} x^{2}} \\
& -\frac{24 \mathrm{~g}^{2} H^{2} m Q^{2} \mathcal{V}^{2} \alpha \gamma_{4} \Delta_{\tau_{4}} \sqrt{\left(2 \mathrm{~g}^{2} Q^{2}+H^{2} x^{2}\right) \tau_{4}}}{\sqrt{2 \mathrm{~g}^{2} Q^{2}+H^{2} x^{2}}} \\
& -\frac{12 H^{4} m x^{2} \mathcal{V}^{2} \alpha \gamma_{4} \Delta_{\tau_{4}} \sqrt{\left(2 \mathrm{~g}^{2} Q^{2}+H^{2} x^{2}\right) \tau_{4}}}{\sqrt{2 \mathrm{~g}^{2} Q^{2}+H^{2} x^{2}}} \\
& -\frac{6 H^{4} m x^{4} \mathcal{V} \alpha \mathcal{V}_{, x, x} \gamma_{4} \Delta_{\tau_{4}} \sqrt{\left(2 \mathrm{~g}^{2} Q^{2}+H^{2} x^{2}\right) \tau_{4}}}{\sqrt{2 \mathrm{~g}^{2} Q^{2}+H^{2} x^{2}}} \\
& -\frac{12 \mathrm{~g}^{2} H^{2} m Q^{2} x^{2} \mathcal{V} \alpha \mathcal{V}_{, x, x} \gamma_{4} \Delta_{\tau_{4}} \sqrt{\left(2 \mathrm{~g}^{2} Q^{2}+H^{2} x^{2}\right) \tau_{4}}}{\sqrt{2 \mathrm{~g}^{2} Q^{2}+H^{2} x^{2}}} \\
& -\frac{6 H^{4} m x^{4} \mathcal{V} \alpha \mathcal{V}_{, x} \gamma_{4} \Delta_{\tau_{4}, x} \sqrt{\left(2 \mathrm{~g}^{2} Q^{2}+H^{2} x^{2}\right) \tau_{4}}}{\sqrt{2 \mathrm{~g}^{2} Q^{2}+H^{2} x^{2}}} \\
& -\frac{12 \mathrm{~g}^{2} H^{2} m Q^{2} x^{2} \mathcal{V} \alpha \mathcal{V}_{, x} \gamma_{4} \Delta_{\tau_{4}, x} \sqrt{\left(2 \mathrm{~g}^{2} Q^{2}+H^{2} x^{2}\right) \tau_{4}}}{\sqrt{2 \mathrm{~g}^{2} Q^{2}+H^{2} x^{2}}} \\
& -\frac{12 \mathrm{~g}^{2} H^{2} m Q^{2} \mathcal{V}^{2} \alpha \gamma_{4} \Delta_{\tau_{4}} \xi_{f} \sqrt{\left(2 \mathrm{~g}^{2} Q^{2}+H^{2} x^{2}\right) \tau_{4}}}{\sqrt{2 \mathrm{~g}^{2} Q^{2}+H^{2} x^{2}}} \\
& -\frac{6 H^{4} m x^{2} \mathcal{V}^{2} \alpha \gamma_{4} \Delta_{\tau_{4}} \xi_{f} \sqrt{\left(2 \mathrm{~g}^{2} Q^{2}+H^{2} x^{2}\right) \tau_{4}}}{\sqrt{2 \mathrm{~g}^{2} Q^{2}+H^{2} x^{2}}} \\
& -\frac{6 H^{4} m x^{3} \mathcal{V} \alpha \nu_{, x} \gamma_{4} \Delta_{\tau_{4}} \xi_{f} \sqrt{\left(2 \mathrm{~g}^{2} Q^{2}+H^{2} x^{2}\right) \tau_{4}}}{\sqrt{2 \mathrm{~g}^{2} Q^{2}+H^{2} x^{2}}} \\
& -\frac{12 \mathrm{~g}^{2} H^{2} m Q^{2} x \mathcal{V} \alpha \mathcal{V}_{, x} \gamma_{4} \Delta_{\tau_{4}} \xi_{f} \sqrt{\left(2 \mathrm{~g}^{2} Q^{2}+H^{2} x^{2}\right) \tau_{4}}}{\sqrt{2 \mathrm{~g}^{2} Q^{2}+H^{2} x^{2}}} \\
& -\frac{6 H^{4} m x^{4} \mathcal{V} \alpha \gamma_{4} \Delta_{\mathcal{V}} \sqrt{\left(2 \mathrm{~g}^{2} Q^{2}+H^{2} x^{2}\right) \tau_{4}} \tau_{4, x, x}}{\sqrt{2 \mathrm{~g}^{2} Q^{2}+H^{2} x^{2}}} \\
& -\frac{12 \mathrm{~g}^{2} H^{2} m Q^{2} x^{2} \mathcal{V} \alpha \gamma_{4} \Delta_{\mathcal{V}} \sqrt{\left(2 \mathrm{~g}^{2} Q^{2}+H^{2} x^{2}\right) \tau_{4}} \tau_{4, x, x}}{\sqrt{2 \mathrm{~g}^{2} Q^{2}+H^{2} x^{2}}} \\
& -\frac{3 H^{4} m x^{4} \mathcal{V}^{2} \alpha \gamma_{4} \Delta_{\tau_{4}} \sqrt{\left(2 \mathrm{~g}^{2} Q^{2}+H^{2} x^{2}\right) \tau_{4}} \tau_{4, x, x}}{\sqrt{2 \mathrm{~g}^{2} Q^{2}+H^{2} x^{2}} \tau_{4}} \\
& -\frac{6 \mathrm{~g}^{2} H^{2} m Q^{2} x^{2} \nu^{2} \alpha \gamma_{4} \Delta_{\tau_{4}} \sqrt{\left(2 \mathrm{~g}^{2} Q^{2}+H^{2} x^{2}\right) \tau_{4}} \tau_{4, x, x}}{\sqrt{2 \mathrm{~g}^{2} Q^{2}+H^{2} x^{2}} \tau_{4}}= \\
& =0 \text {, }
\end{aligned}
$$




$$
\begin{aligned}
& \frac{m Q x^{4} \Delta_{2} H^{5}}{\sqrt{2}\left(2 \mathrm{~g}^{2} Q^{2}+H^{2} x^{2}\right)^{3 / 2}}+\frac{m Q x^{5} \Delta_{2, x} H^{5}}{\sqrt{2}\left(2 \mathrm{~g}^{2} Q^{2}+H^{2} x^{2}\right)^{3 / 2}} \\
& -\frac{m x^{5} Q_{, x} \Delta_{2} H^{5}}{\sqrt{2}\left(2 \mathrm{~g}^{2} Q^{2}+H^{2} x^{2}\right)^{3 / 2}}+\frac{m Q x^{2} \Delta_{2} H^{3}}{\sqrt{2} \sqrt{2 \mathrm{~g}^{2} Q^{2}+H^{2} x^{2}}} \\
& +\frac{\mathrm{g}^{2} m Q^{3} x^{3} \sqrt{2} \Delta_{2, x} H^{3}}{\left(2 \mathrm{~g}^{2} Q^{2}+H^{2} x^{2}\right)^{3 / 2}}-\frac{m x^{3} Q_{, x} \Delta_{2} H^{3}}{\sqrt{2} \sqrt{2 \mathrm{~g}^{2} Q^{2}+H^{2} x^{2}}} \\
& -\frac{m Q x^{3} \Delta_{2, x} H^{3}}{\sqrt{2} \sqrt{2 \mathrm{~g}^{2} Q^{2}+H^{2} x^{2}}}+\frac{\mathrm{g} m Q^{3} x^{2} \Delta_{\tau_{4}} H^{3}}{\left(2 \mathrm{~g}^{2} Q^{2}+H^{2} x^{2}\right) \tau_{4}} \\
& -\frac{\mathrm{g} m Q^{2} x^{3} Q_{, x} \Delta_{\tau_{4}} H^{3}}{\left(2 \mathrm{~g}^{2} Q^{2}+H^{2} x^{2}\right) \tau_{4}}+\frac{4 \sqrt{2} \sqrt{\frac{\tau_{4}}{\zeta}} g_{s} \Delta_{b} H^{2}}{\mathcal{V}} \\
& +\frac{2 x \sqrt{2} \sqrt{\frac{\tau_{4}}{\zeta}} g_{s} \mathcal{V}_{, x} \Delta_{b} H^{2}}{\mathcal{V}^{2}}+\frac{2 x^{2} \sqrt{2} \sqrt{\frac{\tau_{4}}{\zeta}} g_{s} \mathcal{V}_{, x} \Delta_{b, x} H^{2}}{\mathcal{V}^{2}} \\
& +\frac{2 x^{2} \sqrt{2} \sqrt{\frac{\tau_{4}}{\zeta}} b_{, x, x} g_{s} \Delta_{\mathcal{V}} H^{2}}{\mathcal{V}^{2}}+\frac{2 \sqrt{2} \sqrt{\frac{\tau_{4}}{\zeta}} g_{s} \Delta_{b} \xi_{f} H^{2}}{\mathcal{V}} \\
& +\frac{2 x \sqrt{2} \sqrt{\frac{\tau_{4}}{\zeta}} g_{s} \Delta_{b, x} \xi_{f} H^{2}}{\mathcal{V}}+\frac{2 x \sqrt{2} \sqrt{\frac{\tau_{4}}{\zeta}} g_{s} \Delta_{\tau_{4}, x} \xi_{h} H^{2}}{m \mathcal{V}} \\
& +\frac{2 \sqrt{2} \sqrt{\frac{\tau_{4}}{\zeta}} g_{s} \Delta_{\tau_{4}} \xi_{f} \xi_{h} H^{2}}{m \mathcal{V}}+\frac{4 \sqrt{2} \sqrt{\frac{\tau_{4}}{\zeta}} g_{s} \Delta_{\mathcal{V}} \xi_{h} \tau_{4} H^{2}}{m \mathcal{V}^{2}} \\
& +\frac{8 x \sqrt{2} \sqrt{\frac{\tau_{4}}{\zeta}} g_{s} \mathcal{V}_{, x} \Delta_{\mathcal{V}} \xi_{h} \tau_{4} H^{2}}{m \mathcal{V}^{3}}+\frac{4 \sqrt{2} \sqrt{\frac{\tau_{4}}{\zeta}} g_{s} \Delta_{\mathcal{V}} \xi_{f} \xi_{h} \tau_{4} H^{2}}{m \mathcal{V}^{2}} \\
& -\frac{2 \sqrt{2} x^{2} g_{s} \Delta_{b} \sqrt{\frac{\tau_{4}}{\zeta}} H^{2}}{V}-\frac{2 \sqrt{2} \epsilon g_{s} \Delta_{b} \sqrt{\frac{\tau_{4}}{\zeta}} H^{2}}{\mathcal{V}} \\
& -\frac{2 \sqrt{2} x^{2} g_{s} \Delta_{b, x, x} \sqrt{\frac{\tau_{4}}{\zeta}} H^{2}}{\mathcal{V}}-\frac{2 \sqrt{2} g_{s} \Delta_{\tau_{4}} \xi_{h} \sqrt{\frac{\tau_{4}}{\zeta}} H^{2}}{m \mathcal{V}} \\
& -\frac{2 \sqrt{2} x g_{s} \mathcal{V}_{, x} \Delta_{\tau_{4}} \xi_{h} \sqrt{\frac{\tau_{4}}{\zeta}} H^{2}}{m \mathcal{V}^{2}}-\frac{4 \sqrt{2} x g_{s} \Delta_{\mathcal{V}, x} \xi_{h} \tau_{4} \sqrt{\frac{\tau_{4}}{\zeta}} H^{2}}{m \mathcal{V}^{2}} \\
& -\frac{\sqrt{2} x^{2} b_{, x, x} g_{s} \Delta_{\tau_{4}} \sqrt{\frac{\tau_{4}}{\zeta}} H^{2}}{\mathcal{V} \tau_{4}}-\frac{\mathrm{g}^{2} m^{2} Q^{4} x^{2} \Delta_{b} H^{2}}{2 \mathrm{~g}^{2} \tau_{4} Q^{2}+H^{2} x^{2} \tau_{4}} \\
& -2 \mathrm{~g} m Q^{2} \sqrt{2} \Delta_{1} H+2 \mathrm{~g} m Q x \sqrt{2} Q_{, x} \Delta_{1} H \\
& +\mathrm{g} m Q^{2} x \sqrt{2} \Delta_{1, x} H+\frac{2 \mathrm{~g}^{2} m Q^{3} \sqrt{2} \Delta_{2} H}{\sqrt{2 \mathrm{~g}^{2} Q^{2}+H^{2} x^{2}}} \\
& -\frac{2 \sqrt{2} \mathrm{~g}^{2} m Q^{2} x Q_{, x} \Delta_{2} H}{\sqrt{2 \mathrm{~g}^{2} Q^{2}+H^{2} x^{2}}}-\frac{\sqrt{2} \mathrm{~g}^{2} m Q^{3} x \Delta_{2, x} H}{\sqrt{2 \mathrm{~g}^{2} Q^{2}+H^{2} x^{2}}} \\
& -V_{, b, b} \Delta_{b}-V_{, \tau_{1}, b} \Delta_{\tau_{1}}-V_{, b, \tau_{4}} \Delta_{\tau_{4}}-V_{, \nu, b} \Delta_{\mathcal{V}}=0,
\end{aligned}
$$




$$
\begin{aligned}
& \frac{9 H^{2} \mathcal{V}^{2} x^{2} \tau_{1, x}^{2} \Delta_{\tau_{1}}}{\tau_{1}}+8 \mathcal{V}^{2} \tau_{1}^{3} \Delta_{b} V_{, \tau_{1}, b}-6 H^{2} \mathcal{V}^{2} \tau_{1} \Delta_{\tau_{1}}+3 H^{2} \mathcal{V}^{2} \epsilon \tau_{1} \Delta_{\tau_{1}} \\
& -2 H^{2} x^{2} \tau_{1} \Delta_{\tau_{1}} \nu_{, x}^{2}-4 H^{2} \nu x \tau_{1} \Delta_{\tau_{1}} \nu_{, x}+3 H^{2} \nu^{2} x^{2} \tau_{1} \Delta_{\tau_{1}} \\
& +2 H^{2} \mathcal{V} x^{2} \tau_{1} \Delta_{\tau_{1}} \mathcal{V}_{, x, x}+3 H^{2} \mathcal{V}^{2} x^{2} \tau_{1} \Delta_{\tau_{1}, x, x}+4 H^{2} \mathcal{V} \tau_{1}^{2} \Delta_{\mathcal{V}} \\
& -2 H^{2} \mathcal{V}_{\epsilon} \tau_{1}^{2} \Delta_{\mathcal{V}}-\frac{4 H^{2} x^{2} \tau_{1}^{2} \Delta_{\mathcal{V}} \mathcal{\nu}_{, x}^{2}}{\mathcal{V}}+4 H^{2} x^{2} \tau_{1}^{2} \Delta_{\mathcal{V}, x} \mathcal{V}_{, x} \\
& -2 H^{2} \mathcal{V} x^{2} \tau_{1}^{2} \Delta_{\mathcal{V}}-2 H^{2} \mathcal{V} x^{2} \tau_{1}^{2} \Delta_{\mathcal{V}, x, x}+2 H^{2} x^{2} \tau_{1}^{2} \Delta_{\mathcal{V}} \mathcal{V}_{, x, x} \\
& +8 \mathcal{V}^{2} \tau_{1}^{3} \Delta_{\tau_{1}} V_{, \tau_{1}, \tau_{1}}+8 \mathcal{V}^{2} \tau_{1}^{3} V_{, \tau_{1}, \tau_{4}} \Delta_{\tau_{4}}+8 \mathcal{V}^{2} \tau_{1}^{3} \Delta_{\mathcal{V}} V_{, \nu, \tau_{1}} \\
& +6 H^{2} \mathcal{V}^{2} x \tau_{1, x} \Delta_{\tau_{1}}-6 H^{2} \mathcal{V}^{2} x^{2} \tau_{1, x} \Delta_{\tau_{1, x}}-6 H^{2} \mathcal{V}^{2} x^{2} \tau_{1, x, x} \Delta_{\tau_{1}}=0 \text {, }
\end{aligned}
$$

$$
\begin{aligned}
& -\frac{6 H^{2} \alpha \gamma_{4} \Delta_{\nu} \xi_{f} \tau_{4}^{3 / 2}}{\mathcal{V}^{3}}-\frac{3 H^{2} \alpha \gamma_{4} \Delta_{\nu} \xi_{f}^{2} \tau_{4}^{3 / 2}}{2 \mathcal{V}^{3}} \\
& +\frac{8 H^{2} \sqrt{2} \sqrt{\frac{\tau_{4}}{\zeta}} g_{s} \Delta_{\nu} \xi_{h}^{2} \tau_{4}^{2}}{m^{2} \mathcal{V}^{3}}+\frac{4 H^{2} \sqrt{2} \sqrt{\frac{\tau_{4}}{\zeta}} g_{s} \Delta_{b} \xi_{h} \tau_{4}}{m \mathcal{V}^{2}} \\
& +\frac{4 H^{2} x \sqrt{2} \sqrt{\frac{\tau_{4}}{\zeta}} g_{s} \Delta_{b, x} \xi_{h} \tau_{4}}{m \mathcal{V}^{2}}-\frac{2 \sqrt{2} H^{2} g_{s} \Delta_{\tau_{4}} \xi_{h}^{2} \sqrt{\frac{\tau_{4}}{\zeta}} \tau_{4}}{m^{2} \mathcal{V}^{2}} \\
& +\frac{H^{2} x^{2} \Delta_{\mathcal{V}} \tau_{1, x}^{2}}{4 \mathcal{V}^{2} \tau_{1}^{2}}+\frac{H^{2} x^{2} \Delta_{\tau_{1}} \tau_{1, x}^{2}}{2 \mathcal{V} \tau_{1}^{3}}-V_{, \mathcal{\nu}, b} \Delta_{b}-V_{, \mathcal{V}, \tau_{1}} \Delta_{\tau_{1}} \\
& -V_{, \mathcal{V}, \tau_{4}} \Delta_{\tau_{4}}+\frac{3 H^{2} x^{2} \alpha \sqrt{\tau_{4}} \gamma_{4} \Delta_{\tau_{4}}}{4 \mathcal{V}^{2}}+\frac{3 H^{2} \alpha \epsilon \sqrt{\tau_{4}} \gamma_{4} \Delta_{\tau_{4}}}{4 \mathcal{V}^{2}} \\
& +\frac{3 H^{2} x^{2} \alpha \sqrt{\tau_{4}} \gamma_{4} \Delta_{\tau_{4}, x, x}}{4 \mathcal{V}^{2}}-V_{, \mathcal{V}, \mathcal{V}} \Delta_{\mathcal{V}}+\frac{H^{2} x^{2} \mathcal{V}_{, x, x} \Delta_{\mathcal{V}}}{\mathcal{V}^{3}}+\frac{H^{2} \Delta_{\mathcal{V}}}{\mathcal{V}^{2}} \\
& +\frac{H^{2} x^{2} \mathcal{V}_{, x} \Delta_{\mathcal{V}, x}}{\mathcal{V}^{3}}+\frac{3 H^{2} \alpha \sqrt{\tau_{4}} \gamma_{4} \Delta_{\tau_{4}} \xi_{f}}{4 \mathcal{V}^{2}}+\frac{H^{2} x \Delta_{\mathcal{V}} \tau_{1, x}}{2 \mathcal{V}^{2} \tau_{1}} \\
& +\frac{3 H^{2} x^{2} \alpha \gamma_{4} \Delta_{\tau_{4}} \tau_{4, x, x}}{8 \mathcal{V}^{2} \sqrt{\tau_{4}}}-\frac{3 H^{2} \alpha \gamma_{4} \Delta_{\tau_{4}} \sqrt{\tau_{4}}}{2 \mathcal{V}^{2}}-\frac{H^{2} x^{2} \Delta_{\mathcal{V}}}{2 \mathcal{V}^{2}}-\frac{H^{2} \epsilon \Delta_{\mathcal{V}}}{2 \mathcal{V}^{2}} \\
& -\frac{H^{2} x^{2} \Delta_{\mathcal{V}, x, x}}{2 \mathcal{V}^{2}}-\frac{3 H^{2} x \alpha \gamma_{4} \Delta_{\tau_{4}, x} \xi_{f} \sqrt{\tau_{4}}}{4 \mathcal{V}^{2}}-\frac{3 H^{2} \alpha \gamma_{4} \Delta_{\tau_{4}} \xi_{f}^{2} \sqrt{\tau_{4}}}{8 \mathcal{V}^{2}} \\
& -\frac{H^{2} x \mathcal{V}_{, x} \Delta_{\mathcal{V}}}{\mathcal{V}^{3}}-\frac{3 H^{2} x^{2} \alpha \gamma_{4} \Delta_{\mathcal{V}} \sqrt{\tau_{4}} \tau_{4, x, x}}{2 \mathcal{V}^{3}}-\frac{3 H^{2} x^{2} \mathcal{V}_{, x}^{2} \Delta_{\mathcal{V}}}{2 \mathcal{V}^{4}} \\
& -\frac{H^{2} \Delta_{\tau_{1}}}{2 \mathcal{V} \tau_{1}}+\frac{H^{2} x^{2} \Delta_{\tau_{1}}}{4 \mathcal{V} \tau_{1}}+\frac{H^{2} \epsilon \Delta_{\tau_{1}}}{4 \mathcal{V} \tau_{1}}+\frac{H^{2} x^{2} \Delta_{\tau_{1}, x, x}}{4 \mathcal{V} \tau_{1}} \\
& -\frac{H^{2} x^{2} \Delta_{\mathcal{V}} \tau_{1, x, x}}{4 \mathcal{V}^{2} \tau_{1}}-\frac{H^{2} x^{2} \Delta_{\tau_{1, x}} \tau_{1, x}}{2 \mathcal{V} \tau_{1}^{2}}-\frac{H^{2} x^{2} \Delta_{\tau_{1}} \tau_{1, x, x}}{4 \mathcal{V} \tau_{1}^{2}}=0,
\end{aligned}
$$




$$
\begin{aligned}
\Delta_{1, x, x}= & -\Delta_{1}-\frac{4 \mathrm{~g}^{2} Q^{2} \Delta_{1}}{H^{2} x^{2}}+\frac{2 \mathrm{~g} Q \Delta_{1} \xi_{h}}{H x^{2}}+\frac{2 \Delta_{1, x} \xi_{f}}{x}-\frac{4 \mathrm{~g}^{2} Q^{2} \Delta_{2} \xi_{h}}{H x^{2} \sqrt{2 \mathrm{~g}^{2} Q^{2}+H^{2} x^{2}}} \\
& -\frac{2 H \Delta_{2} \xi_{h}}{\sqrt{2 \mathrm{~g}^{2} Q^{2}+H^{2} x^{2}}}+\frac{2 \mathrm{~g} Q \Delta_{2}}{\sqrt{2 \mathrm{~g}^{2} Q^{2}+H^{2} x^{2}}}+\frac{4 \mathrm{~g}^{3} Q^{3} \Delta_{2}}{H^{2} x^{2} \sqrt{2 \mathrm{~g}^{2} Q^{2}+H^{2} x^{2}}} \\
& -\frac{\sqrt{2} \mathrm{~g} m Q^{2} \Delta_{b}}{H x^{2} \tau_{4}}-\frac{\sqrt{2} \mathrm{~g} m Q^{2} \Delta_{b, x}}{H x \tau_{4}}-\frac{2 \sqrt{2} \mathrm{~g}^{2} Q^{3} \Delta_{\tau_{4}}}{H^{2} x^{2} \tau_{4}}+\frac{\sqrt{2} Q_{, x} \Delta_{\tau_{4}}}{x \tau_{4}} \\
& -\frac{\sqrt{2} Q \Delta_{\tau_{4}}}{x^{2} \tau_{4}}+\frac{\sqrt{2} Q \epsilon \Delta_{\tau_{4}}}{x^{2} \tau_{4}}-\frac{\sqrt{2} Q_{, x, x} \Delta_{\tau_{4}}}{\tau_{4}}-\frac{\sqrt{2} Q_{, x} \Delta_{\tau_{4}, x}}{\tau_{4}}+\frac{\sqrt{2} Q \Delta_{\tau_{4}, x}}{x \tau_{4}}
\end{aligned}
$$

and finally

$$
\begin{aligned}
\Delta_{2, x, x}= & \frac{8 \mathrm{~g}^{5} \sqrt{2 \mathrm{~g}^{2} Q^{2}+H^{2} x^{2}} \Delta_{\tau_{4}} Q^{7}}{H^{6} Q \sqrt{2} \tau_{4} x^{6}+4 \mathrm{~g}^{2} H^{4} Q^{3} \sqrt{2} \tau_{4} x^{4}+4 \mathrm{~g}^{4} H^{2} Q^{5} \sqrt{2} \tau_{4} x^{2}} \\
& -\frac{8 \sqrt{2} \mathrm{~g}^{6} \Delta_{2} \tau_{4} Q^{7}}{H^{6} Q \sqrt{2} \tau_{4} x^{6}+4 \mathrm{~g}^{2} H^{4} Q^{3} \sqrt{2} \tau_{4} x^{4}+4 \mathrm{~g}^{4} H^{2} Q^{5} \sqrt{2} \tau_{4} x^{2}} \\
& +\frac{4 \mathrm{~g}^{4} H m \sqrt{2 \mathrm{~g}^{2} Q^{2}+H^{2} x^{2}} \Delta_{b} Q^{6}}{H^{6} Q \sqrt{2} \tau_{4} x^{6}+4 \mathrm{~g}^{2} H^{4} Q^{3} \sqrt{2} \tau_{4} x^{4}+4 \mathrm{~g}^{4} H^{2} Q^{5} \sqrt{2} \tau_{4} x^{2}} \\
& +\frac{4 \mathrm{~g}^{4} H m x \sqrt{2 \mathrm{~g}^{2} Q^{2}+H^{2} x^{2}} \Delta_{b, x} Q^{6}}{H^{6} Q \sqrt{2} \tau_{4} x^{6}+4 \mathrm{~g}^{2} H^{4} Q^{3} \sqrt{2} \tau_{4} x^{4}+4 \mathrm{~g}^{4} H^{2} Q^{5} \sqrt{2} \tau_{4} x^{2}} \\
& +\frac{8 \mathrm{~g}^{5} \sqrt{4 \mathrm{~g}^{2} Q^{2}+2 H^{2} x^{2}} \Delta_{1} \tau_{4} Q^{6}}{H^{6} Q \sqrt{2} \tau_{4} x^{6}+4 \mathrm{~g}^{2} H^{4} Q^{3} \sqrt{2} \tau_{4} x^{4}+4 \mathrm{~g}^{4} H^{2} Q^{5} \sqrt{2} \tau_{4} x^{2}} \\
& -\frac{4 \mathrm{~g}^{3} H^{2} \sqrt{2 \mathrm{~g}^{2} Q^{2}+H^{2} x^{2}} \epsilon \Delta_{\tau_{4}} Q^{5}}{H^{6} Q \sqrt{2} \tau_{4} x^{6}+4 \mathrm{~g}^{2} H^{4} Q^{3} \sqrt{2} \tau_{4} x^{4}+4 \mathrm{~g}^{4} H^{2} Q^{5} \sqrt{2} \tau_{4} x^{2}} \\
& +\frac{4 \mathrm{~g}^{3} H^{2} \sqrt{2 \mathrm{~g}^{2} Q^{2}+H^{2} x^{2}} \Delta_{\tau_{4}} Q^{5}}{H^{6} Q \sqrt{2} \tau_{4} x^{6}+4 \mathrm{~g}^{2} H^{4} Q^{3} \sqrt{2} \tau_{4} x^{4}+4 \mathrm{~g}^{4} H^{2} Q^{5} \sqrt{2} \tau_{4} x^{2}}
\end{aligned}
$$




$$
\begin{aligned}
& +\frac{8 \mathrm{~g}^{3} H^{2} x^{2} \sqrt{2 \mathrm{~g}^{2} Q^{2}+H^{2} x^{2}} \Delta_{\tau_{4}} Q^{5}}{H^{6} Q \sqrt{2} \tau_{4} x^{6}+4 \mathrm{~g}^{2} H^{4} Q^{3} \sqrt{2} \tau_{4} x^{4}+4 \mathrm{~g}^{4} H^{2} Q^{5} \sqrt{2} \tau_{4} x^{2}} \\
& -\frac{4 \mathrm{~g}^{3} H^{2} x \sqrt{2 \mathrm{~g}^{2} Q^{2}+H^{2} x^{2}} \Delta_{\tau_{4}, x} Q^{5}}{H^{6} Q \sqrt{2} \tau_{4} x^{6}+4 \mathrm{~g}^{2} H^{4} Q^{3} \sqrt{2} \tau_{4} x^{4}+4 \mathrm{~g}^{4} H^{2} Q^{5} \sqrt{2} \tau_{4} x^{2}} \\
& -\frac{12 \sqrt{2} \mathrm{~g}^{4} H^{2} x^{2} \Delta_{2} \tau_{4} Q^{5}}{H^{6} Q \sqrt{2} \tau_{4} x^{6}+4 \mathrm{~g}^{2} H^{4} Q^{3} \sqrt{2} \tau_{4} x^{4}+4 \mathrm{~g}^{4} H^{2} Q^{5} \sqrt{2} \tau_{4} x^{2}} \\
& +\frac{8 \mathrm{~g}^{4} H^{2} x \sqrt{2} \Delta_{2, x} \xi_{f} \tau_{4} Q^{5}}{H^{6} Q \sqrt{2} \tau_{4} x^{6}+4 \mathrm{~g}^{2} H^{4} Q^{3} \sqrt{2} \tau_{4} x^{4}+4 \mathrm{~g}^{4} H^{2} Q^{5} \sqrt{2} \tau_{4} x^{2}} \\
& -\frac{8 \sqrt{2} \mathrm{~g}^{4} H \sqrt{2 \mathrm{~g}^{2} Q^{2}+H^{2} x^{2}} \Delta_{1} \xi_{h} \tau_{4} Q^{5}}{H^{6} Q \sqrt{2} \tau_{4} x^{6}+4 \mathrm{~g}^{2} H^{4} Q^{3} \sqrt{2} \tau_{4} x^{4}+4 \mathrm{~g}^{4} H^{2} Q^{5} \sqrt{2} \tau_{4} x^{2}} \\
& +\frac{2 \mathrm{~g}^{2} H^{3} m x^{2} \sqrt{2 \mathrm{~g}^{2} Q^{2}+H^{2} x^{2}} \Delta_{b} Q^{4}}{H^{6} Q \sqrt{2} \tau_{4} x^{6}+4 \mathrm{~g}^{2} H^{4} Q^{3} \sqrt{2} \tau_{4} x^{4}+4 \mathrm{~g}^{4} H^{2} Q^{5} \sqrt{2} \tau_{4} x^{2}} \\
& +\frac{2 \mathrm{~g}^{2} H^{3} m x^{3} \sqrt{2 \mathrm{~g}^{2} Q^{2}+H^{2} x^{2}} \Delta_{b, x} Q^{4}}{H^{6} Q \sqrt{2} \tau_{4} x^{6}+4 \mathrm{~g}^{2} H^{4} Q^{3} \sqrt{2} \tau_{4} x^{4}+4 \mathrm{~g}^{4} H^{2} Q^{5} \sqrt{2} \tau_{4} x^{2}} \\
& -\frac{4 \mathrm{~g}^{3} H^{2} x \sqrt{2 \mathrm{~g}^{2} Q^{2}+H^{2} x^{2}} Q_{, x} \Delta_{\tau_{4}} Q^{4}}{H^{6} Q \sqrt{2} \tau_{4} x^{6}+4 \mathrm{~g}^{2} H^{4} Q^{3} \sqrt{2} \tau_{4} x^{4}+4 \mathrm{~g}^{4} H^{2} Q^{5} \sqrt{2} \tau_{4} x^{2}} \\
& +\frac{4 \mathrm{~g}^{3} H^{2} x^{2} \sqrt{2 \mathrm{~g}^{2} Q^{2}+H^{2} x^{2}} Q_{, x, x} \Delta_{\tau_{4}} Q^{4}}{H^{6} Q \sqrt{2} \tau_{4} x^{6}+4 \mathrm{~g}^{2} H^{4} Q^{3} \sqrt{2} \tau_{4} x^{4}+4 \mathrm{~g}^{4} H^{2} Q^{5} \sqrt{2} \tau_{4} x^{2}} \\
& +\frac{4 \mathrm{~g}^{3} H^{2} x^{2} \sqrt{2 \mathrm{~g}^{2} Q^{2}+H^{2} x^{2}} Q_{, x} \Delta_{\tau_{4}, x} Q^{4}}{H^{6} Q \sqrt{2} \tau_{4} x^{6}+4 \mathrm{~g}^{2} H^{4} Q^{3} \sqrt{2} \tau_{4} x^{4}+4 \mathrm{~g}^{4} H^{2} Q^{5} \sqrt{2} \tau_{4} x^{2}} \\
& +\frac{8 \mathrm{~g}^{3} H^{2} x^{2} \sqrt{2} \sqrt{2 \mathrm{~g}^{2} Q^{2}+H^{2} x^{2}} \Delta_{1} \tau_{4} Q^{4}}{H^{6} Q \sqrt{2} \tau_{4} x^{6}+4 \mathrm{~g}^{2} H^{4} Q^{3} \sqrt{2} \tau_{4} x^{4}+4 \mathrm{~g}^{4} H^{2} Q^{5} \sqrt{2} \tau_{4} x^{2}} \\
& -\frac{2 \mathrm{~g} H^{4} x^{2} \sqrt{2 \mathrm{~g}^{2} Q^{2}+H^{2} x^{2}} \epsilon \Delta_{\tau_{4}} Q^{3}}{H^{6} Q \sqrt{2} \tau_{4} x^{6}+4 \mathrm{~g}^{2} H^{4} Q^{3} \sqrt{2} \tau_{4} x^{4}+4 \mathrm{~g}^{4} H^{2} Q^{5} \sqrt{2} \tau_{4} x^{2}}
\end{aligned}
$$




$$
\begin{aligned}
& +\frac{2 \mathrm{~g} H^{4} x^{4} \sqrt{2 \mathrm{~g}^{2} Q^{2}+H^{2} x^{2}} \Delta_{\tau_{4}} Q^{3}}{H^{6} Q \sqrt{2} \tau_{4} x^{6}+4 \mathrm{~g}^{2} H^{4} Q^{3} \sqrt{2} \tau_{4} x^{4}+4 \mathrm{~g}^{4} H^{2} Q^{5} \sqrt{2} \tau_{4} x^{2}} \\
& +\frac{6 \mathrm{~g} H^{4} x^{2} \sqrt{2 \mathrm{~g}^{2} Q^{2}+H^{2} x^{2}} \Delta_{\tau_{4}} Q^{3}}{H^{6} Q \sqrt{2} \tau_{4} x^{6}+4 \mathrm{~g}^{2} H^{4} Q^{3} \sqrt{2} \tau_{4} x^{4}+4 \mathrm{~g}^{4} H^{2} Q^{5} \sqrt{2} \tau_{4} x^{2}} \\
& -\frac{2 \mathrm{~g} H^{4} x^{3} \sqrt{2 \mathrm{~g}^{2} Q^{2}+H^{2} x^{2}} \Delta_{\tau_{4}, x} Q^{3}}{H^{6} Q \sqrt{2} \tau_{4} x^{6}+4 \mathrm{~g}^{2} H^{4} Q^{3} \sqrt{2} \tau_{4} x^{4}+4 \mathrm{~g}^{4} H^{2} Q^{5} \sqrt{2} \tau_{4} x^{2}} \\
& -\frac{6 \sqrt{2} \mathrm{~g}^{2} H^{4} x^{4} \Delta_{2} \tau_{4} Q^{3}}{H^{6} Q \sqrt{2} \tau_{4} x^{6}+4 \mathrm{~g}^{2} H^{4} Q^{3} \sqrt{2} \tau_{4} x^{4}+4 \mathrm{~g}^{4} H^{2} Q^{5} \sqrt{2} \tau_{4} x^{2}} \\
& -\frac{2 \sqrt{2} \mathrm{~g}^{2} H^{4} x^{2} \Delta_{2} \tau_{4} Q^{3}}{H^{6} Q \sqrt{2} \tau_{4} x^{6}+4 \mathrm{~g}^{2} H^{4} Q^{3} \sqrt{2} \tau_{4} x^{4}+4 \mathrm{~g}^{4} H^{2} Q^{5} \sqrt{2} \tau_{4} x^{2}} \\
& -\frac{2 \sqrt{2} \mathrm{~g}^{2} H^{4} x^{2} \epsilon \Delta_{2} \tau_{4} Q^{3}}{H^{6} Q \sqrt{2} \tau_{4} x^{6}+4 \mathrm{~g}^{2} H^{4} Q^{3} \sqrt{2} \tau_{4} x^{4}+4 \mathrm{~g}^{4} H^{2} Q^{5} \sqrt{2} \tau_{4} x^{2}} \\
& +\frac{4 \mathrm{~g}^{2} H^{4} x^{2} \sqrt{2} \Delta_{2} \xi_{f} \tau_{4} Q^{3}}{H^{6} Q \sqrt{2} \tau_{4} x^{6}+4 \mathrm{~g}^{2} H^{4} Q^{3} \sqrt{2} \tau_{4} x^{4}+4 \mathrm{~g}^{4} H^{2} Q^{5} \sqrt{2} \tau_{4} x^{2}} \\
& +\frac{8 \mathrm{~g}^{2} H^{4} x^{3} \sqrt{2} \Delta_{2, x} \xi_{f} \tau_{4} Q^{3}}{H^{6} Q \sqrt{2} \tau_{4} x^{6}+4 \mathrm{~g}^{2} H^{4} Q^{3} \sqrt{2} \tau_{4} x^{4}+4 \mathrm{~g}^{4} H^{2} Q^{5} \sqrt{2} \tau_{4} x^{2}} \\
& -\frac{8 \sqrt{2} \mathrm{~g}^{2} H^{3} x^{2} \sqrt{2 \mathrm{~g}^{2} Q^{2}+H^{2} x^{2}} \Delta_{1} \xi_{h} \tau_{4} Q^{3}}{H^{6} Q \sqrt{2} \tau_{4} x^{6}+4 \mathrm{~g}^{2} H^{4} Q^{3} \sqrt{2} \tau_{4} x^{4}+4 \mathrm{~g}^{4} H^{2} Q^{5} \sqrt{2} \tau_{4} x^{2}} \\
& +\frac{2 H^{5} m x^{4} \sqrt{2 \mathrm{~g}^{2} Q^{2}+H^{2} x^{2}} \Delta_{b} Q^{2}}{H^{6} Q \sqrt{2} \tau_{4} x^{6}+4 \mathrm{~g}^{2} H^{4} Q^{3} \sqrt{2} \tau_{4} x^{4}+4 \mathrm{~g}^{4} H^{2} Q^{5} \sqrt{2} \tau_{4} x^{2}} \\
& -\frac{10 \mathrm{~g} H^{4} x^{3} \sqrt{2 \mathrm{~g}^{2} Q^{2}+H^{2} x^{2}} Q_{, x} \Delta_{\tau_{4}} Q^{2}}{H^{6} Q \sqrt{2} \tau_{4} x^{6}+4 \mathrm{~g}^{2} H^{4} Q^{3} \sqrt{2} \tau_{4} x^{4}+4 \mathrm{~g}^{4} H^{2} Q^{5} \sqrt{2} \tau_{4} x^{2}}
\end{aligned}
$$




$$
\begin{aligned}
& +\frac{2 \mathrm{~g} H^{4} x^{4} \sqrt{2 \mathrm{~g}^{2} Q^{2}+H^{2} x^{2}} Q_{, x, x} \Delta_{\tau_{4}} Q^{2}}{H^{6} Q \sqrt{2} \tau_{4} x^{6}+4 \mathrm{~g}^{2} H^{4} Q^{3} \sqrt{2} \tau_{4} x^{4}+4 \mathrm{~g}^{4} H^{2} Q^{5} \sqrt{2} \tau_{4} x^{2}} \\
& +\frac{2 \mathrm{~g} H^{4} x^{4} \sqrt{2 \mathrm{~g}^{2} Q^{2}+H^{2} x^{2}} Q_{, x} \Delta_{\tau_{4}, x} Q^{2}}{H^{6} Q \sqrt{2} \tau_{4} x^{6}+4 \mathrm{~g}^{2} H^{4} Q^{3} \sqrt{2} \tau_{4} x^{4}+4 \mathrm{~g}^{4} H^{2} Q^{5} \sqrt{2} \tau_{4} x^{2}} \\
& +\frac{2 \mathrm{~g} H^{4} x^{4} \sqrt{4 \mathrm{~g}^{2} Q^{2}+2 H^{2} x^{2}} \Delta_{1} \tau_{4} Q^{2}}{H^{6} Q \sqrt{2} \tau_{4} x^{6}+4 \mathrm{~g}^{2} H^{4} Q^{3} \sqrt{2} \tau_{4} x^{4}+4 \mathrm{~g}^{4} H^{2} Q^{5} \sqrt{2} \tau_{4} x^{2}} \\
& +\frac{8 \mathrm{~g}^{2} H^{4} x^{3} \sqrt{2} Q_{, x} \Delta_{2} \tau_{4} Q^{2}}{H^{6} Q \sqrt{2} \tau_{4} x^{6}+4 \mathrm{~g}^{2} H^{4} Q^{3} \sqrt{2} \tau_{4} x^{4}+4 \mathrm{~g}^{4} H^{2} Q^{5} \sqrt{2} \tau_{4} x^{2}} \\
& +\frac{2 \mathrm{~g}^{2} H^{4} x^{4} \sqrt{2} Q_{, x, x} \Delta_{2} \tau_{4} Q^{2}}{H^{6} Q \sqrt{2} \tau_{4} x^{6}+4 \mathrm{~g}^{2} H^{4} Q^{3} \sqrt{2} \tau_{4} x^{4}+4 \mathrm{~g}^{4} H^{2} Q^{5} \sqrt{2} \tau_{4} x^{2}} \\
& -\frac{4 \sqrt{2} \mathrm{~g}^{2} H^{4} x^{3} Q_{, x} \Delta_{2} \xi_{f} \tau_{4} Q^{2}}{H^{6} Q \sqrt{2} \tau_{4} x^{6}+4 \mathrm{~g}^{2} H^{4} Q^{3} \sqrt{2} \tau_{4} x^{4}+4 \mathrm{~g}^{4} H^{2} Q^{5} \sqrt{2} \tau_{4} x^{2}} \\
& -\frac{2 H^{5} m x^{5} \sqrt{2 \mathrm{~g}^{2} Q^{2}+H^{2} x^{2}} Q_{, x} \Delta_{b} Q}{H^{6} Q \sqrt{2} \tau_{4} x^{6}+4 \mathrm{~g}^{2} H^{4} Q^{3} \sqrt{2} \tau_{4} x^{4}+4 \mathrm{~g}^{4} H^{2} Q^{5} \sqrt{2} \tau_{4} x^{2}} \\
& +\frac{4 \mathrm{~g} H^{4} x^{4} \sqrt{2 \mathrm{~g}^{2} Q^{2}+H^{2} x^{2}} Q_{, x}^{2} \Delta_{\tau_{4}} Q}{H^{6} Q \sqrt{2} \tau_{4} x^{6}+4 \mathrm{~g}^{2} H^{4} Q^{3} \sqrt{2} \tau_{4} x^{4}+4 \mathrm{~g}^{4} H^{2} Q^{5} \sqrt{2} \tau_{4} x^{2}} \\
& -\frac{\sqrt{2} H^{6} x^{6} \Delta_{2} \tau_{4} Q}{H^{6} Q \sqrt{2} \tau_{4} x^{6}+4 \mathrm{~g}^{2} H^{4} Q^{3} \sqrt{2} \tau_{4} x^{4}+4 \mathrm{~g}^{4} H^{2} Q^{5} \sqrt{2} \tau_{4} x^{2}} \\
& -\frac{6 \sqrt{2} \mathrm{~g}^{2} H^{4} x^{4} Q_{, x}^{2} \Delta_{2} \tau_{4} Q}{H^{6} Q \sqrt{2} \tau_{4} x^{6}+4 \mathrm{~g}^{2} H^{4} Q^{3} \sqrt{2} \tau_{4} x^{4}+4 \mathrm{~g}^{4} H^{2} Q^{5} \sqrt{2} \tau_{4} x^{2}} \\
& -\frac{\sqrt{2} H^{6} x^{4} \epsilon \Delta_{2} \tau_{4} Q}{H^{6} Q \sqrt{2} \tau_{4} x^{6}+4 \mathrm{~g}^{2} H^{4} Q^{3} \sqrt{2} \tau_{4} x^{4}+4 \mathrm{~g}^{4} H^{2} Q^{5} \sqrt{2} \tau_{4} x^{2}} \\
& +\frac{2 H^{6} x^{4} \sqrt{2} \Delta_{2} \tau_{4} Q}{H^{6} Q \sqrt{2} \tau_{4} x^{6}+4 \mathrm{~g}^{2} H^{4} Q^{3} \sqrt{2} \tau_{4} x^{4}+4 \mathrm{~g}^{4} H^{2} Q^{5} \sqrt{2} \tau_{4} x^{2}} \\
& +\frac{2 H^{6} x^{4} \sqrt{2} \Delta_{2} \xi_{f} \tau_{4} Q}{H^{6} Q \sqrt{2} \tau_{4} x^{6}+4 \mathrm{~g}^{2} H^{4} Q^{3} \sqrt{2} \tau_{4} x^{4}+4 \mathrm{~g}^{4} H^{2} Q^{5} \sqrt{2} \tau_{4} x^{2}} \\
& +\frac{2 H^{6} x^{5} \sqrt{2} \Delta_{2, x} \xi_{f} \tau_{4} Q}{H^{6} Q \sqrt{2} \tau_{4} x^{6}+4 \mathrm{~g}^{2} H^{4} Q^{3} \sqrt{2} \tau_{4} x^{4}+4 \mathrm{~g}^{4} H^{2} Q^{5} \sqrt{2} \tau_{4} x^{2}} \\
& -\frac{2 \sqrt{2} H^{5} x^{4} \sqrt{2 \mathrm{~g}^{2} Q^{2}+H^{2} x^{2}} \Delta_{1} \xi_{h} \tau_{4} Q}{H^{6} Q \sqrt{2} \tau_{4} x^{6}+4 \mathrm{~g}^{2} H^{4} Q^{3} \sqrt{2} \tau_{4} x^{4}+4 \mathrm{~g}^{4} H^{2} Q^{5} \sqrt{2} \tau_{4} x^{2}} \\
& -\frac{2 \sqrt{2} H^{6} x^{5} Q_{, x} \Delta_{2} \tau_{4}}{H^{6} Q \sqrt{2} \tau_{4} x^{6}+4 \mathrm{~g}^{2} H^{4} Q^{3} \sqrt{2} \tau_{4} x^{4}+4 \mathrm{~g}^{4} H^{2} Q^{5} \sqrt{2} \tau_{4} x^{2}} \\
& +\frac{H^{6} x^{6} \sqrt{2} Q_{, x, x} \Delta_{2} \tau_{4}}{H^{6} Q \sqrt{2} \tau_{4} x^{6}+4 \mathrm{~g}^{2} H^{4} Q^{3} \sqrt{2} \tau_{4} x^{4}+4 \mathrm{~g}^{4} H^{2} Q^{5} \sqrt{2} \tau_{4} x^{2}} \\
& -\frac{2 \sqrt{2} H^{6} x^{5} Q_{, x} \Delta_{2} \xi_{f} \tau_{4}}{H^{6} Q \sqrt{2} \tau_{4} x^{6}+4 \mathrm{~g}^{2} H^{4} Q^{3} \sqrt{2} \tau_{4} x^{4}+4 \mathrm{~g}^{4} H^{2} Q^{5} \sqrt{2} \tau_{4} x^{2}} .
\end{aligned}
$$


C. FULL PERTURBATION EQUATIONS FOR CHAPTER 5 


\section{References}

[1] Planck Collaboration, P. Ade et al., "Planck 2015 results. XIII. Cosmological parameters", Astron. Astrophys. 594 (2016) A13, arXiv:1502.01589 [astro-ph.CO], ix, 4,5

[2] R. Namba, M. Peloso, M. Shiraishi, L. Sorbo, and C. Unal, "Scale-dependent gravitational waves from a rolling axion", JCAP 1601 (2016) no. 01, 041, arXiv:1509.07521 [astro-ph.C0], ix, 27, 52, 56, 57, 73, 194

[3] E. Dimastrogiovanni, M. Fasiello, and T. Fujita, "Primordial Gravitational Waves from Axion-Gauge Fields Dynamics", JCAP 1701 (2017) no. 01, 019 , arXiv: 1608.04216 [astro-ph.C0], Xv, Xvi, 27, 52, 53, 59, 60, 61, 65, 69, 70, 72, 173, 105, 115, 116, 117, 120, 124, 132, 133, 137, 143, 144, 145, 146, 148, 152, 157, 158, 160, 163, 170, 175, 176, 194, 196

[4] E. McDonough and S. Alexander, "Observable Chiral Gravitational Waves from Inflation in String Theory", JCAP 1811 (2018) no. 11, 030, arXiv: 1806.05684 [hep-th], xv, 116, 117, 132, 133, 137, 159, 160, 173, 174, 199

[5] "Fred Hoyle: An Online Exhibition, Hoyle on the Radio: Creating the 'Big Bang'". HTTPS://WWW. JOH.CAM.AC.UK/LIBRARY/SPECIAL_COLLECTIONS/ HOYLE/EXHIBITION/RADIO/, Accessed: 2020-07-14. 1

[6] H. Kragh, "Big Bang: the etymology of a name", Astronomy and Geophysics 54 $(04,2013), 1$

[7] A. Friedmann, "On the Curvature of space", Z. Phys. 10 (1922) 377-386. 2

[8] E. Hubble, "A relation between distance and radial velocity among extra-galactic nebulae", Proceedings of the National Academy of Sciences $\mathbf{1 5}$ 


\section{REFERENCES}

(1929) no. 3, 168-173, https://www.pnas.org/content/15/3/168.full.pdf. https://www.pnas.org/content/15/3/168, 2

[9] N. A. Bahcall, "Hubble's law and the expanding universe", Proceedings of the National Academy of Sciences 112 (2015) no. 11, 3173-3175, https://www.pnas.org/content/112/11/3173.full.pdf. https://www.pnas.org/content/112/11/3173. 2

[10] Dominique Lambert, "Histoires des sciences: L'hypothèse de l'atome primitif". HTTPS://WWW.POURLASCIENCE.FR/SD/HISTOIRE-SCIENCES/ LHYPOTHESE-DE-LATOME-PRIMITIF-936.PHP, 1999. Accessed: 2020-07-15. 2

[11] R. A. Alpher and R. C. Herman, "On the relative abundance of the elements", Phys. Rev. 74 (Dec, 1948) 1737-1742. https://link.aps.org/doi/10.1103/PhysRev.74.1737. 2

[12] P. J. E. Peebles, "Discovery of the Hot Big Bang: What happened in 1948", Eur. Phys. J. H 39 (2014) 205-223, arXiv:1310.2146 [physics.hist-ph]. 2

[13] R. Mitalas and K. Sills, "On the Photon Diffusion Time Scale for the Sun", The Astrophysical Journal 401 (Dec., 1992) 759. 3

[14] H. Bondi and T. Gold, "The Steady-State Theory of the Expanding Universe", Monthly Notices of the Royal Astronomical Society 108 (Jan., 1948) 252. 3

[15] F. Hoyle, "A New Model for the Expanding Universe", Monthly Notices of the Royal Astronomical Society 108 (Jan., 1948) 372, 3

[16] J. Hansen, Spaceflight Revolution: NASA Langley Research Center from Sputnik to Apollo. National Aeronautics and Space Administration, 1995. 3

[17] A. A. Penzias and R. W. Wilson, "A Measurement of Excess Antenna Temperature at 4080 Mc/s.", The Astrophysical Journal 142 (July, 1965) 419-421. 4

[18] "The 6 billion dollar experiment: Cosmic microwave background discovered", 2007. https://www.bbc.co.uk/programmes/p009xr65. Premiered 01/05/2007 on BBC Two. 4 
[19] R. H. Dicke, P. J. E. Peebles, P. G. Roll, and D. T. Wilkinson, "Cosmic Black-Body Radiation.", The Astrophysical Journal 142 (July, 1965) 414-419. 4

[20] A. McKellar, "Molecular Lines from the Lowest States of Diatomic Molecules Composed of Atoms Probably Present in Interstellar Space", Publications of the Dominion Astrophysical Observatory Victoria 7 (Jan., 1941) 251. 4

[21] C. Bennett, A. Banday, K. Gorski, G. Hinshaw, P. Jackson, P. Keegstra, A. Kogut, G. F. Smoot, D. Wilkinson, and E. Wright, "Four year COBE DMR cosmic microwave background observations: Maps and basic results", Astrophys. J. Lett. 464 (1996) L1-L4, arXiv:astro-ph/9601067, 4

[22] C. Bennett, D. Larson, J. Weiland, N. Jarosik, G. Hinshaw, N. Odegard, K. Smith, R. Hill, B. Gold, M. Halpern, E. Komatsu, M. Nolta, L. Page, D. Spergel, E. Wollack, J. Dunkley, A. Kogut, M. Limon, S. Meyer, and E. Wright, "Nine-year Wilkinson Microwave Anisotropy Probe (WMAP) observations: Final maps and results", The Astrophysical Journal Supplement Series $208(12,2012)$. 4

[23] Planck Collaboration, P. Ade et al., "Planck 2013 results. I. Overview of products and scientific results", Astron. Astrophys. 571 (2014) A1, arXiv:1303.5062 [astro-ph.C0]. 4

[24] Planck Collaboration, Y. Akrami et al., "Planck 2018 results. I. Overview and the cosmological legacy of Planck", arXiv:1807.06205 [astro-ph.CO]. 4

[25] D. Baumann, "Inflation", in Theoretical Advanced Study Institute in Elementary Particle Physics: Physics of the Large and the Small, pp. 523-686. 2011. arXiv:0907.5424 [hep-th], 4, 8, 15, 16

[26] S. Weinberg, Cosmology. 9, 2008. 152, 171, 186

[27] L. Senatore, "Lectures on Inflation", in Theoretical Advanced Study Institute in Elementary Particle Physics: New Frontiers in Fields and Strings, pp. 447-543. 2017. arXiv:1609.00716 [hep-th]. 4 


\section{REFERENCES}

[28] G. Smoot, C. Bennett, A. Kogut, E. Wright, J. Aymon, N. Boggess, E. Cheng, G. de Amici, S. Gulkis, M. Hauser, G. Hinshaw, P. Jackson, M. Janssen, E. Kaita, T. Kelsall, P. Keegstra, C. Lineweaver, K. Loewenstein, P. Lubin, and D. Wilkinson, "Structure in the COBE differential microwave radiometer first-year maps", The Astrophysical Journal 396 (09, 1992) L1-L5. 4

[29] E. Wright, "Theoretical overview of cosmic microwave background anisotropy", in Carnegie Observatories Centennial Symposium. 2. Measuring and Modeling the Universe, pp. 291-308. 5, 2003. arXiv:astro-ph/0305591. 4

[30] A. Challinor, "CMB anisotropy science: a review", IAU Symp. 288 (2013) 42, arXiv:1210.6008 [astro-ph.CO], 4

[31] Planck Collaboration, N. Aghanim et al., "Planck 2018 results. VI. Cosmological parameters", arXiv:1807.06209 [astro-ph.CO]. 8

[32] A. H. Guth, "The Inflationary Universe: A Possible Solution to the Horizon and Flatness Problems", Adv. Ser. Astrophys. Cosmol. 3 (1987) 139-148. 10

[33] A. Riotto, "Inflation and the theory of cosmological perturbations", ICTP Lect. Notes Ser. 14 (2003) 317-413, arXiv:hep-ph/0210162, 14, 15

[34] S. Weinberg, "Can non-adiabatic perturbations arise after single-field inflation?", Phys. Rev. D 70 (2004) 043541, arXiv:astro-ph/0401313. 15

[35] R. L. Arnowitt, S. Deser, and C. W. Misner, "The Dynamics of general relativity", Gen. Rel. Grav. 40 (2008) 1997-2027, arXiv:gr-qc/0405109, 15

[36] T. Bunch and P. Davies, "Quantum Field Theory in de Sitter Space: Renormalization by Point Splitting", Proc. Roy. Soc. Lond. A A360 (1978) 117-134, 18

[37] Planck Collaboration, Y. Akrami et al., "Planck 2018 results. X. Constraints on inflation", arXiv:1807.06211 [astro-ph.C0], 20, 21, 23, 29, 114, 148, 184 193,199

[38] M. Kamionkowski and E. D. Kovetz, "The Quest for B Modes from Inflationary Gravitational Waves", Ann. Rev. Astron. Astrophys. 54 (2016) 227-269, arXiv:1510.06042 [astro-ph.C0], 23 
[39] SPT-3G Collaboration, B. Benson et al., "SPT-3G: A Next-Generation Cosmic Microwave Background Polarization Experiment on the South Pole Telescope", Proc. SPIE Int. Soc. Opt. Eng. 9153 (2014) 91531P, arXiv:1407.2973 [astro-ph. IM], 23, 113, 141, 149, 189, 197

[40] Simons Observatory Collaboration, J. Aguirre et al., "The Simons Observatory: Science goals and forecasts", JCAP 1902 (2019) 056 , arXiv: 1808.07445 [astro-ph.C0]. 149

[41] CMB-S4 Collaboration, K. N. Abazajian et al., "CMB-S4 Science Book, First Edition", arXiv:1610.02743 [astro-ph.C0], 51, 149

[42] T. Essinger-Hileman et al., "CLASS: The Cosmology Large Angular Scale Surveyor", Proc. SPIE Int. Soc. Opt. Eng. 9153 (2014) 91531I, arXiv:1408.4788 [astro-ph.IM], 149

[43] T. Matsumura et al., "Mission design of LiteBIRD", arXiv:1311.2847 [astro-ph.IM]. [J. Low. Temp. Phys.176,733(2014)].

[44] NASA PICO Collaboration, S. Hanany et al., "PICO: Probe of Inflation and Cosmic Origins", arXiv:1902.10541 [astro-ph.IM], 23, 113, 141, 149, 189 , 197

[45] S. Kanno, J. Soda, and M.-a. Watanabe, "Anisotropic Power-law Inflation", JCAP 1012 (2010) 024, arXiv: 1010.5307 [hep-th], 27, 34, 35, 37, 40, 45, 48, 49, 191, 192, 193

[46] E. Silverstein and D. Tong, "Scalar speed limits and cosmology: Acceleration from D-cceleration", Phys. Rev. D70 (2004) 103505, arXiv:hep-th/0310221 [hep-th], 27, 45

[47] T. Q. Do and W. F. Kao, "Anisotropic power-law inflation for the Dirac-Born-Infeld theory", Phys. Rev. D84 (2011) 123009, 27, 33, 35, 39, 45

[48] M. Peloso, L. Sorbo, and C. Unal, "Rolling axions during inflation: perturbativity and signatures", JCAP 09 (2016) 001, arXiv:1606.00459 [astro-ph.C0], 27, 52,73 


\section{REFERENCES}

[49] J. P. Conlon and F. Quevedo, "Kahler moduli inflation", JHEP 01 (2006) 146, arXiv:hep-th/0509012 [hep-th], 27, 51, 75, 77, 79, 86, 103, 116, 117, 128 , 194

[50] M. Cicoli, C. P. Burgess, and F. Quevedo, "Fibre Inflation: Observable Gravity Waves from IIB String Compactifications", JCAP 0903 (2009) 013, arXiv:0808.0691 [hep-th], 27, 76, 79, 80, 84, 86, 105, 108, 109, 110, 112 , $179,180,194,195,199$

[51] J. Holland, S. Kanno, and I. Zavala, "Anisotropic Inflation with Derivative Couplings", Phys. Rev. D97 (2018) no. 10, 103534, arXiv:1711.07450 [hep-th]. 29, 44, 191

[52] J. Martin, C. Ringeval, R. Trotta, and V. Vennin, "The Best Inflationary Models After Planck", JCAP 1403 (2014) 039, arXiv:1312.3529 [astro-ph.CO], 29

[53] J. Kim and E. Komatsu, "Limits on anisotropic inflation from the Planck data", Phys. Rev. D88 (2013) 101301, arXiv:1310.1605 [astro-ph.C0], 29, 30, 32 , 44, 45, 49, 192

[54] R. Emami, Anisotropic Inflation and Cosmological Observations. PhD thesis, Hong Kong U. Sci. Tech., 2015. arXiv:1511.01683 [astro-ph.CO]. https://inspirehep.net/record/1402934/files/arXiv:1511.01683.pdf. 29,31

[55] J. Soda, "Statistical Anisotropy from Anisotropic Inflation", Class. Quant. Grav. 29 (2012) 083001, arXiv:1201.6434 [hep-th], 30, 31, 192,193

[56] E. W. Kolb and M. S. Turner, "The Early Universe", Front. Phys. 69 (1990) 1-547. 30

[57] R. M. Wald, "Asymptotic behavior of homogeneous cosmological models in the presence of a positive cosmological constant", Phys. Rev. D28 (1983) 2118-2120. 30. 
[58] A. Maleknejad, M. M. Sheikh-Jabbari, and J. Soda, "Gauge Fields and Inflation", Phys. Rept. 528 (2013) 161-261, arXiv:1212.2921 [hep-th], 31, 114

[59] M.-a. Watanabe, S. Kanno, and J. Soda, "Inflationary Universe with Anisotropic Hair", Phys. Rev. Lett. 102 (2009) 191302, arXiv:0902.2833 [hep-th], 31, 34, 35

[60] K. Dimopoulos, "Can a vector field be responsible for the curvature perturbation in the Universe?", Phys. Rev. D74 (2006) 083502, arXiv:hep-ph/0607229 [hep-ph], 31

[61] K. Dimopoulos, M. Karciauskas, D. H. Lyth, and Y. Rodriguez, "Statistical anisotropy of the curvature perturbation from vector field perturbations", JCAP 0905 (2009) 013, arXiv:0809.1055 [astro-ph].

[62] K. Dimopoulos, M. Karciauskas, and J. M. Wagstaff, "Vector Curvaton without Instabilities", Phys. Lett. B683 (2010) 298-301, arXiv:0909.0475 [hep-ph]. 31

[63] K. Dimopoulos, "Statistical Anisotropy and the Vector Curvaton Paradigm", Int. J. Mod. Phys. D21 (2012) 1250023, arXiv:1107.2779 [hep-ph]. [Erratum: Int. J. Mod. Phys.D21,1292003(2012)]. 31

[64] K. Dimopoulos, D. Wills, and I. Zavala, "Statistical Anisotropy from Vector Curvaton in D-brane Inflation", Nucl. Phys. B868 (2013) 120-155, arXiv:1108.4424 [hep-th], 32, 33, 45

[65] A. Avgoustidis and I. Zavala, "Warped Wilson Line DBI Inflation", JCAP 0901 (2009) 045, arXiv:0810.5001 [hep-th], 32

[66] C. van de Bruck, T. Koivisto, and C. Longden, "Disformally coupled inflation", JCAP 1603 (2016) 006, arXiv:1510.01650 [astro-ph.CO], 32

[67] X. Chen, Y. Wang, and Z.-Z. Xianyu, "Standard Model Background of the Cosmological Collider", Phys. Rev. Lett. 118 (2017) no. 26, 261302. arXiv: 1610.06597 [hep-th], 32, 38, 48 


\section{REFERENCES}

[68] A. A. Abolhasani, M. Akhshik, R. Emami, and H. Firouzjahi, "Primordial Statistical Anisotropies: The Effective Field Theory Approach", JCAP 1603 (2016) no. 03, 020, arXiv:1511.03218 [astro-ph.C0], 32, 35, 48

[69] T. Rostami, A. Karami, and H. Firouzjahi, "Effective field theory of statistical anisotropies for primordial bispectrum and gravitational waves", JCAP 1706 (2017) no. 06, 039, arXiv:1702.03744 [astro-ph.C0], 32, 48

[70] A. Naruko, E. Komatsu, and M. Yamaguchi, "Anisotropic inflation reexamined: upper bound on broken rotational invariance during inflation", JCAP 1504 (2015) no. 04, 045, arXiv:1411.5489 [astro-ph.C0], 32, 44,192

[71] J. Ohashi, J. Soda, and S. Tsujikawa, "Anisotropic power-law k-inflation", Phys. Rev. D88 (2013) 103517, arXiv:1310.3053 [hep-th]. 33, 35

[72] A. Ito and J. Soda, "Anisotropic Constant-roll Inflation", arXiv:1710.09701 [hep-th]. 35

[73] M.-a. Watanabe, S. Kanno, and J. Soda, "The Nature of Primordial Fluctuations from Anisotropic Inflation", Prog. Theor. Phys. 123 (2010) 1041-1068, arXiv:1003.0056 [astro-ph.C0]. 40

[74] T. Koivisto and F. Urban, "Disformal vectors and anisotropies on a warped brane", JCAP 1503 (2015) 003, arXiv:1407.3445 [astro-ph.C0]. 45

[75] K. Abazajian et al., "CMB-S4 Science Case, Reference Design, and Project Plan", arXiv:1907.04473 [astro-ph.IM], 51

[76] J. J. Blanco-Pillado, D. Buck, E. J. Copeland, M. Gomez-Reino, and N. J. Nunes, "Kahler Moduli Inflation Revisited", JHEP 01 (2010) 081. arXiv: 0906.3711 [hep-th], 51, 75, 76, 79, 80, 86, 88, 93, 94, 103, 117, 128 , 129, 163, 189, 194, 195

[77] M. S. Wang, "Second order action for gravitational waves from inflation", 2017. Lecture notes from the course "Advanced Cosmology", part of Mathematical Tripos Part III, University of Cambridge. 57 
[78] P. Adshead and M. Wyman, "Chromo-Natural Inflation: Natural inflation on a steep potential with classical non-Abelian gauge fields", Phys. Rev. Lett. 108 (2012) 261302, arXiv: 1202.2366 [hep-th], 59, 114, 120, 124, 137

[79] P. Adshead and M. Wyman, "Gauge-flation trajectories in Chromo-Natural Inflation", Phys. Rev. D86 (2012) 043530, arXiv:1203.2264 [hep-th]. 114

[80] E. Dimastrogiovanni and M. Peloso, "Stability analysis of chromo-natural inflation and possible evasion of Lyth's bound", Phys. Rev. D87 (2013) no. 10, 103501, arXiv: 1212.5184 [astro-ph.C0], 65, 69, 124, 143, 144, 152

[81] P. Adshead, E. Martinec, and M. Wyman, "Perturbations in Chromo-Natural Inflation", JHEP 09 (2013) 087, arXiv: 1305.2930 [hep-th], 59, 115, 198

[82] S. Kachru, R. Kallosh, A. D. Linde, and S. P. Trivedi, "De Sitter vacua in string theory", Phys. Rev. D 68 (2003) 046005, arXiv:hep-th/0301240, 77

[83] L. E. Ibanez and A. M. Uranga, String theory and particle physics: An introduction to string phenomenology. Cambridge University Press, 2012. http://www. cambridge.org/de/knowledge/isbn/item6563092/?site_ locale=de_DE. 77, 201

[84] V. Balasubramanian, P. Berglund, J. P. Conlon, and F. Quevedo, "Systematics of moduli stabilisation in Calabi-Yau flux compactifications", JHEP 03 (2005) 007, arXiv:hep-th/0502058 [hep-th], 77, 116, 117

[85] J. P. Conlon, F. Quevedo, and K. Suruliz, "Large-volume flux compactifications: Moduli spectrum and D3/D7 soft supersymmetry breaking", JHEP 08 (2005) 007, arXiv:hep-th/0505076 [hep-th], 77, 79, 80, 81, 116, 117, 127, 190, 195, 197

[86] C. P. Burgess, M. Cicoli, S. de Alwis, and F. Quevedo, "Robust Inflation from Fibrous Strings", JCAP 1605 (2016) no. 05, 032, arXiv: 1603.06789 [hep-th]. 86

[87] V. Gluscevic and M. Kamionkowski, "Testing Parity-Violating Mechanisms with Cosmic Microwave Background Experiments", Phys. Rev. D81 (2010) 123529, arXiv:1002.1308 [astro-ph.C0], 105, 149, 170, 178, 186, 189, 190, 197, 199 


\section{REFERENCES}

[88] J. Holland, I. Zavala, and G. Tasinato, "On chromonatural inflation in string theory", JCAP 12 (2020) 026, arXiv:2009.00653 [hep-th], 113, 118, 162 , 179,195

[89] M. Zaldarriaga and U. Seljak, "An all sky analysis of polarization in the microwave background", Phys. Rev. D 55 (1997) 1830-1840, arXiv: astro-ph/9609170. 113

[90] M. Kamionkowski, A. Kosowsky, and A. Stebbins, "Statistics of cosmic microwave background polarization", Phys. Rev. D 55 (1997) 7368-7388, arXiv:astro-ph/9611125. 113

[91] J.-O. Gong, "Multi-field inflation and cosmological perturbations", Int. J. Mod. Phys. D 26 (2016) no. 01, 1740003, arXiv:1606.06971 [gr-qc]. 114

[92] A. Maleknejad and M. Sheikh-Jabbari, "Gauge-flation: Inflation From Non-Abelian Gauge Fields", Phys. Lett. B 723 (2013) 224-228, arXiv:1102.1513 [hep-ph]. 114

[93] A. Maleknejad and M. Sheikh-Jabbari, "Non-Abelian Gauge Field Inflation", Phys. Rev. D 84 (2011) 043515, arXiv:1102.1932 [hep-ph]. 114

[94] M. Biagetti, M. Fasiello, and A. Riotto, "Enhancing Inflationary Tensor Modes through Spectator Fields", Phys. Rev. D 88 (2013) 103518, arXiv:1305.7241 [astro-ph.CO], 114

[95] M. Biagetti, E. Dimastrogiovanni, M. Fasiello, and M. Peloso, "Gravitational Waves and Scalar Perturbations from Spectator Fields", JCAP 04 (2015) 011, arXiv:1411.3029 [astro-ph.C0].

[96] T. Fujita, J. Yokoyama, and S. Yokoyama, "Can a spectator scalar field enhance inflationary tensor mode?", PTEP 2015 (2015) 043E01, arXiv:1411. 3658 [astro-ph.CO], 114

[97] N. Bartolo et al., "Science with the space-based interferometer LISA. IV:

Probing inflation with gravitational waves", JCAP 1612 (2016) 026 , arXiv:1610.06481 [astro-ph.C0], 114 
[98] K. Freese and W. H. Kinney, "On: Natural inflation", Phys. Rev. D 70 (2004) 083512, arXiv:hep-ph/0404012, 114

[99] P. Adshead, E. Martinec, and M. Wyman, "Gauge fields and inflation: Chiral gravitational waves, fluctuations, and the Lyth bound", Phys. Rev. D 88 (2013) no. 2, 021302, arXiv:1301.2598 [hep-th], 115, 198

[100] I. Obata and J. Soda, "Chiral primordial gravitational waves from dilaton induced delayed chromonatural inflation", Phys. Rev. D93 (2016) no. 12, 123502, arXiv:1602.06024 [hep-th], [Addendum: Phys.

Rev.D95,no.10,109903(2017)]. 115, 119

[101] T. Fujita, R. Namba, and Y. Tada, "Does the detection of primordial gravitational waves exclude low energy inflation?", Phys. Lett. B778 (2018) 17-21, arXiv: 1705.01533 [astro-ph.C0], 115, 116, 117, 124, 125, 134, 137, 140, 163, 175, 176, 190, 198, 199

[102] A. Maleknejad and E. Komatsu, "Production and Backreaction of Spin-2 Particles of $S U(2)$ Gauge Field during Inflation", JHEP 05 (2019) 174, arXiv: 1808.09076 [hep-ph], 116, 117

[103] A. Papageorgiou, M. Peloso, and C. Unal, "Nonlinear perturbations from axion-gauge fields dynamics during inflation", JCAP 07 (2019) 004 , arXiv:1904.01488 [astro-ph.C0], 115, 125, 163

[104] G. Dall'Agata, "Chromo-Natural inflation in Supergravity", Phys. Lett. B782 (2018) 139-142, arXiv: 1804.03104 [hep-th], 115, 116, 117, 173

[105] C. Long, L. McAllister, and P. McGuirk, "Aligned Natural Inflation in String Theory", Phys. Rev. D 90 (2014) 023501, arXiv:1404.7852 [hep-th], 116 , 159, 160,177

[106] I. Ben-Dayan, F. G. Pedro, and A. Westphal, "Towards Natural Inflation in String Theory", Phys. Rev. D 92 (2015) no. 2, 023515, arXiv:1407.2562 [hep-th]. 116 


\section{REFERENCES}

[107] P. Agrawal, J. Fan, and M. Reece, "Clockwork Axions in Cosmology: Is Chromonatural Inflation Chrononatural?", JHEP 10 (2018) 193, arXiv:1806.09621 [hep-th]. 116, 173

[108] R. R. Caldwell and C. Devulder, "Axion Gauge Field Inflation and Gravitational Leptogenesis: A Lower Bound on B Modes from the Matter-Antimatter Asymmetry of the Universe", Phys. Rev. D97 (2018) no. 2, 023532, arXiv:1706.03765 [astro-ph.C0], 120

[109] R. R. Caldwell and C. Devulder, "Gravitational Wave Opacity from Gauge Field Dark Energy", arXiv:1802.07371 [gr-qc]. 120

[110] A. Achucarro, V. Atal, and Y. Welling, "On the viability of $m^{2} \phi^{2}$ and natural inflation", JCAP 07 (2015) 008, arXiv: 1503.07486 [astro-ph.C0]. 121

[111] A. R. Brown, "Hyperbolic Inflation", Phys. Rev. Lett. 121 (2018) no. 25, 251601, arXiv:1705.03023 [hep-th].

[112] S. Garcia-Saenz, S. Renaux-Petel, and J. Ronayne, "Primordial fluctuations and non-Gaussianities in sidetracked inflation", JCAP 07 (2018) 057, arXiv:1804.11279 [astro-ph.CO].

[113] T. Bjorkmo and M. D. Marsh, "Hyperinflation generalised: from its attractor mechanism to its tension with the "swampland conditions"', JHEP 04 (2019) 172, arXiv: 1901.08603 [hep-th].

[114] T. Bjorkmo, "Rapid-Turn Inflationary Attractors", Phys. Rev. Lett. 122 (2019) no. 25, 251301, arXiv: 1902.10529 [hep-th].

[115] V. Aragam, S. Paban, and R. Rosati, "Multi-field Inflation in High-Slope Potentials", JCAP 04 (2020) 022, arXiv:1905.07495 [hep-th]

[116] D. Chakraborty, R. Chiovoloni, O. Loaiza-Brito, G. Niz, and I. Zavala, "Fat inflatons, large turns and the $\eta$-problem", JCAP 01 (2020) 020 , arXiv:1908.09797 [hep-th], 121, 175

[117] A. Maleknejad, "Axion Inflation with an SU(2) Gauge Field: Detectable Chiral Gravity Waves", JHEP 07 (2016) 104, arXiv:1604.03327 [hep-ph], 124 
[118] D. Langlois and S. Renaux-Petel, "Perturbations in generalized multi-field inflation", JCAP 0804 (2008) 017, arXiv:0801.1085 [hep-th], 149

[119] H. Jockers and J. Louis, "The Effective action of D7-branes in $\mathrm{N}=1$

Calabi-Yau orientifolds", Nucl. Phys. B 705 (2005) 167-211, arXiv: hep-th/0409098, 158, 159, 160, 162, 179

[120] H. Jockers and J. Louis, "D-terms and F-terms from D7-brane fluxes", Nucl. Phys. B 718 (2005) 203-246, arXiv:hep-th/0502059, 160, 162, 179

[121] T. W. Grimm, M. Kerstan, E. Palti, and T. Weigand, "On Fluxed Instantons and Moduli Stabilisation in IIB Orientifolds and F-theory", Phys. Rev. D $\mathbf{8 4}$ (2011) 066001, arXiv:1105.3193 [hep-th], 159, 160

[122] B. Heidenreich, M. Reece, and T. Rudelius, "The Weak Gravity Conjecture and Emergence from an Ultraviolet Cutoff", Eur. Phys. J. C 78 (2018) no. 4, 337, arXiv:1712.01868 [hep-th], 173

[123] D. Baumann and L. McAllister, Inflation and String Theory. Cambridge Monographs on Mathematical Physics. Cambridge University Press, 2015. arXiv:1404.2601 [hep-th], http://www.cambridge.org/mw/academic/ subjects/physics/theoretical-physics-and-mathematical-physics/ inflation-and-string-theory?format=HB. 201

[124] D. Rajan, "Complex Spacetimes and the Newman-Janis trick", Master's thesis, Victoria U., Wellington, 2015.

[125] A. Cannas da Silva, Lectures on Symplectic Geometry. Springer-Verlag Berlin Heidelberg, 2008.

[126] E. Flaherty, Hermitian and Kählerian Geometry in Relativity. Springer-Verlag Berlin Heidelberg, 1976. 206

[127] V. Bouchard, "Lectures on complex geometry, Calabi-Yau manifolds and toric geometry", arXiv:hep-th/0702063.

[128] H. Ocampo, E. Pariguan, and S. Paycha, Geometric and Topological Methods for Quantum Field Theory. Cambridge University Press, 6, 2010. 
[129] E. Clader and R. Yongbin, B-Model Gromov-Witten Theory. Birkhäuser Basel.

[130] A. Font and S. Theisen, "Introduction to string compactification", Lect. Notes Phys. 668 (2005) 101-181, 201, 209

[131] E. Calabi, "On Kähler manifolds with vanishing canonical class", in Algebraic geometry and topology: A symposium in honor of S. Lefschetz, R. Fox,

D. Spencer, and A. Tucker, eds., ch. 5, pp. 78 - 89. Princeton University Press, Princeton, 1957. 205

[132] S.-T. Yau, "Calabi's conjecture and some new results in algebraic geometry",

Proceedings of the National Academy of Sciences 74 (1977) no. 5, 1798-1799,

https://www.pnas.org/content/74/5/1798.full.pdf.

https://www.pnas.org/content/74/5/1798, 205 